\title{
Genetic determination and layout rules of visual cortical architecture
}

\author{
Dissertation
}

for the award of the degree

"Doctor rerum naturalium"

Division of Mathematics and Natural Sciences of the

Georg August University Göttingen within the doctoral program GGNB of the Georg August University School of Science (GAUSS) submitted by

\section{Joscha Liedtke}

from Marburg 
Thesis committee:

- Prof. Dr. Fred Wolf

(Thesis supervisor and 1st reviewer)

Department of Nonlinear Dynamics, MPI for Dynamics and Self-Organization

- Prof. Dr. Jörg Enderlein

(2nd reviewer)

Third Institute of Physics, Georg-August-University Göttingen

- Prof. Dr. Siegrid Löwel

Department of Systems Neuroscience, Georg-August-University Göttingen

Further members of the examination board:

- Prof. Dr. Ulrich Parlitz

Biomedical Physics, MPI for Dynamics and Self-Organization

- Prof. Dr. Stefan Klumpp

Department of Nonlinear Dynamics, Georg-August-University Göttingen

- Dr. Andreas Neef

Biophysics of Neural Computation, MPI for Dynamics and Self-Organization

Date of oral examination: $14^{\text {th }}$ August 2017 


\section{Affidavit}

I hereby declare that this dissertation entitled "Genetic determination and layout rules of visual cortical architecture" has been written independently and with no other sources and aids than quoted.

Joscha Liedtke, Göttingen, 31 ${ }^{\text {st }}$ May 2017 



\section{Contents}

1 Introduction 1

2 Fundamentals $\mathbf{5}$

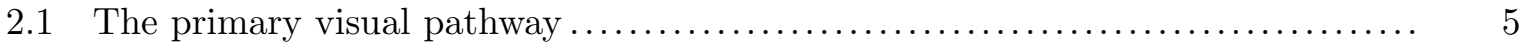

2.2 Functional architecture of the primary visual cortex $\ldots \ldots \ldots \ldots \ldots \ldots \ldots \ldots \ldots \ldots \ldots$

2.3 Development and plasticity of orientation domains ......................... 9

2.4 Modeling development and organization of orientation domains $\ldots \ldots \ldots \ldots \ldots \ldots . \quad 11$

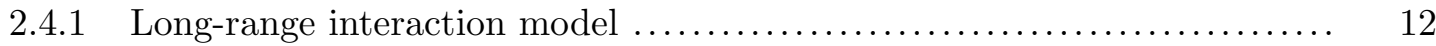

2.4.2 Orientation field ensembles ..................................... 14

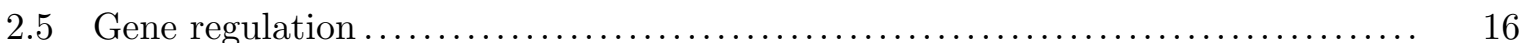

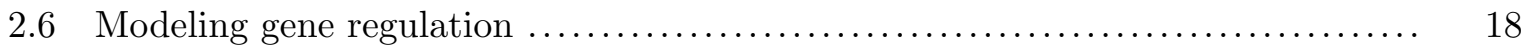

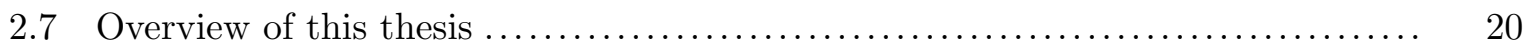

3 Genetic assimilation of visual cortical architecture 23

3.1 Abstract ....................................................... 24

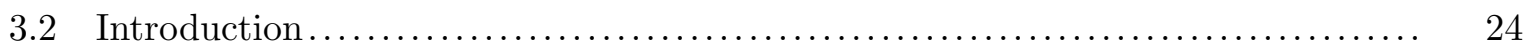

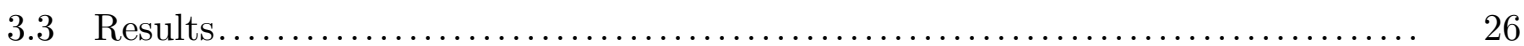

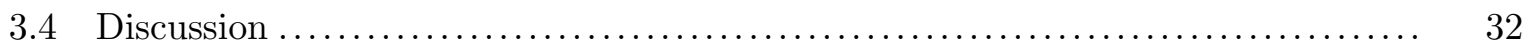

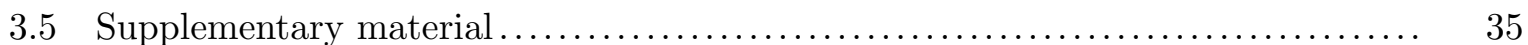

3.5.1 Genetic network construction ................................... 35

3.5.2 Complexity reduction to canonical genetic network ................... 40

3.5.3 Symmetry-confined canonical genetic network ...................... 50

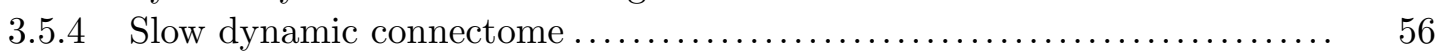

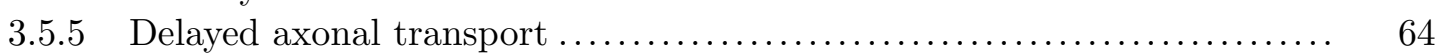

3.5.6 Extended solution set of canonical genetic network .................. 69

3.5.7 Benchmarking orientation fields of canonical genetic network ........... 75

3.5 .8 Numerical methods........................................ 80

3.5.9 Alternative model: positionally specified orientation preference......... 82

3.5.10 Nomenclature ............................................. 87

4 Pinwheel configuration in max. entropy models of orientation domains $\quad 89$

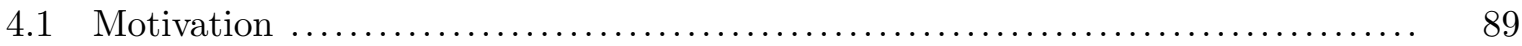

4.2 Maximum entropy models of orientation fields .......................... 89

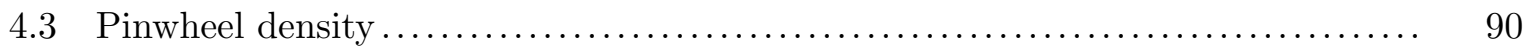

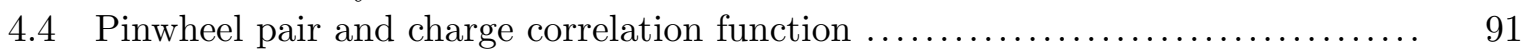

4.4.1 Isotropic and shift-symmetric orientation field ensembles.............. 92 


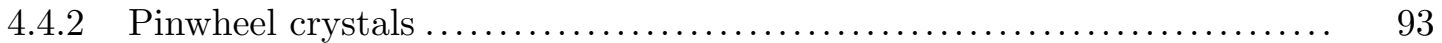

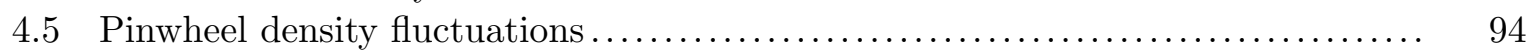

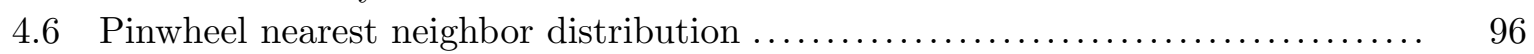

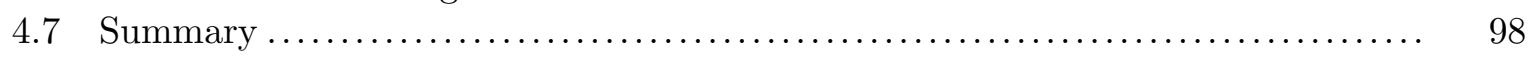

5 Pinwheel configuration: theo. significance and precision measurement 101

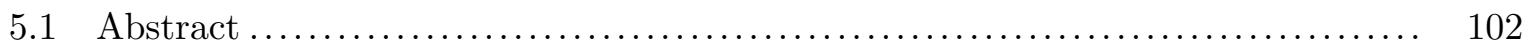

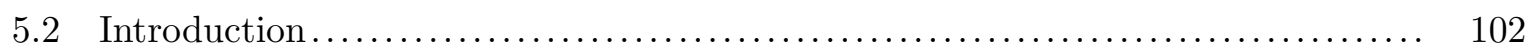

5.3 Results......................................................... 107

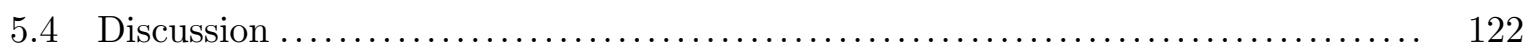

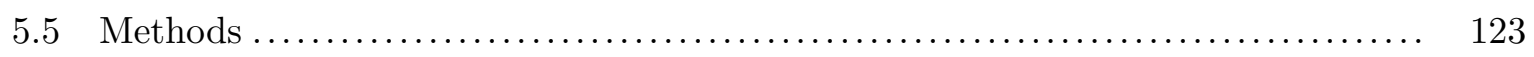

6 Three-dimensional organization of orientation selectivity in cortical tissue 131

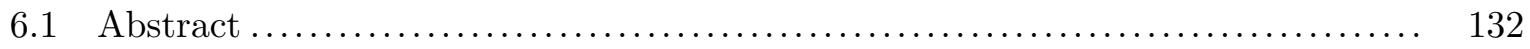

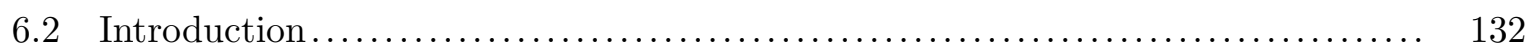

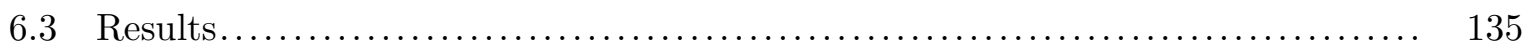

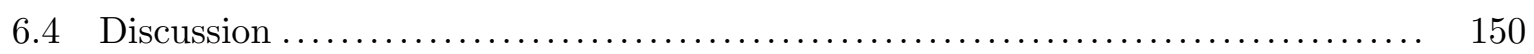

6.5 Methods ........................................................ 152

$\begin{array}{llr}7 & \text { Discussion } & 157\end{array}$

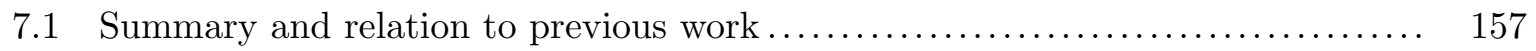

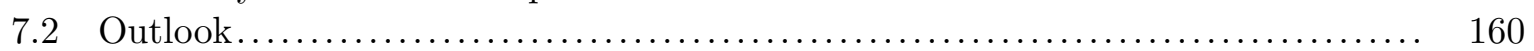

8 Appendix $\quad 163$

8.1 Derivation of pair and charge pinwheel correlation function $\ldots \ldots \ldots \ldots \ldots \ldots \ldots, 163$

$\begin{array}{ll}\text { Bibliography } & 167\end{array}$ 


\title{
Introduction
}

\author{
"Organic chemistry, indeed, in investigating more \\ and more complicated molecules, has come very \\ much nearer to that 'aperiodic crystal' which, in my \\ opinion, is the material carrier of life. And \\ therefore it is small wonder that the organic \\ chemist has already made large and important \\ contributions to the problem of life, whereas the \\ physicist has made next to none."
}

- E. Schrödinger, What is life?

The relative importance of genes and environments on complex human traits remains controversial in fields like medicine, neuroscience, social sciences and evolutionary biology [1]. Coarse morphological traits such as organs and body plan, but also finer structures such as the overall organization of the neocortex are controlled to a large extend by genetic information. The neocortex, in particular, is parceled into cytoarchitectonically defined areas and six laminar layers with stereotypical inter-laminar connectivities that are considered to be mainly shaped by genes, cell fate and cell autonomous developmental programs $[2,3]$. Various functional features, however, are thought to be strongly influenced by neuronal activity. While functional traits such as learning, memory and executive control require neuronal activity, cognitive traits such as bird migration [4], early face recognition [5] and the notion of a universal grammar in language acquisition [6] are granted to be influenced by genes. This indicates that the influence of genetic mechanisms might reach further in the development of functional traits than expected.

The debate of nature versus nurture is perhaps most advanced at the first visual cortical processing stage, the primary visual cortex (V1). The functional architecture of V1 is set up by orientation selective neurons that respond preferentially to visual stimuli with contours of a specific orientation in visual space. In both primates and placental carnivores, orientation preference is arranged into continuous and roughly repetitive iso-orientation domains. Exceptions are pinwheel centers that are topological singularities surrounded by all orientation preferences. Iso-orientation domains are linked by long-range patchy connections [7-16] that are shaped by neuronal activity as they preferentially connect neurons with correlated activity [17]. On the other hand, these long-range horizontal connections are a general feature of cortical architecture and are observed in other cortical areas such as somatosensory and prefrontal cortex [18]. Upper layer neurons and long-range horizontal connections are hence a core machinery of the neocortex. Other aspects of visual cortical architecture are clearly influenced by genes [19, 20], but it is suspected that neuronal activity is an instructive mechanism for the development of its functional architecture [21]. Therefore, visual cortical architecture shares general features of 
the neocortical architecture, but is suspected to be mainly shaped by neuronal activity.

Visual cortical architecture in V1 follows quantitative layout rules across different species and thus constitutes a unique circuit for probing the influence of genetic information on functional traits. In primates and placental carnivores, the pinwheel configuration of the functional architecture adheres to quantitative species-invariant statistics, the common design [22-24], and most strikingly also emerges in the absence of any visual experience [22]. The quantitative layout rules pose an ideal framework for benchmarking putative models. Moreover, the common design likely evolved independently at least twice in the course of the past 65 million years [22], which might indicate a functionally advantageous trait. The possible acquisition of environment-dependent functional traits by genes, the Baldwin effect, makes it conceivable that visual cortical architecture is partially or redundantly encoded by genes. In this conception, genetic mechanisms support the emergence of visual cortical architecture or even establish it under unfavorable environments. Recent experimental evidence in fact hints at the importance of genetic mechanisms for functional cortical development. For instance, a molecular correlate of the neuronal response feature ocular dominance is present weeks before ocular dominance forms [25]. Intriguingly, genetic mechanisms are not confined to local areas as homeoprotein transcription factors get transported from the retina to V1 and are there involved in regulating a period of high cortical plasticity, the critical period [26].

The development of visual cortical architecture raises numerous theoretical questions. Whereas activity-dependent mechanisms have been theoretically examined in depth, genetic mechanisms were excluded from theoretical research, mostly because the information capacity of the genome appeared too small to contain a blueprint for wiring up the cortex [27]. Is it theoretically conceivable that a genetic mechanism may quantitatively prescribe visual cortical architecture? What are the biological prerequisites for such a mechanism? Model benchmarking against the quantitative layout rules is a powerful approach towards answering these questions and is, more generally, required for any model of visual cortical architecture. What is the link between the common design and orientation domain ensembles? In the analysis of experimental pinwheel configurations noise sources are attenuated but not eliminated. What effect has measurement noise on the observed pinwheel configuration? Although two-dimensional functional architectures of primates and placental carnivores adhere to the common design, their three-dimensional layouts are distinct, most notably, in their columnar orientation selectivity profile. What are the implications of three-dimensional functional architectures? Do they contain information about transitions between states of functional architecture?

In this thesis, we utilize analytical and numerical techniques from dynamical systems, pattern formation theory $[28,29]$ and statistical physics $[30,31]$ to examine the capability of genetic mechanisms for encoding visual cortical architecture and to examine the pinwheel configuration under measurement noise as well as in different geometries. For the first time, we provide a biologically plausible scheme for genetically encoding a complex cortical architecture that circumvents the alleged information bottleneck. These findings together with recent experimental advances in transcriptome sequencing [32-36] will allow to systematically seek for such a molecular basis and may help to clarify the relative importance of genes and environments on complex human traits. We analyze orientation domain ensembles and examine their link to the speciesinvariant pinwheel statistics of the common design. We further provide a theoretical analysis of the susceptibility of the pinwheel configuration to measurement noise that facilitates highprecision measurements and enhances benchmarking for devising more accurate models of visual cortical development. Finally, we theoretically investigate three-dimensional functional architectures and find that highly convoluted cortices are prone to non-columnar organizations and are thus expected to be informative about evolutionary transitions. The advent of three-photon imaging makes these regions experimentally accessible [37] and can thus foster the understanding of the evolution of visual cortical architecture. 
In Chapter 2, we briefly summarize the biological and theoretical foundations of this thesis. The first part reviews the development of visual cortical architectures together with their dynamic and descriptive models. The second part summarizes the mechanisms and theoretical modeling of gene regulation and their role in biological tissue patterning such as the neocortex. Finally, the scientific questions addressed in this thesis are motivated and embedded in the current state of research.

In Chapter 3, we devise a transcellular genetic network model that can quantitatively encode functional visual cortical architecture. This mechanism might support neuronal activity and serve as a redundant plan in the absence of instructive neuronal activity. We find that active transport and trans-neuronal signaling as well as joined dynamics of morphogens and connectome are key ingredients for such a genetic mechanism. This theory provides predictions for experimental tests and can thus guide research to identify genetic mechanisms that shape cortical architecture on large scales.

In Chapter 4, we review previous work on the link between pinwheel configurations and orientation domain ensembles and extend it to all observed species-invariant pinwheel statistics of the common design. This examination highlights informative measures of pinwheel configurations and their link to orientation domain ensembles.

In Chapter 5, we first review theoretical arguments for the salience of pinwheels and subsequently examine their configuration under measurement noise. The results give rise to an extrapolation method of pinwheel densities to the zero noise limit and provide an approximated analytical expression for confidence regions of pinwheel centers. The precise quantitative determination of pinwheel positions and their confidence regions provides a paradigm benchmark framework for the development of quantitative models.

In Chapter 6, we examine maximum entropy models of three-dimensional functional visual cortical architectures in different geometries. We show that this approach describes entire model classes in a broad parameter regime and thus provides a profound theoretical understanding of three-dimensional functional architectures. This theory enables the examination of possible evolutionary transitions between different functional architectures for which intermediate organizations might still exist.

Finally, in Chapter 7, we conclude this thesis with a discussion of the main findings, their relation to previous work and an outlook for fruitful prospective research directions. 



\section{Fundamentals}

"Thus the number of critical physical events necessary in the retina in order to produce a visual effect lies between 5 and 7 . ... We must therefore consider them as the actual number of quanta absorbed by the retina for the initiation of a visual act."

- S. Hecht et al., [38]

\subsection{The primary visual pathway}

Visual information sensed by the retina is processed by three major pathways that are associated with different tasks. The primary visual pathway is mainly involved in visual perception and projects from the retina over the lateral geniculate nucleus (LGN) to the primary visual cortex (V1). The second visual pathway conveys visual information to the superior colliculus and is important for eye movement. The third visual pathway is involved in pupillary reflexes and transmits visual signals to the pretectal area of the midbrain. This thesis is exclusively concerned with the primary visual cortex and therefore highlights key aspects of the primary visual pathway in the following.

The primary visual pathway combines visual signals from both eyes by an elaborated connectivity pattern, Fig. 2.1A. The pathway begins in the eyes' retina, where axons from retinal ganglion cells (RGCs) form the optic nerve. Optic nerve fibers extend to the optic chiasm, where axons from the nasal hemiretina cross to the contralateral hemisphere and axons from the temporal hemiretina continue to the ipsilateral hemisphere. Thus, optical signals get separated such that one hemisphere receives only visual inputs from the visual field of its opposite side. After the optic chiasm, the axons from the temporal and nasal hemiretina join in the optic tract that terminates in the LGN. The LGN keeps both eye signals separated and relays the retinal signals to V1 through the optic radiation, where neurons get for the first time input from both eyes, Fig. 2.1B. The output of neurons in V1 are projected over two major pathways to higher cortical areas: the ventral and dorsal pathway, Fig. 2.1C. The ventral pathway is concerned with what objects in the visual world are ("what" pathway) and the dorsal pathway with where objects are located in the visual world ("where" pathway).

The functional properties of neurons vary substantially along the primary visual pathway. Neurons respond to stimuli in localized regions of the visual field termed receptive fields. In general, receptive field sizes vary with visual field eccentricity and position in the visual pathway. Receptive fields with the same eccentricity usually become successively larger with the level of 
A

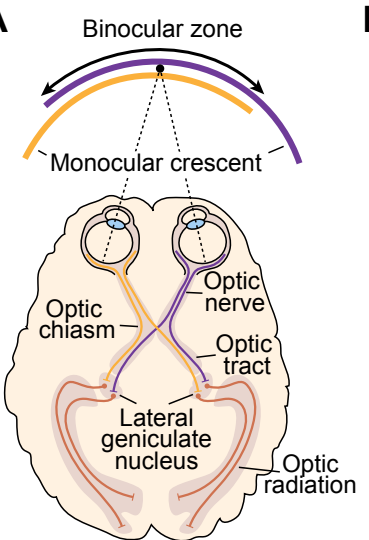

B

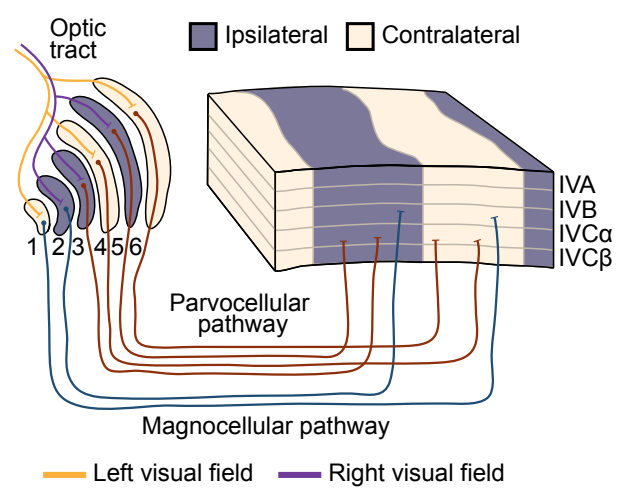

C

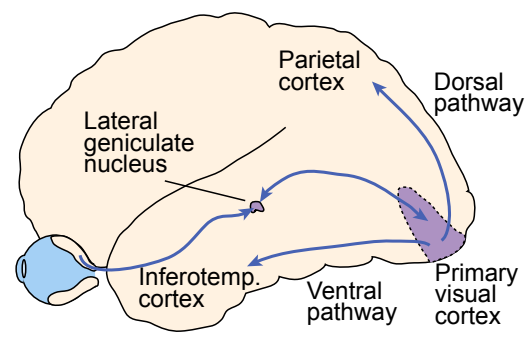

Figure 2.1: Schematic illustration of the primary visual pathway of humans. A, B Signals from retinal ganglion cells (RGC) of both retina are projected along the optic nerve, get partially divided at the optic chiasm and transmitted over the optic tract to the lateral geniculate nucleus (LGN). Relay neurons in the LGN further project the signals to layer IVC of the primary visual cortex (V1) over the optic radiation. C Cortical neurons, for the first time, respond to stimuli of both eyes and project finally into two major pathways: the ventral and dorsal pathway. The dorsal pathway is concerned with the position (where?) and the ventral pathway with the structure or kind (what?) of a visual stimulus. Figure based on [39].

the visual processing stage. At the first stage, RGCs have center-surround receptive fields with either an ON- or OFF-center. At the second stage in the LGN, neurons have similar receptive fields as RGCs and are therefore often called relay cells. At the third stage in V1, neurons for the first time receive input from both eyes. The response dominance of a neuron to input from one specific eye is called ocular dominance. A key organization principle of V1 is that the visual field is systematically represented along its cortical surface, a property called visuotopic (retinotopic) organization. In many mammalian species, neurons in V1 develop for the first time non-concentric receptive fields that allow contour detection, a property called orientation selectivity, Fig. 2.2A.

\subsection{Functional architecture of the primary visual cortex}

Orientation selective neurons in V1 are observed in several mammalian species with different spatial functional organizations. In this thesis, the spatial arrangement of orientation preference is referred to as the functional architecture following [40]. The spatial arrangement of orientation preference is observed in two distinct types of horizontal organizations, Fig. 2.2B. An interspersed, salt-and-pepper, organization of orientation preference is observed in the clade Glires or rodents, e.g. rats [41], gray squirrels [42], mice [43] and rabbits [44-48]. In the clades Laurasiatheria and Euarchonta, or primates and placental carnivores, orientation preference is smoothly organized and segmented into iso-orientation domains or short orientation domains. More precisely, they are observed in species of the primate order such as macaques [49-53], galagos [54], squirrel monkeys [53, 55, 56], owl monkeys [53, 57] and humans [58], in the order carnivora comprising cats [59-67] and ferrets [14, 15, 67,68] as well as in the order scadentia in tree shrews [12]. The typical spacing between orientation domains with the same orientation preference is referred to as the typical scale or column spacing. Both functional architectures are organized on a cellular level, Fig. 2.2C. Orientation domains are additionally organized coarsely into columns such that vertically aligned neurons respond preferentially to similar orientations [69]. They are therefore also called orientation columns in analogy to the columns of 
A

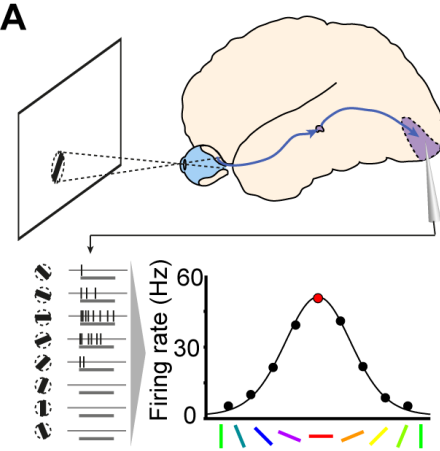

B

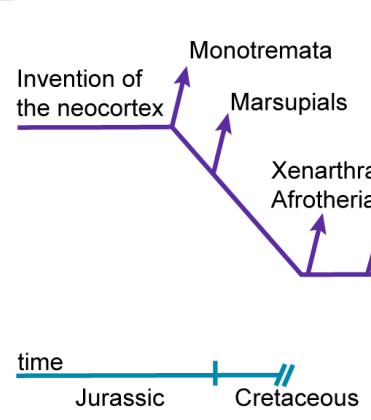

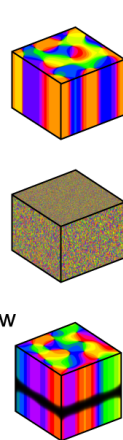

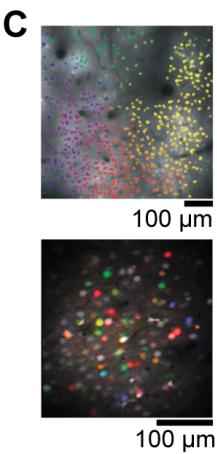

Figure 2.2: Functional visual cortical architecture of different mammalian species. A Visual information are projected from the retina over the LGN to V1. Neuronal responses (ticks on line) to the presentation of oriented bars (solid thick lines) in the receptive field (dashed circle) are summarized by the tuning curve that assigns preferred orientations to neurons. Brain illustration based on [39]. B Orientation preference of neurons in V1 is observed in different mammalian species. The horizontal spatial arrangement of orientation preference divides into two distinct functional architectures: an ordered arrangement into iso-orientation domains found in the evolutionary clades of Laurasiatheria and Euarchonta and an interspersed layout observed in the clade Glires. Reprinted from [24]. C Orientation preference is precisely organized on a cellular level in orientation domains at, e.g. pinwheel centers (top), and in interspersed orientation layouts (bottom) as revealed by two-photon calcium imaging. Reprinted with permission from $[41,71]$.

cat's somatosensory cortex [70].

Orientation domains are typically measured by optical imaging of intrinsic signals ${ }^{1}$. This technique records the change in light reflectance of V1's cortical surface $E\left(\boldsymbol{x} \mid \phi_{j}\right)$ evoked by drifting gratings of different orientations, e.g. $\phi_{j} \in\left\{0^{\circ}, 45^{\circ}, 90^{\circ}, 135^{\circ}\right\}$. The change in light reflectance is due to blood oxygenation changes caused by neural activity. After noise attenuation of the signal, orientation domains are mathematically summarized by the complex orientation director field or short orientation field

$$
\Psi(\boldsymbol{x})=\sum_{j} E\left(\boldsymbol{x} \mid \phi_{j}\right) e^{2 i \phi_{j}}=S(\boldsymbol{x}) e^{2 i \vartheta(\boldsymbol{x})} .
$$

The preferred orientation of the orientation field is then given by $\vartheta(\boldsymbol{x})$ and orientation selectivity by $S(\boldsymbol{x})$, which result from the vector average of the neuronal responses. Orientation preference varies smoothly along the cortical surface except at point singularities called pinwheels that are surrounded by all orientation preferences, Fig. 2.2C and Fig. 2.3A. In the mathematical description, pinwheels are simply defined by the roots of the orientation field $S(\boldsymbol{x})=0$. Depending on the ordering of orientation preference around a pinwheel at position $\boldsymbol{x}_{i}$, different topological charges can be assigned to it by

$$
q_{i}=\frac{1}{2 \pi} \oint_{C_{i}} \mathrm{~d} \boldsymbol{r} \cdot \nabla \vartheta\left(\boldsymbol{x}_{i}\right)
$$

where $C_{i}$ denotes a closed path around only that pinwheel. As orientation preference $\vartheta(\boldsymbol{x})$ is $\pi$-periodic, topological charges can only assume multiples of half integer values $q_{i} \in \mathbb{Z} / 2$. In experiments, only single charged pinwheels $q_{i}= \pm 1 / 2$ are observed, Fig. $\mathbf{2 . 3 C}$, and are highly organized on a cellular level as revealed by two-photon calcium imaging in cats, Fig. 2.2C.

Pinwheel configurations determine a substantial part of orientation domains. However, orientation domains are heterogeneous and their typical scale varies substantially across V1. Comparative quantification of pinwheel configurations that neglected this heterogeneity hence found

\footnotetext{
${ }^{1}$ See [72] for a detailed description of optical imaging of intrinsic signals, [59] for a comparison of this technique to electrode and voltage sensitive dye recording and $[73,74]$ for a discussion of its limitations.
} 
A

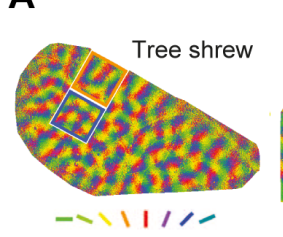

B

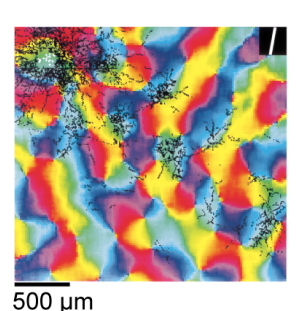

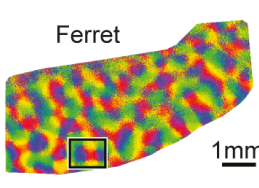

$1 \mathrm{~mm}$

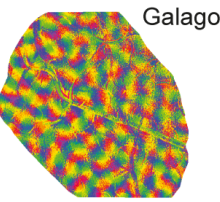

C

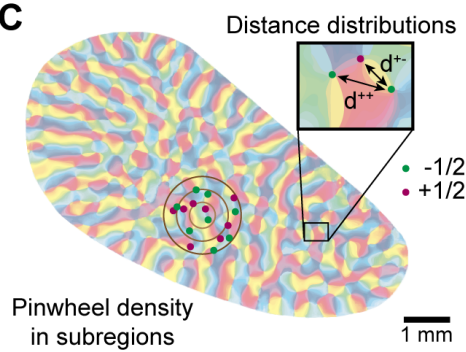

D

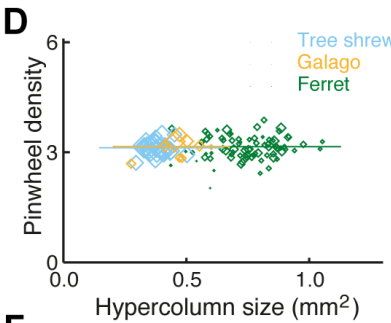

$\mathbf{F}$

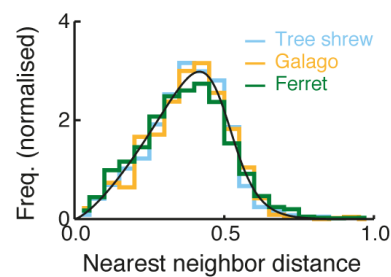

E

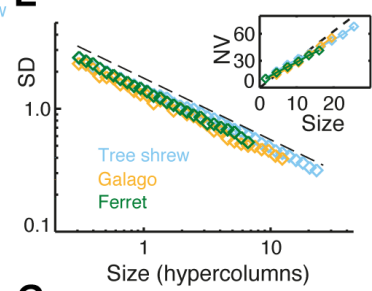

G

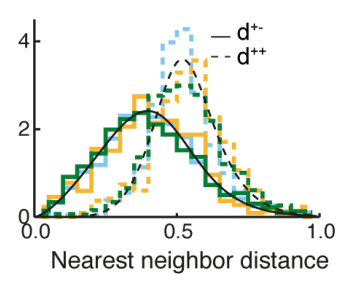

Figure 2.3: Pinwheel configurations of primates and placental carnivores adhere to a common design and are linked to long-range patchy connections. A Orientation domains of tree shrew (Scadentia order), ferret (Carnivora order) and galago (Primate order) obtained by intrinsic signal imaging with subsequent noise filtering (see Eq. (2.1) and main text). B Long-range patchy connections (black dots labeled by biocytin) span several millimeters of cortex and link preferentially neurons with similar orientation preference. C Orientation domains posses pinwheels that are points surrounded by all preferred orientations. Depending on the order of surrounding orientation preference, pinwheels have different topological charges (green and red dots, see Eq. (2.2)). D-G Pinwheel configurations of primates and placental carnivores, more precisely, of tree shrews, galagos, ferrets and cats (not shown) adhere to a common design [22-24]. D Pinwheel density, E Pinwheel density fluctuation of subregions (standard deviation) with inset: number variance versus typical scale area. F Nearest neighbor distribution of pinwheels, G Nearest neighbor distribution of pinwheels with equal $\mathrm{d}^{++}$and opposite $\mathrm{d}^{+-}$topological charge. B, C Adapted with permission from [12]. A, D-G Adapted with permission from [22].

statistically distinct pinwheel patterns with substantial pinwheel density variations across species $[56,67,75]$. The typical scale heterogeneity can be accounted for by a semi-local wavelet analysis. This approach revealed strikingly that the pinwheel configurations in the four species cats, ferrets, galagos and tree shrews adhere to a quantitative species-invariant common design [2224], see Fig. 2.3C-G. This common design is suggested to be an evolutionary convergent trait (see Fig. 2.2B for tree of mammal evolution), because of two main reasons: (i) the last common ancestor of the four species was a small shrew-like mammal and hence, most likely, possessed an interspersed orientation layout in $\mathrm{V} 1[22,76]$. (ii) these mammals have distinct neuronal circuits for orientation selectivity generation $[24,77]$. For instance, tree shrews generate orientation selectivity mostly intracortically [78, 79], whereas cats generate it mostly thalamocortically [69, 80].

An additional visual cortical feature shared by different species is an elaborate horizontal connectome in V1, Fig. 2.3B. These horizontal connections extend over several millimeters up to the entire primary visual cortex and are organized in patches linking neurons with similar preferred orientations [7-16]. Intriguingly, these long-range patchy connections are not only found in $\mathrm{V} 1$, but throughout the neocortex in cortical areas such as the motor area, prefrontal area or inferotemporal area [18]. 


\subsection{Development and plasticity of orientation domains}

Although orientation selectivity and their spatial arrangement is subject to extensive research, the precise underlying mechanisms of orientation domain emergence are yet unresolved. A major question that arose during this research is to what extend functional visual cortical architecture is determined by genetic and activity-depend mechanisms. The initial claim of an innate (genetic) predetermination proposed by Hubel and Wiesel [40] developed into a long debate that is still unsettled [81]. In order to refine the question of a genetic or activity-dependent cause of orientation domains, it is pivotal to differentiate between endogenously and exogenously caused activity-dependent mechanisms as well as activity-independent mechanisms such as cell fate specification by genetic factors.

In the following a brief summary of the major developmental stages of V1 and the primary visual pathway of the two most studied species cats and ferrets will be given (reviewed in e.g. [21, 82-84] and illustrated in Fig. 2.4). Notice that ferrets are more immature at birth than cats and are therefore better suited for early developmental research. In both species, anatomical and physiological maturation of the retina precedes that of LGN, which in turn precedes that of V1 [21]. Axons from LGN grow at an early stage to V1, where they reach the subplate of V1 at E36 in cats [85] and E36 in ferrets [21]. The afferent LGN axons remain in the subplate for a couple of weeks before innervating the cortex. Shortly after LGN afferents reach the subplate around E43 in cats and P0 in ferrets [21], the first functional retinogeniculate synapses emerge. In the following time endogenous retinal waves appear that are accompanied by LGN laminar segregation. Thalamocortical axons begin to innervate V1's input layer 4 at about P0 in cats and P12 in ferrets $[86,87]$. In the following time (P22-P28), spontaneous long-range correlated cortical activity is observed in ferrets [88]. At the same time (P24-P27) in ferrets, LGN's left-eye and right-eye layers exhibit correlated activity that is shown to require cortical feedback [89]. In ferrets, orientation selectivity is already present in V1 at P23 with about $25 \%$ orientation selective cells that gradually matures over the course of a month (P49) to roughly $75 \%$ [90]. A landmark event in visual cortical development is eye-opening at P7 in cats [81] and P31-P36 in ferrets $[68,91]$ from where on the animals can first experience normal exogenously driven neuronal activity. However, as early as two weeks before eye-opening, visual stimuli presented through closed eyelids can already drive neuronal activity in LGN and V1 of ferrets [92]. In both species orientation domains are already observed shortly after eye-opening and are rather robust over the following two weeks $[64,66,68]$. The emergence of orientation domains coincides roughly with the elaboration of long-range horizontal connections to long-range patchy connections $[10$, $14,82,91,93]$. Intriguingly, layer $2 / 3$ neurons in cats finish their neural migration and arrive at their final positions only at P21 [86]. In cats, the visual cortical area increases substantially over the first 14 postnatal weeks [94-97], while basically preserving the typical spacing $[19,20]$ of ocular dominance and orientation selectivity bands during most of that time implying cortical reorganization [97-99].

The influence of experience driven activity on orientation domains is commonly dissected by exposing animals to abnormal visual developmental environments such as dark rearing, lid suture and stripe rearing. Stripe rearing in cats expands orientation domains devoted to the orientation of the striped environment, but is permissive to orientation selectivity emergence of unseen orientations [100]. In ferrets, lid-suture ${ }^{2}$ destroys or even prevents orientation domain emergence [14]. Lid suture in cats does not affect crude clustering of horizontal connections, but prevents their refinement [101]. Most strikingly, the total lack of visual experience leads to weaker orientation selectivity, but generates orientation domains in ferrets that adhere to

\footnotetext{
${ }^{2}$ Notice that as early as 2 weeks before eye-opening, visual stimuli presented through closed eyelids can drive neuronal activity in LGN and V1 of ferrets [92]. Hence lid-sutured animals experience a disturbed visual environment.
} 
A

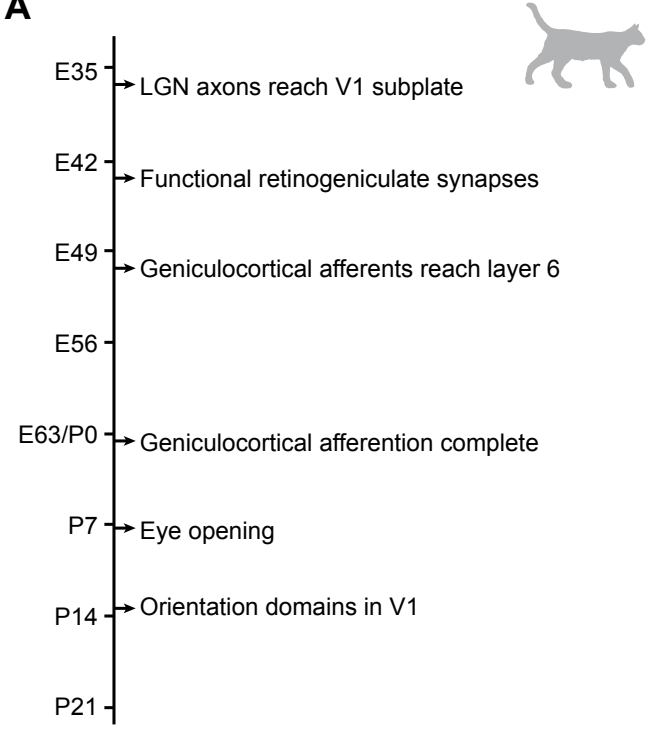

B

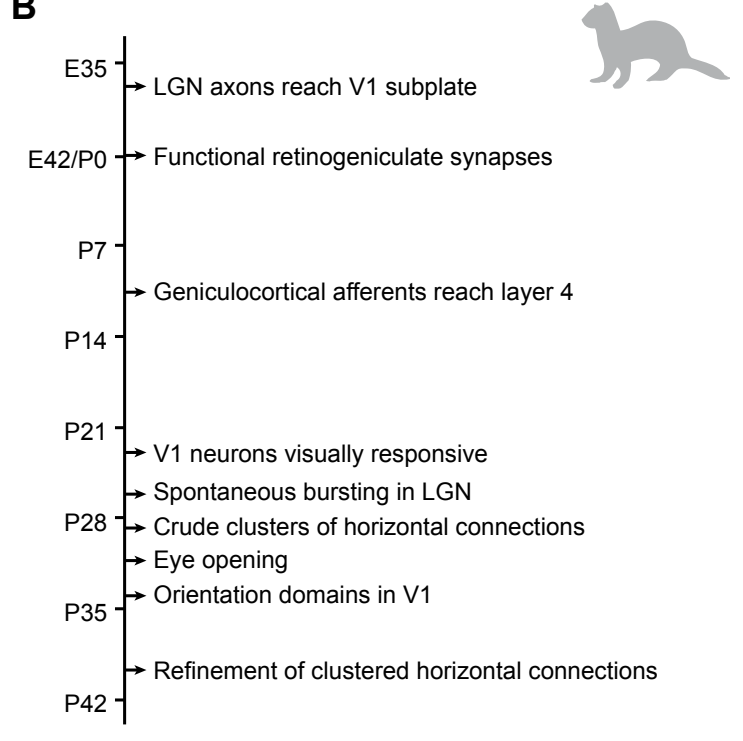

Figure 2.4: Timeline of visual development and plasticity of the primary visual cortex. A and $\mathbf{B}$ list the major developmental stages of cats and ferrets during their embryonic (E) and postnatal (P) age in days, respectively. Ferrets are more immature at birth than cats and are therefore better suited for early development research. Figure based on [21].

the common design $[14,22]$. These results suggest that initial orientation domain emergence is independent of visual experience, while susceptible to inappropriate visual input.

What is the effect of endogenous activity on the formation of orientation domains? Silencing cortical activity of V1 with the neurotoxin TTX in an early developmental stage of ferrets (P21-P28) completely abolishes maturation of orientation selectivity and clustering of horizontal connections [90, 93]. Similarly, blocking NMDA receptors at P21 in ferrets' V1 prevents orientation selectivity maturation [102]. Intriguingly, bilateral enucleation at P21 in ferrets does not prevent clustering of horizontal connections [93]. Subplate ablation prevents formation of functional architecture even if LGN axons already entered V1 [103]. Infusion of TTX in LGN of fetal cats between E42 and E56 prevents topographically organized thalamocortical projections [104]. Stimulation of the optic nerve over a course of two weeks weakens orientation selectivity but does not prevent normal appearing orientation domains [105]. Electric focal stimulation paired with a preceding visual stimulation of V1 in cats changes orientation preference strongly locally and weakly globally [106], see [107]. In the light of these experimental findings, it can be summarized that activity-dependent mechanisms appear necessary for orientation selectivity emergence and elaboration of horizontal connections, but it is unclear whether they are sufficient for this task.

Initial orientation domains have a bias in orientation preference representation towards cardinal orientations. An analysis of more than 4000 recorded neurons of cat's V1 revealed such a cardinal bias [108]. Optical imaging demonstrated a cardinal bias in cats $[67,100,109,110]$ and ferrets [67, 68, 111-113]. A comparison between dark reared and normal raised ferrets suggests that the cardinal bias is endogenous and that normal visual experience equalizes the orientation selectivity distribution [113]. Finally, fMRI recordings show the existence of a cardinal bias also in humans [114].

In total, it can be summarized that (i) visual experience is not necessary for orientation domain emergence, (ii) activity-dependent mechanisms are necessary for orientation selectivity emergence and elaboration of horizontal connections and (iii) a cardinal bias appears to be 
present in initial orientation domains, but equalizes during development. However, it is unclear whether activity-dependent mechanisms are sufficient for the emergence of orientation domains.

\subsection{Modeling development and organization of orientation domains}

Modeling orientation domains has been a challenging task since their first experimental discovery. The main difficulties in modeling are due to the high dimensional nature of the visual cortical architecture and the limited knowledge of their development. First models proposed by Hubel and Wiesel qualitatively argued how orientation selectivity and the spatial arrangement can be generated by genetic mechanisms [40]. This view of genetic predetermination as an explanation for orientation domains emergence changed to activity-dependent models due to various experiments listed in the previous section. Additionally, it was pointed out that the genetic information required for a predetermination of the connectome for orientation domains is insufficient and likely exceeds the information capacity of the $\mathrm{DNA}^{3}$ [27]. Developmental models of orientation domain emergence were therefore based on neuronal activity with mostly Hebbian learning rules. An alternative class of models were formulated more abstract and is neither related to neural activity nor to genetic mechanisms. The merits and shortcomings of these models will be listed in the following.

The development of orientation domains was modeled in various ways in the past. One class of models uses numerous neurons in a network with a dynamic connectome that is typically rearranged by an activity-dependent learning rule, see e.g. [27, 115-121]. These models have typically many parameters, can be mostly evaluated numerically and thus make it difficult to reveal underlying principles of orientation domain emergence. Another model class interprets the emergence of orientation domains as a pattern forming system and models their emergence in an abstract and phenomenological manner [22, 122-131]. Orientation selectivity and preference is typically treated as the only dynamical variable. The dynamics are then constrained by phenomenology and symmetry assumptions. These models are hence relatively simple and often analytically tractable to a high degree thus revealing underlying principles of orientation domain emergence. For instance, it was shown that for a universality class of orientation domain development, long-range interactions are necessary for stabilization of quasiperiodic orientation preference layouts that are in agreement with the common design [22, 128]. The drawback of these models is clearly their lack of information about biological mechanisms and the functional role of orientation domains. The interactions could be for instance mediated by the visual environment, internal neural activity or even by genetic mechanisms.

Throughout this thesis the spatial arrangement of orientation preference will be summarized by an orientation field

$$
\Psi(\boldsymbol{x}, t)=S(\boldsymbol{x}, t) e^{2 i \vartheta(\boldsymbol{x}, t)}
$$

as proposed in $[122,132]$ and analogously to Eq. (2.1), whose phase summarizes the preferred orientation $\vartheta(\boldsymbol{x}, t)$ and the amplitude its orientation selectivity $S(\boldsymbol{x}, t)$. We will devise a dynamic model for their emergence during development and descriptive models for which the basics of both will be elucidated in the following sections.

\footnotetext{
${ }^{3}$ The human brain consists of around $10^{11}$ neurons communicating through about $10^{14}$ synapses. Hardwiring the brain by a blueprint would thus require $10^{15} \approx 10^{14} \log _{2}\left(10^{11}\right)$ bits, however, the maximal information capacity of the human DNA with its 3.2 billion bases is only about $10^{10} \approx 3.2 \cdot 10^{9} \log _{2}(4)$ bits posing an information gap of a factor $10^{5}$.
} 


\subsubsection{Long-range interaction model}

The development of visual cortical architecture is reminiscent of dynamic pattern generation in physical systems $[28,29]$. This analogy inspired a symmetry based model for the dynamic emergence of orientation fields: the long-range interaction model [128]. The long-range interaction model is the only developmental model that is shown to be in good quantitative agreement with the common design. The central concept is based on the idea to constrain all possible variational dynamics

$$
\partial_{t} \Psi(\boldsymbol{x}, t)=\hat{F}[\Psi]
$$

governed by a nonlinear operator $\hat{F}[\Psi]$ through symmetry principles and phenomenology of visual cortical development. Symmetry principles require equivariance of the operator $\hat{F}$ under a set of symmetry operators $\hat{\mathcal{S}}$ that is

$$
\hat{\mathcal{S}} \hat{F}[\Psi]=\hat{F}[\hat{\mathcal{S}} \Psi] .
$$

In the long-range interaction model the system is invariant under translation $\hat{T}_{\boldsymbol{y}}$, rotation $\hat{R}_{\alpha}$, reflection $\hat{P}$, phase shift $\hat{S}_{\theta}$ and conjugation $\hat{C}$ with the symmetry operators defined by

$$
\begin{gathered}
\hat{T}_{\boldsymbol{y}} \Psi(\boldsymbol{x})=\Psi(\boldsymbol{x}+\boldsymbol{y}), \quad \hat{R}_{\alpha} \Psi(\boldsymbol{x})=e^{2 i \alpha} \Psi\left(\Omega_{\alpha} \boldsymbol{x}\right), \quad \hat{P} \Psi(\boldsymbol{x})=\Psi(\tilde{\boldsymbol{x}}), \\
\hat{S}_{\theta} \Psi(\boldsymbol{x})=\Psi(\boldsymbol{x}) e^{i \theta}, \quad \hat{C} \Psi(\boldsymbol{x})=\bar{\Psi}(\boldsymbol{x}),
\end{gathered}
$$

where $\Omega_{\alpha}$ denotes the rotation matrix of angle $\alpha$ and $\tilde{\boldsymbol{x}}=(x,-y)$ the reflection at the $x$ axis. These symmetry principles imply a homogeneous unselective state $\Psi(\boldsymbol{x}, t)=0$ and a power series consisting of odd terms only

$$
\partial_{t} \Psi(\boldsymbol{x}, t)=\hat{L}[\Psi]+\hat{N}_{3}[\Psi, \Psi, \bar{\Psi}]+\mathcal{O}\left(\Psi^{5}\right) .
$$

A linear operator of Swift-Hohenberg type [28] defined by $\hat{L}[\Psi]=\left(r-\left(k_{c}^{2}+\Delta\right)^{2}\right) \Psi(\boldsymbol{x}, t)$ provides the system with a typical scale $\Lambda=2 \pi / k_{c}$ for $r>0$ due to unstable modes on the critical circle at $k_{c}=\|\boldsymbol{k}\|$. Close to the symmetry breaking point of $r=0$, that is the range $0<r \ll 1$, the field dynamics can be analyzed by weakly nonlinear analysis [28, 29]. In weakly nonlinear analysis (see section 3.5.2 for details) the field $\Psi(\boldsymbol{x}, t)$ is expanded in a power series of $\sqrt{r}$ around its homogeneous unselective state leading to dynamics confined to the critical circle. Measuring time in units of the intrinsic timescale $1 / r$ and discretizing the orientation field equidistantly on the critical circle

$$
\Psi(\boldsymbol{x}, t)=\sum_{j=1}^{N} A_{j}(t) e^{i \boldsymbol{k}_{j} \boldsymbol{x}} \quad \text { with } \quad \boldsymbol{k}_{j}=k_{c}\left(\begin{array}{l}
\cos \frac{2 \pi j}{N} \\
\sin \frac{2 \pi j}{N}
\end{array}\right)
$$

leads to the variational amplitude equations

$$
\partial_{t} A_{i}(t)=r A_{i}(t)-A_{i}(t) \sum_{j=1}^{N} g_{i j}\left|A_{j}(t)\right|^{2}-\bar{A}_{i^{-}}(t) \sum_{j=1}^{N} f_{i j} A_{j}(t) A_{j^{-}}(t) .
$$

Here, the notation $i^{-}=i+N / 2$ and the coefficients $g_{i j}, f_{i j}$ that depend on the cubic operator $\hat{N}_{3}$ was used. Without any further symmetry principles stable closed-form solutions of the amplitude equations can cause visual scotoma containing only two preferred orientations. Stable visual scotoma solutions ought to be absent in any biophysically relevant model and indeed become unstable by the additional symmetry principle of permutation-symmetric cubic operators

$$
\hat{N}_{3}[u, v, w]=\hat{N}_{3}[w, u, v] .
$$


A

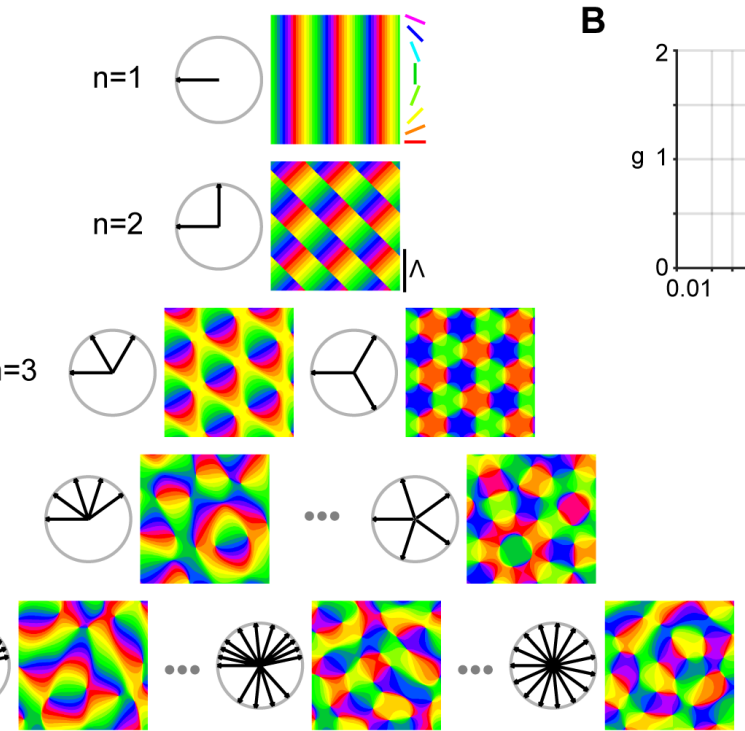

C

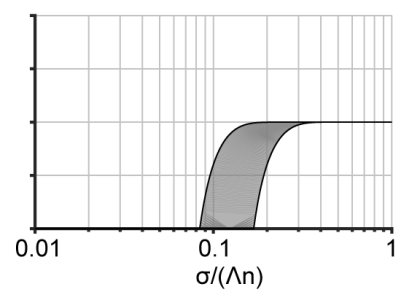

\section{D}

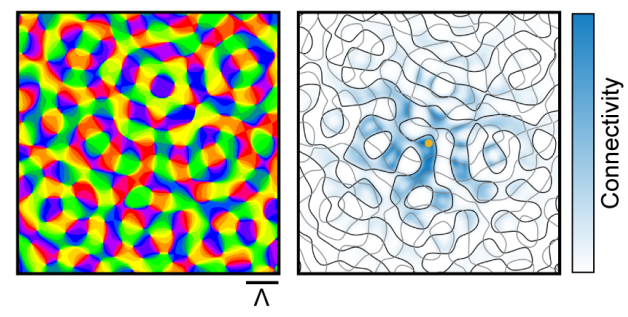

Figure 2.5: Closed-form solutions (ECPs) of the long-range interaction model. A ECP orientation fields and their active mode configurations of example solutions are depicted for different numbers of active modes $n$. B Phase diagram of the minimal energy regions of the ECPs for a Gaussian long-range interaction kernel (color-code serves illustration purposes). C Asymptotic stability region (gray area) for large $n$ is shown for a Gaussian interaction kernel. D Example ECP together with its long-range patchy connectivity of a neuron (orange dot). The interaction kernel is Gaussian.

Notice that this is not a sufficient condition, since example cubic operators can typically be written as both permutation and non-permutation symmetric operators.

Closed-form solutions of permutation symmetric models that contain all preferred orientations are essentially complex planforms (ECPs) defined by $n=N / 2$ active modes of equal amplitude $\mathcal{A} \in \mathbb{R}$

$$
\Psi(\boldsymbol{x})=\mathcal{A} \sum_{j=1}^{n} e^{i\left(l_{j} \boldsymbol{k}_{j} \boldsymbol{x}+\phi_{j}\right)}
$$

with $l_{j} \in\{-1,1\}$ as illustrated in Fig. 2.5A. The energy corresponding to the variational amplitude equations in Eq. (2.9) is degenerated in its phases $\phi_{j}$ of closed-form solutions with $n$ active modes. Thus, ECP closed-form solutions represent $n$-tori $\mathbb{T}^{n}$ in phase space. The number of distinct $n$-tori ${ }^{4}$ is given by $[133,134]$

$$
N_{\mathrm{ECP}}(n)=\frac{1}{4 n} \sum_{2 \nmid k \mid n} \varphi(k) 2^{2 n / k}+2^{\lfloor(n-3) / 2\rfloor}
$$

with $\varphi(\cdot)$ the Euler totient function that scales for large $n$ as $N_{\mathrm{ECP}}(n) \sim 2^{n-2} / n$. The closedform solution set of ECPs are in quantitative agreement with the common design for large numbers of active modes [22]. In fact, the closed-form solutions predicted a mean pinwheel density close to $\pi$ for large $n$ that was later discovered in distinct species. The mean pinwheel densities cluster around $\pi$ for small $n$ and converge to it from below for large $n$ as verified by a random field approximation (see next section and Fig. 2.6D) yielding a pinwheel density of $\rho(\zeta)=\sqrt{\pi^{2}-8 \zeta^{2}}$ and $\zeta=\left\|\sum_{j} l_{j} \boldsymbol{k}_{j}\right\| /(4 n) \leq 1[128,135]$.

\footnotetext{
${ }^{4}$ Solutions defined by $\mathbf{l}_{1}$ and $\mathbf{l}_{2}$ are distinct if they cannot be transformed to each other by symmetries. For instance, the solutions $\mathbf{l}=(1,-1,1)$ can be transformed by a rotation of $\pi / 3$ to $\mathbf{l}=(-1,1,-1)$, but is distinct to the solution $\mathbf{l}=(1,1,1)$.
} 
In order to stabilize ECPs, an example permutation symmetric cubic operator was phenomenologically motivated. In the primary visual cortex, neurons with similar preferred orientation preferences are preferentially linked by long-range patchy connections. This motivated an orientation preference selective connectivity $W(\boldsymbol{x}, \boldsymbol{y}) \propto K(\|\boldsymbol{x}-\boldsymbol{y}\|) \exp \left(-|\Psi(\boldsymbol{x})-\Psi(\boldsymbol{y})|^{2} / \sigma_{z}^{2}\right)$ with variable interaction range $K(\|\boldsymbol{x}\|)=\exp \left(-\|\boldsymbol{x}\|^{2} / 2 \sigma^{2}\right)$, see Fig. 2.5D. The long-range interactions together with local interactions result in the relevant third order terms for orientation field selection

$$
\hat{N}_{3}[\Psi, \Psi, \bar{\Psi}]=(1-g) \Psi(\boldsymbol{x})|\Psi(\boldsymbol{x})|^{2}-\frac{2-g}{2 \pi \sigma^{2}} \int \mathrm{d}^{2} y e^{-\|\boldsymbol{x}-\boldsymbol{y}\|^{2} / 2 \sigma^{2}}\left(|\Psi(\boldsymbol{y})|^{2} \Psi(\boldsymbol{x})+\frac{1}{2} \Psi(\boldsymbol{y})^{2} \bar{\Psi}(\boldsymbol{x})\right)
$$

and only depends on the interaction range $\sigma$ and the weighting between local and non-local interactions $g$. The example operator was generalized in [136] by different long-range interaction kernels $K(\|x\|)$. The region of minimal energy of an ECP is centered at large interaction ranges for a large number of active modes as shown in Fig. 2.5B for the Gaussian kernel. The closed-form ECP solutions are multistable in regions of their minimal energy and converge asymptotically to the stability regions depicted in Fig. 2.5C for large $n$.

This orientation domain model type was generalized to models with higher-order interaction terms of Eq. (2.7) [137], orientation domains lacking shift-symmetry $\hat{S}_{\theta}$ (see Eq. (2.6)) [138, 139], ocular dominance coupled orientation domains [129-131, 137] and models producing interspersed orientation layouts [140].

\subsubsection{Orientation field ensembles}

Statistical features of orientation field ensembles can be analyzed by descriptive models. In this framework orientation field ensembles are described by a probability functional $\mathcal{P}[\Psi]$ that assigns a statistical weight to the occurrence of a specific orientation field $\Psi(\boldsymbol{x})$. Orientation fields thus become infinite dimensional random variables. The challenge for an accurate description of orientation field ensembles consists in the specification of the probability functional. Sampling this distribution from experimentally measured orientation domains without any further constraints is not feasible.

Maximum entropy models provide a systematic and agnostic approach for approximating probability functionals under the constraint of limited knowledge [141, 142]. The resulting maximum entropy distribution is the least structured distribution that fulfills a set of provided statistical quantities. For instance, the maximum entropy distribution of a random variable $\boldsymbol{u}=\left(\Psi\left(\boldsymbol{x}_{1}\right), \Psi\left(\boldsymbol{x}_{2}\right), \ldots, \Psi\left(\boldsymbol{x}_{d}\right)\right)^{T}$ with known mean $\boldsymbol{\mu}=\langle\boldsymbol{u}\rangle$ and covariance $\boldsymbol{\Sigma}=\left\langle\boldsymbol{u} \boldsymbol{u}^{T}\right\rangle$ is a multivariate Gaussian distribution

$$
p(\boldsymbol{u})=\frac{1}{\sqrt{(2 \pi)^{d} \operatorname{det} \boldsymbol{\Sigma}}} e^{-\frac{1}{2}(\boldsymbol{u}-\boldsymbol{\mu})^{T} \boldsymbol{\Sigma}^{-1}(\boldsymbol{u}-\boldsymbol{\mu})} .
$$

In the limit of an infinite dimensional random variable $\Psi(\boldsymbol{x})$, the field is called Gaussian process or Gaussian (random) field [143]. Marginal distributions of a Gaussian random field for $\Psi\left(\boldsymbol{x}_{1}\right)$ and $\Psi\left(\boldsymbol{x}_{2}\right)$ are then given by a multivariate Gaussian distribution.

Historically, Gaussian random fields as a proxy for orientation fields became known under the name of band-pass filtered white noise [144-146]. In these models, white noise was bandpass filtered with a kernel $K_{\mathrm{bp}}(\boldsymbol{k})$ and subsequently Fourier transformed. Thus, these models are Gaussian random fields with a vanishing mean $\langle\Psi(\boldsymbol{x})\rangle$, vanishing first correlation function $\langle\Psi(\boldsymbol{x}) \Psi(\boldsymbol{x})\rangle$ and a translation invariant second correlation function $C(\boldsymbol{x})=\mathcal{F}^{-1}\left[\left|K_{\mathrm{bp}}\right|^{2}\right](\boldsymbol{x})=$ $\langle\Psi(0) \bar{\Psi}(\boldsymbol{x})\rangle$ due to the Wiener-Khinchin theorem. These descriptive models were shown to be equivalent to developmental dynamics of orientation fields in their transient linear regime 
and frozen noise models [56, 147]. Hence, these descriptive models account for developmental dynamic models such as $[122,148]$. Gaussian random fields as descriptive models are particularly powerful and are extensively used in singular optics [149] as well as in quantum billiards [150$152]$ and allow us to derive various analytical expectations of intrinsic features.

The pinwheel configuration can be quantified analytically in these descriptive models. Pinwheels are points in two-dimensional fields and lines in three-dimensional fields defined by vanishing amplitudes $|\Psi(\boldsymbol{x})|=0$. In the Gaussian random field literature, these points are known as topological defects, dislocations, nodal points or singularities. The topological charge $q_{i}$ of a pinwheel (see Eq. (2.2)) in Gaussian random fields is a positive or negative half integer and typically $+1 / 2$ or $-1 / 2[30,153]$. Notice that in the Gaussian random field literature the topological charges are full integers, since the phase is defined as $2 \pi$ periodic. For a two-dimensional system, statistics of pinwheel centers $\boldsymbol{x}_{i}$ (roots of the complex field) can be evaluated by expressing these centers in terms of the real and imaginary part of the orientation field $\Psi(\boldsymbol{x})=\xi(\boldsymbol{x})+i \eta(\boldsymbol{x})$ as

$$
\sum_{i} \delta\left(\boldsymbol{x}-\boldsymbol{x}_{i}\right)=\delta(\xi(\boldsymbol{x})) \delta(\eta(\boldsymbol{x})) \underbrace{\left|\frac{\partial(\xi(\boldsymbol{x}), \eta(\boldsymbol{x}))}{\partial(x, y)}\right|}_{\nu(\boldsymbol{x})} .
$$

The orthogonality measure $\nu(\boldsymbol{x})=\left|\partial_{x} \xi(\boldsymbol{x}) \partial_{y} \eta(\boldsymbol{x})-\partial_{y} \xi(\boldsymbol{x}) \partial_{x} \eta(\boldsymbol{x})\right|$ that measures the area of the spanned parallelogram of $\nabla \xi(\boldsymbol{x})$ and $\nabla \eta(\boldsymbol{x})$ is required for normalization. The ensemble average of this quantity results in a Kac-Rice type formula and counts the average number of pinwheels per area that is the pinwheel density

$$
\rho(\boldsymbol{x})=\langle\delta(\xi(\boldsymbol{x})) \delta(\eta(\boldsymbol{x})) \nu(\boldsymbol{x})\rangle=\langle\tilde{\rho}(\boldsymbol{x})\rangle .
$$

The ensemble average $\langle\cdot\rangle$ can be obtained by averaging over the Gaussian multivariate distribution with the state vector composed of real and imaginary parts $\xi(\boldsymbol{x}), \eta(\boldsymbol{x})$ and their gradients $\nabla \xi(\boldsymbol{x}), \nabla \eta(\boldsymbol{x})$. Topological defect averages can be obtained by

$$
\langle F[\Psi(\boldsymbol{x})]\rangle_{\text {pinwheel }}=\frac{1}{\rho(\boldsymbol{x})}\langle F[\Psi(\boldsymbol{x})] \tilde{\rho}(\boldsymbol{x})\rangle .
$$

In three-dimensional systems the normalizing orthogonality measure is simply replaced by $|\nabla \xi(\boldsymbol{x}) \times \nabla \eta(\boldsymbol{x})|$. The topological defect average can be further extended by incorporating higher topological defect correlations. The resulting $n$-point topological defect correlation functions are then given by

$$
\rho^{(n)}\left(\boldsymbol{x}_{1}, \boldsymbol{x}_{2}, \ldots, \boldsymbol{x}_{n}\right)=\left\langle\tilde{\rho}\left(\boldsymbol{x}_{1}\right) \tilde{\rho}\left(\boldsymbol{x}_{2}\right) \cdots \tilde{\rho}\left(\boldsymbol{x}_{n}\right)\right\rangle .
$$

Weighting every topological defect in the $n$-point correlation function with its topological charge gives the charge correlation function. The pair correlation function $(n=2)$ is cumbersome to solve and was calculated for isotropic Gaussian fields in [30, 154]. Derivation of the charge correlation function is much simpler and is solved for arbitrary $n$-point charge correlations of isotropic Gaussian fields in [155]. The pair charge correlation function $(n=2)$ is solved for general Gaussian fields with vanishing mean $[30,156]$. The $n$-point correlation functions are tightly related to the nearest neighbor distribution of topological defects [157-159]. The effect of boundaries on topological defect distributions was examined in [160-165]. The statistics of topological defects in Euclidean geometry was extended to e.g. extremal point statistics [166, 167], possible temporal dynamics of topological defects [168] and to topological defect statistics on a sphere [169]. A review of topological defect statistics in Gaussian fields is provided in [31] and a widespread collection of corresponding calculations in [30]. 
A

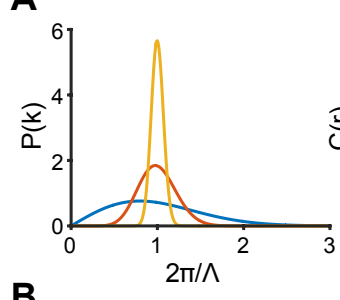

B

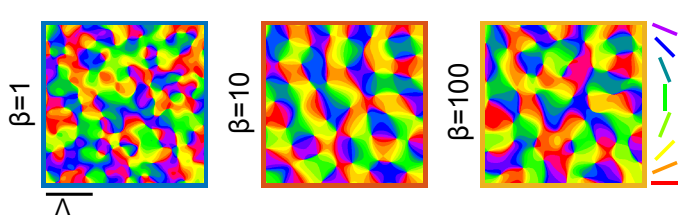

C

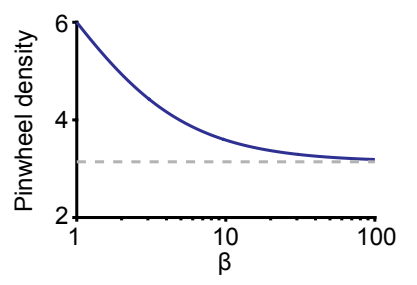

D

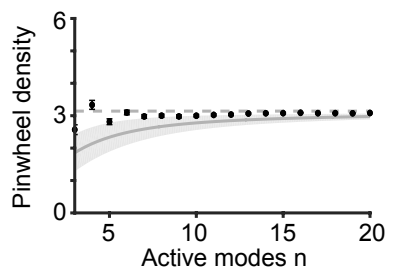

Figure 2.6: Maximum entropy model of orientation domain ensembles. A The correlation function $C(r)=\left\langle\Psi\left(\boldsymbol{x}_{1}\right) \bar{\Psi}\left(\boldsymbol{x}_{2}\right)\right\rangle$ with $r=\left\|\boldsymbol{x}_{1}-\boldsymbol{x}_{2}\right\|$ of an orientation domain ensemble [171] together with the radial part of its Fourier transform $P(k)=\mathcal{F}[C](k)$ for different power-spectral density widths $\sim 1 / \beta$. B Synthesized orientation domain examples corresponding to the correlation functions of $\mathbf{A}$. C Analytical pinwheel density of the orientation domain ensemble in A-B from [171] (solid line) that converges to a pinwheel density of $\pi$ (dashed line) for small power-spectral density widths. D Pinwheel density of closed-form ECP solutions (black dots) is depicted together with its maximum entropy model, that is a Gaussian random field, (gray area) and its limit for $n \rightarrow \infty$ of $\pi$ (dashed line) [135].

The pinwheel density of isotropic orientation domains was found to be bounded from below by $\pi$ that lead to the prediction of pinwheel annihilation during visual development [147]. Notice that the lower bound is not valid for anisotropic orientation domains that can assume any pinwheel density as shown in [170]. A descriptive model with variable power-spectral density width that mimics orientation domains in the transient linear regime is provided in [171] and depicted together with its correlation function and pinwheel density in Fig. 2.6A-C. The pinwheel density of the anisotropic ECP was calculated and shown to converge to $\pi$ from below for an increased number of active modes [135] as shown in Fig. 2.6D. The variance of the typical scale in orientation domains was calculated in a similar manner in [172]. Orientation domains and pinwheel densities were further studied in hyperbolic and spherical geometries [173].

In all these models centered Gaussian random fields with vanishing mean were considered. In three-dimensional geometries the analysis was restricted to isotropic Gaussian random fields. In this thesis, we extend the examination of Gaussian random fields to ensembles with nonvanishing, spatially heterogeneous means and to non-isotropic three-dimensional geometries.

\subsection{Gene regulation}

Proteins, the molecular machinery and structuring molecules of biological tissue, are encoded by specific DNA sequences called genes. Protein-encoding genes are decoded in two major steps: (i) RNA-polymerase docks to the promoter of a gene and transcribes the DNA sequence to an mRNA polymer and (ii) Ribosomes translate the mRNA to a sequence of amino acids that folds into a specific conformation and thus constitutes the protein. Although almost all cells carry the same DNA, their protein composition and their function varies substantially in different body areas and within the same tissue. This well-orchestrated control of gene regulation is influenced on various processing stages, see Fig. 2.7. While the paramount control point lies at the regulation of transcription [174-176], gene activity can be altered by RNA processing, translational and transport control, mRNA degradation control and protein activity control $[174,177]$. A class of proteins called transcription factors bind to specific DNA sequences in 


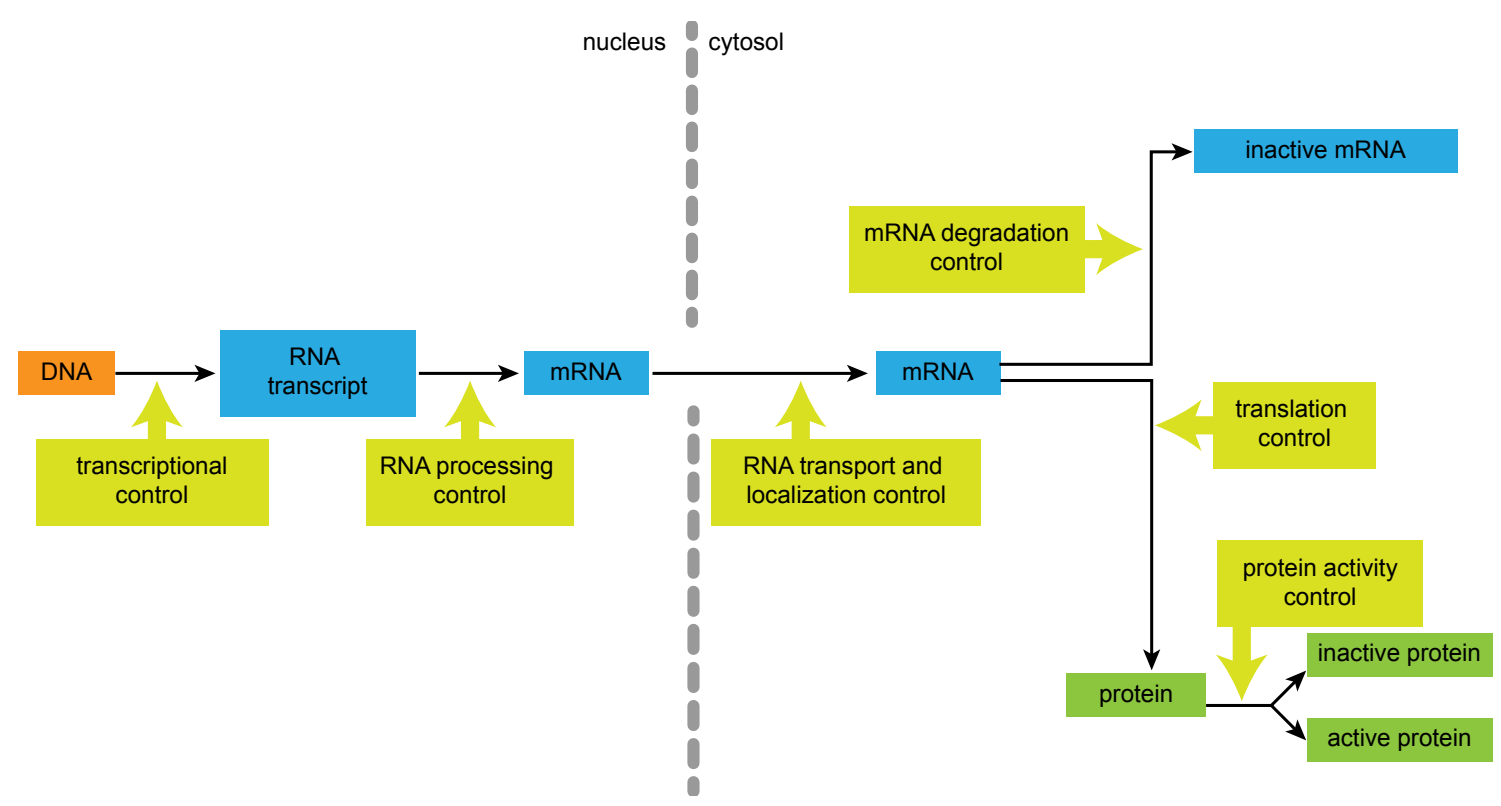

Figure 2.7: Cellular mechanisms for regulating gene expression in eukaryotes. In eukaryotes, gene regulatory mechanisms can be grouped into six stages. The first and major mechanism controls transcription of DNA directly (see main text). For further details on the other gene regulation mechanisms consult e.g. [174]. Figure based on [174].

the regulatory region of a gene that influences RNA-polymerase transcription and thus protein expression. In humans, $10 \%$ transcription factor encoding genes of about 21000 protein-coding genes indicates gene regulation as a key driving force in evolution [174, 178]. In fact, gene regulation is thought to be one of the main evolutionary driving forces for phenotypic variation between eukaryotic species rather than the protein code [179].

Transcription factors are grouped by their structural motifs into families and are called activator or repressor depending on their transcriptional control [174, 178]. In prokaryotes, the ability of RNA-polymerase to initiate transcription is nonrestrictive, in contrast to eukaryotes where DNA is packed into chromatin templates restricting the access of RNA-polymerase to DNA [180]. Transcriptional regulation is typically controlled by a few transcription factors in prokaryotes, whereas in multicellular eukaryotes the regulation is commonly combinatorial and requires the coordinated interaction of multiple transcription factors [178]. Regulatory regions are correspondingly comprised by only a few binding sites in prokaryotes in contrast to multicellular eukaryotes, where 10-50 binding sites in the regulatory regions, called cis-regulatory elements, for 5-15 different transcription factors is not unusual [181]. Transcription factor binding affinities are weak in eukaryotes, but increase by transcription factor dimerization, oligomerization and agglomeration through cooperative binding. Multiple transcription factors usually assemble in groups at their cis-regulatory-elements together with proteins called coactivators and co-repressors. Through the combinatorial regulation of the co-activators and co-repressors, a single transcription factor can be involved in both activation and repression.

Mutual gene regulation of transcription factors paves the way for patterning biological tissue both in space and time. In bacteria, gene regulation can be precisely organized in temporal cascades, for instance, during cell cycles [182] or the assembly of flagella in E. coli [183]. Regulation in combination with diffusion can generate spatial patterns during morphogenesis that is known as Turing mechanism [184]. The mechanism relies on transcription factors called morphogens with a rapidly diffusing repressor and a slowly diffusing activator. However, the required diffusion coefficients for such a mechanism appeared biologically implausible [185-187], but see [188]. 
Although the Turing mechanism might be implausible in many biological systems, a Turing type mechanism is suspected to shape, for instance, skin patterning [186, 189-193]. Candidate morphogens such as Bicoid, Decapentaplegic and Activin were later discovered in the fruit fly Drosophila melanogaster [194] and the frog Xenopus laevis [195]. Blastoderm patterning of Drosophila melanogaster during embryogenesis is probably the most famous example for body segmentation caused by genes [196]. A suspected universal design principle for body segmentation is the segmentation clock that temporally oscillates and thus segments growing tissue [197, 198]. The most famous temporal oscillation is probably the circadian clock [199] that coordinates the day-night cycle. A spectacular effect of this rhythm is discovered in spiders, where light sensitivity during night is increased by a factor of 1000 [200]. Remarkably, the circadian clock is very robust as in the cyanobacterium Synechococcus elongatus it appears to function without transcriptional and protein degradation feedback for at least several days [201].

In the neocortex, many developmental aspects are thought to be mainly shaped by genes. Neocortical parcellation into Brodmann areas, the "organs of the brain", but also the organization into six laminar layers and the stereotypical wiring between laminar layers are thought to be mostly genetically specified $[2,3]$. Similarly, sensory projections that contribute to define Brodmann areas by innervation are guided by genes [202,203]. Long-range patchy connections, a large-scale circuit motif within the neocortex, that in the primary visual cortex is tightly linked to the functional architecture, is suspected to be shaped by genes [18]. Axon guidance by chemical cues was already early proposed for topographic mapping in the developing nervous system [204] and later supported by various experiments [205-211], although the role of spontaneous activity is still a matter of debate, see [83]. While contact-dependent exchange over several cell bodies expands the repertoire of diffusive and systemic signaling, the spatial complexity and specificity of the neuronal connectome and its contribution to cell fate is unique in the animal body [212]. This complexity in principle additionally allows for a genetic encoding of neural response properties. The arguably most spectacular example is homeobox protein Otx2 that is transported from the retina to V1 and involved in regulating the critical period of high cortical plasticity [26]. Only recently, a molecular correlate for eye-specific neuronal response patterns in the primary visual cortex was discovered [25]. These findings indicate that genetic mechanisms can be an important ingredient for structuring complex cortical architectures.

\subsection{Modeling gene regulation}

Gene regulation is typically modeled as transcriptional regulation on different levels of details depending on the scientific question. The major model classes are logical, continuous and single-molecule models that increase in the level of modeled details [213-217]. In multicellular eukaryotic organisms, transcription factors are expressed at high concentrations of about 10000 300000 molecules/cell, which suggests to neglect stochastic molecule fluctuations and to use continuous models [218].

A paradigm continuous model of genetic control is a thermodynamic model with pair-wise interactions [219]. Thermodynamic models assume thermodynamic equilibrium during binding of transcription factors and RNA polymerase onto DNA. The rate of expression is then taken to be proportional to the promoter occupancy of RNA polymerase in the thermodynamic equilibrium

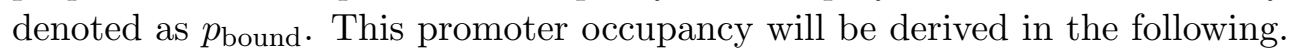

Consider a single gene with a promoter for RNA polymerase and $n$ binding regions for transcription factors. Each binding energy of the molecules is composed of a specific energy $E_{i}$ and a contribution from the chemical potential $\mu_{i}=\mu_{i}^{0}+\ln \left(c_{i} / \tilde{c}_{i}\right) / \beta$, where $\mu_{i}^{0}$ is a concentrationindependent contribution to the potential, $\beta=k_{B} T$ and $c_{i} / \tilde{c}_{i}$ the ratio of the concentration of a substance $c_{i}$ to a reference concentration $\tilde{c}_{i}$. The total energy of a molecule required to bind 
on the region is therefore without any interaction between molecules given by

$$
E_{i}^{\text {tot }}=E_{i}+\mu_{i} .
$$

Including pair-wise interactions between the bounded substances $i$ and $j$ extends the energy by $E_{i j}$. Introducing the promoter occupancy vector $\boldsymbol{\sigma}=\left(\sigma_{0}, \sigma_{1}, \ldots, \sigma_{n}\right)$ for which each entry can assume the value 0 for unoccupied or 1 for occupied, the total energy for a specific configuration of occupancy can be written as

$$
\mathcal{H}(\boldsymbol{\sigma})=\sum_{i=0}^{n} \sigma_{i} E_{i}^{\mathrm{tot}}+\sum_{i<j} \sigma_{i} \sigma_{j} E_{i j} .
$$

The probability that RNA polymerase is bound to its promoter is given by the probability of all possible occupancy configurations, where RNA polymerase occupies its promoter $\sigma_{0}=1$. The RNA polymerase binding probability is then given by

$$
p_{\text {bound }}=\frac{Z_{\mathrm{ON}}}{Z_{\mathrm{OFF}}+Z_{\mathrm{ON}}}=\frac{Z_{\mathrm{ON}}}{Z}
$$

with the partition function $Z_{\mathrm{ON}}$ of bound RNA polymerase and $Z_{\mathrm{OFF}}$ of unbound RNA polymerase

$$
Z_{\mathrm{ON}}=\sum_{\left\{\boldsymbol{\sigma} \mid \sigma_{0}=1\right\}} e^{-\beta \mathcal{H}(\boldsymbol{\sigma})}, \quad \quad Z_{\mathrm{OFF}}=\sum_{\left\{\boldsymbol{\sigma} \mid \sigma_{0}=0\right\}} e^{-\beta \mathcal{H}(\boldsymbol{\sigma})}
$$

Notice that this model class of transcriptional regulation is equivalent to a Boltzmann machine [220]. In the simplest case of a single RNA polymerase binding to a promoter without transcription factors, i.e. $n=0$, the probability of RNA polymerase bound to the promoter is given by

$$
p_{\text {bound }}=\frac{e^{-\beta\left(E-\mu_{P}\right)}}{1+e^{-\beta\left(E-\mu_{P}\right)}}=\frac{c_{P} / K}{1+c_{P} / K}
$$

where $E$ defines the binding energy of RNA polymerase to the promoter, $\mu_{P}=\mu_{0}+\ln \left(c_{P} / \tilde{c}_{P}\right) / \beta$ is the chemical potential, $c_{P}$ the concentration of RNA polymerase and $K=\tilde{c}_{P} e^{\beta\left(E-\mu_{0}\right)}$ the effective equilibrium dissociation constant of RNA polymerase. This function is the well-known Hill function that was already derived for oxygen binding on hemoglobin [221]. The key conceptual outcome of the consideration of more complex interacting transcription factors and RNA polymerase is that the simple case of RNA polymerase binding is altered only by a single, so called, regulation factor $F_{\text {reg }}$

$$
p_{\text {bound }}=\frac{F_{\text {reg }} c_{P} / K}{1+F_{\text {reg }} c_{P} / K} .
$$

An increase of the regulation factor $F_{\text {reg }}>1$ leads to an effective increase in RNA polymerase molecules and thus to an increase in gene expression and vice versa. In this conceptual framework various cases can be examined based on the regulation factor. A simple activator $A$ results in the regulation factor

$$
F_{\text {reg }}=\frac{1+f c_{A} / K_{A}}{1+c_{A} / K_{A}}
$$

where the factor $f=e^{-\beta E_{A P}}$ gives a measure of the interaction between RNA polymerase and transcription factor by their interaction energy $E_{A P}$. A simple repressor $R$ gives

$$
F_{\text {reg }}=\frac{1}{1+c_{R} / K_{R}} .
$$


The combinatorial control of $N$ independently acting activators or repressors gives a regulation factor of

$$
F_{\mathrm{reg}}=\prod_{i=1}^{N} F_{\mathrm{reg}}^{i},
$$

where $F_{\text {reg }}^{i}$ denotes the regulation factor of the $i$ th activator/repressor. The combinatorial regulation effects are not limited to these cases and it can be shown that all logical operation can be implemented within this scheme [220] as also experimentally observed in, e.g. sea urchin [222].

Notice that this model is equivalent to the Monod-Wyman-Changeaux (MWC) model [223$225]$ in the case where transcription factors only interact with RNA polymerase. In the limit of high transcription factor binding affinity and strong interactions between $n$ transcription factors and RNA polymerase the binding probability of RNA polymerase becomes

$$
p_{\text {bound }}==\frac{c_{P} / K\left(c_{A} / K_{A}\right)^{n}}{1+c_{P} / K\left(c_{A} / K_{A}\right)^{n}},
$$

which is again a Hill function.

Typical protein-protein interaction energies are in the range $N_{A} E_{i j} \approx 1-4 \mathrm{kcal} / \mathrm{mol}$ [220], which gives a pair-wise interaction of $f=e^{-\beta E_{i j}} \approx 5-786$. The effective dissociation constant of transcription factors to bind on DNA is in the range of $K_{i} \approx 1-10000 \mathrm{nM}$ for bacteria [220]. As the number of regulating transcription factors is very high in eukaryotes, the regulatory control on protein expression can be fairly complex.

\subsection{Overview of this thesis}

This thesis is concerned with the development and precise quantification of functional visual cortical architecture. The work is divided into four chapters: Chapter 3 Genetic assimilation of visual cortical architecture, Chapter 4 Pinwheel configuration in maximum entropy models of orientation domains, Chapter 5 The pinwheel configuration: theoretical significance and precision measurement and Chapter 6 Three-dimensional organization of orientation selectivity in cortical tissue. The motivation of these projects will be briefly summarized by contextualizing the afore-reviewed fundamentals.

\section{Genetic assimilation of visual cortical architecture}

Despite a vast collection of experimental findings, the underlying biological mechanisms for visual cortical architecture development remain unclear. In primates and placental carnivores, functional architectures adhere to a common design that likely evolved independently in the course of evolution. Convergent evolution might indicate a functional advantageous trait that can be genetically assimilated. This so called Baldwin effect makes it conceivable that visual cortical architecture is at least partially encoded genetically. In fact, experience driven neuronal activity is not necessary for the emergence of the common design [14, 22], but can impair visual cortical architecture [100]. Thus, the initially raised question of nature versus nurture refines to how genes initiate the emergence of functional visual cortical architecture, i.e. by endogenous, spontaneous neuronal activity or by direct genetic mechanisms. Although neuronal activity is critical for its development, it is unclear whether it has an instructive or merely a permissive role for the functional architecture [21, 226]. Recent findings indicate that neuronal activity is an important, but not the only mechanism involved in shaping the visual cortical architecture, e.g. a molecular correlate for ocular dominance bands [25] and the homeoprotein Otx2 that 
is actively transported from the retina to the primary visual cortex and involved in regulating the critical period, a period of high cortical plasticity [26]. From a theoretical point of view, a genetic mechanism was excluded due to an apparent limited information capacity of the genome for hardwiring the cortex [27]. However, utilizing findings of the long-range interaction model, we show in Chapter 3 that this information bottleneck can be circumvented by a transcellular genetic network.

\section{Pinwheel configuration in maximum entropy models of orientation domains}

The pinwheel configuration of primates and placental carnivores follows species-invariant quantitative laws [22-24]. A theoretical understanding of these invariant statistics is crucial for a deeper understanding of the design principles of functional visual cortical architecture. However, the theoretical link between pinwheel configuration statistics and orientation domain ensembles is so far only studied for the pinwheel density [135, 147, 170, 171]. In Chapter 4, we extend the theoretical analysis of orientation domain ensembles to an analytical treatment of all pinwheel configuration statistics of the common design.

\section{The pinwheel configuration: theoretical significance and precision measurement}

The common design of functional visual cortical architecture in primates and placental carnivores shows the experimental significance of the pinwheel configuration. In the experimental analysis of pinwheel configurations, measurement noise was reduced by semi-local wavelet filtering. This filtering method leaves noise components similar to the ground truth signal unchanged. In order to establish a high precision analysis of functional visual cortical architectures and a model benchmarking framework, it is inevitable to examine the effect of measurement noise on the pinwheel configuration. In Chapter 5, we first review the theoretical significance of the pinwheel configuration and subsequently examine their configurations under measurement noise by utilizing non-centered Gaussian random fields.

\section{Three-dimensional organization of orientation selectivity in cortical tissue}

Columnar structure of neocortical tissue is a mammalian organization principle found in many species and different brain areas. In the primary visual cortex (V1) of primates and carnivores, the functional architecture is composed of orientation selective neurons that are horizontally organized into orientation domains. In cats, orientation domains are present in all laminar layers, while the preferred orientation selectivity is the same along association fibers. In contrast to cats, a substantially varying columnar orientation selectivity profile was observed in macaques. In rodents, functional architecture of $\mathrm{V} 1$ is also composed of orientation selective neurons but they lack any spatial organization principle. These different organization principles of orientation selectivity in V1 are most remarkable in the light of evolution, since they originated from one single species, the last common ancestor, more than 65 millions years ago. To shed light on possible evolutionary paths of V1's functional architecture, the full three-dimensional organization ought to be considered. In Chapter 6, we examine maximum entropy ensembles of three-dimensional functional visual cortical architectures in different geometries. 



\title{
Genetic assimilation of visual cortical architecture
}

\author{
"Nothing in biology makes sense except in the light \\ of evolution" \\ - C. T. Dobzhansky, Evolution versus Creationism
}

\section{Original contribution}

J. Liedtke, W. Keil and F. Wolf conceived and designed the study. J. Liedtke performed the theoretical analysis and numerical simulations. M. Schottdorf provided analyzed experimental data. J. Liedtke wrote the main manuscript and supplementary material. 


\subsection{Abstract}

Although genetic information is critically important for brain development and structure, it is widely believed that neocortical functional architecture is largely shaped by activity-dependent mechanisms. The information capacity of the genome appears too small to contain a blueprint for hardwiring the cortex. We found theoretically that genetic mechanisms can circumvent this apparent information bottleneck. Using our prior research on universality classes of circuit self-organization in the visual cortex, we devised mathematical models of genetic networks of neurons interacting by long-range axonal morphogen transport. Neurons dynamically generate morphogen patterns that prescribe the layout of orientation domains as experimentally observed in the primary visual cortex (V1) of primates and placental carnivores. We find that active transport and trans-neuronal signaling as well as joined dynamics of morphogens and the connectome are crucial for orientation domain development. Furthermore, we scan for specific genetic networks that produce orientation domains, which are the best compatible with experimental data. Our theory, for the first time, shows how a complex cortical processing architecture can be specified using a genetic mechanism of small bandwidth and opens a novel perspective on the experimentally observed robustness of V1's architecture against radically abnormal developmental conditions.

\subsection{Introduction}

The evolution of the neocortex, the seat of our conscious experience, cognition, memory and executive control capabilities constitutes a critical innovation in mammalian history. Its parcellation into Brodmann areas, the "organs of the brain", but also the organization into six laminar layers and the stereotypical wiring between and across regions are thought to be mostly genetically specified $[2,3]$. Similarly, sensory projections that contribute to define Brodmann areas by innervation $[202,203]$ as well as the presence of long-range patchy connections [18], a large-scale circuit motif within the neocortex, are guided by genes.

In the primary visual cortex of primates and placental carnivores, Brodmann area 17, these long-range patchy connections are tightly linked to a specific spatial arrangement of feature selective neurons [12, 227-229]. This functional architecture fulfills several species invariant quantitative layout rules that likely evolved independently in several branches of the mammalian tree over the past 65 million years indicating a functional advantage of this trait [22-24]. Whether this trait is acquired during development or genetically specified is a long-standing debate. While the refinement of circuits and development of the functional architecture critically depends on neural activity [90, 93, 104], several reports discovered a normal initial development of feature selective neurons without visual input $[14,66]$, stereotypical visual responses in abnormal visual environments [100], a molecular correlate for eye-specific neuronal response patterns [25] and a tight coupling of molecular and neuronal dynamics [230-233]. Therefore, the early development of visual cortical architecture is hard to reconcile with a single, activity-dependent cause. Additionally, acquired functionally advantageous traits can become genetically encoded, as if they "sunk into the hereditary substance", a mechanism known as the Baldwin effect, and it is therefore conceivable that this functional architecture is at least in parts encoded genetically. A genetic cause was typically excluded as the limited information capacity of the genome appeared too small to contain a blueprint for hardwiring the cortex [27]. However, here we show how this information bottleneck can be overcome by an area-spanning genetic network.

Cell types, their morphology and position in the central nervous system are mostly specified genetically both by intrinsic factors and by molecular exchange of neurotransmitters and signaling cues across synapses. While contact-dependent exchange over several cell bodies is observed 
A

Gradient read-out cell fates

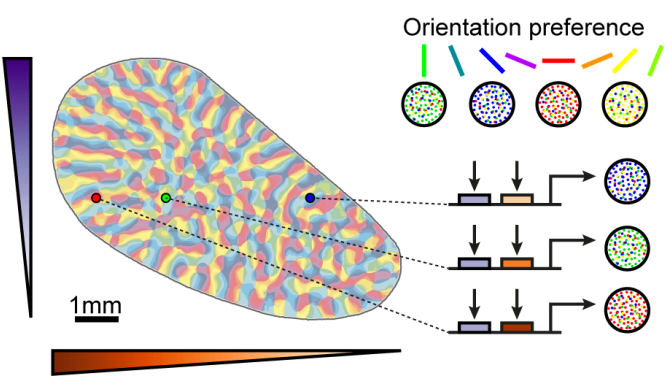

B Self-organizing cell fates

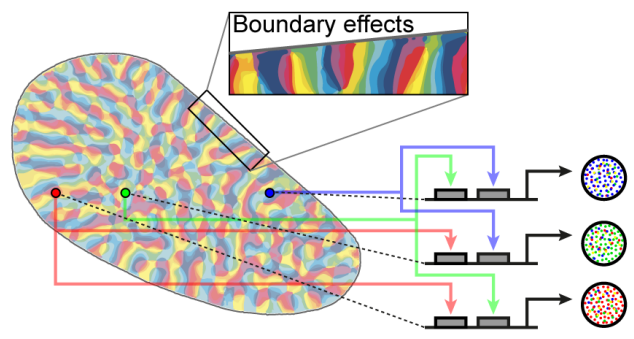

Figure 3.1: The spatial organization of feature selectivity can be determined by gradient read-out or self-organizing genetic networks. A Orientation preference, a key aspect of neuronal feature selectivity, is determined by the read-out of gradient concentrations. Thus, gradients determine cortical positions that control expression of orientation preference encoding morphogens. The number of morphogens and their concentration is indicated by colored dots. B Orientation preference of cells is determined via a self-organizing genetic network with interacting genes. Cells co-regulate their orientation preference encoding morphogen expressions and form patterns via a Turing type instability. A typical feature of pattern forming systems are boundary effects as experimentally observed in orientation preference layouts [12]. Orientation domains adapted with permission from [12].

in other instances, like wing disc and the air sac primordium of Drosophila melanogaster [234] expanding the repertoire of diffusive and systemic signaling observed in blastoderm patterning [196], the spatial complexity and specificity of the neuronal connectome and its contribution to cell fate is unique in the animal body. This complexity in principle additionally allows for a genetic encoding of neural response properties. The arguably most spectacular example is homeobox protein Otx2 that is transported from the retina to the primary visual cortex and involved in the regulation of the critical period, a period of high neural plasticity [26]. Diffusive gradients and the exchange of signaling molecules over an elaborated connectome might therefore be key ingredients to genetically determine functional visual cortical architecture.

In a first scenario, neurons can determine their feature preference by extracellular gradient read-out, Fig. 3.1A, similar to blastoderm patterning in developing Drosophila melanogaster. While viable in small systems $[235,236]$ gradient read-out has a number of drawbacks (see methods): (i) Heterogeneous feature layouts require typically a high number of genes, (ii) Cell fate determination is highly susceptible to gradient fluctuations, (iii) Experimentally observed boundary effects [12] together with a conserved typical scale, the distance between neurons with similar feature preferences, during ontogenesis [98] is difficult to reconcile in this model class. Alternatively, the layout of feature selective neurons can be determined by a self-organizing genetic network with inter-cellular interactions, Fig. 3.1B. Key intrinsic characteristics of selforganizing pattern forming systems are boundary effects and a typical scale invariant to cortical area growth. Thus, the second scenario of self-organizing genetic networks appears to be more compatible with the experimental findings, but adds a level of mathematical complexity.

Recent progress in quantitative characterization of functional visual cortical architecture allows us to benchmark models with high precision [22-24]. Here we show that an area-spanning transcellular genetic network is capable of specifying key ingredients of this architecture: the spatial organization of the selective neuronal response to contour orientations and its recurrent connectome. We construct a self-organizing genetic network class with a dynamic connectome that can quantitatively encode orientation preference layouts in the concentration of morphogens. This genetic network class requires contact dependent active transport and transneuronal signaling as well as joined dynamics of connectome and morphogens. It circumvents the information bottleneck by self-organization and makes specific predictions for experimental 
A

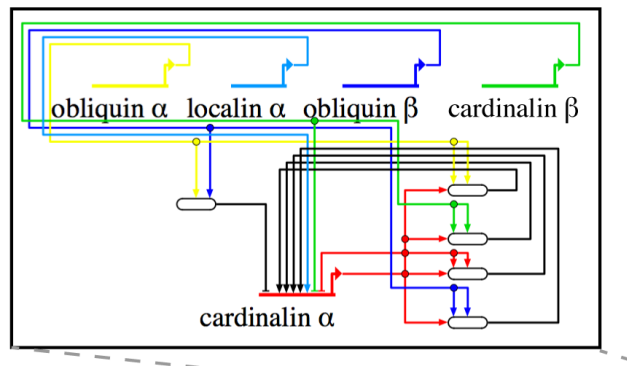

B

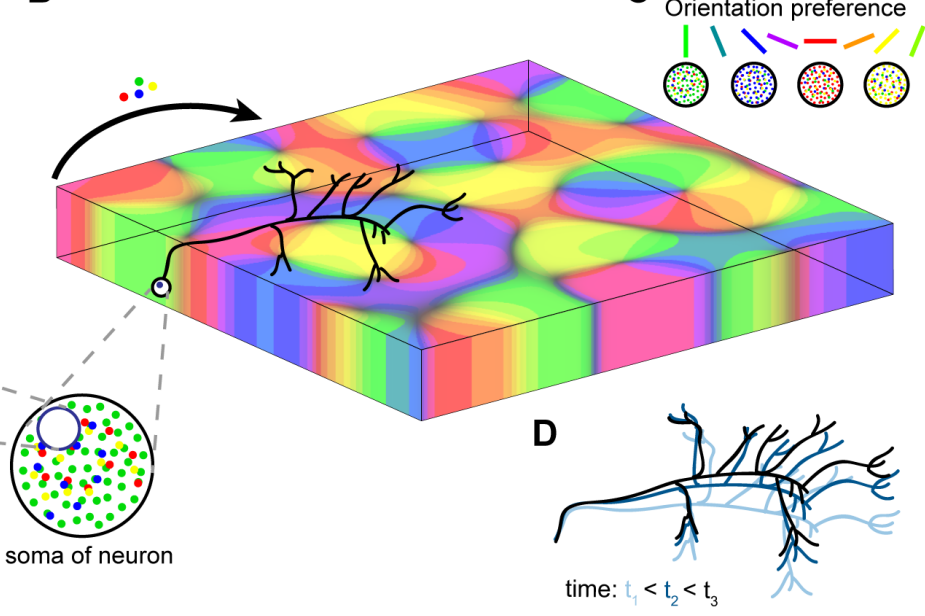

Figure 3.2: Genetic network class encoding visual cortical architecture. A Example genetic subnetwork illustrating how morphogens mutually regulate their expression rates in nuclei of neurons. Interneuronal translocation of morphogens induces a signaling cascade, which exerts regulatory control on morphogen expression. B Neurons actively transport morphogens and translocate them over dynamic axons. $\mathbf{C}$ The number of morphogens in a neuron is illustrated by the colored dots. The concentration difference of two morphogen pairs encode the selectivity strength to cardinal and oblique orientations, respectively. D Dynamic axons preferentially link neurons with similar morphogen complements. See main text and section 3.5.1 for details.

tests to identify these genetic networks and to distinguish between different genetic determination types.

\section{$3.3 \quad$ Results}

\section{Genetic network class encoding visual cortical architecture}

How can the neuronal molecular inventory encode visual cortical architecture of V1? We propose a minimal model comprising four orientation preference encoding morphogens that are transcription factors, see methods for details. The concentration difference of two morphogen pairs encode the selectivity for cardinal and oblique orientations, Fig. 3.2C, respectively. On a neuronal level, morphogens mutually regulate their gene expression according to a genetic network, Fig. 3.2A. The repertoire of gene regulatory control can encompass activation, repression and combinatorial control that is expanded to an effective regulation. In adults, neurons are interconnected over short-range isotropic and long-range patchy connections extending several millimeters [12]. Morphogens utilize these connections to translocate either directly between different neurons or by exchanging signaling cues, Fig. 3.2B. As both mechanisms are mathematically identical in a biologically plausible regime, we use morphogen transport as the representing mechanism in the following. In the course of cortical development, the presence of molecular cues can initiate molecular processes in axons, for instance, mRNA localized in axons gets locally translated in response to molecular cues [237, 238]. This mechanism is incorporated by a signaling cascade generating proteins upon interneuronal morphogen transport. These proteins can exert an effective negative transport feedback on the morphogen concentration by their gene regulatory control. Long-range patchy connections are dynamic and elaborate in the course of ontogenesis [82]. Therefore, the connectome consisting of short- and long-range connections is dynamic in our model. Short-range connections dynamically contact their surrounding isotropically, while long-range patchy connections target neurons with similar morphogen complements, Fig. 3.2D. 

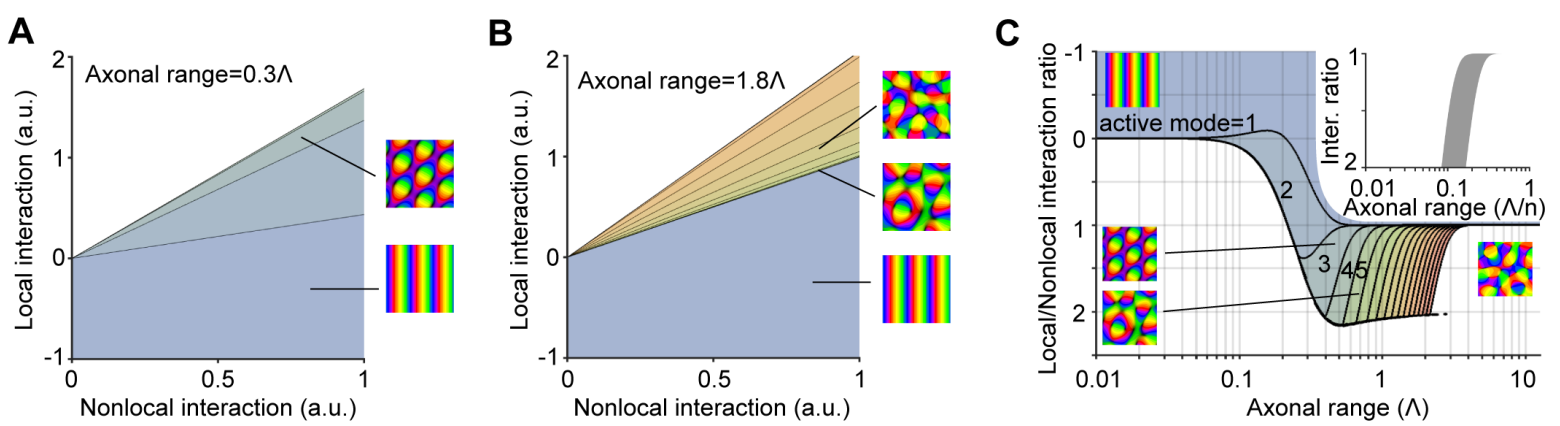

Figure 3.3: Axonal range of patchy connections organize closed-form solution selection in symmetry-confined canonical genetic network. A For short-range patchy connections the parameter space spanned by a gene regulatory related local effective parameter and a morphogen transport related nonlocal effective parameter parcels into regions of repetitive Turing patterns with a typical scale $\Lambda$. B Long-range patchy connections expand the repertoire to spatially complex quasiperiodic orientation preference layouts. C Orientation domains are entirely determined by the ratio of the local and nonlocal parameter. Phase diagram indicates ground states of orientation domain closed-form solutions. Inset: The asymptotic stability region of closed-form solutions with many active modes $n$. See main text and method section 3.5.3 for details. Parameters, see Eq. (3.120): Gaussian kernel with axonal range $\sigma$ and local and nonlocal interactions $\gamma_{6}$ and $\gamma_{1}$, respectively.

Thus, transport and exchange of morphogens through long-range connections is combined with long-range connections targeting cells with similar morphogen concentration profiles. The connectome and visual cortical architecture is known to be modulated by neuronal activity [17, 100], but survives in the absence of visual experience under dark rearing [14, 22]. Activity dependence of axonal morphogenesis [14] and axonal trafficking [26] might thus be a component to couple activity dependent mechanisms to circuit wide morphogen dynamics.

\section{Long-range transport stabilizes realistic orientation domains}

The complexity of the genetic network class can be reduced by biologically plausible assumptions resulting in a model that we term canonical genetic network model. First, we simplify the system by rapid rearrangements of the connectome in comparison to morphogen concentration dynamics. Thus, the model state is fully determined by the total concentration and concentration difference of morphogen pairs. The concentration difference determines the layout of orientation preference. Second, weak anisotropy of patchy connections and orientation encoding dynamics that are independent of total concentrations result in the analytically tractable canonical genetic network. The canonical genetic network still depends on several parameters that determine for instance regulatory control, transport ranges and transport rates. Varying these parameters alters steady-state morphogen concentrations and encoded orientation domains. Symmetry principles reduce the number of parameters drastically. We demand symmetries that guarantee the presence of all orientation preferences and an equal occurrence of orientation domains, where cardinal and oblique encoding morphogens are interchanged. The dynamics of orientation preference encoding morphogen concentration difference is then mathematically equivalent to a universality class of pattern forming systems [128]. The remaining parameters determine the total morphogen concentrations and the emergence of a non-uniform morphogen pattern with typical scale $\Lambda$. Near the transition to a non-uniform morphogen pattern we obtain closed-form orientation domain solutions. The closed-form solutions are composed of active modes (Fourier components) and are highly multistable. The closed-form solutions parcel the parameter space spanned by a gene regulatory related local and a morphogen transport related nonlocal parameter into regions of ground states. Varying the range of patchy connections to short ranges results 


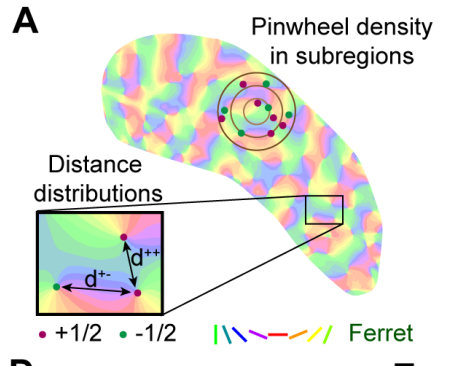

D

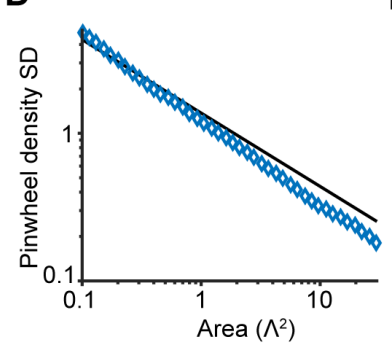

B

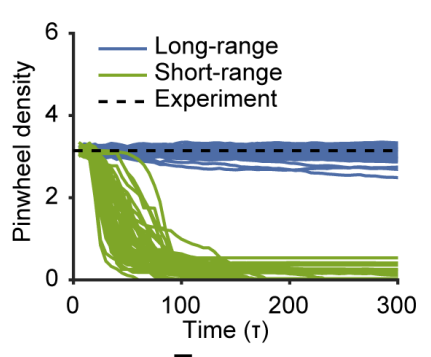

$\mathbf{F}$
E

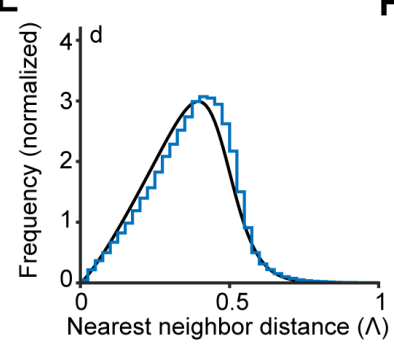

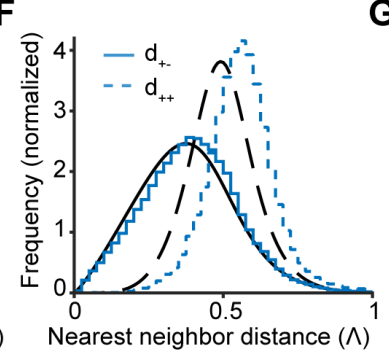

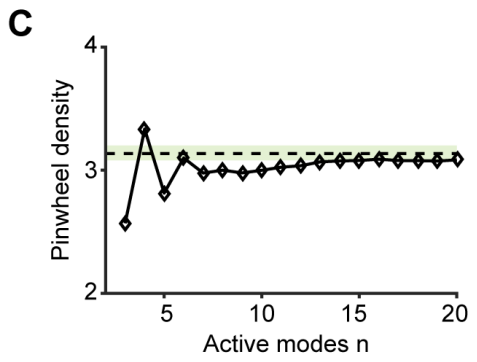

$\mathbf{G}$

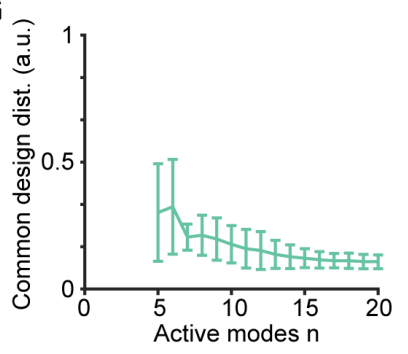

Figure 3.4: Orientation domains of symmetry-confined canonical genetic network model adhere to the common design. A The common design is defined by statistics of the pinwheel configuration of orientation domains. Pinwheels are points surrounded by all preferred orientations and have a positive or negative topological charge (green and red dots) depending on the arrangement of the surrounding orientation preference. Species invariant pinwheel configuration statistics are pinwheel density, pinwheel density standard deviation (SD) scaling with area and nearest neighbor distribution of pinwheels. B Temporal evolution of pinwheel densities for short- and long-range axons. For long-range axons, pinwheel densities cluster around the experimentally observed density (dashed line), whereas for short-range axons pinwheel-free solutions emerge. See method section 3.5.8 for numerical details. C Average pinwheel density of closed-form solutions from symmetry-confined canonical genetic network model. For a large number of active modes $n$ the pinwheel density converges to $\pi$ (dashed line: experimental mean, green area: confidence interval from ferret). D, E and $\mathbf{F}$ Pinwheel density SD and nearest neighbor distributions of solution set with $n=9$ active modes (blue diamonds and lines) together with experimentally fitted curves (black lines) from [22]. G Pinwheel configuration statistics of model solutions are combined to a single distance measure to the configuration of ferrets, see section 3.5.7 for details.

in a segmentation of ground states with a typical repetitive Turing patterns and a low number of active modes, Fig. 3.3A. Long-range patchy connections expand the repertoire to spatially complex quasiperiodic orientation domains with large numbers of active modes, Fig. 3.3B. In fact, the ground states of the closed-form solutions are organized by the range of patchy connections and the ratio of the local and nonlocal parameter, Fig. 3.3D. Thus long-range patchy connections are essential for quasiperiodic orientation preference layouts that resemble experimentally observed orientation domains.

\section{Canonical genetic network adheres to common design}

Orientation domains of the symmetry-confined canonical genetic network resemble experimentally observed orientation domains of V1 not only qualitatively, but also quantitatively. In primates and placental carnivores, the spatial organization of points surrounded by all orientations called pinwheels follow species-invariant quantitative statistics [22-24]. This common design consists of the pinwheel density, the pinwheel density fluctuation in cortical subareas and nearest neighbor distributions between pinwheel pairs of equal, opposite and independent of topological charge, Fig. 3.4A. The temporal evolution of pinwheel densities in the symmetryconfined canonical model reveals for short-range patchy connections annihilation of pinwheel 
A

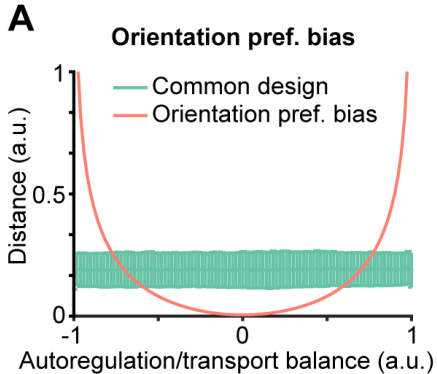

Autoregulation/transport balance (a.u.)

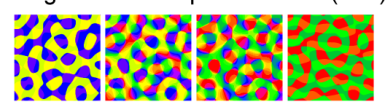

B Insensitive kinetic parameters
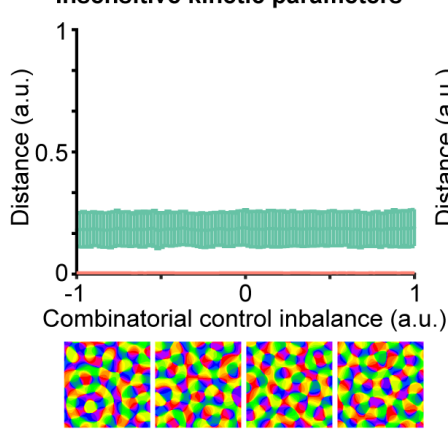

C Sensitive kinetic parameters D

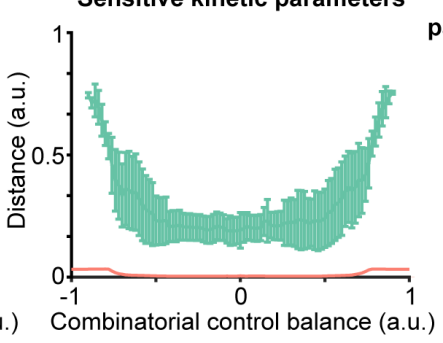

Common design parameter susceptibility

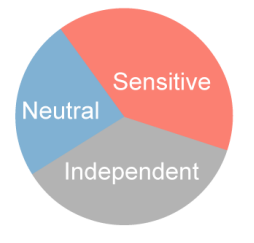

Figure 3.5: Orientation domain layout sensitivity on kinetic parameters. The distance to the common design pinwheel configuration from ferrets (mean and 95\% confidence interval of 1000 bootstrapped pinwheel configurations from 30 initial conditions) and the KL divergence between the orientation preference representation and a uniform distribution is computed for varying effective parameters originating around a working point of $n=9$ active modes, see section 3.5.8 for numerical details. A Neutral common design parameter direction that generates and orientation preference bias. B Neutral parameter direction that retains the pinwheel configuration and orientation preference representation. $\mathbf{C}$ Sensitive parameter direction that generates hexagonal biased orientation domains that are incompatible with the experimentally observed pinwheel configurations. D Overview of common design susceptibility to 25 free parameters. Neutral parameters (6) result in the same or similar pinwheel configurations (A and $\mathbf{B})$, sensitive parameters (10) exhibit a clear tendency to different pinwheel configurations $(\mathbf{C}$ and axonal range) and independent parameters (9) are not involved in orientation encoding, but control the total concentration of morphogens.

pairs and for long-range patchy connections a clustering around the experimentally observed value 3.14, Fig. 3.4B. In fact, the average pinwheel density of closed-form solutions converges from below to $\pi$ for large numbers of active modes [135] and is statistically indistinguishable from the experimental value $[22]$, Fig. 3.4C. The pinwheel density fluctuation in subareas obeys a power law with an exponent of approximately $1 / 2$ for experimentally observed pinwheel configurations and a slightly higher exponent for closed-form solutions with $n=9$ active modes, Fig. 3.4D. The pinwheel nearest neighbor distributions of pinwheel pairs independent of topological charge and of opposite topological charge are virtually identical for the closedform solutions with $n=9$ active modes and experimentally observed pinwheel configurations, Fig. 3.4E,F. However the nearest neighbor distribution of pinwheel pairs with equal topological charge of the closed-form solutions with $n=9$ active modes shares the same shape with the experimentally observed nearest neighbor distribution, but is shifted to larger pinwheel pair distances, Fig. 3.4F. The quantitative agreement of the model pinwheel configuration with the common design can be condensed to a single quantity that we term common design distance (see methods). The agreement of the closed-form solutions successively improves with increasing active modes, see Fig. 3.4G. In total, the closed-form solutions quantitatively adhere to the common design to a large extent already for a small number of active modes.

\section{Evolution of genetic networks}

How much genetic pressure is exerted on the molecular mechanisms? In the course of evolution proteins can acquire multiple and independent functional roles in different cell types a property called mosaic pleiotropism. Essential for the acquisition of novel functional traits is the evolution of the regulatory control element [179]. In the genetic network we can vary these regulatory control elements and additionally other molecular mechanisms. We utilize the common design 
distance as a proxy for inverse fitness to directly probe the evolutionary fitness landscape of the canonical genetic network. For that we investigate the parameter space of the canonical genetic network around the previously investigated working point of closed-form solutions with $n=9$ active modes. From the 25 free parameters, 9 parameters are independent from orientation domain specification and are involved in regulation of the total steady-state concentrations. Varying a parameter that can be associated with the balance between morphogen autoregulation and transport rate leads to orientation domains with invariant pinwheel configurations, but with an orientation bias, Fig. 3.5A. Such an orientation bias is experimentally observed in juvenile ferrets, but disappears during development [113]. Varying a parameter that is associated with the imbalance of a combinatorial control between morphogens is neutral and retains pinwheel configuration and orientation preference representation, Fig. 3.5B. However, there exist sensitive effective parameter directions that drastically departure from the common design and the orientation preference distribution, Fig. 3.5C. From an evolutionary perspective, there exist therefore neutral and even independent directions on the evolutionary fitness landscape that can mutate without functional disadvantage, but also sensitive directions where strong genetic pressure must be present for preserving functionality.

\section{Dynamic connectome and active transport is essential for visual cortical architecture}

A dynamic, co-evolving connectome and active transport is essential for realistic orientation domain layouts. In the previously analyzed symmetry-confined canonical genetic network, the connectome rapidly rearranges to the dynamic morphogen concentration profiles. This idealization allowed the derivation of closed-form orientation domain solutions. Reducing the rearrangement time of the connectome slightly retains the orientation field solutions, but destabilizes them for sufficiently slow dynamic connectomes. In the limiting case of a frozen long-range morphogen-dependent connectome that is isotropic the impact on orientation domain emergence is detrimental. In fact, this scenario is mathematically equivalent to diverging local/nonlocal interaction ratios in Fig. 3.3C. Thus the morphogen concentrations are either diverging or encode unrealistic pinwheel free orientation fields for positive and negative local/nonlocal interaction ratios, Fig. 3.6A, respectively. Freezing synapses to a long-range patchy static connectome yields frustrated orientation domains with a stripy organization, Fig. 3.6B. In the intermediate regime with finite connectome rearrangement time, orientation fields retain stability up to a critical connectome rearrangement time of about half of the regulation time scale, Fig. 3.6C. In this stable regime perturbations around the closed-form solutions lead to damped oscillations of morphogen concentrations and the connectome, Fig. 3.6D,E,F. For rapid connectomes, but fixed long-range transport delay closed-form solutions destabilize for transport delays larger than about 0.8 of the regulation time scale, Fig. 3.6G. Transport delays larger than the critical transport delay result in sudden orientation preference shifts, Fig. $\mathbf{3 . 6 H}$, and oscillations in morphogen concentrations and connectome, Fig. 3.6I,J. This shows the importance of joined dynamics of morphogens and the connectome as well as the requirement of sufficiently fast active transport for establishing realistic orientation domains.

\section{Perspectives on predictions for experimental tests}

Although many details of the genetic network model are variable, generic features exists and are suitable for experimental tests and predictions. Four morphogens for the genetic determination of visual cortical architecture is likely neither the largest nor lowest number of morphogens for this task. A probable lower bound are two morphogens, since a single orientation encoding morphogen concentration is necessarily discontinuous due to orientation preference singularities 
A

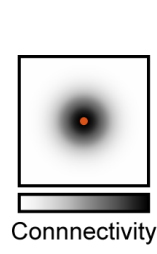

C Slow dynamic connectome

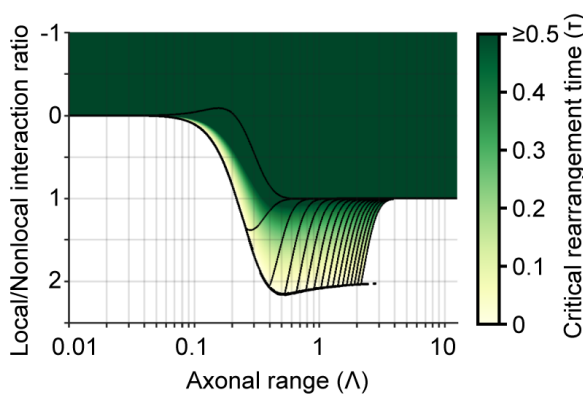

G

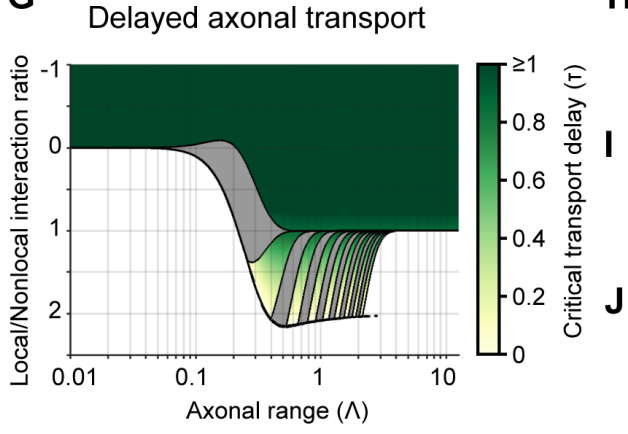

D

H
B
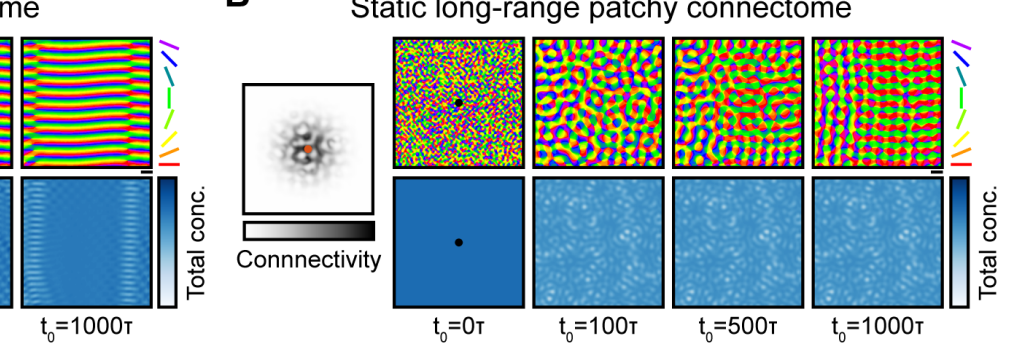
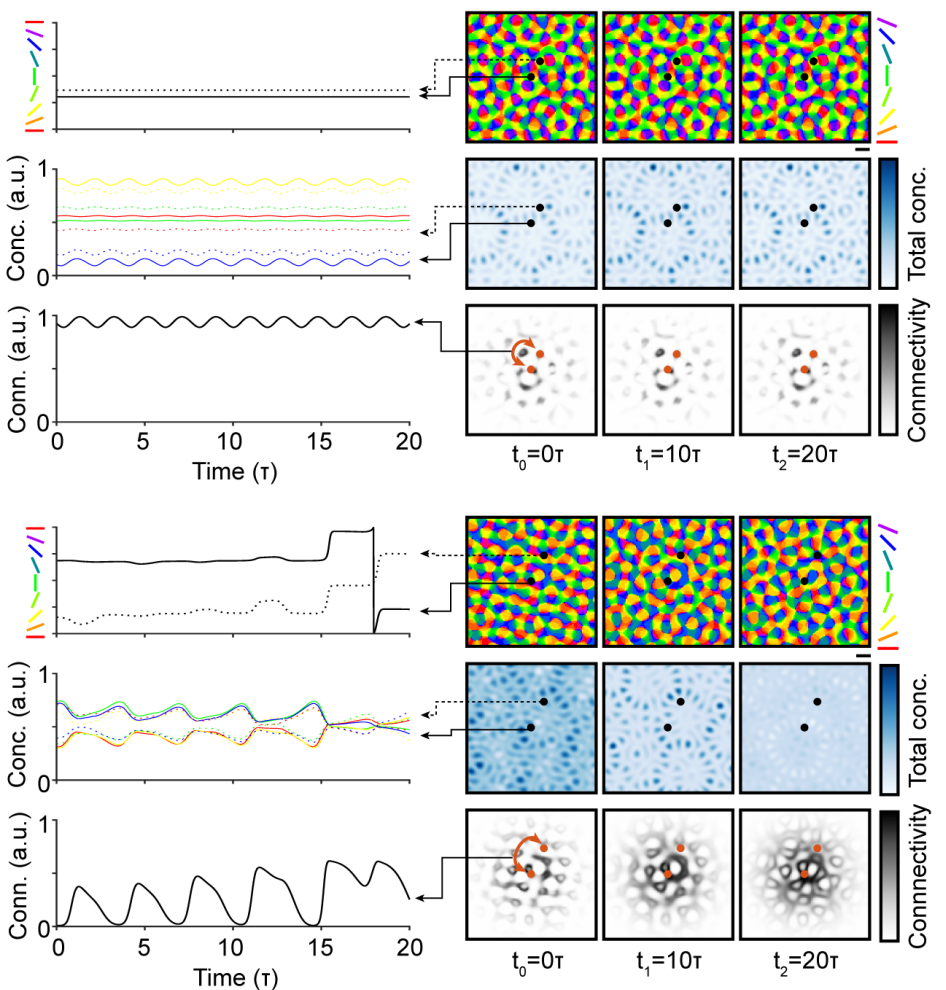

Figure 3.6: Rapid dynamic connectome and fast axonal transport is essential for stable visual cortical architecture. A Static isotropic long-range connectome leads to artificial orientation domains consisting of plane waves. B Static long-range patchy connectome generates orientation domains with stripy orientation preference layouts. C, G Critical rearrangement times and transport delays (color code) in symmetry-confined canonical genetic model solutions of minimal energy (solid black lines separate minimal energy solutions, see Fig. 3.3C, respectively. Exceeding critical times destabilizes the orientation domain solutions. Grey areas indicate regions that eluded a direct calculation of critical transport delays, but is numerically found to be similar to its surrounding. D-F, H-J Left: Orientation preference, morphogen concentration and connection dynamics for critical rearrangement time and slightly above critical transport delay of neurons indicated by dots on the right, respectively. Right: Orientation domains, total concentration and connectome of neuron in the center for $t_{0}=0 \tau, t_{1}=10 \tau, t_{2}=20 \tau$. Scale bar indicates the typical scale $\Lambda$ of the orientation domain. See section 3.5.8 for numerical details. 
A
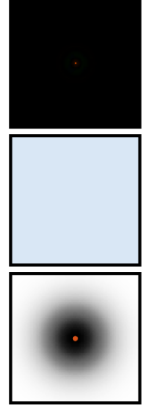

$t_{0}=0.1 \mathrm{~T}$
Focal overexpression
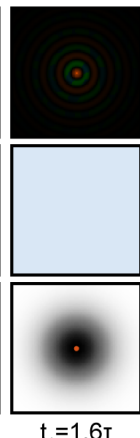

$t_{1}=1.6 \mathrm{~T}$
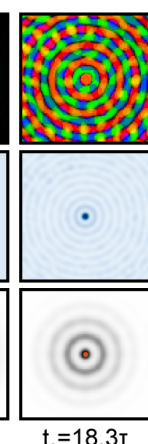

$t_{2}=18.3 T$
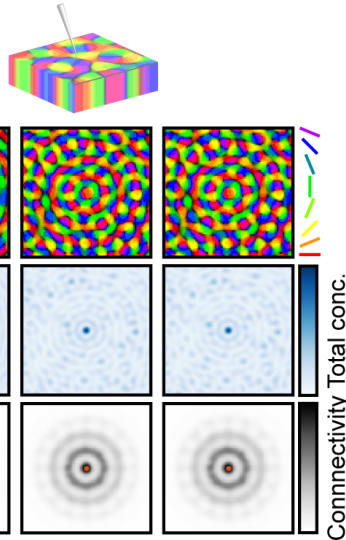

$\mathrm{t}_{4}=292 \mathrm{~T}$
B

Dual site overexpression
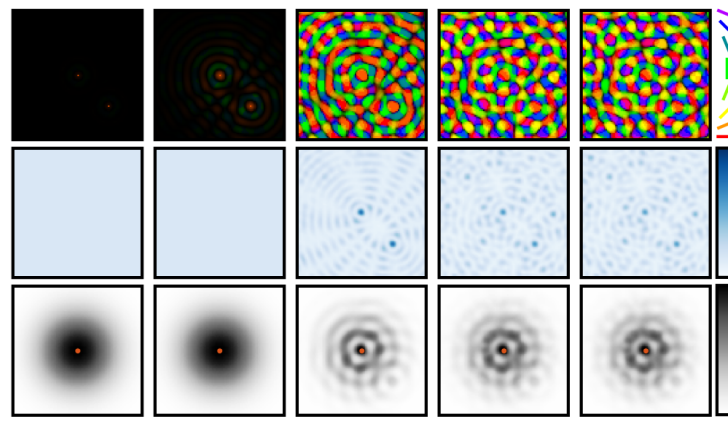

$\mathrm{t}_{0}=0.1 \mathrm{~T}$
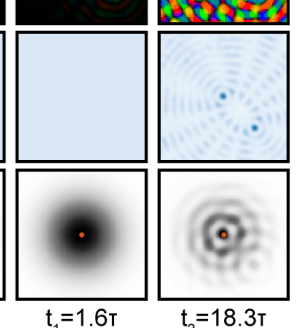

$t_{2}=18.3 T$

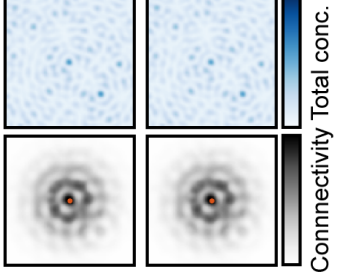

$t_{4}=292 T$

Figure 3.7: Focal and dual transfections leading to localized morphogens overexpression. A A focal overexpression of a single morphogen determines the emerging orientation preference at this spot due to the increased concentration of the morphogen. Reorganization of the surrounding to the increased central morphogen concentration leads to a transient concentric orientation preference pattern and connectome. Eventually, the system converges to quasiperiodic orientation domain layouts. See method section 3.5.8 for numerical details. B A dual overexpression of a single morphogen determines the emerging orientation preference at these spots due to the increased concentration of the morphogen. After a transient, the system converges to quasiperiodic orientation domain layouts. See section 3.5.8 for numerical details.

at pinwheels. An essential feature of this model is long-range active transport that suggests to experimentally dissect the signaling endosome proteome for putative candidate morphogens. Additionally, the coding scheme that concentrations determine feature neuronal response properties suggests to experimentally examine functionally targeted transcriptomes. Qualitatively, they key characteristic of this genetic network model is that cell fates are neither specified by a single source as bicoid gradient in Drosophila nor are they predetermined by their cortical positions. Instead, cell fates are dynamically self-organized and collectively react to perturbations. For instance, an early focal transfection of neurons leading to an overexpression of one morphogen leads to orientation domains that are organized around this spot, Fig. 3.7A. A dual site transfection resulting in an overexpression of a morphogen also reorganizes the orientation domains, while retaining the quasiperiodic intrinsic orientation domain layout, Fig. 3.7B. This result qualitatively distinguishes genetic cell fate predetermination by position from selforganizing genetic networks.

\subsection{Discussion}

In this article, we showed that a self-organizing genetic network can determine functional visual cortical architecture that is in quantitative agreement with experimental data. This shows for the first time how to circumvent the information bottleneck for genetically determining complex cortical processing architecture. We find that active long-range transport and trans-neuronal signaling as well as joined dynamics of connectome and morphogens is decisive for functional visual cortical architecture determination. Finally, we propose experiments for differentiation between possible genetic networks that specify functional visual cortical architecture.

What kind of genetic cell fate determination is proposed here? In our proposed genetic network, neuronal cell fates are not inherited in detail by for instance their cortical position as observed in blastoderm patterning in Drosophila melanogaster [196], but are dynamically generated by the collective molecular interactions in V1. This dynamical self-organization of cell 
fates circumvents the genetic information bottleneck of specifying every neuron and connection by a blueprint [27]. A surplus is that the salient boundary features of experimentally observed orientation domains [12] are generated naturally in these types of pattern forming models [28].

What is the relation to activity-dependent mechanisms? It is intriguing to speculate that such genetic networks might contribute to maintenance of memories. A key feature of such a mechanism is to preserve favorable functional architectures even under unfavorable developmental environments. This view of a redundant mechanism for functional architecture specification is supported by the robustness of V1 under abnormal developmental environments. Under stripe rearing the cortical area devoted to the orientation of the environment is overrepresented, but shows also orientation preferences of unseen orientations with an overall normal appearing orientation layout [100]. More strikingly, ferrets raised in darkness generate orientation domains that adhere to the common design of primates and placental carnivores [14, 22, 24]. Nevertheless, neural activity is indispensable for development of visual cortical architecture as silencing experiments prevent the ermergence of or even destroy the functional architecture [90, 93, 104]. On the other hand, active axonal transport mechanisms can be regulated by neural activity suggesting activity as permissive control in the genetic network model [26, 239]. Guidance molecules can be modulated by neural activity $[230,231]$, but guidance molecules can also influence neural activity $[232,233]$. In the presence of instructive neuronal activity, the genetic network might support targeting and pruning of axons similar to synapse formation by chemoaffinity $[2,83$, 204]. Hence, this genetic mechanism might serve as a redundant plan or as a supportive agent for neuronal activity in visual cortical development.

Which cells are potentially involved in such a genetic mechanism? An indispensable trait of neurons that encode orientation preference with the proposed genetic mechanism are longrange patchy connections. A promising candidate are upper layer pyramidal neurons that are interconnected via clustered horizontal connections that elaborate during development to link neurons with similar response profiles $[10,12]$. Another possible candidate are transient subplate neurons with their long-ranging horizontal connections [84]. Ablation of subplate neurons prevent formation of functional architecture even if LGN axons already entered V1 [103]. Potentially both neuron types that are interconnected during ontogenesis build the substrate for the proposed genetic mechanism.

What is the evolutionary perspective on neocortical development? As a consequence of the possibility that visual cortical architecture can be genetically determined, the long-standing nature versus nurture view should be called into question. Instead, genetic assimilation of acquired traits known under the name Baldwin effect should be considered [240]. As the genetic network depends on the connectome it does not overrule activity, but serves as redundant mechanism for visual cortical architecture generation in the absence of instructive developmental environments. This is particularly interesting for the evolution of the common design that likely evolved independently at least two or three times [22]. Our theory indicates that the cause is rather convergent evolution than developmental constraints.

Emerging pinwheel arrangements depend on the organization of gene-regulatory circuits. Molecular mechanisms need to be adjusted to fulfill the quantitative conditions of the common design. Without selective pressure on the precise cortical architecture such tuning is not expected to emerge. The invariant architecture of orientation domains, pinwheel centers and long-range connectome is realized by different cells and synaptic circuits in different mammalian lineages. If the development of the common design in these cells is canalized by tissue wide genetic networks, it is thus expected that distinct systems of morphogens are recruited for the genetic circuit.

What causes the initiation of orientation domain emergence? In the genetic network model a single parameter determines the onset of pattern formation. This parameter can be interpreted as the concentration of a master organizing morphogen. Thus, upon the presence of this master morphogen, orientation domains begin to emerge. It is intriguing to hypothesise that this 
master morphogen is not expressed in the primary visual cortex, but subcortically and actively transported to V1 such as Otx2. Active afferent transport of the master morphogen hence leads to neocortical identification of the inputs that ought to be processed and initiates orientation domain development. This mechanism neatly meets the experimental observation of inducing orientation domains in the primary auditory cortex by rewiring of the primary visual pathway [241].

How can the neurons acquire orientation preferences as prescribed by the morphogen concentrations? A prerequisite for establishing orientation selective cells is a topographic mapping from the retina over the LGN to V1, a property called retinotopy. The development of retinotopy is initially guided by molecular cues that are gradually expressed and subsequently refined by neural activity in the primary visual cortex [83]. Thus, afferent fibers already project to their local vicinity of their correct retinotopic cortical positions. Orientation selectivity can be established by selective pruning of the local initial projections according to the morphogen concentrations and the retinotopic positions. This mechanism can be potentially combined with activity-dependent mechanisms and eye-specific segregation into ocular dominance columns as pioneered in [242]. 


\subsection{Supplementary material}

\section{Table of contents}

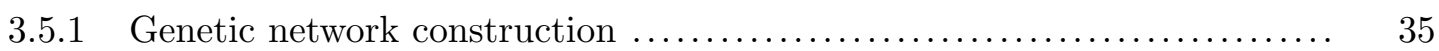

3.5.2 Complexity reduction to canonical genetic network ................... 40

3.5.3 Symmetry-confined canonical genetic network $\ldots \ldots \ldots \ldots \ldots \ldots \ldots \ldots \ldots \ldots \ldots$

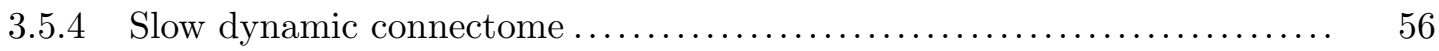

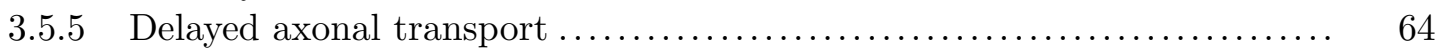

3.5.6 Extended solution set of canonical genetic network .................. 69

3.5.7 Benchmarking orientation fields of canonical genetic network ........... 75

3.5 .8 Numerical methods ......................................... 80

3.5.9 Alternative model: positionally specified orientation preference......... 82

3.5 .10 Nomenclature $\ldots \ldots \ldots \ldots \ldots \ldots \ldots \ldots \ldots \ldots \ldots \ldots \ldots \ldots \ldots \ldots \ldots \ldots, \quad 87$

\subsubsection{Genetic network construction}

The underlying concept of determining visual cortical architecture genetically lies in encoding orientation preference in the concentration of four morphogens denoted by $c_{i}$ with $i \in\{1,2,3,4\}$. Typical sizes of the primary visual cortex (V1) are on the order of hundreds to thousands of $\mathrm{mm}^{2}$, while neurons have a diameter of $\sim 10 \mathrm{\mu m}^{2}$, suggesting continuous cortical positions $\boldsymbol{x}=(x, y)^{T}$. The morphogen concentration is modeled as a continuous variable due to the fact that most animal transcription factors are expressed at 10 000-300 000 molecules/cell [218]. Thus, the morphogen concentrations constitute a continuous dynamic morphogen concentration field $c_{i}(\boldsymbol{x}, t) \in \mathbb{R}$ with $t$ denoting time. In order to encode the neuronal response feature of a preferred orientation $\vartheta(\boldsymbol{x}, t)$ in a concentration field, it should be noted that $\vartheta(\boldsymbol{x}, t)$ is a cyclic, $\pi$-periodic, variable. Hence, orientation preference can be conveniently encoded by the difference of concentrations leading to the orientation director or short orientation field

$$
\Psi(\boldsymbol{x}, t)=\left(c_{1}(\boldsymbol{x}, t)-c_{2}(\boldsymbol{x}, t)\right)+i\left(c_{3}(\boldsymbol{x}, t)-c_{4}(\boldsymbol{x}, t)\right) .
$$

Orientation preference $\vartheta(\boldsymbol{x}, t)$ is then given by $\Psi(\boldsymbol{x}, t)=S(\boldsymbol{x}, t) e^{2 i \vartheta(\boldsymbol{x}, t)}$. The morphogen concentration fields are fully specified by the orientation field together with the total concentration field

$$
\Sigma(\boldsymbol{x}, t)=\left(c_{1}(\boldsymbol{x}, t)+c_{2}(\boldsymbol{x}, t)\right)+i\left(c_{3}(\boldsymbol{x}, t)+c_{4}(\boldsymbol{x}, t)\right) .
$$

In the following, dynamics of the morphogen concentrations will be specified.

\section{Inter-neuronal transport}

On a cellular level, diffusion is a key mechanism for protein transportation. Typical diffusion constants of proteins are on the order of $10 \mathrm{\mu m}^{2} / \mathrm{s}\left(0.05-18.9 \mathrm{\mu m}^{2} / \mathrm{s}\right)$ [243]. Therefore, diffusion is efficient for neurotransmitters to overcome nanometer wide synaptic clefts in microseconds or proteins to traverse micrometer thick cells in seconds. In morphogenesis, diffusion is well suited for establishing short-range gradients in developmental time scales, but not for transporting proteins over long ranges such as a $1 \mathrm{~mm}$ distance that takes about half a day [185]. This makes diffusion as transport mechanism inappropriate for large distances and active transport mechanisms can take over to circumvent this temporal bottleneck.

Neuronal active transport comes in two different flavors, the retrograde transport goes from post- to pre-synaptic neurons (mediated by dynein in the axon) and anterogradely in the opposite 
direction (mediated by kinesin in the axon). In axons and dendrites, it is widely accepted that vesicular-based transport of a signaling endosome is the predominant signaling mechanism [244]. Endosomes are small vesicles build by lipid bilayers with molecules in their interior. They are transported by kinesin and dynein along microtubuli with a speed of about $1-5 \mu \mathrm{m} / \mathrm{s}[238,245$, 246]. Thus, the time to transport cargo over a distance of $1 \mathrm{~mm}$ is dramatically reduced to around 3-15 min in contrast to the diffusion time of $14 \mathrm{~h}$. In comparison to the transport speed, peripheral axonal growth rates after an injury can reach $0.02-0.06 \mu \mathrm{m} / \mathrm{s}[247]$.

Active transport is typically highly selective for particular proteins. For instance, dynein intermediate chain isoforms transport is highly selective for specific cargo like TrkB signalling endosomes [248]. Dynein selectivity of La is mediated by SUMO, which attaches to the RNAbinding protein La and gets thus retrogradely transported [249]. In contrast, La in absence of SUMO gets anterogradely transported in axons. It is hypothesized that this mechanism plays a role in targeting other proteins for retrograde transport [244, 249].

Retrograde transport conveys proteins such as growth factors, neurotrophins and transcription factors that regulate, for instance, apoptosis. An intriguing example for anterograde transport is the transcription factor Otx2, which is transported from the retina to V1. Interestingly, Otx2 plays a crucial role for the regulation of the critical period in V1 [26].

An upper bound for the transport rate can be estimated by assuming that vesicles are transported densely in the axon as a chain. The typical vesicle diameter is around $50 \mathrm{~nm}[250]$, which would yield a transport rate of 20-100 vesicles/s. In fact, measured transport rates in fruit flies range between $2-100$ molecules/min [251].

In the model, active transport is assumed to be mediated over axons and dendrites. It can be described by a dynamic connectivity $w(\boldsymbol{x}, \boldsymbol{y}, t)$ and a transport rate $\nu$. The outgoing flux of a substance with concentration $c(\boldsymbol{x}, t)$ from $\boldsymbol{x}$ to $\boldsymbol{y}$ at time $t$ is given by

$$
j_{\boldsymbol{x} \rightarrow \boldsymbol{y}}^{\text {out }}(t)=\nu w(\boldsymbol{y}, \boldsymbol{x}, t) c(\boldsymbol{x}, t) .
$$

The incoming flux from $\boldsymbol{y}$ to $\boldsymbol{x}$ at time $t$ is given by the outgoing flux from $\boldsymbol{y}$ to $\boldsymbol{x}$ at time $t$ subtracted by the transportation time of substances over the distance $\|\boldsymbol{x}-\boldsymbol{y}\|$ with velocity $v$

$$
j_{\boldsymbol{y} \rightarrow \boldsymbol{x}}^{\text {in }}(t)=j_{\boldsymbol{y} \rightarrow \boldsymbol{x}}^{\text {out }}\left(t-\frac{\|\boldsymbol{x}-\boldsymbol{y}\|}{v}\right) .
$$

Hence, the change in concentration is given by

$$
\begin{aligned}
\partial_{t} c(\boldsymbol{x}, t) & =\int \mathrm{d}^{2} y j_{\boldsymbol{y} \rightarrow \boldsymbol{x}}^{\text {in }}-\int \mathrm{d}^{2} y j_{\boldsymbol{x} \rightarrow \boldsymbol{y}}^{\text {out }}(t) \\
& =\nu \int \mathrm{d}^{2} y w\left(\boldsymbol{x}, \boldsymbol{y}, t-\frac{\|\boldsymbol{x}-\boldsymbol{y}\|}{v}\right) c\left(\boldsymbol{y}, t-\frac{\|\boldsymbol{x}-\boldsymbol{y}\|}{v}\right)-\nu \int \mathrm{d}^{2} y w(\boldsymbol{y}, \boldsymbol{x}, t) c(\boldsymbol{x}, t)
\end{aligned}
$$

The retarded part of the equation is negligible if the remaining dynamics act on a timescale $\tau$ that is larger than the transportation time $\|\boldsymbol{x}-\boldsymbol{y}\| / v \ll \tau$. The typical transport time over $1 \mathrm{~mm}$ axon is about $3-15 \mathrm{~min}$. In the limit of instantaneous transport the expression simplifies to

$$
\partial_{t} c(\boldsymbol{x}, t)=-\nu c(\boldsymbol{x}, t) \int \mathrm{d}^{2} y w(\boldsymbol{y}, \boldsymbol{x}, t)+\nu \int \mathrm{d}^{2} y w(\boldsymbol{x}, \boldsymbol{y}, t) c(\boldsymbol{y}, t)
$$

In our model, two possible scenarios of how inter-neuronal transport might be implemented exist: (i) morphogens get directly transported over synapses or (ii) morphogens trigger signaling cues that effectively mediate morphogen transport. In both scenarios, transport is mediated 
over a symmetric connectome $w(\boldsymbol{x}, \boldsymbol{y})=w(\boldsymbol{y}, \boldsymbol{x})$ with individual transport rats $\nu_{i}>0$ for $i \in\{1,2,3,4\}$. In the first scenario, the transport of morphogens results in the transport terms

$$
\tau_{s} \partial_{t} c_{i}(\boldsymbol{x}, t) \propto \nu_{i} \int \mathrm{d}^{2} y\left(c_{i}(\boldsymbol{y})-c_{i}(\boldsymbol{x})\right) w(\boldsymbol{x}, \boldsymbol{y}, t) .
$$

In the second scenario, signaling cues $s_{i}(\boldsymbol{x}, t)$ trigger a signaling of

$$
\tau_{s} \partial_{t} c_{i}(\boldsymbol{x}, t) \propto \nu_{i} \int \mathrm{d}^{2} y\left(s_{i}(\boldsymbol{y})-s_{i}(\boldsymbol{x})\right) w(\boldsymbol{x}, \boldsymbol{y}, t)
$$

that results in the same dynamics if signaling cues adapt rapidly to the concentration of morphogens $c_{i}(\boldsymbol{x}, t)$.

\section{Dynamic connectome}

We assume that the four morphogens get transported along two different types of synaptic connections an isotropic connection $w_{+}(\boldsymbol{x}-\boldsymbol{y}, t)$ and an anisotropic connection $w_{-}(\boldsymbol{x}, \boldsymbol{y}, t)$. The anisotropic connections depend on the morphogen concentration profiles. Both connection types are symmetric $w(\boldsymbol{x}, \boldsymbol{y})=w(\boldsymbol{y}, \boldsymbol{x})$ such that retrograde and anterograde transport is indistinguishable. The synaptic connections are dynamic

$$
\begin{aligned}
\tau_{m} \partial_{t} w_{+}(\boldsymbol{x}-\boldsymbol{y}, t) & =w_{+}^{\infty}(\boldsymbol{x}-\boldsymbol{y})-w_{+}(\boldsymbol{x}-\boldsymbol{y}, t) \\
\tau_{m} \partial_{t} w_{-}(\boldsymbol{x}, \boldsymbol{y}, t) & =w_{-}^{\infty}(\boldsymbol{x}, \boldsymbol{y}, t)-w_{-}(\boldsymbol{x}, \boldsymbol{y}, t)
\end{aligned}
$$

with a typical rearrangement constant of $\tau_{m}<1 \mathrm{~h}[252,253]$. Synapse rearrange exponentially to their asymptotic steady-state connection profiles

$$
\begin{aligned}
w_{+}^{\infty}(\boldsymbol{x}-\boldsymbol{y}) & =j_{u}^{+} K_{+}(\|\boldsymbol{x}-\boldsymbol{y}\|), \\
w_{-}^{\infty}(\boldsymbol{x}, \boldsymbol{y}, t) & =j_{u}^{-} K_{-}(\|\boldsymbol{x}-\boldsymbol{y}\|) \\
& +j^{-} K(\|\boldsymbol{x}-\boldsymbol{y}\|) e^{-\frac{\left(c_{1}(\boldsymbol{x}, t)-c_{2}(\boldsymbol{x}, t)-c_{1}(\boldsymbol{y}, t)+c_{2}(\boldsymbol{y}, t)\right)^{2}}{\sigma_{\Psi}^{2}}} e^{-\frac{\left(c_{3}(\boldsymbol{x}, t)-c_{4}(\boldsymbol{x}, t)-c_{3}(\boldsymbol{y}, t)+c_{4}(\boldsymbol{y}, t)\right)^{2}}{\sigma_{\Psi}^{2}}} .
\end{aligned}
$$

The morphogen-dependent part of the connectivity is phenomenologically motivated from patchy connections in V1 that preferentially link neurons with similar orientation preferences. The connectivity range can be given by different types of synaptic kernels $K(\|\boldsymbol{x}\|)$ that must have the properties of (i) positive connections $K(\|\boldsymbol{x}\|), K_{ \pm}(\|\boldsymbol{x}\|) \geq 0$ for all $\boldsymbol{x}$, (ii) normalized connections $\tilde{K}(0)=\tilde{K}_{ \pm}(0)=1$ with the Fourier transform $\tilde{K}(\|\boldsymbol{k}\|)=\mathcal{F}\{K\}(\|\boldsymbol{k}\|)$ and (iii) positive Fourier transform $\tilde{K}(\|\boldsymbol{k}\|), \tilde{K}_{ \pm}(\|\boldsymbol{k}\|) \geq 0$ for all $\boldsymbol{k}$ and a maximum at $\boldsymbol{k}=0$. A typical choice for the connections is a Gaussian profile

$$
K_{+}(\|\boldsymbol{x}\|)=\frac{1}{2 \pi \rho_{u}^{2}} e^{-\frac{\|\boldsymbol{x}\|^{2}}{2 \rho_{u}^{2}}}, \quad K_{-}(\|\boldsymbol{x}\|)=\frac{1}{2 \pi \sigma_{u}^{2}} e^{-\frac{\|\boldsymbol{x}\|^{2}}{2 \sigma_{u}^{2}}}, \quad K(\|\boldsymbol{x}\|)=\frac{1}{2 \pi \sigma^{2}} e^{-\frac{\|\boldsymbol{x}\|^{2}}{2 \sigma^{2}}} .
$$

\section{Transportation triggers local protein synthesis}

Transcription factors may have a dual function of an additional translational control. A prominent example for a dual function of transcription factors is the homeodomain protein Bicoid. It is found that Bicoid exerts translational control on Caudal by the binding of its homeodomain to the Caudal mRNA [254-256]. It has been proposed that over 200 homeodomain proteins, which are normally thought to regulate transcription, can additionally regulate translation [257]. Translation control is also observed in distal-axon NGF stimulation that induces axonal CREB translation. The axonal derived transcription factor CREB is retrogradely trafficked to the 
soma, where it is responsible for NGF-dependent neuronal survival [237]. As this mechanism appears to be crucial in neurogenesis, we include this mechanism in our model.

In the first scenario, morphogens translocating synapses of $w_{-}(\boldsymbol{x}, \boldsymbol{y}, t)$ cause local synthesis of proteins that subsequently get transported to the soma and degraded over time

$$
\begin{aligned}
& \tau_{f} \partial_{t} g_{c_{1}}(\boldsymbol{x}, t)=\int \mathrm{d}^{2} y\left[\mu_{1}^{2} c_{2}(\boldsymbol{y}, t)+\mu_{1}^{1} c_{1}(\boldsymbol{x}, t)\right] w_{-}(\boldsymbol{x}, \boldsymbol{y}, t)-g_{c_{1}}(\boldsymbol{x}, t) / \tau_{1} \\
& \tau_{f} \partial_{t} g_{c_{2}}(\boldsymbol{x}, t)=\int \mathrm{d}^{2} y\left[\mu_{2}^{2} c_{1}(\boldsymbol{y}, t)+\mu_{2}^{1} c_{2}(\boldsymbol{x}, t)\right] w_{-}(\boldsymbol{x}, \boldsymbol{y}, t)-g_{c_{2}}(\boldsymbol{x}, t) / \tau_{2} \\
& \tau_{f} \partial_{t} g_{c_{3}}(\boldsymbol{x}, t)=\int \mathrm{d}^{2} y\left[\mu_{3}^{2} c_{4}(\boldsymbol{y}, t)+\mu_{3}^{1} c_{3}(\boldsymbol{x}, t)\right] w_{-}(\boldsymbol{x}, \boldsymbol{y}, t)-g_{c_{3}}(\boldsymbol{x}, t) / \tau_{3} \\
& \tau_{f} \partial_{t} g_{c_{4}}(\boldsymbol{x}, t)=\int \mathrm{d}^{2} y\left[\mu_{4}^{2} c_{3}(\boldsymbol{y}, t)+\mu_{4}^{1} c_{4}(\boldsymbol{x}, t)\right] w_{-}(\boldsymbol{x}, \boldsymbol{y}, t)-g_{c_{4}}(\boldsymbol{x}, t) / \tau_{4}
\end{aligned}
$$

The degradation rates and the translation efficiencies are positive by definition, i.e. $\mu_{i}^{1}, \mu_{i}^{2}, \tau_{i} \in$ $\mathbb{R}_{+}$for $i \in\{1,2,3,4\}$. The time $\tau_{f}$ of a ribosome to translate a typical gene is approximately 1-6 min (translation rate is $1-8 \mathrm{aa} / \mathrm{s}$ [258] with median protein length of 375 aa [259]). In the second scenario, where signaling cues mediate effective transport, the signaling cues act either in the same way as transported morphogens or they exert additionally an effective regulatory control as the locally synthesized proteins $g_{c_{i}}$.

\section{Local gene regulation}

Genetic networks can be modeled in various ways that are most distinct by the level of detail considered, reviewed in [213-217]. Three major genetic network modeling approaches are (i) Logical models: These are the simplest models with discrete states and logic-based circuits (ii) Continuous models: These models assume continuous states and continuous interactions and (iii) Single-molecule models: Continuous models extended with stochastic effects.

Here, we model gene regulation of the four morphogens as a continuous model in a very general way that ensures positive definiteness of concentrations. We expand possible dynamics caused by a combination of Hill function in the unsaturated concentration regime, see section 3.5.1, by

$$
\tau_{s} \partial_{t} c_{i}(\boldsymbol{x}, t) \propto \xi_{i} g_{c_{i}}(\boldsymbol{x}, t)+c_{i}(\boldsymbol{x}, t)\left(\rho_{i}+\boldsymbol{\kappa}_{i} \cdot \boldsymbol{c}(\boldsymbol{x}, t)+\frac{1}{2} \boldsymbol{c}(\boldsymbol{x}, t)^{T} \boldsymbol{Q}_{i} \boldsymbol{c}(\boldsymbol{x}, t)\right)
$$

with

$$
\boldsymbol{c}(\boldsymbol{x}, t)=\left(\begin{array}{c}
c_{1}(\boldsymbol{x}, t) \\
c_{2}(\boldsymbol{x}, t) \\
c_{3}(\boldsymbol{x}, t) \\
c_{4}(\boldsymbol{x}, t)
\end{array}\right), \quad \boldsymbol{\kappa}_{i}=\left(\begin{array}{c}
\kappa_{i}^{1} \\
\kappa_{i}^{2} \\
\kappa_{i}^{3} \\
\kappa_{i}^{4}
\end{array}\right) \quad \text { and } \quad \boldsymbol{Q}_{i}=\left(\begin{array}{cccc}
2 \omega_{i}^{1} & \omega_{i}^{2} & \omega_{i}^{3} & \omega_{i}^{4} \\
\omega_{i}^{2} & 2 \omega_{i}^{5} & \omega_{i}^{6} & \omega_{i}^{7} \\
\omega_{i}^{3} & \omega_{i}^{6} & 2 \omega_{i}^{8} & \omega_{i}^{9} \\
\omega_{i}^{4} & \omega_{i}^{7} & \omega_{i}^{9} & 2 \omega_{i}^{10}
\end{array}\right)
$$

The linear coefficient $\rho_{i}$ is the combined degradation and regulation coefficient. All coefficients can assume positive and negative values except for the positive $\xi_{i}$. Generically, the power of the single terms in concentrations indicates the degree of cooperativity of the morphogen in regulation.

The time for RNA polymerase to transcribe a typical mammalian gene takes about $2-3 \mathrm{~min}$ and up to $16 \mathrm{~h}$ for long intron containing human genes [260]. Incorporating the typical exportation time from the nucleus to the soma and translation time, the typical time for regulation is about $\tau_{s}>1 \mathrm{~h}[261]$. 


\section{Genetic network model}

The full genetic network model is composed of local regulation, a dynamic connectome and locally synthesized substances. The dynamics of the morphogen concentration $c_{i}(\boldsymbol{x})$ with $i \in$ $\{1,2,3,4\}$ are composed of local regulation and of active axonal transport

$$
\begin{aligned}
\tau_{s} \partial_{t} c_{i}(\boldsymbol{x}, t)= & \xi_{i} g_{c_{i}}(\boldsymbol{x}, t)+c_{i}(\boldsymbol{x}, t)\left(\rho_{i}+\boldsymbol{\kappa}_{i} \cdot \boldsymbol{c}(\boldsymbol{x}, t)+\frac{1}{2} \boldsymbol{c}(\boldsymbol{x}, t)^{T} \boldsymbol{Q}_{i} \boldsymbol{c}(\boldsymbol{x}, t)\right) \\
& +\nu_{i} \int \mathrm{d}^{2} y\left[c_{i}(\boldsymbol{y}, t)-c_{i}(\boldsymbol{x}, t)\right]\left(w_{+}(\boldsymbol{x}-\boldsymbol{y}, t)+w_{-}(\boldsymbol{x}, \boldsymbol{y}, t)\right) .
\end{aligned}
$$

The dynamic connectome is symmetric and composed of two synaptic connection types. A local isotropic connectivity $w_{+}(\boldsymbol{x}-\boldsymbol{y}, t)$ and a morphogen concentration specific connectivity $w_{-}(\boldsymbol{x}, \boldsymbol{y}, t)$ with the dynamics

$$
\begin{aligned}
\tau_{m} \partial_{t} w_{+}(\boldsymbol{x}-\boldsymbol{y}, t) & =w_{+}^{\infty}(\boldsymbol{x}-\boldsymbol{y})-w_{+}(\boldsymbol{x}-\boldsymbol{y}, t), \\
\tau_{m} \partial_{t} w_{-}(\boldsymbol{x}, \boldsymbol{y}, t) & =w_{-}^{\infty}(\boldsymbol{x}, \boldsymbol{y}, t)-w_{-}(\boldsymbol{x}, \boldsymbol{y}, t) .
\end{aligned}
$$

Active transport over the connections of $w_{-}(\boldsymbol{x}, \boldsymbol{y}, t)$ leads to a local translation of mRNA and a subsequent transportation of the products to the soma

$$
\begin{aligned}
& \tau_{f} \partial_{t} g_{c_{1}}(\boldsymbol{x}, t)=\int \mathrm{d}^{2} y\left[\mu_{1}^{2} c_{2}(\boldsymbol{y}, t)+\mu_{1}^{1} c_{1}(\boldsymbol{x}, t)\right] w_{-}(\boldsymbol{x}, \boldsymbol{y}, t)-g_{c_{1}}(\boldsymbol{x}, t) / \tau_{1}, \\
& \tau_{f} \partial_{t} g_{c_{2}}(\boldsymbol{x}, t)=\int \mathrm{d}^{2} y\left[\mu_{2}^{2} c_{1}(\boldsymbol{y}, t)+\mu_{2}^{1} c_{2}(\boldsymbol{x}, t)\right] w_{-}(\boldsymbol{x}, \boldsymbol{y}, t)-g_{c_{2}}(\boldsymbol{x}, t) / \tau_{2}, \\
& \tau_{f} \partial_{t} g_{c_{3}}(\boldsymbol{x}, t)=\int \mathrm{d}^{2} y\left[\mu_{3}^{2} c_{4}(\boldsymbol{y}, t)+\mu_{3}^{1} c_{3}(\boldsymbol{x}, t)\right] w_{-}(\boldsymbol{x}, \boldsymbol{y}, t)-g_{c_{3}}(\boldsymbol{x}, t) / \tau_{3}, \\
& \tau_{f} \partial_{t} g_{c_{4}}(\boldsymbol{x}, t)=\int \mathrm{d}^{2} y\left[\mu_{4}^{2} c_{3}(\boldsymbol{y}, t)+\mu_{4}^{1} c_{4}(\boldsymbol{x}, t)\right] w_{-}(\boldsymbol{x}, \boldsymbol{y}, t)-g_{c_{4}}(\boldsymbol{x}, t) / \tau_{4}
\end{aligned}
$$

The model has in total 64 regulation coefficients, 8 coefficient of the local translation rate, 4 degradation times, 4 transport efficiencies, 3 transport rates, 3 transport ranges, 1 coefficient for selectiveness of axonal connections and 3 timescales - in total 90 parameters. Note that the concentrations of all fields are positive by construction for positive time constants and parameters of $\xi_{i}, \mu_{i}^{j}, \nu_{i}>0$ for $i \in\{1,2,3,4\}$.

\section{Appendix: Modeling transcriptional gene regulation phenomenologically}

In eukaryotic genetic circuits, the combinatorial regulation involves more complex regulatory mechanisms than simple activation or repression of a gene. Since in thermodynamical models typically only pair-wise interactions of transcription factors (TFs) are considered, another, phenomenological, approach used in [224] will be pursuit here that allows a higher complexity of combinatorial control.

The regulation of a gene with relative concentration $c$ is assumed to have the shape of a Hill function, which results for an activator and inhibitor in

$$
h_{n}(c)=\frac{c^{n}}{c^{n}+K^{n}}, \quad h_{n}(c)=\frac{K^{n}}{c^{n}+K^{n}},
$$

respectively, where $n$ indicates the number of binding sites or cooperativity and $K$ is the halfoccupancy concentration leading to the interpretation of the free binding energy $F=-k_{B} T \ln K$. In order to account for the combinatorial regulation of two TFs with concentrations $c_{1}$ and $c_{2}$, regulation can be modeled as a logical AND or as a logical OR

$$
h_{n m}\left(c_{1}, c_{2}\right)=h_{n}\left(c_{1}\right) \cdot h_{m}\left(c_{2}\right), \quad h_{n m}\left(c_{1}, c_{2}\right)=h_{n}\left(c_{1}\right)+h_{m}\left(c_{2}\right),
$$


respectively. This phenomenological combination of TFs generalizes naturally to larger genetic networks.

If binding affinities $K$ are equal, the concentration can be measured in units of $K$ leading to the simplified Hill functions

$$
h_{n}(c)=\frac{c^{n}}{c^{n}+1}, \quad g_{n}(c)=\frac{1}{c^{n}+1}=1-h_{n}(c),
$$

which can be combined by multiplication and addition. Given a polynomial

$$
f(c)=\alpha c+\beta c^{2}+\gamma c^{3}
$$

and assuming that the concentrations are small $c<1$, the aim is to find Hill functions producing this polynomial. The Hill functions can be approximated by

$$
\begin{aligned}
h_{1}(c) & =c-c^{2}+c^{3}+\mathcal{O}\left(c^{4}\right) \\
h_{2}(c) & =c^{2}+\mathcal{O}\left(c^{4}\right) \\
h_{3}(c) & =c^{3}+\mathcal{O}\left(c^{4}\right) \\
h_{n>3}(c) & =\mathcal{O}\left(c^{4}\right) .
\end{aligned}
$$

Multiplication of the Hill functions gives only leading terms for $h_{1} h_{1}, h_{1} h_{1} h_{1}$ and $h_{1} h_{2}$ which will not be considered here. Assuming a superposition of Hill functions $f=a h_{1}+b h_{2}+c h_{3}$, the coefficients of $f$ can be chosen arbitrarily by using the monomial as basis for a vector space. This results in the linear equation

$$
\left(\begin{array}{l}
\alpha \\
\beta \\
\gamma
\end{array}\right)=\left(\begin{array}{ccc}
1 & 0 & 0 \\
-1 & 1 & 0 \\
1 & 0 & 1
\end{array}\right)\left(\begin{array}{l}
a \\
b \\
c
\end{array}\right)
$$

The generalization to multiple TFs is analogous. Thus an explicit combination of Hill functions can be assigned to an expanded genetic network. However, the combination is not unique as an activator and inhibitor regulate gene expression similarly.

\subsubsection{Complexity reduction to canonical genetic network}

The complexity of the genetic network will be reduced here for analytical tractability. This complexity reduction results in an analyzable genetic network that we term canonical. The robustness of the findings in the canonical genetic network to assumptions used in the complexity reduction will be examined subsequently.

\section{Separation of time scales}

The first step in reducing the complexity of the genetic network is to notice three different time scales: local regulation (on the order of $\tau_{s}>1 \mathrm{~h}$ ), axonal rearrangement (on the order of $\tau_{m}<1 \mathrm{~h}$ ) and local translation (on the order of $\tau_{f}=5 \mathrm{~min}$ ). Separating timescales $\tau_{f}, \tau_{m} \ll \tau_{s}$ simplifies the model to four concentration fields

$$
\begin{aligned}
\tau_{s} \partial_{t} c_{1}(\boldsymbol{x}, t) & =\hat{F}^{c_{1}}\left[c_{1}, c_{2}, c_{3}, c_{4}\right], & & \tau_{s} \partial_{t} c_{2}(\boldsymbol{x}, t)=\hat{F}^{c_{2}}\left[c_{1}, c_{2}, c_{3}, c_{4}\right], \\
\tau_{s} \partial_{t} c_{3}(\boldsymbol{x}, t) & =\hat{F}^{c_{3}}\left[c_{1}, c_{2}, c_{3}, c_{4}\right], & \tau_{s} \partial_{t} c_{4}(\boldsymbol{x}, t) & =\hat{F}^{c_{4}}\left[c_{1}, c_{2}, c_{3}, c_{4}\right] .
\end{aligned}
$$


As the factor $\xi_{i} \tau_{i}$ always emerges as a product the factor will be absorbed as $\xi_{i} \tau_{i} \rightarrow \xi_{i}$. In the following the dynamics of the concentration fields will be analyzed by the convenient choice of two complex fields - an orientation (encoding) field $\Psi$ and a total concentration field $\Sigma$

$$
\begin{aligned}
& \Psi(\boldsymbol{x}, t)=\left(c_{1}(\boldsymbol{x}, t)-c_{2}(\boldsymbol{x}, t)\right)+i\left(c_{3}(\boldsymbol{x}, t)-c_{4}(\boldsymbol{x}, t)\right) \\
& \Sigma(\boldsymbol{x}, t)=\left(c_{1}(\boldsymbol{x}, t)+c_{2}(\boldsymbol{x}, t)\right)+i\left(c_{3}(\boldsymbol{x}, t)+c_{4}(\boldsymbol{x}, t)\right),
\end{aligned}
$$

where the dynamics are given by

$$
\tau_{s} \partial_{t} \Psi(\boldsymbol{x}, t)=\hat{F}^{\Psi}[\Psi, \Sigma], \quad \tau_{s} \partial_{t} \Sigma(\boldsymbol{x}, t)=\hat{F}^{\Sigma}[\Psi, \Sigma]
$$

The operators $\hat{F}^{\Psi}$ and $\hat{F}^{\Sigma}$ are omitted here for the sake of clarity. The derivation of the operators is basic algebra.

\section{Weak anisotropy of selective axonal connections}

The morphogen selective connections are assumed to be weakly anisotropic due to a small concentration difference compared to $\sigma_{\Psi}$, which leads to the approximation of

$$
\begin{aligned}
w_{-}^{\infty}(\boldsymbol{x}, \boldsymbol{y}, t) & =j_{u}^{-} K_{-}(\|\boldsymbol{x}-\boldsymbol{y}\|)+j^{-} K(\|\boldsymbol{x}-\boldsymbol{y}\|) e^{-\frac{|\Psi(\boldsymbol{x}, t)-\Psi(\boldsymbol{y}, t)|^{2}}{\sigma_{\Psi}^{2}}} \\
& \simeq j_{u}^{-} K_{-}(\|\boldsymbol{x}-\boldsymbol{y}\|)+j^{-} K(\|\boldsymbol{x}-\boldsymbol{y}\|)\left(1-\frac{|\Psi(\boldsymbol{x}, t)-\Psi(\boldsymbol{y}, t)|^{2}}{\sigma_{\Psi}^{2}}\right) .
\end{aligned}
$$

This approximation truncates the operators of the dynamics up to third order

$$
\begin{aligned}
\hat{F}^{\Psi}[\Psi, \Sigma]= & \hat{\tilde{L}}_{1}^{\Psi}[\Psi]+\hat{\tilde{L}}_{2}^{\Psi}[\Sigma]+\hat{\tilde{Q}}_{1}^{\Psi}[\Psi, \Psi]+\hat{\tilde{Q}}_{2}^{\Psi}[\Psi, \Sigma]+\hat{\tilde{Q}}_{3}^{\Psi}[\Sigma, \Sigma]+ \\
& \hat{\tilde{C}}_{1}^{\Psi}[\Psi, \Psi, \Psi]+\hat{\tilde{C}}_{2}^{\Psi}[\Psi, \Psi, \Sigma]+\hat{\tilde{C}}_{3}^{\Psi}[\Psi, \Sigma, \Sigma]+\hat{\tilde{C}}_{4}^{\Psi}[\Sigma, \Sigma, \Sigma], \\
\hat{F}^{\Sigma}[\Psi, \Sigma]= & \hat{L}_{1}^{\Sigma}[\Psi]+\hat{L}_{2}^{\Sigma}[\Sigma]+\hat{Q}_{1}^{\Sigma}[\Psi, \Psi]+\hat{Q}_{2}^{\Sigma}[\Psi, \Sigma]+\hat{Q}_{3}^{\Sigma}[\Sigma, \Sigma] \\
& +\hat{C}_{1}^{\Sigma}[\Psi, \Psi, \Psi]+\hat{C}_{2}^{\Sigma}[\Psi, \Psi, \Sigma]+\hat{C}_{3}^{\Sigma}[\Psi, \Sigma, \Sigma]+\hat{C}_{4}^{\Sigma}[\Sigma, \Sigma, \Sigma] .
\end{aligned}
$$

For the sake of clarity the operators are omitted again as the calculation involves only basic algebraic manipulations.

\section{Decoupling orientation encoding from total concentration}

The orientation field $\Psi(\boldsymbol{x}, t)$ is assumed to decouple from the field of total concentrations $\Sigma(\boldsymbol{x}, t)$. In this case, the encoding strategy is more robust to global factors impairing the dynamics of genetic expression, e.g. global heat fluctuations. The decoupling, which is defined by

$$
\hat{\tilde{L}}_{2}^{\Psi}[\Sigma]=\hat{\tilde{Q}}_{2}^{\Psi}[\Psi, \Sigma]=\hat{\tilde{Q}}_{3}^{\Psi}[\Sigma, \Sigma]=\hat{\tilde{C}}_{2}^{\Psi}[\Psi, \Psi, \Sigma]=\hat{\tilde{C}}_{3}^{\Psi}[\Psi, \Sigma, \Sigma]=\hat{\tilde{C}}_{4}^{\Psi}[\Sigma, \Sigma, \Sigma]=0,
$$


results in the identities

$$
\begin{array}{lll}
\kappa_{4}^{1}=\kappa_{3}^{1}, & \kappa_{1}^{2}=\kappa_{2}^{1}-2 \kappa_{1}^{1}, & \kappa_{2}^{2}=-\kappa_{1}^{1}, \\
\kappa_{3}^{2}=-\kappa_{3}^{1}, & \kappa_{4}^{2}=-\kappa_{3}^{1}, & \kappa_{2}^{3}=\kappa_{1}^{3}, \\
\kappa_{1}^{4}=-\kappa_{1}^{3}, & \kappa_{2}^{4}=-\kappa_{1}^{3}, & \kappa_{3}^{4}=\kappa_{4}^{3}-2 \kappa_{3}^{3}, \\
\kappa_{4}^{4}=-\kappa_{3}^{3}, & \nu_{2}=\nu_{1}, & \nu_{4}^{1}=\omega_{3}^{1}, \\
\rho_{2}=\rho_{1}, & \rho_{4}=\rho_{3}, & \omega_{1}^{4}=-\omega_{1}^{3}, \\
\omega_{3}^{2}=-2 \omega_{3}^{1}, & \omega_{4}^{2}=-2 \omega_{4}^{1}, & \omega_{1}^{5}=3 \omega_{1}^{1}-2 \omega_{1}^{10}+2 \omega_{2}^{10}+\omega_{2}^{2}, \\
\omega_{3}^{4}=\omega_{4}^{3}-2 \omega_{3}^{3}, & \omega_{4}^{4}=-\omega_{3}^{3}, & \omega_{4}^{5}=\omega_{4}^{1}, \\
\omega_{2}^{5}=\omega_{1}^{1}-\omega_{1}^{10}+\omega_{2}^{10}, & \omega_{3}^{5}=\omega_{3}^{1}, & \omega_{3}^{6}=-\omega_{3}^{3}, \\
\omega_{1}^{6}=\omega_{2}^{3}-2 \omega_{1}^{3}, & \omega_{2}^{6}=-\omega_{1}^{3}, & \omega_{3}^{7}=2 \omega_{3}^{3}+\omega_{4}^{6}, \\
\omega_{1}^{7}=2 \omega_{1}^{3}+\omega_{2}^{4}, & \omega_{2}^{7}=\omega_{1}^{3}, & \omega_{2}^{8}=\omega_{2}^{10}, \\
\omega_{4}^{7}=\omega_{3}^{3}, & \omega_{1}^{8}=\omega_{1}^{10}, & \omega_{2}^{9}=-2 \omega_{2}^{10}, \\
\omega_{3}^{8}=\omega_{3}^{1}-\omega_{4}^{1}+\omega_{4}^{10}, & \omega_{1}^{9}=-2 \omega_{1}^{10}, & \omega_{2}^{10}=\omega_{1}^{10}, \\
\omega_{3}^{9}=-2 \omega_{3}^{1}+2 \omega_{4}^{1}-3 \omega_{4}^{10}+\omega_{4}^{8}, & \omega_{4}^{9}=-\omega_{3}^{1}+\omega_{4}^{1}+\omega_{3}^{10}-3 \omega_{4}^{10}, & \mu_{2}^{2}=\frac{\mu_{1}^{2} \xi_{1}}{\xi_{2}}, \\
\mu_{2}^{1}=\frac{\mu_{1}^{1} \xi_{1}}{\xi_{2}}, & \mu_{4}^{1}=\frac{\mu_{3}^{1} \xi_{3}}{\xi_{4}}, & \\
\mu_{4}^{2}=\frac{\mu_{3}^{2} \xi_{3}}{\xi_{4}} & &
\end{array}
$$

\section{Pattern formation in orientation field}

The linear operators separate into a local part that depends only on $\boldsymbol{x}$ and a non-local part that also depends on the surrounding of $\boldsymbol{x}$. The non-local linear operators of the orientation field $\Psi(\boldsymbol{x}, t)$ are given by

$$
\begin{aligned}
\hat{L}_{\text {non-local }}^{\Psi}[\Psi]= & \frac{1}{2}\left[-\left(\mu_{1}^{1} \xi_{1}+\mu_{3}^{1} \xi_{3}\right)\left(j_{u}^{-} K_{-}+j^{-} K\right)\right. \\
& \left.+\left(\nu_{1}+\nu_{3}\right)\left(j_{u}^{-} K_{-}+j_{u}^{+} K_{+}+j^{-} K\right)\right] \star \Psi(\boldsymbol{y}, t) \\
\hat{L}_{\text {non-local }}^{\Psi}[\bar{\Psi}]= & \frac{1}{2}\left[-\left(\mu_{1}^{1} \xi_{1}-\mu_{3}^{1} \xi_{3}\right)\left(j_{u}^{-} K_{-}+j^{-} K\right)\right. \\
& \left.+\left(\nu_{1}-\nu_{3}\right)\left(j_{u}^{-} K_{-}+j_{u}^{+} K_{+}+j^{-} K\right)\right] \star \bar{\Psi}(\boldsymbol{y}, t) .
\end{aligned}
$$

For the sake of simplicity we assume that $\hat{L}_{n l}^{\Psi}[\bar{\Psi}]=0$, which leads to the parameter identities

$$
\mu_{1}^{1} \xi_{1}=\mu_{3}^{1} \xi_{3}, \quad \nu_{3}=\nu_{1} .
$$

This choice of parameters fixes a single critical wavelength of the Turing instability for the real and imaginary part of the orientation field $\Psi(\boldsymbol{x}, t)$. With the additional parameter choice, the orientation encoding field is given by

$$
\tau_{s} \partial_{t} \Psi(\boldsymbol{x}, t)=\hat{L}^{\Psi}[\Psi]+\hat{Q}^{\Psi}[\Psi, \Psi]+\hat{C}^{\Psi}[\Psi, \Psi, \Psi]
$$

with the operators that can be decomposed into

$$
\begin{aligned}
\hat{L}^{\Psi}[\Psi] & =\hat{L}_{1}^{\Psi}[\Psi]+\hat{L}_{2}^{\Psi}[\bar{\Psi}], \\
\hat{Q}^{\Psi}[\Psi, \Psi] & =\hat{Q}_{1}^{\Psi}[\Psi, \Psi]+\hat{Q}_{2}^{\Psi}[\Psi, \bar{\Psi}]+\hat{Q}_{3}^{\Psi}[\bar{\Psi}, \bar{\Psi}], \\
\hat{C}^{\Psi}[\Psi, \Psi, \Psi] & =\hat{C}_{1}^{\Psi}[\Psi, \Psi, \bar{\Psi}]+\hat{C}_{2}^{\Psi}[\Psi, \bar{\Psi}, \bar{\Psi}]+\hat{C}_{3}^{\Psi}[\bar{\Psi}, \bar{\Psi}, \bar{\Psi}]+\hat{C}_{4}^{\Psi}[\Psi, \Psi, \Psi] .
\end{aligned}
$$


The individual operators are given by the linear operators

$$
\begin{aligned}
& \hat{L}_{1}^{\Psi}[\Psi]=\alpha_{1} \Psi(\boldsymbol{x}, t)-\gamma_{1} \sigma_{\Psi}^{2}\left(\frac{j_{u}^{-}}{j^{-}} K_{-}+K\right) \star \Psi(\boldsymbol{y}, t)+\nu_{1} j_{u}^{+} K_{+} \star \Psi(\boldsymbol{y}, t), \\
& \hat{L}_{2}^{\Psi}[\bar{\Psi}]=\alpha_{2} \bar{\Psi}(\boldsymbol{x}, t),
\end{aligned}
$$

the quadratic operators

$$
\begin{aligned}
& \hat{Q}_{1}^{\Psi}[\Psi, \Psi]=-\left(\beta_{1}+i \zeta_{1}\right) \Psi(\boldsymbol{x}, t)^{2}, \\
& \hat{Q}_{2}^{\Psi}[\Psi, \bar{\Psi}]=\left(\beta_{2}-\beta_{1}+i\left(\zeta_{1}-\zeta_{2}\right)\right)|\Psi(\boldsymbol{x}, t)|^{2}, \\
& \hat{Q}_{3}^{\Psi}[\bar{\Psi}, \bar{\Psi}]=\left(\beta_{2}+i \zeta_{2}\right) \bar{\Psi}(\boldsymbol{x}, t)^{2}
\end{aligned}
$$

and the cubic operators

$$
\begin{aligned}
& \hat{C}_{1}^{\Psi}[\Psi, \Psi, \bar{\Psi}]=K \star\left(\gamma_{1} \Psi(\boldsymbol{y}, t)-\gamma_{2} \Psi(\boldsymbol{x}, t)\right)|\Psi(\boldsymbol{x}, t)-\Psi(\boldsymbol{y}, t)|^{2}+\left(\gamma_{6}-i \eta_{1}\right) \Psi(\boldsymbol{x}, t)|\Psi(\boldsymbol{x}, t)|^{2}, \\
& \hat{C}_{2}^{\Psi}[\Psi, \bar{\Psi}, \bar{\Psi}]=K \star \gamma_{3} \bar{\Psi}(\boldsymbol{x})|\Psi(\boldsymbol{x}, t)-\Psi(\boldsymbol{y}, t)|^{2}+\left(\gamma_{5}+i \eta_{2}\right) \bar{\Psi}(\boldsymbol{x}, t)|\Psi(\boldsymbol{x}, t)|^{2}, \\
& \hat{C}_{3}^{\Psi}[\bar{\Psi}, \bar{\Psi}, \bar{\Psi}]=\left(\gamma_{4}+i \eta_{1}\right) \bar{\Psi}(\boldsymbol{x}, t)^{3}, \\
& \hat{C}_{4}^{\Psi}[\Psi, \Psi, \Psi]=\left(\gamma_{7}-i \eta_{2}\right) \Psi(\boldsymbol{x}, t)^{3},
\end{aligned}
$$

with the substitutions

$$
\begin{aligned}
\alpha_{1}=\frac{\rho_{1}+\rho_{3}}{2}+\frac{j^{-}+j_{u}^{-}}{2}\left(\mu_{1}^{2} \xi_{1}+\mu_{3}^{2} \xi_{3}\right)-\nu_{1}\left(j^{-}+j_{u}^{-}+j_{u}^{+}\right), & \\
\alpha_{2}=\frac{\rho_{1}-\rho_{3}}{2}+\frac{j^{-}+j_{u}^{-}}{2}\left(\mu_{1}^{2} \xi_{1}-\mu_{3}^{2} \xi_{3}\right), & \\
\beta_{1}=-\frac{1}{4}\left(\kappa_{1}^{1}+\kappa_{3}^{1}\right), & \beta_{2}=\frac{1}{4}\left(\kappa_{1}^{1}-\kappa_{3}^{1}\right), \\
\zeta_{1}=\frac{1}{4}\left(\kappa_{1}^{3}+\kappa_{3}^{3}\right), & \zeta_{2}=\frac{1}{4}\left(\kappa_{1}^{3}-\kappa_{3}^{3}\right), \\
\eta_{1}=\frac{1}{8}\left(\omega_{1}^{3}-\omega_{3}^{3}\right), & \eta_{2}=\frac{1}{8}\left(\omega_{1}^{3}+\omega_{3}^{3}\right), \\
\gamma_{1}=\frac{j^{-}}{\sigma_{\Psi}^{2}}\left(\mu_{3}^{1} \xi_{3}-\nu_{1}\right), & \gamma_{2}=\frac{j^{-}}{2 \sigma_{\Psi}^{2}}\left(\mu_{1}^{2} \xi_{1}+\mu_{3}^{2} \xi_{3}-2 \nu_{1}\right), \\
\gamma_{3}=-\frac{j^{-}}{2 \sigma_{\Psi}^{2}}\left(\mu_{1}^{2} \xi_{1}-\mu_{3}^{2} \xi_{3}\right), & \gamma_{4}=\frac{1}{8}\left(\left(\omega_{1}^{1}+\omega_{4}^{10}\right)-\left(\omega_{3}^{1}+\omega_{1}^{10}\right)\right), \\
\gamma_{5}=\frac{1}{8}\left(3\left(\omega_{1}^{1}-\omega_{4}^{10}\right)-\left(\omega_{3}^{1}-\omega_{1}^{10}\right)\right), & \gamma_{6}=\frac{1}{8}\left(3\left(\omega_{1}^{1}+\omega_{4}^{10}\right)+\left(\omega_{3}^{1}+\omega_{1}^{10}\right)\right), \\
\gamma_{7}=\frac{1}{8}\left(\left(\omega_{1}^{1}-\omega_{4}^{10}\right)+\left(\omega_{3}^{1}-\omega_{1}^{10}\right)\right) . &
\end{aligned}
$$

Notice that all 15 effective parameters are independent and can be chosen arbitrarily.

In order to get a wavelength instability of the orientation field, consider the eigenspectrum of the linear operator in Fourier space

$$
\begin{aligned}
\mathcal{F}\left\{\hat{L}_{1}[\Psi]\right\} & =\left(\alpha_{1}+\lambda_{\hat{L}}(\boldsymbol{k})\right) \tilde{\Psi}(\boldsymbol{k}, t) \\
\lambda_{\hat{L}}(\boldsymbol{k}) & =-\gamma_{1} \sigma_{\Psi}^{2}\left(\frac{j_{u}^{-}}{j^{-}} \tilde{K}_{-}(\|\boldsymbol{k}\|)+\tilde{K}(\|\boldsymbol{k}\|)\right)+\nu_{1} j_{u}^{+} \tilde{K}_{+}(\|\boldsymbol{k}\|) .
\end{aligned}
$$

Since all coefficients except for $\gamma_{1}$ are positive, $\tilde{K}(\|\boldsymbol{k}\|), \tilde{K}_{ \pm}(\|\boldsymbol{k}\|)>0$ and $\tilde{K}(\|\boldsymbol{k}\|), \tilde{K}_{ \pm}(\|\boldsymbol{k}\|)$ have a maximum for $\boldsymbol{k}=0$, a necessary but not sufficient condition to obtain a non-trivial 
wavelength instability is $\gamma_{1}>0$. For a suitable parameter choice the critical wavelength is given by

$$
\boldsymbol{k}_{c}=\operatorname{argmax}\left\{\lambda_{\hat{L}}(\boldsymbol{k})\right\}
$$

and the parameter $\alpha_{1}$ will be chosen as

$$
\alpha_{1}=\lambda_{\hat{L}}\left(\boldsymbol{k}_{c}\right)+r
$$

\section{Stability of total concentration field}

In this section, it will be shown that the dynamics of the total concentration field can be chosen to be stable for arbitrary choices of the effective parameters except for $\gamma_{1}$.

Dynamics of the total concentration field $\Sigma$ for a vanishing orientation field $\Psi=0$ is given by

$$
\tau_{s} \partial_{t} \Sigma(\boldsymbol{x}, t)=\sum_{i=1}^{2} \hat{L}_{i}^{\Sigma}+\sum_{i=1}^{3} \hat{Q}_{i}^{\Sigma}+\sum_{i=1}^{4} \hat{C}_{i}^{\Sigma}
$$

with the individual operators defined by the linear operators

$$
\begin{aligned}
\hat{L}_{1}^{\Sigma}[\Sigma]= & \alpha_{1} \Sigma(\boldsymbol{x}, t)+\nu_{1}\left(2 j^{-} K+2 j_{u}^{-} K_{-}+j_{u}^{+} K_{+}\right) \star \Sigma(\boldsymbol{y}, t) \\
& +\gamma_{1} \sigma_{\Psi}^{2}\left(K+\frac{j_{u}^{-}}{j^{-}} K_{-}\right) \star \Sigma(\boldsymbol{y}, t), \\
\hat{L}_{2}^{\Sigma}[\bar{\Sigma}]= & \alpha_{2} \bar{\Sigma}(\boldsymbol{x}, t)
\end{aligned}
$$

the quadratic operators

$$
\begin{aligned}
& \hat{Q}_{1}^{\Sigma}[\Sigma, \Sigma]=\frac{1}{8}\left(2 \beta_{1}-2 \beta_{2}+\kappa_{2}^{1}+i\left(2 \zeta_{1}-2 \zeta_{2}-\kappa_{4}^{3}\right)\right) \Sigma(\boldsymbol{x}, t)^{2}, \\
& \hat{Q}_{2}^{\Sigma}[\Sigma, \bar{\Sigma}]=\frac{1}{4}\left(2 \beta_{1}-2 \beta_{2}+\kappa_{2}^{1}-i\left(2 \zeta_{1}-2 \zeta_{2}-\kappa_{4}^{3}\right)\right)|\Sigma(\boldsymbol{x}, t)|^{2}, \\
& \hat{Q}_{3}^{\Sigma}[\bar{\Sigma}, \bar{\Sigma}]=\frac{1}{8}\left(2 \beta_{1}-2 \beta_{2}+\kappa_{2}^{1}+i\left(2 \zeta_{1}-2 \zeta_{2}-\kappa_{4}^{3}\right)\right) \bar{\Sigma}(\boldsymbol{x}, t)^{2}
\end{aligned}
$$

and the cubic operators

$$
\begin{aligned}
\hat{C}_{1}^{\Sigma}[\Sigma, \Sigma, \Sigma]= & \frac{1}{32}\left[3\left(\gamma_{4}+\gamma_{6}\right)-\left(\gamma_{5}+\gamma_{7}\right)\right. \\
& \left.+\left(\omega_{2}^{1}-\omega_{3}^{10}+\omega_{2}^{2}-\omega_{4}^{8}\right)-i\left(\omega_{2}^{3}+\omega_{4}^{3}+\omega_{2}^{4}+\omega_{4}^{6}\right)\right] \Sigma(\boldsymbol{x}, t)^{3}, \\
\hat{C}_{2}^{\Sigma}[\Sigma, \bar{\Sigma}, \bar{\Sigma}]= & \frac{1}{32}\left[9\left(\gamma_{4}+\gamma_{6}\right)-3\left(\gamma_{5}+\gamma_{7}\right)\right. \\
& \left.+3\left(\omega_{2}^{1}-\omega_{3}^{10}+\omega_{2}^{2}-\omega_{4}^{8}\right)+i\left(\omega_{2}^{3}+\omega_{4}^{3}+\omega_{2}^{4}+\omega_{4}^{6}\right)\right] \bar{\Sigma}(\boldsymbol{x}, t)|\Sigma(\boldsymbol{x}, t)|^{2}, \\
\hat{C}_{3}^{\Sigma}[\Sigma, \Sigma, \bar{\Sigma}]= & \frac{1}{32}\left[-3\left(\gamma_{4}+\gamma_{6}\right)+9\left(\gamma_{5}+\gamma_{7}\right)\right. \\
& \left.+3\left(\omega_{2}^{1}+\omega_{3}^{10}+\omega_{2}^{2}+\omega_{4}^{8}\right)-i\left(\omega_{2}^{3}-\omega_{4}^{3}+\omega_{2}^{4}-\omega_{4}^{6}\right)\right] \Sigma(\boldsymbol{x}, t)|\Sigma(\boldsymbol{x}, t)|^{2}, \\
\hat{C}_{4}^{\Sigma}[\bar{\Sigma}, \bar{\Sigma}, \bar{\Sigma}]= & \frac{1}{32}\left[-\left(\gamma_{4}+\gamma_{6}\right)+3\left(\gamma_{5}+\gamma_{7}\right)\right. \\
& \left.+\left(\omega_{2}^{1}+\omega_{3}^{10}+\omega_{2}^{2}+\omega_{4}^{8}\right)+i\left(\omega_{2}^{3}-\omega_{4}^{3}+\omega_{2}^{4}-\omega_{4}^{6}\right)\right] \bar{\Sigma}(\boldsymbol{x}, t)^{3} .
\end{aligned}
$$

The parameters $\omega_{i}^{j}$ and $\kappa_{i}^{j}$ that appear in these operators can be chosen arbitrarily without changing the pattern of the orientation field. However, the dynamics of the total concentration 
field determine the total concentration of the pattern and can lead to substantially different dynamics of the concentrations. The choice of the model guarantees that the concentrations are always positive and hence $\arg \{\Sigma\} \in[0, \pi / 2]$. An important model class consists of choosing the remaining parameters in such a way that $\Sigma$ has a stable single fixed point with $|\Sigma|>0$ and $\arg \{\Sigma\} \in(0, \pi / 4)$ for $\Psi=0$. This leads to finite concentration dynamics.

A convenient way to construct such a stable fixed point is to choose the parameters such that

$$
\hat{C}_{i}^{\Sigma}=0
$$

for all $i \in\{1,2,3,4\}$. The fixed points for the choice of vanishing cubic order of $\Sigma$ and $\Psi=0$ separates real and imaginary part of $\Sigma_{j}=A_{j}+i B_{j}$ and results in the fixed points

$$
\Sigma_{1}=0, \quad \Sigma_{2}=\frac{a_{1}+a_{2}}{b_{1}}, \quad \Sigma_{3}=i \frac{a_{1}-a_{2}}{b_{2}}, \quad \Sigma_{4}=\frac{a_{1}+a_{2}}{b_{1}}+i \frac{a_{1}-a_{2}}{b_{2}}
$$

with the parameters

$$
\begin{aligned}
& a_{1}=\alpha_{1}+\nu_{1}\left(2 j^{-}+2 j_{u}^{-}+j_{u}^{+}\right)+\gamma_{1} \sigma_{\Psi}^{2}\left(1+\frac{j_{u}^{-}}{j^{-}}\right) \\
& a_{2}=\alpha_{2} \\
& b_{1}=-\left(2 \beta_{1}-2 \beta_{2}+\kappa_{2}^{1}\right) / 2 \\
& b_{2}=\left(2 \zeta_{1}-2 \zeta_{2}-\kappa_{4}^{3}\right) / 2 .
\end{aligned}
$$

The stability of the fixed points is determined by the eigenvalues of the linearized dynamics of the real and imaginary part $\left(\partial_{t} A_{i} \propto e^{\lambda_{i} t}\right.$ and $\left.\partial_{t} B_{i} \propto e^{\mu_{i} t}\right)$ and gives

$$
\lambda_{1}=-\lambda_{2}=\lambda_{3}=-\lambda_{4}=a_{1}+a_{2}, \quad \mu_{1}=\mu_{2}=-\mu_{3}=-\mu_{4}=\left(a_{1}-a_{2}\right) .
$$

Thus, the fixed points are stable for $a_{1}<0$ and $\left|a_{2}\right|<\left|a_{1}\right|, a_{2}>0$ and $\left|a_{1}\right|<\left|a_{2}\right|, a_{2}<0$ and $\left|a_{1}\right|<\left|a_{2}\right|, a_{1}>0$ and $\left|a_{2}\right|<\left|a_{1}\right|$ for the first, second, third and fourth fixed point, respectively. Notice that for the coefficient $a_{1}$ a lower bound can be calculated

$$
a_{1}=\alpha_{1}+\nu_{1}\left(2 j^{-}+2 j_{u}^{-}+j_{u}^{+}\right)+\gamma_{1} \sigma_{\Psi}^{2}\left(1+\frac{j_{u}^{-}}{j^{-}}\right) \geq \nu_{1}\left(2 j^{-}+2 j_{u}^{-}\right)+r>r .
$$

Thus, upon symmetry breaking $r>0$ the first trivial fixed point becomes unstable. Depending on the specific parameters the non-trivial fixed points of the total concentration field $\Sigma$ remain stable in linear order since $\Sigma$ does not influence $\Psi$ and $\Psi$ is small. As stability of the total concentration field is guaranteed and the fixed point can be chosen to arbitrary total concentrations in this specific setting, the exact dynamics of the total concentration field will be omitted here for the sake of clarity.

\section{Weakly nonlinear analysis of third order field dynamics}

In order to dissect the patterns that emerge from the orientation field dynamics, we use a perturbation method, called weakly nonlinear analysis [28, 29]. This allows an analytical treatment of a homogeneous solution close to its instability of pattern formation, see Fig. 3.8. The dynamics of the orientation field can be written as

$$
\tau_{s} \partial_{t} \Psi(\boldsymbol{x}, t)=\hat{L}^{\Psi}[\Psi]+\hat{Q}^{\Psi}[\Psi, \Psi]+\hat{C}^{\Psi}[\Psi, \Psi, \Psi],
$$


A

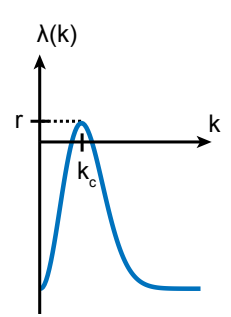

B

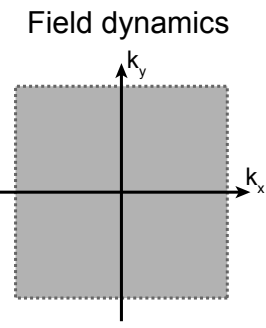

C

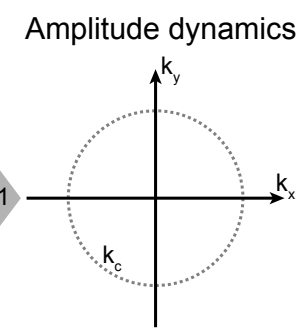

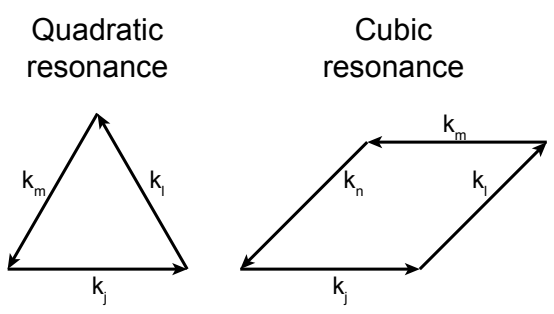

Figure 3.8: Scheme of weakly nonlinear analysis of pattern forming field dynamics. A Field dynamics are initially governed by a linear operator with an rotation symmetric eigenspectrum. The eigenspectrum $\lambda(\|\boldsymbol{k}\|)$ of the linear operator possesses a single maximum of amplitude $r$. The amplitude of the maximal eigenvalue is $0<r \ll 1$ at the wave vector $\boldsymbol{k}$ with $k_{c}=\|\boldsymbol{k}\|$ defines the critical circle. $\mathbf{B}$ Expanding the field dynamics (gray area) in powers of the maximal eigenvalue $r$ simplifies the dynamics to amplitudes on the critical circle (gray dashed circle). $\mathbf{C}$ Resonant quadratic and cubic modes on the critical circle fulfill the geometrical constraints of $\boldsymbol{k}_{j}$ forming a triangle and parallelogram, respectively. See main text for further details.

where the linear, quadratic and cubic operators can be decomposed into

$$
\begin{aligned}
\hat{L}^{\Psi}[\Psi] & =\hat{L}_{1}^{0}[\Psi]+r \Psi(\boldsymbol{x}, t)+\alpha_{2} \bar{\Psi}(\boldsymbol{x}, t), \\
\hat{Q}^{\Psi}[\Psi, \Psi] & =\hat{Q}_{1}^{\Psi}[\Psi, \Psi]+\hat{Q}_{2}^{\Psi}[\Psi, \bar{\Psi}]+\hat{Q}_{3}^{\Psi}[\bar{\Psi}, \bar{\Psi}], \\
\hat{C}^{\Psi}[\Psi, \Psi, \Psi] & =\hat{C}_{1}^{\Psi}[\Psi, \Psi, \bar{\Psi}]+\hat{C}_{2}^{\Psi}[\Psi, \bar{\Psi}, \bar{\Psi}]+\hat{C}_{3}^{\Psi}[\bar{\Psi}, \bar{\Psi}, \bar{\Psi}]+\hat{C}_{4}^{\Psi}[\Psi, \Psi, \Psi],
\end{aligned}
$$

respectively. The eigenspectrum $\lambda(\boldsymbol{k})$ of the linear operator $\hat{L}_{1}^{0}$, Fig. $\mathbf{3 . 8 A}$, is assumed to be rotation symmetric and to possess a wavelength instability with vanishing maximal eigenvalue, i.e. $\max \lambda(\|\boldsymbol{k}\|)=\lambda\left(k_{c}\right)=0$. At pattern onset, i.e. $0<r \ll 1$, the field dynamics can be analyzed analytically by expanding the control parameter $r$, the field $\Psi(\boldsymbol{x}, t)$ and the time constant $\tau_{s}$ in powers of a small positive parameter $0<\epsilon \ll 1$ as

$$
\begin{aligned}
r & =\epsilon r_{1}+\epsilon^{2} r_{2}+\mathcal{O}\left(\epsilon^{3}\right) \\
\Psi(\boldsymbol{x}, t) & =\sqrt{\epsilon} \Psi_{1}(\boldsymbol{x}, t)+\sqrt{\epsilon}^{2} \Psi_{2}(\boldsymbol{x}, t)+\sqrt{\epsilon}^{3} \Psi_{3}(\boldsymbol{x}, t)++\mathcal{O}\left(\sqrt{\epsilon}^{4}\right) \\
\tau_{s} & =\epsilon \tau_{1}+\epsilon^{2} \tau_{2}+\mathcal{O}\left(\epsilon^{3}\right)
\end{aligned}
$$

Here, the additional simplifying assumption of small quadratic terms $\beta_{i} \rightarrow \sqrt{\epsilon} \beta_{i}, \zeta_{i} \rightarrow \sqrt{\epsilon} \zeta_{i}$ and small linear terms $\alpha_{2} \rightarrow \epsilon \alpha_{2}$ is made. Plugging the ansatz into the field dynamics and sorting by the order of $\sqrt{\epsilon}$ leads to

$$
\begin{aligned}
0= & \sqrt{\epsilon} \hat{L}_{1}^{0}\left[\Psi_{1}\right]+\epsilon \hat{L}_{1}^{0}\left[\Psi_{2}\right] \\
& +\sqrt{\epsilon}^{3}\left(\left(r_{1}-\tau_{1} \partial_{t}\right) \Psi_{1}(\boldsymbol{x}, t)+\hat{L}_{1}^{0}\left[\Psi_{3}\right]+\alpha_{2} \bar{\Psi}_{1}(\boldsymbol{x}, t)+\hat{Q}^{\Psi}\left[\Psi_{1}, \Psi_{1}\right]+\hat{C}^{\Psi}\left[\Psi_{1}, \Psi_{1}, \Psi_{1}\right]\right) \\
& +\mathcal{O}\left(\sqrt{\epsilon}^{4}\right) .
\end{aligned}
$$

In order to fulfill this equation, each order in $\sqrt{\epsilon}$ must vanish. This leads to the finding that the first two orders of the orientation field must be in the kernel of the linear operator, i.e. $\Psi_{1}(\boldsymbol{x}, t), \Psi_{2}(\boldsymbol{x}, t) \in \operatorname{ker} \hat{L}_{1}^{0}$. The condition for the third order term is more conveniently written as

$$
-\hat{L}_{1}^{0}\left[\Psi_{3}\right]=-\tau_{1} \partial_{t} \Psi_{1}(\boldsymbol{x}, t)+\alpha_{2} \bar{\Psi}_{1}(\boldsymbol{x}, t)+r_{1} \Psi_{1}(\boldsymbol{x}, t)+\hat{Q}^{\Psi}\left[\Psi_{1}, \Psi_{1}\right]+\hat{C}^{\Psi}\left[\Psi_{1}, \Psi_{1}, \Psi_{1}\right] .
$$


This equation can be analyzed by the Fredholm alternative that corresponds to a projection onto the kernel of the linear operator ker $\hat{L}_{1}^{0}$. Denoting the projection operator by $\hat{P}_{c}$ results in

$$
0=-\tau_{1} \partial_{t} \Psi_{1}(\boldsymbol{x}, t)+\alpha_{2} \bar{\Psi}_{1}(\boldsymbol{x}, t)+r_{1} \Psi_{1}(\boldsymbol{x}, t)+\hat{P}_{c} \hat{Q}^{\Psi}\left[\Psi_{1}, \Psi_{1}\right]+\hat{P}_{c} \hat{C}^{\Psi}\left[\Psi_{1}, \Psi_{1}, \Psi_{1}\right]
$$

Measuring time in units of $\tau_{1} / r_{1}$ and the amplitude of $\Psi(\boldsymbol{x}, t)$ in units of $r_{1}$ leads to the equation

$$
\partial_{t} \Psi_{1}(\boldsymbol{x}, t)=\Psi_{1}(\boldsymbol{x}, t)+\alpha_{2} \bar{\Psi}_{1}(\boldsymbol{x}, t)+\hat{P}_{c} \hat{Q}^{\Psi}\left[\Psi_{1}, \Psi_{1}\right]+\hat{P}_{c} \hat{C}^{\Psi}\left[\Psi_{1}, \Psi_{1}, \Psi_{1}\right],
$$

where all coefficients were scaled by $1 / r_{1}$. By construction, the solution of $\Psi_{1}(\boldsymbol{x}, t)$ is an element of the kernel ker $\hat{L}_{1}^{0}$ and can therefore be written as a superposition of Fourier modes

$$
\Psi_{1}(\boldsymbol{x}, t)=\sum_{j=0}^{2 n-1} A_{j}(t) e^{i \boldsymbol{k}_{j} \boldsymbol{x}}
$$

with the wave vector $\boldsymbol{k}=k_{c}(\cos (\pi j / n), \sin (\pi j / n))^{T}$. Thus the field dynamics are reduced to dynamics of the amplitudes $A_{j}(t)$, see Fig. 3.8B. Explicit amplitude dynamics can be derived through projection onto the $j$ th mode by the operator $\hat{P}_{j}$. This results in

$$
\partial_{t} A_{j}(t)=A_{j}(t)+\alpha_{2} A_{j^{-}}(t)+e^{-i \boldsymbol{k}_{j} \boldsymbol{x}} \hat{P}_{j} \hat{Q}^{\Psi}\left[\Psi_{1}, \Psi_{1}\right]+e^{-i \boldsymbol{k}_{j} \boldsymbol{x}} \hat{P}_{j} \hat{C}^{\Psi}\left[\Psi_{1}, \Psi_{1}, \Psi_{1}\right],
$$

where $j^{-}=j-n$ denotes the index of the Fourier mode on the opposite side of the critical circle to the $j$ th Fourier mode. Plugging the solution into the operators gives for the quadratic term

$$
\begin{aligned}
& \hat{Q}_{1}^{\Psi}\left[\Psi_{1}, \Psi_{1}\right]=\sum_{l m} A_{l}(t) A_{m}(t) q_{l m}^{1} e^{i\left(\boldsymbol{k}_{l}+\boldsymbol{k}_{m}\right) \boldsymbol{x}} \\
& \hat{Q}_{2}^{\Psi}\left[\Psi_{1}, \bar{\Psi}_{1}\right]=\sum_{l m} A_{l}(t) \bar{A}_{m}(t) q_{l m}^{2} e^{i\left(\boldsymbol{k}_{l}-\boldsymbol{k}_{m}\right) \boldsymbol{x}} \\
& \hat{Q}_{3}^{\Psi}\left[\bar{\Psi}_{1}, \bar{\Psi}_{1}\right]=\sum_{l m} \bar{A}_{l}(t) \bar{A}_{m}(t) q_{l m}^{3} e^{-i\left(\boldsymbol{k}_{l}+\boldsymbol{k}_{m}\right) \boldsymbol{x}}
\end{aligned}
$$

and for the cubic term

$$
\begin{aligned}
\hat{C}_{1}^{\Psi}\left[\Psi_{1}, \Psi_{1}, \bar{\Psi}_{1}\right] & =\sum_{l m n} A_{l}(t) A_{m}(t) \bar{A}_{n}(t) c_{l m n}^{1} e^{i\left(\boldsymbol{k}_{l}+\boldsymbol{k}_{m}-\boldsymbol{k}_{n}\right) \boldsymbol{x}} \\
\hat{C}_{2}^{\Psi}\left[\Psi_{1}, \bar{\Psi}_{1}, \bar{\Psi}_{1}\right] & =\sum_{l m n} A_{l}(t) \bar{A}_{m}(t) \bar{A}_{n}(t) c_{l m n}^{2} e^{i\left(\boldsymbol{k}_{l}-\boldsymbol{k}_{m}-\boldsymbol{k}_{n}\right) \boldsymbol{x}} \\
\hat{C}_{3}^{\Psi}\left[\bar{\Psi}_{1}, \bar{\Psi}_{1}, \bar{\Psi}_{1}\right] & =\sum_{l m n} \bar{A}_{l}(t) \bar{A}_{m}(t) \bar{A}_{n}(t) c_{l m n}^{3} e^{-i\left(\boldsymbol{k}_{l}+\boldsymbol{k}_{m}+\boldsymbol{k}_{n}\right) \boldsymbol{x}} \\
\hat{C}_{4}^{\Psi}\left[\Psi_{1}, \Psi_{1}, \Psi_{1}\right] & =\sum_{l m n} A_{l}(t) A_{m}(t) A_{n}(t) c_{l m n}^{4} e^{i\left(\boldsymbol{k}_{l}+\boldsymbol{k}_{m}+\boldsymbol{k}_{n}\right) \boldsymbol{x}}
\end{aligned}
$$

with amplitude coefficients $q_{l m}^{i}, c_{l m n}^{i}$ that depend on the quadratic and cubic operators, respectively. For the quadratic terms, the projections onto the $j$ th mode selects only wave vector combinations that span a triangle, Fig. 3.8C, giving the resonance conditions

$$
\begin{aligned}
& { }_{j} \Delta_{l m}^{q 1}=e^{-i \boldsymbol{k}_{j} \boldsymbol{x}} \hat{P}_{j} e^{i\left(\boldsymbol{k}_{l}+\boldsymbol{k}_{m}\right) \boldsymbol{x}}=\delta_{l, j-\nu} \delta_{m, j+\nu}+\delta_{l, j+\nu} \delta_{m, j-\nu} \\
& { }_{j} \Delta_{l m}^{q 2}=e^{-i \boldsymbol{k}_{j} \boldsymbol{x}} \hat{P}_{j} e^{i\left(\boldsymbol{k}_{l}-\boldsymbol{k}_{m}\right) \boldsymbol{x}}=\delta_{l, j-\nu} \delta_{m, j-2 \nu}+\delta_{l, j+\nu} \delta_{m, j+2 \nu} \\
& { }_{j} \Delta_{l m}^{q 3}=e^{-i \boldsymbol{k}_{j} \boldsymbol{x}} \hat{P}_{j} e^{-i\left(\boldsymbol{k}_{l}+\boldsymbol{k}_{m}\right) \boldsymbol{x}}=\delta_{l, j+2 \nu} \delta_{m, j-2 \nu}+\delta_{l, j-2 \nu} \delta_{m, j+2 \nu}
\end{aligned}
$$


with $\nu=n / 3$. For the cubic terms, the projections onto the $j$ th mode selects only wave vector combinations that span a parallelogram, Fig. 3.8C, giving the resonance condition

$$
\begin{aligned}
{ }_{j} \square_{l m n}^{c 1} & =e^{-i \boldsymbol{k}_{j} \boldsymbol{x}} \hat{P}_{j} e^{i\left(\boldsymbol{k}_{l}+\boldsymbol{k}_{m}-\boldsymbol{k}_{n}\right) \boldsymbol{x}} \\
& =\delta_{j m} \delta_{l n}\left(1-\frac{\delta_{j l}}{2}\right)+\delta_{j l} \delta_{m n}\left(1-\frac{\delta_{j n}}{2}\right)+\delta_{j n^{-}} \delta_{m l^{-}}\left(1-\delta_{j l}-\delta_{j l^{-}}\right) \\
{ }_{j} \square_{l m n}^{c 2} & =e^{-i \boldsymbol{k}_{j} \boldsymbol{x}} \hat{P}_{j} e^{i\left(\boldsymbol{k}_{l}-\boldsymbol{k}_{m}-\boldsymbol{k}_{n}\right) \boldsymbol{x}}={ }_{j} \square_{l m}^{c 1} n \\
{ }_{j} \square_{l m n}^{c 3} & =e^{-i \boldsymbol{k}_{j} \boldsymbol{x}} \hat{P}_{j} e^{-i\left(\boldsymbol{k}_{l}+\boldsymbol{k}_{m}+\boldsymbol{k}_{n}\right) \boldsymbol{x}}={ }_{j} \square_{l^{-} m^{-} n}^{c 1} \\
{ }_{j} \square_{l m n}^{c 4} & =e^{-i \boldsymbol{k}_{j} \boldsymbol{x}} \hat{P}_{j} e^{i\left(\boldsymbol{k}_{l}+\boldsymbol{k}_{m}+\boldsymbol{k}_{n}\right) \boldsymbol{x}}={ }_{j} \square_{l m n^{-}}^{c 1} .
\end{aligned}
$$

The full amplitude equations are then given by

$$
\begin{aligned}
\partial_{t} A_{j}= & A_{j}+\alpha_{2} \bar{A}_{j^{-}}+q_{1} A_{j-\nu} A_{j+\nu}+q_{2}\left(A_{j+\nu} \bar{A}_{j+2 \nu}+A_{j-\nu} \bar{A}_{j-2 \nu}\right)+q_{3} \bar{A}_{j-2 \nu} \bar{A}_{j+2 \nu} \\
& +A_{j} \sum_{k=0}^{2 n-1} g_{j k}^{1}\left|A_{k}\right|^{2}+\bar{A}_{j^{-}} \sum_{k=0}^{2 n-1} g_{j k}^{2} A_{k} A_{k^{-}}+A_{j} \sum_{k=0}^{2 n-1} f_{j k}^{1} \bar{A}_{k^{-}} \bar{A}_{k}+\bar{A}_{j^{-}} \sum_{k=0}^{2 n-1} f_{j k}^{2}\left|A_{k}\right|^{2} \\
& +\bar{A}_{j^{-}} \sum_{k=0}^{2 n-1} h_{j k} \bar{A}_{k^{-}} \bar{A}_{k}+A_{j} \sum_{k=0}^{2 n-1} s_{j k} A_{k^{-}} A_{k},
\end{aligned}
$$

where the temporal dependence of the amplitudes $A_{j}(t)$ is omitted for the sake of brevity. The quadratic coefficients are given by

$$
q_{1}=-2\left(\beta_{1}+i \zeta_{1}\right), \quad q_{2}=\beta_{2}-\beta_{1}+i\left(\zeta_{1}-\zeta_{2}\right), \quad q_{3}=2\left(\beta_{2}+i \zeta_{2}\right)
$$

and the cubic coefficients in terms of the coefficients by

$$
\begin{aligned}
g_{j k}^{1} & =\left(1-\frac{\delta_{j k}}{2}\right)\left(c_{k j k}^{1}+c_{j k k}^{1}\right) \\
g_{j k}^{2} & =\left(1-\delta_{j k}-\delta_{j k^{-}}\right) c_{k k^{-} j^{-}}^{1} \\
f_{j k}^{1} & =\left(1-\frac{\delta_{j k}}{2}\right) c_{j k^{-} k}^{2} \\
f_{j k}^{2} & =\left(1-\frac{\delta_{j k}}{2}\right) c_{k j^{-} k}^{2}+\left(1-\delta_{j k}-\delta_{j k^{-}}\right) c_{k k j^{-}}^{2} \\
h_{j k}= & \left(1-\frac{\delta_{j k}}{2}\right)\left(c_{k j^{-} k^{-}}^{3}+c_{j^{-} k^{-} k}^{3}\right)+\left(1-\delta_{j k}-\delta_{j k^{-}}\right) c_{k k^{-} j^{-}}^{3} \\
s_{j k}= & \left(1-\frac{\delta_{j k}}{2}\right)\left(c_{k j k^{-}}^{4}+c_{j k^{-} k}^{4}\right)+\left(1-\delta_{j k}-\delta_{j k^{-}}\right) c_{k k^{-} j}^{4}
\end{aligned}
$$


that gives the final angle-interaction matrices of

$$
\begin{aligned}
& g_{j k}^{1}=\left(1-\frac{\delta_{j k}}{2}\right)\left(2\left(\gamma_{6}-i \eta_{1}\right)-\left(\gamma_{1}+3 \gamma_{2}\right)+4\left(\gamma_{1}+\gamma_{2}\right) \tilde{K}\left(k_{c}\right)-\right. \\
&\left.2 \gamma_{1} \tilde{K}\left(\left\|\boldsymbol{k}_{j}+\boldsymbol{k}_{k}\right\|\right)-\left(\gamma_{1}+\gamma_{2}\right) \tilde{K}\left(\left\|\boldsymbol{k}_{j}-\boldsymbol{k}_{k}\right\|\right)\right) \\
& g_{j k}^{2}=\left(1-\delta_{j k}-\delta_{j k^{-}}\right)\left(\left(\gamma_{6}-i \eta_{1}\right)-\left(\gamma_{1}+\gamma_{2}\right)+2\left(\gamma_{1}+\gamma_{2}\right) \tilde{K}\left(k_{c}\right)-\right. \\
&\left.\frac{\gamma_{1}+\gamma_{2}}{2}\left(\tilde{K}\left(\left\|\boldsymbol{k}_{j}-\boldsymbol{k}_{k}\right\|\right)+\tilde{K}\left(\left\|\boldsymbol{k}_{j}+\boldsymbol{k}_{k}\right\|\right)\right)\right) \\
& f_{j k}^{1}=\left(1-\frac{\delta_{j k}}{2}\right)\left(\gamma_{5}+i \eta_{2}+\gamma_{3}-2 \gamma_{3} \tilde{K}\left(k_{c}\right)+\gamma_{3} \tilde{K}\left(\left\|\boldsymbol{k}_{j}+\boldsymbol{k}_{k}\right\|\right)\right) \\
& f_{j k}^{2}=\left(1-\delta_{j k}-\delta_{j k^{-}}\right)\left(\gamma_{5}+i \eta_{2}-2 \gamma_{3} \tilde{K}\left(k_{c}\right)+2 \gamma_{3}\right) \\
& h_{j k}=3\left(1-\frac{\delta_{j k}}{2}-\frac{\delta_{j k^{-}}}{2}\right)\left(\gamma_{4}+i \eta_{1}\right) \\
& s_{j k}=3\left(1-\frac{\delta_{j k}}{2}-\frac{\delta_{j k^{-}}}{2}\right)\left(\gamma_{7}-i \eta_{2}\right) .
\end{aligned}
$$

\section{Shift- and conjugation-symmetric model}

Orientation field dynamics can be constrained by symmetry assumptions of the dynamics. If orientation field dynamics are symmetric under a phase shift $\phi$ and conjugation, the operator $\hat{F}^{\Psi}[\Psi]$ must be equivariant to these symmetries, that is

$$
\hat{F}^{\Psi}\left[e^{i \phi} \Psi\right]=e^{i \phi} \hat{F}^{\Psi}[\Psi] \quad \text { and } \quad \hat{F}^{\Psi}[\bar{\Psi}]=\overline{\hat{F}^{\Psi}[\Psi]}
$$

Shift-symmetry leads to a vanishing of all operators that if multiplied by $\Psi$ have not the same number of $\Psi$ and $\bar{\Psi}$ arguments. In our case, only odd operators $\hat{L}_{1}^{\Psi}[\Psi]$ and $\hat{C}_{1}^{\Psi}[\Psi, \Psi, \bar{\Psi}]$ fulfill this criterion. Conjugation symmetry leads to a vanishing of complex parameters in the operators and gives the amplitude equations

$$
\partial_{t} A_{j}=A_{j}+A_{j} \sum_{k=0}^{2 n-1} g_{j k}^{1}\left|A_{k}\right|^{2}+\bar{A}_{j^{-}} \sum_{k=0}^{2 n-1} g_{j k}^{2} A_{k} A_{k^{-}},
$$

where the temporal dynamics are again omitted. The angle-interaction matrices are given by

$$
\begin{aligned}
& g_{j k}^{1}=\gamma_{1}\left(1-\frac{\delta_{j k}}{2}\right)\left(2 \gamma_{6} / \gamma_{1}-(6 \chi-2)+8 \chi \tilde{K}\left(k_{c}\right)-2\left(\tilde{K}\left(\left\|\boldsymbol{k}_{j}+\boldsymbol{k}_{k}\right\|\right)+\chi \tilde{K}\left(\left\|\boldsymbol{k}_{j}-\boldsymbol{k}_{k}\right\|\right)\right)\right) \\
& g_{j k}^{2}=\gamma_{1}\left(1-\delta_{j k}-\delta_{j k^{-}}\right)\left(\gamma_{6} / \gamma_{1}-2 \chi+4 \chi \tilde{K}\left(k_{c}\right)-\chi\left(\tilde{K}\left(\left\|\boldsymbol{k}_{j}-\boldsymbol{k}_{k}\right\|\right)+\tilde{K}\left(\left\|\boldsymbol{k}_{j}+\boldsymbol{k}_{k}\right\|\right)\right)\right)
\end{aligned}
$$

with the permutation-symmetry breaking coefficient $2 \chi=1+\gamma_{2} / \gamma_{1}$. For $\chi=1$ the cubic operator $\hat{C}_{1}^{\Psi}[\Psi, \Psi, \bar{\Psi}]$ can be written as permutation symmetric in its field arguments, i.e. $\hat{C}_{1}^{\Psi}\left[\Psi_{1}, \Psi_{2}, \bar{\Psi}_{3}\right]=\hat{C}_{1}^{\Psi}\left[\Psi_{3}, \Psi_{1}, \bar{\Psi}_{2}\right]$ and belongs to the universality class [128]. As the amplitude of the angle-interaction matrices can be scaled out by rescaling the amplitudes $A_{j} \rightarrow A_{j} / \sqrt{\gamma_{1}}$, only the shape of the matrices is relevant for pattern selection. Therefore, the angle-interaction 
matrices can be simplified to

$$
\begin{aligned}
& g_{j k}^{1}=\left(1-\frac{\delta_{j k}}{2}\right)\left(2 \gamma_{6} / \gamma_{1}-(6 \chi-2)+8 \chi \tilde{K}\left(k_{c}\right)-2\left(\tilde{K}\left(\left\|\boldsymbol{k}_{j}+\boldsymbol{k}_{k}\right\|\right)+\chi \tilde{K}\left(\left\|\boldsymbol{k}_{j}-\boldsymbol{k}_{k}\right\|\right)\right)\right) \\
& g_{j k}^{2}=\left(1-\delta_{j k}-\delta_{j k^{-}}\right)\left(\gamma_{6} / \gamma_{1}-2 \chi+4 \chi \tilde{K}\left(k_{c}\right)-\chi\left(\tilde{K}\left(\left\|\boldsymbol{k}_{j}-\boldsymbol{k}_{k}\right\|\right)+\tilde{K}\left(\left\|\boldsymbol{k}_{j}+\boldsymbol{k}_{k}\right\|\right)\right)\right)
\end{aligned}
$$

leaving only two relevant parameter $\gamma_{6} / \gamma_{1}$ and $\chi$ for pattern selection.

\subsubsection{Symmetry-confined canonical genetic network}

Confining the canonical genetic network by symmetries will be called symmetry-confined canonical genetic network. An advantage of this example genetic network is its analytical tractability. The dynamics of the orientation field are assumed to be shift- and conjugation-symmetric and the cubic operator should be permutation-symmetric in its arguments, that is

$$
\hat{F}^{\Psi}\left[e^{i \phi} \Psi\right]=e^{i \phi} \hat{F}^{\Psi}[\Psi], \quad \hat{F}^{\Psi}[\bar{\Psi}]=\overline{\hat{F}^{\Psi}[\Psi]}, \quad \hat{C}_{1}^{\Psi}\left[\Psi_{1}, \Psi_{2}, \bar{\Psi}_{3}\right]=\hat{C}_{1}^{\Psi}\left[\Psi_{3}, \Psi_{1}, \bar{\Psi}_{2}\right],
$$

respectively. As previously, shift-symmetry leads to a vanishing of all operators that if multiplied by $\Psi$ have not the same number of $\Psi$ and $\bar{\Psi}$ arguments. In our case, only odd operators $\hat{L}_{1}^{\Psi}[\Psi]$ and $\hat{C}_{1}^{\Psi}[\Psi, \Psi, \bar{\Psi}]$ fulfill this criterion. Conjugation symmetry leads to a vanishing of complex parameters in the operators. Finally, permutation symmetry of the cubic operator leads to $\gamma_{1}=\gamma_{2}$ resulting in the orientation field dynamics of

$$
\begin{aligned}
\tau_{s} \partial_{t} \Psi(\boldsymbol{x}, t)= & \hat{L}_{1}^{0}[\Psi]+r \Psi(\boldsymbol{x}, t)+\gamma_{1} K \star(\Psi(\boldsymbol{y}, t)-\Psi(\boldsymbol{x}, t))|\Psi(\boldsymbol{x}, t)-\Psi(\boldsymbol{y}, t)|^{2} \\
& +\gamma_{6} \Psi(\boldsymbol{x}, t)|\Psi(\boldsymbol{x}, t)|^{2} .
\end{aligned}
$$

and thus belongs to the universality class introduced in [128].

The total concentration field still exhibits various free parameters that lead to potentially very distinct morphogen concentration dynamics. A simple shape of total concentration dynamics can be obtained by the parameter set of section 3.5.2 leading to the total concentration dynamics

$$
\tau_{s} \partial_{t} \Sigma(\boldsymbol{x}, t)=\hat{L}^{\Sigma}[\Sigma]+\hat{Q}_{1}^{\Sigma}[\Sigma, \Sigma]+\hat{Q}_{2}^{\Sigma}[\Psi, \Psi]+\hat{C}^{\Sigma}[\Psi, \bar{\Psi}, \Sigma] .
$$

with the operators

$$
\begin{aligned}
\hat{L}^{\Sigma}[\Sigma] & =\alpha_{1} \Sigma(\boldsymbol{x}, t)+\left(\left(2 \nu_{1} j^{-}+\gamma_{1} \sigma_{\Psi}^{2}\right) K+j_{u}^{-}\left(2 \nu_{1}+\frac{\gamma_{1} \sigma_{\Psi}^{2}}{j^{-}}\right) K_{-}+\nu_{1} j_{u}^{+} K_{+}\right) \star \Sigma(\boldsymbol{y}, t), \\
\hat{Q}_{1}^{\Sigma}[\Sigma, \Sigma]= & -b_{1} \Re\{\Sigma(\boldsymbol{x}, t)\}^{2}-i b_{2} \Im\{\Sigma(\boldsymbol{x}, t)\}^{2} \\
\hat{Q}_{2}^{\Sigma}[\Psi, \Psi]= & b_{1} \Re\{\Psi(\boldsymbol{x}, t)\}^{2}+i b_{2} \Im\{\Psi(\boldsymbol{x}, t)\}^{2} \\
\hat{C}^{\Sigma}[\Psi, \bar{\Psi}, \Sigma]= & \gamma_{6} \Sigma(\boldsymbol{x}, t)|\Psi(\boldsymbol{x}, t)|^{2} \\
& -K \star\left(\left(\gamma_{1}+2 \frac{j^{-} \nu_{1}}{\sigma_{\Psi}^{2}}\right) \Sigma(\boldsymbol{y}, t)+\gamma_{1} \Sigma(\boldsymbol{x}, t)\right)|\Psi(\boldsymbol{x}, t)-\Psi(\boldsymbol{y}, t)|^{2} .
\end{aligned}
$$

The additional parameters are given by

$$
\omega_{2}^{3}=\omega_{4}^{3}=0, \quad \omega_{2}^{1}=\omega_{3}^{10}=\gamma_{6}, \quad b_{1}=-\kappa_{2}^{1} / 2, \quad b_{2}=-\kappa_{4}^{3} / 2 .
$$

The fixed points of the total concentration field in the absence of the orientation field is given by

$$
\Sigma_{1}=0, \quad \Sigma_{2}=\frac{a_{1}}{b_{1}}, \quad \Sigma_{3}=i \frac{a_{1}}{b_{2}}, \quad \Sigma_{4}=\frac{a_{1}}{b_{1}}+i \frac{a_{1}}{b_{2}},
$$

with $a_{1}=\hat{L}^{\Sigma}[1]$ and where only the last fixed point retains stability if $r>0$, see section 3.5.2. 


\section{Amplitude equations}

Amplitude equations of the symmetry-confined canonical genetic network can be derived by setting $\chi=1$ in the shift- and conjugation-symmetric model of Eq. (3.114) that leads to

$$
\partial_{t} A_{j}(t)=A_{j}(t)+A_{j}(t) \sum_{k=0}^{2 n-1} g_{j k}^{1}\left|A_{k}(t)\right|^{2}+\bar{A}_{j^{-}}(t) \sum_{k=0}^{2 n-1} g_{j k}^{2} A_{k}(t) A_{k^{-}}(t)
$$

with the simplified angle-interaction matrices

$$
g_{j k}^{1}=\left(1-\frac{\delta_{j k}}{2}\right) g\left(\alpha_{j k}\right), \quad \quad g_{j k}^{2}=\left(1-\delta_{j k}-\delta_{j k^{-}}\right) \frac{g\left(\alpha_{j k}\right)}{2}
$$

and the angle-interaction function

$$
g\left(\alpha_{j k}\right)=2 \gamma_{1}\left(\frac{\gamma_{6}}{\gamma_{1}}-2+4 \tilde{K}\left(k_{c}\right)-\tilde{K}\left(k_{c} \sqrt{2\left(1+\cos \alpha_{j k}\right)}\right)-\tilde{K}\left(k_{c} \sqrt{2\left(1-\cos \alpha_{j k}\right)}\right)\right)
$$

with $\alpha_{j k}=\pi(j-k) / n$. Notice that the angle-interaction function is $\pi$ periodic, i.e. $g(\alpha+\pi)=$ $g(\alpha)$. The amplitude dynamics can be written as gradient descend dynamics with the energy function

$$
E(t)=-\sum_{i=0}^{2 n-1}\left|A_{i}(t)\right|^{2}-\frac{1}{2} \sum_{i, j=0}^{2 n-1}\left(\left|A_{i}(t)\right|^{2} g_{i j}^{1}\left|A_{j}(t)\right|^{2}+A_{i}(t) A_{i^{-}}(t) g_{i j}^{2} \bar{A}_{j}(t) \bar{A}_{j^{-}}(t)\right)
$$

The amplitude dynamics are then given by the functional derivative of the energy functional

$$
\partial_{t} A_{j}(t)=-\frac{\partial E(t)}{\partial \bar{A}_{j}(t)}
$$

where the functional derivative with respect to the complex amplitude $\bar{A}_{j}(t)$ denotes the Wirtinger derivative. Thus, the amplitude dynamics minimize the energy functional

$$
\mathrm{d} E(t)=\sum_{j=0}^{2 n-1}\left(\frac{\partial E(t)}{\partial A_{j}(t)} \partial_{t} A_{j}(t) \mathrm{d} t+\frac{\partial E(t)}{\partial \bar{A}_{j}(t)} \partial_{t} \bar{A}_{j}(t) \mathrm{d} t\right)=-2 \sum_{j=0}^{2 n-1}\left|\frac{\partial E(t)}{\partial A_{j}(t)}\right|^{2} \mathrm{~d} t \leq 0 .
$$

\section{Stationary orientation field solutions}

The symmetry-confined amplitude dynamics allow us to derive closed-form solutions. In particular, permutation-symmetry guarantees that visual scotoma solutions, that is orientation fields with only two orientations, become unstable. In order to derive the closed-form solutions of essentially complex planforms (ECP), we introduce the amplitude representation

$$
\Psi(\boldsymbol{x}, t)=\sum_{j=0}^{n-1} A_{j}^{+}(t) e^{i l_{j} \boldsymbol{k}_{j} \boldsymbol{x}}+\sum_{j=0}^{n-1} A_{j}^{-}(t) e^{-i l_{j} \boldsymbol{k}_{j} \boldsymbol{x}}
$$

with $l_{j} \in\{-1,1\}$ and $\boldsymbol{k}_{j}=k_{c}(\cos \pi j / n, \sin \pi j / n)^{T}$. Temporal dependence of the amplitudes will be omitted in the following for the sake of brevity. The amplitude equations of the active $A_{j}^{+}$and inactive $A_{j}^{-}$modes are obtained by plugging the representation into Eq. (3.128) that gives

$$
\partial_{t} A_{j}^{ \pm}=A_{j}^{ \pm}+A_{j}^{ \pm}\left(\sum_{j=0}^{n-1} g_{j k}^{1}\left|A_{k}^{ \pm}\right|^{2}+\sum_{j=0}^{n-1} g_{j k}^{1}\left(1+\delta_{j k}\right)\left|A_{j}^{\mp}\right|^{2}\right)+2 \bar{A}_{j}^{\mp} \sum_{j=0}^{n-1} g_{j k}^{2} A_{k}^{+} A_{k}^{-} .
$$


A

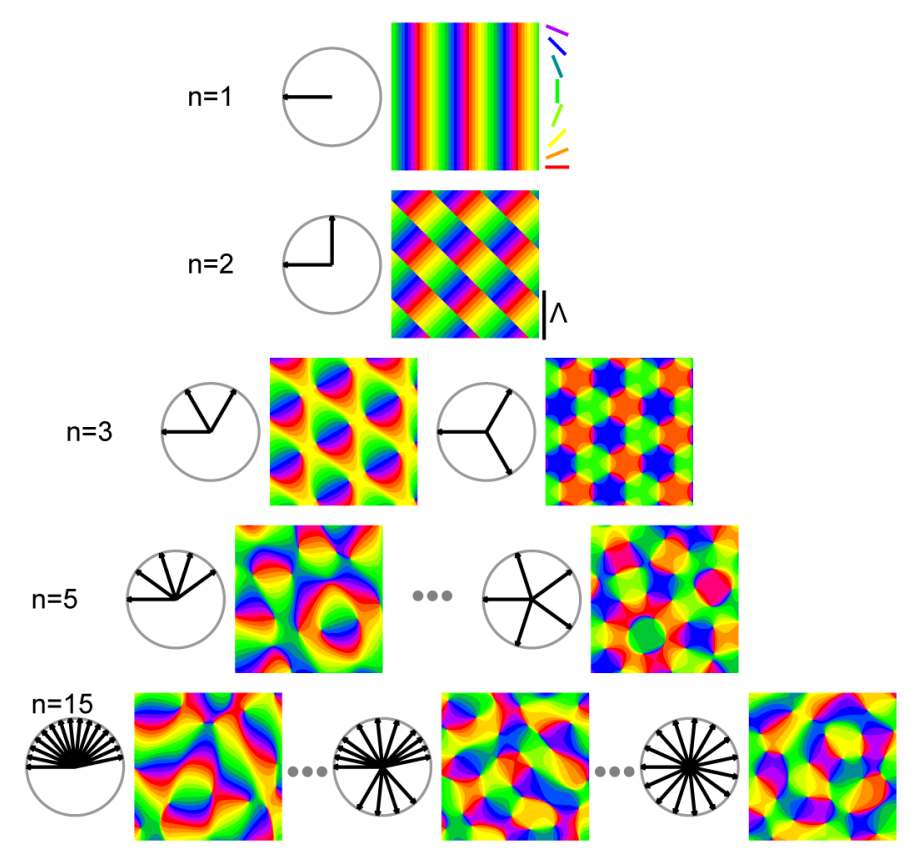

B

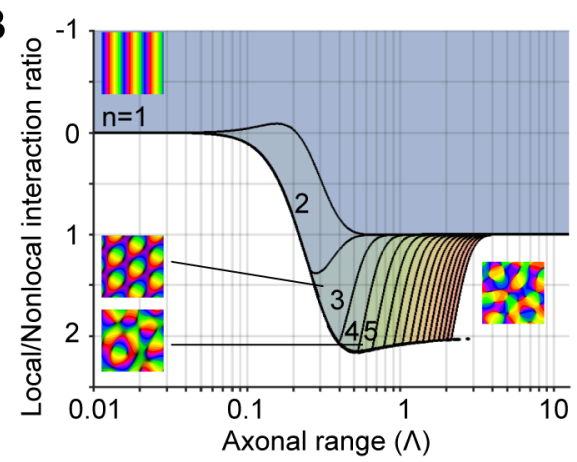

C

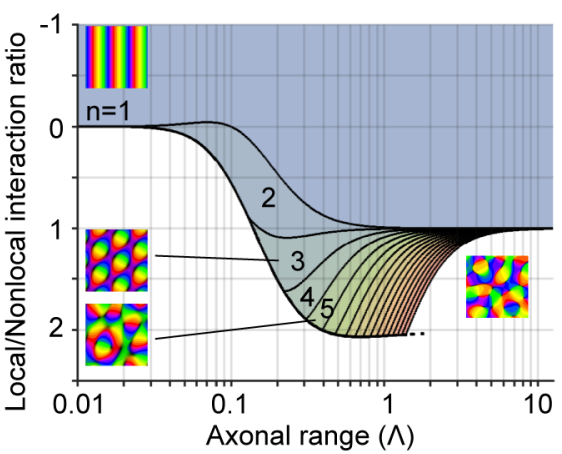

Figure 3.9: Essentially complex planform solutions and their minimal energy phase space diagram. A The essentially complex planform (ECP) solution set ordered by the number of active modes $n$, see Eq. (3.134) and Eq. (3.138). Circle with arrows represent the wave vectors of active modes in Fourier space. Orientation fields are synthesized examples of the wave vectors with random phases. B, C Phase diagram of minimal energy regions of ECPs solutions (see Eq. (3.139)) for a Gaussian and exponential synaptic kernel $K(\|\boldsymbol{x}\|)$, respectively. The axonal range $\sigma$ is given in units of the typical scale $\Lambda$ and the local/nonlocal interaction ratio by $\gamma_{6} / \gamma_{1}$.

Decomposing the amplitudes into a magnitude and a phase $A_{j}^{ \pm} \rightarrow \mathcal{A}_{j}^{ \pm} e^{i \phi_{j}^{ \pm}}$leads to the dynamics

$$
\begin{aligned}
\partial_{t} \mathcal{A}_{j}^{ \pm}= & \mathcal{A}_{j}^{ \pm}+\mathcal{A}_{j}^{ \pm}\left(\sum_{j=0}^{n-1} g_{j k}^{1} \mathcal{A}_{k}^{ \pm 2}+\sum_{j=0}^{n-1} g_{j k}^{1}\left(1+\delta_{j k}\right) \mathcal{A}_{j}^{\mp 2}\right) \\
& +2 \mathcal{A}_{j}^{\mp} \sum_{j=0}^{n-1} g_{j k}^{2} \mathcal{A}_{k}^{+} \mathcal{A}_{k}^{-} \cos \left(\phi_{k}^{+}+\phi_{k}^{-}-\phi_{j}^{+}-\phi_{j}^{-}\right) \\
\mathcal{A}_{j}^{ \pm} \partial_{t} \phi_{j}^{ \pm}= & 2 \mathcal{A}_{j}^{\mp} \sum_{j=0}^{n-1} g_{j k}^{2} \mathcal{A}_{k}^{+} \mathcal{A}_{k}^{-} \sin \left(\phi_{k}^{+}+\phi_{k}^{-}-\phi_{j}^{+}-\phi_{j}^{-}\right) .
\end{aligned}
$$

Assuming that the inactive modes vanish $\mathcal{A}_{j}^{-}=0$ and that the active modes have a uniform, non-vanishing amplitude $\mathcal{A}_{j}^{+}=\mathcal{A}$ gives the amplitude

$$
\mathcal{A}^{2}=-\left(\sum_{j=0}^{n-1} g_{0 k}^{1}\right)^{-1}
$$

with degenerated phases $\phi_{k}^{+}+\phi_{k}^{-}=\Phi_{0}$. The closed-form solutions are depicted in Fig. 3.9A and are termed essentially complex planforms (ECP). The energy of ECPs with $n$ active modes 
is degenerated in the phases and given by

$$
E=-\frac{1}{2} n \mathcal{A}^{2}
$$

The regions of minimal energy are depicted in Fig. 3.9B,C for a Gaussian $K(r)=$ $\exp \left(-r^{2} /\left(2 \sigma^{2}\right)\right)$ and exponential $K(r)=\exp (-r / \sigma)$ morphogen selective transport kernel, respectively.

\section{Stability of stationary orientation field}

The stability consideration of ECPs can be decomposed into three separated problems (see $[128,136,262]$ for details) as illustrated in Fig. 3.10A. The reason for the separating stability conditions is that perturbations in the active modes, inactive modes and phases mutually couple only quadratically.

Intrinsic stability Linearizing the dynamics around ECP solutions and perturbing the active mode by $\mathcal{A}_{j}^{+} \rightarrow \mathcal{A}^{+}+a_{j}^{+}$leads to dynamics of the active mode perturbation

$$
\partial_{t} a_{j}^{+}=2 \mathcal{A}^{2} \sum_{k=0}^{n-1} g_{j k}^{1} a_{k}^{+} .
$$

The stability condition is that the matrix $g_{j k}^{1}$ is negative definite.

Inactive mode stability Linearizing the dynamics around the ECP solutions and perturbing the inactive mode by $\mathcal{A}_{j}^{-} \rightarrow a_{j}^{-}$leads to the dynamics of the inactive mode perturbation

$$
\partial_{t} a_{j}^{-}=\mathcal{A}^{2} \sum_{k=0}^{n-1} g_{j k}^{1} a_{k}^{-} .
$$

The stability condition is again that the matrix $g_{j k}^{1}$ is negative definite.

Extrinsic stability Introducing an additional mode at $\boldsymbol{k}=k_{c}(\cos \alpha, \sin \alpha)^{T}$ with amplitude $b$ and linearizing the resulting dynamics around ECP solutions leads to

$$
\partial_{t} b=\left(1+\mathcal{A}^{2} \sum_{k=0}^{n-1} g\left(\alpha-\frac{\pi k}{n}\right)\right) b .
$$

The function $\sum_{k=0}^{n-1} g\left(\alpha-\frac{\pi j}{n}\right)$ is $\pi / n$ periodic and therefore stability must only be considered for the interval $[0, \pi / n)$. The function assumes its maximum for $\alpha=\pi / 2 n$ and hence the stability condition is given by

$$
1+\mathcal{A}^{2} \sum_{k=0}^{n-1} g\left(\frac{\pi}{2 n}-\frac{\pi j}{n}\right)<0 .
$$

Notice that a combination of additional modes would decouple and reduce to the problem considered here. The stability region of ECP solutions is depicted in Fig. 3.10B-D for $n=1,3,5$ active modes, respectively. For $n>1$ active modes, the stability region occupies a larger area than the minimal energy. 
A

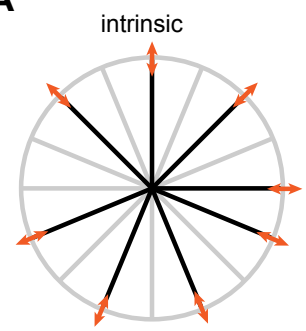

C

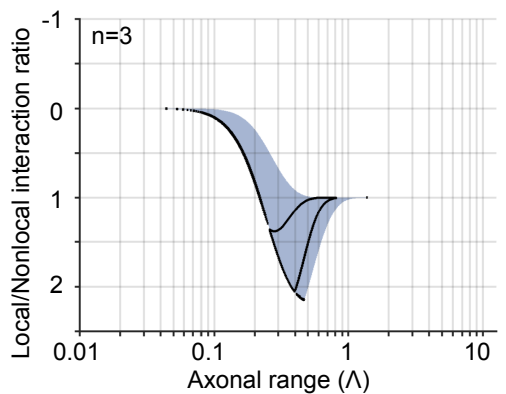

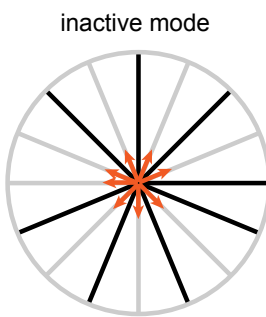

D
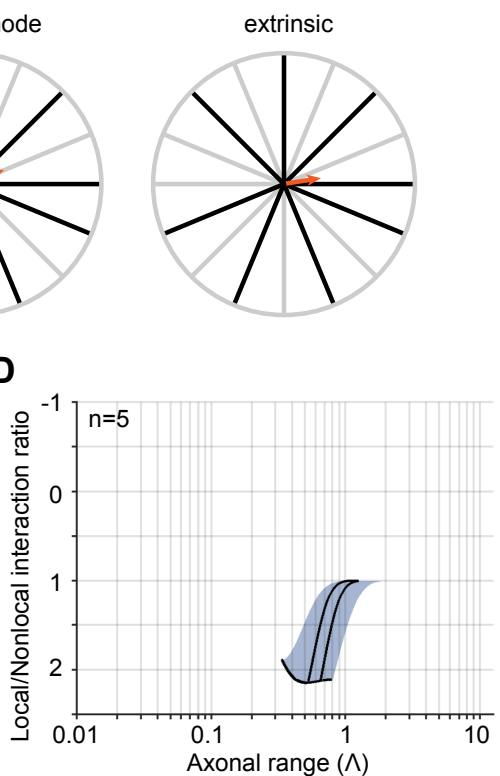

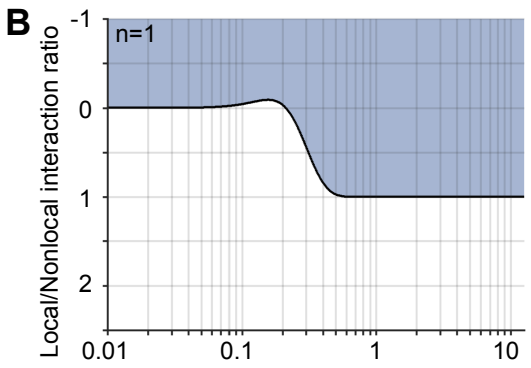

$\mathbf{E}$

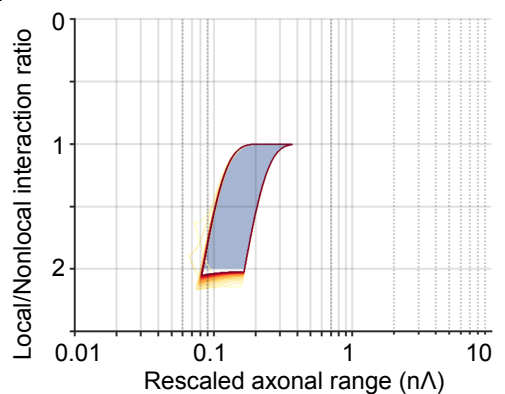

Figure 3.10: Stability of essentially complex planforms. A ECP amplitudes illustrated by radial extend of black lines. Red arrows represent the perturbations of three distinct types of instabilities: intrinsic stability, inactive mode stability and extrinsic stability. B, C, D Stability region (blue area, see text for details) of $n=1,3,5$ active modes and their minimal energy region (black lines, see Eq. (3.139) and Fig. 3.9C) for a Gaussian kernel, respectively. For $n=1$ stability and minimal energy regions coincide, whereas for larger $n$ the stability region typically overlaps the minimal energy region. The axonal range $\sigma$ is given in units of the typical scale $\Lambda$ and the local/nonlocal interaction ratio by $\gamma_{6} / \gamma_{1}$. E Asymptotic stability region (blue area, see text for details) for large $n$ together with stability boundaries of $n=4 \ldots 20$ (lines with colors from yellow over red to black for increasing $n$ ).

\section{Asymptotic stability of essentially complex planforms}

As shown in the previous section, the stability consideration can be reduced to intrinsic and extrinsic stability. Here the stability for large $n$ will be derived, which follows closely $[128,136$, 262].

For long-range transport in comparison to the typical scale $\sigma \gg \Lambda$, the angle-interaction function can be approximated by

$$
g\left(\alpha_{j k}\right)=2 \gamma_{1}\left(\frac{\gamma_{6}}{\gamma_{1}}-2-\tilde{K}\left(k_{c} \alpha_{j k}\right)-\tilde{K}\left(k_{c}\left(\pi-\alpha_{j k}\right)\right)\right) .
$$

Positive amplitude The first condition is that the amplitude must be positive that is given by

$$
0<\mathcal{A}^{2}=\left(\frac{g(0)}{2}-\sum_{j=0}^{n-1} g\left(\frac{\pi j}{n}\right)\right)^{-1} \simeq\left(\frac{g(0)}{2}-2 \gamma_{1} n\left(\frac{\gamma_{6}}{\gamma_{1}}-2\right)\right)^{-1} \simeq \frac{1}{2 \gamma_{1} n\left(2-\frac{\gamma_{6}}{\gamma_{1}}\right)} .
$$

Hence, the first condition for a large number of active modes $n$ is given by

$$
\frac{\gamma_{6}}{\gamma_{1}}<2
$$


Intrinsic stability ECPs are intrinsically stable if the eigenspectrum of the circulant matrix $g_{i j}$ is positive definite. The $l$ th eigenvalue of the angle-interaction matrix is given by

$$
\begin{aligned}
\lambda_{g}^{l} & =\sum_{j=0}^{n-1} \cos \left(\frac{2 \pi j}{n} l\right) g_{0 j} \\
& \simeq-\frac{g(0)}{2}+2 n \gamma_{1}\left(\frac{\gamma_{6}}{\gamma_{1}}-2\right) \delta_{0 l}-2 \gamma_{1} \sum_{j=0}^{n-1} \cos \left(\frac{2 \pi j}{n} l\right)\left(\tilde{K}\left(k_{c} \frac{\pi j}{n}\right)+\tilde{K}\left(k_{c} \frac{\pi}{n}(n-j)\right)\right) \\
& \simeq-\gamma_{1}\left(\frac{\gamma_{6}}{\gamma_{1}}-3\right)+2 n \gamma_{1}\left(\frac{\gamma_{6}}{\gamma_{1}}-2\right) \delta_{0 l}-2 \gamma_{1} \sum_{j=-\infty}^{\infty} \cos \left(\frac{2 \pi j}{n} l\right) \tilde{K}\left(k_{c} \frac{\pi j}{n}\right) .
\end{aligned}
$$

Thus, the eigenvalues have a maximum for $l=n / 2$ and a minimum for $l=0$. The condition for intrinsic stability is hence given by $0>\lambda_{g}^{n / 2}$ that is equivalent to

$$
\frac{\gamma_{6}}{\gamma_{1}}>3-2 \sum_{j=-\infty}^{\infty}(-1)^{j} \tilde{K}\left(k_{c} \frac{\pi j}{n}\right) \simeq 3-2\left(1-2 \tilde{K}\left(k_{c} \frac{\pi}{n}\right)\right) .
$$

Extrinsic stability For extrinsic stability the condition for stability can be rewritten as

$$
\begin{aligned}
0 & >\mathcal{A}^{-2}+\sum_{j=0}^{n-1} g\left(\frac{\pi}{2 n}(1-2 j)\right) \\
& =\frac{g(0)}{2}-\sum_{j=0}^{n-1}\left(g\left(\frac{\pi j}{n}\right)-g\left(\frac{\pi}{2 n}(1-2 j)\right)\right) \\
& \simeq \gamma_{1}\left(\frac{\gamma_{6}}{\gamma_{1}}-3\right)+2 \gamma_{1} \sum_{j=-\infty}^{\infty}\left(\tilde{K}\left(k_{c} \frac{\pi j}{n}\right)-\tilde{K}\left(k_{c} \frac{\pi}{n}\left(\frac{1}{2}-j\right)\right)\right)
\end{aligned}
$$

The final condition for extrinsic stability is hence given by

$$
\frac{\gamma_{6}}{\gamma_{1}}<3-2 \sum_{j=-\infty}^{\infty}\left(\tilde{K}\left(k_{c} \frac{\pi j}{n}\right)-\tilde{K}\left(k_{c} \frac{\pi}{n}\left(\frac{1}{2}-j\right)\right)\right) \simeq 1+2\left(2 \tilde{K}\left(k_{c} \frac{\pi}{2 n}\right)-2 \tilde{K}\left(k_{c} \frac{\pi}{n}\right)\right) .
$$

The asymptotic stability region is shown in Fig. 3.10E.

\section{Linear response of total concentration field}

For $0<r \ll 1$ the dynamics of the orientation field become arbitrary slow with a time constant of $\tau=1 / r$. Since the damping term of the dynamics is cubic, the field is of order $|\Psi| \sim \sqrt{r}$, see also weakly nonlinear analysis in section 3.5.2. As the total concentration field is coupled to a quadratic term in $\Psi$, a perturbation of $\Sigma$ is on the order of $r$. Therefore, the fields will be expanded around the homogeneous equilibrium point $(\Psi, \Sigma)=\left(0, \Sigma_{0}\right)$ as

$$
\begin{aligned}
& \Sigma(\boldsymbol{x}, t) \approx \Sigma_{0}+r \Sigma_{1}(\boldsymbol{x}, t) \\
& \Psi(\boldsymbol{x}, t) \approx \sqrt{r} \Psi(\boldsymbol{x}, t),
\end{aligned}
$$

where the equilibrium point is given by

$$
\Sigma_{0}=a_{1}\left(\frac{1}{b_{1}}+i \frac{1}{b_{2}}\right) .
$$


The dynamics of the orientation field is not altered by this transformation due to its independence of the total concentration field. The expansion of $\Sigma$ is given by

$$
\tau_{s} \partial_{t}\left(\Sigma_{0}+r \Sigma_{1}(\boldsymbol{x}, t)\right)=r(\underbrace{\hat{L}^{\Sigma}\left[\Sigma_{1}\right]+2 \hat{Q}_{1}^{\Sigma}\left[\Sigma_{1}, \Sigma_{0}\right]}_{\hat{L}^{\Sigma_{0}}}+\underbrace{\hat{Q}_{2}^{\Sigma}\left[\Psi_{1}, \Psi_{1}\right]+\hat{C}^{\Sigma}\left[\bar{\Psi}, \Psi, \Sigma_{0}\right]}_{\hat{L}^{\Sigma_{0}, \Psi}})+\mathcal{O}\left(\sqrt{r}^{3}\right)
$$

For small $r$, the equation can be solved in Fourier space by the Green function resulting in

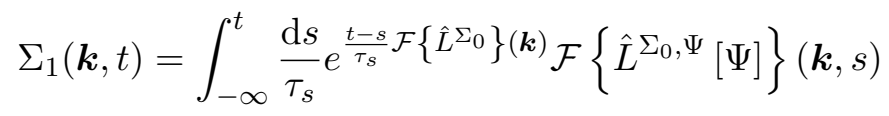

with the steady-state solution of

$$
\Sigma_{1}(\boldsymbol{x}, t)=-\left(\hat{L}^{\Sigma_{0}}\right)^{-1} \hat{L}^{\Sigma_{0}, \Psi}[\Psi] .
$$

The operators are given by

$$
\begin{aligned}
\mathcal{F}\left\{\hat{L}^{\Sigma_{0}}\right\}= & \alpha_{1}+\nu_{1}\left(2 j^{-} \tilde{K}(\|\boldsymbol{k}\|)+2 j_{u}^{-} \tilde{K}_{-}(\|\boldsymbol{k}\|)+j_{u}^{+} \tilde{K}_{+}(\|\boldsymbol{k}\|)\right) \\
& +\gamma_{1} \sigma_{\Psi}^{2}\left(\tilde{K}(\|\boldsymbol{k}\|)+\frac{j_{u}^{-}}{j^{-}} \tilde{K}_{-}(\|\boldsymbol{k}\|)\right)-2 a_{1} \\
\hat{L}^{\Sigma_{0}, \Psi}[\Psi]= & b_{1} \Re\{\Psi(\boldsymbol{x}, t)\}^{2}+i b_{2} \Im\{\Psi(\boldsymbol{x}, t)\}^{2}+\gamma_{6} \Sigma_{0}|\Psi(\boldsymbol{x}, t)|^{2} \\
& -\left(2 \gamma_{1}+2 \frac{j^{-} \nu_{1}}{\sigma_{\Psi}^{2}}\right) \Sigma_{0} K \star|\Psi(\boldsymbol{x}, t)-\Psi(\boldsymbol{y}, t)|^{2} .
\end{aligned}
$$

\subsubsection{Slow dynamic connectome}

The previously employed separation of time scales of the local regulation (on the order of $\tau_{s}>1 \mathrm{~h}$ ) and axonal rearrangement (on the order of $\tau_{m}<1 \mathrm{~h}$ ) is obviously an idealization. What morphogen dynamics can be expected if we loosen this idealization and slow down the rearrangement time of the connectome? In order to shed light on the impact of a slow connectome, morphogen dynamics of the symmetry-confined canonical genetic network together with a variable rearrangement time of the connectome will be analyzed in the following.

The connectome dynamics of the isotropic and morphogen concentration profile specific synapses are given by

$$
\begin{aligned}
\tau_{m} \partial_{t} w_{+}(\boldsymbol{x}-\boldsymbol{y}, t) & =w_{+}^{\infty}(\boldsymbol{x}-\boldsymbol{y})-w_{+}(\boldsymbol{x}-\boldsymbol{y}, t), \\
\tau_{m} \partial_{t} w_{-}(\boldsymbol{x}, \boldsymbol{y}, t) & =w_{-}^{\infty}(\boldsymbol{x}, \boldsymbol{y}, t)-w_{-}(\boldsymbol{x}, \boldsymbol{y}, t) .
\end{aligned}
$$

If the initial time lies sufficiently far in the past, i.e. $t_{0} \rightarrow-\infty$, the isotropic synaptic connections are already given by their asymptotic time-independent steady-state $w_{+}^{\infty}(\boldsymbol{x}-\boldsymbol{y})$. Thus, the analysis reduces to a variable rearrangement time of the morphogen selective connections

$$
\tau_{m} \partial_{t} w_{-}(\boldsymbol{x}, \boldsymbol{y}, t)=w_{-}^{\infty}(\boldsymbol{x}, \boldsymbol{y}, t)-w_{-}(\boldsymbol{x}, \boldsymbol{y}, t)
$$

with the time-dependent steady-state selective connectome

$$
w_{-}^{\infty}(\boldsymbol{x}, \boldsymbol{y}, t)=j_{u}^{-} K_{-}(\|\boldsymbol{x}-\boldsymbol{y}\|)+j^{-} K(\|\boldsymbol{x}-\boldsymbol{y}\|) e^{-\frac{|\Psi(\boldsymbol{x}, t)-\Psi(\boldsymbol{y}, t)|^{2}}{\sigma_{\Psi}^{2}}} .
$$


The dynamics of the selective connectome can be formally solved by its Green function. Thus the connectome is given by

$$
w_{-}(\boldsymbol{x}, \boldsymbol{y}, t)=\int_{-\infty}^{t} \frac{\mathrm{d} s}{\tau_{m}} e^{-\frac{t-s}{\tau_{m}}} w_{-}^{\infty}(\boldsymbol{x}, \boldsymbol{y}, s)=\hat{M}_{s}^{t} w_{-}^{\infty}(\boldsymbol{x}, \boldsymbol{y}, s),
$$

where the operator $\hat{M}_{s}^{t}$ is a short-hand notation and endows the connectivity with a memory. The assumption of a weakly anisotropic connectome leads to an approximation of the selective connectome up to second order in the orientation field

$$
\hat{M}_{s}^{t} K(\|\boldsymbol{x}-\boldsymbol{y}\|) e^{-\frac{|\Psi(\boldsymbol{x}, s)-\Psi(\boldsymbol{y}, s)|^{2}}{\sigma_{\Psi}^{2}}} \approx K(|\boldsymbol{x}-\boldsymbol{y}|)\left(1-\hat{M}_{s}^{t} \frac{|\Psi(\boldsymbol{x}, s)-\Psi(\boldsymbol{y}, s)|^{2}}{\sigma_{\Psi}^{2}}\right) .
$$

This time-dependent connectome is plugged into the field equations of the symmetry-confined canonical genetic network in Eq. (3.120), which leads to

$$
\tau_{s} \partial_{t} \Psi(\boldsymbol{x}, t)=\hat{L}[\Psi]+\hat{C}_{1}^{\text {slow }}[\Psi, \Psi, \bar{\Psi}]+\hat{C}_{2}^{\text {slow }}[\Psi, \Psi, \bar{\Psi}]
$$

with the modified nonlinear operators

$$
\begin{aligned}
& \hat{C}_{1}^{\text {slow }}[\Psi, \Psi, \bar{\Psi}]=\gamma_{1} \int \mathrm{d}^{2} y[\Psi(\boldsymbol{y}, t)-\Psi(\boldsymbol{x}, t)] K(\|\boldsymbol{x}-\boldsymbol{y}\|) \hat{M}_{s}^{t}|\Psi(\boldsymbol{x}, s)-\Psi(\boldsymbol{y}, s)|^{2} \\
& \hat{C}_{2}^{\text {slow }}[\Psi, \Psi, \bar{\Psi}]=\gamma_{6} \Psi(\boldsymbol{x}, t)|\Psi(\boldsymbol{x}, t)|^{2} .
\end{aligned}
$$

\section{Amplitude equations}

The amplitude equations can be derived by weakly nonlinear analysis as already shown in section 3.5.2. However, in the case considered here, the time dependence of the amplitudes must be taken into account. The amplitude equations are given by

$$
\begin{aligned}
\partial_{t} A_{j}(t)= & A_{j}(t)+\sum_{k=0}^{2 n-1} \tilde{g}_{j k}^{1}\left(A_{k}(t) \hat{M}_{s}^{t} A_{j}(s) \bar{A}_{k}(s)+A_{j}(t) \hat{M}_{s}^{t}\left|A_{k}(s)\right|^{2}\right) \\
& +\sum_{k=0}^{2 n-1} \tilde{g}_{j k}^{2} A_{k}(t) \hat{M}_{s}^{t} A_{k^{-}}(s) \bar{A}_{j^{-}}(s) \\
& +2 \gamma_{6} \sum_{k=0}^{2 n-1}\left(1-\delta_{j k} / 2\right) A_{j}(t)\left|A_{k}(t)\right|^{2}+\gamma_{6} \sum_{k=0}^{2 n-1}\left(1-\delta_{j k}-\delta_{j k^{-}}\right) A_{k}(t) A_{k^{-}}(t) \bar{A}_{j^{-}}(t) .
\end{aligned}
$$

with the angle-interaction matrices

$$
\tilde{g}_{j k}^{1}=\left(1-\frac{\delta_{j k}}{2}\right) \tilde{g}\left(\alpha_{j k}\right), \quad \quad \tilde{g}_{j k}^{2}=\left(1-\delta_{j k}-\delta_{j k^{-}}\right) \tilde{g}\left(\alpha_{j k}\right)
$$

and the modified angle-interaction function

$$
\begin{aligned}
\tilde{g}\left(\alpha_{j k}\right) & =\frac{1}{2} g\left(\alpha_{j k}\right)-\gamma_{6} \\
& =\gamma_{1}\left(-2+4 \tilde{K}\left(k_{c}\right)-\tilde{K}\left(k_{c} \sqrt{2\left(1+\cos \alpha_{j k}\right)}\right)-\tilde{K}\left(k_{c} \sqrt{2\left(1-\cos \alpha_{j k}\right)}\right)\right) .
\end{aligned}
$$




\section{Stationary orientation field solutions}

Transforming to the essentially complex planform representation

$$
\Psi(\boldsymbol{x}, t)=\sum_{j=0}^{n-1} A_{j}^{+}(t) e^{i l_{j} \boldsymbol{k}_{j} \boldsymbol{x}}+\sum_{j=0}^{n-1} A_{j}^{-}(t) e^{-i l_{j} \boldsymbol{k}_{j} \boldsymbol{x}}
$$

gives by the use of the $\pi$-periodicity of $\tilde{g}(\alpha)$

$$
\begin{aligned}
\partial_{t} A_{j}^{ \pm}(t)= & A_{j}^{ \pm}(t)+\sum_{k=0}^{n-1} \tilde{g}_{j k}^{1}\left(A_{k}^{ \pm}(t) \hat{M}_{s}^{t} A_{j}^{ \pm}(s) \bar{A}_{k}^{ \pm}(s)+A_{j}^{ \pm}(t) \hat{M}_{s}^{t}\left|A_{k}^{ \pm}(s)\right|^{2}\right) \\
& +\sum_{k=0}^{n-1}\left(1+\delta_{j k}\right) \tilde{g}_{j k}^{1}\left(A_{k}^{\mp}(t) \hat{M}_{s}^{t} A_{j}^{ \pm}(s) \bar{A}_{k}^{\mp}(s)+A_{j}^{ \pm}(t) \hat{M}_{s}^{t}\left|A_{k}^{\mp}(s)\right|^{2}\right) \\
& +\sum_{k=0}^{n-1} \tilde{g}_{j k}^{2}\left(A_{k}^{ \pm}(t) \hat{M}_{s}^{t} A_{k}^{\mp}(s) \bar{A}_{j}^{\mp}(s)+A_{k}^{\mp}(t) \hat{M}_{s}^{t} A_{k}^{ \pm}(s) \bar{A}_{j}^{\mp}(s)\right) \\
& +2 \gamma_{6} \sum_{k=0}^{n-1}\left(1-\delta_{j k} / 2\right) A_{j}^{ \pm}(t)\left|A_{k}^{ \pm}(t)\right|^{2}+2 \gamma_{6} \sum_{k=0}^{n-1} A_{j}^{ \pm}(t)\left|A_{k}^{\mp}(t)\right|^{2} \\
& +2 \gamma_{6} \sum_{k=0}^{n-1}\left(1-\delta_{j k}\right) A_{k}^{ \pm}(t) A_{k}^{\mp}(t) \bar{A}_{j}^{\mp}(t) .
\end{aligned}
$$

The absolute values of the amplitudes and phases can be separated by $A_{j}^{ \pm} \rightarrow \mathcal{A}_{j}^{ \pm} e^{i \phi_{j}^{ \pm}}$leading to the amplitude dynamics

$$
\begin{aligned}
\partial_{t} \mathcal{A}_{j}^{ \pm}(t)= & \mathcal{A}_{j}^{ \pm}(t)+\sum_{k=0}^{n-1} \tilde{g}_{j k}^{1} \mathcal{A}_{k}^{ \pm}(t) \hat{M}_{s}^{t} \mathcal{A}_{j}^{ \pm}(s) \mathcal{A}_{k}^{ \pm}(s) \cos \left(\phi_{k}^{ \pm}(t)-\phi_{k}^{ \pm}(s)+\phi_{j}^{ \pm}(s)-\phi_{j}^{ \pm}(t)\right) \\
& +\sum_{k=0}^{n-1} \tilde{g}_{j k}^{1} \mathcal{A}_{j}^{ \pm}(t) \hat{M}_{s}^{t} \mathcal{A}_{k}^{ \pm}(s)^{2}+\sum_{k=0}^{n-1}\left(1+\delta_{j k}\right) \tilde{g}_{j k}^{1} \mathcal{A}_{j}^{ \pm}(t) \hat{M}_{s}^{t} \mathcal{A}_{k}^{\mp}(s)^{2} \\
& +\sum_{k=0}^{n-1}\left(1+\delta_{j k}\right) \tilde{g}_{j k}^{1} \mathcal{A}_{k}^{\mp}(t) \hat{M}_{s}^{t} \mathcal{A}_{j}^{ \pm}(s) \mathcal{A}_{k}^{\mp}(s) \cos \left(\phi_{k}^{\mp}(t)-\phi_{k}^{\mp}(s)+\phi_{j}^{ \pm}(s)-\phi_{j}^{ \pm}(t)\right) \\
& +\sum_{k=0}^{n-1} \tilde{g}_{j k}^{2} \mathcal{A}_{k}^{ \pm}(t) \hat{M}_{s}^{t} \mathcal{A}_{k}^{\mp}(s) \mathcal{A}_{j}^{\mp}(s) \cos \left(\phi_{k}^{ \pm}(t)+\phi_{k}^{\mp}(s)-\phi_{j}^{\mp}(s)-\phi_{j}^{ \pm}(t)\right) \\
& +\sum_{k=0}^{n-1} \tilde{g}_{j k}^{2} \mathcal{A}_{k}^{\mp}(t) \hat{M}_{s}^{t} \mathcal{A}_{k}^{ \pm}(s) \mathcal{A}_{j}^{\mp}(s) \cos \left(\phi_{k}^{\mp}(t)+\phi_{k}^{ \pm}(s)-\phi_{j}^{\mp}(s)-\phi_{j}^{ \pm}(t)\right) \\
& +2 \gamma_{6} \sum_{k=0}^{n-1}\left(1-\delta_{j k} / 2\right) \mathcal{A}_{j}^{ \pm}(t) \mathcal{A}_{k}^{ \pm}(t)^{2}+2 \gamma_{6} \sum_{k=0}^{n-1} \mathcal{A}_{j}^{ \pm}(t) \mathcal{A}_{k}^{\mp}(t)^{2} \\
& +2 \gamma_{6} \sum_{k=0}^{n-1}\left(1-\delta_{j k}\right) \mathcal{A}_{k}^{ \pm}(t) \mathcal{A}_{k}^{\mp}(t) \mathcal{A}_{j}^{\mp}(t) \cos \left(\phi_{k}^{ \pm}(t)+\phi_{k}^{\mp}(t)-\phi_{j}^{\mp}(t)-\phi_{j}^{ \pm}(t)\right)
\end{aligned}
$$


and the phase dynamics

$$
\begin{aligned}
\mathcal{A}_{j}^{ \pm}(t) \partial_{t} \phi_{j}^{ \pm}(t)= & \sum_{k=0}^{n-1} \tilde{g}_{j k}^{1} \mathcal{A}_{k}^{ \pm}(t) \hat{M}_{s}^{t} \mathcal{A}_{j}^{ \pm}(s) \mathcal{A}_{k}^{ \pm}(s) \sin \left(\phi_{k}^{ \pm}(t)-\phi_{k}^{ \pm}(s)+\phi_{j}^{ \pm}(s)-\phi_{j}^{ \pm}(t)\right) \\
& +\sum_{k=0}^{n-1}\left(1+\delta_{j k}\right) \tilde{g}_{j k}^{ \pm} \mathcal{A}_{k}^{\mp}(t) \hat{M}_{s}^{t} \mathcal{A}_{j}^{ \pm}(s) \mathcal{A}_{k}^{\mp}(s) \sin \left(\phi_{k}^{\mp}(t)-\phi_{k}^{\mp}(s)+\phi_{j}^{ \pm}(s)-\phi_{j}^{ \pm}(t)\right) \\
& +\sum_{k=0}^{n-1} \tilde{g}_{j k}^{2} \mathcal{A}_{k}^{ \pm}(t) \hat{M}_{s}^{t} \mathcal{A}_{k}^{\mp}(s) \mathcal{A}_{j}^{\mp}(s) \sin \left(\phi_{k}^{ \pm}(t)+\phi_{k}^{\mp}(s)-\phi_{j}^{\mp}(s)-\phi_{j}^{ \pm}(t)\right) \\
& +\sum_{k=0}^{n-1} \tilde{g}_{j k}^{2} \mathcal{A}_{k}^{\mp}(t) \hat{M}_{s}^{t} \mathcal{A}_{k}^{ \pm}(s) \mathcal{A}_{j}^{\mp}(s) \sin \left(\phi_{k}^{\mp}(t)+\phi_{k}^{ \pm}(s)-\phi_{j}^{\mp}(s)-\phi_{j}^{ \pm}(t)\right) \\
& +2 \gamma_{6} \sum_{k=0}^{n-1}\left(1-\delta_{j k}\right) \mathcal{A}_{k}^{ \pm}(t) \mathcal{A}_{k}^{\mp}(t) \mathcal{A}_{j}^{\mp}(t) \sin \left(\phi_{k}^{ \pm}(t)+\phi_{k}^{\mp}(t)-\phi_{j}^{\mp}(t)-\phi_{j}^{ \pm}(t)\right) .
\end{aligned}
$$

The ECP solutions $\mathcal{A}_{j}^{+}=\mathcal{A}, \mathcal{A}_{j}^{-}=0$ and $\phi_{j}^{+}+\phi_{j}^{-}=\Phi_{0}$ are retained by a slow connectome as the memory kernel becomes $\hat{M}_{s}^{t} \rightarrow 1$ for constant arguments. The amplitudes are given by

$$
\mathcal{A}^{2}=-\left(2 \sum_{k=0}^{n-1} \tilde{g}_{j k}^{1}+2 \gamma_{6} \sum_{k=0}^{n-1}\left(1-\delta_{j k} / 2\right)\right)^{-1}=-\left(\sum_{k=0}^{n-1} g_{j k}^{1}\right)^{-1}
$$

and the phases by

$$
\phi_{k}^{+}+\phi_{k}^{-}-\left(\phi_{j}^{+}+\phi_{j}^{-}\right)=\mathbb{Z} \pi
$$

\section{Stability of linear equations with memory kernel}

The stability conditions of the ECP solutions are derived from the linearized equations that are of the type

$$
\partial_{t} \boldsymbol{a}(t)=\boldsymbol{D} \boldsymbol{a}(t)+\boldsymbol{H} \hat{M}_{s}^{t} \boldsymbol{a}(s)
$$

with $\boldsymbol{a} \in \mathbb{R}^{d}$ and $\boldsymbol{D}, \boldsymbol{H} \in \mathbb{R}^{d \times d}$. The matrices $\boldsymbol{D}$ and $\boldsymbol{H}$ are circulant and have therefore a common eigenbasis. The transformation onto these eigenbasis leads to a scalar stability problem, which is in Laplace space (or equivalently for a test mode $\|\boldsymbol{a}\| \propto e^{\lambda t}$ ) given by

$$
\lambda_{D}^{l}+\lambda_{H}^{l} \frac{1}{1+\lambda \tau_{m}}-\lambda=0,
$$

where $\lambda_{X}^{l}$ denotes the $l$ th eigenvalue of the matrix $X$. The eigenvalue $\lambda$ is given by

$$
\lambda_{1 / 2}^{l}=-\frac{\frac{1}{\tau_{m}}-\lambda_{D}^{l}}{2}\left(1 \pm \sqrt{1+4 \frac{\lambda_{D}^{l}+\lambda_{H}^{l}}{\left(\lambda_{D}^{l}-\frac{1}{\tau_{m}}\right)^{2}}}\right) .
$$

Decomposing the eigenvalue into real and imaginary part $\lambda^{l}=\alpha^{l}+i \beta^{l}$ and solving for the critical time $\tau_{c}^{l}$ and imaginary amplitude $\beta^{l}$ for which $\alpha^{l}=0$ leads to

$$
\tau_{c}^{l}=\frac{1}{\lambda_{D}^{l}}, \quad \quad \beta^{l}= \pm \sqrt{-\lambda_{D}^{l}\left(\lambda_{D}^{l}+\lambda_{H}^{l}\right)} .
$$


Hence, the system is stable if

$$
\tau_{m}<\min _{l, \tau_{c}^{l}>0}\left\{\tau_{c}^{l}\right\} \quad \text { and } \quad \max _{l}\left\{\lambda_{D}^{l}+\lambda_{H}^{l}\right\}<0 .
$$

Notice that the latter condition is exactly the same condition as previously derived for rapid axonal rearrangement $\left(\tau_{m}=0\right)$, because the eigenvalues of $\boldsymbol{D}$ and $\boldsymbol{H}$ sum up to the eigenvalue of the angle-interaction matrix $g_{i j}^{1}$ from Eq. (3.129), i.e. $\lambda_{D}^{l}+\lambda_{H}^{l}=\lambda_{g}^{l}$.

\section{Stability of stationary orientation field}

The linearized equations of a perturbation around the essentially complex planforms of $\mathcal{A}_{j}^{+}(t) \rightarrow$ $\mathcal{A}(t)+a_{j}^{+}(t)$ and $\mathcal{A}_{j}^{-}(t) \rightarrow a_{j}^{-}(t)$ decouple from the phases and are given by

$$
\partial_{t} \boldsymbol{a}^{ \pm}(t)=\boldsymbol{D}^{ \pm} \boldsymbol{a}^{ \pm}(t)+\boldsymbol{H}^{ \pm} \hat{M}_{s}^{t} \boldsymbol{a}^{ \pm}(s)
$$

for the amplitudes and

$$
\partial_{t} \boldsymbol{\varphi}^{+}(t)=\boldsymbol{D}_{\varphi}^{+} \boldsymbol{\varphi}^{+}(t)+\boldsymbol{H}_{\varphi}^{+} \hat{M}_{s}^{t} \boldsymbol{\varphi}^{+}(s)
$$

for the phases $\left(\varphi^{ \pm}\right)_{j}=\phi_{j}^{ \pm}$. The stability consideration of the phases $\varphi^{-}$can be neglected due to the vanishing amplitudes $\mathcal{A}_{j}^{-}$.

Intrinsic stability For intrinsic stability the matrices are given by

$$
\begin{aligned}
\left(\boldsymbol{D}^{+}\right)_{j k} & =\delta_{j k}\left(\frac{1}{2}+\mathcal{A}^{2} \gamma_{6}(n-2)\right)+\mathcal{A}^{2}\left(\frac{g_{j k}^{1}}{2}+3 \gamma_{6}\right) \\
\left(\boldsymbol{H}^{+}\right)_{j k} & =-\delta_{j k}\left(\frac{1}{2}+\mathcal{A}^{2} \gamma_{6}(n-2)\right)+3 \mathcal{A}^{2}\left(\frac{g_{j k}^{1}}{2}-\gamma_{6}\right) .
\end{aligned}
$$

Diagonalizing the matrices and considering the $l$ th circulant eigenbasis leads to

$$
\lambda_{D}^{l}=\frac{1}{2}+\mathcal{A}^{2} \gamma_{6}(n-2)+\mathcal{A}^{2}\left(\frac{\lambda_{g}^{l}}{2}+3 \delta_{0 l} n \gamma_{6}\right), \quad \lambda_{D}^{l}+\lambda_{H}^{l}=2 \mathcal{A}^{2} \lambda_{g}^{l},
$$

where $\lambda_{g}^{l}$ denotes the $l$ th eigenvalue of $g_{i j}^{1}$. The last stability condition of $\lambda_{D}^{l}+\lambda_{H}^{l}<0$ is hence equal to the case of rapid axonal rearrangement, i.e. $\tau_{m}=0$.

Inactive mode stability For inactive mode stability, the stability matrices are given by

$$
\begin{aligned}
\left(\boldsymbol{D}^{-}\right)_{j k} & =\delta_{j k}\left(\frac{1}{2}+\mathcal{A}^{2} \gamma_{6}(n-1)\right)+\mathcal{A}^{2}\left(\frac{g_{j k}^{1}}{2}+\gamma_{6}\right) \\
\left(\boldsymbol{H}^{-}\right)_{j k} & =-\delta_{j k}\left(\frac{1}{2}+\mathcal{A}^{2} \gamma_{6}(n-1)\right)+\mathcal{A}^{2}\left(\frac{g_{j k}^{1}}{2}-\gamma_{6}\right)
\end{aligned}
$$

with the $l$ th eigenvalues

$$
\lambda_{D}^{l}=\frac{1}{2}+\mathcal{A}^{2} \gamma_{6}(n-1)+\mathcal{A}^{2}\left(\frac{\lambda_{g}^{l}}{2}+\delta_{0 l} n \gamma_{6}\right), \quad \lambda_{D}^{l}+\lambda_{H}^{l}=\mathcal{A}^{2} \lambda_{g}^{l} .
$$

The last stability condition of $\lambda_{D}^{l}+\lambda_{H}^{l}<0$ is hence equal to the case of rapid axonal rearrangement. 
Extrinsic stability In the case of an arbitrary plane wave, the stability of the system

$$
\Psi(\boldsymbol{x}, t)=\sum_{j=1}^{n} A_{j}^{+}(t) e^{i l_{j} \boldsymbol{k}_{j} \boldsymbol{x}}+B(t) e^{i \boldsymbol{h} \boldsymbol{x}}
$$

for $B=0,\left|A_{j}\right|=\mathcal{A}$ and $\boldsymbol{h}=k_{c}(\cos \alpha, \sin \alpha)^{T}$ will be considered. The linearization around the fixed point is similar as above and given by

$$
\begin{aligned}
\partial_{t} b(t)= & \left(1+\mathcal{A}^{2} \sum_{k=0}^{n-1}\left(\frac{1}{2} g\left(\alpha-\frac{\pi k}{n}\right)-\gamma_{6}\left(1-\delta_{j k} / 2\right)\right)+2 \gamma_{6} \mathcal{A}^{2} n\right) b(t) \\
& +\mathcal{A}^{2} \sum_{k=0}^{n-1}\left(\frac{1}{2} g\left(\alpha-\frac{\pi k}{n}\right)-\gamma_{6}\left(1-\delta_{j k} / 2\right)\right) \hat{M}_{s}^{t} b(s),
\end{aligned}
$$

which leads to the stability scalars

$$
D=1+\frac{1}{2} \mathcal{A}^{2} \sum_{j=0}^{n-1} g\left(\alpha-\frac{\pi j}{n}\right)+\mathcal{A}^{2} \gamma_{6} n, \quad H=\frac{1}{2} \mathcal{A}^{2} \sum_{j=0}^{n-1} g\left(\alpha-\frac{\pi j}{n}\right)-\mathcal{A}^{2} \gamma_{6} n .
$$

For $\alpha=\pi /(2 n)$ the scalars assume their maximum and therefore only this scalar has to be evaluated.

Phase stability In contrast to the case of rapid axonal rearrangement, a slow connectome requires examination of the phase stability leading to the stability matrices

$$
\begin{aligned}
& \left(\boldsymbol{D}_{\varphi}^{+}\right)_{j k}=\mathcal{A}^{2}\left(\delta_{j k}\left(\frac{\gamma_{6}}{2}-1\right)+\frac{g_{j k}^{1}}{2}-\gamma_{6}\right) \\
& \left(\boldsymbol{H}_{\varphi}^{+}\right)_{j k}=-\left(\boldsymbol{D}_{\varphi}^{+}\right)_{j k} .
\end{aligned}
$$

The eigenvalues of the matrices are given by

$$
\lambda_{D}^{l}=\mathcal{A}^{2}\left(\left(\frac{\gamma_{6}}{2}-1\right)+\frac{\lambda_{g}^{l}}{2}-\delta_{0 l} \gamma_{6} n\right), \quad \lambda_{D}^{l}+\lambda_{H}^{l}=0 .
$$

The result of critical delays is depicted in Fig. 3.11A,B,C,F .

\section{Asymptotic stability of essentially complex planforms}

The condition for the critical delay of the rearrangement of axonal connections is given by

$$
\tau_{c}=\min _{l, \lambda_{D}^{l}>0}\left\{\frac{1}{\lambda_{D}^{l}}\right\}
$$

where $D$ is the matrix corresponding to the different stability considerations. In this section a stability criterion for the limit of large $n$ will be derived. The asymptotic stability region for rapid axonal rearrangement is retained, that is

$$
\lambda_{g}^{l}<0, \quad 1+\mathcal{A}^{2} \sum_{j=0}^{n-1} g\left(\frac{\pi}{2 n}-\frac{\pi j}{n}\right)<0,
$$

with the $l$ th eigenvalue $\lambda_{g}^{l}$ of the angle-interaction matrix $g_{j k}^{1}$. The stability region is hence restricted to $1<\gamma_{6} / \gamma_{1}<2$. As shown previously in the symmetry-confined genetic network, $\lambda_{g}^{l}$ has a minimum for $l=0$ and a maximum for $l=n / 2$. For $l \neq 0$, the eigenvalues of $\boldsymbol{D}$ are proportional to the eigenvalues $\lambda_{g}^{l}$ except for a constant shift. For $l=0$, the eigenvalue of $\boldsymbol{D}$ is additionally shifted. Since extrema are invariant to constant shifts, the maximal eigenvalue of $\boldsymbol{D}$ is for all stability conditions either given by the $l=0$ or $l=n / 2$ eigenvalue. 


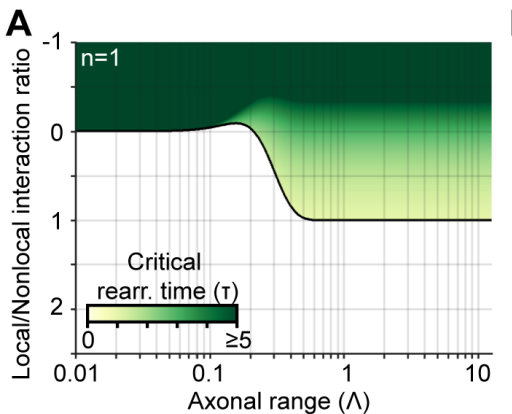

D

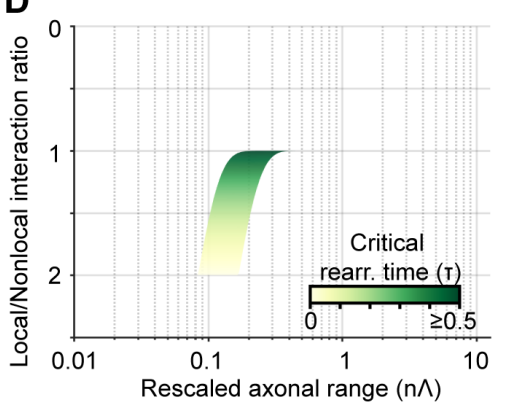

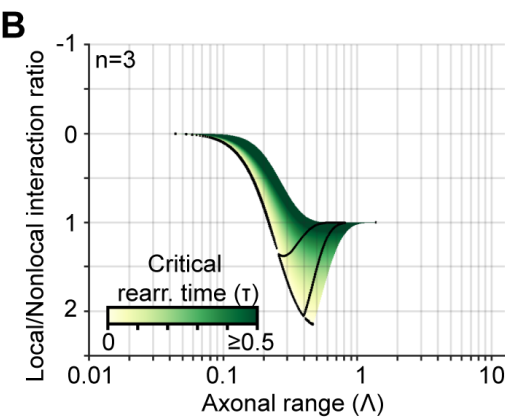

E

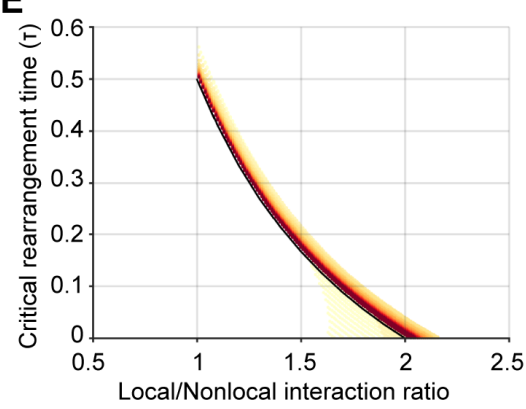

C

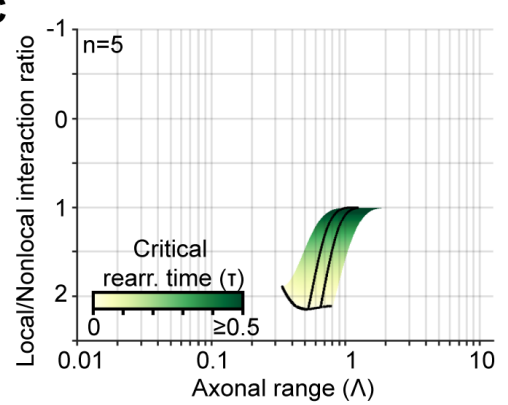

$\mathbf{F}$

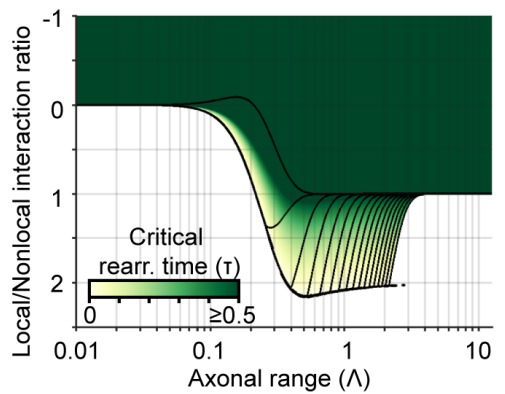

Figure 3.11: Stability of essentially complex planforms with slow dynamic connectome A, B, C Critical rearrangement times of connectome for $n=1,3,5$ active modes (see text for details) and their minimal energy region (black lines, see Eq. (3.139) and Fig. 3.9C) for a Gaussian kernel, respectively. D Asymptotic critical rearrangement times for large $n$, see Eq. (3.205). E Cross-section of asymptotic critical rearrangement time (black line, see Eq. (3.205)) for large $n$ together with critical rearrangement times of $n=4 \ldots 20$ (dots with colors from yellow over red to black for increasing $n$ ). $\mathbf{E}$ Critical rearrangement times of minimal energy ECP solutions.

Intrinsic stability The eigenvalues for intrinsic stability are given by

$$
\begin{aligned}
\lambda_{D}^{0} & =\frac{1}{2}+\mathcal{A}^{2} \gamma_{6}(n-2)+\mathcal{A}^{2}\left(\frac{\lambda_{g}^{0}}{2}+3 n \gamma_{6}\right)=\mathcal{A}^{2} \gamma_{6}(4 n-2) \simeq \frac{2 \frac{\gamma_{6}}{\gamma_{1}}}{2-\frac{\gamma_{6}}{\gamma_{1}}}, \\
\lambda_{D}^{n / 2} & =\frac{1}{2}+\mathcal{A}^{2} \gamma_{6}(n-2)+\mathcal{A}^{2} \frac{\lambda_{g}^{n / 2}}{2} \leq \frac{1}{2}+\mathcal{A}^{2} \gamma_{6}(n-2) \simeq \frac{1}{2-\frac{\gamma_{6}}{\gamma_{1}}} \leq \lambda_{D}^{0},
\end{aligned}
$$

where again the asymptotic amplitude of Eq. (3.145) was used and the fact that $\lambda_{g}^{0}=-\mathcal{A}^{-2}$.

Inactive mode stability The eigenvalues for inactive mode stability are given by

$$
\begin{aligned}
\lambda_{D}^{0} & =\frac{1}{2}+\mathcal{A}^{2} \gamma_{6}(n-1)+\mathcal{A}^{2}\left(\frac{\lambda_{g}^{0}}{2}+n \gamma_{6}\right)=\mathcal{A}^{2} \gamma_{6}(2 n-2) \simeq \frac{\frac{\gamma_{6}}{\gamma_{1}}}{2-\frac{\gamma_{6}}{\gamma_{1}}}, \\
\lambda_{D}^{n / 2} & =\frac{1}{2}+\mathcal{A}^{2} \gamma_{6}(n-1)+\mathcal{A}^{2} \frac{\lambda_{g}^{n / 2}}{2} \leq \frac{1}{2}+\mathcal{A}^{2} \gamma_{6}(n-1) \simeq \frac{1}{2-\frac{\gamma_{6}}{\gamma_{1}}} \leq \lambda_{D}^{0},
\end{aligned}
$$

where the asymptotic amplitude of Eq. (3.145) was used and the fact that $\lambda_{g}^{0}=-\mathcal{A}^{-2}$.

Extrinsic stability The eigenvalue for extrinsic stability is given by

$$
D=1+\frac{1}{2} \mathcal{A}^{2} \sum_{j=0}^{n-1} g\left(\frac{\pi}{2 n}-\frac{\pi j}{n}\right)+\mathcal{A}^{2} \gamma_{6} n, \leq \frac{1}{2}+\mathcal{A}^{2} \gamma_{6} n \simeq \frac{1}{2-\frac{\gamma_{6}}{\gamma_{1}}}
$$


Phase stability The eigenvalues for phase stability are given by

$$
\lambda_{D}^{l} \leq \mathcal{A}^{2}\left(\left(\frac{\gamma_{6}}{2}-1\right)-\delta_{0 l} \gamma_{6} n\right) \simeq-\frac{\delta_{0 l}}{2-\frac{\gamma_{6}}{\gamma_{1}}}
$$

Therefore the critical $\tau_{c}$ is given by the intrinsic instability of the zeroth mode

$$
\lambda_{D}^{0} \simeq \frac{2 \frac{\gamma_{6}}{\gamma_{1}}}{2-\frac{\gamma_{6}}{\gamma_{1}}}
$$

such that the critical delay is simply given by

$$
\tau_{c}=\frac{\gamma_{1}}{\gamma_{6}}-\frac{1}{2}
$$

The result and the comparison to the explicitly calculated critical delays is depicted in Fig. 3.11D,E.

\section{Linear response of total concentration field}

The only operator for the total concentration field dynamics that changes in the case of a slow connectome is the cubic operator

$$
\begin{aligned}
\hat{C}^{\Sigma, \text { slow }}[\Psi, \bar{\Psi}, \Sigma]= & \gamma_{6} \Sigma(\boldsymbol{x}, t)|\Psi(\boldsymbol{x}, t)|^{2}- \\
& \gamma_{1} K \star\left(\left(\gamma_{1}+2 \frac{j^{-} \nu_{1}}{\sigma_{\Psi}^{2}}\right) \Sigma(\boldsymbol{y})+\gamma_{1} \Sigma(\boldsymbol{x})\right) \hat{M}_{s}^{t}|\Psi(\boldsymbol{x}, s)-\Psi(\boldsymbol{y}, s)|^{2} .
\end{aligned}
$$

For $0<r \ll 1$ the dynamics of the $\Psi$ field become arbitrary slow with a time constant of $\tau=1 / r$. Since the damping term in the $\Psi$ dynamics are cubic, the field is of order $|\Psi| \sim \sqrt{r}$. As the $\Sigma$ field is forced by a quadratic factor in $\Psi$, a perturbation of $\Sigma$ is of the order $r$. Therefore the fields will be expanded around the equilibrium point $(\Psi, \Sigma)=\left(0, \Sigma_{0}\right)$ as

$$
\Sigma(\boldsymbol{x}, t) \approx \Sigma_{0}+r \Sigma_{1}(\boldsymbol{x}, t), \quad \Psi(\boldsymbol{x}, t) \approx \sqrt{r} \Psi(\boldsymbol{x}, t),
$$

where the equilibrium point is given by

$$
\Sigma_{0}=a_{1}\left(\frac{1}{b_{1}}+i \frac{1}{b_{2}}\right) .
$$

The equation for the $\Psi$ field are not altered by this transformation. The expansion of $\Sigma$ is given by

$$
\tau_{s} \partial_{t}\left(\Sigma_{0}+r \Sigma_{1}(\boldsymbol{x}, t)\right)=r(\underbrace{\hat{L}^{\Sigma}\left[\Sigma_{1}\right]+2 \hat{Q}_{1}^{\Sigma}\left[\Sigma_{1}, \Sigma_{0}\right]}_{\hat{L}^{\Sigma_{0}}}+\underbrace{\hat{Q}_{2}^{\Sigma}\left[\Psi_{1}, \Psi_{1}\right]+\hat{C}^{\Sigma, \text { slow }}\left[\bar{\Psi}, \Psi, \Sigma_{0}\right]}_{\hat{L}^{\Sigma_{0}, \Psi}})+\mathcal{O}\left(\sqrt{r}^{3}\right)
$$

For small $r$, the equation can be solved in Fourier space by the Green function resulting in

$$
\Sigma_{1}(\boldsymbol{k}, t)=\int_{-\infty}^{t} \frac{\mathrm{d} s}{\tau_{s}} e^{\frac{t-s}{\tau_{s}} \mathcal{F}\left\{\hat{L}^{\Sigma_{0}}\right\}(\boldsymbol{k})} \mathcal{F}\left\{\hat{L}^{\Sigma_{0}, \Psi}[\Psi]\right\}(\boldsymbol{k}, s)
$$

with the steady-state solution of

$$
\Sigma_{1}(\boldsymbol{x}, t)=-\left(\hat{L}^{\Sigma_{0}}\right)^{-1} \hat{L}^{\Sigma_{0}, \Psi}[\Psi] .
$$


The operators are given by

$$
\begin{aligned}
\mathcal{F}\left\{\hat{L}^{\Sigma_{0}}\right\}= & \alpha_{1}+\nu_{1}\left(2 j^{-} \tilde{K}(\|\boldsymbol{k}\|)+2 j_{u}^{-} \tilde{K}_{-}(\|\boldsymbol{k}\|)+j_{u}^{+} \tilde{K}_{+}(\|\boldsymbol{k}\|)\right) \\
& +\gamma_{1} \sigma_{\Psi}^{2}\left(\tilde{K}(\|\boldsymbol{k}\|)+\frac{j_{u}^{-}}{j^{-}} \tilde{K}_{-}(\|\boldsymbol{k}\|)\right)-2 a_{1} \\
\hat{L}^{\Sigma_{0}, \Psi}[\Psi]= & b_{1} \Re\{\Psi(\boldsymbol{x}, t)\}^{2}+i b_{2} \Im\{\Psi(\boldsymbol{x}, t)\}^{2}+\gamma_{6} \Sigma_{0}|\Psi(\boldsymbol{x}, t)|^{2} \\
& -\left(2 \gamma_{1}+2 \frac{j^{-} \nu_{1}}{\sigma_{\Psi}^{2}}\right) \Sigma_{0} K \star|\Psi(\boldsymbol{x}, s)-\Psi(\boldsymbol{y}, s)|^{2} .
\end{aligned}
$$

\subsubsection{Delayed axonal transport}

The neglected delay of axonal transport of about $\tau=15 \mathrm{~min}$ in comparison to the local regulation time scale $\tau_{s}>1 \mathrm{~h}$ is rather justified. Nevertheless, it is essential that the genetic network is robust against small axonal delay times. Therefore the morphogen dynamics with a delayed axonal transport of the symmetry-confined canonical genetic network is analyzed in the following.

The connectome is given by short-range isotropic and rather long-range morphogen selective connections. We assume that the delay over short-range isotropic connections is negligible. A realistic account for delays in axonal transport of velocity $v$ is given by Eq. (3.5) that gives for the individual morphogen transport

$$
\tau_{s} \partial_{t} c_{i}(\boldsymbol{x}, t) \propto \int \mathrm{d} y^{2} c_{i}\left(\boldsymbol{y}, t-\frac{\|\boldsymbol{x}-\boldsymbol{y}\|}{v}\right) w_{-}\left(\boldsymbol{x}, \boldsymbol{y}, t-\frac{\|\boldsymbol{x}-\boldsymbol{y}\|}{v}\right)
$$

and the thus retarded local protein synthesis

$$
\begin{aligned}
\tau_{f} \partial_{t} g_{c_{1}}(\boldsymbol{x}, t)= & \int \mathrm{d} y^{2} \mu_{1}^{2} c_{2}\left(\boldsymbol{y}, t-\frac{\|\boldsymbol{x}-\boldsymbol{y}\|}{v}\right) w_{-}\left(\boldsymbol{x}, \boldsymbol{y}, t-\frac{\|\boldsymbol{x}-\boldsymbol{y}\|}{v}\right) \\
& +\mu_{1}^{1} c_{1}(\boldsymbol{x}, t) \int \mathrm{d} y^{2} w_{-}(\boldsymbol{x}, \boldsymbol{y}, t)-g_{c_{1}}(\boldsymbol{x}, t) / \tau_{1} .
\end{aligned}
$$

The remaining local protein synthesis terms of Eq. (3.26) are analogously defined. The orientation field with delayed transport and rapid connectome rearrangement has then the altered third order term

$$
\partial_{t} \Psi(\boldsymbol{x}, t) \propto-\int \mathrm{d}^{2} \boldsymbol{y} \Psi\left(\boldsymbol{y}, t-\frac{\|\boldsymbol{x}-\boldsymbol{y}\|}{v}\right) w_{-}^{\infty}\left(\boldsymbol{x}, \boldsymbol{y}, t-\frac{\|\boldsymbol{x}-\boldsymbol{y}\|}{v}\right)+\Psi(\boldsymbol{x}, t) \int \mathrm{d}^{2} \boldsymbol{y} w_{-}^{\infty}(\boldsymbol{x}, \boldsymbol{y}, t),
$$

where the connectome is given by

$$
w_{-}^{\infty}(\boldsymbol{x}, \boldsymbol{y}, t)=j_{u}^{-} K_{-}(\|\boldsymbol{x}-\boldsymbol{y}\|)+j^{-} K(\|\boldsymbol{x}-\boldsymbol{y}\|) \exp \left(\frac{-|\Psi(\boldsymbol{x}, t)-\Psi(\boldsymbol{y}, t)|^{2}}{\sigma_{\Psi}^{2}}\right) .
$$

In the following calculation three assumptions will be made:

1. The delay of the long-range transport $K(\|\boldsymbol{x}\|)$ is approximated by a constant delay $\frac{\|\boldsymbol{x}-\boldsymbol{y}\|}{v} \rightarrow \tau$.

2. Only terms up to third order in $\Psi$ will be considered.

3. Range of long-range transport is larger than the typical wavelength $\exp \left(-\sigma^{2} k_{c}^{2} / 2\right) \ll 1$. 
Introducing the notation $s=t-\tau$, the first three assumptions yield the equation

$$
\begin{aligned}
\partial_{t} \Psi(\boldsymbol{x}, t)= & \hat{\tilde{L}}[\Psi]+\gamma_{6} \Psi(\boldsymbol{x}, t)|\Psi(\boldsymbol{x}, t)|^{2} \\
& -\gamma_{1} \sigma_{\Psi}^{2} \int \mathrm{d}^{2} \boldsymbol{y} \Psi(\boldsymbol{y}, s) K(\|\boldsymbol{x}-\boldsymbol{y}\|)\left(1-\frac{|\Psi(\boldsymbol{x}, s)-\Psi(\boldsymbol{y}, s)|^{2}}{\sigma_{\Psi}^{2}}\right) \\
& +\gamma_{1} \sigma_{\Psi}^{2} \Psi(\boldsymbol{x}, t) \int \mathrm{d}^{2} \boldsymbol{y} K(\|\boldsymbol{x}-\boldsymbol{y}\|)\left(1-\frac{|\Psi(\boldsymbol{x}, t)-\Psi(\boldsymbol{y}, t)|^{2}}{\sigma_{\Psi}^{2}}\right) .
\end{aligned}
$$

The linear term in $\Psi(\boldsymbol{y}, s)$ will be neglected due to assumption three. The second linear term resulting from the long-range transported will be absorbed in the linear operator by $\hat{\tilde{L}} \rightarrow \hat{L}$. The final equation is given by

$$
\partial_{t} \Psi(\boldsymbol{x}, t)=\hat{L}[\Psi]+\hat{C}_{1}^{\text {delay }}[\Psi, \Psi, \bar{\Psi}]+\hat{C}_{2}^{\text {delay }}[\Psi, \Psi, \bar{\Psi}] .
$$

The cubic operators can be decomposed to

$$
\begin{aligned}
& \hat{C}_{1}^{\text {delay }}[\Psi, \Psi, \bar{\Psi}]=\gamma_{1} \int \mathrm{d}^{2} \boldsymbol{y} \Psi(\boldsymbol{y}, s) K(\|\boldsymbol{x}-\boldsymbol{y}\|)|\Psi(\boldsymbol{x}, s)-\Psi(\boldsymbol{y}, s)|^{2} \\
& \hat{C}_{2}^{\text {delay }}[\Psi, \Psi, \bar{\Psi}]=\gamma_{6} \Psi(\boldsymbol{x}, t)|\Psi(\boldsymbol{x}, t)|^{2}-\gamma_{1} \Psi(\boldsymbol{x}, t) \int \mathrm{d}^{2} \boldsymbol{y} K(\|\boldsymbol{x}-\boldsymbol{y}\|)|\Psi(\boldsymbol{x}, t)-\Psi(\boldsymbol{y}, t)|^{2}
\end{aligned}
$$

\section{Amplitude equations}

The amplitude equations can be derived analogously to the calculation in section 3.5.2. However, the time dependence of the amplitudes must be taken into account here. The resulting amplitude equations are given by

$$
\begin{aligned}
\partial_{t} A_{j}(t)= & A_{j}(t)+A_{j}(t) \sum_{k=0}^{2 n-1} g_{j k}^{t}\left|A_{k}(t)\right|^{2}+\bar{A}_{j^{-}}(t) \sum_{k=0}^{2 n-1} f_{j k}^{t} A_{k}(t) A_{k^{-}}(t) \\
& +A_{j}(s) \sum_{k=0}^{2 n-1} g_{j k}^{s}\left|A_{k}(s)\right|^{2}+\bar{A}_{j^{-}}(s) \sum_{k=0}^{2 n-1} f_{j k}^{s} A_{k}(s) A_{k^{-}}(s)
\end{aligned}
$$

with the angle-interaction matrices

$$
\begin{aligned}
& g_{j k}^{t}=\gamma_{1}\left(1-\frac{\delta_{j k}}{2}\right)\left(-3+2 \frac{\gamma_{6}}{\gamma_{1}}+4 \tilde{K}\left(k_{c}\right)-\tilde{K}\left(\left\|\boldsymbol{k}_{j}-\boldsymbol{k}_{k}\right\|\right)\right) \\
& f_{j k}^{t}=\gamma_{1}\left(1-\delta_{j k}-\delta_{j k^{-}}\right)\left(-1+\frac{\gamma_{6}}{\gamma_{1}}+2 \tilde{K}\left(k_{c}\right)-\tilde{K}\left(\left\|\boldsymbol{k}_{j}-\boldsymbol{k}_{k}\right\|\right)\right) \\
& g_{j k}^{s}=\gamma_{1}\left(1-\frac{\delta_{j k}}{2}\right)\left(-1-\tilde{K}\left(\left\|\boldsymbol{k}_{j}-\boldsymbol{k}_{k}\right\|\right)-2 \tilde{K}\left(\left\|\boldsymbol{k}_{j}+\boldsymbol{k}_{k}\right\|\right)+4 \tilde{K}\left(k_{c}\right)\right) \\
& f_{j k}^{s}=\gamma_{1}\left(1-\delta_{j k}-\delta_{j k^{-}}\right)\left(-1-\tilde{K}\left(\left\|\boldsymbol{k}_{j}+\boldsymbol{k}_{k}\right\|\right)+2 \tilde{K}\left(k_{c}\right)\right) .
\end{aligned}
$$

Rescaling the amplitudes by $A \rightarrow A / \sqrt{\gamma_{1}}$ cancels the dependence on $\gamma_{1}$. The quotient $\gamma_{6} / \gamma_{1}$ remains for pattern selection.

\section{Stationary orientation field solutions}

The amplitude equations are transformed to the representation

$$
\Psi(\boldsymbol{x}, t)=\sum_{j=0}^{n-1} A_{j}^{+}(t) e^{i l_{j} \boldsymbol{k}_{j} \boldsymbol{x}}+\sum_{j=0}^{n-1} A_{j}^{-}(t) e^{-i l_{j} \boldsymbol{k}_{j} \boldsymbol{x}}
$$


with $l_{j} \in\{-1,1\}$ and $\boldsymbol{k}_{j}=k_{c}(\cos (\pi j / n), \sin (\pi j / n))^{T}$. The amplitude equations for this representation are given by

$$
\begin{aligned}
\partial_{t} A_{j}^{ \pm}(t)= & A_{j}^{ \pm}(t)+A_{j}^{ \pm}(t)\left(\sum_{k=0}^{n-1} g_{ \pm m_{j}, \pm m_{k}}^{t}\left|A_{k}^{ \pm}(t)\right|^{2}+\sum_{k=0}^{n-1} g_{ \pm m_{j}, \mp m_{k}}^{t}\left|A_{k}^{\mp}(t)\right|^{2}\right) \\
& +\bar{A}_{j}^{\mp}(t) \sum_{k=0}^{n-1}\left(f_{ \pm m_{j}, m_{k}}^{t}+f_{ \pm m_{j},-m_{k}}^{t}\right) A_{k}^{+}(t) A_{k}^{-}(t) \\
& +A_{j}^{ \pm}(s)\left(\sum_{k=0}^{n-1} g_{ \pm m_{j}, \pm m_{k}}^{s}\left|A_{k}^{ \pm}(s)\right|^{2}+\sum_{k=0}^{n-1} g_{ \pm m_{j}, \mp m_{k}}^{s}\left|A_{k}^{\mp}(s)\right|^{2}\right) \\
& +\bar{A}_{j}^{\mp}(s) \sum_{k=0}^{n-1}\left(f_{ \pm m_{j}, m_{k}}^{s}+f_{ \pm m_{j},-m_{k}}^{s}\right) A_{k}^{+}(s) A_{k}^{-}(s),
\end{aligned}
$$

where the short-hand notation of $\pm m_{j}=j^{ \pm l_{j}}$ was used. Separating the amplitudes and phases by $A_{j}^{ \pm} \rightarrow \mathcal{A}_{j}^{ \pm} e^{i \phi_{j}^{ \pm}}$leads to the dynamics of the amplitudes

$$
\begin{aligned}
\partial_{t} \mathcal{A}_{j}^{ \pm}(t)= & \mathcal{A}_{j}^{ \pm}(t)+\mathcal{A}_{j}^{ \pm}(t)\left(\sum_{k=0}^{n-1} g_{ \pm m_{j}, \pm m_{k}}^{t} \mathcal{A}_{k}^{ \pm}(t)^{2}+\sum_{k=0}^{n-1} g_{ \pm m_{j}, \mp m_{k}}^{t} \mathcal{A}_{k}^{\mp}(t)^{2}\right) \\
& +\mathcal{A}_{j}^{\mp}(t) \sum_{k=0}^{n-1}\left(f_{ \pm m_{j}, m_{k}}^{t}+f_{ \pm m_{j},-m_{k}}^{t}\right) \mathcal{A}_{k}^{+}(t) \mathcal{A}_{k}^{-}(t) \cos \left(\Omega_{k j}^{ \pm}(t, t)+\Omega_{k j}^{\mp}(t, t)\right) \\
& +\mathcal{A}_{j}^{ \pm}(s)\left(\sum_{k=0}^{n-1} g_{ \pm m_{j}, \pm m_{k}}^{s} \mathcal{A}_{k}^{ \pm}(s)^{2}+\sum_{k=0}^{n-1} g_{ \pm m_{j}, \mp m_{k}}^{s} \mathcal{A}_{k}^{\mp}(s)^{2}\right) \cos \left(\Omega_{j j}^{ \pm}(s, t)\right) \\
& +\mathcal{A}_{j}^{\mp}(s) \sum_{k=0}^{n-1}\left(f_{ \pm m_{j}, m_{k}}^{s}+f_{ \pm m_{j},-m_{k}}^{s}\right) \mathcal{A}_{k}^{+}(s) \mathcal{A}_{k}^{-}(s) \cos \left(\Omega_{k j}^{ \pm}(s, s)+\Omega_{k j}^{\mp}(s, t)\right)
\end{aligned}
$$

and the dynamics of phases

$$
\begin{aligned}
\mathcal{A}_{j}^{ \pm}(t) \partial_{t} \phi_{j}^{ \pm}(t)= & \mathcal{A}_{j}^{\mp}(t) \sum_{k=0}^{n-1}\left(f_{ \pm m_{j}, m_{k}}^{t}+f_{ \pm m_{j},-m_{k}}^{t}\right) \mathcal{A}_{k}^{+}(t) \mathcal{A}_{k}^{-}(t) \sin \left(\Omega_{k j}^{ \pm}(t, t)+\Omega_{k j}^{\mp}(t, t)\right) \\
& +\mathcal{A}_{j}^{ \pm}(s)\left(\sum_{k=0}^{n-1} g_{ \pm m_{j}, \pm m_{k}}^{s} \mathcal{A}_{k}^{ \pm}(s)^{2}+\sum_{k=0}^{n-1} g_{ \pm m_{j}, \mp m_{k}}^{s} \mathcal{A}_{k}^{\mp}(s)^{2}\right) \sin \left(\Omega_{j j}^{ \pm}(s, t)\right) \\
& +\mathcal{A}_{j}^{\mp}(s) \sum_{k=0}^{n-1}\left(f_{ \pm m_{j}, m_{k}}^{s}+f_{ \pm m_{j},-m_{k}}^{s}\right) \mathcal{A}_{k}^{+}(s) \mathcal{A}_{k}^{-}(s) \sin \left(\Omega_{k j}^{ \pm}(s, s)+\Omega_{k j}^{\mp}(s, t)\right)
\end{aligned}
$$

with the abbreviation $\Omega_{j k}^{ \pm}(s, t)=\phi_{j}^{ \pm}(s)-\phi_{k}^{\mp}(t)$. The fixed point of $\mathcal{A}_{j}^{+}=\mathcal{A}$ and $\mathcal{A}_{j}^{-}=0$ is given by

$$
\mathcal{A}^{2}=-\left(\sum_{k=0}^{n-1} g_{m_{j}, m_{k}}^{t}+g_{m_{j}, m_{k}}^{s}\right)^{-1}=-\left(\sum_{k=0}^{n-1} g_{0 k}^{1}\right)^{-1}
$$

and the phases are

$$
\phi_{k}^{+}+\phi_{k}^{-}-\left(\phi_{j}^{+}+\phi_{j}^{-}\right)=\mathbb{Z} \pi
$$

Note that the function $g_{j k}=g_{m_{j}, m_{k}}^{t}+g_{m_{j}, m_{k}}^{s}$ is $\pi$-periodic and hence the fixed points are independent of the choice of $l_{j}$. 


\section{Stability of linear delayed differential equations}

The general type of linear equation that have to be analyzed is

$$
\partial_{t} \boldsymbol{a}(t)=\boldsymbol{D} \boldsymbol{a}(t)+\boldsymbol{H a}(s) .
$$

Plugging in the ansatz $\boldsymbol{a}(t)=\boldsymbol{v} e^{\lambda t}$ or equivalently applying a Laplace transform to the equation leads to

$$
0=\left(-\lambda \mathbf{1}+\boldsymbol{D}+\boldsymbol{H} e^{-\lambda \tau}\right) \boldsymbol{v}
$$

The stability criterion of the equation is given by $\operatorname{det}\left(-\lambda \mathbf{1}+\boldsymbol{D}+\boldsymbol{H} e^{-\lambda \tau}\right)=0$ only for $\lambda$ with negative real part, that is $\Re\{\lambda\}<0$. The equation has typically infinitely many solutions for $\lambda$ and therefore the simplified case of a scalar equation will be considered.

$$
0=-\lambda+D+H e^{-\lambda \tau} .
$$

Decomposing this equation into real and imaginary part, where $\lambda=\alpha+i \beta$ and solving for the delay time for which $\alpha=0$ leads to the critical delay time of

$$
\tau_{c}=-\frac{\cos ^{-1}\left(-\frac{D}{H}\right)+2 \mathbb{Z} \pi}{H \sqrt{1-\left(\frac{D}{H}\right)^{2}}} .
$$

In general, the transformation of $\boldsymbol{D}$ and $\boldsymbol{H}$ to a common eigenbasis is not possible for delayed transport. In contrast to the previously discussed cases, $\boldsymbol{D}$ and $\boldsymbol{H}$ are not circulant matrices, because the angle-interaction function is not $\pi$-periodic. However, the special case of isotropic ECP solutions with $l_{j}=(-1)^{j}$ and $n=2 \mathbb{Z}+1$ transforms the matrices $\boldsymbol{D}$ and $\boldsymbol{H}$ of the intrinsic and inactive mode suppression discussion to circulant matrices. For circulant matrices the eigenvectors of $\boldsymbol{D}$ and $\boldsymbol{H}$ are identical and the stability consideration can be transformed onto them reducing the equations to $n$ independent scalar equations. These equations can then be treated by the equation above.

\section{Stability of stationary orientation field}

The linearized equations around the ECP solution with a perturbation of $\mathcal{A}_{j}^{+}(t) \rightarrow \mathcal{A}(t)+a_{j}^{+}(t)$ and $\mathcal{A}_{j}^{-}(t) \rightarrow a_{j}^{-}(t)$ decouple from the phases and are given by

$$
\partial_{t} \boldsymbol{a}^{ \pm}(t)=\boldsymbol{D}^{ \pm} \boldsymbol{a}^{ \pm}(t)+\boldsymbol{H}^{ \pm} \boldsymbol{a}^{ \pm}(s)
$$

for the amplitudes and

$$
\partial_{t} \boldsymbol{\varphi}^{+}(t)=\boldsymbol{D}_{\varphi}^{+} \boldsymbol{\varphi}^{+}(t)+\boldsymbol{H}_{\varphi}^{+} \boldsymbol{\varphi}^{+}(s)
$$

for the phases. The phases $\varphi^{-}$can be neglected due to the vanishing amplitudes $\mathcal{A}_{j}^{-}$.

Intrinsic stability The stability matrices for intrinsic stability are given by

$$
\begin{aligned}
& \left(\boldsymbol{D}^{+}\right)_{j k}=\delta_{j k}\left(1+\mathcal{A}^{2} \sum_{f=0}^{n-1} g_{m_{j}, m_{f}}^{t}\right)+2 \mathcal{A}^{2} g_{m_{j}, m_{k}}^{t} \\
& \left(\boldsymbol{H}^{+}\right)_{j k}=\delta_{j k}\left(\mathcal{A}^{2} \sum_{f=0}^{n-1} g_{m_{j}, m_{f}}^{s}\right)+2 \mathcal{A}^{2} g_{m_{j}, m_{k}}^{s}
\end{aligned}
$$



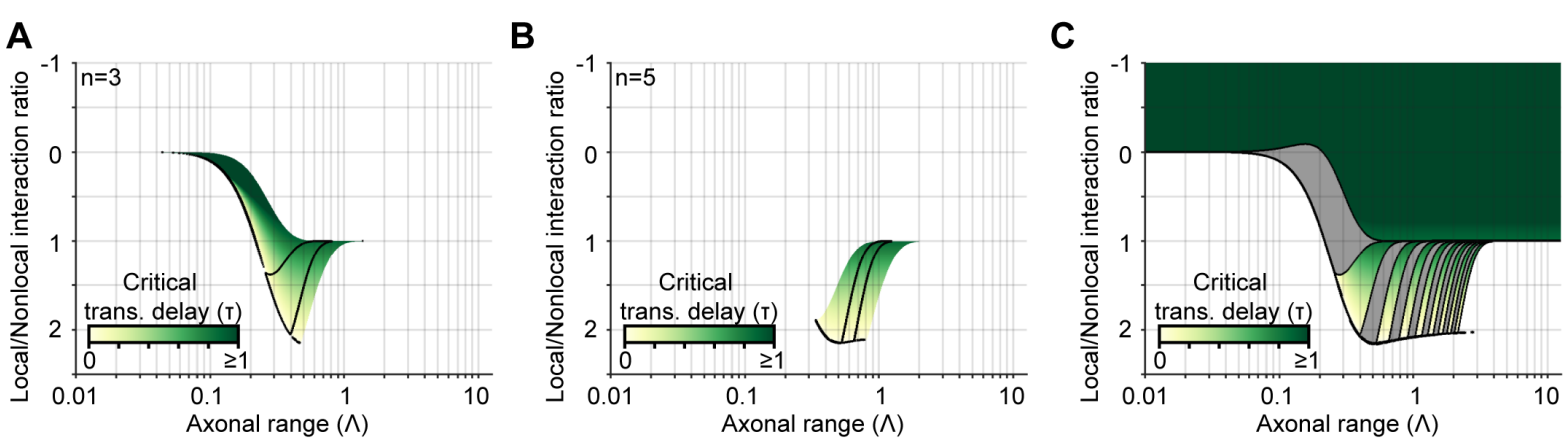

Figure 3.12: Stability of essentially complex planforms with delayed axonal transport A, B Critical transport delays of $n=3,5$ active modes (see text for details) and their minimal energy region (black lines, see Eq. (3.139) and Fig. 3.9C) for a Gaussian kernel, respectively. C Critical transport delays of minimal energy ECP solutions. Notice that the transport delay of the minimal energy regions for an even number of active modes (gray regions) eluded a calculation, but are numerically found to be similar to their surrounding critical delay times from uneven number active mode solutions.

Inactive mode suppression The stability matrices for inactive mode stability are given by

$$
\begin{aligned}
& \left(\boldsymbol{D}^{-}\right)_{j k}=\delta_{j k}\left(1+\mathcal{A}^{2} \sum_{f=0}^{n-1} g_{-m_{j}, m_{f}}^{t}\right)+\mathcal{A}^{2}\left(f_{-m_{j}, m_{k}}^{t}+f_{-m_{j},-m_{k}}^{t}\right) \\
& \left(\boldsymbol{H}^{-}\right)_{j k}=\delta_{j k}\left(\mathcal{A}^{2} \sum_{f=0}^{n-1} g_{-m_{j}, m_{f}}^{s}\right)+\mathcal{A}^{2}\left(f_{-m_{j}, m_{k}}^{s}+f_{-m_{j},-m_{k}}^{s}\right)
\end{aligned}
$$

Extrinsic stability The linearized equations for extrinsic stability are given by

$$
\partial_{t} b(t)=D b(t)+H b(s)
$$

with the scalars

$$
D=1+A^{2} \sum_{f=0}^{n-1} \tilde{g}_{x, m_{f}}^{t}, \quad H=A^{2} \sum_{f=0}^{n-1} \tilde{g}_{x, m_{f}}^{s} .
$$

Here $x$ denotes the direction of the test mode, which can point in an arbitrary direction. The most unstable direction has to be determined numerically.

Phase stability The stability matrices of the phases are given by

$$
\left(\boldsymbol{D}_{\varphi}^{+}\right)_{j k}=-\delta_{j k} \mathcal{A}^{2} \sum_{k=0}^{n-1} g_{ \pm m_{j}, \pm m_{k}}^{s}, \quad\left(\boldsymbol{H}_{\varphi}^{+}\right)_{j k}=-\left(\boldsymbol{D}_{\varphi}^{+}\right)_{j k}
$$

Notice that for the phase stability no critical delay time exists. The result of critical delays is depicted in Fig. 3.12.

\section{Linear response of total concentration field}

The only operator of the total concentration field that changes in the case of delayed transport is the cubic operator

$$
\begin{aligned}
\hat{C}^{\Sigma, \text { delay }}[\Psi, \bar{\Psi}, \Sigma]= & \gamma_{6} \Sigma(\boldsymbol{x}, t)|\Psi(\boldsymbol{x}, t)|^{2}-K \star\left(\left(\gamma_{1}+2 \frac{j^{-} \nu_{1}}{\sigma_{\Psi}^{2}}\right) \Sigma(\boldsymbol{y}, s)|\Psi(\boldsymbol{x}, s)-\Psi(\boldsymbol{y}, s)|^{2}\right. \\
& \left.+\gamma_{1} \Sigma(\boldsymbol{x}, t)|\Psi(\boldsymbol{x}, t)-\Psi(\boldsymbol{y}, t)|^{2}\right) .
\end{aligned}
$$


For $0<r \ll 1$ the dynamics of the orientation field become arbitrary slow with a time constant of $\tau=1 / r$. Since the damping term in the orientation field dynamics is cubic, the field is of order $\Psi \sim \sqrt{r}$. As the total concentration field is coupled to a quadratic factor in $\Psi$, a perturbation of $\Sigma$ is of the order $r$. Therefore, the fields will be expanded around the equilibrium point $(\Psi, \Sigma)=\left(0, \Sigma_{0}\right)$ as

$$
\Sigma(\boldsymbol{x}, t) \approx \Sigma_{0}+r \Sigma_{1}(\boldsymbol{x}, t), \quad \Psi(\boldsymbol{x}, t) \approx \sqrt{r} \Psi(\boldsymbol{x}, t),
$$

where the equilibrium point is given by

$$
\Sigma_{0}=a_{1}\left(\frac{1}{b_{1}}+i \frac{1}{b_{2}}\right)
$$

The dynamics of the $\Psi$ field are not altered by this transformation. The expansion of $\Sigma$ is given by

$$
\begin{aligned}
\tau_{s} \partial_{t}\left(\Sigma_{0}+r \Sigma_{1}(\boldsymbol{x}, t)\right)= & r(\underbrace{\hat{L}^{\Sigma}\left[\Sigma_{1}\right]+2 \hat{Q}_{1}^{\Sigma}\left[\Sigma_{1}, \Sigma_{0}\right]}_{\hat{L}^{\Sigma_{0}}}+\underbrace{\hat{Q}_{2}^{\Sigma}\left[\Psi_{1}, \Psi_{1}\right]+\hat{C}^{\Sigma, \text { delay }}\left[\bar{\Psi}, \Psi, \Sigma_{0}\right]}_{\hat{L}^{\Sigma_{0}, \Psi}}) \\
& +\mathcal{O}\left(\sqrt{r}^{3}\right)
\end{aligned}
$$

For small $r$, the equation can be solved in Fourier space by the Green function resulting in

$$
\Sigma_{1}(\boldsymbol{k}, t)=\int_{-\infty}^{t} \frac{\mathrm{d} s}{\tau_{s}} e^{\frac{t-s}{\tau_{s}}} \mathcal{F}\left\{\hat{L}^{\Sigma_{0}}\right\}(\boldsymbol{k}) \mathcal{F}\left\{\hat{L}^{\Sigma_{0}, \Psi}[\Psi]\right\}(\boldsymbol{k}, s)
$$

with the steady-state solution of

$$
\Sigma_{1}(\boldsymbol{x}, t)=-\left(\hat{L}^{\Sigma_{0}}\right)^{-1} \hat{L}^{\Sigma_{0}, \Psi}[\Psi] .
$$

The operators are given by

$$
\begin{aligned}
\mathcal{F}\left\{\hat{L}^{\Sigma_{0}}\right\}= & \alpha_{1}+\nu_{1}\left(2 j^{-} \tilde{K}(\|\boldsymbol{k}\|)+2 j_{u}^{-} \tilde{K}_{-}(\|\boldsymbol{k}\|)+j_{u}^{+} \tilde{K}_{+}(\|\boldsymbol{k}\|)\right)+ \\
& \gamma_{1} \sigma_{\Psi}^{2}\left(\tilde{K}(\|\boldsymbol{k}\|)+\frac{j_{u}^{-}}{j^{-}} \tilde{K}_{-}(\|\boldsymbol{k}\|)\right)-2 a_{1} \\
\hat{L}^{\Sigma_{0}, \Psi}[\Psi]= & b_{1} \Re\{\Psi(\boldsymbol{x}, t)\}^{2}+i b_{2} \Im\{\Psi(\boldsymbol{x}, t)\}^{2}+\gamma_{6} \Sigma_{0}|\Psi(\boldsymbol{x}, t)|^{2}- \\
& \left(\gamma_{1}+2 \frac{j^{-} \nu_{1}}{\sigma_{\Psi}^{2}}\right) \Sigma_{0} K \star|\Psi(\boldsymbol{x}, s)-\Psi(\boldsymbol{y}, s)|^{2}-\gamma_{1} \Sigma_{0} K \star|\Psi(\boldsymbol{x}, t)-\Psi(\boldsymbol{y}, t)|^{2} .
\end{aligned}
$$

\subsubsection{Extended solution set of canonical genetic network}

In order to appreciate the potential of the canonical genetic network model for visual cortical architecture specification, the full amplitude equations, Eq. (3.99), for orientation field emergence needs to be considered. Here, the linear perturbation of the orientation field

$$
\partial_{t} A_{j}=A_{j}+\alpha_{2} \bar{A}_{j^{-}}+A_{j} \sum_{k=0}^{2 n-1} g_{j k}^{1}\left|A_{k}\right|^{2}+\bar{A}_{j^{-}} \sum_{k=0}^{2 n-1} g_{j k}^{2} A_{k} A_{k^{-}}
$$

by its complex conjugate is analyzed analytically, see [139] for a similar calculation. The time dependence is and will be omitted in the following for the sake of clarity. 


\section{Stationary orientation field solutions}

In order to diagonalize the linear part of the amplitude equations the transformed amplitudes of ECPs from Eq. (3.134)

$$
B_{j}^{ \pm}=\frac{1}{2}\left(A_{j}^{+} e^{-i \phi} \pm \bar{A}_{j}^{-} e^{i \phi}\right)
$$

are introduced with the phase of the perturbation $\alpha_{2}=a e^{2 i \phi}$ and $a>0$. The amplitude equations are then given by

$$
\begin{aligned}
\partial_{t} B_{j}^{ \pm}= & (1 \pm a) B_{j}^{+}+B_{j}^{ \pm} \sum_{k=0}^{n-1} g_{j k}^{1}\left(2+\delta_{j k}\right)\left(\left|B_{k}^{+}\right|^{2}+\left|B_{k}^{-}\right|^{2}\right)-g_{00}^{1} B_{j}^{\mp}\left(B_{j}^{+} \bar{B}_{j}^{-}+\bar{B}_{j}^{+} B_{j}^{-}\right) \\
& \pm 2 B_{j}^{ \pm} \sum_{k=0}^{n-1} g_{j k}^{2}\left(\left|B_{k}^{+}\right|^{2}-\left|B_{k}^{-}\right|^{2}\right) \mp 2 B_{j}^{\mp} \sum_{k=0}^{n-1} g_{j k}^{2}\left(\left|B_{k}^{+}\right|^{2}-\left|B_{k}^{-}\right|^{2}\right)
\end{aligned}
$$

Separating the amplitudes and the phases of a single mode $B_{j}^{ \pm}=\mathcal{B}_{j}^{ \pm} e^{i \phi_{j}^{ \pm}}$and introducing the phase difference variable $\Omega_{j}=\phi_{j}^{+}-\phi_{j}^{-}$leads to an equation of motion for the amplitudes and the phases

$$
\begin{aligned}
\partial_{t} \mathcal{B}_{j}^{ \pm}= & (1 \pm a) \mathcal{B}_{j}^{ \pm}+\mathcal{B}_{j}^{ \pm} \sum_{k=0}^{n-1} g_{j k}^{1}\left(2+\delta_{j k}\right)\left(\mathcal{B}_{k}^{+2}+\mathcal{B}_{k}^{-2}\right)-g_{00}^{1} \mathcal{B}_{j}^{ \pm} \mathcal{B}_{j}^{\mp 2}\left(1+\cos 2 \Omega_{j}\right) \\
& \pm 2 \mathcal{B}_{j}^{ \pm} \sum_{k=0}^{n-1} g_{j k}^{2}\left(\mathcal{B}_{k}^{+2}-\mathcal{B}_{k}^{-2}\right)+4 \mathcal{B}_{j}^{\mp} \sin \Omega_{j} \sum_{k=0}^{n-1} g_{j k}^{2} \mathcal{B}_{k}^{+} \mathcal{B}_{k}^{-} \sin \Omega_{k} \\
\partial_{t} \Omega_{j}= & g_{00}^{1}\left(\mathcal{B}_{j}^{+2}+\mathcal{B}_{j}^{-2}\right) \sin 2 \Omega_{j}+4\left(\mathcal{B}_{j}^{-} \mathcal{B}_{j}^{+-1}+\mathcal{B}_{j}^{+} \mathcal{B}_{j}^{-1}\right) \cos \Omega_{j} \sum_{k=0}^{n-1} g_{j k}^{2} \mathcal{B}_{k}^{+} \mathcal{B}_{k}^{-} \sin \Omega_{k}
\end{aligned}
$$

Stationary solutions of the transformed amplitude equations can be easily found by assuming $\mathcal{B}_{j}^{ \pm}=\mathcal{B}^{ \pm}$. The condition on the phases is then given by

$$
\partial_{t} \Omega_{j}=0=\left(\mathcal{B}^{+2}+\mathcal{B}^{-2}\right)\left(g_{00}^{1} \sin 2 \Omega_{j}+4 \cos \Omega_{j} \sum_{k=0}^{n-1} g_{j k}^{2} \sin \Omega_{k}\right)
$$

which can be solved by $\Omega_{j}=\mathbb{Z} \pi$. This results in the fixed point equations for the amplitudes

$$
\begin{aligned}
\partial_{t} \mathcal{B}_{j}^{ \pm}=0= & (1 \pm a) \mathcal{B}^{ \pm}+\mathcal{B}^{ \pm}\left(\mathcal{B}^{+2}+\mathcal{B}^{-2}\right) \sum_{k=0}^{n-1} g_{0 k}^{1}\left(2+\delta_{j k}\right)-2 g_{00}^{1} \mathcal{B}^{ \pm} \mathcal{B}^{\mp 2} \\
& \pm 2 \mathcal{B}^{ \pm}\left(\mathcal{B}^{+2}-\mathcal{B}^{-2}\right) \sum_{k=0}^{n-1} g_{0 k}^{2}
\end{aligned}
$$

leading with the stationary amplitude of an ECP denoted by $\mathcal{A}$ (see Eq. (3.138)) to three distinct solutions

$$
\mathcal{B}^{+}=\mathcal{A} \cdot\left\{\begin{array}{ll}
\sqrt{(1+2 a) / 4} & , a \in[0,1 / 2] \\
\sqrt{(1+a) / 3} & , a \in[0, \infty) \\
0 & , a \in[0,1]
\end{array} \quad, \quad \mathcal{B}^{-}=\mathcal{A} \cdot \begin{cases}\sqrt{(1-2 a) / 4} & , a \in[0,1 / 2] \\
0 & , a \in[0, \infty) \\
\sqrt{(1-a) / 3} & , a \in[0,1]\end{cases}\right.
$$


The validity range of the solutions result from the fact that the amplitudes must be real numbers and $a \geq 0$. From these solutions the amplitudes of the orientation field can be obtained by noting

$$
\left|A_{j}^{ \pm}\right|^{2}=\left|B_{j}^{+} \pm B_{j}^{-}\right|^{2}=\left(\mathcal{B}_{j}^{+}\right)^{2}+\left(\mathcal{B}_{j}^{-}\right)^{2} \pm 2 \mathcal{B}_{j}^{+} \mathcal{B}_{j}^{-} \cos \Omega_{j},
$$

which gives for the non-trivial first case

$$
\left|A_{j}^{ \pm}\right|^{2}=\frac{\mathcal{A}^{2}}{2}\left(1 \pm \cos \Omega_{j} \sqrt{1-(2 a)^{2}}\right) .
$$

We require that for $a \rightarrow 0$ only the active mode $A^{+}$remains that results in the phase condition $\Omega_{j}=2 \mathbb{Z} \pi$. In order to obtain the phases of the amplitudes corresponding to the orientation field for the non-trivial case the pair-wise correlation is considered

$$
A_{j}^{+} A_{j}^{-}=\left|A_{j}^{+}\right| e^{i \psi_{j}^{+}}\left|A_{j}^{-}\right| e^{i \psi_{j}^{-}}=e^{4 i \phi}\left(\mathcal{B}_{j}^{+2}-\mathcal{B}_{j}^{-2}\right)=e^{4 i \phi} a \mathcal{A}^{2} .
$$

This leads to the phase condition of $\psi_{j}^{+}+\psi_{j}^{-}=4 \phi$. To sum up, the closed-form solution of the orientation field is given by

$$
A_{j}^{ \pm}=\mathcal{A} e^{2 i\left(\phi \pm \psi_{j}\right)} \cdot \begin{cases}\sqrt{\left(1 \pm \sqrt{1-(2 a)^{2}}\right) / 2} & , a \in[0,1 / 2] \\ \sqrt{(1+a) / 3} & , a \in[0, \infty) \\ i \sqrt{(1-a) / 3} & , a \in[0,1]\end{cases}
$$

with $\psi_{j}=\left(\psi_{j}^{+}-\psi_{j}^{-}\right) / 2$. An explicit solution of the orientation field, Fig. 3.13A, is given by

$$
\Psi(\boldsymbol{x})=2 e^{2 i \phi} \mathcal{A} \sum_{j=0}^{n-1}\left(\xi \cos \left(l_{j} \boldsymbol{k}_{j} \boldsymbol{x}+2 \psi_{j}\right)+i \eta \sin \left(l_{j} \boldsymbol{k}_{j} \boldsymbol{x}+2 \psi_{j}\right)\right)
$$

with

$$
\xi=\left\{\begin{array}{ll}
\left(\gamma_{+}+\gamma_{-}\right) / \sqrt{2} & , a \in[0,1 / 2] \\
\sqrt{(1+a) / 3} & , a \in[0, \infty) \\
i \sqrt{(1-a) / 3} & , a \in[0,1]
\end{array} \quad, \quad \eta= \begin{cases}\left(\gamma_{+}-\gamma_{-}\right) / \sqrt{2} & , a \in[0,1 / 2] \\
0 & , a \in[0, \infty) \\
0 & , a \in[0,1]\end{cases}\right.
$$

and

$$
\gamma_{ \pm}=\sqrt{1 \pm \sqrt{1-(2 a)^{2}}}
$$

\section{Stability of stationary orientation field}

The stability of the stationary solutions can be decomposed into an intrinsic and extrinsic stability. For intrinsic stability consider the perturbation around the stationary state

$$
\mathcal{B}_{j}^{ \pm} \rightarrow \mathcal{B}^{ \pm}+b_{j}^{ \pm} \quad \text { and } \quad \Omega_{j} \rightarrow 2 \mathbb{Z} \pi+\omega_{j} .
$$

Linearizing the amplitude Eq. (3.257) and the phase Eq. (3.258) around the closed-form solution leads to a decoupling of the linearized amplitude equations from the phase equations

$$
\begin{aligned}
\partial_{t} b_{j}^{ \pm} & =\left(1 \pm a-\left(3 \mathcal{B}^{ \pm 2}+\mathcal{B}^{\mp 2}\right) \mathcal{A}^{-2}\right) b_{j}^{ \pm}+6 \mathcal{B}^{ \pm 2} \sum_{k=0}^{n-1} g_{j k}^{1} b_{k}^{ \pm}+2 \mathcal{B}^{ \pm} \mathcal{B}^{\mp} \sum_{k=0}^{n-1} g_{j k}^{1} b_{k}^{\mp} \\
\partial_{t} \omega_{j} & =2\left(\mathcal{B}^{+2}+\mathcal{B}^{-2}\right) \sum_{k=0}^{n-1} g_{j k}^{1} \omega_{k} .
\end{aligned}
$$


The system is intrinsically stable if all eigenvalues of the system are negative. Transforming the phase equation in the circulant eigenbasis of $g_{j k}^{1}$ and denoting its $l$ th eigenvalue by $\lambda_{g}^{l}$ leads to the phase stability condition of $\lambda_{g}^{l}<0$. The amplitude equations can be written as

$$
\partial_{t}\left(\begin{array}{l}
\mathbf{b}^{+} \\
\mathbf{b}^{-}
\end{array}\right)=\left(\begin{array}{ll}
\mathbf{M}_{1} & \mathbf{M}_{2} \\
\mathbf{M}_{2} & \mathbf{M}_{3}
\end{array}\right)\left(\begin{array}{l}
\mathbf{b}^{+} \\
\mathbf{b}^{-}
\end{array}\right)=\mathbf{M}\left(\begin{array}{l}
\mathbf{b}^{+} \\
\mathbf{b}^{-}
\end{array}\right)
$$

with the vectors $\mathbf{b}^{ \pm}=\left(b_{1}^{ \pm}, b_{2}^{ \pm}, \ldots, b_{n}^{ \pm}\right)^{T}$ and the matrices

$$
\begin{aligned}
\left(\mathbf{M}_{1}\right)_{j k} & =6 \mathcal{B}^{+2} g_{j k}^{1}+\delta_{j k} \lambda^{+} \\
\left(\mathbf{M}_{2}\right)_{j k} & =2 \mathcal{B}^{+} \mathcal{B}^{-} g_{j k}^{1} \\
\left(\mathbf{M}_{3}\right)_{j k} & =6 \mathcal{B}^{-2} g_{j k}^{1}+\delta_{j k} \lambda^{-} \\
\lambda^{ \pm} & =1 \pm a-\left(3 \mathcal{B}^{ \pm}+\mathcal{B}^{\mp^{2}}\right) \mathcal{A}^{-2} .
\end{aligned}
$$

Since the matrix $\mathbf{M}$ is symmetric, there exists an orthogonal transformation matrix, which diagonalizes the matrix such that on the diagonal there are formally the two eigenmatrices

$$
\lambda_{M}^{ \pm}=\frac{1}{2}\left(\mathbf{M}_{1}+\mathbf{M}_{3} \pm \sqrt{\left(\mathbf{M}_{1}-\mathbf{M}_{3}\right)^{2}+4 \mathbf{M}_{2}^{2}}\right)
$$

This formal solving onto eigenmatrices is justified, because $g_{j k}^{1}$ is a circulant matrix and hence the matrices $\mathbf{M}_{i}$ for $i=1,2,3$ have all the same eigenbasis. Denoting the $l$ th eigenvalue of $\mathbf{M}_{i}$ as $\lambda_{i}^{l}$ the eigenvalues of the matrix $\mathbf{M}$ finally become

$$
\lambda_{M}^{ \pm l}=\frac{1}{2}\left(\lambda_{1}^{l}+\lambda_{3}^{l} \pm \sqrt{\left(\lambda_{1}^{l}-\lambda_{3}^{l}\right)^{2}+4 \lambda_{2}^{l}}\right) .
$$

The stability condition for the amplitudes is given by

$$
\lambda_{1}^{l}+\lambda_{3}^{l} \leq 0, \quad \lambda_{1}^{l} \lambda_{3}^{l}-\lambda_{2}^{l^{2}} \geq 0
$$

or equivalently expressed by the eigenvalues of $g_{j k}^{1}$ by

$$
32 \lambda_{g}^{l}{ }^{2} \mathcal{B}^{+2} \mathcal{B}^{-2}+6 \lambda_{g}^{l}\left(\mathcal{B}^{+^{2}} \lambda^{-}+\mathcal{B}^{-} \lambda^{+^{2}}\right)+\lambda^{+} \lambda^{-}>0, \quad 6 \lambda_{g}^{l}\left(\mathcal{B}^{+^{2}}+\mathcal{B}^{-2}\right)+\lambda^{+}+\lambda^{-}<0
$$

The coefficient $\lambda^{ \pm}$of the three solutions is given by

$$
\lambda^{+}=\left\{\begin{array}{ll}
0 & , a \in[0,1 / 2] \\
0 & , a \in[0, \infty) \\
2(1+2 a) / 3 & , a \in[0,1]
\end{array} \quad, \quad \lambda^{-}= \begin{cases}0 & , a \in[0,1 / 2] \\
2(1-2 a) / 3 & , a \in[0, \infty) \\
0 & , a \in[0,1]\end{cases}\right.
$$

The first stability condition gives $a<1 / 2, a>1 / 2$ and $a>1$ for the three closed-form solutions, respectively. The second stability condition on the amplitudes of the three closed-form solutions is

$$
\lambda_{g}^{l}<0, \quad \quad \lambda_{g}^{l}<\frac{2 a-1}{3 \mathcal{A}^{2}(a+1)} \quad \lambda_{g}^{l}<\frac{2 a+1}{3 \mathcal{A}^{2}(a-1)} .
$$

that is always fulfilled by plugging in the first stability condition and the phase stability condition. 
For extrinsic stability it suffices to consider an additional mode at the angle $\pi /(2 n)$ to an active mode. The linearized equation for such a mode $b^{ \pm}$is given by

$$
\partial_{t} b^{ \pm}=\left(1 \pm a+\left((2 \pm 1) \mathcal{B}^{+2}+(2 \mp 1) \mathcal{B}^{-2}\right) \sum_{k=0}^{n-1} \bar{g}_{0 k}\right) b^{ \pm}=\lambda^{ \pm} b^{ \pm}
$$

with the angle-interaction function

$$
\bar{g}_{0 k}=g\left(\frac{\pi}{2 n}-\frac{\pi k}{n}\right) .
$$

The stability condition is hence

$$
\lambda^{+}=(1+a)\left(1+\mathcal{A}^{2} \sum_{k=0}^{n-1} \bar{g}_{0 k}\right)<0 \quad \text { and } \quad \lambda^{-}=(1-a)\left(1+\mathcal{A}^{2} \sum_{k=0}^{n-1} \bar{g}_{0 k}\right)<0
$$

for the first solution,

$$
\lambda^{+}=(1+a)\left(1+\mathcal{A}^{2} \sum_{k=0}^{n-1} \bar{g}_{0 k}\right)<0 \quad \text { and } \quad \lambda^{-}=(1-a)+(1+a) \frac{\mathcal{A}^{2}}{3} \sum_{k=0}^{n-1} \bar{g}_{0 k}<0
$$

for the second solution and

$$
\lambda^{+}=(1+a)+(1-a) \frac{\mathcal{A}^{2}}{3} \sum_{k=0}^{n-1} \bar{g}_{0 k} \quad \text { and } \quad \lambda^{-}=(1-a)\left(1+\mathcal{A}^{2} \sum_{k=0}^{n-1} \bar{g}_{0 k}\right)<0
$$

for the third solution. If extrinsic stability of ECPs is assumed, see Eq. (3.143), the resulting conditions on $a$ are given by $a<1, a>1 / 2$ and $a<-1 / 2$ for the three solutions, respectively. To conclude, the first solution is stable for $a<1 / 2$, the second for $a>1 / 2$ and the third is always unstable since $a \geq 0$, see Fig. 3.13B.

\section{Pinwheel stability in stationary orientation field}

Notice that the pinwheel configuration is stable over the range of the parameter $a \in[0,1 / 2)$. This stems from the fact that the real and imaginary part at pinwheels vanish, which is for the explicit stable solution given by the condition

$$
\sum_{j=0}^{n-1} \cos \left(l_{j} \boldsymbol{k}_{j} \boldsymbol{x}+2 \psi_{j}\right)=0 \quad \text { and } \quad \sum_{j=0}^{n-1} \sin \left(l_{j} \boldsymbol{k}_{j} \boldsymbol{x}+2 \psi_{j}\right)=0 .
$$

However, for $a \in[1 / 2, \infty)$ the orientation field generated visual scotoma without any pinwheels.

\section{Orientation preference bias distribution}

The orientation field solutions exhibit a progressively increasing orientation bias for increasing linear shift-symmetry breaking perturbations that eventually leads to visual scotoma with only two preferred orientations, Fig. 3.13A. How strong is the orientation bias with respect to the linear perturbation?

The stable orientation field with no visual scotoma is given by

$$
\Psi(\boldsymbol{x})=\sqrt{2} \mathcal{A} e^{i 2 \phi} \sum_{j=0}^{n-1}\left(\left(\gamma_{+}+\gamma_{-}\right) \cos \left(l_{j} \boldsymbol{k}_{j} \boldsymbol{x}+2 \psi_{j}\right)+i\left(\gamma_{+}-\gamma_{-}\right) \sin \left(l_{j} \boldsymbol{k}_{j} \boldsymbol{x}+2 \psi_{j}\right)\right) .
$$


A

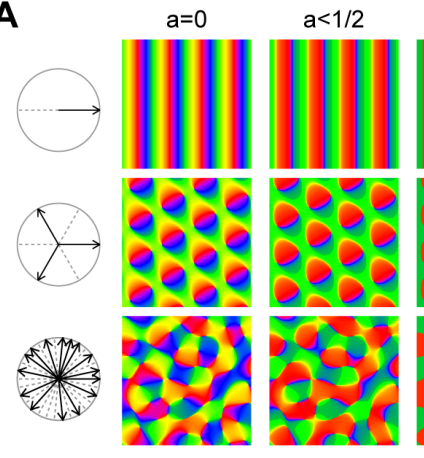

$a>1 / 2$

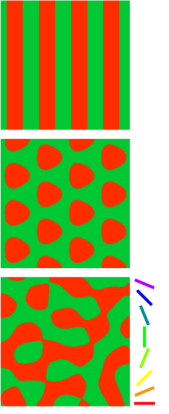

B

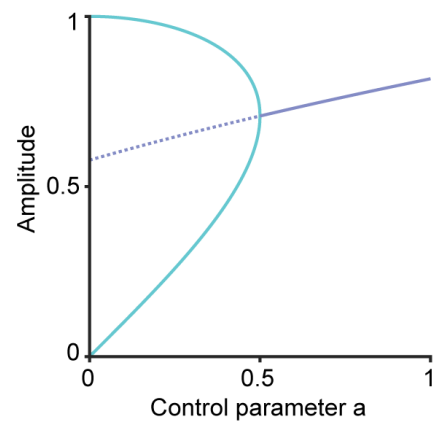

C

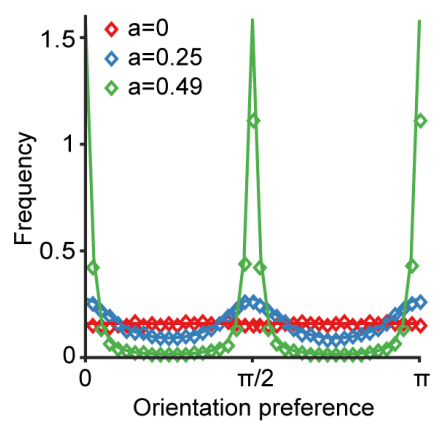

Figure 3.13: Linear perturbation in symmetry-confined canonical genetic network. A Solutions of amplitude Eq. (3.254) that originate from ECP solutions $(a=0)$ with different numbers of active modes $n=1,3,15$ to an orientation field solution with only two preferred orientations, see Eq. (3.266). Varying the phase of the linear perturbation leads to an orientation bias towards this phase. B Amplitude solutions from Eq. (3.265) of diametrically placed amplitudes on the critical circle. Stable amplitude solutions are indicated by solid lines and unstable amplitude solutions by a dotted line. C Orientation preference distribution (Eq. (3.292)) for varying linear perturbation amplitudes $a$ and fixed phases (Diamonds: measurement of synthesized orientation fields with $n=8$ active modes, $17 \Lambda \times 17 \Lambda$ and $256 \times 256$ pixels, solid lines: Gaussian random field approximation).

For a large number of active modes $n$, the field can be approximated by a Gaussian random field, since the phases $\psi_{j} \in[0, \pi)$ are randomly selected by the dynamics. Decomposing the real and imaginary part of the orientation field $\Psi(\boldsymbol{x})=R(\boldsymbol{x})+i I(\boldsymbol{x})$, the moments are calculated for the special case $\phi=0$. Other cases can be obtained by a global phase-shift of the orientation field $\Psi \rightarrow \Psi e^{2 i \phi}$. The average over the random phases $\psi_{j}$ leads to vanishing first moments $\langle R(\boldsymbol{x})\rangle=\langle I(\boldsymbol{x})\rangle=0$, vanishing cross-correlations $\langle R(\boldsymbol{x}) I(\boldsymbol{x})\rangle=0$ and constant second moments

$$
\sigma_{R}^{2}=\left\langle R(\boldsymbol{x})^{2}\right\rangle=4 \pi n \mathcal{A}^{2}\left(\gamma_{+}+\gamma_{-}\right)^{2}, \quad \sigma_{I}^{2}=\left\langle I(\boldsymbol{x})^{2}\right\rangle=4 \pi n \mathcal{A}^{2}\left(\gamma_{+}-\gamma_{-}\right)^{2} .
$$

The diagonal covariance matrix defines the probability distribution of the orientation field

$$
p(\Psi)=\frac{1}{2 \pi \sigma_{R} \sigma_{I}} e^{-\frac{R^{2}}{2 \sigma_{R}^{2}}-\frac{I^{2}}{2 \sigma_{I}^{2}}}
$$

The distribution of orientation preference representation is given by a wrapped Cauchy distribution

$$
\begin{aligned}
P(\theta) & =\left\langle\delta\left(\frac{1}{2} \arctan \left(\frac{R}{I}\right)-\theta\right)\right\rangle \\
& =\frac{1}{2 \pi \sigma_{R} \sigma_{I}} \int \mathrm{d} R \int \mathrm{d} I \exp \left(-\frac{R^{2}}{2 \sigma_{R}^{2}}-\frac{I^{2}}{2 \sigma_{I}^{2}}\right) \delta\left(\frac{1}{2} \arctan \left(\frac{R}{I}\right)-\theta\right) \\
& =\frac{1}{2 \pi} \int \mathrm{d} \phi \delta\left(\frac{1}{2} \arctan \left(\frac{\sigma_{R}}{\sigma_{I}} \tan \phi\right)-\theta\right) \\
& =\frac{1}{\pi} \frac{1}{\frac{\sigma_{R}}{\sigma_{I}} \cos ^{2} 2 \theta+\frac{\sigma_{I}}{\sigma_{R}} \sin ^{2} 2 \theta} .
\end{aligned}
$$

The orientation preference distribution for arbitrary $\phi$ is obtained by the transformation $P(\theta-\phi)$. The ratio of the real and imaginary standard deviation can be simplified to

$$
\frac{\sigma_{R}}{\sigma_{I}}=\sqrt{\frac{1+2 a}{1-2 a}} .
$$

The comparison between the numerical and the random field approximation is depicted in Fig. 3.13C for $n=8$ active modes. 
A

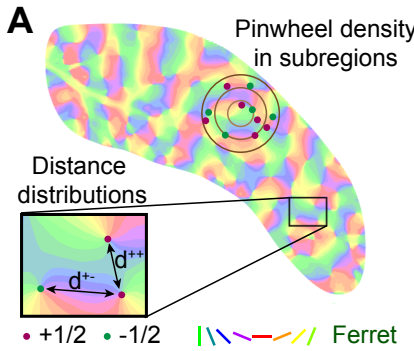

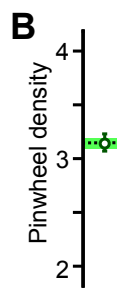

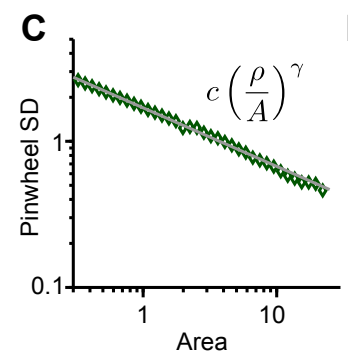

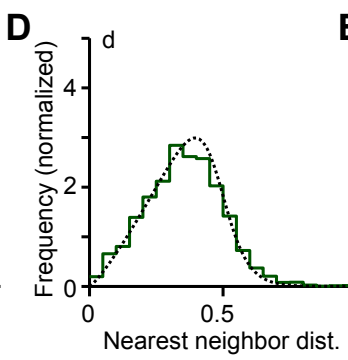

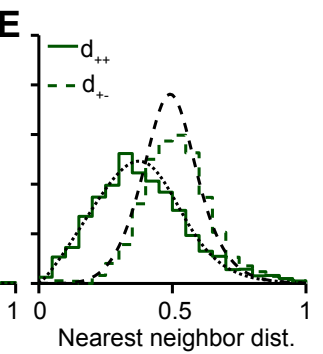

Figure 3.14: Common design of pinwheel configuration. A Orientation field is reduced to pinwheels of two different topological charges (green and red dots). The number of pinwheel per typical scale area gives the pinwheel density in $\mathbf{B}$. The standard deviation of pinwheel densities in subregions has a power law scaling that can be summarized by the fitting parameter $c$ and $\gamma$. The nearest neighbor distances between pinwheels, pinwheels of opposite topological charge and between pinwheels with equal topological charge (inset) are summarized by their distribution in $\mathbf{D}$ and $\mathbf{E}$. Data of ferrets (green lines) and fits (dotted and dashed lines) from [24].

\subsubsection{Benchmarking orientation fields of canonical genetic network}

Pinwheel configurations of orientation fields from the symmetry-confined canonical genetic network were shown to be compatible to experimentally observed pinwheel configurations of the common design [22]. In contrast, many other models converge to pinwheel crystals that are incompatible to the experimentally observed pinwheel configurations $[27,126,129-131,263-$ 266]. This raises the questions whether the parameters of the symmetry-confined model are fine-tuned. Hence, we will explore the possible pinwheel configurations in the full parameter space and benchmark the orientation layouts.

In order to benchmark orientation fields, the layout of orientation preference is reduced to their pinwheel configuration. Pinwheels are points in orientation fields which are surrounded by all orientation preferences and come in two different topological charges depending on the surrounding of the pinwheel, Fig. 3.14A. The number of pinwheels per typical scale area is called the pinwheel density, Fig. 3.14B. The standard deviation scaling of the pinwheel density with typical scale area is called pinwheel density SD, Fig. 3.14C. The nearest neighbor distribution between pinwheels can be decomposed by consideration of the topological charge. It can be decomposed into nearest neighbor distributions between pinwheels independent of topological charge, with equal topological charge and with opposite topological charge, Fig. 3.14D,E.

\section{Distance measure for common design}

The common design is summarized by bootstrapping the pinwheel density, pinwheel density standard deviation scaling and the first four cumulants of the nearest neighbor distribution, Fig. 3.15A-O. This results in distributions of 15 parameters $\chi \in \mathbb{R}^{15}$ for the description of the common design. Notice that not all species possess a unimodal probability distribution due to the limit amount of orientation layouts. Therefore, we use the smooth distribution of ferrets, Fig. 3.16A-O, as a representative distribution for the common design. The distribution of the parameters $\boldsymbol{\chi}$ is approximated by the maximum entropy model with fixed mean $\boldsymbol{\mu}=\langle\boldsymbol{\chi}\rangle$ and covariance $\boldsymbol{\Sigma}=\left\langle\chi \chi^{T}\right\rangle$ that is equal to the multivariate Gaussian distribution

$$
P(\boldsymbol{\chi})=\frac{1}{\sqrt{(2 \pi)^{15} \operatorname{det} \boldsymbol{\Sigma}}} e^{-\frac{1}{2}(\boldsymbol{\chi}-\boldsymbol{\mu})^{T} \boldsymbol{\Sigma}^{-1}(\boldsymbol{\chi}-\boldsymbol{\mu})} .
$$

In order to measure the compatibility of model orientation fields with the common design, the distribution was rotated onto the principal vectors such that the inverse of the covariance 
becomes diagonal $\boldsymbol{\Lambda}=\boldsymbol{U}^{T} \boldsymbol{\Sigma}^{-1} \boldsymbol{U}$ with $\boldsymbol{\Lambda}=\operatorname{diag}\left(\lambda_{1}, \lambda_{2}, \ldots, \lambda_{15}\right)$. The multivariate Gaussian distribution is hence in every principal direction $\tilde{\boldsymbol{\chi}}=\boldsymbol{U}^{T} \boldsymbol{\chi}$ reduced to an independent univariate Gaussian distribution. The two-tailed p-value in the $i$ th principal direction of a point $\chi$ with the distance vector to the mean $\boldsymbol{\nu}=\boldsymbol{U}^{T}(\boldsymbol{\chi}-\boldsymbol{\mu})$ is given by

$$
p_{i}=1-\int_{-\left|\nu_{i}\right|}^{\left|\nu_{i}\right|} \mathrm{d} \tilde{\chi}_{i} \sqrt{\frac{\lambda_{i}}{2 \pi}} e^{-\frac{1}{2} \tilde{\chi}_{i} \lambda_{i} \tilde{\chi}_{i}}=\operatorname{erfc}\left(\sqrt{\frac{\lambda_{i}}{2}}\left|\nu_{i}\right|\right) .
$$

The compatibility of model orientation layouts was assessed by the average log p-value along the principal directions

$$
p=-\frac{1}{15} \sum_{i=1}^{15} \log p_{i}
$$

This measure weights pinwheel configurations according to the common design and is independent on the number of dimensions. In fact, pinwheel configurations drawn from the distribution $P(\chi)$ generate an average $\log \mathrm{p}$-value of $p=\log 2$. Departure from the mean leads to higher values of the $\log$ p-value.

The pinwheel configuration statistics of closed-form ECP solutions are depicted in Fig. 3.17.

\section{Distance measure for orientation bias}

The orientation bias, which is is not captured by the pinwheel configuration, is measured by the Kullback-Leibler (KL) divergence

$$
D\left(P(\theta) \| \pi^{-1}\right)=\int_{0}^{\pi} \mathrm{d} \theta P(\theta) \log \pi P(\theta) .
$$

In the case of the extended closed-form solutions with an orientation bias, see Eq. (3.292), the divergence is given by

$$
D\left(P(\theta) \| \pi^{-1}\right)=\log \left(1-e^{-2 \operatorname{arcsec}(2 a)}\right) .
$$

Thus for $a=0$, this measure is 0 and diverges for the visual scotoma solutions at $a=1 / 2$.

\section{Probing the canonical genetic network}

In order to benchmark the canonical genetic network model, we scan the free parameters and quantify the quality of their solutions by the afore-mentioned distance measures.

Notice that in the canonical genetic network model of Eq. (3.48) and Eq. (3.60) there are 25 free parameters. Two parameters are required for a wavelength instability on the critical circle at a specific spatial frequency. As this condition for the wavelength stability can not be solved analytically, we assume that $\sigma_{u}$ and $\rho_{u}$ suffice for that task. The 25 free parameters are then: (i) 16 orientation encoding parameters: $\alpha_{1}, \alpha_{2}, \beta_{1}, \beta_{2}, \zeta_{1}, \zeta_{2}, \eta_{1}, \eta_{2}, \gamma_{1}, \gamma_{2}, \gamma_{3}, \gamma_{4}, \gamma_{5}$, $\gamma_{6}, \gamma_{7}, \sigma$ and (ii) 9 orientation encoding independent parameters: $a_{1}, b_{1}, b_{2}, \nu_{1}, j^{-}, j_{u}^{-}, j_{u}^{+}$, $\sigma_{\Psi}^{2}, \tau_{s}$. Alternatively, the number of free parameters can be calculated by starting with the 90 free parameters of the full model and subtract the fixed parameters resulting from the reduction to the canonical genetic network: (i) separation of timescales 2, (ii) weak anisotropy 0, (iii) decoupling orientation encoding from total concentration 42, (iv) symmetry breaking 2 and (v) pattern onset 1.

The parameter $\alpha_{1}$ only rescales the amplitude dynamics and hence leaves the encoded orientation domains invariant. For non-permutation symmetric cases $\gamma_{1} \neq \gamma_{2}$, orientation domains 

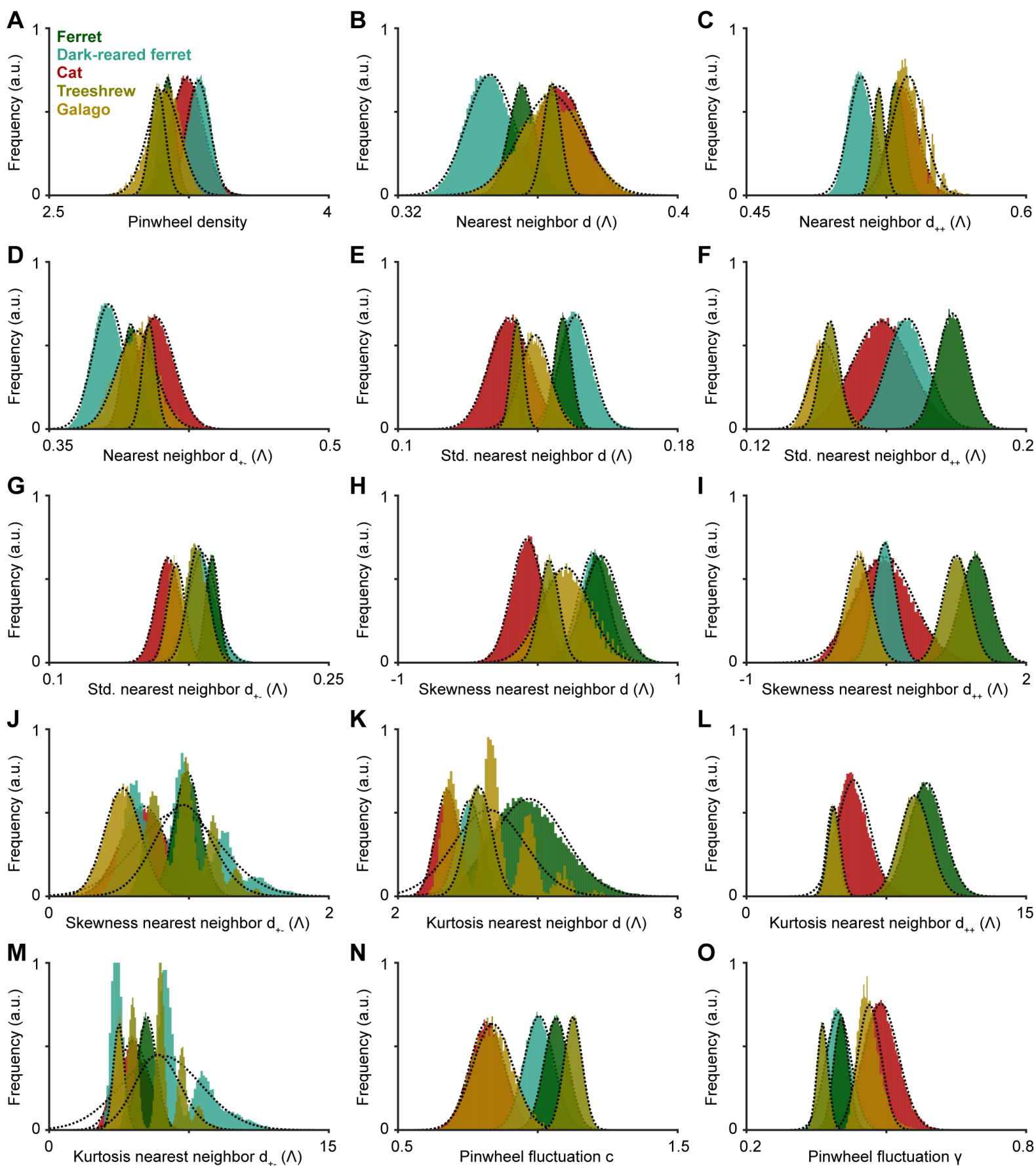

Figure 3.15: Distribution of common design cumulants from five species. Distribution of 10000 bootstrapped common design cumulants of five species from [24], see text for details. Dotted lines: Gaussian distribution corresponding to the experimentally observed cumulants. A Pinwheel density, see Fig. 3.14A, B-M the first four cumulants of pinwheel nearest neighbor distributions, see Fig. 3.14D-E. N, O Parameters that summarize the pinwheel density standard deviation scaling with cortical area, see Fig. 3.14C. Data from [24]. 

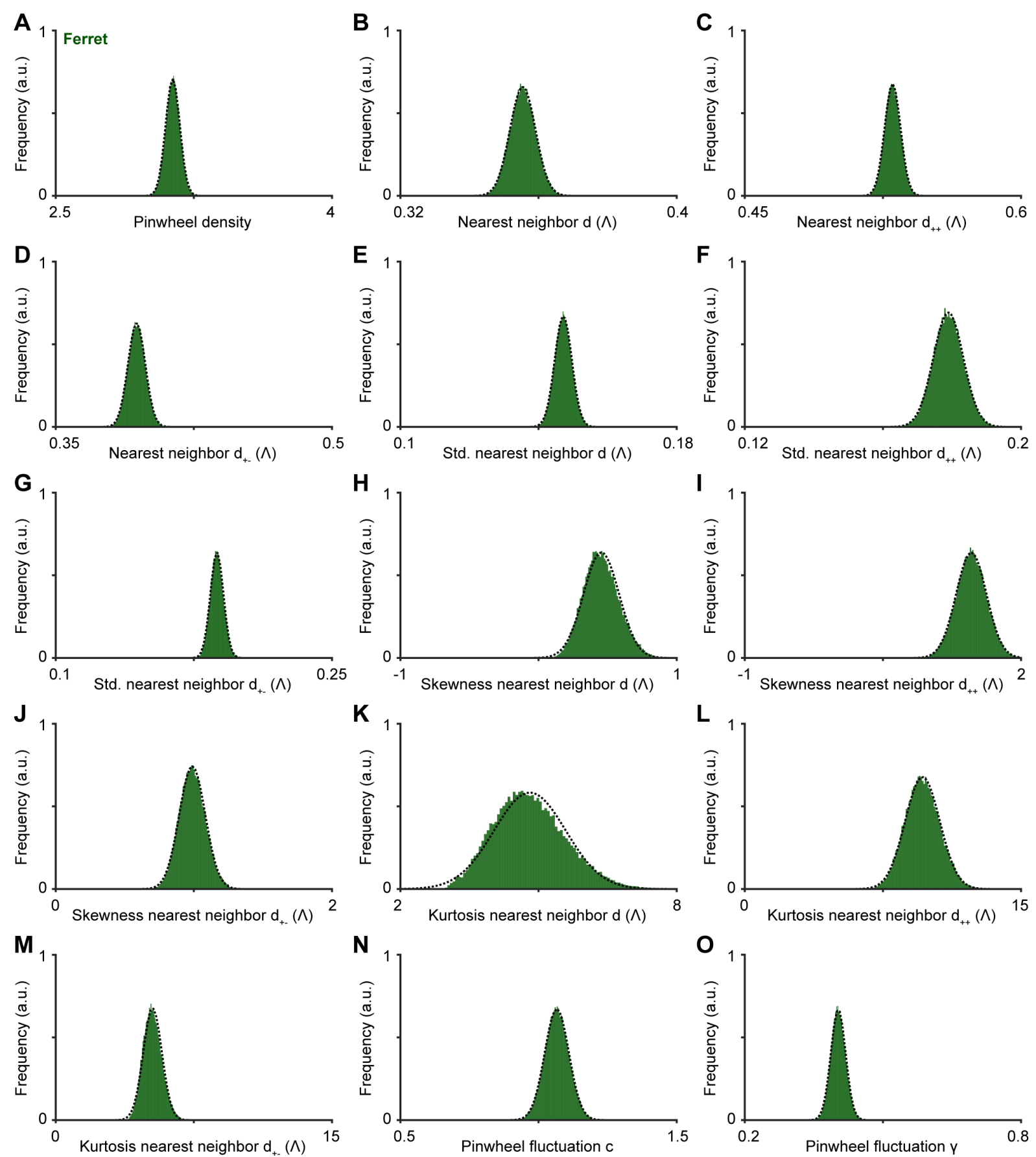

Figure 3.16: Distribution of common design cumulants from ferrets. Distribution of 10000 bootstrapped common design cumulants of ferrets from [24]. Dotted lines: Gaussian distribution corresponding to the experimentally observed cumulants. A Pinwheel density, see Fig. 3.14A, B-M the first four cumulants of pinwheel nearest neighbor distributions, see Fig. 3.14D-E. N, O Parameters that summarize the pinwheel density standard deviation scaling with cortical area, see Fig. 3.14C. Data from $[24]$. 
A

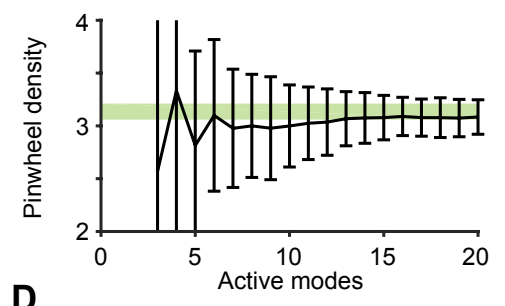

D

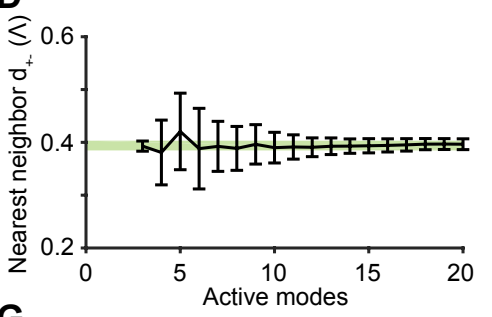

G

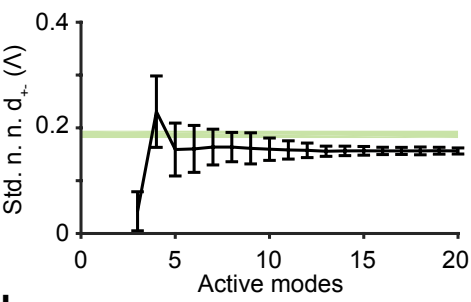

J

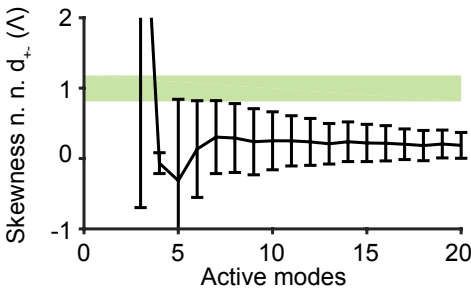

M

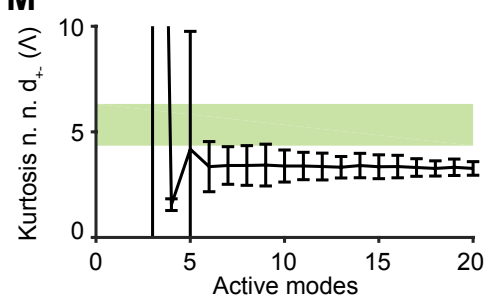

B

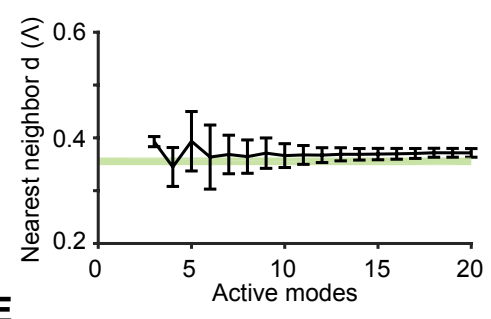

E

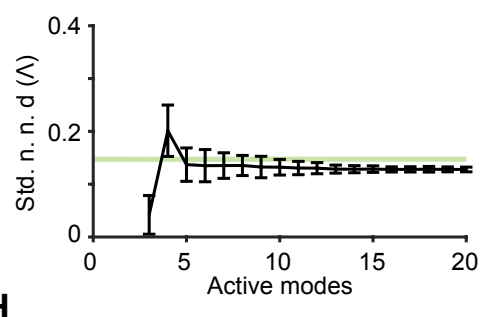

H

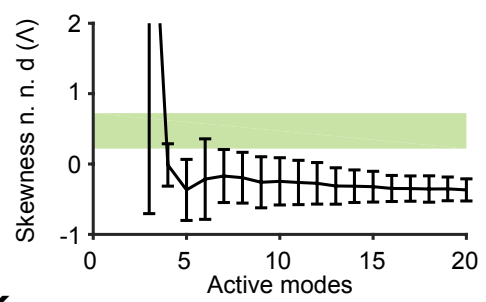

K

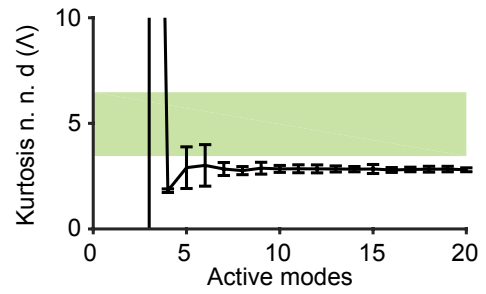

N

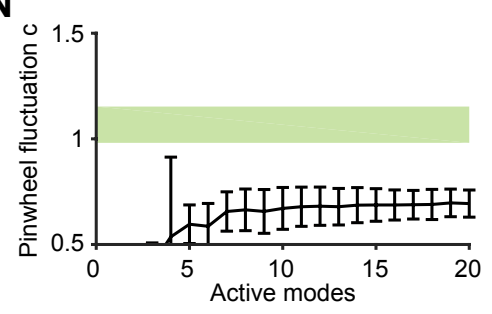

C

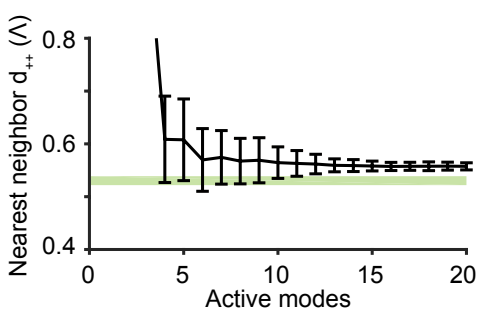

$\mathbf{F}$

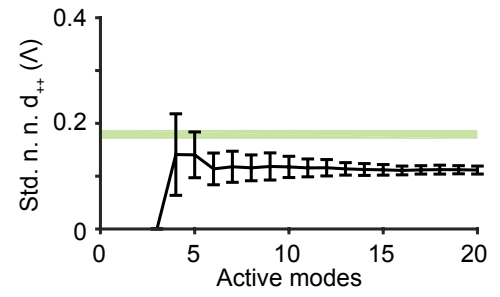

I

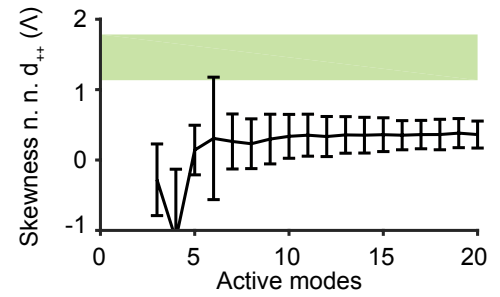

$\mathbf{L}$

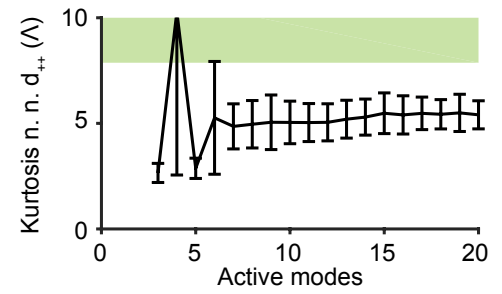

0

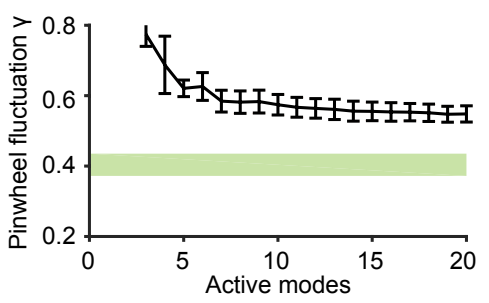

Figure 3.17: Pinwheel configuration statistics of ECPs. Pinwheel configuration statistics of ECP solutions with random anisotropies $l_{j}$. Dark lines indicate mean and standard deviation of 100 synthesized samples on a grid of $2048 \times 2048$ pixels with $22 \times 22$ typical scales. Green area indicates $95 \%$ confidence interval from ferrets pinwheel distributions. A Pinwheel density, see Fig. 3.14A, B-M the first four cumulants of pinwheel nearest neighbor distributions, see Fig. 3.14D-E. N, O Parameters that summarize the pinwheel density standard deviation scaling with cortical area, see Fig. 3.14C.

develop visual scotoma, i.e. only two orientation preferences, that we exclude from our analysis [137]. Varying the parameter from the amplitude dynamics of the canonical genetic network around the point $\gamma_{1}=\gamma_{2}=1$ and $\gamma_{6} / \gamma_{1} \approx 1$ and $\sigma=1.7 \Lambda$ with a Gaussian selective transport kernel leads to 11 free parameters for pattern selection. The results are shown in Fig. 3.18. We group these parameters into orientation encoding: (i) sensitive: $\beta_{1}, \beta_{2}, \eta_{1}, \eta_{2}, \gamma_{3}, \gamma_{4}, \gamma_{5}, \zeta_{1}, \zeta_{2}$, $\sigma, \gamma_{2}$, (ii) neutral $\alpha_{1}, \alpha_{2}, \eta_{1}, \gamma_{1}, \gamma_{6}, \gamma_{7}$ and the remaining (iii) 9 insensitive parameters. 
A

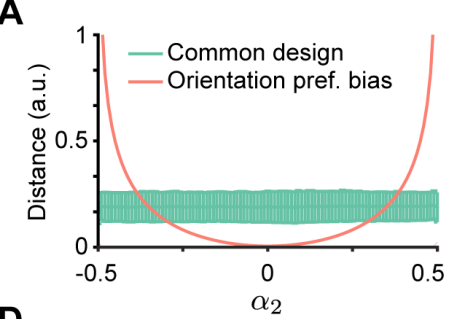

D

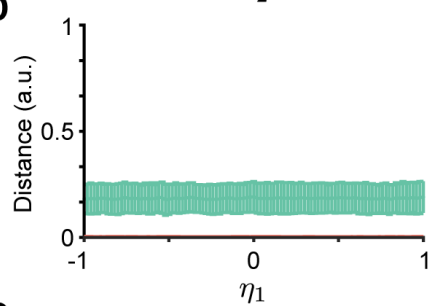

G

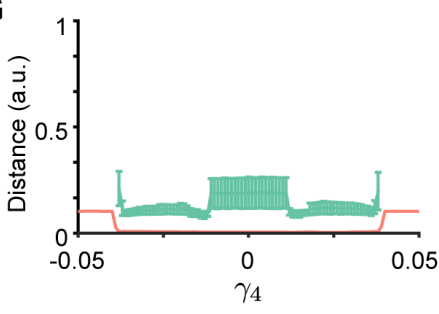

$\mathbf{J}$

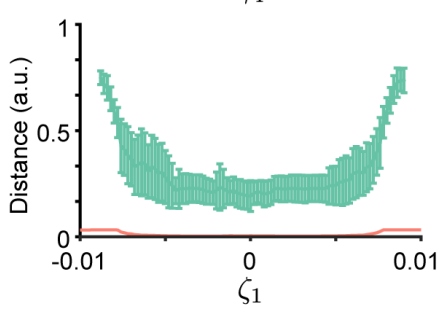

B

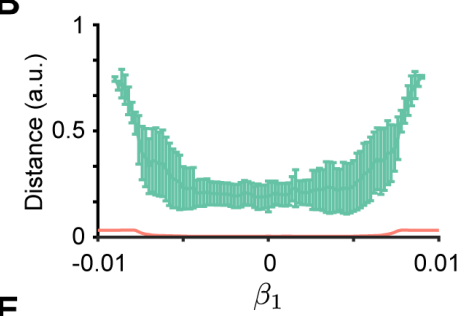

E

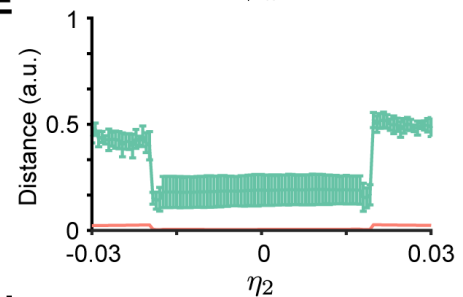

H

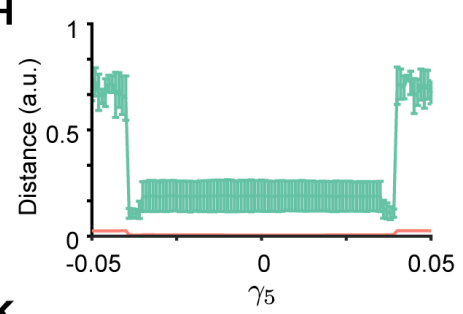

K

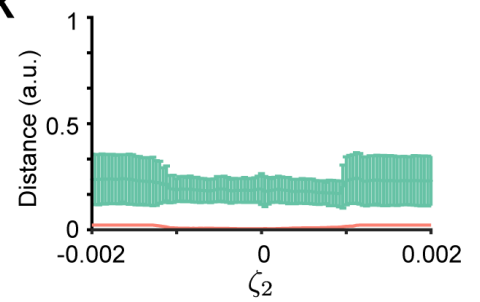

C

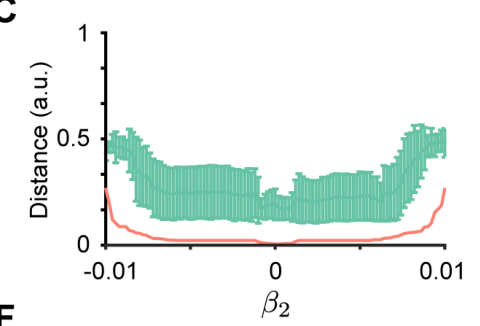

F

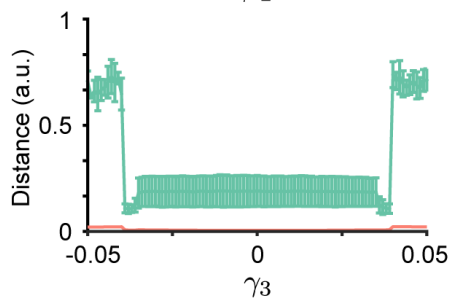

I

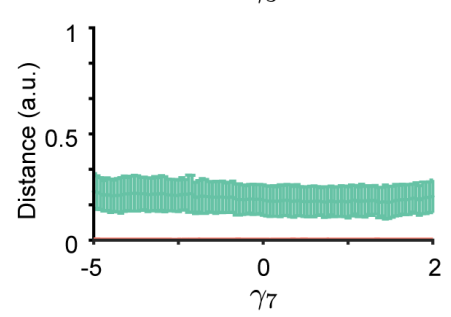

Figure 3.18: Orientation layout sensitivity on kinetic parameters. Varying the parameter from the amplitude dynamics of the canonical genetic network around the point $\gamma_{1}=\gamma_{2}=1, \gamma_{6} / \gamma_{1} \approx 1$ and $\sigma=1.7 \Lambda$ with a Gaussian selective transport kernel leads to 11 free parameters for pattern selection, see text for details. The distance to the common design of ferrets (mean and $95 \%$ confidence interval of 1000 bootstrapped pinwheel configurations from 30 initial conditions) and KL divergence to an equally distributed orientation preference is shown in $\mathbf{A}-\mathbf{K}$ for the 11 different free parameters. Simulation time was $T=10^{5} \tau$.

\subsubsection{Numerical methods}

The simulation of the coupled field dynamics followed the scheme developed for uncoupled fields in [267]. The orientation field equations are stiff partial differential equations of SwiftHohenberg type that we simulate by a fully implicit integration scheme to avoid impractically small integration time steps that arise in explicit schemes [268]. The field dynamics

$$
\begin{aligned}
& \partial_{t} \Psi(\boldsymbol{x}, t)=\hat{L}_{\Psi}[\Psi]+N_{\Psi}[\Psi] \\
& \partial_{t} \Sigma(\boldsymbol{x}, t)=\hat{L}_{\Sigma}[\Sigma]+N_{\Sigma}[\Sigma]
\end{aligned}
$$

with their linear $\hat{L}$ and non-linear operators $\hat{N}$ are discretized in time $\Delta t$ and space $\Delta x$ such that time and space is given by an integer $t$ and $i$, respectively. The discretized field dynamics 
are given in the Crank-Nicolson integration scheme as

$$
\begin{aligned}
& \frac{\Psi_{t+1}^{i}-\Psi_{t}^{i}}{\Delta t}=\frac{1}{2}\left(\hat{L}_{\Psi}^{i}\left[\Psi_{t+1}\right]+\hat{N}_{\Psi}^{i}\left[\Psi_{t+1}\right]+\hat{L}_{\Psi}^{i}\left[\Psi_{t}\right]+\hat{N}_{\Psi}^{i}\left[\Psi_{t}\right]\right) \\
& \frac{\Sigma_{t+1}^{i}-\Sigma_{t}^{i}}{\Delta t}=\frac{1}{2}\left(\hat{L}_{\Sigma}^{i}\left[\Sigma_{t+1}\right]+\hat{N}_{\Sigma}^{i}\left[\Sigma_{t+1}\right]+\hat{L}_{\Sigma}^{i}\left[\Sigma_{t}\right]+\hat{N}_{\Sigma}^{i}\left[\Sigma_{t}\right]\right),
\end{aligned}
$$

where the operators are discretized as well. The operators are evaluated in Fourier space and real space depending on their computational efficiency. The Crank-Nicolson scheme is iteratively solved by finding the root of the function $\boldsymbol{G}(t+1)=\left(\boldsymbol{G}_{\Psi}\left[\Psi_{t+1}\right], \boldsymbol{G}_{\Sigma}\left[\Sigma_{t+1}\right]\right)^{T}$, where

$$
\begin{aligned}
G_{\Psi}^{i}\left[\Psi_{t+1}\right] & =\hat{L}_{\Psi}^{i}\left[\Psi_{t+1}\right]+\hat{N}_{\Psi}^{i}\left[\Psi_{t+1}\right]-\frac{2}{\Delta t} \Psi_{t+1}^{i}+\hat{L}_{\Psi}^{i}\left[\Psi_{t}\right]+\hat{N}_{\Psi}^{i}\left[\Psi_{t}\right]+\frac{2}{\Delta t} \Psi_{t}^{i} \\
G_{\Sigma}^{i}\left[\Sigma_{t+1}\right] & =\hat{L}_{\Sigma}^{i}\left[\Sigma_{t+1}\right]+\hat{N}_{\Sigma}^{i}\left[\Sigma_{t+1}\right]-\frac{2}{\Delta t} \Sigma_{t+1}^{i}+\hat{L}_{\Sigma}^{i}\left[\Sigma_{t}\right]+\hat{N}_{\Sigma}^{i}\left[\Sigma_{t}\right]+\frac{2}{\Delta t} \Sigma_{t}^{i}
\end{aligned}
$$

with the Newton method. The $k$ th Newton iteration is then given by

$$
D \boldsymbol{G}(t+1)\left(\boldsymbol{u}^{k}\right)\left(\boldsymbol{u}^{k+1}-\boldsymbol{u}^{k}\right)=-G\left(\boldsymbol{u}^{k}\right)
$$

with $D \boldsymbol{G}(t+1)$ the Jacobian of $\boldsymbol{G}(t+1)$ and $\boldsymbol{u}$ the discretized fields. The Jacobian is calculated by a matrix-free method using finite differences. The remaining linear system of the Newton iteration is then solved by the Generalized Minimum Residual (GMRES) algorithm that utilizes the Arnoldi process to efficiently approximate Krylov subspaces. The convergence of the integrator is additionally improved by preconditioning the system with the inverse of its shifted linear operator. As the convergence of Newton's method is only guaranteed in the vicinity of the solution a line search algorithm is used to ensure global convergence [269]. Finally, an adaptive step size control was implemented to improve convergence in the neighborhood of an attractor as described in [270]. The integrator is implemented in $C++$ using the PetSc library http://www.mcs.anl.gov/petsc/petsc-as/.

Field dynamics were only simulated for the symmetry-confined canonical genetic network of section 3.5.3 and slightly modified versions of it. The parameters of the linear kernel $\hat{L}_{\Psi}$ were chosen such that the eigenspectrum has a maximum of $r>0$ for wave vectors $\boldsymbol{k}$ on the critical circle $k_{c}=\|\boldsymbol{k}\|$. In order to achieve this parametrization, the scaling parameters of the kernels $K_{+}, K_{-}$and $K$ in Eq. (3.120) were fixed to 4, 10 and 1, respectively. Setting $\alpha_{1}=r$, $\sigma_{u}=1$ and $\sigma$ to the desired axonal range leaves the last free parameter $\rho_{u}$ of the linear operator undetermined. This parameter was then automatically adjusted such that the afore-mentioned condition was met. Notice that the parametrization is permitted as sufficient free parameters exist in the field dynamics of Eq. (3.120).

In Fig. 3.4, Eq. (3.120) was integrated by the described algorithm with Gaussian kernels, $r=1 / \tau=0.05, \gamma_{6} \approx 0.56$ and for long-range $\gamma_{1}=0.51, \sigma=1.7 \Lambda$ and short-range with no patchy connections $\gamma_{1}=0$. The field was discretized to $256 \times 256$ pixels with an aspect ratio of $22 \Lambda \times 22 \Lambda$. Two of 20 simulations were excluded for illustration purposes. In Fig. 3.5, the amplitude Eq. (3.99) were integrated by the MATLAB internal Runge-Kutta $(4,5)$ formula with adaptive step-size control ode45. Fixed parameters were $\sigma=1.7 \Lambda, n=9, \gamma_{6} / \gamma_{1}=1.5$ and $b_{1}=b_{2}=15$. Simulation time was $T=10^{5} \tau$ and number of modes $N=18$. In Fig. 3.6A,B, the connectome was fixed to an isotropic Gaussian and patchy connectome from a previous simulation, respectively. The parameters used to integrate modified Eq. (3.120) were for both Gaussian kernels, $r=1 / \tau=0.05, \sigma=1.7 \Lambda$ and $b_{1}=b_{2}=15$ with the additional parameters for $\mathbf{A}$ of $\gamma_{6} \approx 0.56, \gamma_{1}=0.51$ and for $\mathbf{B}$ of $\gamma_{6} \approx-0.25, \gamma_{1}=0.25$ and $\sigma=1.7 \Lambda$. The field was in both cases discretized to $128 \times 128$ pixels with an aspect ratio of $22 \Lambda \times 22 \Lambda$. In Fig. 3.6D-F, the amplitude Eq. (3.168) were integrated again by ode 45 as for Fig. 3.5 with a connectome 
timescale equal to the critical timescale $\tau_{m}=\tau_{c}$. The total concentration field was obtained by linear response of $\Sigma$ to $\Psi$. In Fig. 3.6H-J, the delay differential amplitude Eq. (3.226) were integrated by the MATLAB internal method dde23 with delay slightly larger than the critical delay $\tau=1.001 \tau_{c}$. The total concentration field was obtained by linear response of $\Sigma$ to $\Psi$. In Fig. 3.7, Eq. (3.120) was modified by a focal and dual overexpression of a single morphogen with strength $0.1 r$ and simulated with the same parameters as for Fig. 3.4 with the additional parameter of $b_{1}=b_{2}=15$.

\subsubsection{Alternative model: positionally specified orientation preference}

An alternative mechanism for cell fate specification is a position-dependent cell fate. In contrast to self-organizing models, cells are typically not interacting and simply read out their position by concentration gradients. We will first approximate the number of required gradients for determining orientation domains by information theory. Subsequently, we devise an explicit model for that task and increase gradually the level of complexity.

In order to encode the spatial organization of orientation preference uniquely, every isoorientation domain must be determined by morphogen gradients. The number of iso-orientation domains increases with the number of pinwheels $N$. The number of pinwheels scales with the area $A$ of $\mathrm{V} 1$ as $N \approx \pi A / \Lambda^{2}[22-24]$ with $\Lambda$ the typical scale. If four different iso-orientation domains are required to be encoded per pinwheel, the information content required to encode orientation preference in V1 is given by the logarithm of the number of encoded states

$$
I_{V 1}(A)=\log _{2}\left(4 \pi A / \Lambda^{2}\right) \text { bit. }
$$

The typical scale is typically about $\Lambda \approx 1 \mathrm{~mm}$ [23]. Cortical areas of V1 vary substantially between different species, for instance, for macaque $A_{m}=1200 \mathrm{~mm}^{2}$ and for human $A_{h}=3000 \mathrm{~mm}^{2}$, see e.g. [271]. The required information is then about $I_{m} \approx 14$ bit and $I_{h} \approx 15$ bit. This information content must be provided by morphogen gradients. In fruit flies, it was shown that morphogen gradients during embryogenesis carry 1-2 bit of information [235]. Thus the number of morphogen gradients should be at least on the order of $7-15$. This range of required morphogen gradients is in a biologically realistic regime. In order to examine how such a mechanism could be biologically realized, we examine an explicit model in the following.

In a two-dimensional cortical tissue two orthogonally oriented gradients

$$
\begin{array}{ll}
g_{1}(\boldsymbol{x})=x & g_{2}(\boldsymbol{x})=y
\end{array}
$$

suffice to specify cortical positions $\boldsymbol{x} \in[0,1]^{2}$, see Fig. 3.19A. Orientation preference is as before encoded by four morphogens through the difference in concentrations

$$
\Psi(\boldsymbol{x})=\left(c_{1}(\boldsymbol{x})-c_{2}(\boldsymbol{x})\right)+i\left(c_{3}(\boldsymbol{x})-c_{4}(\boldsymbol{x})\right) .
$$

In order to encode morphogen concentrations by their cortical position or equivalently gradient concentration, the expression of morphogens must be controlled by the gradient concentrations. The simplest expression control is given by a single gene regulatory element whose regulatory control can be phenomenologically modeled by Hill functions, see section 3.5.1. A more flexible model that incorporates combinatorial control is given by the logistic function

$$
\phi_{\beta, \mu}[c(\boldsymbol{x})]=\frac{1}{1+e^{-\beta(c(\boldsymbol{x})-\mu)}}
$$

that possesses a similar shape to Hill functions, but is mathematically more convenient. The parameter $\mu$ and $\beta$ are similar to the binding affinity and the cooperativity of the gene regulatory element, respectively. 


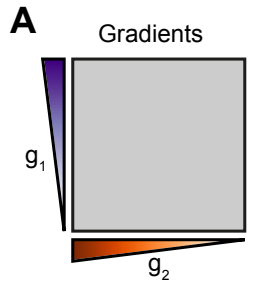

C

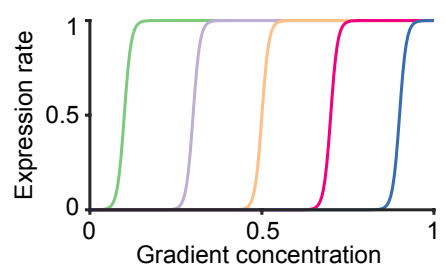

B

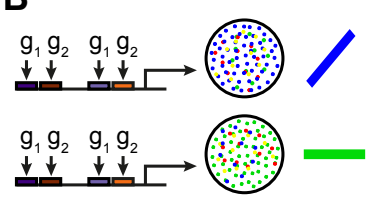

D

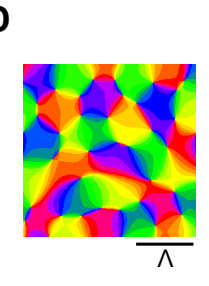

$\mathbf{F}$

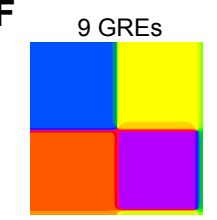

$\mathbf{E}$
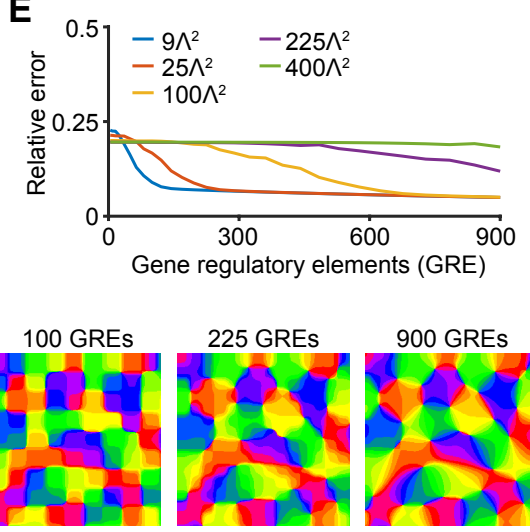

900 GREs

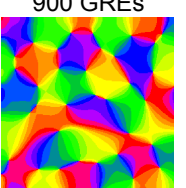

Figure 3.19: Orientation encoding by cortical position and non-interacting genes. A Cell fates are specified by their cortical position, which can be read out from gradients. B Orientation encoding morphogens expression is controlled by gene regulatory element affinities to gradient concentrations. C The expression rates are combinatorial Hill functions with equidistantly placed affinities for both gradients, see text for more details. D Target orientation domain that gene regulatory elements specify by their cortical positions. E Relative error of morphogen concentrations versus the number of gene regulatory elements and for different orientation domain sizes. F Optimal orientation domain specification by cortical position of the orientation domain in $\mathbf{D}$.

\section{Orientation encoding by non-interacting genes}

The simplest genetic network that encodes orientation preference by cortical position can be solved analytically. In order to encode the morphogen concentrations $c_{i}(\boldsymbol{x})$ with $i \in\{1,2,3,4\}$, a genetic expression response to the gradients is required that exactly reproduce the morphogen concentrations at every cortical position. The simplest control over a gene regulatory element is given by combinatorial Hill functions with different binding affinities, Fig. 3.19B, given by

$$
\phi_{j}^{i}(\boldsymbol{x})=\phi_{\beta, \mu_{j}}\left[g_{1}(\boldsymbol{x})\right] \phi_{\beta, \tilde{\mu}_{j}}\left[g_{2}(\boldsymbol{x})\right] .
$$

A superposition of Hill functions with different binding affinities results in the equilibrium morphogen concentration of

$$
c_{i}(\boldsymbol{x})=\sum_{j=0}^{M} \omega_{j}^{i} \phi_{j}^{i}(\boldsymbol{x}) .
$$

For a given concentration field at positions $\boldsymbol{x}_{k}$ the coupling parameters $\omega_{j}$ can be derived by minimizing the error function

$$
E=\frac{1}{2} \sum_{k=0}^{N}\left(c_{i}\left(\boldsymbol{x}_{k}\right)-\sum_{j=0}^{M} \omega_{j}^{i} \phi_{j}^{i}\left(\boldsymbol{x}_{k}\right)\right)^{2}
$$

This is mathematically equivalent to linear regression with the basis functions $\phi_{j}^{i}(\boldsymbol{x})$. The solution for the weights is given by the multiplication of the orientation encoding concentration with the design matrix, i.e.

$$
\boldsymbol{\omega}^{i}=\left(\phi^{i T} \phi^{i}\right)^{-1} \phi^{i T} \boldsymbol{c}_{i}
$$


with the abbreviations $\left(\boldsymbol{\omega}^{i}\right)_{j}=\omega_{j}^{i},\left(\boldsymbol{c}_{i}\right)_{k}=c_{i}\left(\boldsymbol{x}_{k}\right)$ and

$$
\boldsymbol{\phi}^{i}=\left(\begin{array}{cccc}
\phi_{0}^{i}\left(\boldsymbol{x}_{0}\right) & \phi_{0}^{i}\left(\boldsymbol{x}_{1}\right) & \ldots & \phi_{0}^{i}\left(\boldsymbol{x}_{N}\right) \\
\phi_{1}^{i}\left(\boldsymbol{x}_{0}\right) & \phi_{1}^{i}\left(\boldsymbol{x}_{1}\right) & \ldots & \phi_{1}^{i}\left(\boldsymbol{x}_{N}\right) \\
\vdots & \vdots & \ddots & \vdots \\
\phi_{M}^{i}\left(\boldsymbol{x}_{0}\right) & \phi_{M}^{i}\left(\boldsymbol{x}_{1}\right) & \ldots & \phi_{M}^{i}\left(\boldsymbol{x}_{N}\right)
\end{array}\right)
$$

For a simple orientation field specified by an ECP with $n=8$ active modes, Fig. 3.19D, with equidistantly spaced Hill functions for both gradients, Fig. 3.19C, the relative error of the morphogen concentrations declines rapidly for small orientation field areas by increasing the number of gene regulatory elements, Fig. 3.19F. However, for biologically plausible visual cortical areas of the size of a thousand $\Lambda^{2}[272,273]$ in macaques, the number of gene regulatory elements per morphogen is exceedingly high and unrealistic, Fig. 3.19E. This shows that orientation preference specification by cortical position read out requires a biologically unrealistic number of gene regulatory elements in this type of genetic networks.

\section{Orientation encoding by interacting genes}

The required large number of gene regulatory elements can be circumvented by including additional genes that combinatorially control the morphogen concentrations. Here, the concentration of genes are mutually regulated in a hierarchical scheme. The first stage is composed of the gradient concentrations $g_{1}(\boldsymbol{x})$ and $g_{2}(\boldsymbol{x})$ and the last stage of the morphogen concentrations $c_{i}(\boldsymbol{x})$, Fig. 3.20A. Thus at every regulatory level $l$ the $i$ th concentration $c_{i}^{l}(\boldsymbol{x})$ is given by the equilibrium concentration through a superposition of gene regulatory elements that are controlled by the equilibrium concentrations of the previous regulatory level $c_{j}^{l-1}(\boldsymbol{x})$

$$
c_{i}^{l}(\boldsymbol{x})=\sum_{j=0}^{M} \omega_{i j}^{l} \phi_{\beta, \mu_{i j}^{l}}\left[c_{j}^{l-1}(\boldsymbol{x})\right]
$$

as illustrated in Fig. 3.20B. As the binding affinities $\mu_{i j}^{l}$ and cooperative control $\beta$ are implicitly determined by the superposition of weights $\omega_{i j}^{l}$, the model is simplified to $\mu_{i j}^{l}=0$ and $\beta=1$. Thus the gene regulatory element affinities are not equidistantly distributed, but are specifically selected. This is similar to the previous model, but with additional regularization though without combinatorial control of the gradients. Notice that for a generic superposition of weights, the concentrations can become negative. However, negative concentrations can be cured by assuming an additional baseline gene expression and adapted binding affinities of that concentration. This would compensate for the negative concentration while retaining the regulatory control of that gene. Thus the superposition of weights can be chosen to any value without loosing biological meaning.

The final genetic network is given by

$$
c_{i}^{l}(\boldsymbol{x})=\sum_{j=0}^{M} \frac{\omega_{i j}^{l}}{1+e^{-c_{j}^{l-1}(\boldsymbol{x})}}
$$

and is equivalent to an artificial neural network. These networks can be trained to a specific output for a given input by a backpropagation algorithm. This algorithm minimizes the squared error between the network output and the desired output by gradient descent. Normal gradient descent is intrinsically unsuited to minimize a complex energy landscape as it often stucks in local minima. Therefore, backpropagation algorithms were extensively enhanced by various techniques that resulted in a fast minimization of the squared error between desired and network 
A

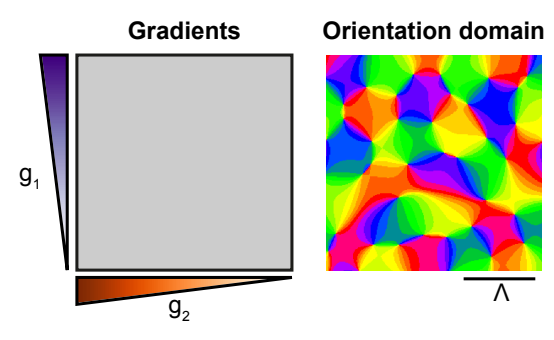

C

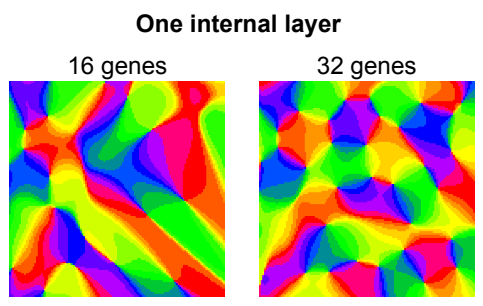

Two internal layers

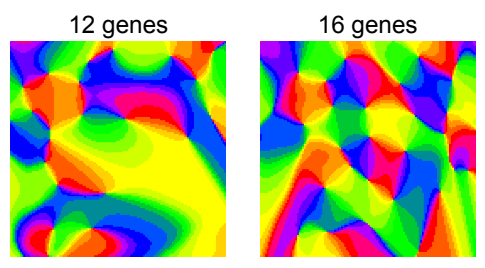

B

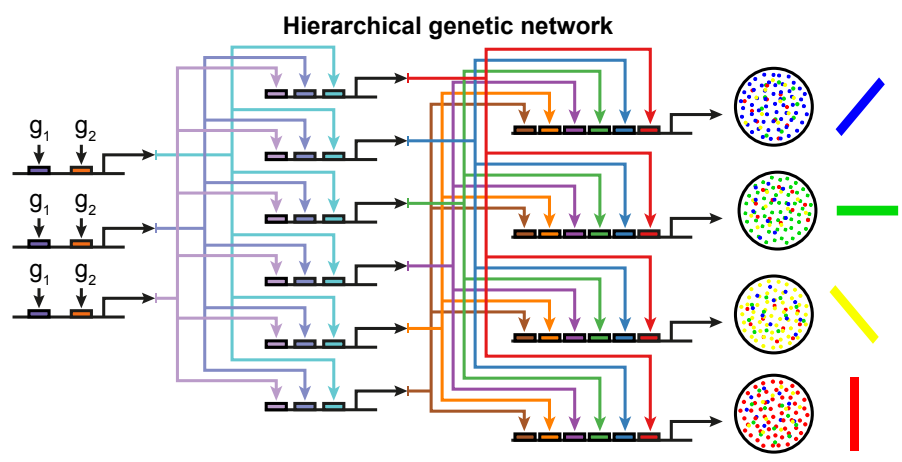

D

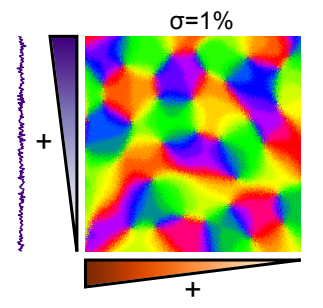

E
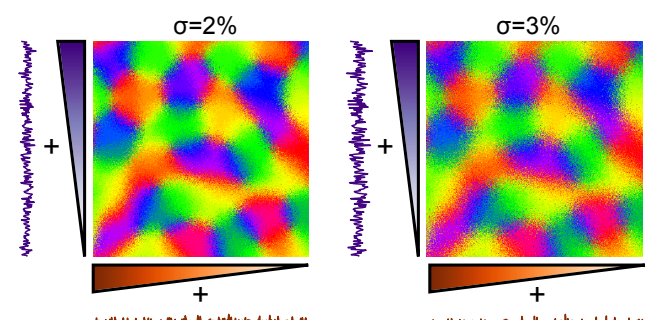

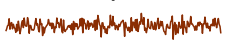

$g_{1}$

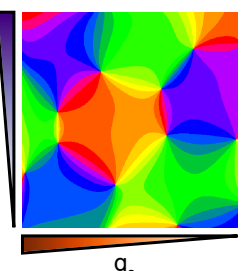

$g_{2}$
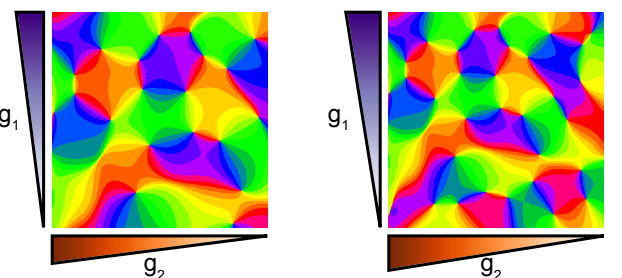

Figure 3.20: Hierarchical genetic network encoding orientation preference by cortical position. A Two orthogonally oriented cortical gradients determine cortical position by their concentrations. Morphogens are expressed according to the gradient concentration and encode orientation preference by their difference in concentrations, see text for details. B The hierarchical genetic network consists of genes that are organized in layers with gradient concentration as input in the first (internal) layer and morphogen concentrations encoding orientation preference in the last layer. The number of genes and inner layers are adjustable. C For one internal layer, orientation domains can be encoded by about 30 genes. Two internal layers reduce the number of genes required for orientation domain encoding to about 15 genes. D Gradient fluctuations with standard deviation of a few percentage of the maximal gradient amplitude severely affects the encoded orientation domains (Network of one layer with 32 genes). E Varying the gradient magnitude as expected during ontogenesis changes the typical scale of the orientation domains (Network of one layer with 32 genes).

output without stucking in every local minimum, see e.g. [274-276]. Here we use the iRprop+ algorithm with standard values to efficiently train the superposition weights [274]. The genetic networks are trained until the minimal error of 500 learning iterations drops less than $10^{-3}$ of the minimal error of the last 500 learning iterations.

As the number of additional genes increases, the encoded orientation domain appears more and more realistic, Fig. 3.20C. Increasing the area of orientation domains requires certainly more genes, but this could be in a biologically realistic regime. Using only two morphogens $c_{i}$ slightly reduces the number of genes, while retaining orientation domain specification. Thus a hierarchical genetic network might be able to encode orientation domains with a high though realistic number of genes and gene regulatory elements under ideal conditions. Including small shot noise fluctuations to the gradient of the order of a few percentage of the maximal gradient amplitude severely affects the smooth progression of orientation preference, Fig. 3.20D. 
Asumming noise integration from the dendritic tree of about $\Lambda / 10 \approx 100 \mu \mathrm{m}$ leads to correlated noise with a correlation length of about the dendritic tree that also severly affects orientation preference layout for small noise strengths (not shown). Generically, the noise sensitivity further increases with visual cortical area if the maximal gradient concentration and the noise level are retained. This is in stark conflict to the experimentally observed highly ordered cellular arrangement of orientation preference around pinwheels [71]. Increasing the gradient amplitude changes the typical scale of the orientation domain, Fig. 3.20E. Thus the effect on the typical scale of a growing visual cortical area accompanied with a gradient increase could compensate such that the typical scale is conserved during ontogenesis as experimentally observed [98]. However, this scheme is difficult to reconcile with typical boundary effects of orientation domains [12] as only two boundaries can be fixed in this hierarchical genetic network scheme, Fig. 3.20E. From an evolutionary perspective it is hard to explain similar typical scales in different visual cortical area sizes [23] with such a static mechanism. In total, the high number of required genes, the noise susceptibility and the inability of a fixed typical scale with fixed boundary orientation preference layouts makes this type of genetic network with non-interacting cells unlikely to account for orientation domain specification. 


\subsubsection{Nomenclature}

\section{Genetic network}

$\Lambda \quad$ Typical scale of orientation domains

$\Psi(\boldsymbol{x}, t) \quad$ Orientation encoding field or short orientation field

$\sigma \quad$ Range of morphogen selective connections

$\Sigma(\boldsymbol{x}, t) \quad$ Total concentration field

$\Sigma_{0} \quad$ Total concentration field fixed point

$c_{i}(\boldsymbol{x}, t) \quad$ Morphogen concentration field

\section{Mathematical symbols}

$\mathcal{F} \quad$ Two dimensional Fourier transformation $\mathcal{F}\{K\}=\int \mathrm{d}^{2} y K(\boldsymbol{y}) e^{-i \boldsymbol{k} \boldsymbol{y}}$

$\Re, \Im \quad$ Real and imaginary part of a complex variable $\Re\{a+i b\}=a$ and $\Im\{a+i b\}=b$, respectively

$\star \quad$ Two dimensional convolution operator $K \star \Psi(\boldsymbol{y})=\int \mathrm{d}^{2} y K(\boldsymbol{x}-\boldsymbol{y}) \Psi(\boldsymbol{y})$

$\|\cdot\| \quad$ Euclidean norm $\|\boldsymbol{x}\|=\sqrt{\boldsymbol{x}^{T} \boldsymbol{x}}$ 



\section{Pinwheel configuration in maximum entropy models of orientation domains}

\subsection{Motivation}

The pinwheel configuration of orientation domains observed in primates and placental carnivores adhere to quantitative species-invariant statistics. This common design is composed of the pinwheel density, pinwheel density fluctuations in subareas and nearest neighbor distributions of pinwheels with equal, opposite and independent of topological charge, see Fig. 2.3. The common design is a non-trivial mammalian trait as shown by comparisons to surrogate data and theoretical analysis of pinwheel densities in orientation field ensembles. Other statistical measures of the pinwheel configuration were so far not examined theoretically. In this chapter, we derive an analytical expression for the pinwheel density fluctuations and an approximated analytical expression for the nearest neighbor distribution for maximum entropy models of orientation domains.

\subsection{Maximum entropy models of orientation fields}

In order to investigate pinwheel configurations in maximum entropy models of orientation fields, the preferred orientation $\vartheta(\boldsymbol{x})$ together with its selectivity $S(\boldsymbol{x})$ of a neuron at cortical position $\boldsymbol{x}=(x, y)^{T}$ is combined to an orientation director field

$$
\Psi(\boldsymbol{x})=S(\boldsymbol{x}) e^{2 i \vartheta(\boldsymbol{x})}
$$

or short orientation field with a real $\xi(\boldsymbol{x})$ and imaginary $\eta(\boldsymbol{x})$ part. Maximum entropy models of orientation field ensembles with vanishing mean are then given by centered Gaussian random fields. Marginal distributions of orientation field components, for instance, $\chi=\left(\xi, \eta, \xi_{x}, \xi_{y}, \eta_{x}, \eta_{y}\right)^{T}$ with the derivative $\partial_{x} \xi=\xi_{x}$ and omitted spatial dependence are described by a multivariate Gaussian distribution

$$
p(\chi)=\frac{1}{\sqrt{(2 \pi)^{6} \operatorname{det} \boldsymbol{\Sigma}}} e^{-\frac{1}{2} \chi^{T} \boldsymbol{\Sigma}^{-1} \chi}
$$

with the covariance $\boldsymbol{\Sigma}=\left\langle\chi \chi^{T}\right\rangle$. In the following, we will focus exclusively on orientation fields with a vanishing mean $\langle\boldsymbol{\chi}\rangle=\mathbf{0}$.

The pinwheel configuration statistics constituting the common design will be derived analytically in the following for maximum entropy models. For the simplest quantity, the pinwheel density, a formula for generic centered Gaussian random fields will be derived. For all other 
pinwheel configuration statistics that depend on the distance between pinwheels, the analysis will be restricted to isotropic and shift-symmetric maximum entropy models. Shift-symmetric orientation field ensembles are defined by invariant statistics to global phase shifts of the orientation field $\Psi(\boldsymbol{x}) \rightarrow e^{i \phi} \Psi(\boldsymbol{x})$. Thus, the mean and one of the correlation functions of the orientation field vanishes

$$
\langle\Psi(\boldsymbol{x})\rangle=e^{i \phi}\langle\Psi(\boldsymbol{x})\rangle=0 \quad\left\langle\Psi\left(\boldsymbol{x}_{1}\right) \Psi\left(\boldsymbol{x}_{2}\right)\right\rangle=e^{2 i \phi}\left\langle\Psi\left(\boldsymbol{x}_{1}\right) \Psi\left(\boldsymbol{x}_{2}\right)\right\rangle=0 .
$$

Shift-symmetric and isotropic orientation field ensembles are defined by the additional invariance to rotations and translations resulting in the correlation function

$$
C(r)=\left\langle\Psi\left(\boldsymbol{x}_{1}\right) \bar{\Psi}\left(\boldsymbol{x}_{2}\right)\right\rangle
$$

with $r=\left\|\boldsymbol{x}_{1}-\boldsymbol{x}_{2}\right\|$.

Translation invariant Gaussian random fields can be efficiently synthesized. The correlation function $C\left(\boldsymbol{x}_{1}-\boldsymbol{x}_{2}\right)=\left\langle\Psi\left(\boldsymbol{x}_{1}\right) \bar{\Psi}\left(\boldsymbol{x}_{2}\right)\right\rangle$ is diagonal in Fourier space and given by $P(\boldsymbol{k})$. The Fourier transform of an orientation layout $\tilde{\Psi}(\boldsymbol{k})$ with the specified correlation function is synthesized by drawing Gaussian random numbers $u, v \sim \mathcal{N}(0,1)$ for all $\boldsymbol{k}$ and scaling them by $\tilde{\Psi}(\boldsymbol{k})=$ $\sqrt{P(\boldsymbol{k})}(u+i v)$.

\subsection{Pinwheel density}

The total number of pinwheel centers $\boldsymbol{x}_{i}$ in an area $A$ is given by the spatial integral over

$$
\sum_{i} \delta\left(\boldsymbol{x}-\boldsymbol{x}_{i}\right)=\delta(\eta(\boldsymbol{x})) \delta(\xi(\boldsymbol{x}))\left|\operatorname{det} \frac{\partial(\eta(\boldsymbol{x}), \xi(\boldsymbol{x}))}{\partial(x, y)}\right|
$$

For the sake of brevity, the dependence on the spatial position will be omitted in the following. The pinwheel density is given by the ensemble average $\langle\cdot\rangle$ over the pinwheel center positions

$$
\rho=\left\langle\delta(\xi) \delta(\eta)\left|\xi_{x} \eta_{y}-\xi_{y} \eta_{x}\right|\right\rangle=\left\langle\delta(\xi) \delta(\eta)\left|\chi^{T} \boldsymbol{A} \boldsymbol{\chi}\right|\right\rangle .
$$

with the orientation field components $\chi=\left(\xi, \eta, \xi_{x}, \xi_{y}, \eta_{x}, \eta_{y}\right)^{T}$ and the matrices

$$
\boldsymbol{A}=\left(\begin{array}{cc}
0 & 0 \\
0 & \boldsymbol{A}_{0}
\end{array}\right) \in \mathbb{R}^{6 \times 6}, \quad \boldsymbol{A}_{0}=\left(\begin{array}{cccc}
0 & 0 & 0 & 1 / 2 \\
0 & 0 & -1 / 2 & 0 \\
0 & -1 / 2 & 0 & 0 \\
1 / 2 & 0 & 0 & 0
\end{array}\right)
$$

Utilizing a convenient representation for the absolute value, the pinwheel density can be written as

$$
\rho=\frac{1}{\pi} \int \frac{\mathrm{d} t}{t^{2}}\left(T(0)-\frac{T(t)+T(-t)}{2}\right) \quad \text { with } \quad T(t)=\left\langle\delta(\xi) \delta(\eta) e^{i t \boldsymbol{\chi}^{T} \boldsymbol{A} \boldsymbol{\chi}}\right\rangle .
$$

The ensemble average thus reduces to a simple Gaussian integral that can be analytically calculated [277] by introducing $\boldsymbol{C}=\left(\boldsymbol{\Sigma}^{-1}\right)_{i>2, j>2}$ yielding

$$
T( \pm t)=\frac{1}{2 \pi} \sqrt{\frac{\operatorname{det} \boldsymbol{\Sigma}^{-1}}{\operatorname{det} \boldsymbol{C}}} \frac{1}{\sqrt{\operatorname{det} \boldsymbol{M}_{\mp}(t)}}
$$


A
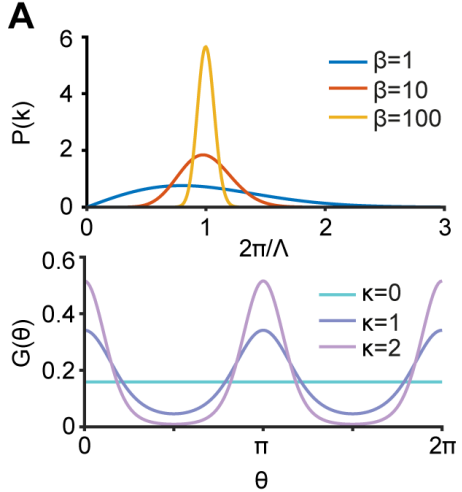

B

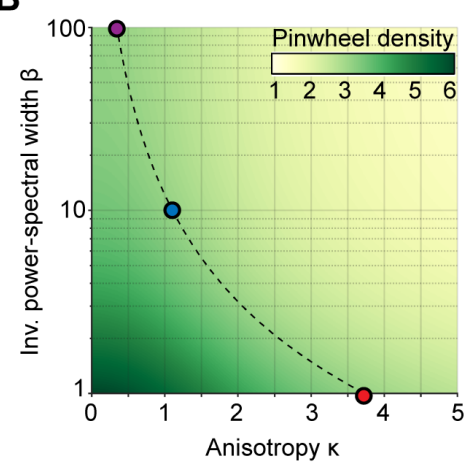

C
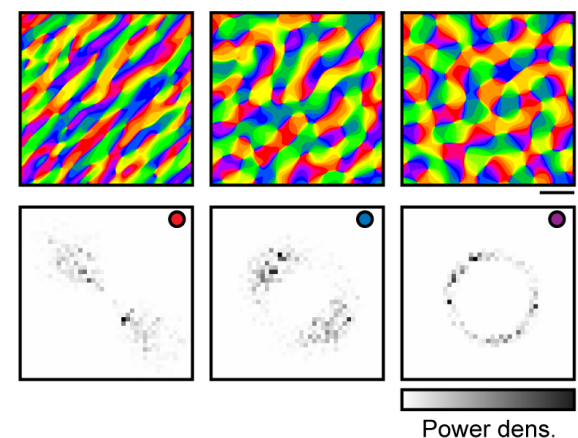

Figure 4.1: Pinwheel density of anisotropic orientation fields with finite power-spectral bandwidth. A The Fourier transform of the translation invariant correlation function separates into a radial and angular term $\mathcal{F}[C(\boldsymbol{x})]=P(k) G(\theta)$. The radial term $P(k)$ is a phenomenological model with a power-spectral density centered around a typical wave vector $k=1=2 \pi / \Lambda$ from [171]. The angular component $G(\theta)=\exp (\kappa \cos \theta) / 2 \pi I_{0}(\kappa)$ is chosen to be a von Mises distribution with $\kappa$ its measure of concentration or anisotropy. B Pinwheel densities in units of $\Lambda^{2}$ can be analytically calculated with the formula provided in [22]. The dashed line indicates a pinwheel density of $\rho=\pi / \Lambda^{2}$. C Synthesized orientation fields with a pinwheel density of $\rho=\pi / \Lambda^{2}$ together with their inverse power-spectral density $\beta=1,10,100$ and anisotropies indicated by colored dots in $\mathbf{B}(1024 \times 1024$ pixels and aspect ratio $5 \Lambda \times 5 \Lambda)$.

with $\boldsymbol{M}_{ \pm}(t)=\mathbf{1} \pm 2 i t \boldsymbol{C}^{-1} \boldsymbol{A}_{0}$. The general solution of the pinwheel density for centered Gaussian field ensembles hence reads

$$
\rho=\frac{1}{2 \pi^{2}} \sqrt{\frac{\operatorname{det} \boldsymbol{\Sigma}^{-1}}{\operatorname{det} \boldsymbol{C}}} \int \frac{\mathrm{d} t}{t^{2}}\left(1-\frac{1}{2 \sqrt{\operatorname{det} \boldsymbol{M}_{+}(t)}}-\frac{1}{2 \sqrt{\operatorname{det} \boldsymbol{M}_{-}(t)}}\right) .
$$

Notice that, without further assumptions, the pinwheel density still depends on the spatial position $\boldsymbol{x}$. Translation invariance of the orientation field ensemble reliefs this property and makes the pinwheel density independent of the spatial position. Various special cases of pinwheel densities for translation invariant orientation fields have been considered previously. In isotropic and shift- or shift-twist-symmetric orientation fields, the pinwheel density was found to be bounded from below by $\rho \geq \pi / \Lambda^{2}$ with the typical scale $\Lambda[147,171]$. In shift-symmetric anisotropic orientation fields the pinwheel density can assume any positive value as shown in $[135,170]$. As the experimental pinwheel density is found to be close to $\pi / \Lambda^{2}$, anisotropic orientation fields models from $[170,171]$ with a pinwheel density of $\pi / \Lambda^{2}$ are illustrated in Fig. 4.1.

\subsection{Pinwheel pair and charge correlation function}

Pinwheel configuration statistics constituting the common design comprise, apart from the pinwheel density, additionally pinwheel densities in subareas and nearest neighbor distributions. In these statistics, higher order pinwheel correlations are implicitly summarized, which we will examine in detail later. The second order pinwheel correlations partially capture these statistics and will be calculated for maximum entropy models of orientation field ensembles and pinwheel crystals in the following.

Pinwheel correlations at positions $\boldsymbol{x}$ and $\boldsymbol{x}^{\prime}$ of a translation invariant pinwheel distribution with pinwheel density $\rho$, pinwheel centers located at $\boldsymbol{x}_{i}$ and topological charges of $q_{i}$ can be 
quantified by the pinwheel pair and charge correlation function

$$
\begin{aligned}
g\left(\boldsymbol{x}-\boldsymbol{x}^{\prime}\right) & =\frac{1}{\rho^{2}}\left\langle\left(\sum_{i} \delta\left(\boldsymbol{x}-\boldsymbol{x}_{i}\right)\right)\left(\sum_{j} \delta\left(\boldsymbol{x}^{\prime}-\boldsymbol{x}_{j}\right)\right)\right\rangle \\
g_{Q}\left(\boldsymbol{x}-\boldsymbol{x}^{\prime}\right) & =\frac{1}{\rho^{2}}\left\langle\left(\sum_{i} \operatorname{sgn}\left(q_{i}\right) \delta\left(\boldsymbol{x}-\boldsymbol{x}_{i}\right)\right)\left(\sum_{j} \operatorname{sgn}\left(q_{j}\right) \delta\left(\boldsymbol{x}^{\prime}-\boldsymbol{x}_{j}\right)\right)\right\rangle,
\end{aligned}
$$

respectively. The pair correlation function converges to unity for large distances and the charge correlation function to zero if the total topological charges of the pinwheel configuration vanish. Notice that the pinwheel pair correlation functions are radially symmetric for isotropic Gaussian random fields. Hence, we will focus only on the radial part $r=\left\|\boldsymbol{x}-\boldsymbol{x}^{\prime}\right\|$ of the pinwheel pair correlation functions in the following. The pair correlation function between pinwheels of same charge $g_{++}(r)$ and pinwheels of opposite charges $g_{+-}(r)$ are given by the relations

$$
g_{++}(r)=g(r)+g_{Q}(r), \quad g_{+-}(r)=g(r)-g_{Q}(r)
$$

which can be verified by plugging in the definitions of the pinwheel pair correlation functions. In order to appreciate possible differences in the pinwheel correlation functions, we examine orientation domain ensembles and pinwheel crystals in the following.

\subsubsection{Isotropic and shift-symmetric orientation field ensembles}

The pair and charge correlation functions of pinwheels in isotropic and shift-symmetric orientation field ensembles can be obtained by noting the identity of the pinwheel positions in Eq. (4.5) and taking the ensemble average over the resulting terms

$$
\begin{aligned}
g(r) & =\frac{1}{\rho^{2}}\left\langle\delta(\eta(\boldsymbol{x})) \delta(\xi(\boldsymbol{x}))|\nu(\boldsymbol{x})| \delta\left(\eta\left(\boldsymbol{x}^{\prime}\right)\right) \delta\left(\xi\left(\boldsymbol{x}^{\prime}\right)\right)\left|\nu\left(\boldsymbol{x}^{\prime}\right)\right|\right\rangle \\
g_{Q}(r) & =\frac{1}{\rho^{2}}\left\langle\delta(\eta(\boldsymbol{x})) \delta(\xi(\boldsymbol{x})) \nu(\boldsymbol{x}) \delta\left(\eta\left(\boldsymbol{x}^{\prime}\right)\right) \delta\left(\xi\left(\boldsymbol{x}^{\prime}\right)\right) \nu\left(\boldsymbol{x}^{\prime}\right)\right\rangle,
\end{aligned}
$$

with $r=\left\|\boldsymbol{x}-\boldsymbol{x}^{\prime}\right\|$ and the orthogonality measure $\nu(\boldsymbol{x})=\xi_{x}(\boldsymbol{x}) \eta_{y}(\boldsymbol{x})-\xi_{y}(\boldsymbol{x}) \eta_{x}(\boldsymbol{x})$ between $\nabla \xi(\boldsymbol{x})$ and $\nabla \eta(\boldsymbol{x})$ that is given by the signed area of their spanned parallelogram. A formula for $g(r)$ and $g_{Q}(r)$ was derived previously for isotropic and shift-symmetric Gaussian random fields [30]. An explicit formula for $g(r)$ can be derived, but is cumbersome due to its lengthy expression [154]. A detailed derivation of the pair correlation functions is provided in section 8.1. Notice that the calculation of the pair correlation function is tedious in contrast to the charge correlation function, which can be calculated for $n$-point correlations [155].

The pair correlation function is given in its simplest representation by

$$
g(r)=\frac{2 c_{1}^{2}\left(c_{4}\left(c_{1}^{2}-c_{2}^{2}\right)-c_{1} c_{3}^{2}\right)}{\pi c_{4}\left(c_{1}^{2}-c_{2}^{2}\right)^{2}} \int_{0}^{\infty} \mathrm{d} t \frac{2 t^{4} Z+t^{2}\left(-\frac{Y^{2}}{2}+Z+3\right)+\frac{Y^{2}}{2}-Z+3}{\left(t^{2}+1\right)^{3} \sqrt{t^{4} Z+t^{2}\left(-\frac{Y^{2}}{4}+Z+1\right)+1}}
$$


where the spatial dependence of the parameters

$$
\begin{array}{rlrlrl}
Y & =\frac{2 c_{6}\left(c_{5}\left(c_{1}^{2}-c_{2}^{2}\right)-c_{2} c_{3}^{2}\right)}{\left(c_{1}^{2}-c_{2}^{2}\right) c_{4}^{2}-c_{1} c_{3}^{2} c_{4}}, & Z & =\frac{\left(c_{4}^{2}-c_{6}^{2}\right)\left(c_{1}^{2}-c_{2}^{2}\right) \operatorname{det} \boldsymbol{M}_{1}}{\left(\left(c_{1}^{2}-c_{2}^{2}\right) c_{4}^{2}-c_{1} c_{3}^{2} c_{4}\right)^{2}}, & \boldsymbol{M}_{1} & =\left(\begin{array}{cccc}
c_{1} & c_{2} & 0 & -c_{3} \\
c_{2} & c_{1} & c_{3} & 0 \\
0 & c_{3} & c_{4} & c_{5} \\
-c_{3} & 0 & c_{5} & c_{4}
\end{array}\right) \\
c_{1} & =\frac{1}{2} C(0), & c_{3} & =\frac{1}{2} \partial_{r} C(r) \\
c_{4} & =-\frac{1}{2} \partial_{r}^{2} C(0), & c_{6} & =-\frac{1}{2 r} \partial_{r} C(r), & c_{6} &
\end{array}
$$

is omitted for the sake of brevity. The charge correlation function reads

$$
g_{Q}(r)=\frac{2 c_{6} c_{1}^{2}\left(c_{5}\left(c_{1}^{2}-c_{2}^{2}\right)-c_{2} c_{3}^{2}\right)}{\left(c_{1}^{2}-c_{2}^{2}\right)^{2} c_{4}^{2}}
$$

The pair and charge correlation function for maximum entropy models of orientation domains [171] are in good agreement with numerically calculated correlation functions as shown in Fig. 4.2.

\subsubsection{Pinwheel crystals}

In pinwheel crystals, pinwheels are arranged in a unit cell that repeats upon translation by vectors $\boldsymbol{v}_{1}$ and $\boldsymbol{v}_{2}$. In every unit cell there exist $n_{\text {cell }}$ pinwheels which are separated by the vector $\boldsymbol{u}_{k}$ from one particular pinwheel. The pair correlation function of pinwheel crystals with pinwheels that are indistinguishable by rotation and translation is then given by

$$
g(r)=\frac{1}{\rho} \sum_{i, j=-\infty}^{\infty} \sum_{k=\delta_{i 0} \delta_{j 0}}^{n_{\text {cell }}-1} \frac{\delta\left(r-r_{i j k}\right)}{2 \pi r_{i j k}} \quad \text { with } \quad r_{i j k}=\left\|i \boldsymbol{v}_{1}+j \boldsymbol{v}_{2}+k \boldsymbol{u}_{k}\right\|
$$

where the pinwheel correlation for zero distance was excluded. In the following, the pair correlation function will be calculated for hexagonal and rhombic pinwheel crystals.

In the case of a hexagonal orientation layout

$$
\Psi_{ \pm}(\boldsymbol{x})=e^{i \boldsymbol{k}_{1} \boldsymbol{x}}+e^{ \pm i \boldsymbol{k}_{2} \boldsymbol{x}}+e^{i \boldsymbol{k}_{3} \boldsymbol{x}} \quad \text { with } \quad \boldsymbol{k}_{j}=\frac{2 \pi}{\Lambda}\left(\begin{array}{c}
\cos (\pi j / 3) \\
\sin (\pi j / 3)
\end{array}\right)
$$

convenient translation vectors are given by $\boldsymbol{v}_{1}=\Lambda(2 / \sqrt{3}, 0)^{T}$ and $\boldsymbol{v}_{2}=\Lambda(1 / \sqrt{3}, 1)^{T}$. The hexagonal pinwheel crystal with $+\boldsymbol{k}_{2}$, Fig. $4.2 \mathrm{D}$, has only one additional pinwheel in its unit cell at position $\boldsymbol{u}_{1}=\Lambda / 3(-1 / \sqrt{3}, 1)^{T}$ and therefore a low pinwheel density of $\rho=\sqrt{3} / \Lambda^{2}$, see also supplementary material of [22]. In the other hexagonal pinwheel crystal with $-\boldsymbol{k}_{2}$, Fig. 4.2E, there exist five additional pinwheels in a unit cell at positions

$$
\boldsymbol{u}_{1}=\frac{\boldsymbol{v}_{1}}{3}, \quad \boldsymbol{u}_{2}=\frac{\Lambda}{3}\left(\begin{array}{c}
-1 / \sqrt{3} \\
1
\end{array}\right), \quad \boldsymbol{u}_{3}=\boldsymbol{u}_{1}+\frac{\boldsymbol{v}_{2}}{3}, \quad \boldsymbol{u}_{4}=\boldsymbol{u}_{2}+\frac{\boldsymbol{v}_{2}}{3}, \quad \boldsymbol{u}_{5}=\boldsymbol{u}_{4}+\boldsymbol{u}_{3}
$$

and therefore the layout has a high pinwheel density of $\rho=3 \sqrt{3} / \Lambda^{2}$. In the case of a rhombic orientation layout with a variable parameter $\alpha, \mathbf{F i g} . \mathbf{4 . 2 F}, \mathbf{G}$, the orientation field reads

$$
\Psi_{\alpha}(\boldsymbol{x})=\sin \left(\frac{2 \pi}{\Lambda} x\right)+i \sin \left(\frac{2 \pi}{\Lambda}(x \cos \alpha+y \sin \alpha)\right) .
$$


A
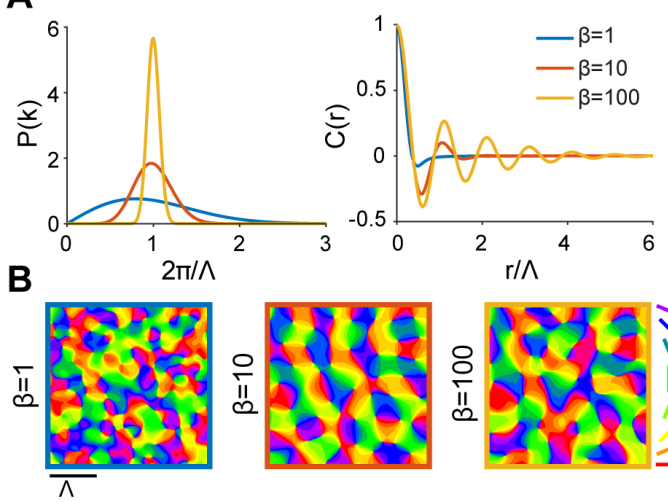

D

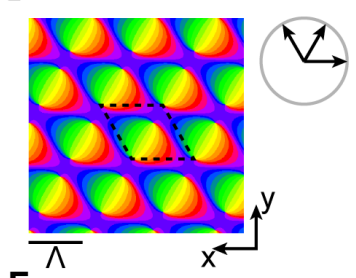

$\mathbf{F}$

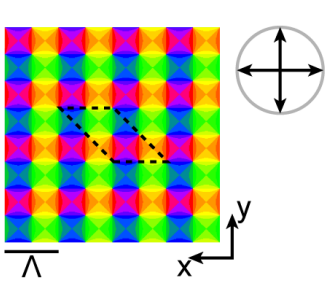

E

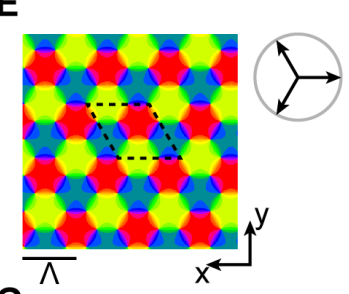

G

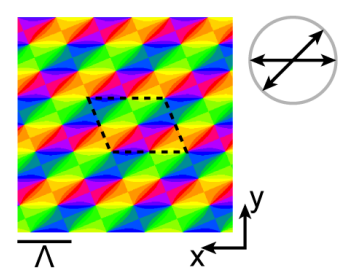

C

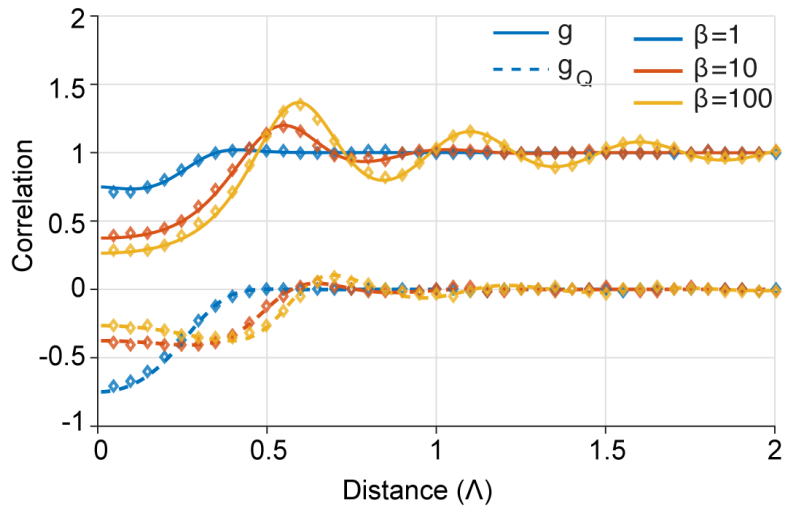

H

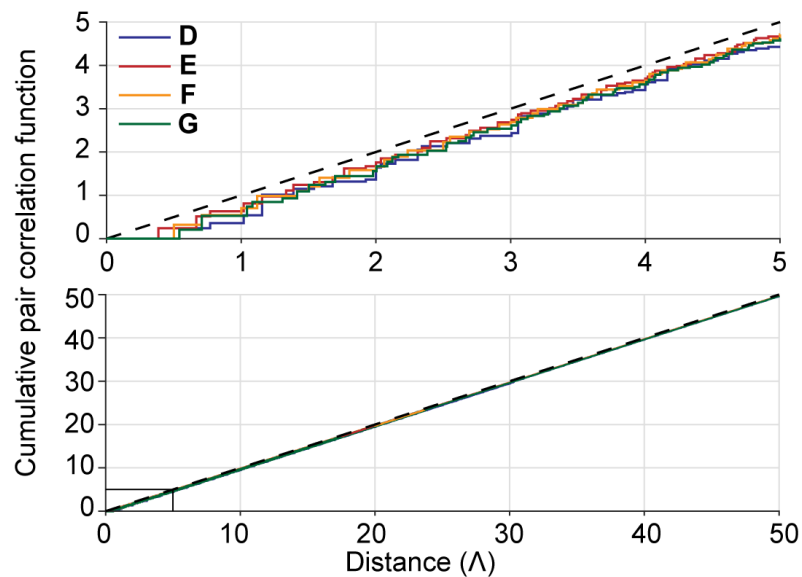

Figure 4.2: Pair and charge correlation function of orientation domain ensembles and pinwheel crystals. A The correlation function $C(r)$ and the radial part of its Fourier transform $P(k)$ of the maximum entropy model of isotropic and shift-symmetric orientation domain ensembles in [171] is depicted for varying power-spectral density widths $\sim 1 / \beta$. B Synthesized orientation domains corresponding to the correlation functions of $\mathbf{A}$. $\mathbf{C}$ The numerically obtained pair and charge correlation function from synthesized examples (diamonds) is compared to the analytical result from Eq. (4.16) and Eq. (4.18) (solid and dashed lines). D, E, F and G Pinwheel crystal layouts are depicted with their corresponding arrangement of wave vectors on a circle in Fourier space. The dashed rhomboids mark the unit cells of the pinwheel crystals. H The cumulative pair correlation function is shown for short and long distances. On short distances the non-smooth step function is clearly visible, whereas on long distances the cumulative distribution scales as the identity function, which is indicated by the dash line.

Convenient translation vectors are given by $\boldsymbol{v}_{1}=\Lambda\left(1, \frac{1-\cos \alpha}{\sin \alpha}\right)^{T}$ and $\boldsymbol{v}_{2}=\Lambda\left(0, \frac{1}{\sin \alpha}\right)^{T}$ with three additional pinwheels per unit cells at positions

$$
\boldsymbol{u}_{1}=\frac{\boldsymbol{v}_{1}}{2}, \quad \boldsymbol{u}_{2}=\frac{\boldsymbol{v}_{2}}{2}, \quad \boldsymbol{u}_{3}=\boldsymbol{u}_{1}+\boldsymbol{u}_{3}
$$

and hence a pinwheel density of $\rho=4 \sin \alpha / \Lambda^{2}$. The obtained cumulative pair correlation functions of these pinwheel crystal are depicted in Fig. $\mathbf{4 . 2 H}$.

\subsection{Pinwheel density fluctuations}

The pinwheel density fluctuation of the common design is a measure of the regularity of pinwheel configuration and was studied earlier in other contexts such as in randomly distributed points, called point processes. Previously, a generic formula for the scaling of number fluctuations of 
a $d$ dimensional point process in a regular window centered around a point was derived [278, 279]. The density standard deviation $\mathrm{SD}$ in a regular window scales as $\operatorname{SD}_{\text {rect }}(A) \propto A^{-\gamma}$ for large areas $A$ and is generically smaller than $\gamma=3 / 4$ except for pathological cases [280, 281]. In the case of $\gamma=3 / 4$ the point patterns are termed hyperuniform in contrast to hyposurficial point patterns with an exponent of $\gamma=1 / 2$ [278]. For crystals, formulas for $\mathrm{SD}_{\text {rect }}$ are provided in $[278,282,283]$. Although the examination of the scaling behavior for rectangular windows should hold for large areas, there has been no derivation of SD in a disk around an arbitrary point as used in the common design.

Given a disk or radius $r$ what is the standard deviation of pinwheel density in that disk? In order to answer this question, it should be noted that the derived pair correlations excluded the self-correlation component of single pinwheels, i.e. at zero distance. The general formula of the pair correlation function is given by

$$
g_{\mathrm{tot}}(r)=\frac{1}{\rho} \delta(r)+g(r) .
$$

The correlation function is related to the second moment of the pinwheel number in an area $A$ by

$$
\left\langle N(A)^{2}\right\rangle=\rho^{2} \int_{A} \mathrm{~d}^{2} y^{\prime} \int_{A} \mathrm{~d}^{2} x^{\prime} g_{\mathrm{tot}}(r)
$$

The variance of the pinwheel density in a disk of area $A=\pi R^{2}$ becomes

$$
\mathrm{SD}^{2}(A)=\frac{\left\langle N(A)^{2}\right\rangle-\langle N(A)\rangle^{2}}{A^{2}}=\frac{\rho}{A}+\frac{\rho^{2}}{A^{2}} \int_{A} \mathrm{~d}^{2} y^{\prime} \int_{A} \mathrm{~d}^{2} x^{\prime} h\left(r^{\prime}\right),
$$

where the total correlation function $h(r)=g(r)-1$ is introduced. If a pinwheel is at the center of the disk, the pinwheel variance simplifies to

$$
\mathrm{SD}^{2}(A)=\frac{\rho}{A}+\frac{2 \pi \rho^{2}}{A} \int_{0}^{R} \mathrm{~d} r^{\prime} h\left(r^{\prime}\right) r^{\prime}
$$

which is connected to Ripley's $K$ function [284, 285]. For an arbitrary disk center, the integral can be evaluated by utilizing the probability density function of distances $l \in(0,2 R]$ between two uniformly distributed random points in a disk of radius $R[286,287]$ that is given by

$$
f_{R}(l)=\frac{2 l}{\pi R^{2}}\left(2 \cos ^{-1}\left(\frac{l}{2 R}\right)-\sin \left(2 \cos ^{-1}\left(\frac{l}{2 R}\right)\right)\right) .
$$

The variance of the pinwheel density is then

$$
\mathrm{SD}^{2}(A)=\frac{\rho}{A}+\rho^{2} \int_{0}^{2 R} \mathrm{~d} r^{\prime} h\left(r^{\prime}\right) f_{R}\left(r^{\prime}\right),
$$

which leads for Poisson distributed pinwheels with a pair correlation function of $g(r)=1$ to the exact power law $\operatorname{SD}(A)=\sqrt{\rho / A}$. For the orientation domain model introduced in [171] and for pinwheel crystals the pinwheel standard deviation with its corresponding scaling is depicted in Fig. 4.3. Notice that the pinwheel density fluctuations for crystals can be written in an elegant way by using reciprocal lattices [278].

Depending on the total correlation function different scaling behaviors were derived in [278, 279]. For periodic pinwheel and random pinwheel layouts the total correlation function decays exponentially $h(r) \sim \exp (-\lambda r)$ leading to an asymptotic scaling of the pinwheel density variation

$$
\mathrm{SD}(A) \sim \begin{cases}A^{-1 / 2} & , 0 \neq 1+2 \pi \rho \int_{0}^{\infty} \mathrm{d} r h(r) r \\ A^{-3 / 4} & , \text { else }\end{cases}
$$


A
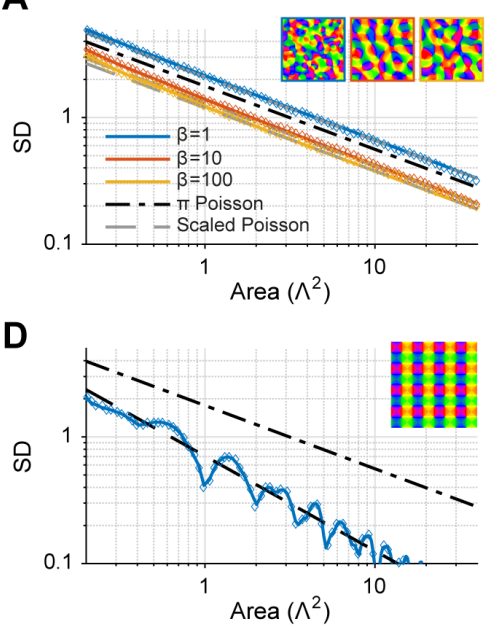

B

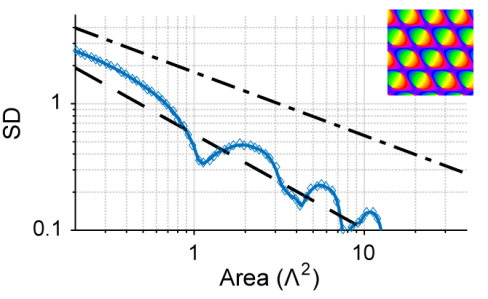

E

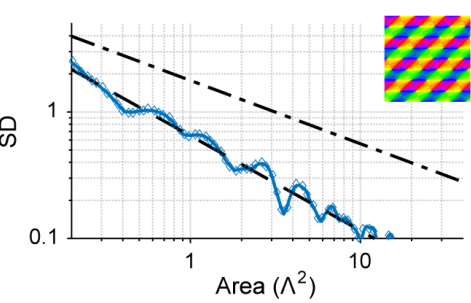

C

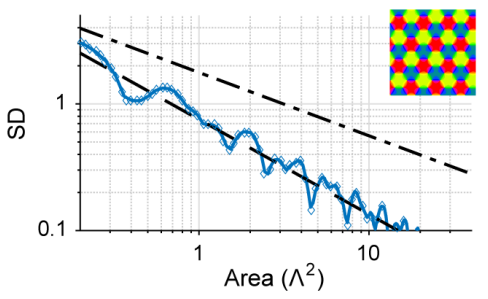

$\mathbf{F}$

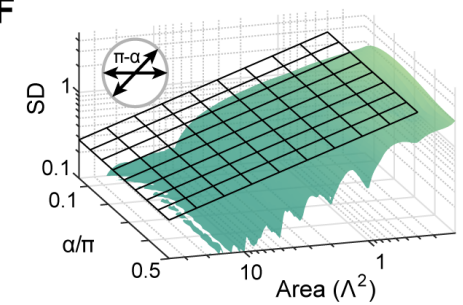

Figure 4.3: Pinwheel density fluctuation for random orientation domains and pinwheel crystals. A SD of random orientation domains [171] for varying power-spectral density width, see Fig. 4.2A,B, is numerically obtained by synthesized examples (diamonds) together with the analytical result from Eq. (4.29) (solid lines) and their limits of a scaled Poisson power law (gray dashed line). Black dashed line corresponds to a Poisson distributed pinwheel pattern with density $\pi$. Inset shows corresponding orientation layouts. B, C, D and $\mathbf{E}$ SD is depicted for various pinwheel crystals obtained numerically by synthesized examples (diamonds) together with their analytical result from Eq. (4.29) (solid lines), their limiting scaling (dashed line) and a reference Poisson pinwheel pattern with density $\pi$. Inset shows corresponding orientation layout. F Theoretically obtained SD is shown for rhombic pinwheel crystals with variable intersection angle $\alpha$ of their wave vectors (see inset). Black solid lines indicate Poisson distributed pinwheels with density $\pi$.

If the total correlation function is fat tailed $h(r) \sim-1 / r^{2+\xi}$ with $\xi \in(0,1]$, the pinwheel density variation asymptotically scales as

$$
\mathrm{SD}(A) \sim \begin{cases}A^{-3 / 4} \sqrt{\ln A} & , \xi=1 \\ A^{-1 / 2-\xi / 4} & , \xi \in(0,1)\end{cases}
$$

and for a scaling of $h(r) \sim 1 / r^{2+\xi}$ with $\xi \in(0,1]$, the pinwheel density variation has the asymptotic form of

$$
\operatorname{SD}(A) \sim\left\{\begin{array}{ll}
A^{-1 / 2} & , \xi \in(0, \infty) \\
A^{-1 / 2} \sqrt{\ln A} & , \xi=0 \\
A^{-1 / 2+|\xi| / 4} & , \xi \in(-2,0)
\end{array} .\right.
$$

\subsection{Pinwheel nearest neighbor distribution}

Already in 1909, Paul Hertz derived a formula for the nearest neighbor distribution of randomly distributed points, more precisely Poisson distributed points, with fixed density in a volume [288]. Later, the relation between the $n$-point correlation functions and the nearest neighbor distribution was discovered [157]. More recentely, a Poisson and Bernoulli approach was derived to approximate the nearest neighbor distribution from the pair correlation function [158]. Finally, the Poisson concept was generalized to a hierarchical scheme for estimating the $n$th nearest neighbor distributions from the pair correlation function [159]. In the following, the approximation of the nearest neighbor distribution by the pair correlation function will be derived and the approximated $n$th nearest neighbor distributions will be stated according to [159]. 
A

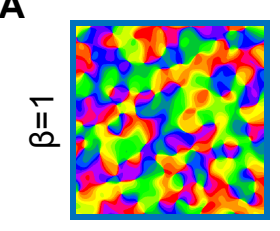

C

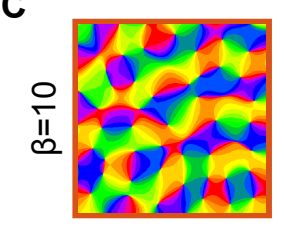

E

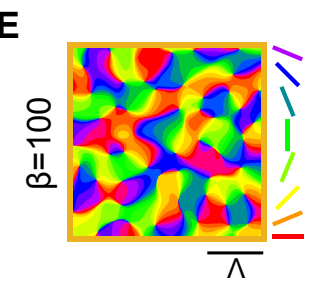

B

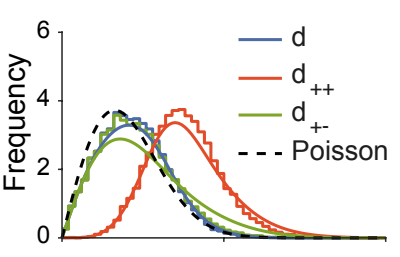

D

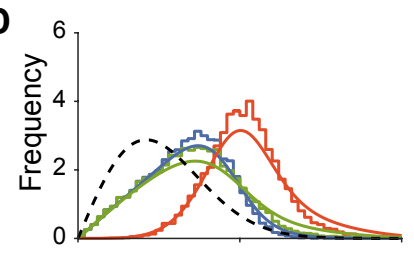

$\mathbf{F}$

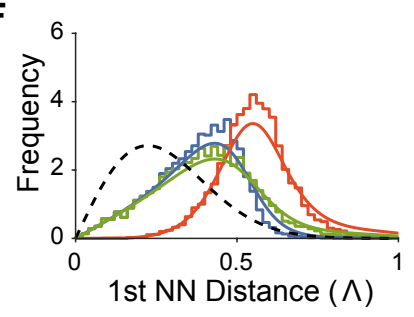

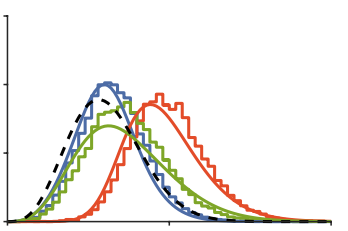
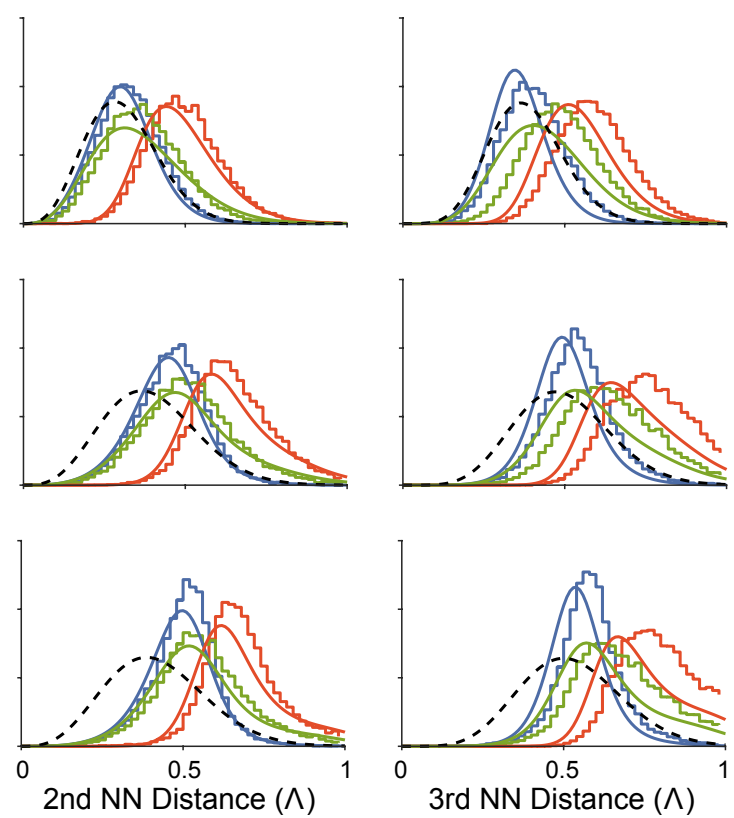

Figure 4.4: Nearest neighbor distribution estimate from pinwheel pair correlation function. Nearest neighbor distributions of pinwheels independent of charge $d$, of same charge $d_{++}$and of opposite charge $d_{+-}$for random orientation domains [171] with varying inverse power-spectral density width $\beta$, see Fig. 4.2A,B. A-C, D-F, G-I First, second and third nearest neighbor distribution is computed numerically (histogram) from synthesized orientation domains and theoretically (solid lines) by Eq. (4.34) and Eq. (4.36) with $g(r), g_{++}(r)$ and $g_{+-}(r)$ for varying inverse power-spectral desnity widths $\beta$, respectively. The nearest neighbor distributions of Poisson distributed pinwheels (dashed lines) serve as reference.

The pinwheel nearest neighbor distribution function $W(r)$ in two-dimensional orientation domains is defined as the probability density that the nearest pinwheel pairs are separated by a distance $r$. Thus, the probability to find an additional pinwheel between $r$ and $r+\mathrm{d} r$ around a pinwheel is given by the probability of observing no additional pinwheel at distance smaller $r$ times the probability to find a pinwheel in that interval. Approximating the probability of observing no pinwheel by not observing two pinwheels in a disk of radius $r$, the nearest neighbor distribution is given by

$$
W(r) \mathrm{d} r=\left(1-\int_{0}^{r} \mathrm{~d} r^{\prime} r^{\prime} W\left(r^{\prime}\right)\right) \cdot \rho g(r) r \mathrm{~d} r
$$

where $g(r)$ is the pair correlation function and $\rho$ the pinwheel density. This equation can be solved and leads to the nearest neighbor distribution of

$$
W(r)=2 \pi r \rho g(r) \exp \left(-2 \pi \rho \int_{0}^{r} \mathrm{~d} r^{\prime} r^{\prime} g\left(r^{\prime}\right)\right) .
$$

In the special case of Poisson distributed pinwheels the pair correlation function is unity $g(r)=1$ and the nearest neighbor distribution

$$
W(r)=2 \pi r \rho \exp \left(-\pi r^{2} \rho\right)
$$

validating the result derived by Hertz [288]. For the $n$th nearest neighbor distribution $W(n, r)$ with $W(1, r)=W(r)$ a hierarchical scheme can be derived for its approximation [159] and results 
for a two-dimensional system in

$$
W(n, r)=2 \pi r \rho g(r) F(n-1, r) \exp \left(-2 \pi \rho \int_{0}^{r} \mathrm{~d} r^{\prime} r^{\prime} g\left(r^{\prime}\right) F\left(n-1, r^{\prime}\right)\right)
$$

with the approximated probability of observing $n$ pinwheels in a disk of radius $r$

$$
F(n, r)=\int_{0}^{r} \mathrm{~d} r^{\prime} W\left(n, r^{\prime}\right)
$$

Notice that this hierarchical scheme has to be interpreted in a mean field sense, since no explicit pinwheel correlation functions of higher order than two are considered. However, the approach yields reasonable results for low $n$ in Gaussian random fields as shown in Fig. 4.4. In the first nearest neighbor distribution the tails get typically overestimated, whereas for higher nearest neighbor distributions the theoretical description leads to a shift to smaller distances.

\subsection{Summary}

In this chapter, we analytically examined the pinwheel configuration statistics that constitute the common design [22] in maximum entropy models of orientation domain ensembles and partially in pinwheel crystals. The common design is composed of the pinwheel density, pinwheel density fluctuations in subareas and nearest neighbor distributions of pinwheels with equal, opposite and independent of topological charge. We extend the systematic analytical understanding of the species-invariant pinwheel statistics by:

- Deriving a general pinwheel density of maximum entropy models for orientation domains with vanishing mean.

- Recapitulating the derivation of the pinwheel pair and charge correlation function of these ensembles [30].

- Based on the pair correlation functions, providing and exact and approximated analytical expression for the pinwheel density fluctuations in subareas and nearest neighbor distributions, respectively.

Thus, all species-invariant pinwheel statistics that have been observed so far can be exactly or approximately related to the correlation function $C(r)$ of maximum entropy models of orientation field ensembles. This extends previous analytical work that only considered the pinwheel density $[135,147,170,171]$.

We reviewed that the scaling of pinwheel number fluctuations has an upper bound of $3 / 4$ for hyperuniform and $1 / 2$ for hyposurficial point processes [278, 280, 281], while experimentally observed exponents are around 0.4 [24]. These small exponents are probably due to a smoothing of pinwheels before calculating their pinwheel density fluctuations (not shown here). In fact, small exponents vanish in the case of less smoothing and appear to approach an exponent of $1 / 2[22]$.

The difference in the analysis algorithm exemplifies the challenge of the systematic noise reduction in experimental data of orientation domains. If this problem is settled, pinwheel configurations should be interpreted as a point process and analyzed by standard methods to infer their statistical nature [284]. Typical measures are the density, nearest neighbor distribution, void nearest neighbor distribution, pair and charge correlation function. The advantages are that (i) for the density and (void) nearest neighbor distributions analytical results are known for standard point processes such as Poisson, Cox and Nexman-Scott processes, (ii) the pair and 
charge correlation function is related to the pinwheel density in subregions and Ripley's $K$ or $L$ function by $2 \pi r g(r)=\partial_{r} K(r)$ and (iii) a simple parameter for quantifying short-range order is given by $M=\left(g\left(r_{1}\right)-g\left(r_{2}\right)\right) /\left(r_{2}-r_{1}\right)$ with $r_{1}$ the first maximum and $r_{2}$ the first minimum of $g(r)[284]$.

In total, these results extends the systematic understanding of the statistical properties of pinwheel configurations that is crucial for model testing. 



\section{The pinwheel configuration: theoretical significance and precision measurement}

\section{Original contribution}

J. Liedtke and F. Wolf conceived and designed the study. J. Liedtke performed the theoretical analysis and numerical simulations. J. Liedtke wrote the manuscript. 


\subsection{Abstract}

The functional architecture of the primary visual cortex (V1) of primates and placental carnivores consists of iso-orientation domains. The organizational centers of iso-orientation domains are pinwheels that are surrounded by all preferred orientations. The pinwheel configuration in primates and placental carnivores follows species-invariant quantitative statistics. Measurement noise can be only partially attenuated and thus obscures the precise determination of pinwheel configurations. Hence, precision measurement of pinwheel configurations requires theoretical understanding of the impact of measurement noise. Here we derive an analytical pinwheel observation probability for ground truth orientation domains with additive Gaussian measurement noise. We examine frequently observed orientation layout motifs such as linear zones, pinwheels and pinwheel pairs. Subsequently, we dissect the impact of measurement noise on crystalline, quasiperiodic, random orientation domains and finally on high orientation selectivity layouts. We find strong suppression of noise effects for orientation motifs, a linear scaling of the pinwheel density on the noise variance for heterogeneous orientation domains and a strong suppression of noise effects on high orientation selectivity and heterogeneous orientation domains. The results give rise to an extrapolation method of pinwheel densities to their zero noise limit. They provide an approximated analytical expression for confidence regions of pinwheel centers. Finally, the results quantitatively favor recording techniques capturing spiking activity for precise pinwheel configuration measurements. This precise quantitative determination of pinwheel positions and their confidence regions provides a paradigm benchmark framework for the development of quantitative models of canonical circuits.

\subsection{Introduction}

After the greatest mass extinction in earth's history about 250 million years ago, early mammals branched off the therapsids and developed a neocortex with about 20 cortical areas that have been retained over the course of more than 170 million years up to most present living mammals [289]. During that period neocortices changed size and concomitantly the number of higher cortical areas [271]. Interestingly, many cortical areas and structures scale allometrically with other brain structures [290-294]. For instance, primary visual cortex (V1) size scales with the power of $3 / 2$ of its efferent brain structure (LGN) size hypothesized to maintain visual resolution [292]. Most intriginguely, primary sensory area sizes scale with neocortex size, where V1 grows the most and independent of nocturnality or diurnality [294]. The neuronal inventory of cortical areas share remarkable commonalities between different areas and species suggesting a notion of a canonical circuit $[295,296]$. Key features of a canonical circuit are excitatory principal neurons and inhibitory interneurons that are recurrently connected dependent on the feature they encode. For instance, in V1 principal neurons respond to visual features such as edge orientations $[69,297]$ and are preferentially connected to regions that respond to similar visual features [12, 227-229, 298]. The orientation preference feature in V1 of primates and placental carnivores is spatially smoothly organized with point singularities called pinwheels that are surrounded by all orientation preferences [49, 60-62]. Pinwheels are organizational centers of orientation domains and their configuration determine a substantial part of orientation domains, see Fig. 5.1. Strikingly the pinwheel configuration layout in the four species cats, ferrets, galagos and tree shrews adhere to a quantitative species-invariant common design [2224]. This common design is suggested to be an evolutionary convergent trait, because of two main reasons: (i) The last common ancestor of the four species was a small shrew-like mammal that is likely to have possessed an interspersed orientation layout $[22,76]$. (ii) These mammals possess distinct neuronal circuits for orientation selectivity generation [24, 77]. For instance, tree shrews 

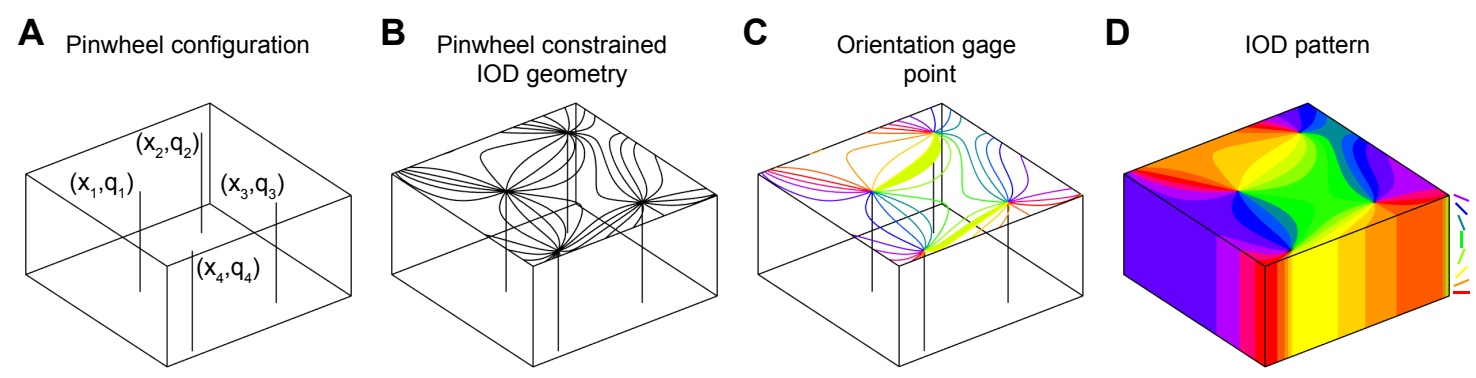

Figure 5.1: Pinwheel configurations contain substantial information about iso-orientation domains (IOD). A The pinwheel configuration is composed of the pinwheel center positions and their topological charges. B Including iso-orientation contour lines reveals the spatial arrangement of isoorientation domains except for a global change of orientation preference. $\mathbf{C}$ The additional knowledge of a single iso-orientation domain is sufficient to complete the entire iso-orientation domain (short orientation domain) of $\mathbf{D}$.

generate orientation selectivity mostly intracortically [78, 79], whereas cats generate it mostly thalamocortically [69, 80]. The facts of cortical organizational principles such as allometric scaling, the notion of a canonical circuit and a convergent common design in V1 suggests that evolution optimizes this primary visual processing stage and possibly other primary areas with respect to an unknown cost function. If cortical structure were genetically determined, then optimization is exerted by genetic variation. However, optimization may also occur during the ontogenetic maturation of organisms by activity-dependent mechanisms. This is in line with the finding that V1 implements an optimal internal model adapting gradually during development to the statistical structure of natural visual environment [299]. Most likely none of these views are entirely correct instead nature and nurture shape V1 in collusion [300].

From a theoretical point of view, the optimization of V1 might be modeled by a dynamical system that settles onto an attracting invariant manifold, Fig. 5.2A,B, as suggested in [301]. In a finite dimensional system, the attracting invariant manifold becomes an inertial manifold [302]. V1 is a high dimensional system constituted on a coarse scale on the order of 10 thousand synapses per neuron of about 140 million in a single human brain hemisphere [303]. The initial dynamics are clearly confined to a subspace of possible connections summarized by the concept of a canonical circuit. In fact, after ferrets open their eyes long-range connections elaborate over the course of months and preferentially connect regions with similar orientation preferences [14, 82]. Orientation selectivity is already present at eye-opening and gradually matures over the course of a month in ferrets, in contrast to direction selectivity that emerges after eye-opening and matures in about two weeks $[82,304-306]$. Orientation domains are rather robust in the first two weeks after eye-opening [68] arguing for slow long-term dynamics. In cats, the visual cortical area increases substantially over the first 14 postnatal weeks [94-97], while preserving the typical spacing $[19,20]$ of ocular dominance and orientation selectivity bands during most of that time implying cortical reorganization [97-99]. In macaques a reorganization of orientation selectivity and ocular dominance with age was suggested based on a smaller dataset [52]. More evidence for long-term dynamics is found in rodents. In mice, neurons are already orientation selective at eye-opening in the second postnatal week [307-309]. However, the maturation of orientation selectivity is controversial: a gradual increase in responsiveness and selectivity over two postnatal months was observed [308, 310-313], whereas other studies found almost mature orientation selectivity at eye-opening [314-316]. The period of juvenile plasticity extends beyond the third postnatal month [309, 317, 318] or even further in an enriched environment [319]. The alignment of orientation selectivity in the binocular zone, called binocular matching, continues up to five weeks postnatally [307]. In juvenile mice the distribution of preferred directions 
A

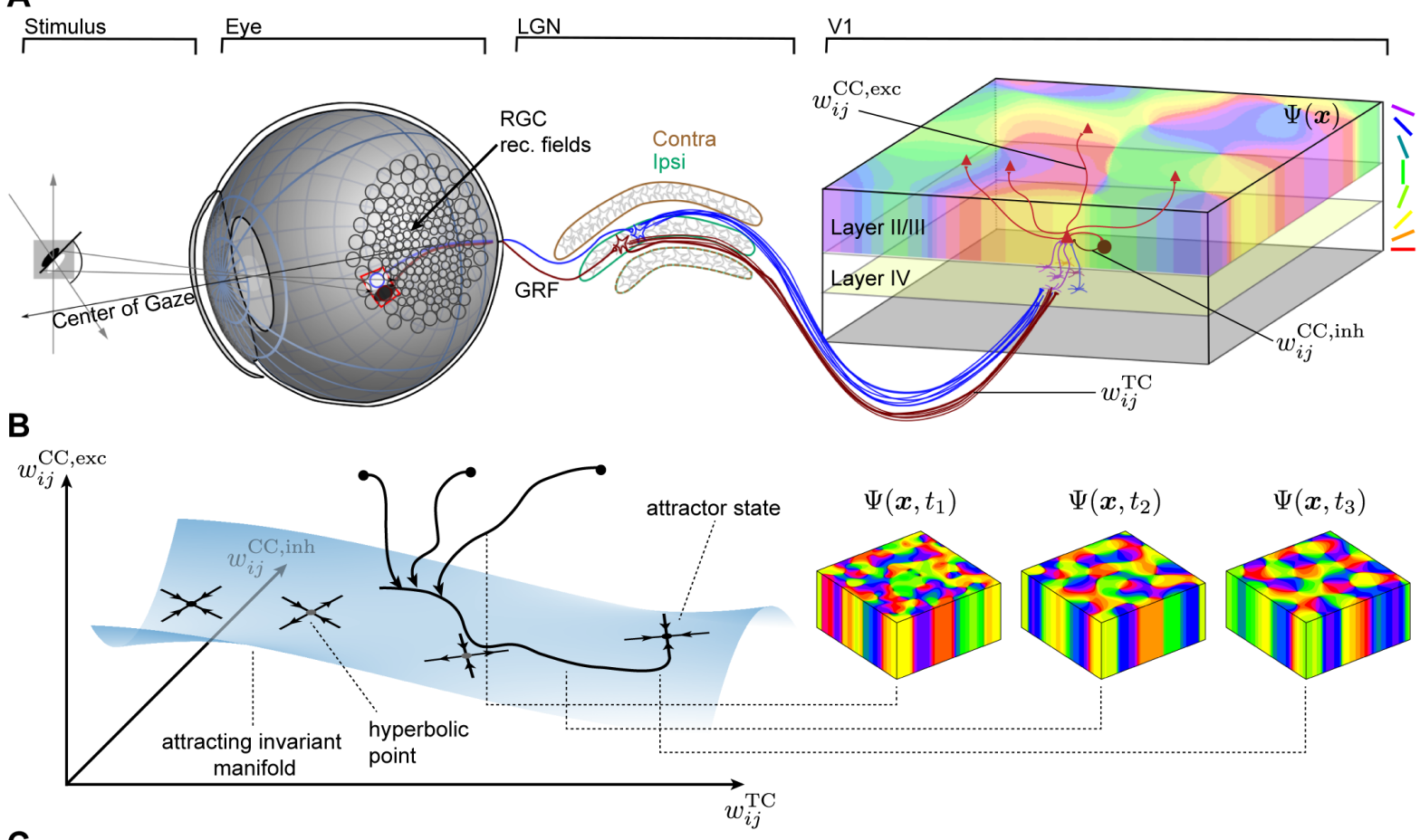

C
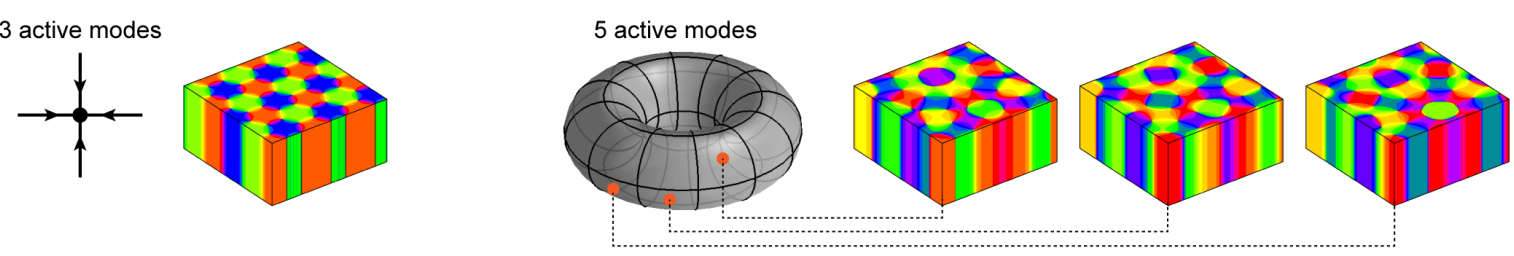

Figure 5.2: Primary visual cortex (V1) as a dynamical system and feature states of V1. A The primary visual pathway of a cat. Retinal ganglion cells in the eye project signals from the visual stimulus over the lateral geniculate nucleus (LGN) and the subsequent optic radiation fibers $w^{\mathrm{TC}}$ to the primary visual cortex (V1). Principal neurons with a feature orientation preference response $\Psi$ interact with interneurons recurrently over connectivities $w^{\mathrm{CC} \text {,exc }}$ and $w^{\mathrm{CC} \text {,inh }}$. Adapted from [24]. B The dynamical system of V1 is spanned by connectivities. Optimization of V1 corresponds to a settling on the attractive invariant manifold and a subsequent convergence to the attractor state. The feature field changes along the trajectory. C Example attractor states (ECP solution class, see Eq. (5.26)) of the long-range interaction model [22, 128]. Left: A point attractor composed of three active modes, right: a two-dimensional torus attractor composed of five active modes.

and orientations is biased at eye-opening and equalizes in the subsequent days [308, 316]. The equalization can be due to (i) an increase of responsive neurons as [308], (ii) a reorganization of responsive neurons [316] or (iii) a mixture of (i) and (ii). Finally, in the barrel cortex the preferred direction of whisker deflection emerges in juvenile rats and reorganizes to a single pinwheel over the course of the first four postnatal months [320]. Together these experimental findings support the proposed view of rapid dynamic convergence to an attracting invariant manifold with subsequent slow dynamics on this manifold. On the theory side, the convergence to optimal orientation domains and long-term reorganization was proposed based on long-range order of orientation domains [321]. This optimal solution might be given by a single point attractor that exists under a set of symmetries [127], but can be also a more complex attractor state. A dynamical model based on symmetries and assuming an optimization process of V1 correctly predicts orientation layouts that are in quantitative agreement with the common design 
$[22,128,135]$. More strikingly it supports the view of an attractive invariant manifold composed of high dimensional tori and thus allowing numerous visual cortical states, Fig. 5.2C.

An attractive invariant manifold greatly reduces the high dimensional dynamical system while retaining most of its dynamics. Feature parameter fields are a characteristic component of these manifolds and are hence convenient for parametrization. But which feature parameter field is the best suitable for parametrization? A candidate criterion for the natural feature field is homotopy theory [322], because every feature is mapped continuously on the surface of V1 in primates and placental carnivores. As the feature space has a certain topology, only specific defects are topologically stable. A defect is topologically stable if a surrounding path on the cortical surface can not be continuously deformed to a single point in the feature space. Candidate features in V1 are orientation preference, direction preference or the more subtle feature of the phase of a simple cell receptive field [323, 324], i.e. the relative position of its ON and OFF receptive fields, together with orientation preference, Fig. 5.3A. The simplest topological defects are single charged pinwheels for orientation preference, double charged pinwheels for direction preference and single charged pinwheels and phase defects for the phase and orientation feature, Fig. 5.3B. Single charged pinwheels are the only experimentally observed topological defects, Fig. 5.3C. Double charged pinwheels are incompatible with the experimentally observed well separated single charged pinwheels and topologically unstable direction fractures [41, 325-327], see Fig. 5.3C. Phase defects also disagree with the experimental finding of strong spatial phase differences between neighboring V1 neurons in cats [328, 329] and a bias to OFF-dominated simple cell receptive fields in tree shrews [330] and cats [331, 332], see Fig. 5.3C. In fact, preferred orientation is locally more smoothly organized than preferred direction and spatial frequency [333]. In total this favors orientation preference as an informative feature parameter field and questions models that predict phase ordering and phase maps $[118,334,335]$ rather than periodicity of orientation preference. Mathematically, the preferred orientation $\vartheta(\boldsymbol{x})$ of a neuron at cortical position $\boldsymbol{x}=(x, y)^{T}$ is a cyclic, $\pi$-periodic quantity and is hence conveniently represented together with its selectivity $S(\boldsymbol{x})$ by a complex field $\Psi(\boldsymbol{x})=S(\boldsymbol{x}) e^{2 i \vartheta(\boldsymbol{x})}$ as already suggested in $[122,132]$. This representation where pinwheels are roots of $\Psi(\boldsymbol{x})$ reveals that topological stability does not imply dynamic stability. In fact, arbitrary pinwheel configurations can be stabilized by relaxing exponentially against the fixed point $\Psi(\boldsymbol{x})=\prod_{i}\left(x-x_{i}+\operatorname{sign}\left(q_{i}\right) i\left(y-y_{i}\right)\right)$, where $q_{i}$ denotes the topological charge of a pinwheel located at position $\boldsymbol{x}_{i}=\left(x_{i}, y_{i}\right)^{T}$. If pinwheels are at the same position, multiple charged pinwheels can be stabilized. However, many theoretical models only have single charged pinwheels as a dynamical solution and in random field models only single charged pinwheels exist. In total, topological and dynamical stability argues for orientation domains as the natural feature parameter field to parametrize the attractive invariant manifold.

Pinwheel configurations already contain substantial information about the layout of orientation domains [321, 336] and are convenient for benchmarking orientation domain models. In contrast to experimentally observed quasiperiodic pinwheel configurations that are also found in some models, e.g. [22, 128, 337], many other models converge to pinwheel crystals $[27,126,129-$ 131, 263-266], including models of orientation domains seeded by a retinal bias [24, 338-340] that quantitatively disagree with the common design. The pinwheel configuration constrains orientation layouts, but in general not uniquely defines them and thus not uniquely defines the attractive invariant manifold. Instead, the pinwheel configuration determines a subset of possible orientation domains and attractive invariant manifolds. However, there exists perfectly reducible model solutions $[128,137]$ for which pinwheel configurations uniquely define orientation domains except for a global orientation shift and they in turn uniquely determine the network state except for a coupling ratio. Hence, there is a one-to-one mapping from an equivalence class in the network state to a feature field and a one-to-one mapping from an equivalence class of a feature field to a pinwheel configuration, Fig. 5.1. 
A
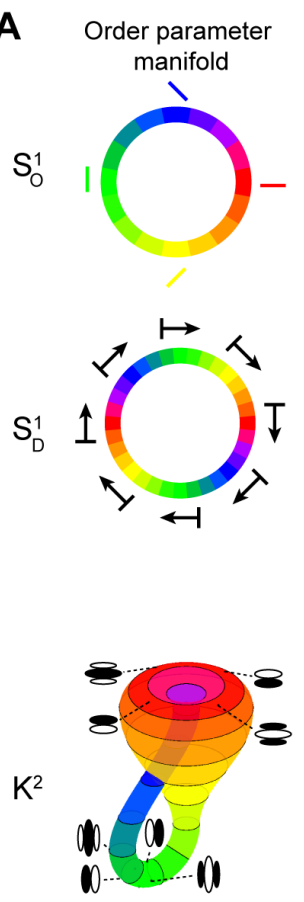

B

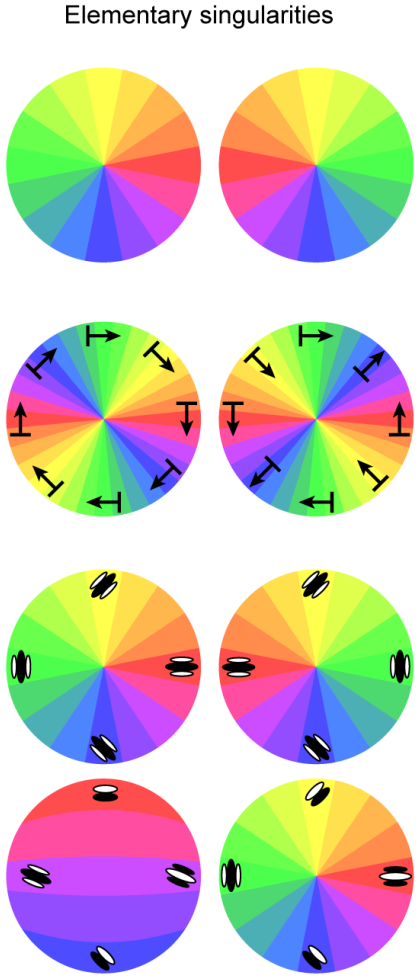

\section{Experimentally observed singularities}
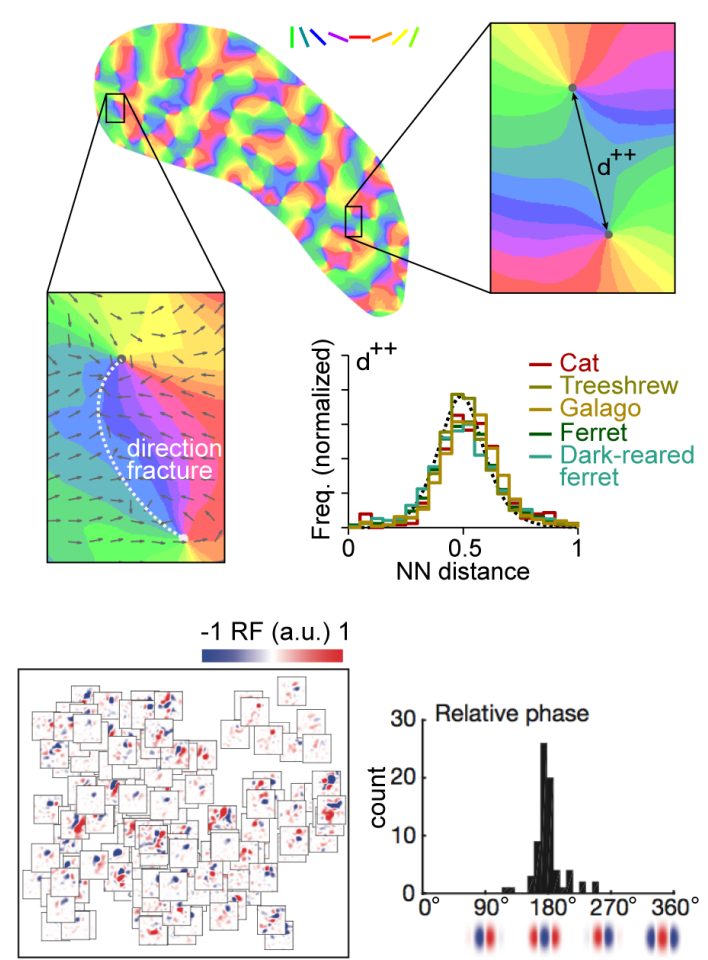

Figure 5.3: Topologically stable feature singularities and their experimental evidence in the primary visual cortex. A The topology of the order parameter manifold. Orientation and direction preference is $\pi$ - and $2 \pi$-periodic with a circle manifold $S_{\mathrm{O}}^{1}$ and $S_{\mathrm{D}}^{1}$, respectively. The union of orientation preference and receptive field phase is $\pi$-periodic in both quantities and its manifold is topologically equivalent to a Klein bottle $K^{2}$. B The elementary singularities are the simplest topological defects that arise if the order parameter manifold is continuously mapped on a two-dimensional cortex (see main text for details). For orientation and direction preference the elementary singularities correspond to a closed clockwise and counter-clockwise path in the order parameter manifold. The upper elementary singularities of the Klein bottle correspond to paths along its tube, the lower left singularity corresponds to a path perimetric to the tube and the lower right to the combination of both. C Experimentally observed singularities. Only topological defects of orientation preference (pinwheels) are experimentally observed. Direction fractures and a strong repulsion of equally charged pinwheels questions the direction preference as order parameter. The diversity of receptive field phases and its OFF domination argues against the union of orientation preference and receptive field phase as order parameter. (We thank David Whitney for providing experimental data of orientation and direction preference from ferret. Pinwheel nearest neighbor distribution adapted from [24]. Receptive field figures are adapted with permission from [330].)

The approach of reducing dimensionality of the system description while keeping the crucial information relies heavily on the exact determination of pinwheel positions in experiments. The conventional measuring technique for orientation domain recording is optical imaging of intrinsic signals. Optical imaging of intrinsic signals records the change in light reflectance of neural tissue caused by a number of biological mechanisms [72, 341]. The predominant contribution of intrinsic signals is believed to be activity-induced oxygen delivery of blood vessels [342]. Thus, the signal is additionally composed of activity-independent hemodynamic artifacts including heart beat, respiration rate, vasomotor signal [343] and others such as ongoing activity from an internal state of the cortical tissue [344]. These rather low temporal frequency noise components can be reduced (e.g. by averaging over multiple trials, temporal filtering or synchronized recording 
with noise frequencies), but can not be entirely removed [72]. A high temporal frequency noise component is the stochastic fluctuation of light emission called shot noise [72]. A theory for measurement errors is only partially available. For instance, the spatial resolution of intrinsic signal imaging was claimed to be about $50-100 \mu \mathrm{m}$ by Grinvald, colleagues and others $[50,62,72$, $345,346]$ based on reproducibility of orientation domains [50,62] and the enhancement of spatial resolution by differential maps [62]. However, an inaccurate measurement caused by a systematic error can be exactly reproducible. In fact, measurements and theoretical estimates of photon scatter and absorption in brain tissue poses the spatial resolution of optical imaging to about $200 \mu \mathrm{m}[73,74,347]$. Differential maps generically increase signal-to-noise ratios, but spatial resolution improvement can only be achieved by deconvolution methods [73]. The measured orientation domains are thus smoothed versions of the ground truth orientation domains [73]. Apart from this systematic error, the overall orientation layout is precisely reproducible, e.g. root-mean-square error of difference between two total recordings (about 50 single condition response recordings) is about $6.5 \%$ of the total range of the recording [62]. Therefore, pinwheel densities were measured with high precision by a variable spatial local bandpass approach [2224]. This analysis observed a pinwheel density plateau for a broad range of spatial low-pass filter positions, but kept spatial noise components with similar frequencies to the signal. This remaining noise did not allow the determination of the exact pinwheel positions. In order to quantify orientation domains more rigorously, insight on the impact of the remaining noise on the pinwheel density and exact pinwheel center positions is crucial.

The presentation of our results is organized as follows. First, we derive a pinwheel observation probability for orientation domains with additive isotropic and shift-symmetric Gaussian measurement noise. We identify three general noise effects on pinwheels: displacement of pinwheels, pinwheels hidden by noise and the generation of spurious pinwheels. Second, we dissect the contribution of these effects on three abundant orientation layout motifs: (i) linear zones of progressive orientation preference change along a line, (ii) single pinwheels and (iii) pinwheel pairs of same and opposite topological charge. In the third part, we proceed to crystalline, quasiperiodic and random orientation domains and show how the pinwheel observation probability scales in the small noise limit. Finally, orientation domains with spatially uniform orientation selectivity are examined. The results shed light on the confidence regions of pinwheel positions, give rise to an improved scheme for pinwheel density analysis and favor measuring techniques that record neuronal activity directly for a precise determination of pinwheel configurations.

\subsection{Results}

\section{Observation of pinwheels and orientation domains with additive noise}

The preferred orientation $\vartheta(\boldsymbol{x})$ of a neuron at cortical position $\boldsymbol{x}=(x, y)^{T}$ is a cyclic, $\pi$-periodic quantity and is hence conveniently represented together with its selectivity $S(\boldsymbol{x})$ by a complex orientation preference director field

$$
\Psi(\boldsymbol{x})=S(\boldsymbol{x}) e^{2 i \vartheta(\boldsymbol{x})}
$$

or short orientation field. In measurements, the observed orientation field $\Psi(\boldsymbol{x})=\xi(\boldsymbol{x})+i \eta(\boldsymbol{x})$ is in general a mixture of the ground truth orientation field $\Psi_{0}(\boldsymbol{x})=\xi_{0}(\boldsymbol{x})+i \eta_{0}(\boldsymbol{x})$ and a noise field $\Psi_{n}(\boldsymbol{x})=\xi_{n}(\boldsymbol{x})+i \eta_{n}(\boldsymbol{x})$. We consider the impact of additive noise on orientation fields in measurements

$$
\Psi(\boldsymbol{x})=\Psi_{0}(\boldsymbol{x})+\Psi_{n}(\boldsymbol{x})
$$


The ground truth orientation field $\Psi_{0}(\boldsymbol{x})$ is presumably composed of isotropic spatial frequencies centered around a typical scale of $\Lambda=0.6-1 \mathrm{~mm}$ [23]. The composition of the noise field depends on the specific measurement technique.

Usually, orientation fields are measured with optical imaging of intrinsic signals. Optical imaging of intrinsic signals measures the change in light reflectance of neural tissue predominantly caused by activity-induced oxygen delivery of blood vessels $[72,341,342]$. The signal is however more correlated to membrane potential changes than to the activity of neurons [346]. The merit of this technique is a large field of view on the order of $100 \mathrm{~mm}^{2}$. The spatial resolution is limited by light scatter and absorption of brain tissue and debated in the literature. Grinvald and colleagues claimed a spatial resolution of 50-100 $\mathrm{um}[50,62,72,345,346]$, whereas others estimated it to be $200 \mu \mathrm{m}[73,74,347]$. Calcium imaging measures intra-neuronal calcium concentration by fluorescent calcium indicators that correlates stronger with the activity of neurons than their subthreshold membrane potential change [348-351]. The resolution, field of view and noise sensitivities depend on the imaging device used. Two-photon imaging allows single-cell recordings on submicrometer resolutions in small field of views of about one $\mathrm{mm}^{2}$. In contrast, epi-fluorescence imaging records field of views of about $10 \mathrm{~mm}^{2}$ with a spatial resolution that is similar to intrinsic signal imaging and limited by light scattering of cortical tissue [352]. In order to obtain smooth orientation fields, two-photon recordings must be additionally low-pass filtered due to the submicrometer resolution.

The different measurement techniques are all subject to artifacts from an internal state of the cortical tissue [344] and movement artifacts such as heart beat and breathing related vibrations [72, 344, 352]. For instance, stronger pulsation of high density blood vessels introduce spatially correlated noise. Intrinsic signals are additionally composed of noise originating from activity-independent hemodynamic artifacts such as vasomotor signals [343]. An additional noise component dominant on small spatial scales and limited by the Nyquist frequency of the camera is photon shot noise that is substantial in intrinsic signal imaging due to the small signal strength. Shot noise is rather small in calcium imaging [352].

All these different noise sources are superimposed culminating in a noise field $\Psi_{n}(\boldsymbol{x})$. For uncorrelated or weakly correlated noise sources with finite variance the noise ensemble has Gaussian statistics due to the central limit theorem. The noise ensemble is called shift-invariant and isotropic if the noise is statistically equal for all measured orientations and at every cortical position, respectively. Without further assumptions, the noise field is a centered Gaussian random field entirely defined by its non-vanishing and vanishing correlation function (see methods for further details):

$$
\begin{aligned}
& C_{1}\left(\left\|\boldsymbol{x}_{1}-\boldsymbol{x}_{2}\right\|\right)=\left\langle\Psi_{n}\left(\boldsymbol{x}_{1}\right) \bar{\Psi}_{n}\left(\boldsymbol{x}_{2}\right)\right\rangle=2\left\langle\xi_{n}\left(\boldsymbol{x}_{1}\right) \xi_{n}\left(\boldsymbol{x}_{2}\right)\right\rangle=2\left\langle\eta_{n}\left(\boldsymbol{x}_{1}\right) \eta_{n}\left(\boldsymbol{x}_{2}\right)\right\rangle \\
& C_{2}\left(\left\|\boldsymbol{x}_{1}-\boldsymbol{x}_{2}\right\|\right)=\left\langle\Psi_{n}\left(\boldsymbol{x}_{1}\right) \Psi_{n}\left(\boldsymbol{x}_{2}\right)\right\rangle=0 .
\end{aligned}
$$

A fraction of noise components can be efficiently removed by locking measurements to intrinsic noise frequencies [72, 353], Fourier analysis of continuous recording [344] and high-pass filtering [22]. The dominant remaining noise component is a white noise floor resulting from shot noise that can be removed by low-pass filtering. Gaussian low-pass filtered white noise introduces a spatial noise correlation length and might be defined by the correlation function

$$
C_{1}(r)=\sigma^{2} e^{-r^{2} /\left(2 l_{c}^{2}\right)} .
$$

As the filter is applied to both the ground truth signal and noise, Gaussian filters are particularly unsuitable as they impair the signal significantly in contrast to Fermi filters with a sharp low-pass frequency [22]. Infinitely sharp Fermi filters for both low- and high-pass frequency of $k_{1}=2 \pi / \Lambda_{1}$ 
A

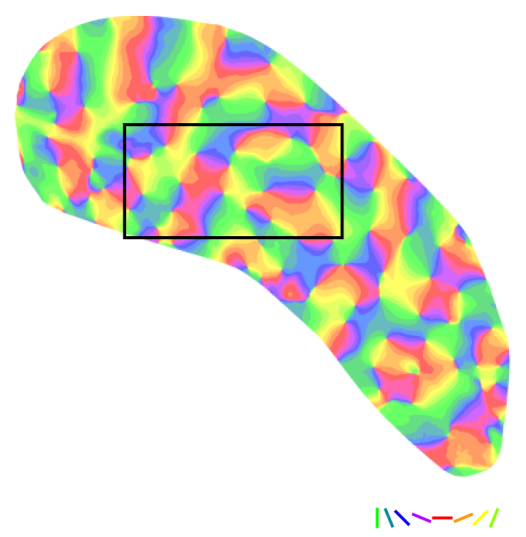

B

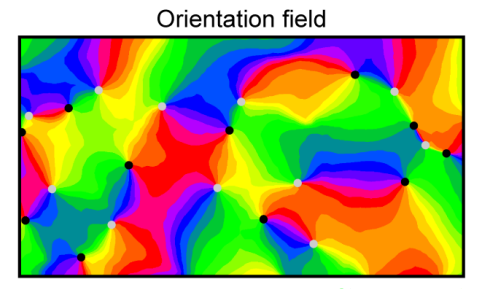

|\ーーー|

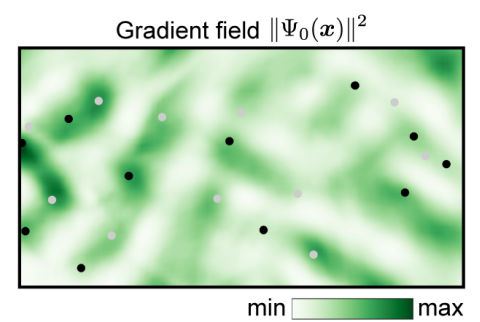

Orientation selectivity field $\left|\Psi_{0}(\boldsymbol{x})\right|^{2}$

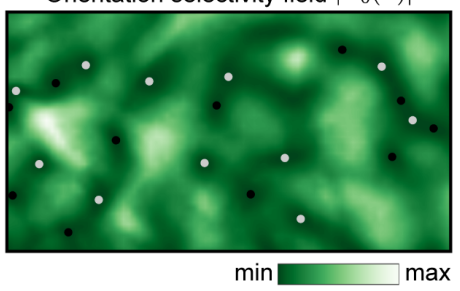

Orthogonality field $\nu_{0}(\boldsymbol{x})$

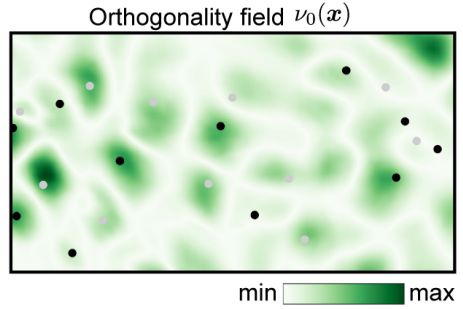

Figure 5.4: Ground truth orientation field properties determine pinwheel observation probability map. A Measured orientation field of ferret used as ground truth orientation field for illustration. B Ground truth orientation field properties that determine noise sensitivity of pinwheel observation probability. White indicates weak noise sensitivity regions and dark green strong noise sensitivity regions. In small orientation selectivity $\left|\Psi_{0}(\boldsymbol{x})\right|^{2}$ regions that are typically centered around pinwheels, the pinwheel observation probability is high. Strong gradients $\left\|\nabla \Psi_{0}(\boldsymbol{x})\right\|^{2}$ increase the pinwheel observation probability. The pinwheel observation probability increases with the orthogonality measure $\nu_{0}(\boldsymbol{x})$. This shows a non-redundant contribution in large areas of the three fields to the pinwheel observation probability.

and $k_{0}=2 \pi / \Lambda_{0}$, respectively, lead to the correlation function

$$
C_{1}(r)=\frac{2 \sigma^{2}}{r\left(k_{1}^{2}-k_{0}^{2}\right)}\left(k_{1} J_{1}\left(k_{1} r\right)-k_{0} J_{1}\left(k_{0} r\right)\right)
$$

For noise without an obvious correlation an effective spatial correlation length of $l_{c}^{\text {eff }}=$ $\sqrt{-2 C_{1}(0) / \Delta C_{1}(0)}$ can be assigned that leads to $l_{c}^{\text {eff }}=2 / \sqrt{k_{0}^{2}+k_{1}^{2}}$ and $l_{c}^{\text {eff }}=l_{c}$ for the band-pass and low-pass filtered noise, respectively. In both noise examples, the noise strength or variance $\left\langle\left|\Psi_{n}(\boldsymbol{x})\right|^{2}\right\rangle=\sigma^{2}$ is controlled by a single parameter and added on orientation domains of average orientation selectivity $\left\langle\left|\Psi_{0}(\boldsymbol{x})\right|^{2}\right\rangle=1$. Intrinsic signal imaging measurement noise from [62] suggests a noise strength of about $\sigma^{2} \approx 0.2$ in units of the average orientation selectivity. Data size scaling utilizes the technique-independent approach of repetitive measurements of trials with the same stimulus set. Considering subsets of trials increases the amount of noise in an observed orientation field. For independent inter-trial noise, the variance of the noise scales inversely with the number of trials $\sigma^{2} \propto 1 / N_{\text {trial }}$.

\section{The pinwheel observation probability map}

The pinwheel configuration is given by the roots $\boldsymbol{x}_{i}$ of the orientation field $\Psi(\boldsymbol{x})$. The microscopic pinwheel density can therefore be written as

$$
\hat{\rho}(\boldsymbol{x})=\sum_{i} \delta\left(\boldsymbol{x}-\boldsymbol{x}_{i}\right)=\delta(\eta(\boldsymbol{x})) \delta(\xi(\boldsymbol{x}))\left|\operatorname{det} \frac{\partial(\eta(\boldsymbol{x}), \xi(\boldsymbol{x}))}{\partial(x, y)}\right|
$$

and is a random variable due to the additive measurement noise. The pinwheel observation probability map is obtained by the expected microscopic pinwheel density over the ensemble of 
noise realizations $\Psi_{n}(\boldsymbol{x})$

$$
\rho(\boldsymbol{x})=\left\langle\delta(\eta(\boldsymbol{x})) \delta(\xi(\boldsymbol{x}))\left|\operatorname{det} \frac{\partial(\eta(\boldsymbol{x}), \xi(\boldsymbol{x}))}{\partial(x, y)}\right|\right\rangle .
$$

For a specific position $\boldsymbol{x}$ and a small surrounding area $\mathrm{d} A$ the probability of observing a pinwheel in that area is thus given by $\rho(\boldsymbol{x}) \mathrm{d} A$. The apparent pinwheel density in an area $A$ is given by

$$
\rho=\frac{1}{A} \int_{A} \mathrm{~d}^{2} x \rho(\boldsymbol{x})
$$

with the total number of pinwheels $N=\rho A$. The noise average of Eq. (5.8) can be evaluated by the multivariate Gaussian probability density function of the noise and its spatial derivatives (see methods for further details) leading to

$$
\rho(\boldsymbol{x})=\frac{c_{G}}{2 \pi c_{A}} e^{-\frac{\left|\Psi_{0}(\boldsymbol{x})\right|^{2}}{2 c_{A}}} \frac{1}{\pi} \int \frac{\mathrm{d} t}{t^{2}}\left(1-\frac{1}{t^{2}+1} e^{-\frac{t^{2}}{t^{2}+1} \frac{\left\|\nabla \Psi_{0}(\boldsymbol{x})\right\|^{2}}{2 c_{G}}} \cos \left(\frac{t}{t^{2}+1} \frac{\nu_{0}(\boldsymbol{x})}{c_{G}}\right)\right) .
$$

Here $c_{A}=C_{1}(0) / 2$ is proportional to the noise strength, $c_{G}=-\Delta C_{1}(0) / 4$ proportional to the spatial noise correlation decay and $\nu_{0}(\boldsymbol{x})=\left|\partial_{x} \xi_{0}(\boldsymbol{x}) \partial_{y} \eta_{0}(\boldsymbol{x})-\partial_{x} \eta_{0}(\boldsymbol{x}) \partial_{y} \xi_{0}(\boldsymbol{x})\right|$ an orthogonality measure between $\nabla \xi_{0}(\boldsymbol{x})$ and $\nabla \eta_{0}(\boldsymbol{x})$ that is given by the area of their spanned parallelogram. In total, three fields $\left|\Psi_{0}(\boldsymbol{x})\right|^{2} / c_{A},\left\|\nabla \Psi_{0}(\boldsymbol{x})\right\|^{2} / c_{G}$ and $\nu(\boldsymbol{x}) / c_{G}$ determine the pinwheel observation probability map. The pinwheel observation probability grows with a decrease of the first and an increase of the latter two fields in a broad parameter regime. The ground truth orientation field reveals non-redundant large areas that increase the pinwheel observation probability, Fig. 5.4.

In the absence of ground truth orientation fields the pinwheel observation probability map is independent of the spatial position and given by the noise pinwheel density $\rho_{n}=c_{G} /\left(2 \pi c_{A}\right)$. This expression was derived already earlier in other contexts, see [30, 147, 171]. The low- and band-pass correlation functions yield the same parameter $c_{A}=\sigma^{2} / 2$, but different $c_{G}=\sigma^{2} /\left(2 l_{c}^{2}\right)$ and $c_{G}=\sigma^{2}\left(k_{0}^{2}+k_{1}^{2}\right) / 8$, respectively. Notice that the noise pinwheel density of band-pass filtered noise is similar to the ground truth signal due to similar spatial frequencies.

Interestingly, the pinwheel observation probability only depends on two parameters of the noise correlation function and is independent of its specific shape. Noise ensembles can hence be described by $c_{A}$ and $c_{G}$, by the noise strength $\sigma^{2}$ and (effective) correlation length $l_{c}$ or by the noise strength $\sigma^{2}$ and noise pinwheel density $\rho_{n}$.

The effect of measurement noise on the observed layout of orientation domains and pinwheel centers consists of three components illustrated in Fig. 5.5. Pinwheels are generally displaced by noise, noise can generate spurious pinwheel pairs of topologically opposite charge and pinwheels can be hidden by noise. Furthermore, the probability of spurious pinwheel creation and annihilation of pinwheel pairs can be approximated for time-varying measurement noise and identifies similar orientation field regions that are susceptible to noise (see methods for details).

\section{Generation of spurious pinwheels in pinwheel free orientation layouts}

An important concern in the assessment of measured orientation domain arrangements is the potential presence of spurious pinwheels, i.e. pinwheel centers caused by measurement noise in a genuine pinwheel free region. Purely random orientation layouts generically possess more pinwheels than ordered orientation layouts [147]. This observation and common intuition suggests that spurious pinwheels generically proliferate monotonically with noise strength. To test this hypothesis, we examine the impact of noise on ground truth orientation fields with spatially uniform selectivity $\left|\Psi_{0}(\boldsymbol{x})\right|^{2}=s^{2}$ and hence without any pinwheels. Typical pinwheel free motifs with spatially uniform selectivity are experimentally observed linear zones $[12,51,354]$ 
A

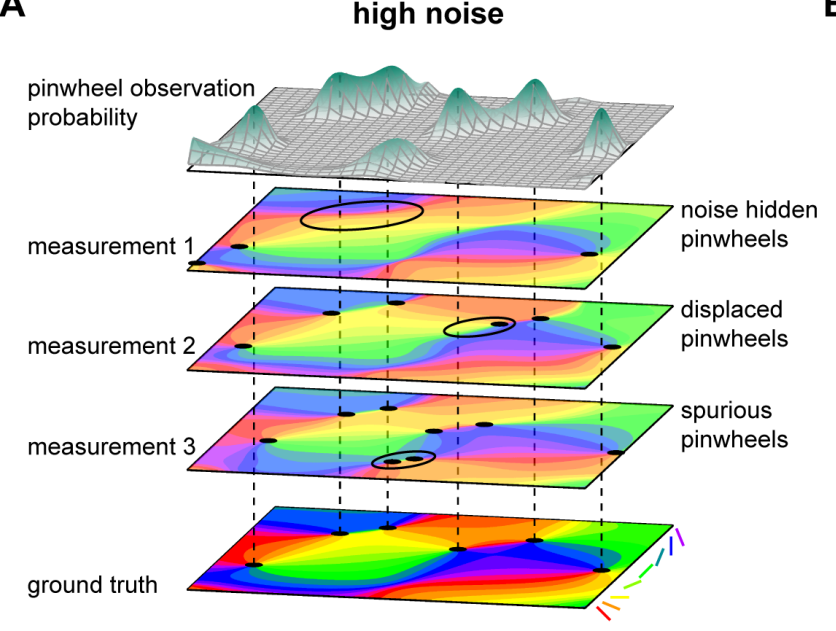

B

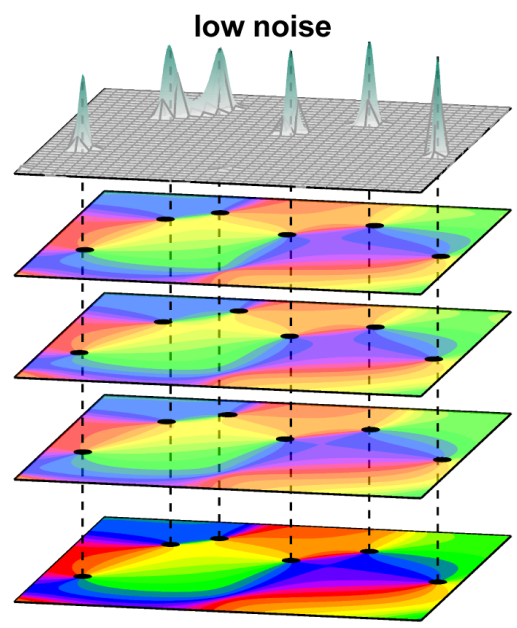

Figure 5.5: Spurious, displaced and hidden pinwheels in orientation domain measurements. Observed orientation fields can exhibit noise induced spurious pinwheels that consist of pinwheel pairs with opposite topological charge. Ground truth pinwheels are typically displaced by noise. Two ground truth pinwheels of opposite topological charge that are displaced to the same location by noise are hidden in measurements. The pinwheel observation probability summarizes the combination of the three effects for a given noise ensemble and a ground truth orientation field. A In high noise regimes spurious pinwheels can emerge, while ground truth pinwheels can be hidden and displaced. Varying the noise strength and correlation length alters the pinwheel observation probability. B In low noise regimes pinwheel displacements are mostly observed. Hence, the pinwheel observation probability map is peaked around ground truth pinwheels and converges to the ground truth pinwheel positions.

characterized by a progressive change in orientation preference along a single direction. Linear zones can appear with different selectivity $s$ and typical wavelengths $\Lambda$ in orientation fields. The simplest model of a linear zone is a plane wave

$$
\Psi_{0}(\boldsymbol{x})=s e^{i \boldsymbol{k}_{\phi} \boldsymbol{x}}
$$

with the wave vector $\boldsymbol{k}_{\phi}=k_{0}(\cos \phi, \sin \phi)^{T}$ and $k_{0}=2 \pi / \Lambda$, Fig. 5.6A. For Eq. (5.11) the orthogonality measure $\nu_{0}$ vanishes and the gradient is given by $\left\|\nabla \Psi_{0}(\boldsymbol{x})\right\|^{2}=s^{2} k_{0}^{2}$. The vanishing orthogonality $\nu_{0}$ is valid for all constant orientation selectivity layouts and simplifies the pinwheel observation probability to

$$
\rho(\boldsymbol{x})=\frac{c_{G}}{2 \pi c_{A}} e^{-\frac{\left|\Psi_{0}(\boldsymbol{x})\right|^{2}}{2 c_{A}}} e^{-\beta(\boldsymbol{x})}\left(I_{0}(\beta(\boldsymbol{x}))+2 \beta(\boldsymbol{x})\left(I_{0}(\beta(\boldsymbol{x}))+I_{1}(\beta(\boldsymbol{x}))\right)\right),
$$

where $\beta(\boldsymbol{x})=\left\|\nabla \Psi_{0}(\boldsymbol{x})\right\|^{2} /\left(4 c_{G}\right)$ and $I_{l}$ denotes the modified Bessel function of the $l$ th kind (see methods). In the plane wave case, the pinwheel observation probability map is constant and therefore equal to the pinwheel density

$$
\rho(\boldsymbol{x})=\rho_{n} e^{-\frac{s^{2}}{\sigma^{2}}\left(1+\frac{2 \pi^{2}}{\lambda^{2}}\right)}\left(I_{0}\left(\frac{2 \pi^{2}}{\lambda^{2}} \frac{s^{2}}{\sigma^{2}}\right)+\frac{4 \pi^{2}}{\lambda^{2}} \frac{s^{2}}{\sigma^{2}}\left(I_{0}\left(\frac{2 \pi^{2}}{\lambda^{2}} \frac{s^{2}}{\sigma^{2}}\right)+I_{1}\left(\frac{2 \pi^{2}}{\lambda^{2}} \frac{s^{2}}{\sigma^{2}}\right)\right)\right) .
$$

with the noise pinwheel density $\rho_{n}$, the signal-to-noise ratio $s^{2} / \sigma^{2}$ and the dimensionless parameter $\lambda=\Lambda / l_{c}$. Spurious pinwheels are strongly suppressed in the small noise regime $\sigma^{2} / s^{2} \lesssim 0.5$ such that the pinwheel density decreases with the non-analytic function $\rho \sim e^{-s^{2} / \sigma^{2}} / \sigma$, see Fig. 5.6B inset. In the intermediate noise strength regime $0.5 \lesssim \sigma^{2} / s^{2} \lesssim 2$ the pinwheel density monotonically increases. For large noise regimes $2 \lesssim \sigma^{2} / s^{2}$ the pinwheel density converges to the noise pinwheel density, see Fig. 5.6B dashed line. Noise with correlation lengths smaller 
A

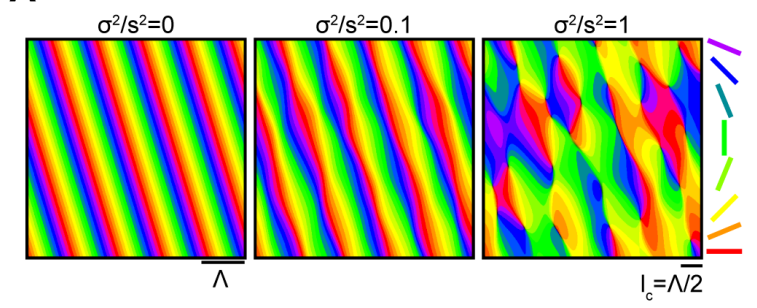

B

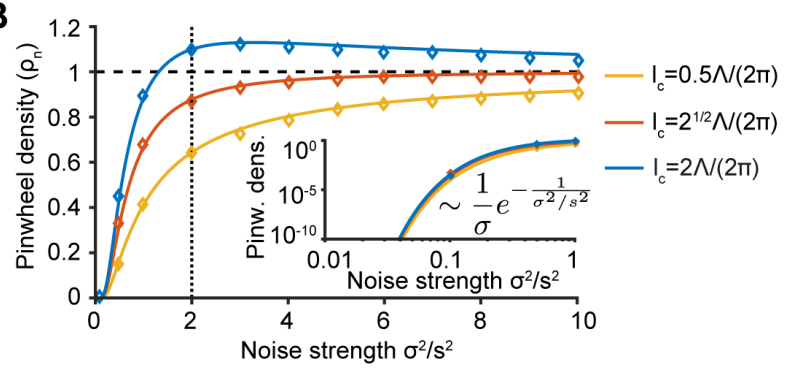

Figure 5.6: Measurement noise induces exclusively spurious pinwheels in spatially uniform selectivity layouts. A Three linear zone examples (Eq. (5.11)) of spatially uniform orientation selectivity $s$ and different additive noise strengths $\sigma^{2} / s^{2}$. B The pinwheel observation probability map of linear zones (Eq. (5.13)) is constant and hence equals its pinwheel density. The pinwheel density is strongly suppressed for small noise strengths (inset). For noise correlation lengths $l_{c} \leq \Lambda /(\sqrt{2} \pi)$ the pinwheel density increases monotonically and for $l_{c}>\Lambda /(\sqrt{2} \pi)$ non-monotonically with noise strength. In the limit of strong noise strength, the pinwheel density converges to the noise pinwheel density $\rho_{n}=1 /\left(2 \pi l_{c}^{2}\right)$. The maximal pinwheel density for large noise correlation lengths is assumed at a strength $\sigma^{2} / s^{2}=2$. (solid line: pinwheel observation probability, diamonds: numerical results).

than $l_{c}<\Lambda /(\sqrt{2} \pi)$ induce monotonically increasing pinwheel densities with noise strength $\sigma^{2} / s^{2}$. For larger noise correlation lengths the number of spurious pinwheels can decrease with noise strength such that the pinwheel density is greater than the noise pinwheel density in a noise strength regime. The maximum pinwheel density is reached at the noise strength $\sigma^{2} / s^{2}=2$ in the limit of large correlation lengths $l_{c} \gg \Lambda$, see Fig. 5.6B dotted line. The strong suppression of spurious pinwheels in the small noise regime and its subsequent increase in the intermediate noise regime makes linear zones in the small noise regime particularly favorable in measurements of pinwheel configurations.

\section{Displaced and spurious pinwheels near true pinwheel centers}

To understand conditions for precise localization of pinwheel centers and the proliferation/suppression of spurious pinwheels near genuine pinwheel centers, we examine isolated ground truth pinwheels, Fig. 5.7A,B, under measurement noise of different correlation lengths. In order to dissect the noise effect on pinwheels, we note that any pinwheel can be represented up to a coordinate system rotation as

$$
\begin{aligned}
\Psi_{0}(\boldsymbol{x}) & =\xi_{0}(\boldsymbol{x})+i \eta_{0}(\boldsymbol{x}) \\
\left(\xi_{0}(\boldsymbol{x}), \eta_{0}(\boldsymbol{x})\right)^{T} & =s \Omega_{\phi}^{T} \Sigma \Omega_{\phi} \boldsymbol{x}
\end{aligned}
$$

with the rotation matrix $\Omega_{\phi}$, selectivity scale $s$ and the matrix $\Sigma=\operatorname{diag}(1, a)$ with the anisotropy parameter $a$. This is found by linearizing $\Psi_{0}(\boldsymbol{x})$ in the vicinity of a pinwheel center. For $a=1$ pinwheels are isotropic, Fig. 5.7A, and for $a \neq 1$ anisotropic, Fig. 5.7B. For $\sigma^{2} \neq 0$ the ground truth pinwheel is typically displaced by noise and additional spurious pinwheels can emerge, Fig. 5.7C. The pinwheel observation probability map is given by

$$
\rho(\boldsymbol{x})=\frac{1}{2 \pi l_{c}^{2}} e^{-\frac{x^{T} \Omega_{\phi}^{T} \Sigma^{T} \Sigma \Omega_{\phi} \boldsymbol{x}}{\sigma^{2} / s^{2}}} \frac{1}{\pi} \int \frac{\mathrm{d} t}{t^{2}}\left(1-\frac{1}{t^{2}+1} e^{-\frac{t^{2}}{t^{2}+1} \frac{l_{c}^{2}\left(1+a^{2}\right)}{\sigma^{2} / s^{2}}} \cos \left(\frac{t}{t^{2}+1} \frac{2 l_{c}^{2} a}{\sigma^{2} / s^{2}}\right)\right)
$$

and composed of the ground truth pinwheel and additional spurious pinwheels with a total Gaussian profile that is for $\phi=0$ proportional to $\exp \left(-\left(x^{2}+a^{2} y^{2}\right) /\left(\sigma^{2} / s^{2}\right)\right)$, Fig. 5.7D. The 
A

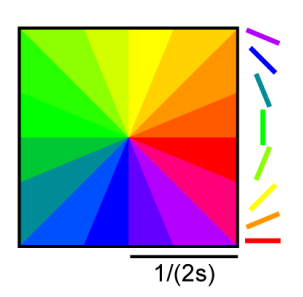

B

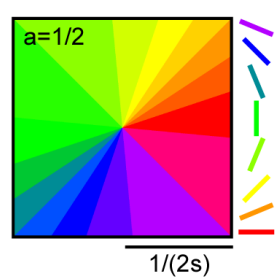

C
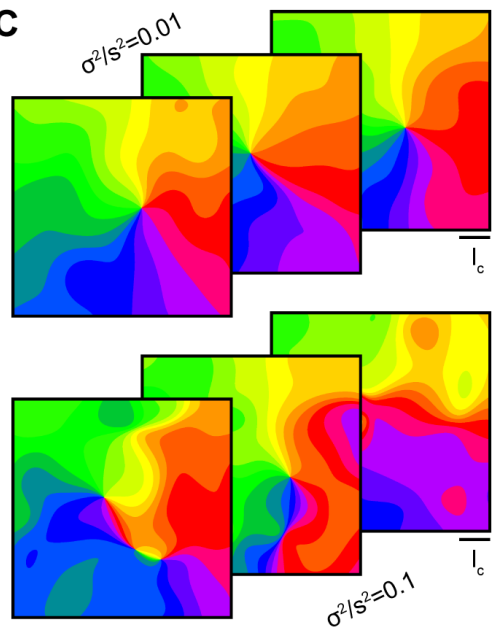

D

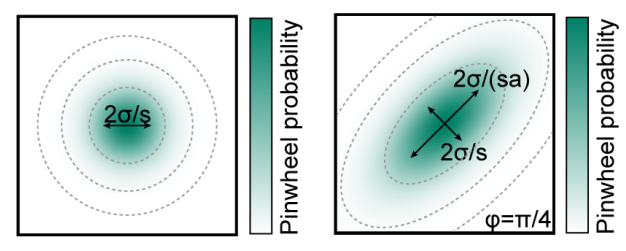

$\mathbf{E}$

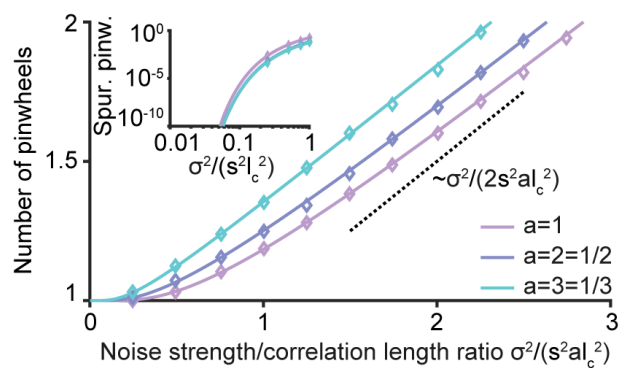

Figure 5.7: Measurement noise displaces pinwheels and induces spurious pinwheels. A and $\mathbf{B}$ depicts an isotropic $a=1$ and anisotropic $a=1 / 2$ pinwheel with selectivity scale $s$ (Eq. (5.15)), respectively. $\mathbf{C}$ Measurement noise typically displaces the isotropic pinwheel of $\mathbf{A}$ for small noise strengths $\sigma^{2} / s^{2}$ (top) and induces additional spurious pinwheels for stronger noise strengths (bottom). D The spatial dependence of the pinwheel observation probability map is given by an isotropic and anisotropic Gaussian profile for A and B, respectively. E The total number of pinwheels (Eq. (5.17)) increases monotonically with the noise strength $\sigma^{2} / s^{2}$ and inversely with the pinwheel anisotropy $a$ and the noise correlation length $l_{c}$. For small noise strengths, spurious pinwheels are strongly suppressed (inset). (solid line: pinwheel observation probability, diamonds: numerical results).

total number of pinwheels is given by the integral over the pinwheel observation probability map and yields

$$
N=\frac{\alpha}{\pi} \int \frac{\mathrm{d} t}{t^{2}}\left(1-\frac{1}{t^{2}+1} e^{-\frac{t^{2}}{t^{2}+1} \frac{(1 / a+a)}{2 \alpha}} \cos \left(\frac{t}{t^{2}+1} \frac{1}{\alpha}\right)\right)
$$

with the dimensionless parameter $\alpha=\frac{\sigma^{2} / s^{2}}{2 a l_{c}^{2}}$, Fig. 5.7E. In the special case of isotropic pinwheels $a=1$, the expression simplifies to $N=1+\alpha \exp (-1 /(2 \alpha))$. For every $\alpha$ the number of pinwheels in the isotropic case $a=1$ is a critical point and numerical analysis indicate that it is a minimum everywhere. This suggests that the number of pinwheels of isotropic pinwheels is a lower bound to the number of pinwheels of anisotropic pinwheels. In general, spurious pinwheels increase monotonically, Fig. 5.7E, and are strongly suppressed for small noise strengths $\sigma^{2} / s^{2}$, see Fig. 5.7E inset. Asymptotically, spurious pinwheels scale with the noise strength and inversely with the anisotropy and noise correlation length as $N \sim \alpha$. In total, we find favorable conditions near isolated ground truth pinwheels of strong suppression of spurious pinwheels. The displaced ground truth pinwheel and the spurious pinwheels form a Gaussian cloud and concentrate at the ground truth pinwheel position. The total number of observed pinwheels can decrease for pinwheel free orientation layouts in a certain noise regime, but can only increase for single ground truth pinwheels.

\section{Noise hidden pinwheels and spurious pinwheel generation at pinwheel pairs}

Absolute pinwheel densities are typically $\sim 3$ pinwheels $/ \Lambda^{2}$ such that pinwheels are often not isolated but in the vicinity of neighboring pinwheels. This opens the possibility of more complex noise effects. We thus used Eq. (5.10) to examine the impact of noise on two distinct pinwheel pair configurations of the same $(++)$ and opposite $(+-)$ topological charge. A pair of isotropic 

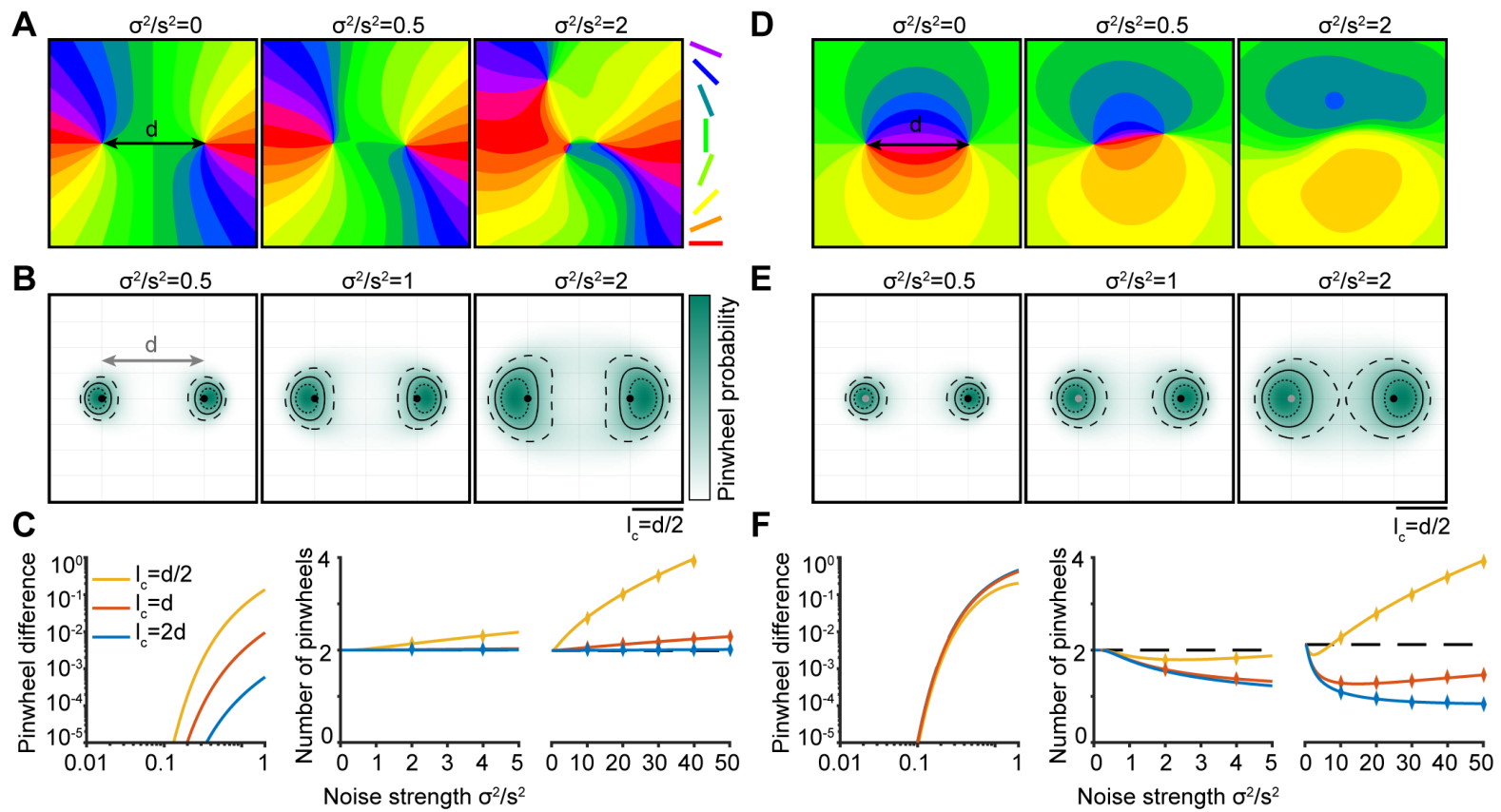

Figure 5.8: Noise hidden pinwheels and spurious pinwheel generation near pinwheel pairs. $\mathbf{A}$ and $\mathbf{D}$ Three examples of pinwheel pairs of equal and opposite topological charge separated by the distance $d$ for different noise strengths $\sigma^{2} / s^{2}$, respectively. B and $\mathbf{E}$ Pinwheel observation probability for three different noise strengths for the pinwheel pairs of $\mathbf{A}$ and $\mathbf{D}$, respectively. Dots mark the positions of ground truth pinwheel centers and the color their topological charge. Dotted, solid and dashed lines indicate the pinwheel observation probability contour lines of $25 \%, 50 \%$ and $75 \%$ of the maximum, respectively. $\mathbf{C}$ and $\mathbf{F}$ Pinwheel number of $\mathbf{A}$ and $\mathbf{D}$ for three different correlation lengths and over three different scales of noise strengths (solid line: pinwheel observation probability, diamonds: numerical results).

pinwheels separated by the distance $d$ and with a selectivity scale $s$ can be modeled by

$$
\begin{aligned}
& \Psi_{0}^{++}(\boldsymbol{x})=s\left(-\frac{d}{2}+x+i y\right)\left(\frac{d}{2}+x+i y\right), \\
& \Psi_{0}^{+-}(\boldsymbol{x})=s\left(-\frac{d}{2}+x+i y\right)\left(\frac{d}{2}+x-i y\right)
\end{aligned}
$$

as illustrated in Fig. 5.8A, D, respectively. The pinwheel observation probability maps in the coordinates with radial distance $r$ and angle $\phi$ from the center of the pinwheel pair are given by

$$
\begin{aligned}
\rho^{++}(r, \phi)= & \frac{1}{2 \pi l_{c}^{2}} e^{-\frac{d^{4}-\frac{1}{2} d^{2} r^{2} \cos 2 \phi+r^{4}}{\sigma^{2} / s^{2}}}\left(\frac{8 l_{c}^{2} r^{2}}{\sigma^{2} / s^{2}}+e^{-\frac{4 l_{c^{2}}^{2}}{\sigma^{2} / s^{2}}}\right), \\
\rho^{+-}(r, \phi)= & \frac{1}{2 \pi l_{c}^{2}} e^{-\frac{d^{4}-\frac{1}{1} d^{2} r^{2} \cos 2 \phi+r^{4}}{\sigma^{2} / s^{2}}} \\
& \frac{1}{\pi} \int \frac{\mathrm{d} t}{t^{2}}\left(1-\frac{1}{t^{2}+1} e^{-\frac{t^{2}}{t^{2}+1} \frac{l_{c}^{2}\left(d^{2}+4 r^{2}\right)}{\sigma^{2} / s^{2}}} \cos \left(\frac{t}{t^{2}+1} \frac{4 l_{c}^{2} d r \cos (\phi)}{\sigma^{2} / s^{2}}\right)\right),
\end{aligned}
$$

respectively. The pinwheel observation probability is spatially localized around the ground truth pinwheel centers for $(++)$ and $(+-)$ pinwheel pair configurations. With increasing noise strength $\sigma^{2} / s^{2}$ the two highest probability peaks increase in distance as shown in Fig. 5.8B, E. In the small noise regime, the noise effect on the pinwheel observation probability is strongly 
suppressed, Fig. 5.8C, F inset. For a $(++)$ pinwheel pair configuration, increasing noise strength induces a monotonic proliferation of spurious pinwheels on all noise strength regimes, Fig. 5.8C. In contrast, for small noise strengths the observed number of pinwheels in a $(+-)$ pinwheel pair configuration first decreases with increasing noise strength indicating that the pinwheel pair is hidden by noise, Fig. 5.8F. For large noise strengths the total number of pinwheels increases for the $(+-)$ pinwheel pair configuration similar to the $(++)$ configuration.

Pinwheel pairs that are very close to each other can be idealized by a vanishing distance $d \rightarrow 0$ for which the pinwheel observation probability map can be derived exactly. In that case the pinwheel observation probability is radially symmetric and becomes

$$
\begin{aligned}
\rho^{++}(r)= & \frac{1}{2 \pi l_{c}^{2}} e^{-\frac{r^{4}}{\sigma^{2} / s^{2}}}\left(\frac{8 l_{c}^{2} r^{2}}{\sigma^{2} / s^{2}}+e^{-\frac{4 l_{c}^{2} r^{2}}{\sigma^{2} / s^{2}}}\right) \\
\rho^{+-}(r)= & \frac{1}{2 \pi l_{c}^{2}} e^{-\frac{r^{4}}{\sigma^{2} / s^{2}}} e^{-\frac{2 l_{c}^{2} r^{2}}{\sigma^{2} / s^{2}}} \\
& \left(I_{0}\left(\frac{2 l_{c}^{2} r^{2}}{\sigma^{2} / s^{2}}\right)+\frac{4 l_{c}^{2} r^{2}}{\sigma^{2} / s^{2}}\left(I_{0}\left(\frac{2 l_{c}^{2} r^{2}}{\sigma^{2} / s^{2}}\right)+I_{1}\left(\frac{2 l_{c}^{2} r^{2}}{\sigma^{2} / s^{2}}\right)\right)\right) .
\end{aligned}
$$

The total number of pinwheels is then given by

$$
\begin{aligned}
N^{++}= & +\frac{\sqrt{\pi} \sigma / s}{4 l_{c}^{2}} e^{\frac{4 \mathrm{ll}^{4}}{\sigma^{2} / s^{2}}} \operatorname{erfc}\left(\frac{2 l_{c}^{2}}{\sigma / s}\right) \\
N^{+-}= & -\frac{2 l_{c}^{4}}{\sigma^{2} / s^{2}}{ }_{2} F_{2}\left(\frac{5}{4}, \frac{7}{4} ; \frac{3}{2}, \frac{5}{2} ; \frac{4 l_{c}^{4}}{\sigma^{2} / s^{2}}\right)+{ }_{2} F_{2}\left(\frac{1}{4}, \frac{3}{4} ; \frac{1}{2}, \frac{1}{2} ; \frac{4 l_{c}^{4}}{\sigma^{2} / s^{2}}\right) \\
& -\frac{1}{2}{ }_{2} F_{2}\left(\frac{3}{4}, \frac{5}{4} ; \frac{3}{2}, \frac{3}{2} ; \frac{4 l_{c}^{4}}{\sigma^{2} / s^{2}}\right)+\frac{\sqrt{\pi} l_{c}^{2}}{2 \sigma / s}{ }_{2} F_{2}\left(\frac{3}{4}, \frac{5}{4} ; \frac{1}{2}, 2 ; \frac{4 l_{c}^{4}}{\sigma^{2} / s^{2}}\right) \\
& -\frac{\sqrt{\pi} l_{c}^{2}}{\sigma / s}{ }_{2} F_{2}\left(\frac{3}{4}, \frac{5}{4} ; 1, \frac{3}{2} ; \frac{4 l_{c}^{4}}{\sigma^{2} / s^{2}}\right)+\frac{\sqrt{\pi} \sigma / s}{4 l_{c}^{2}}{ }_{2} F_{2}\left(\frac{1}{4}, \frac{3}{4} ; \frac{1}{2}, 1 ; \frac{4 l_{c}^{4}}{\sigma^{2} / s^{2}}\right)
\end{aligned}
$$

where ${ }_{2} F_{2}$ is the generalized hypergeometric function.

\section{Spurious and noise hidden pinwheels in hexagonal pinwheel crystals}

The examination of specific orientation motifs revealed strong suppression of pinwheels in noise strength regimes that are small compared to the selectivity scale. In orientation domain layouts these motifs are combined with different selectivity scales to a total pinwheel configuration. Orientation fields with a finite repertoire of motifs are crystalline layouts. Crystalline layouts are interesting because they pose an orientation field of intermediate complexity and they are stable dynamically generated solutions of a very general model class, e.g. [24, 27, 126, 129-131, 263-266]. While at current, experimental analysis [22-24] provides clear evidence against these models, it is interesting to examine how ground truth pinwheel crystals might be transformed by measurement noise. Example pinwheel crystals are given by special cases of essentially complex planforms (ECP) solutions defined by the superposition of $n$ active modes

$$
\Psi_{0}(\boldsymbol{x})=\frac{1}{\sqrt{n}} \sum_{j=1}^{n} e^{i\left(l_{j} \boldsymbol{k}_{j} \boldsymbol{x}+\phi_{j}\right)}
$$

with the wave vectors $\boldsymbol{k}_{j}=k_{0}(\cos (\pi j / n), \sin (\pi j / n))^{T}$ and $k_{0}=2 \pi / \Lambda$, phases $\phi_{j}$ and the binary variables $l_{j} \in\{-1,1\}$. These are closed-form solutions of a universality class for visual cortical 
A
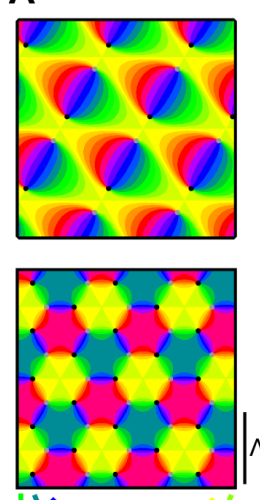

IIーーー
B
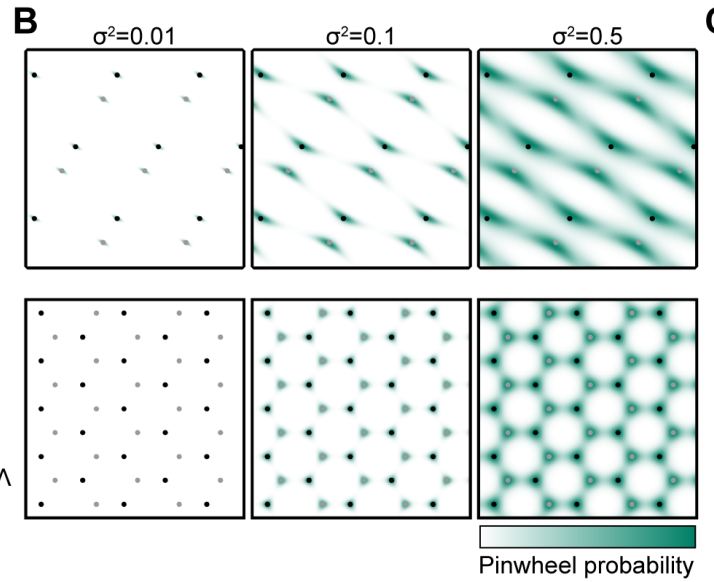

C
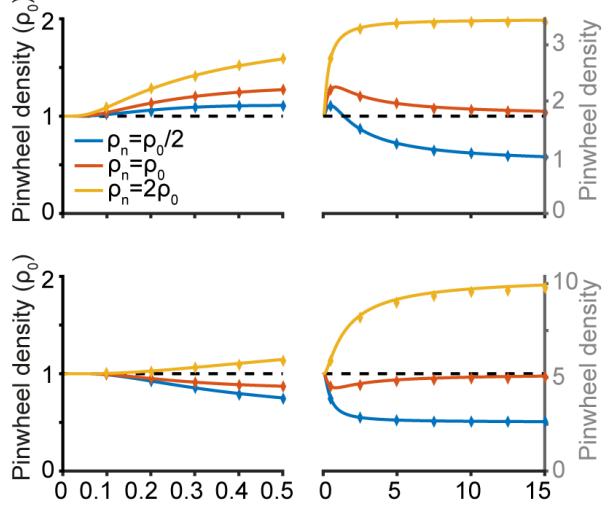

Noise strength $\sigma^{2}$

Figure 5.9: Spurious and noise hidden pinwheels in crystalline orientation layouts. A Examples of two orientation preference crystals with a low (upper, $\sqrt{3}$ ) and high (lower, $3 \sqrt{3}$ ) pinwheel number per $\Lambda^{2}$. B Pinwheel observation probability for both crystals and three different noise strengths with noise pinwheel density $\rho_{n}=\rho_{0}$. C Pinwheel density of observed orientation domains as a function of the noise strength $\sigma^{2}$ and three different noise correlation lengths (solid line: pinwheel observation probability, diamonds: numerical results).

development $[22,128,135]$. Gradient and orthogonality of these orientation fields are

$$
\begin{aligned}
\left\|\nabla \Psi_{0}(\boldsymbol{x})\right\|^{2} & =\frac{k_{0}^{2}}{n} \sum_{j, m=1}^{n} l_{j} l_{m} \cos \left(\frac{\pi(j-m)}{n}\right) \cos \left(\left(l_{j} \boldsymbol{k}_{j}-l_{m} \boldsymbol{k}_{m}\right) \boldsymbol{x}+\phi_{j}-\phi_{m}\right) \\
\nu_{0}(\boldsymbol{x}) & =\frac{k_{0}^{2}}{n}\left|\sum_{j, m=1}^{n} l_{j} l_{m} \sin \left(\frac{\pi(j-m)}{n}\right) \sin \left(l_{j} \boldsymbol{k}_{j} \boldsymbol{x}+\phi_{j}\right) \cos \left(l_{m} \boldsymbol{k}_{m} \boldsymbol{x}+\phi_{m}\right)\right| .
\end{aligned}
$$

For $n=3$ active modes there are two distinct ECP solutions with high and low pinwheel density defined by $\left(l_{1}, l_{2}, l_{3}\right)=(1,1,1)$ and $\left(l_{1}, l_{2}, l_{3}\right)=(1,-1,1)$ shown in the upper and lower part of Fig. 5.9A, respectively. The crystals are most notably distinguished by their number of pinwheels per $\Lambda^{2}$ of $\sqrt{3}$ and $3 \sqrt{3}$ for the first and second case, respectively. Notice that all pinwheels have the same selectivity $s=\sqrt{3} \pi / \Lambda$ and anisotropies of $a=1 / 3$ and $a=1$ in the first and second crystal, respectively.

The pinwheel observation probability is centered around pinwheel centers with a non-elliptic profile already for a noise strength of $\sigma^{2}=0.1$, Fig. 5.9B. This indicates that the linear approximation discussed above is insufficient for small noise regimes on the order of $\sigma^{2}=0.1$. The non-elliptic pinwheel observation probability profile is in both cases caused by low orientation selectivity regions and additionally mainly shaped by the orthogonality or gradient in the low and high pinwheel density crystal, respectively. In the low pinwheel density crystal, pinwheels of opposite topological charge are separated by high orientation selectivity barriers such that there are no hidden pinwheel pairs for small noise strengths. Only spurious pinwheels are present for small noise strengths and noise pinwheel densities similar to the ground truth pinwheel density, Fig. 5.9C. In the high pinwheel density crystal, some pinwheel pairs of opposite topological charge are closely spaced such that the pinwheel density decreases for small noise strengths with noise pinwheel densities similar to the ground truth crystal pinwheel density, Fig. 5.9C. In general, the pinwheel density shows a strong suppression of spurious and noise hidden pinwheels for noise strengths smaller than $\sigma^{2}<0.1$, Fig. 5.9C, for these arrangements. 
A
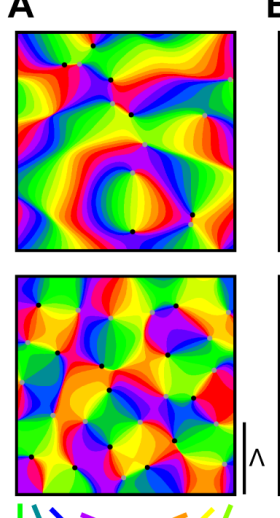

|IIーーーा
B
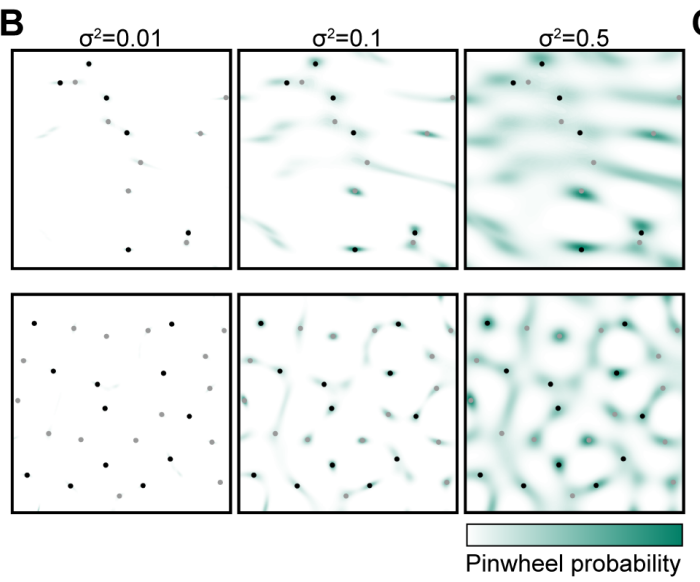
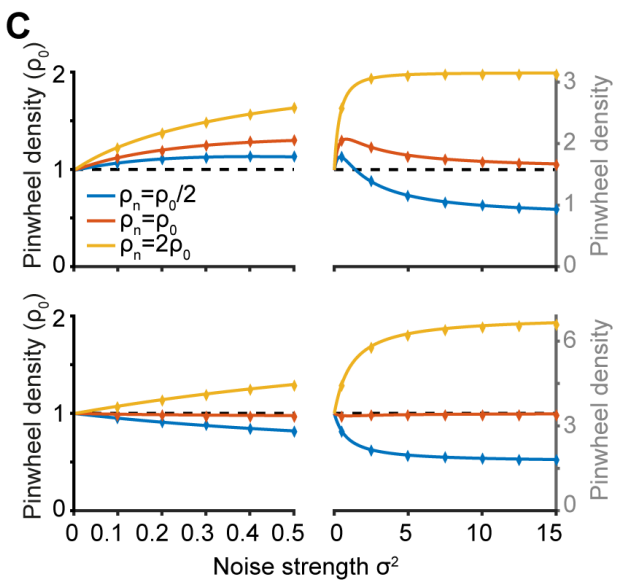

Figure 5.10: Spurious and noise hidden pinwheels in quasiperiodic orientation layouts. A Examples of an anisotropic (upper) and isotropic (lower) quasiperiodic orientation domain. B The pinwheel observation probability for both orientation domains and three different noise strengths with noise pinwheel density $\rho_{n}=\rho_{0}$. $\mathbf{C}$ Pinwheel density of observed orientation domains as a function of the noise strength $\sigma^{2}$ and three different noise correlation lengths (solid line: pinwheel observation probability, diamonds: numerical results).

\section{Spurious and noise hidden pinwheels in quasiperiodic orientation fields}

A more thorough account for the experimentally observed orientation fields are quasiperiodic orientation fields. ECP orientation fields of Eq. (5.26) with $n>3$ active modes are qausiperiodic and account quantitatively for experimentally observed pinwheel configuration with $n \approx 10$ and random $l_{j}[22]$. The pinwheel configuration is only weakly dependent on $n$ and sensitive to $l_{j}$. Here we examine low $l_{j}=1$ and high $l_{j}=(-1)^{j}$ pinwheel density ECP orientation fields with $n=8$ active modes depicted in Fig. 5.10A.

The pinwheel observation probability is already for small noise strengths of $\sigma^{2}<0.1$ centered around pinwheels and present at low orientation selectivity and high orthogonality and gradient regions, Fig. 5.10B. This effect becomes more severe for larger noise strengths and is stronger for low pinwheel density quasiperiodic orientation fields. The total effect on the low pinwheel density orientation field is an increase in the pinwheel density for small noise strengths and noise pinwheel densities similar to the ground truth pinwheel density, Fig. 5.10C, similar to the low pinwheel density hexagonal pinwheel crystal. In the high pinwheel density field, spurious pinwheels emerge and pinwheels are hidden by noise such that for small noise strengths and noise pinwheel densities similar to the ground truth pinwheel density, the pinwheel density appears to increase or decrease, Fig. 5.10C, similar to the crystalline case. However, the noise dependence of quasiperiodic orientation fields is substantially different from hexagonal pinwheel crystals. For small noise strengths, quasiperiodic orientation fields depend linear on the noise strength, whereas crystals revealed a strong nonlinear suppression.

\section{Spatial averaging of pinwheel heterogeneity linearizes low noise suppression of noise artifacts}

Strong suppression of spurious and noise hidden pinwheels should in principle generalize to arbitrary finite domains as only ground truth pinwheel centers contribute for very low noise strengths and the pinwheel observation probability is at every position proportional to $\propto \exp \left(-\left|\Psi_{0}(\boldsymbol{x})^{2}\right| / \sigma^{2}\right)$. For hexagonal pinwheel crystals this generalization holds true, while for quasiperiodic orientation fields there is no apparent strong suppression. A key difference 
A

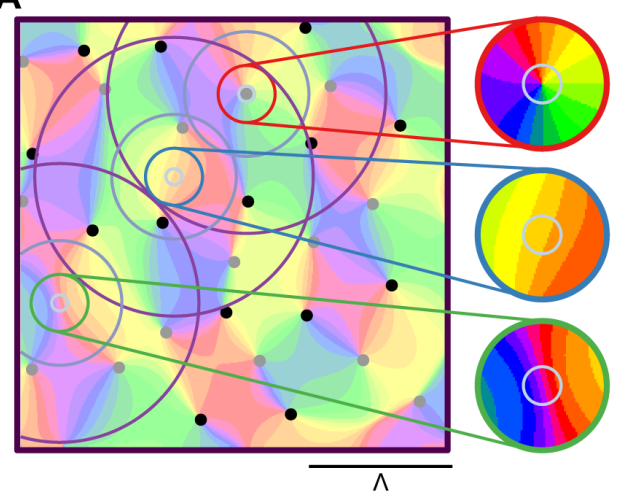

B
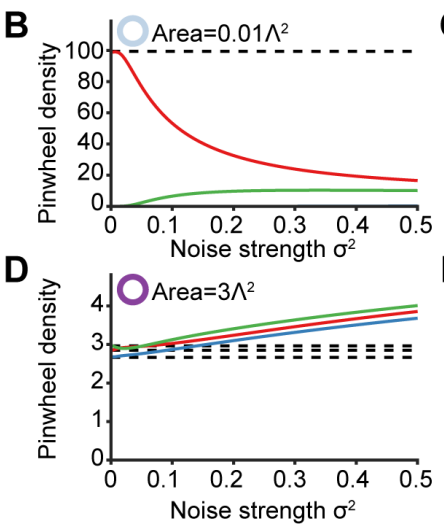
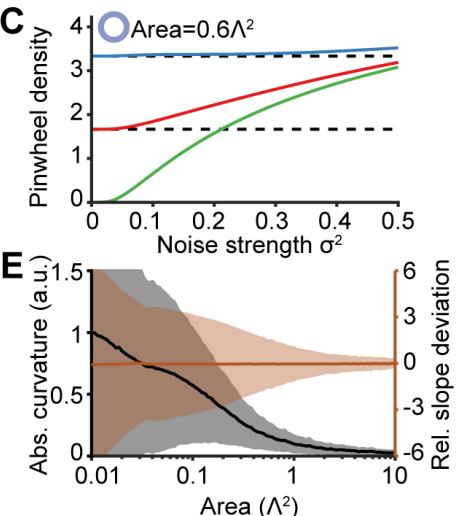

Figure 5.11: Area dependence of pinwheel density in heterogeneous orientation domains. A Quasiperiodic ground truth orientation domain with a pinwheel and a linear zone of low and high progressive phase change in the center of concentric circles of different areas. B, C, D The observed pinwheel densities in areas of different sizes with the pinwheel or one of the linear zones in their center. The four areas correspond to the concentric circles of $\mathbf{A}$ and the last area to the depicted rectangular orientation domain. Line colors correspond to the orientation layout motifs of $\mathbf{A}$. Dashed line marks the ground truth pinwheel density $\rho_{0}$ of that area. E The total absolute curvature (Eq. (5.29)) and relative slope deviation (Eq. (5.30)) of pinwheel densities over the range of noise strengths $\sigma^{2} \leq 1 / 2$ (solid line: mean, shaded area: standard deviation). The curvature of pinwheel density noise scaling decays for increasing areas indicating a linear scaling. The relative slope deviation is on average constant, while its standard deviation decreases with increasing area size (Noise pinwheel density is $\rho_{n}=2 \rho_{0}$ ).

of hexagonal pinwheel crystals and quasiperiodic orientation field is the repertoire of different orientation motifs. Most notably hexagonal pinwheel crystals comprise up to a coordinate rotation and an orientation shift only one distinguished pinwheel, in contrast to a large repertoire of different pinwheels in quasiperiodic orientation fields. This suggests pinwheel heterogeneity as the crucial ingredient for the apparent lack of strong suppression.

In order to dissect the role of heterogeneity on the low noise strength regime, heterogeneity was varied by measuring pinwheel densities in different area sizes, Fig. 5.11A. For small areas around a pinwheel or a linear zone, pinwheel densities are substantially different in small noise regimes due to the presence or absence of a ground truth pinwheel and converge eventually to the noise pinwheel density in the strong noise limit, Fig. 5.11B. Noise artifacts are generically strongly suppressed in low noise regimes, but the extent of these regimes varies depending on the motif, Fig. 5.11C. In particular, the noise artifacts of the second motif of Fig. 5.11A is strongly suppressed over the entire noise range $\sigma^{2}<0.5$, Fig. 5.11B. Increasing the area leads to a convergence of ground truth pinwheel densities to the ground truth pinwheel density of the entire orientation field, Fig. 5.11C, D. The standard deviation of the ground truth pinwheel density scales approximately as $\sigma_{\rho} \propto A^{-\gamma}$ with $\gamma \leq 3 / 4$ except for pathological cases [280, 281]. The scaling depends on the pinwheel configuration and is typically $\gamma=3 / 4$ for pinwheel crystals, $\gamma=1 / 2$ for random pinwheel patterns and $1 / 2<\gamma<3 / 4$ for quasiperiodic pinwheel patterns $[22,278,279]$. The noise artifact scaling becomes linear with noise strength for increasing areas in the regime $\sigma^{2} \leq 0.5$, Fig. $5.11 B, C, D$. The increase in linearity is quantified by the total absolute curvature

$$
\kappa(A)=\int_{0}^{1 / 2} \mathrm{~d} \sigma^{2}\left|\partial_{\sigma^{2}}^{2} \rho\left(\sigma^{2}, A\right)\right|
$$

of observed pinwheel densities $\rho\left(\sigma^{2}, A\right)$ from different areas $A$ with respect to the noise strength 
$\sigma^{2}$ that declines for increasing areas, Fig. 5.11E. The relative deviation of the average slope

$$
m(A)=\int_{0}^{1 / 2} \mathrm{~d} \sigma^{2} \partial_{\sigma^{2}} \rho\left(\sigma^{2}, A\right)
$$

of the pinwheel densities with respect to noise strength is on average constant with decreasing standard deviation. The apparent linear noise artifact scaling of pinwheel densities is hence due to the spatial heterogeneity of orientation fields and is typically a good approximation for areas on the order of $10 \Lambda^{2}$.

\section{Linear noise scaling for Gaussian random orientation fields}

The examined strong suppression of noise artifacts for single orientation motifs culminating in an apparent linear suppression for heterogeneous superpositions of these motifs remain quantitatively paradoxic. In order to elucidate this paradox, we dissect noise artifacts in an exact solvable class of orientation fields, that is Gaussian random fields.

Isotropic and shift-symmetric orientation field ensembles are defined by a single correlation function $C_{1}^{0}(r)$ (see methods for further details). We notice that additive noise leads to an altered correlation function specified by $C_{1}(r)=C_{1}^{0}(r)+C_{1}^{n}(r)$. Denoting the pinwheel density of the ground truth orientation field by $\rho_{0}$ and of the noise field by $\rho_{n}$, the observed pinwheel density becomes (see methods section for further details)

$$
\rho=\rho_{0}+\frac{\sigma^{2}}{1+\sigma^{2}}\left(\rho_{n}-\rho_{0}\right)
$$

For vanishing noise $\sigma \rightarrow 0$ the observed pinwheel density converges to the ground truth pinwheel density $\rho=\rho_{0}$. A lower bound for the pinwheel density $\rho \geq \rho_{0} /\left(1+\sigma^{2}\right)$ is given by a spatially uniform noise offset, that is noise with vanishing pinwheel density $\rho_{n}=0$. In small noise regimes $\sigma^{2} \ll 1$, the observed pinwheel density is approximately given by

$$
\rho=\rho_{0}+\sigma^{2}\left(\rho_{n}-\rho_{0}\right)
$$

revealing the exact linear noise scaling.

We confirmed this result numerically by synthesis of the ECP solution class (Eq. (5.26)) with $l_{j}=(-1)^{j}, n=101$ active modes and random phases $\phi_{j}$ as shown in Fig. 2.6A. For large numbers of active modes $n$, ECPs converge to Gaussian random fields due to the central limit theorem (see methods). The pinwheel observation probability map, Fig. 2.6B, appears qualitatively similar to the high pinwheel density quasiperiodic orientation field of Fig. 5.10B. The pinwheel density, Fig. 5.12C, is again similar to the high pinwheel density quasiperiodic orientation field and fits the analytically predicted pinwheel density from Eq. (5.31). This resemblance to the high pinwheel density quasiperiodic orientation field suggests that the Gaussian random field approximation for noise artifacts is already valid for a few active modes $n \approx 10$.

In small noise regimes, only the vicinity of pinwheel centers should contribute to the pinwheel observation probability. The pinwheel observation probability at a pinwheel center position $\boldsymbol{x}_{i}$ can be derived analytically (see methods) by noting that the amplitude is given by

$$
\Psi_{0}(\boldsymbol{x})=\left(\nabla \Psi_{0}\left(\boldsymbol{x}_{i}\right)\right)^{T}\left(\boldsymbol{x}-\boldsymbol{x}_{i}\right)
$$

and averaging the pinwheel observation probability (Eq. (5.10)) over the distribution of gradients at the pinwheel centers. The pinwheel observation probability map is radially symmetric around pinwheel centers with a long tail that increases with noise strength $\sigma^{2}$, Fig. 5.12D. The amplitude of the pinwheel probability map declines and the full width at half max increases with 
A
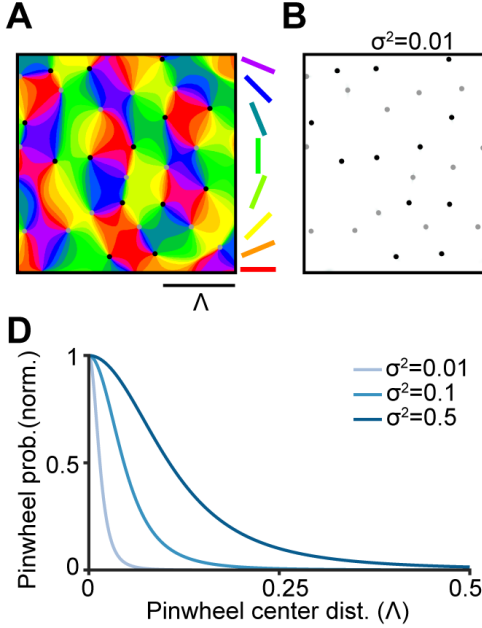

B

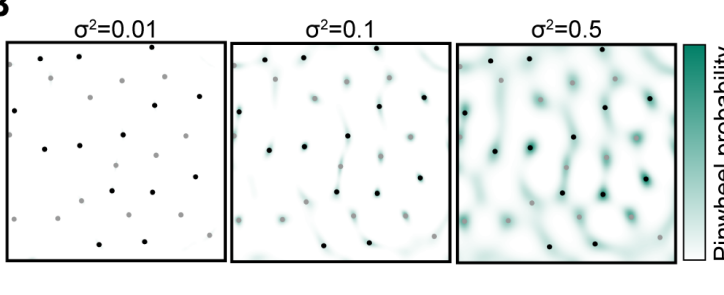

E

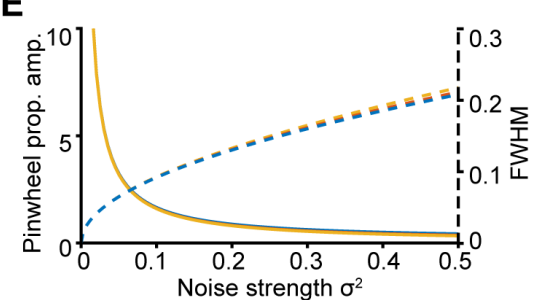

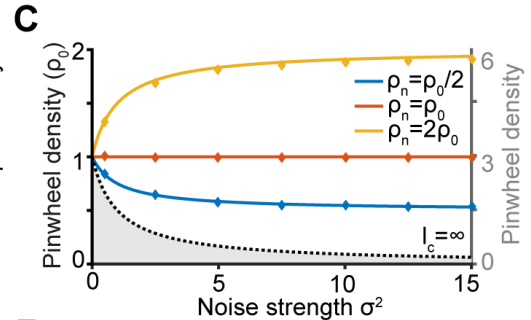

$\mathbf{F}$

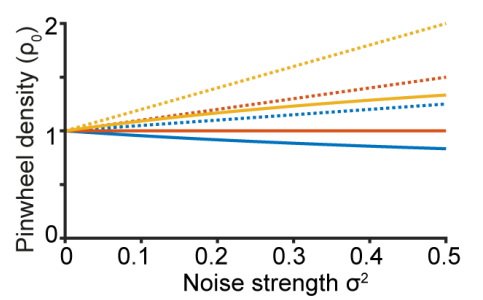

Figure 5.12: Measurement noise in orientation domains with Gaussian selectivity statistics. A An example orientation domain drawn from an isotropic ECP solution (see Eq. (5.26)) with $n=101$ modes. B The pinwheel observation probability for the orientation domain and three different noise strengths with noise pinwheel density $\rho_{n}=\rho_{0}$. C Pinwheel density of observed orientation domains as a function of the noise strength $\sigma^{2}$ and three different noise correlation lengths (solid line: theory see Eq. (5.31), diamonds: numerical results). The lower bound on the pinwheel density is given by $\rho \geq \rho_{0} /\left(1+\sigma^{2}\right)$. D The average pinwheel observation probability profile at a pinwheel center is radially symmetric with a long tail $\left(\rho_{0}=\rho_{n}\right)$. E The average pinwheel observation probability amplitude at the pinwheel center (solid lines) together with the full width at half max (FWHM) of the average pinwheel observation probability at a pinwheel center (dashed lines). Line colors correspond to C. F Pinwheel density (solid line) and linear pinwheel approximation (dotted lines) for small noise strengths.

noise strength, Fig. 5.12E, respectively. The total number of observed pinwheels per ground truth pinwheel was derived earlier and given by Eq. (5.17). For isotropic shift-symmetric Gaussian random fields the joint distribution of the anisotropy $a \geq 1$ and selectivity $s^{2}$ at pinwheel centers can be derived (see methods) and yields

$$
P\left(s^{2}, a\right)=\frac{a\left(a^{2}-1\right) s^{4}}{2 \pi^{3} \rho_{0}^{3}} e^{-\frac{1+a^{2}}{2 \pi \rho_{0}} s^{2}} .
$$

The average number of observed pinwheels at a ground truth pinwheel is given by the average of Eq. (5.17) leading to the pinwheel density

$$
\rho=\rho_{0}\langle N\rangle=\rho_{0}+\sigma^{2} \rho_{n}
$$

and is depicted in Fig. 5.12E. This shows that the generation of spurious pinwheels near ground truth pinwheel centers is an upper bound on the pinwheel density for Gaussian random fields in the small noise regime. However, the linear approximation does not account for noise hidden pinwheels in low noise regimes. In total, the paradoxical linear noise scaling can be attributed partially to the heterogeneity of isolated and linear approximated pinwheels for small noise strengths. Pinwheels that are hidden by noise are presumably due to the non-linear orientation field in the vicinity of pinwheels.

\section{Strong pinwheel suppression in high orientation selectivity layouts}

Previously, orientation selectivity varied across the visual cortex reminiscent of intrinsic signal imaging that measures mostly signals correlated to membrane potential changes [346]. However, 

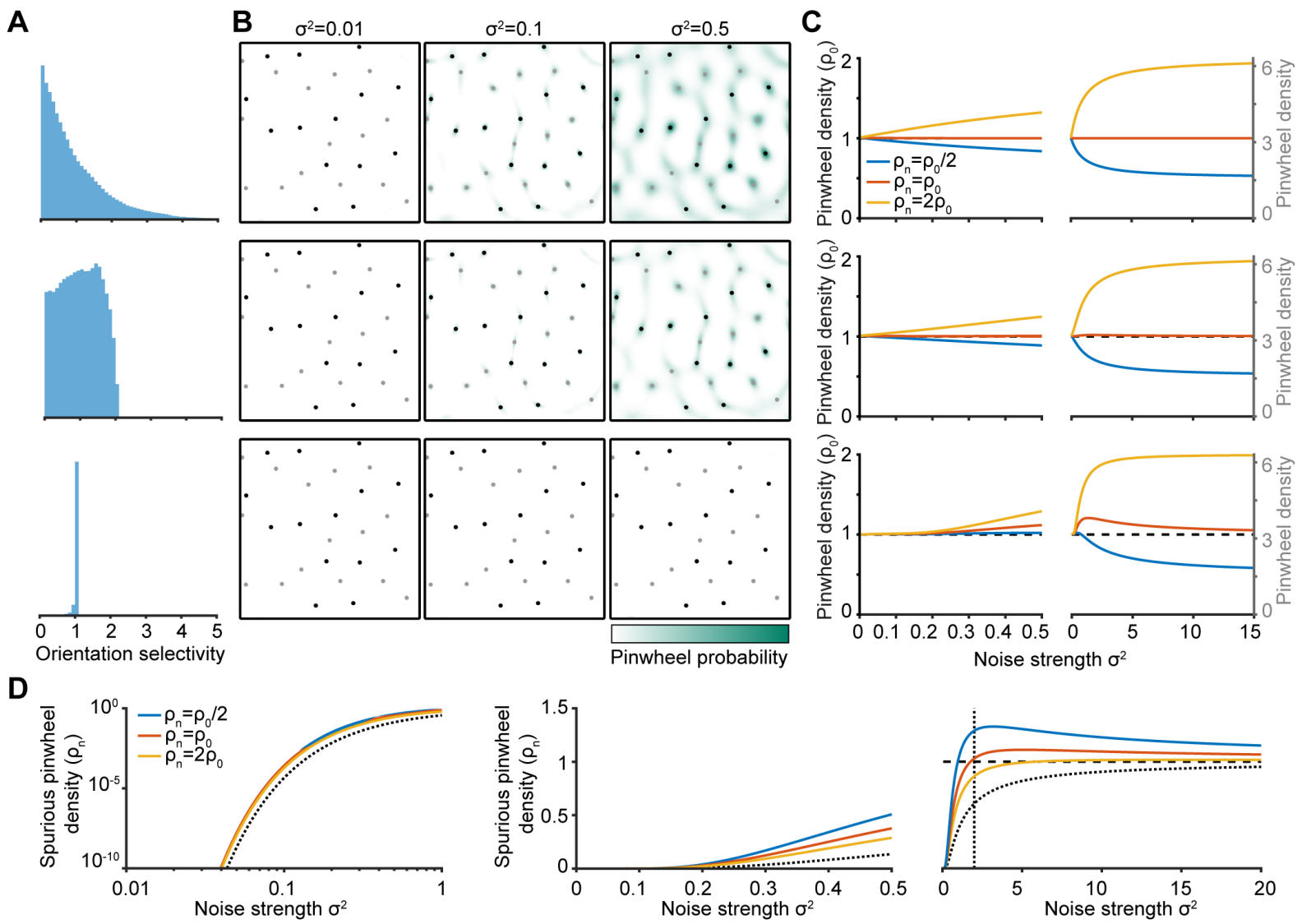

Figure 5.13: Strong pinwheel suppression in high orientation selectivity layouts. A Orientation selectivity distribution of orientation domain layout in Fig. 5.12A that is modified by Eq. (5.36) to increase orientation selectivity globally (upper: $\lambda=0.1$, middle: $\lambda=1$, lower: $\lambda=5$ ). B Pinwheel observation probability maps corresponding to orientation selectivity distribution from $\mathbf{A}$ for three different noise strengths with noise pinwheel density $\rho_{n}=\rho_{0}$. Pinwheel observation probability localize stronger around pinwheel centers for increasing $\lambda$. $\mathbf{C}$ Pinwheel densities corresponding to the rows of $\mathbf{A}$ and $\mathbf{B}$ show a stronger suppression of spurious pinwheels and pinwheels hidden by noise for larger $\lambda$. $\mathbf{D}$ In the limit of uniform orientation selectivity, spurious pinwheel generation is given by Eq. (5.38) and shows a strong suppression of spurious pinwheels similar to the linear zones shown in Fig. 5.6. The vertical dashed line indicates the peak of spurious pinwheels in the limit of small noise pinwheel densities. The dashed horizontal curve shows the limiting spurious pinwheel density for high noise pinwheel densities.

the spiking activities of neurons are sharply tuned at pinwheels and their selectivity is statistically indistinguishable from iso-orientation domains [71, 355-357], but see [358]. What effect does a constant orientation selectivity have on the pinwheel observation probability?

The transition to uniform distributions of orientation selectivity is examined on the orientation layout $\Psi_{0}(\boldsymbol{x})$ of the previous section, Fig. 5.12. The orientation selectivity is altered by an orientation selectivity strength parameter $\lambda$

$$
\Psi_{0}^{\prime}(\boldsymbol{x})=\frac{1}{\lambda} \tanh \left(\lambda\left|\Psi_{0}(\boldsymbol{x})\right|\right) e^{i \arg \Psi_{0}(\boldsymbol{x})}
$$

and subsequently normalized leading to an orientation selectivity distribution shown in Fig. 5.13A. Thus for $\lambda \rightarrow 0$ orientation domains are given by $\Psi_{0}^{\prime}(\boldsymbol{x})=\Psi_{0}(\boldsymbol{x})$ and for $\lambda \rightarrow \infty$ by $\Psi_{0}^{\prime}(\boldsymbol{x})=\Theta\left(\left|\Psi_{0}(\boldsymbol{x})\right|\right) e^{i \arg \Psi_{0}(\boldsymbol{x})}$ with $\Theta(\cdot)$ denoting the Heaviside function. The pinwheel observation probability map localizes to pinwheel centers for increasing orientation selectivity, Fig. 5.13B. This results in a stronger suppression for small noise regimes, Fig. 5.13C. 
In the limiting regime of constant orientation selectivity $\Psi_{0}^{\prime}(\boldsymbol{x})=\Theta\left(\left|\Psi_{0}(\boldsymbol{x})\right|\right) e^{i \vartheta(\boldsymbol{x})}$, the pinwheel observation probability in pinwheel free regions is given by

$$
\begin{aligned}
\rho(\boldsymbol{x})= & \rho_{n} e^{-\frac{1}{\sigma^{2}}} e^{-\frac{\|\nabla \vartheta(\boldsymbol{x})\|^{2}}{4 \pi \sigma^{2} \rho_{n}}} \\
& \left(I_{0}\left(\frac{\|\nabla \vartheta(\boldsymbol{x})\|^{2}}{4 \pi \sigma^{2} \rho_{n}}\right)+\frac{\|\nabla \vartheta(\boldsymbol{x})\|^{2}}{2 \pi \sigma^{2} \rho_{n}}\left(I_{0}\left(\frac{\|\nabla \vartheta(\boldsymbol{x})\|^{2}}{4 \pi \sigma^{2} \rho_{n}}\right)+I_{1}\left(\frac{\|\nabla \vartheta(\boldsymbol{x})\|^{2}}{4 \pi \sigma^{2} \rho_{n}}\right)\right)\right)
\end{aligned}
$$

with the noise pinwheel density $\rho_{n}$. As the pinwheel observation probability is monotonically increasing with $\|\nabla \vartheta(\boldsymbol{x})\|$, it is particularly noise sensitive in the vicinity of phase singularities, i.e. pinwheels. The average over the phase gradient can be computed analytically (see methods) and gives

$$
\begin{aligned}
& \rho=\rho_{n} e^{-\frac{1}{\sigma^{2}}} \\
& \left(\frac{1}{\sqrt{\pi}} \frac{\sigma^{2} \rho_{n}}{\rho_{0}} G_{2,3}^{2,2}\left(\frac{\rho_{0}}{\sigma^{2} \rho_{n}} \mid \begin{array}{c}
1, \frac{5}{2} \\
2,2,2
\end{array}\right)+\frac{\sqrt{\pi}}{2}\left(1+\frac{\rho_{0}}{\sigma^{2} \rho_{n}}\right) U\left(\frac{1}{2}, 0, \frac{\rho_{0}}{\sigma^{2} \rho_{n}}\right)\right)
\end{aligned}
$$

with the ground truth pinwheel density $\rho_{0}, G$ denoting the Meijer $\mathrm{G}$ function and $U$ the confluent hypergeometric function. Spurious pinwheels are thus strongly suppressed by spatially uniform orientation selectivity in orientation domains, Fig. 5.13D, similar to the linear zone motif, Fig. 5.6.

\subsection{Discussion}

In this article, we presented the theoretical significance of pinwheel configurations, developed an analytical approach of their susceptibility to measurement noise and examined different orientation layouts under measurement noise. We identified three distinct noise effects on pinwheels: displacement of pinwheels, pinwheels hidden by noise and the generation of spurious pinwheels. In small noise regimes, we found strong suppression of spurious and noise hidden pinwheels for pinwheel free orientation layouts, single pinwheels, pinwheel pairs, crystalline orientation layouts and orientation layouts with constant selectivity. In contrast, pinwheel densities of quasiperiodic orientation domains scaled virtually linear in small noise regimes of strength $\sigma^{2}<0.2$ and noise pinwheel densities that are similar to the ground truth pinwheel density. Finally, orientation domains composed of infinitely many independent random active modes revealed an exact linear scaling of pinwheel density in small noise regimes.

The observed linear scaling behavior in small noise regimes allows to improve the pinwheel density analysis of experimentally observed orientation domains [22-24] by an extrapolation to the zero noise limit. In this analysis, orientation domains are bandpass filtered such that all spatial noise frequencies except for frequencies similar to the ground truth signal are removed. The remaining noise has thus a similar pinwheel density as the ground truth signal and a noise strength on the order of $\sigma^{2}=0.2$. Although strong suppression of spurious and noise hidden pinwheels in principle generalizes to arbitrary finite domains as only ground truth pinwheel centers contribute in the small noise limit, already simple quasiperiodic orientation domains scale virtually linear in small noise regimes of strength $\sigma^{2}<0.2$ and noise pinwheel densities that are similar to the ground truth pinwheel density. The range of linear scaling is even larger for isotropic layouts that are more similar to experimentally observed layouts. This approximately linear behavior is due to the diversity of spatial orientation selectivity organization and holds for cortical area sizes on the order of $10 \Lambda^{2}$ and sufficiently quasiperiodic orientation domains, Fig. 5.11B. Thus, for sufficiently large, bandpass filtered orientation domains a zero noise limit extrapolation can be achieved by: (i) Estimating pinwheel densities of bandpass filtered orientation domains from subsets of stimulus repetitions. (ii) Plotting estimated pinwheel densities 
against the inverse number of stimulus repetitions $1 / N$ (this scales with $\sigma^{2}$ for independent noise). (iii) Linearly extrapolate the pinwheel density to obtain the zero noise limit $1 / N \rightarrow 0$.

This theory sheds light on the confidence regions of pinwheel positions under additive noise and thus contributes to an improved benchmark scheme for models of orientation domains. Generically, pinwheel observation probability scales with the noise pinwheel density $\rho_{n}$, with the scaled gradient $l_{c}\left\|\nabla \Psi_{0}\right\| / \sigma$ and orthogonality measure $l_{c}^{2}\left|\nu_{0}\right| / \sigma^{2}$ and inversely with the scaled orientation selectivity $\left|\Psi_{0}\right| / \sigma$. Thus pinwheels observed in low selectivity regions and high gradients should be handled with care, whereas pinwheels surrounded by high selectivity regions should be trusted the most. The confidence regions of pinwheel positions can be quantitatively approximated by calculating the mean orientation domain of a given set of stimulus repetition recordings, estimate noise strength and correlation length by either bootstrapping or similar techniques and finally use Eq. (5.10) to calculate the pinwheel observation probability map.

The strong suppression of spurious and noise hidden pinwheels for spatially uniform orientation selectivity domains suggests the recording of activity responses to obtain precise pinwheel configurations. Conventional intrinsic signal imaging allows us to image large cortical areas, but suffers from signal recordings that are rather related to the membrane potential than to neuronal activity [346]. However, the membrane potential shows only weak selectivity in the vicinity of pinwheel centers, whereas orientation selectivity measured by neuronal activity showed statistically indistinguishable selectivity in iso-orientation domains and in the vicinity of pinwheel centers [71, 355, 356], but see [358]. Epi-florescence imaging of neurons expressing fluorescent dyes such as GCaMP6 offers an alternative that overcomes this noise sensitivity.

Apart from the theoretical significance of the pinwheel configuration, the precise determination of their position provides additionally robust anchors for a chronic comparison of orientation layouts. In [147] pinwheels are predicted to annihilate and therefore move during development. An experimental confirmation of this prediction requires a profound understanding of the noise impact on pinwheel positions. In total, precise pinwheel configurations are the first step of introducing a benchmark system for the development of quantitative models of a paradigm canonical circuit.

\subsection{Methods}

\section{Pinwheel observation probability map for a general Gaussian ensemble}

The layout of orientation domains can be completely specified by a complex field $\Psi(\boldsymbol{x})=\xi(\boldsymbol{x})+$ $i \eta(\boldsymbol{x})$ with $\boldsymbol{x}=(x, y)^{T}$. The total number of pinwheel centers $\boldsymbol{x}_{i}$ in an area $A$ is given by the spatial integral over

$$
\sum_{i} \delta\left(\boldsymbol{x}-\boldsymbol{x}_{i}\right)=\delta(\eta(\boldsymbol{x})) \delta(\xi(\boldsymbol{x}))\left|\operatorname{det} \frac{\partial(\eta(\boldsymbol{x}), \xi(\boldsymbol{x}))}{\partial(x, y)}\right| .
$$

For the sake of brevity, the dependence on the spatial position will be omitted in the following. If the complex field has a random component, the pinwheel observation probability is given by the ensemble average $\langle\cdot\rangle$ over the pinwheel center positions

$$
\rho=\left\langle\delta(\xi) \delta(\eta)\left|\xi_{x} \eta_{y}-\xi_{y} \eta_{x}\right|\right\rangle
$$

where derivatives are abbreviated by $\partial_{x} \xi=\xi_{x}$. A convenient notation for the pinwheel density can be achieved by introducing the matrix

$$
\boldsymbol{A}=\left(\begin{array}{cc}
0 & 0 \\
0 & \boldsymbol{A}_{0}
\end{array}\right) \in \mathbb{R}^{6 \times 6}, \quad \boldsymbol{A}_{0}=\left(\begin{array}{cccc}
0 & 0 & 0 & 1 / 2 \\
0 & 0 & -1 / 2 & 0 \\
0 & -1 / 2 & 0 & 0 \\
1 / 2 & 0 & 0 & 0
\end{array}\right)
$$


that allows to rewrite the averaging term as $\left|\xi_{x} \eta_{y}-\xi_{y} \eta_{y}\right|=\left|\chi^{T} \boldsymbol{A} \boldsymbol{\chi}\right|$ with the vector $\boldsymbol{\chi}=$ $\left(\xi, \eta, \xi_{x}, \xi_{y}, \eta_{x}, \eta_{y}\right)^{T}$. The pinwheel observation probability can thus be written as

$$
\rho=\frac{1}{\pi} \int \frac{\mathrm{d} t}{t^{2}}\left(T(0)-\frac{T(t)+T(-t)}{2}\right) \quad \text { with } \quad T(t)=\left\langle\delta(\xi) \delta(\eta) e^{i t \boldsymbol{\chi}^{T} \boldsymbol{A} \boldsymbol{\chi}}\right\rangle .
$$

Evaluation of the average requires the knowledge of the ensemble probability density function $P(\chi)$. The least structured probability density function $P(\chi)$ that fixes a set of statistical quantities is the maximum entropy ensemble [141, 142]. For fixed one- and two-point correlation functions $\boldsymbol{\mu}=\langle\boldsymbol{\chi}(\boldsymbol{x})\rangle$ and $\boldsymbol{\Sigma}=\left\langle\delta \boldsymbol{\chi}(\boldsymbol{x}) \delta \boldsymbol{\chi}(\boldsymbol{x})^{T}\right\rangle$ it is given by a Gaussian multivariate distribution

$$
P(\chi)=\frac{1}{\sqrt{(2 \pi)^{6} \operatorname{det} \Sigma}} e^{-\frac{1}{2}(\chi-\mu)^{T} \Sigma^{-1}(\chi-\mu)}
$$

with at most 27 parameters. The ensemble average is then a simple Gaussian integral that can be calculated [277] by introducing $\boldsymbol{C}=\left(\boldsymbol{\Sigma}^{-1}\right)_{i>2, j>2}$ and $\boldsymbol{\mu}_{0}=(\boldsymbol{\mu})_{i>2}$, which gives

$$
T( \pm t)=\frac{e^{-\frac{1}{2} \boldsymbol{\mu}^{T} \boldsymbol{\Sigma}^{-1} \boldsymbol{\mu}}}{2 \pi} \sqrt{\frac{\operatorname{det} \boldsymbol{\Sigma}^{-1}}{\operatorname{det} \boldsymbol{C}}} \frac{e^{\frac{1}{2} \boldsymbol{\mu}_{0}^{T} \boldsymbol{M}_{\mp}^{-1}(t) \boldsymbol{C} \boldsymbol{\mu}_{0}}}{\sqrt{\operatorname{det} \boldsymbol{M}_{\mp}(t)}}
$$

with $\boldsymbol{M}_{ \pm}(t)=\mathbf{1} \pm 2 i t \boldsymbol{C}^{-1} \boldsymbol{A}_{0}$. This leads to the most general solution of the pinwheel observation probability of Gaussian field ensembles

$$
\rho=\frac{e^{-\frac{1}{2} \boldsymbol{\mu}^{T} \boldsymbol{\Sigma}^{-1} \boldsymbol{\mu}}}{2 \pi^{2}} \sqrt{\frac{\operatorname{det} \boldsymbol{\Sigma}^{-1}}{\operatorname{det} \boldsymbol{C}}} \int \frac{\mathrm{d} t}{t^{2}}\left(e^{\frac{1}{2} \boldsymbol{\mu}_{0}^{T} \boldsymbol{C} \boldsymbol{\mu}_{0}}-\frac{e^{\frac{1}{2} \boldsymbol{\mu}_{0}^{T} \boldsymbol{M}_{+}^{-1}(t) \boldsymbol{C} \boldsymbol{\mu}_{0}}}{2 \sqrt{\operatorname{det} \boldsymbol{M}_{+}(t)}}-\frac{e^{\frac{1}{2} \boldsymbol{\mu}_{0}^{T} \boldsymbol{M}_{-}^{-1}(t) \boldsymbol{C} \boldsymbol{\mu}_{0}}}{2 \sqrt{\operatorname{det} \boldsymbol{M}_{-}(t)}}\right) .
$$

The pinwheel observation probability reduces drastically in complexity by assuming a ground truth orientation field with a rotation-, translation- and shift-symmetric additive noise field. The mean of the multivariate Gaussian distribution is then equal to the ground truth orientation field and the covariance matrix is given by

$$
\boldsymbol{\Sigma}=\operatorname{diag}\left(c_{A}, c_{A}, c_{G}, c_{G}, c_{G}, c_{G}\right)
$$

where $c_{A}=C_{1}(0) / 2$ is the noise strength and $c_{G}=-\Delta C_{1}(0) / 4$ the noise correlation decay. The pinwheel observation probability is given by

$$
\rho=\frac{c_{G}}{2 \pi c_{A}} e^{-\frac{\left|\Psi_{0}\right|^{2}}{2 c_{A}}} \frac{1}{\pi} \int \frac{\mathrm{d} t}{t^{2}}\left(1-e^{-\frac{t^{2}}{t^{2}+1} \frac{\left\|\nabla \Psi_{0}\right\|^{2}}{2 c_{G}}} \cos \left(\frac{t}{t^{2}+1} \frac{\nu_{0}}{c_{G}}\right) /\left(t^{2}+1\right)\right),
$$

with $\nu_{0}=\left|\partial_{x} \xi_{0} \partial_{y} \eta_{0}-\partial_{x} \eta_{0} \partial_{y} \xi_{0}\right|$ the orthogonality between $\nabla \xi_{0}$ and $\nabla \eta_{0}$ given by the area of their spanned parallelogram. A small noise approximation is given by

$$
\rho^{\mathrm{SN}}=\frac{e^{-\frac{\left|\Psi_{0}\right|^{2}}{2 c_{A}}}}{2 \pi c_{A}}\left(\nu_{0} \operatorname{erf}\left(\frac{\nu_{0}}{\sqrt{2 c_{G}\left\|\nabla \Psi_{0}\right\|^{2}}}\right)+\frac{1}{\sqrt{\pi}} e^{-\frac{\nu 0^{2}}{2 c_{G}\left\|\nabla \Psi_{0}\right\|^{2}}} \sqrt{2 c_{G}\left\|\nabla \Psi_{0}\right\|^{2}}\right),
$$

where terms of order $\mathcal{O}\left(\xi_{n}^{2}\right)$ and $\mathcal{O}\left(\eta_{n}^{2}\right)$ and their derivatives in $\left|\xi_{x} \eta_{y}-\xi_{y} \eta_{x}\right|$ are neglected during the derivation.

In the case of vanishing orthogonality $\nu_{0}=0$, the integral can be solved analytically. Using the abbreviation $a=\frac{\left\|\nabla \Psi_{0}\right\|^{2}}{2 c_{G}}$ and transforming the coordinate of the integral in Eq. (5.47) by 
$t=\tan u$ leads to

$$
\begin{aligned}
\frac{2 \pi \rho c_{A}}{c_{G}} e^{\frac{\left|\Psi_{0}\right|^{2}}{2 c_{A}}} & =\int_{-\pi / 2}^{\pi / 2} \frac{\mathrm{d} u}{\pi} \frac{1}{\sin ^{2} u}\left(1-e^{-a \sin ^{2} u} \cos ^{2} u\right) \\
& =\left(1+\partial_{a}\right) \int_{-\pi / 2}^{\pi / 2} \frac{\mathrm{d} u}{\pi} \frac{1}{\sin ^{2} u}\left(1-e^{-a \sin ^{2} u}\right) \\
& =\left(1+\partial_{a}\right) \int_{0}^{a} \mathrm{~d} x \int_{-\pi / 2}^{\pi / 2} \frac{\mathrm{d} u}{\pi} e^{-x \sin ^{2} u} \\
& =\left(1+\partial_{a}\right) \int_{0}^{a} \mathrm{~d} x e^{-x / 2} I_{0}\left(\frac{x}{2}\right) \\
& =e^{-\frac{a}{2}}\left(I_{0}\left(\frac{a}{2}\right)+a\left(I_{0}\left(\frac{a}{2}\right)+I_{1}\left(\frac{a}{2}\right)\right)\right),
\end{aligned}
$$

where $I_{l}$ denotes the modified Bessel function of the $l$ th kind. The pinwheel observation probability for vanishing orthogonality is hence given by

$$
\begin{aligned}
\rho^{\nu_{0}=0}= & \frac{c_{G}}{2 \pi c_{A}} e^{-\frac{\left|\Psi_{0}\right|^{2}}{2 c_{A}}} e^{-\frac{\left\|\nabla \Psi_{0}\right\|^{2}}{4 c_{G}}} \\
& \cdot\left(I_{0}\left(\frac{\left\|\nabla \Psi_{0}\right\|^{2}}{4 c_{G}}\right)+\frac{\left\|\nabla \Psi_{0}\right\|^{2}}{2 c_{G}}\left(I_{0}\left(\frac{\left\|\nabla \Psi_{0}\right\|^{2}}{4 c_{G}}\right)+I_{1}\left(\frac{\left\|\nabla \Psi_{0}\right\|^{2}}{4 c_{G}}\right)\right)\right) .
\end{aligned}
$$

\section{Pinwheel creation/annihilation rate}

The event of a spurious pinwheel generation and a ground truth pinwheel that is hidden by noise corresponds to pinwheel creation and annihilation events of pinwheel pairs, respectively. In the following, this rate will be derived in the small noise approximation for spatio-temporal measurement noise

$$
\Psi(\boldsymbol{x}, t)=\Psi_{0}(\boldsymbol{x})+\Psi_{n}(\boldsymbol{x}, t) .
$$

At points where pinwheels create or annihilate, the real and imaginary part of the orientation field must vanish and the gradients of the real and imaginary part must be co-linear, i.e. the orthogonality measure $\nu=\left|\partial_{x} \xi \partial_{y} \eta-\partial_{y} \xi \partial_{x} \eta\right|$ must vanish as illustrated in Fig. 5.14. Thus, the number of creation/annihilation events at position $\boldsymbol{x}_{i}$ and times $t_{j}$ is given by the integral over

$$
\sum_{i, j} \delta\left(\boldsymbol{x}-\boldsymbol{x}_{i}\right) \delta\left(t-t_{j}\right)=\delta(\eta(\boldsymbol{x}, t)) \delta(\xi(\boldsymbol{x}, t)) \delta(\nu(\boldsymbol{x}, t))\left|\operatorname{det} \frac{\partial(\eta(\boldsymbol{x}, t), \xi(\boldsymbol{x}, t), \nu(\boldsymbol{x}, t))}{\partial(x, y, t)}\right|,
$$

where the determinant serves as a normalization factor. Notice that the normalization term is of quartic order in the noise field components in contrast to the quadratic order of the normalization term of the pinwheel observation probability. The spatio-temporal dependence of the field components will be omitted in the following for the sake of brevity. The pinwheel creation/annihilation rate $\rho_{\mathrm{c} / \mathrm{a}}(\boldsymbol{x})$ is given by the expectation over the noise field ensemble

$$
\rho_{\mathrm{c} / \mathrm{a}}(\boldsymbol{x}, t)=\left\langle\delta(\eta) \delta(\xi) \delta(\nu)\left|\operatorname{det} \frac{\partial(\eta, \xi, \nu)}{\partial(x, y, t)}\right|\right\rangle .
$$

The relevant noise field components appearing in this formula are

$$
\begin{aligned}
\chi^{T} & =\left(\xi_{n}, \partial_{x} \xi_{n}, \partial_{y} \xi_{n}, \partial_{t} \xi_{n}, \partial_{x} \partial_{t} \xi_{n}, \partial_{y} \partial_{t} \xi_{n}\right) \\
\boldsymbol{\nu}^{T} & =\left(\eta_{n}, \partial_{x} \eta_{n}, \partial_{y} \eta_{n}, \partial_{t} \eta_{n}, \partial_{x} \partial_{t} \eta_{n}, \partial_{y} \partial_{t} \eta_{n}\right) .
\end{aligned}
$$


A

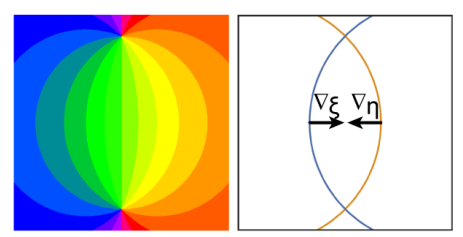

B

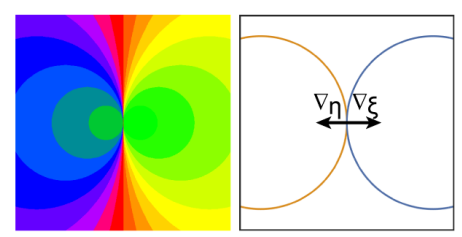

C

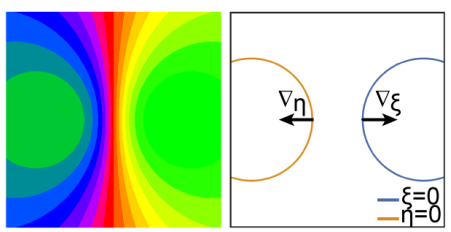

Figure 5.14: Normal form of two oppositely charged pinwheels that annihilate/create.A Separated pinwheels of opposite topological charges are depicted and defined by the intersections of the contour lines of vanishing real and imaginary amplitude. B At the creation/annihilation event, the zero-amplitude contour lines meet in a single point that is equivalent to the gradients of the real and imaginary part to be co-linear. These events are counted by the creation/annihilation rate in Eq. (5.53). C Pinwheels are annihilated and zero-amplitude contour lines have no intersection.

The noise field ensemble is chosen to be isotropic in space, translation invariant in time and shift-symmetric that leads to the maximum entropy distribution of

$$
p(\boldsymbol{\chi}, \boldsymbol{\nu})=\frac{1}{\sqrt{(2 \pi)^{12} \operatorname{det} \boldsymbol{\Sigma}}} \exp \left(-\frac{1}{2}(\boldsymbol{\chi}, \boldsymbol{\nu})^{T} \boldsymbol{\Sigma}^{-1}(\boldsymbol{\chi}, \boldsymbol{\nu})\right)
$$

with the covariance matrix

$$
\boldsymbol{\Sigma}=\operatorname{diag}\left(c_{A}, c_{G}, c_{G}, c_{C}, c_{T}, c_{T}, c_{A}, c_{G}, c_{G}, c_{C}, c_{T}, c_{T}\right)
$$

and the parameters

$$
c_{A}=\frac{1}{2} C(0), \quad c_{G}=-\frac{1}{4} \Delta C(0), \quad c_{C}=-\frac{1}{2} \partial_{t}^{2} C(0), \quad c_{T}=\frac{1}{4} \Delta \partial_{t}^{2} C(0)
$$

with the 2-point correlation function $C(\boldsymbol{x}, t)=\left\langle\Psi_{n}(0,0) \bar{\Psi}_{n}(\boldsymbol{x}, t)\right\rangle$. Due to the translation invariance in time, the pinwheel creation/annihilation rate is stationary in time and thus only depends on the spatial position $\rho_{c / a}(\boldsymbol{x})$. The exact pinwheel creation/annihilation rate eluded a direct calculation and therefore a small noise approximation will be derived here. For that, the orthogonality measure can be expanded and approximated by

$$
\begin{aligned}
\nu= & \left|\partial_{x} \xi \partial_{y} \eta-\partial_{y} \xi \partial_{x} \eta\right| \\
= & \mid \underbrace{\partial_{x} \xi_{0} \partial_{y} \eta_{0}-\partial_{y} \xi_{0} \partial_{x} \eta_{0}}_{=\nu_{0}}+\underbrace{\partial_{x} \xi_{0} \partial_{y} \eta_{n}-\partial_{y} \xi_{0} \partial_{x} \eta_{n}+\partial_{x} \xi_{n} \partial_{y} \eta_{0}-\partial_{y} \xi_{n} \partial_{x} \eta_{0}}_{=\omega} \\
& +\underbrace{\partial_{x} \xi_{n} \partial_{y} \eta_{n}-\partial_{y} \xi_{n} \partial_{x} \eta_{n}}_{=\nu_{n}} \mid \\
\doteq & \left|\nu_{0}+\omega\right|,
\end{aligned}
$$

where in the last expression all terms of quadratic order or higher in the noise field $\mathcal{O}\left(\xi_{n}^{2}\right)$ and $\mathcal{O}\left(\eta_{n}^{2}\right)$ are neglected. The normalization factor simplifies in the small noise regime to

$$
\begin{aligned}
\left|\operatorname{det} \frac{\partial(\eta, \xi, \nu)}{\partial(x, y, t)}\right| & =\left|\nu \partial_{t} \nu+\partial_{t} \xi_{n} \operatorname{det}\left(\begin{array}{cc}
\partial_{x} \eta & \partial_{x} \nu \\
\partial_{y} \eta & \partial_{y} \nu
\end{array}\right)-\partial_{t} \eta_{n} \operatorname{det}\left(\begin{array}{cc}
\partial_{x} \xi & \partial_{x} \nu \\
\partial_{y} \xi & \partial_{y} \nu
\end{array}\right)\right| \\
& \doteq|\nu_{0} \partial_{t} \omega+\partial_{t} \xi_{n} \underbrace{\left(\partial_{x} \eta_{0} \partial_{y} \nu_{0}-\partial_{y} \eta_{0} \partial_{x} \nu_{0}\right)}_{=\alpha}-\partial_{t} \eta_{n} \underbrace{\left(\partial_{x} \xi_{0} \partial_{y} \nu_{0}-\partial_{y} \xi_{0} \partial_{x} \nu_{0}\right)}_{=\beta}| \\
& =\left|\nu_{0} \partial_{t} \omega+\alpha \partial_{t} \xi_{n}-\beta \partial_{t} \eta_{n}\right| .
\end{aligned}
$$


With this small noise approximation, the argument of the pinwheel creation/annihilation rate is linear in the random noise field

$$
\rho_{\mathrm{c} / \mathrm{a}}^{\mathrm{SN}}(\boldsymbol{x})=\left\langle\delta(\eta) \delta(\xi) \delta\left(\nu_{0}+\omega\right)\left|\nu_{0} \partial_{t} \omega+\alpha \partial_{t} \xi_{n}-\beta \partial_{t} \eta_{n}\right|\right\rangle,
$$

allowing a direct evaluation with the average over the probability density function to

$$
\begin{aligned}
\rho_{\mathrm{c} / \mathrm{a}}^{\mathrm{SN}}(\boldsymbol{x})= & \sqrt{\frac{2}{\pi} \sqrt{c_{C}\left(\alpha(\boldsymbol{x})^{2}+\beta(\boldsymbol{x})^{2}\right)+c_{T} \nu_{0}(\boldsymbol{x})^{2}\left|\nabla \Psi_{0}(\boldsymbol{x})\right|^{2}}} \\
& \frac{1}{\sqrt{2 \pi c_{G}\left|\nabla \Psi_{0}(\boldsymbol{x})\right|^{2}}} e^{-\frac{\nu_{0}(\boldsymbol{x})^{2}}{2 c_{G}\left|\nabla \Psi_{0}(\boldsymbol{x})\right|^{2}}} \frac{1}{2 \pi c_{A}} e^{-\frac{\left|\Psi_{0}(\boldsymbol{x})\right|^{2}}{2 c_{A}}} .
\end{aligned}
$$

The pinwheel creation/annihilation rate is positive and measured in units of one over area and time as expected, since it counts creation/annihilation events per area and time. Notice the similarity to the small noise approximation of the pinwheel observation probability of Eq. (5.48).

\section{Pinwheel density of orientation domain ensembles}

Dynamically generated orientation domains in the linear regime are Gaussian random fields [359] and can be parameterized by the model of [171]. After the transient linear regime nonlinearities select specifically organized orientation domains such as essentially complex planforms (ECP) $[22,128]$ or circular phase progression (CPP) solutions [137]. The noise effect on the pinwheel density can be computed exactly for the transient regime and approximated for ECPs and CPPs in the limit of many modes.

In the transient linear regime, isotropic and shift-symmetric orientation domain ensembles can be parametrized by their power-spectral density width. A useful model ensemble is defined by a parameter $\beta$ inversely scaling with power-spectral density width and the correlation function

$$
C_{1}^{0}(r)={ }_{1} F_{1}\left(\frac{2+\beta}{2} ; 1 ;-\frac{r^{2}}{4 B}\right) \quad \text { with } \quad B=\Gamma\left(\frac{2+\beta}{2}\right)^{2} / \Gamma\left(\frac{1+\beta}{2}\right)^{2} .
$$

The ECP solution class is defined by $n$ equidistantly placed plane waves on a semi-circle in Fourier space $\boldsymbol{k}_{j}=(\cos (j \pi / n), \sin (j \pi / n))^{T}$ with random phases $\phi_{j}$ and binary spin-like variables $l_{j} \in\{-1,1\}$. The normalized orientation domains and their correlation function are given by

$$
\Psi_{0}(\boldsymbol{x})=\frac{1}{\sqrt{n}} \sum_{j=0}^{n-1} e^{i\left(l_{j} \boldsymbol{k}_{j} \boldsymbol{x}+\phi_{j}\right)} \quad \text { and } \quad C_{1}^{0}(\boldsymbol{x})=\frac{1}{n} \sum_{j=0}^{n-1} e^{i l_{j} \boldsymbol{k}_{j} \boldsymbol{x}} .
$$

The CPP solution set is defined by the superposition of $2 n$ equidistantly placed plane waves on a circle in Fourier space with random phases $\phi_{j}$ and the phase progression of $\theta_{j}=\pi j d / n+\vartheta_{0}$ with $d \in\{0,1, \ldots, n-1\}$. The normalized orientation domains and their correlation function are given by

$$
\Psi_{0}(\boldsymbol{x})=\frac{1}{\sqrt{n}} \sum_{j=0}^{n-1} 2 e^{i \theta_{j}} \cos \left(\boldsymbol{k}_{j} \boldsymbol{x}+\phi_{j}\right) \quad \text { and } \quad C_{1}^{0}(\boldsymbol{x})=\frac{1}{n} \sum_{j=0}^{n-1} \cos \left(\boldsymbol{k}_{j} \boldsymbol{x}\right) .
$$

The number of pinwheels per typical scale area $\Lambda^{2}=(2 \pi)^{2}$ of the transient random field and specific solutions with many modes $n$ is given by

$$
\rho_{0}^{\text {trans }}=\pi \frac{(2+\beta) \Gamma\left(\frac{1+\beta}{2}\right)^{2}}{2 \Gamma\left(\frac{2+\beta}{2}\right)^{2}}, \quad \rho_{0}^{\mathrm{CPP}}=\pi, \quad \rho_{0}^{\mathrm{ECP}}=\pi \sqrt{1-2 \zeta^{2}}
$$


with the anisotropy parameter $\boldsymbol{\zeta}=\frac{1}{n} \sum_{j} l_{j} \boldsymbol{k}_{j}[22,171]$. Notice that for the CPP solutions, the random field approximation only holds for $d \neq 0$ in the large $n$ limit. The anisotropy of ECP solutions $|\boldsymbol{\zeta}|$ is bounded for many modes by $2 / \pi$ and vanishes for large $n$ on average leading to the same result as for CPPs.

In the presence of noise the pinwheel density $\rho_{0}$ of these fields changes to

$$
\rho=\rho_{0}\left(1-\sigma^{2} \frac{1-\rho_{n} / \rho_{0}}{1+\sigma^{2}}\right) \doteq \rho_{0}\left(1-\sigma^{2}\left(1-\frac{\rho_{n}}{\rho_{0}}\right)\right)
$$

in the isotropic case of the transient and CPP orientation domains with the noise pinwheel density $\rho_{n}$ and strength $\sigma^{2}$. In the anisotropic case of ECPs the pinwheel density $\rho_{0}$ is altered to

$$
\begin{aligned}
\rho & =\rho_{0}\left(1-\sigma^{2}\left(1-\sqrt{1-2 \zeta^{2}} \frac{\rho_{n}}{\rho_{0}}\right)\right) \\
& \sqrt{1+\frac{2 \zeta^{2}}{1-2 \zeta^{2}} \frac{\sigma^{2}\left(1+\frac{\rho_{n}}{\rho_{0}} \sqrt{1-2 \zeta^{2}}\right)+\sigma^{4} \frac{\rho_{n}}{\rho_{0}} \sqrt{1-2 \zeta^{2}}}{\left(1+\sigma^{2} \frac{\rho_{n}}{\rho_{0}} \sqrt{1-2 \zeta^{2}}\right)\left(1+\sigma^{2}\right)}} \\
& \doteq \rho_{0}\left(1-\frac{\sigma^{2}}{1-2 \zeta^{2}}\left(1-\zeta^{2}-\sqrt{1-2 \zeta^{2}}\left(1-3 \zeta^{2}\right) \frac{\rho_{n}}{\rho_{0}}\right)\right) .
\end{aligned}
$$

\section{Pinwheel observation probability at pinwheel centers in Gaussian random fields}

The spatial average of a functional $F\left[\Psi_{0}(\boldsymbol{x})\right]$ at pinwheel centers $\boldsymbol{x}_{i}$ with pinwheel density $\rho_{0}$ is defined by the spatial average over

$$
\frac{1}{\rho_{0}} \sum_{i} F\left[\Psi_{0}(\boldsymbol{x})\right] \delta\left(\boldsymbol{x}-\boldsymbol{x}_{i}\right)=\frac{1}{\rho_{0}} F\left[\Psi_{0}(\boldsymbol{x})\right] \delta\left(\xi_{0}(\boldsymbol{x})\right) \delta\left(\eta_{0}(\boldsymbol{x})\right) \nu_{0}(\boldsymbol{x}) .
$$

Notice that $\nu_{0}(\boldsymbol{x})$ accounts for correct normalization under the change of variables in the integral. Isotropic and shift-symmetric Gaussian random fields can be described by the multivariate Gaussian distribution

$$
P\left(\xi_{0}(\boldsymbol{x}), \eta_{0}(\boldsymbol{x}), \nabla \Psi_{0}(\boldsymbol{x})\right)=\frac{e^{-\xi_{0}(\boldsymbol{x})^{2}-\eta_{0}(\boldsymbol{x})^{2}}}{\pi} \frac{e^{-\frac{\left\|\nabla \Psi_{0}(\boldsymbol{x})\right\|^{2}}{2 \pi \rho_{0}}}}{\left(2 \pi^{2} \rho_{0}\right)^{2}} .
$$

At pinwheel centers $\boldsymbol{x}_{i}$ the amplitude vanishes such that the linear approximation around a pinwheel center is given by

$$
\Psi_{0}(\boldsymbol{x})=\left(\nabla \Psi_{0}\left(\boldsymbol{x}_{i}\right)\right)^{T}\left(\boldsymbol{x}-\boldsymbol{x}_{i}\right) .
$$

The probability distribution at a pinwheel center is hence given by

$$
P\left(\nabla \Psi_{0}\right)=\frac{1}{4 \pi^{5} \rho_{0}^{3}} \nu_{0} e^{-\frac{\left\|\nabla \Psi_{0}\right\|^{2}}{2 \pi \rho_{0}}}=\frac{1}{4 \pi^{5} \rho_{0}^{3}} e^{-\frac{\left\|\nabla \Psi_{0}\right\|^{2}}{2 \pi \rho_{0}}} \frac{1}{\pi} \int \frac{\mathrm{d} s}{s^{2}}\left(1-\frac{e^{i \nu_{0}}+e^{-i \nu_{0}}}{2}\right) .
$$

The average of the pinwheel observation probability map (Eq. (5.10)) at pinwheel centers is then a Gaussian integral that can be evaluated (see $[30,154]$ for a similar calculation used for pair correlation functions) and leads to a radially symmetric observation probability map

$$
\langle\rho(r)\rangle=\rho_{0} X \int_{0}^{\infty} \mathrm{d} t \frac{2 t^{4} Z+t^{2}(-2 Y+Z+3)+2 Y-Z+3}{\left(t^{2}+1\right)^{3} \sqrt{t^{4} Z+t^{2}(-Y+Z+1)+1}}
$$


with the dimensionless parameters

$$
\begin{aligned}
X & =\frac{2 \sigma^{2} \sqrt{\left(1+\sigma^{2} \rho_{n} / \rho_{0}\right)\left(1+\sigma^{2} \rho_{n} / \rho_{0}+2 \pi \rho_{n} r^{2}\right)}}{\pi\left(2 \pi \rho_{0} r^{2}+\sigma^{2}\right)^{2}}, \\
Y & =\frac{1}{\left(1+\sigma^{2} \rho_{n} / \rho_{0}\right)\left(1+\sigma^{2} \rho_{n} / \rho_{0}+2 \pi \rho_{n} r^{2}\right)}, \\
Z & =\frac{\sigma^{2}\left(2 \pi \rho_{0} r^{2}+\sigma^{2}\right) \rho_{n}^{2} / \rho_{0}^{2}}{\left(1+\sigma^{2} \rho_{n} / \rho_{0}\right)\left(1+\sigma^{2} \rho_{n} / \rho_{0}+2 \pi \rho_{n} r^{2}\right)} .
\end{aligned}
$$

This integral can be solved by a complicated sum of elliptic functions provided by [154].

The total number of pinwheels can be computed by utilizing Eq. (5.17) that requires the distribution of anisotropy and selectivity at pinwheel centers. The probability distribution for the spatial average at pinwheel centers in terms of $\nu_{0}(\boldsymbol{x})$ and $\left\|\nabla \Psi_{0}(\boldsymbol{x})\right\|^{2}$ is given by

$$
P(\nu, g)=\frac{1}{\rho_{0}}\left\langle\delta\left(\xi_{0}(\boldsymbol{x})\right) \delta\left(\eta_{0}(\boldsymbol{x})\right) \nu_{0}(\boldsymbol{x}) \delta\left(\nu-\nu_{0}(\boldsymbol{x})\right) \delta\left(g-\left\|\nabla \Psi_{0}(\boldsymbol{x})\right\|^{2}\right)\right\rangle .
$$

The Fourier representation of the $\delta$ distribution for the last two terms leads to the solution of

$$
P(\nu, g)=\frac{\nu}{2 \pi^{3} \rho_{0}^{3}} e^{-\frac{g}{2 \pi \rho_{0}}} \Theta(g-2 \nu)
$$

with $\Theta(\cdot)$ denoting the Heaviside function. As the linear approximation of a single pinwheel can be transformed to $\nu=s^{2} a$ and $\left\|\nabla \Psi_{0}(\boldsymbol{x})\right\|^{2}=s^{2}\left(1+a^{2}\right)$ the probability distribution can be transformed to these coordinates that gives

$$
P\left(s^{2}, a\right)=\frac{a\left(a^{2}-1\right) s^{4}}{2 \pi^{3} \rho_{0}^{3}} e^{-\frac{1+a^{2}}{2 \pi \rho_{0}} s^{2}}
$$

with the anisotropy $a \geq 1$ and selectivity $s^{2}$, see [360]. The average number of spurious pinwheels around a pinwheel center given by Eq. (5.17) is then a standard integral.

\section{Pinwheel observation probability of spatially uniform selectivity layouts}

The gradient of the phase $\theta=\arg \Psi_{0}$ of an orientation domain ensemble is distributed as

$$
P\left(\|\nabla \vartheta\|^{2}\right)=\left\langle\delta\left(\|\nabla \vartheta\|^{2}-\|\nabla \theta\|^{2}\right)\right\rangle,
$$

where the phase gradient is given by

$$
\|\nabla \theta\|^{2}=\frac{1}{\left|\Psi_{0}\right|^{2}}\left(\left(\partial_{x} \eta_{0} \cos \theta-\partial_{x} \xi_{0} \sin \theta\right)^{2}+\left(\partial_{y} \eta_{0} \cos \theta-\partial_{y} \xi_{0} \sin \theta\right)^{2}\right)=\frac{f^{2}}{\left|\Psi_{0}\right|^{2}} .
$$

For isotropic and shift-symmetric orientation domains the average over the gradient with the probability distribution $\left.p\left(\left\|\nabla \Psi_{0}\right\|\right)=\exp \left(-\frac{1}{2 c_{G}}\left\|\nabla \Psi_{0}\right\|^{2}\right) /\left((2 \pi)^{2} c_{G}^{2}\right)\right)$ can be separated from the amplitude. Thus, the integral over the amplitude can be evaluated as

$$
\begin{aligned}
P\left(\|\nabla \vartheta\|^{2}\right) & =\frac{1}{2 \pi c_{A}} \int_{0}^{2 \pi} \mathrm{d} \theta\left\langle\int_{0}^{\infty} \mathrm{d}\left|\Psi_{0} \| \Psi_{0}\right| \delta\left(\|\nabla \vartheta\|^{2}-\frac{f^{2}}{\left|\Psi_{0}\right|^{2}}\right) e^{-\frac{\left|\Psi_{0}\right|^{2}}{2 c_{A}}}\right\rangle_{\nabla \Psi_{0}} \\
& =\frac{1}{2 \pi c_{A}} \int_{0}^{2 \pi} \mathrm{d} \theta\left\langle\frac{f^{2}}{2\|\nabla \vartheta\|^{4}} e^{-\frac{f^{2}}{2 c_{A}\|\nabla \vartheta\|^{2}}}\right\rangle_{\nabla \Psi_{0}} \\
& =\frac{\partial_{\|\nabla \vartheta\|^{2}}}{2 \pi} \int_{0}^{2 \pi} \mathrm{d} \theta\left\langle e^{-\frac{f^{2}}{2 c_{A}\|\nabla \vartheta\|^{2}}}\right\rangle_{\nabla \Psi_{0}}
\end{aligned}
$$


The remaining integrals over derivatives of the field is a multi-dimensional Gaussian integral that reads

$$
P\left(\|\nabla \vartheta\|^{2}\right)=\frac{c_{A} \partial_{\|\nabla \vartheta\|^{2}}}{2 \pi} \int_{0}^{2 \pi} \mathrm{d} \theta \frac{\|\nabla \vartheta\|^{2}}{c_{A}\|\nabla \vartheta\|^{2}+c_{G}}=\frac{c_{A} c_{G}}{\left(c_{A}\|\nabla \vartheta\|^{2}+c_{G}\right)^{2}}
$$

and is a Student's t-distribution with three degrees of freedom. As the orthogonality vanishes $\nu_{0}=0$ for spatially constant selectivity layouts, the pinwheel observation probability is given by

$$
\begin{aligned}
\rho & =\left\langle\rho\left(\left|\Psi_{0}(\boldsymbol{x})\right|^{2}=1,\left\|\nabla \Psi_{0}(\boldsymbol{x})\right\|^{2}=\|\nabla \vartheta\|^{2}, \nu_{0}=0\right)\right\rangle_{\|\nabla \vartheta\|^{2}} \\
& =\rho_{n} e^{-\frac{1}{\sigma^{2}}} \\
& \left(\frac{1}{\sqrt{\pi}} \frac{\sigma^{2} \rho_{n}}{\rho_{0}} G_{2,3}^{2,2}\left(\frac{\rho_{0}}{\sigma^{2} \rho_{n}} \mid \begin{array}{c}
1, \frac{5}{2} \\
2,2,2
\end{array}\right)+\frac{\sqrt{\pi}}{2}\left(1+\frac{\rho_{0}}{\sigma^{2} \rho_{n}}\right) U\left(\frac{1}{2}, 0, \frac{\rho_{0}}{\sigma^{2} \rho_{n}}\right)\right)
\end{aligned}
$$

with $G$ denoting the Meijer $\mathrm{G}$ function and $U$ the confluent hypergeometric function.

\section{Numerical evaluation of pinwheel observation probability}

For single pinwheels, the pinwheel observation probability Eq. (5.10) was numerically evaluated by the MATLAB 2015b internal integral function that attempts to keep integration errors smaller than the absolute error of $10^{-10}$ and the relative error of $10^{-6}$ [361]. In the case of pinwheel pairs, the three-dimensional integral was computed in polar coordinates by the MATLAB 2015b internal integral3 function with the same error bounds [361,362]. Due to the oscillatory nature of orientation domains, a direct spatial integration of them was not feasible. Instead, orientation domains were numerically approximated on a grid of $4096 \times 4096$ pixels with $22 \Lambda \times 22 \Lambda$ for quasiperiodic orientation domains, $32768 \times 32768$ pixels with $22 \Lambda \times 22 \Lambda$ for orientation domains with high selectivity and on the discretized unit cell with 745 pixels per $\Lambda$ for the pinwheel crystal. The gradient and orthogonality was calculated by a first-order divided difference scheme and subsequently binned by the MATLAB 2015b internal and automated histogram2 function. For each bin value, the integral of the pinwheel observation probability was computed in the same way as for the single pinwheel. Finally, the total pinwheel observation probability was calculated by summing over the non-integral part of Eq. (5.10) in every pixel and scaling with the corresponding integral value of the binned data.

\section{Numerical synthesis of random orientation layouts}

For translation invariant orientation layouts the correlation function $C\left(\boldsymbol{x}_{1}-\boldsymbol{x}_{2}\right)=\left\langle\Psi\left(\boldsymbol{x}_{1}\right) \bar{\Psi}\left(\boldsymbol{x}_{2}\right)\right\rangle$ is diagonal in Fourier space and given by $P(\boldsymbol{k})$. The Fourier transform of a random orientation layout $\tilde{\Psi}(\boldsymbol{k})$ with the specified correlation function is synthesized by drawing Gaussian random numbers $u, v \sim \mathcal{N}(0,1)$ for all $\boldsymbol{k}$ and scaling them by $\tilde{\Psi}(\boldsymbol{k})=\sqrt{P(\boldsymbol{k})}(u+i v)$. 


\title{
Three-dimensional organization of orientation selectivity in cortical tissue
}

\author{
"Names are a menace in evolutionary history. \\ ... The obsession with discrete names is an example \\ of what I call the tyranny of the discontinuous \\ mind." \\ — R. Dawkins, [363]
}

\section{Original contribution}

J. Liedtke and F. Wolf conceived and designed the study. J. Liedtke performed the theoretical analysis and numerical simulations. M. Schottdorf analyzed power-spectra of experimentally measured orientation domains. J. Liedtke wrote the manuscript. 


\subsection{Abstract}

Columnar structure is a neocortical organization principle present in many species and across brain areas. In primates and placental carnivores, the functional architecture of the primary visual cortex (V1) is composed of orientation selective neurons that are horizontally grouped into (iso-) orientation domains and vertically into columns. In cats, orientation selectivity is constant along association fibers, while a substantially varying columnar orientation selectivity profile was observed in macaques. In rodents, functional architecture lack any apparent spatial structure and is organized in an interspersed layout. These different organization principles of orientation selectivity in V1 are most remarkable in the light of evolution, since they originated from a single species, the last common ancestor, more than 65 million years ago. To shed light on possible evolutionary paths of V1's functional architecture, examination of the full three-dimensional organization of orientation selectivity is inevitable. We provide the theoretical basis for a systematic examination of orientation selectivity in three-dimensional geometries that captures an entire model class of random wiring and frozen noise models in a broad parameter regime. Key characteristics of orientation domains are pinwheel centers that are surrounded by all preferred orientations. In three dimensions, pinwheel points generalize to pinwheel strings. We provide an exact formula for the average pinwheel string length in flat cortices and show that it is invariant to a columnar orientation selectivity profile such as an unselective layer $4 \mathrm{C}$. A curved cortex with a fixed typical scale bends pinwheel strings and favors U shaped pinwheel string motifs. Finally, we provide two different scenarios for the transition between an interspersed layout and orientation domains. This work sets the stage for a profound theoretical understanding of three-dimensional functional architectures of $\mathrm{V} 1$ and putative transitions between interspersed and organized functional layouts for which intermediate organizations might still exist.

\subsection{Introduction}

The neocortex is a $1-3 \mathrm{~mm}$ thick cortical tissue composed of 6 horizontal layers and vertical columns [364]. Cortical columns range from the pial surface to the white matter by traversing horizontal layers orthogonally. The concept of cortical columns goes back to Mountcastle's discovery in the 50s, who measured, for the first time, that neurons in the somatosensory cortex respond either to superficial or deep stimulation in one vertical electrode penetration [70]. This columnar organization was subsequently discovered for different neuronal response specificities such as ocular dominance, light contrast and orientation selectivity in V1 [365, 366]. Orientation selective neurons respond preferentially to contours of specific orientations in their receptive fields. More specifically, orientation selectivity was found to be organized in orientation domain columns in V1 of cats [69, 80, 367-372], macaques [297, 373], ferrets [374], tree-shrews [375, 376], minks [377] and perhaps sheep [378], Fig. 6.1A. Orientation domains with the same orientation are separated by a typical column spacing or typical scale. The columnar organization of orientation domains is not uniform across laminar layers, for instance, a weakly selective or even unselective layer $4 \mathrm{C}$ was found in macaques [297, 379, 380] and tree shrews [79, 375], Fig. 6.1A. Although details of this observation are debated [381, 382], all findings have a substantially varying orientation selectivity profile in the columnar direction in common. A drastic deviation of columnar organization was found in rodents with the total absence of orientation preference organization [41], Fig. 6.1A, but see [383]. Most remarkable, the lack of organization seems not to correlate with function, because no apparent organization of orientation selectivity was found in highly visual gray squirrels [42]. Despite the variety of different functional architectures in V1, the columnar organization of orientation selectivity was neglected in theoretical models except for [120]. From an evolutionary point of view, the distinct organizations raise the 


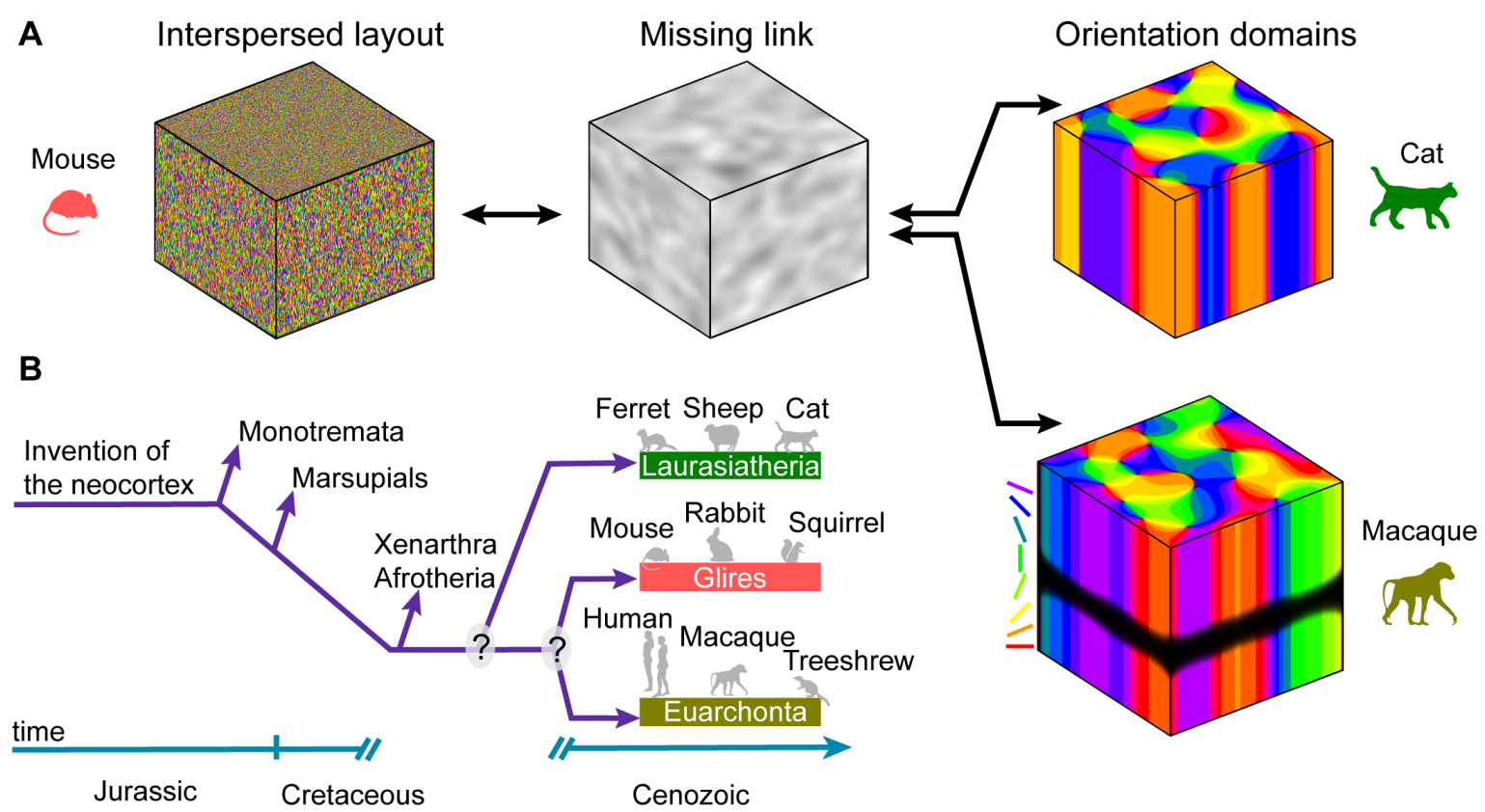

Figure 6.1: Evolution of the functional architecture in the primary visual cortex (V1). A Three distinct three-dimensional functional architectures composed of orientation selective neurons in V1 are observed in different mammalian species. Mice, members of the clade Glires, have an interspersed layout, while cats, members of the clade Laurasiatheria, have orientation domains in all laminar layers and macaques, members of the clade Euarchonta, have orientation domains in all layers but layer $4 \mathrm{C}$. The functional architecture of their last common ancestor's V1 is unknown and suspected to be an interspersed layout. The transition between these different functional architectures is unclear. B The cladistic tree of mammals splits into Laurasiatheria and Euarchonta with orientation domains and Glires with an interspersed layout. The functional architecture of intermediate species is unknown. Adapted from [24].

question: what type of functional architecture might have served as an intermediate (missing link) organization principle, Fig. 6.1A?

In primates and placental carnivores, the horizontal organization of orientation selectivity in V1 adheres to a common design. Orientation preference varies smoothly across the surface except at point singularities termed pinwheels that are surrounded by all preferred orientations. The configuration of pinwheels follows species-invariant quantitative statistics [22-24]. The emergence of this common design is suggested to be an evolutionary convergent trait, because of two main reasons: (i) The last common ancestor of the investigates species cat, ferret, treeshrew and galago, Fig. 6.1B, was a small shrew-like mammal that therefore likely possessed an interspersed orientation layout $[22,76]$. (ii) Mammals from different evolutionary branches generate orientation selectivity through distinct neuronal core circuits [24, 77], see Fig. 6.2. Thus, at least two likely transitions of visual functional architecture occurred in the course of Laurasiatheria and Euarchonta branching, see Fig. 6.1B.

Distinct neuronal precursor cells and core circuits are involved in orientation selectivity generation of macaques, cats and mice. During early ontogenesis of the central nervous system, the brain is constituted of three primary brain vesicles: the forebrain, midbrain and hindbrain. In later embryonic development the anterior and posterior part of the forebrain specializes into the telencephalon and diencephalon, respectively. The diencephalon compartmentalizes into functionally distinct domains such as the thalamus and retina. Neurons generated in the thalamic domains subsequently cluster to form nuclei such as the dorsal lateral geniculate nucleus 
A
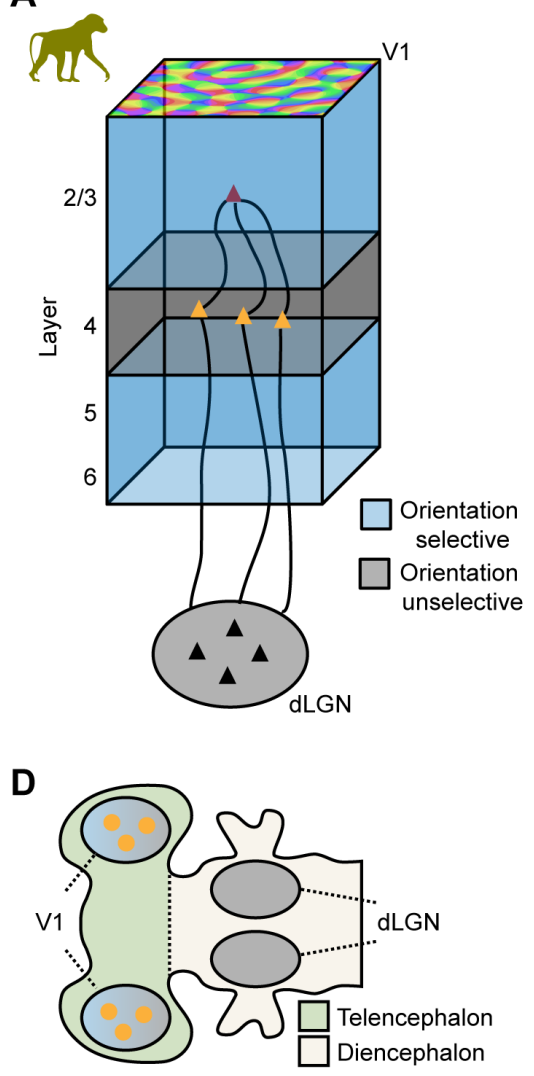

B

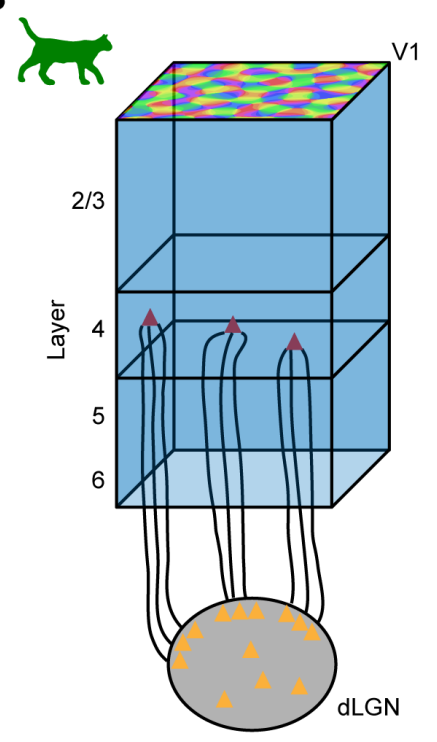

E

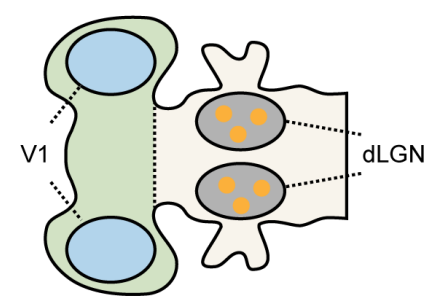

C

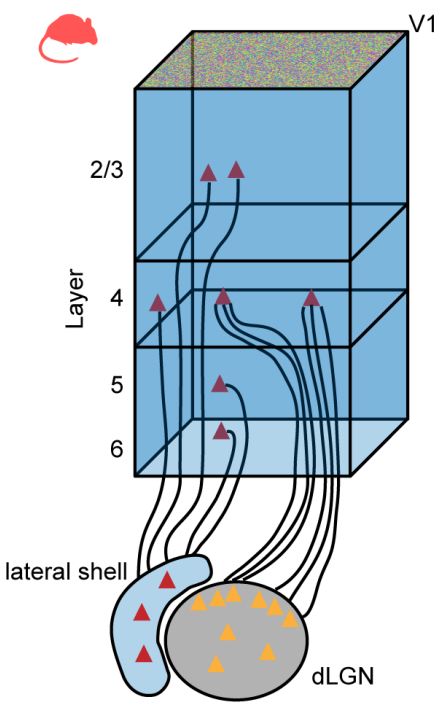

$\mathbf{F}$

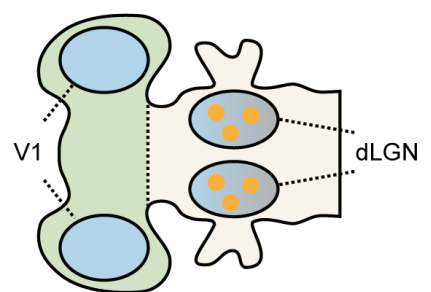

Figure 6.2: Core circuits for orientation selectivity generation and their developmental precursors. A-C Illustration of core circuits for orientation selectivity generation in macaques, cats and mice, respectively. A In macaques, orientation selectivity is generated by intracortical projections from layer 4 to layer $2 / 3$ in the primary visual cortex (V1). The dorsal lateral geniculate nucleus (dLGN) and layer $4 \mathrm{C}$ of $\mathrm{V} 1$ are orientation unselective or weakly selective. B In cats, orientation selectivity is generated by thalamocortical projections. Layer $4 \mathrm{C}$ is orientation selective, while dLGN is orientation unselective. C In mice, direction selective and hence orientation selective retinal ganglion cells project di-synaptically over the lateral shell of dLGN to V1 neurons [384]. Additionally, orientation selectivity is generated by thalamocortical projections. D-F Precursor cells of neurons building core circuits for orientation selectivity generation in species from A-C, respectively. D In macaques, precursor cells generating orientation selectivity are developing in the telencephalon, while dLGN precursor cells are not involved. E In cats, orientation selectivity generating precursor cells develop in the diencephalon. F In mice, orientation selectivity generating precursor cells develop as for cats in the diencephalon.

(dLGN), see [385, 386] for details on the genetic mechanisms involved in patterning of the diencephalon. Thalamocortical and corticothalamic projections between V1 and dLGN involves multiple combinatorially acting genes $[387,388]$. In macaques, orientation selectivity is generated intracortically by the projection of layer 4 neurons, Fig. 6.2A. In contrast, orientation selectivity generation originates in thalamocortical projections in cats and mice, Fig. 6.2B-C, with an additional separate pathway in mice that relays direction selective responses from retinal ganglion cell to V1 [384]. The distinct mechanisms of orientation selectivity generation become more striking in the light of ontogenesis. If the generation of orientation selectivity is determined by genetic mechanisms, it poses a major theoretical challenge to elucidate how precursor cells from the telencephalon in macaques and diencephalon in cats can generate orientation domains that adhere both to the common design, Fig. 6.2D-E. In contrast, precursor cells in the diencephalon of mice generate an interspersed orientation layout. How can such an evolutionary 
transition of cell fate specification occur?

Neocortical columnar organization becomes more accessible with the advent of non-invasive imaging techniques such as two/three photon-excited fluorescence laser-scanning microscopy (2PLSM/3PLSM), optical coherence tomography (OCT) and high-resolution fMRI. Early dissections of columnar organizations commonly used electrode penetrations and 2-deoxyglucose imaging with poor spatial resolution of about $100 \mu \mathrm{m}$ in comparison to the $\sim 1 \mathrm{~mm}$ column spacing of orientation domains. Thus, precision measurements of the columnar organization were not feasible with these techniques. More recent techniques such as intrinsic signal imaging and fluorescence imaging provide better axial resolution, but lack a good depth resolution. In contrast to traditional linear fluorescence microscopy, where the maximal imaging depth is limited to less than $100 \mu \mathrm{m}$ due to scattering, 2PLSM/3PLSM techniques allow imaging at depths of a mm scale [37, 389-392]. The recording technique OCT allows an imaging depth of 2-3 mm. It is widely used for imaging internal structure of biological systems and materials, but can also be used for functional imaging by utilizing the absorption change of oxy- and deoxy-hemoglobin for recording analogously to intrinsic signal imaging [393-395]. Lastly, high-resolution fMRI characterized by a sub-millimeter resolution has already resolved orientation domains in cats $[120,396]$ as well as in humans [58] and is capable to record their columnar organization. These three techniques with an imaging depth of about the neocortical thickness are suitable to dissect the detailed three-dimensional functional architecture of V1.

Key questions arising in the consideration of three-dimensional cortical organizations are: what is the effect of curvature on the functional organization? Do evolutionary intermediate organizations still exist and how could they be organized? Here, we devise maximum entropy models for the three-dimensional spatial organization of orientation selectivity and show that (i) pinwheel strings (the three-dimensional generalization of pinwheels) are generally curved, (ii) pinwheel string length is invariant to different columnar orientation selectivity profiles (iii) for large curvatures closed loops and reconnecting pinwheel strings appear and (iv) for small correlation lengths novel transitions to a rodent-like interspersed organization emerge. The maximum entropy model for orientation domain ensembles is a generic description for a whole model class in the early phase of orientation selectivity emergence and for random wiring schemes. This approach was used previously in two-dimensional orientation domains for the analytical calculation of the pinwheel density $[135,171]$.

\subsection{Results}

\section{Orientation director fields}

In order to examine the spatial arrangement of orientation selectivity in three-dimensional geometries, the spatial position will be treated continuously and the neuronal response properties will be summarized as suggested in $[122,132]$. More specifically, the preferred orientation $\vartheta(\boldsymbol{x})$ of a neuron at cortical position $\boldsymbol{x}=(x, y, z)^{T}$ is a cyclic, $\pi$-periodic, quantity and is hence conveniently represented together with its selectivity $S(\boldsymbol{x})$ as an orientation director field

$$
\Psi(\boldsymbol{x})=S(\boldsymbol{x}) e^{2 i \vartheta(\boldsymbol{x})}
$$

or short orientation field. In the following, the real and imaginary part of the complex field will be denoted by $\xi(\boldsymbol{x})$ and $\eta(\boldsymbol{x})$, respectively.

Previous models suggest different types of orientation selectivity emergence. For instance, random wiring schemes suggest that the spatial arrangement of orientation selectivity emerges by feed-forward connections. In contrast, self-organizing models suggest that the spatial arrangement of orientation selectivity is generated dynamically. In the following, we show that 


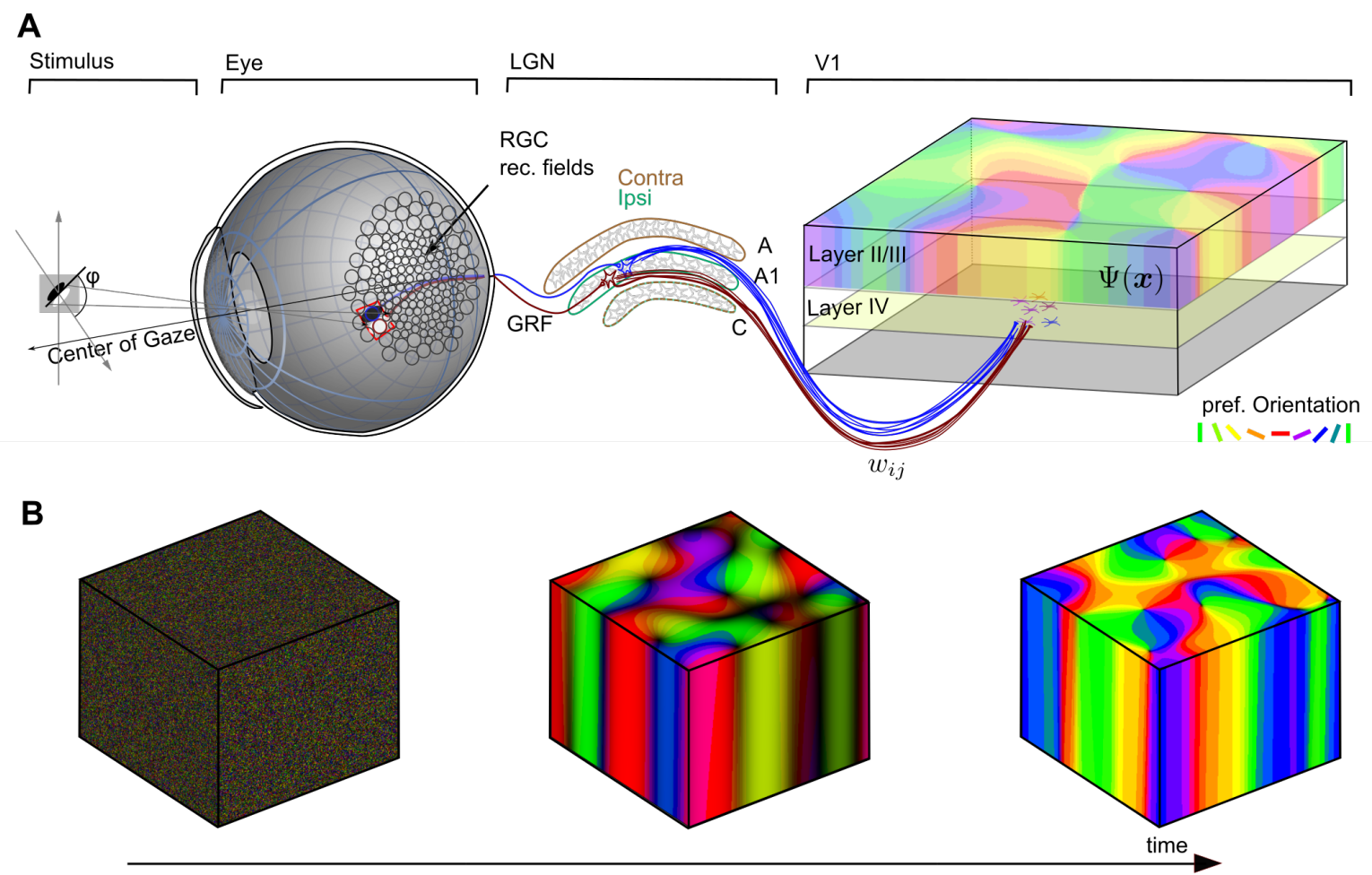

Figure 6.3: Developmental models generating orientation domains. A Schematic illustration of the visual pathway in a cat. In a random wiring scheme the ensemble of orientation selectivity in V1 has maximal entropy (see main text for details). Adapted from [24]. B Orientation domains produced by a Turing-type instability. If dynamics are frozen in the linear regime, the ensemble of orientation selectivity has maximal entropy (see main text for details).

both model types can be described by maximum entropy models of orientation domain ensembles in a broad parameter regime, Fig. 6.3. These maximum entropy models are Gaussian random fields (see methods for details).

\section{Orientation fields seeded by random wiring}

A model class that favors a feed-forward type of orientation field emergence similar to the Hubel and Wiesel scheme is a random wiring scheme examined in [24, 338, 339, 397] and illustrated in Fig. 6.3A.

The receptive field of a retinal ganglion cell at position $\boldsymbol{x}_{j}=\left(x_{j}, y_{j}\right)^{T}$ in retinal space can be modeled by a radially symmetric function $f$

$$
\operatorname{GRF}_{j}(\boldsymbol{x})= \pm f\left(\left\|\boldsymbol{x}-\boldsymbol{x}_{j}\right\|\right),
$$

with a global maximum of $f$ at the origin. This function is weighted by a plus or minus for $\mathrm{ON}$ or OFF center retinal ganglion cells, respectively. A shape used for the retinal ganglion cell receptive field in $[24,338,397]$ is a Gaussian function

$$
f\left(\left\|\boldsymbol{x}-\boldsymbol{x}_{j}\right\|\right)=\exp \left(-\frac{\left\|\boldsymbol{x}-\boldsymbol{x}_{j}\right\|^{2}}{2 \sigma_{r}^{2}}\right) .
$$

The receptive field of a visual cortical neuron at position $\boldsymbol{y}$ can be obtained by pooling over 
several retinal ganglion cell receptive fields

$$
\mathrm{RF}_{\boldsymbol{y}_{i}}(\boldsymbol{x})=\sum_{j} w_{i j} \operatorname{GRF}_{j}(\boldsymbol{x})
$$

where $w_{i j}$ is a positive variable with a distribution that decays with distance $\left\|\boldsymbol{y}_{i}-\boldsymbol{x}_{j}\right\|$. The received input of neurons in cat's V1 can be estimated to about 10-25 retinal ganglion cells [24] by the findings of about 20-40 cortical inputs from LGN X-relay cells [398, 399] and an expansion of 1.5-2 from X-cells in the retina to X-relay cells in the LGN [400, 401]. The response of a visual cortical neuron to a stimulus $I(\boldsymbol{x})$ can be described by an energy model

$$
\mathcal{R}_{\boldsymbol{y}_{i}}=\left|\int \mathrm{d}^{2} x \operatorname{RF}_{\boldsymbol{y}_{i}}(\boldsymbol{x}) I(\boldsymbol{x})\right|^{2} .
$$

For a stimulus of oriented bars with the wave vector $\boldsymbol{k}$ the response is given by the Fourier transformation

$$
\mathcal{R}_{\boldsymbol{y}_{i}}(\boldsymbol{k})=\left|\int \mathrm{d}^{2} x \mathrm{RF}_{\boldsymbol{y}_{i}}(\boldsymbol{x}) e^{-i \boldsymbol{k} \boldsymbol{x}}\right|^{2} \propto|\tilde{f}(\|\boldsymbol{k}\|)|^{2} \sum_{j, l} w_{i j} w_{i l} \cos \boldsymbol{k}\left(\boldsymbol{x}_{j}-\boldsymbol{x}_{l}\right) .
$$

If the number of retinal ganglion cells with uncorrelated positions is large at distances where the average strength of $w$ is sufficiently large, the response $\mathcal{R}_{\boldsymbol{y}}(\boldsymbol{k})$ is Gaussian distributed. To be more precise, if the correlation length of retinal positions $L$ and the average support area $A$ of $w$ fulfills the condition $\sqrt{A} \gg L$ then $\mathcal{R}_{\boldsymbol{y}}(\boldsymbol{k})$ is Gaussian distributed. Alternatively, the response $\mathcal{R}_{\boldsymbol{y}_{i}}(\boldsymbol{k})$ is filtered by a smoothing kernel that leads to Gaussian statistics even for small support of $w$ as in $[24,338,339]$. For a support area size $B$ of the smoothing kernel, the previous condition generalizes to $\sqrt{A^{2}+B^{2}} \gg L$.

In the random wiring scheme, orientation domains can be obtained by

$$
\Psi\left(\boldsymbol{y}_{i}\right) \propto \int_{0}^{\infty} \mathrm{d} k k \int_{0}^{\pi} \mathrm{d} \theta \mathcal{R}_{\boldsymbol{y}_{i}}(\boldsymbol{k}) e^{2 i \theta}
$$

and measure the average response to oriented bars with wave vector $\boldsymbol{k}=k(\cos \theta, \sin \theta)^{T}$. Since the integral over different orientations of the stimulus is a linear operator and sums over Gaussian distributed variables, the orientation field $\Psi(\boldsymbol{y})$ is a Gaussian random field in the limit of continuous space, i.e. the ensemble is described by the first and second non-vanishing cumulants. If the distance distribution of RGC positions is translation invariant and radially symmetric, the mean of the orientation domains vanishes $\langle\Psi(\boldsymbol{y})\rangle=0$. Furthermore, the 2-point correlation becomes rotation and translation invariant $\left\langle\Psi\left(\boldsymbol{y}_{1}\right) \bar{\Psi}\left(\boldsymbol{y}_{2}\right)\right\rangle=C\left(\left\|\boldsymbol{y}_{1}-\boldsymbol{y}_{2}\right\|\right)$. Three-dimensional models can be obtained by assuming identical or similar visual inputs to V1 neurons along a vertical tract.

\section{Dynamically generated orientation fields in the transient linear regime}

In the following, we will show that for a large class of dynamic orientation domain models, the transient from an orientation unselective to selective state can be described by a Gaussian random field as already proposed for two-dimensional models in $[147,359]$. Here, we extend the two-dimensional consideration to three-dimensional models.

The dynamics of a three-dimensional orientation field $\Psi(\boldsymbol{x}, t)$ with $\boldsymbol{x}=(x, y, z)^{T}$ might be given by

$$
\partial_{t} \Psi(\boldsymbol{x}, t)=\hat{F}[\Psi]+\zeta(\boldsymbol{x}, t)
$$


where $\hat{F}$ denotes a nonlinear operator and $\zeta(\boldsymbol{x}, t)$ spatiotemporal noise fluctuations. Orientation fields in which orientation preference contain no information about spatial arrangement might be obtained by the equivariance of $\hat{F}$ under shift symmetry by an angle $\phi$, that is $\hat{F}\left[e^{i \phi} \Psi\right]=e^{i \phi} \hat{F}[\Psi]$. This symmetry implies an unselective stationary orientation field $\Psi(\boldsymbol{x})=0$ in the absence of noise and suggests a first order approximation around a small amplitude random initial state $\Psi(\boldsymbol{x}, 0)=\Psi_{0}(\boldsymbol{x})$ by

$$
\partial_{t} \Psi(\boldsymbol{x}, t)=\hat{L}[\Psi]+\zeta(\boldsymbol{x}, t) .
$$

This approximation is valid for time scales in which the dynamics are dominated by the linear operator. For the sake of clarity, we assume that the linear operator $\hat{L}$ is translation invariant and separable in horizontal and columnar directions, i.e. $\hat{L}=\hat{L}_{c}+\hat{L}_{h}$.

The phenomenological emergence of orientation fields can be specified by the eigenspectrum of the linear operator. In order to gain isotropic orientation fields with a typical scale $\Lambda$ in horizontal layers, the horizontal eigenspectrum $\mu_{h}$ of the linear operator $\hat{L}_{h}$ must be rotation invariant $\mu_{h}(k)$ with $k=\left\|\left(k_{x}, k_{y}\right)^{T}\right\|$ and positive in an interval $\left[k_{l}, k_{r}\right]$ with a peak at the typical wave vector $k_{c}=2 \pi / \Lambda$. Columnar organization is achieved by requiring the columnar eigenspectrum of $\mu_{c}\left(k_{z}\right)$ to be positive in a region around the origin $k_{z} \in\left[-k_{z}^{0}, k_{z}^{0}\right]$. A specific model example with these eigenspectra is a mexican hat interaction in the horizontal layer and a Gaussian interaction in the columnar direction

$$
\begin{aligned}
& \hat{L}_{h}[\Psi]=\int \mathrm{d} x^{\prime} \int \mathrm{d} y^{\prime}\left(e^{-\frac{\left(x-x^{\prime}\right)^{2}+\left(y-y^{\prime}\right)^{2}}{2 \sigma_{1}^{2}}}-e^{-\frac{\left(x-x^{\prime}\right)^{2}+\left(y-y^{\prime}\right)^{2}}{2 \sigma_{2}^{2}}}\right) \Psi\left(\left(x^{\prime}, y^{\prime}, z\right)^{T}\right) \\
& \hat{L}_{c}[\Psi]=\int \mathrm{d} z^{\prime} e^{-\frac{\left(z-z^{\prime}\right)^{2}}{2 \sigma_{z}^{2}}} \Psi\left(\left(x, y, z^{\prime}\right)^{T}\right)
\end{aligned}
$$

with the interaction ranges $\sigma_{1}<\sigma_{2}$ and $\sigma_{z}$.

In the linear regime, the orientation field dynamics can be solved by Green's functions. Due to translational symmetry of the linear operator, the Green's function is a convolution operator that separates to $G(\boldsymbol{x}, t)=G_{h}(r, t) G_{c}(z, t)$ with

$$
G_{h}(r, t)=\frac{1}{2 \pi} \int \mathrm{d} k k J_{0}(k r) e^{\mu_{h}(k) t} \quad \text { and } \quad G_{c}(z, t)=\frac{1}{2 \pi} \int \mathrm{d} k_{z} e^{-i k_{z} z+\mu_{c}\left(k_{z}\right) t}
$$

with the horizontal radial coordinate $r=\sqrt{x^{2}+y^{2}}$ and $J_{0}$ denoting the Bessel function of first kind. The characteristic timescale of the dynamics is the fastest growth rate and given by

$$
\tau=\min _{\mu_{h}>0, \mu_{c}>0}\left\{\frac{1}{\mu_{h}(k)+\mu_{c}\left(k_{z}\right)}\right\} .
$$

The time-dependent orientation field is then solved by

$$
\Psi\left(\boldsymbol{x}_{1}, t\right)=\int \mathrm{d}^{3} x \int_{0}^{t} \mathrm{~d} s G\left(\boldsymbol{x}_{1}-\boldsymbol{x}, t-s\right)\left(\zeta(\boldsymbol{x}, s)+\Psi_{0}(\boldsymbol{x}) \delta(s)\right) .
$$

Therefore, the orientation field is a random variable at every cortical position and time that depends on the statistics of the spatiotemporal noise $\zeta(\boldsymbol{x}, t)$ and the initial field $\Psi_{0}(\boldsymbol{x})$.

Specifying noise statistics and initial field statistics determine the statistics of the orientation field. The temporal noise fluctuations and the initial random field are assumed to possess a vanishing mean and second correlation function and are defined by horizontally isotropic, stationary and separable correlation functions

$$
\begin{aligned}
C_{\zeta}\left(\boldsymbol{x}_{1}, \boldsymbol{x}_{2}, t_{1}, t_{2}\right) & =\left\langle\zeta\left(\boldsymbol{x}_{1}, t_{1}\right) \bar{\zeta}\left(\boldsymbol{x}_{2}, t_{2}\right)\right\rangle=C_{\zeta}^{h}(r) C_{\zeta}^{c}\left(z_{1}, z_{2}\right) C_{\zeta}^{t}\left(t_{2}-t_{1}\right) \\
C_{0}\left(\boldsymbol{x}_{1}, \boldsymbol{x}_{2}\right) & =\left\langle\Psi_{0}\left(\boldsymbol{x}_{1}\right) \bar{\Psi}_{0}\left(\boldsymbol{x}_{2}\right)\right\rangle=C_{0}^{h}(r) C_{0}^{c}\left(z_{1}, z_{2}\right)
\end{aligned}
$$


with the distance $r=\sqrt{\left(x_{2}-x_{1}\right)^{2}+\left(y_{2}-y_{1}\right)^{2}}$. The moments of the orientation field are related to the noise field and lead to a vanishing mean and second moment

$$
\langle\Psi(\boldsymbol{x}, t)\rangle=\left\langle\Psi\left(\boldsymbol{x}_{1}, t_{1}\right) \Psi\left(\boldsymbol{x}_{2}, t_{2}\right)\right\rangle=0 .
$$

The remaining correlation function is given by

$$
\begin{aligned}
C\left(\left\|\Delta \boldsymbol{x}_{h}\right\|, z_{1}, z_{2}, t\right)= & \left\langle\Psi\left(\boldsymbol{x}_{1}, t\right) \bar{\Psi}\left(\boldsymbol{x}_{2}, t\right)\right\rangle \\
= & \int \mathrm{d}^{3} x^{\prime} \int \mathrm{d}^{3} x \int_{0}^{t} \mathrm{~d} s^{\prime} \int_{0}^{t} \mathrm{~d} s G\left(\boldsymbol{x}^{\prime}, t-s^{\prime}\right) \bar{G}(\boldsymbol{x}, t-s) \\
& \left(C_{\zeta}^{h}\left(\left\|\Delta \boldsymbol{x}_{h}-\boldsymbol{x}_{h}+\boldsymbol{x}_{h}^{\prime}\right\|\right) C_{\zeta}^{c}\left(z_{1}-z^{\prime}, z_{2}-z\right) C_{\zeta}^{t}\left(s-s^{\prime}\right)+\right. \\
& \left.C_{0}^{h}\left(\left\|\Delta \boldsymbol{x}_{h}-\boldsymbol{x}_{h}+\boldsymbol{x}_{h}^{\prime}\right\|\right) C_{0}^{c}\left(z_{1}-z^{\prime}, z_{2}-z\right)\right)
\end{aligned}
$$

with the horizontal vector $\boldsymbol{x}_{h}=(x, y)^{T}$ and horizontal distance vector $\Delta \boldsymbol{x}_{h}=\left(x_{2}-x_{1}, y_{2}-y_{1}\right)^{T}$. Notice that the correlation function is radially symmetric in the horizontal layer due to the coordinate choice of $\boldsymbol{x}_{h}^{\prime}-\boldsymbol{x}_{h}$ pointing in the direction of $\Delta \boldsymbol{x}_{h}$ together with the radially symmetric horizontal Green's function. This correlation function is in general not separable into a horizontal and columnar correlation function due to sustained interactions of neurons within and between horizontal layers.

The correlation function is separable into a horizontal and columnar correlation function in the limiting case where the columnar eigenvalue spectrum is constant $\mu_{c}\left(k_{z}\right)=\mu_{c}$. If the columnar eigenspectrum is constant for a range of wave vectors $k_{z} \in\left[-k_{z}^{0}, k_{z}^{0}\right]$ and zero elsewhere, the correlation function can be decomposed to

$$
\begin{aligned}
C_{h}\left(\left\|\Delta \boldsymbol{x}_{h}\right\|, t\right)= & \int \mathrm{d}^{2} x_{h}^{\prime} \int \mathrm{d}^{2} x_{h} \int_{0}^{t} \mathrm{~d} s^{\prime} \int_{0}^{t} \mathrm{~d} s G_{h}\left(\left\|\boldsymbol{x}_{h}^{\prime}\right\|, t-s^{\prime}\right) e^{\mu_{c}\left(t-s^{\prime}\right)} G_{h}\left(\left\|\boldsymbol{x}_{h}\right\|, t-s\right) \\
& e^{\mu_{c}(t-s)}\left(C_{\zeta}^{h}\left(\left\|\Delta \boldsymbol{x}_{h}-\boldsymbol{x}_{h}+\boldsymbol{x}_{h}^{\prime}\right\|\right) C_{\zeta}^{t}\left(s-s^{\prime}\right)+C_{0}^{h}\left(\left\|\Delta \boldsymbol{x}_{h}-\boldsymbol{x}_{h}+\boldsymbol{x}_{h}^{\prime}\right\|\right)\right)
\end{aligned}
$$

and for $k_{z}^{0} \rightarrow \infty$ and $k_{z}^{0} \rightarrow 0$ to the columnar correlation function

$$
\begin{aligned}
& C_{c}\left(z_{1}, z_{2}\right)=C_{\zeta}^{c}\left(z_{1}, z_{2}\right)+C_{0}^{c}\left(z_{1}, z_{2}\right) \\
& C_{c}\left(z_{1}, z_{2}\right)=\left(\frac{k_{z}^{0}}{\pi}\right)^{2} \int \mathrm{d} z^{\prime} \int \mathrm{d} z\left(C_{\zeta}^{c}\left(z^{\prime}, z\right)+C_{0}^{c}\left(z^{\prime}, z\right)\right),
\end{aligned}
$$

respectively. This shows that for weak columnar organization, the correlation is dictated by the noise correlation and for strong columnar organization, the columnar correlation function is constant indicating perfect columnar organization.

The field $\Psi(\boldsymbol{x}, t)$ is a Gaussian random field in a broad range of parameters as long as the dynamics are dominated by the linear operator. To make this more explicit, we assume that the correlation functions of the noise can be approximated by

$$
C_{\zeta}^{h}(r) \sim e^{-r / L}, \quad C_{\zeta}^{c}(z) \sim e^{-|z| / L_{z}}, \quad C_{\zeta}^{t}(t) \sim e^{-t / \tau_{\zeta}}
$$

and of the random initial field by

$$
C_{0}^{h}(r) \sim e^{-r / L_{0}}, \quad C_{0}^{c}(z) \sim e^{-|z| / L_{z, 0}} .
$$

Thus the orientation field is composed of many independent random components if the orientation field is mainly composed of: (i) temporal noise for $\tau_{\zeta} \ll \tau, L \ll \frac{1}{\left|k_{l}-k_{r}\right|}$ or $L_{z} \ll \frac{1}{2 k_{z}^{0}}$ and 
(ii) the random initial field for $L_{0} \ll \sqrt{t / \tau} /\left|k_{l}-k_{r}\right|$ or $L_{z, 0} \ll \sqrt{t / \tau} / k_{z}^{0}$, see [359] for the twodimensional case. Due to the central limit theorem, the field $\Psi(\boldsymbol{x})$ is a Gaussian random field in these parameter regimes, i.e. the ensemble is described by the first and second non-vanishing cumulants. The first condition is biologically plausible by noting that the afferent activity patterns can be correlated over timespans of a few hundred milliseconds, whereas the layout of orientation domains is formed on the timescale of hours to days. In this case the contribution of the noise fluctuations is likely to be large, which results in the statistical properties of $\Psi(\boldsymbol{x}, t)$ being a Gaussian distribution [359].

In total, dynamically generated orientation fields are Gaussian random fields in the transient linear and, in particular, in the frozen noise regime (dynamics freeze in the linear regime) for a broad range of model parameters.

\section{Model unification: Symmetry constrained maximum entropy orientation field ensembles}

The previous models assume explicit mechanisms for the emergence of orientation selectivity. An agnostic approach for the description of orientation fields is to consider ensembles and utilize symmetry principles. Experimentally observed orientation domains exhibit an astonishing diversity of different spatial layouts, while retaining specific layout characteristics such as a typical scale and pinwheels. The diversity and characteristics of orientation domains suggests to view single orientation domain observations as a randomly chosen example of an orientation domain ensemble, see [171]. Thus, the probability of observing a specific orientation domain layout $\Psi(\boldsymbol{x})$ can be described by a probability functional $\mathcal{P}[\Psi]$. This functional is in general unknown, but can be equivalently defined by all its moments

$$
c_{m}^{n}\left(\boldsymbol{x}_{1}, \boldsymbol{x}_{2}, \ldots, \boldsymbol{x}_{n+m}\right)=\left\langle\Psi\left(\boldsymbol{x}_{1}\right) \Psi\left(\boldsymbol{x}_{2}\right) \ldots \Psi\left(\boldsymbol{x}_{m}\right) \bar{\Psi}\left(\boldsymbol{x}_{m+1}\right), \ldots \bar{\Psi}\left(\boldsymbol{x}_{n+m}\right)\right\rangle,
$$

or cumulants, where $\langle\cdot\rangle$ represents the ensemble average. The representation of the probability functional in terms of its moments suggests an approximation by specifying only a subset of moments or cumulants. Maximum entropy models are the least structured probability distributions that determine a set of statistical quantities (see methods). If only first and second moments are determined in an maximum entropy ensemble, the individual realization of orientation domains are known as Gaussian random fields. These statistical descriptions are exact for the afore-mentioned limiting cases of transient pattern formation regimes and random wiring schemes in a broad parameter regime.

Moments of the probability functional can be further confined by symmetries principles. The assumption that no distinguished preferred orientation exists on average can be incorporated by the invariance of moments under a shift transformation of a fixed angle $\phi$

$$
\Psi(\boldsymbol{x}) \rightarrow \Psi(\boldsymbol{x}) e^{i \phi} .
$$

Due to this shift-symmetry the first and one of the second moments vanish

$$
\begin{array}{cccc}
\langle\Psi(\boldsymbol{x})\rangle=e^{i \phi}\langle\Psi(\boldsymbol{x})\rangle & \rightarrow & \langle\xi(\boldsymbol{x})\rangle=\langle\eta(\boldsymbol{x})\rangle=0 \\
\left\langle\Psi\left(\boldsymbol{x}_{1}\right) \Psi\left(\boldsymbol{x}_{2}\right)\right\rangle=e^{2 i \phi}\left\langle\Psi\left(\boldsymbol{x}_{1}\right) \Psi\left(\boldsymbol{x}_{2}\right)\right\rangle & \rightarrow & \left(\begin{array}{c}
\left\langle\xi\left(\boldsymbol{x}_{1}\right) \xi\left(\boldsymbol{x}_{2}\right)\right\rangle \\
\left\langle\xi\left(\boldsymbol{x}_{1}\right) \eta\left(\boldsymbol{x}_{2}\right)\right\rangle
\end{array}\right)=\left(\begin{array}{c}
\left\langle\eta\left(\boldsymbol{x}_{1}\right) \eta\left(\boldsymbol{x}_{2}\right)\right\rangle \\
-\left\langle\eta\left(\boldsymbol{x}_{1}\right) \xi\left(\boldsymbol{x}_{2}\right)\right\rangle
\end{array}\right),
\end{array}
$$

where $\Psi(\boldsymbol{x})=\xi(\boldsymbol{x})+i \eta(\boldsymbol{x})$. The remaining second moment can be simplified by noting the identities obtained from the vanishing second moment

$$
C_{\Psi}\left(\boldsymbol{x}_{1}, \boldsymbol{x}_{2}\right)=\left\langle\Psi\left(\boldsymbol{x}_{1}\right) \bar{\Psi}\left(\boldsymbol{x}_{2}\right)\right\rangle=2\left\langle\xi\left(\boldsymbol{x}_{1}\right) \xi\left(\boldsymbol{x}_{2}\right)\right\rangle=2\left\langle\eta\left(\boldsymbol{x}_{1}\right) \eta\left(\boldsymbol{x}_{2}\right)\right\rangle .
$$


For flat cortical geometries, we assume that the correlation function separates into an horizontal and a columnar part

$$
C\left(\boldsymbol{x}_{1}, \boldsymbol{x}_{2}\right)=\tilde{C}_{h}\left(x_{1}, x_{2}, y_{1}, y_{2}\right) C_{c}\left(z_{1}, z_{2}\right)
$$

In the horizontal direction, we further assume that the statistics of the field are invariant under a translation by a fixed displacement $(\Delta x, \Delta y)$

$$
\tilde{C}_{h}\left(x_{1}, x_{2}, y_{1}, y_{2}\right)=\tilde{C}_{h}\left(x_{1}+\Delta x, x_{2}+\Delta x, y_{1}+\Delta y, y_{2}+\Delta y\right)
$$

reflecting the implicit assumption that no special location exists in orientation domains on average. By choosing $(\Delta x, \Delta y)=-\left(x_{1}, y_{1}\right)$ one coordinate of the horizontal correlation function can be eliminated, thus reducing the parameters of the correlation function to a difference of coordinates

$$
C_{h}^{*}(x, y)=\tilde{C}_{h}(0, x, 0, y) .
$$

Finally, we assume that the statistics of the fields are invariant under a rotation in the horizontal plane by a fixed angle $\beta$

$$
C_{h}^{*}(x, y)=C_{h}^{*}(r \cos (\alpha+\beta), r \sin (\alpha+\beta)),
$$

where $\alpha=\tan ^{-1}(y / x)$ and $r=\sqrt{x^{2}+y^{2}}$. By choosing $\beta=-\alpha$ the horizontal correlation function simplifies to

$$
C_{h}(r)=C_{h}^{*}(r, 0)
$$

and is termed an isotropic orientation field ensemble. The simplified correlation function thus reads

$$
C\left(r, z_{1}, z_{2}\right)=C_{h}(r) C_{c}\left(z_{1}, z_{2}\right)
$$

Notice that for discretized space, the covariance matrix $C\left(\boldsymbol{x}_{1}, \boldsymbol{x}_{2}\right)$ can not be chosen arbitrarily, but instead must be a symmetric and semi-definite matrix.

\section{Pinwheel string density for flat geometries}

Experimentally observed two-dimensional orientation fields of primates and placental carnivores adhere to a common design [22-24]. The common design is defined by a species-invariant set of pinwheel statistics, most strikingly, by the common pinwheel density. In three-dimensional orientation fields, pinwheels generalize to pinwheel strings and pinwheel densities to pinwheel string densities. What is the expectation of the pinwheel string density in maximum entropy models of orientation domain ensembles?

The pinwheel string density of orientation fields $\Psi(\boldsymbol{x})=\xi(\boldsymbol{x})+i \eta(\boldsymbol{x})$ with a columnar dimension can be calculated analytically for Gaussian random fields. The mean length of a pinwheel string per unit volume is given by the pinwheel string density

$$
\rho_{3}(\boldsymbol{x})=\langle\delta(\xi(\boldsymbol{x})) \delta(\eta(\boldsymbol{x}))\|\nabla \xi(\boldsymbol{x}) \times \nabla \eta(\boldsymbol{x})\|\rangle,
$$

where the last term accounts for the length of a segment along a pinwheel string [30]. The unit of the pinwheel string density is length per volume and therefore one over area.

In order to evaluate the pinwheel string density, the joint probability distribution of the field variables

$$
\begin{aligned}
\chi^{T}(\boldsymbol{x}) & =\left(\xi(\boldsymbol{x}), \partial_{x} \xi(\boldsymbol{x}), \partial_{y} \xi(\boldsymbol{x}), \partial_{z} \xi(\boldsymbol{x})\right) \\
\boldsymbol{\nu}^{T}(\boldsymbol{x}) & =\left(\eta(\boldsymbol{x}), \partial_{x} \eta(\boldsymbol{x}), \partial_{y} \eta(\boldsymbol{x}), \partial_{z} \eta(\boldsymbol{x})\right)
\end{aligned}
$$


summarized by $\boldsymbol{\mu}(\boldsymbol{x})^{T}=\left(\boldsymbol{\chi}^{T}(\boldsymbol{x}), \boldsymbol{\nu}^{T}(\boldsymbol{x})\right)$ is required. For Gaussian random fields with shiftsymmetry the mean vanishes and the joint probability distribution is given by

$$
P(\boldsymbol{\mu})=\frac{1}{\sqrt{(2 \pi)^{8} \operatorname{det} \boldsymbol{\Sigma}(\boldsymbol{x})}} e^{-\frac{1}{2} \boldsymbol{\mu}^{T}(\boldsymbol{x}) \boldsymbol{\Sigma}^{-1}(\boldsymbol{x}) \boldsymbol{\mu}(\boldsymbol{x})}
$$

with the covariance matrix

$$
\boldsymbol{\Sigma}(\boldsymbol{x})=\operatorname{diag}\left(\left\langle\chi(\boldsymbol{x}) \chi^{T}(\boldsymbol{x})\right\rangle,\left\langle\boldsymbol{\nu}(\boldsymbol{x}) \boldsymbol{\nu}^{T}(\boldsymbol{x})\right\rangle\right) .
$$

For isotropic orientation domains in the horizontal plane with separating horizontal and columnar correlation functions, the covariance is independent of the horizontal position and therefore given by

$$
\left\langle\chi(z) \chi^{T}(z)\right\rangle=\left\langle\boldsymbol{\nu}(z) \boldsymbol{\nu}^{T}(z)\right\rangle=\frac{1}{2}\left(\begin{array}{cccc}
c_{A}(z) & 0 & 0 & c_{D}(z) \\
0 & c_{G}(z) & 0 & 0 \\
0 & 0 & c_{G}(z) & 0 \\
c_{D}(z) & 0 & 0 & c_{C}(z)
\end{array}\right)
$$

with the parameters

$$
\begin{aligned}
c_{A}(z) & =C_{h}(0) C_{c}(z, z), & c_{D}(z) & =\frac{1}{2} C_{h}(0) \partial_{z} C_{c}(z, z), \\
c_{C}(z) & =\left.C_{h}(0) \partial_{z_{1}} \partial_{z_{2}} C_{c}\left(z_{1}, z_{2}\right)\right|_{z_{1}=z_{2}=z}, & c_{G}(z) & =-\frac{1}{2} C_{c}(z, z) \Delta C_{h}(0),
\end{aligned}
$$

where $\Delta$ denotes the Laplace operator. In the special case of straight pinwheel strings $\partial_{z} \xi(\boldsymbol{x})=$ $\partial_{z} \eta(\boldsymbol{x})=0$, the pinwheel string density is called pinwheel density and counts the number of pinwheel strings piercing a layer of unit area. Isotropic orientation domains in flat geometries have a constant pinwheel density of

$$
\rho_{2}=-\frac{\Delta C_{h}(0)}{4 \pi C_{h}(0)}
$$

see $[30,147,171]$. The pinwheel density of isotropic orientation domains with a typical scale $\Lambda$ is bounded from below by $\rho_{2} \Lambda^{2} \geq \pi$ [147]. This lower bound on the pinwheel density suggests to measure pinwheel densities per typical scale area $\hat{\rho}_{2}=\rho_{2} \Lambda^{2}$ that is a unit-less quantity.

In general, plugging the covariance matrix into the probability distribution and evaluating the integral of the pinwheel string density over $\xi(\boldsymbol{x}), \eta(\boldsymbol{x})$ and the radial parts of the Gaussian distribution leads to the integral

$$
\begin{aligned}
\rho_{3}(z)= & \rho_{2} \frac{2^{5}}{\pi^{2}} \kappa(z) \int_{0}^{\pi} \mathrm{d} \theta_{1} \int_{0}^{\pi} \mathrm{d} \theta_{2} \int_{0}^{2 \pi} \mathrm{d} \phi\left(1-\frac{\phi}{2 \pi}\right) \sin \theta_{1} \sin \theta_{2} \\
& \frac{\sqrt{\sin ^{2} \theta_{2}\left(\sin ^{2} \theta_{1} \sin ^{2} \phi+\cos ^{2} \theta_{1}\right)-\frac{1}{2} \sin 2 \theta_{1} \sin 2 \theta_{2} \cos \phi+\sin ^{2} \theta_{1} \cos ^{2} \theta_{2}}}{\left((\kappa(z)-1) \cos 2 \theta_{1}+\kappa(z)+1\right)^{2}\left((\kappa(z)-1) \cos 2 \theta_{2}+\kappa(z)+1\right)^{2}}
\end{aligned}
$$

that depends on the single dimensionless parameter

$$
\kappa(z)=-\frac{C_{c}(z, z) \Delta C_{h}(0)}{2 C_{h}(0)}\left(\left.\partial_{z_{1}} \partial_{z_{2}} C_{c}\left(z_{1}, z_{2}\right)\right|_{z_{1}=z_{2}=z}-\frac{\left(\partial_{z} C_{c}(z, z)\right)^{2}}{4 C_{c}(z, z)}\right)^{-1} .
$$

The pinwheel string density can be evaluated for isotropic ensembles in all directions where $\kappa(z)=1$ giving a constant pinwheel string density of $\rho_{3}=2 \rho_{2}$. For short columnar correlation lengths $\kappa(z) \ll 1$, the pinwheel string density scales as $\rho_{3}(z) \sim \rho_{2} / \sqrt{\kappa(z)}$. A lower bound for the pinwheel string density is given by the pinwheel density $\rho_{3}(z) \geq \rho_{2}$ and is reached for long columnar correlation length $\kappa(z) \gg 1$. 
A

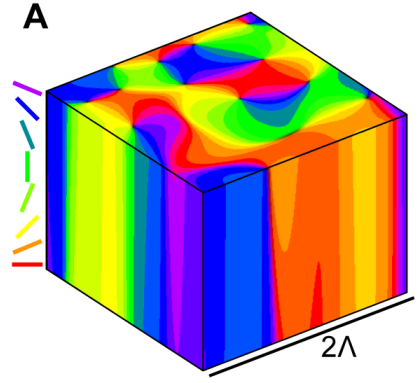

B

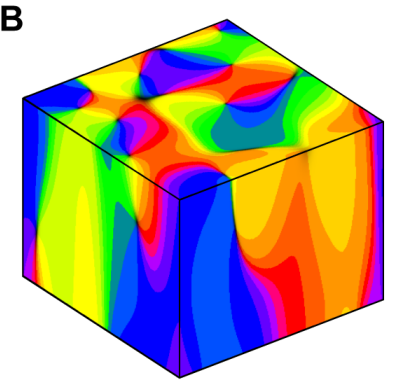

C

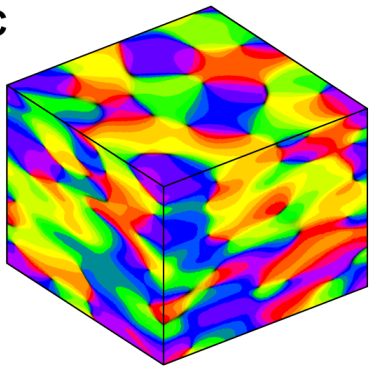

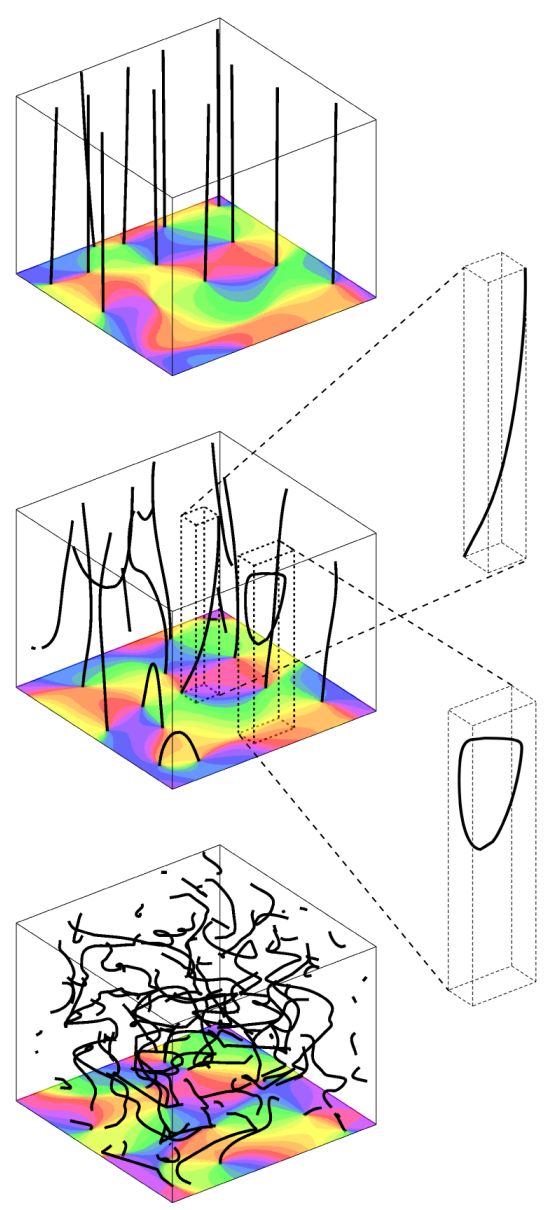

D

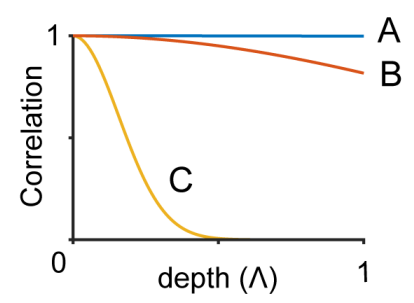

E

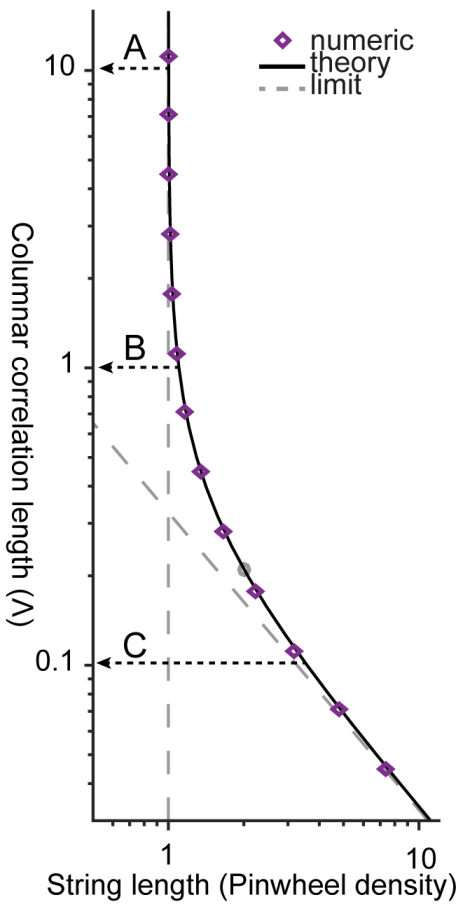

Figure 6.4: Orientation domains in three-dimensional flat geometries with finite columnar correlation length. A-C Left: Synthesized example orientation domains (see Eq. (6.45) and Eq. (6.51)) with finite horizontal typical scale $\Lambda$, cortical thickness $1.6 \Lambda$ and varying finite columnar correlation lengths of $\lambda=10 \Lambda, \lambda=\Lambda$ and $\lambda=0.1 \Lambda$, respectively. Right: Pinwheel strings from left orientation domain examples. Pinwheel strings typically penetrate the entire cortex for large columnar correlation lengths and increase to bend (upper inset, I-motif) and eventually form loops (lower inset, O-motif) with declining columnar correlation length. D Columnar correlation functions $C_{c}(z)$ from Eq. (6.45) for synthesized orientation domain examples of A-C. E Pinwheel string density or mean string length in units of pinwheel density versus columnar correlation length in units of typical scale $\Lambda, \beta=10$ (solid black line: theory from Eq. (6.43), diamonds: measured pinwheel string length of synthesized example orientation domains, see methods, dashed gray lines and gray circle: analytical limits, see main text). 


\section{Orientation domains in three-dimensional flat cortices}

Orientation domains are highly organized in a columnar fashion, but what effect can be expected by relaxing this organization? In order to dissect these effects, a columnar correlation length $\lambda$ is introduced by the translation invariant columnar correlation function

$$
C_{c}\left(z_{1}-z_{2}\right)=e^{-\left(z_{1}-z_{2}\right)^{2} / 2 \lambda^{2}} .
$$

In the horizontal layer, a phenomenological orientation domain model defined by a typical scale $\Lambda$ and a measure of power-spectral density width $\sim 1 / \beta$ from [171] is used, see methods for details. The parameter is fixed to $\beta=10$ in the following. In Fig. 6.4A-C, explicit realizations of three-dimensional orientation domains and their pinwheel strings are depicted for nearly perfect, good and almost vanishing columnar organizations (see Fig. 6.4D for corresponding correlation lengths). The pinwheel strings are straight lines (I-motif) for perfect columnar organization, but are generically curved and even form loops (O-motif) for finite correlation lengths.

The dependence of the pinwheel string length on the columnar organization can be evaluated with the previously derived formula by noting the identities

$$
\hat{\rho}_{2}=\Lambda^{2} \rho_{2}=\pi \frac{(\beta+2) \Gamma\left(\frac{\beta+1}{2}\right)^{2}}{2 \Gamma\left(\frac{\beta+2}{2}\right)^{2}} \quad \text { and } \quad \kappa=\frac{\lambda^{2}(\beta+2) \Gamma\left(\frac{\beta+1}{2}\right)^{2}}{4 \Gamma\left(\frac{\beta+2}{2}\right)^{2}} .
$$

The numerically integrated analytical expression for the pinwheel string density together with the pinwheel string density obtain from synthesized examples is shown in Fig. 6.4E.

\section{Orientation domains in flat cortices with nearly unselective layer $4 \mathrm{C}$}

In macaques and tree shrews, it was observed that neurons in layer $4 \mathrm{C}$ of the primary visual cortex are weakly or even not orientation selective [79, 297, 375, 379-382]. This raises the question of how three-dimensional organization of orientation domains is altered by a variable columnar orientation selectivity profile. Here we consider the extreme case of an orientation unselective layer $4 \mathrm{C}$.

Hence, we extend the previous model by a variable orientation selectivity profile $\sigma(z)$ in the columnar correlation function

$$
C_{c}\left(z_{1}, z_{2}\right)=\sigma\left(z_{1}\right) \sigma\left(z_{2}\right) e^{-\left(z_{1}-z_{2}\right)^{2} / 2 \lambda^{2}}
$$

and investigate the limiting-case scenario of the total absence of orientation selectivity in layer $4 \mathrm{C}$. The combination of the unselective layer $4 \mathrm{C}$ with three different columnar correlation lengths (see Fig. 6.5D) shows an evident difference of orientation domains and their columnar covariance in the synthesized examples, Fig. 6.5A-C, but a seemingly invariant behavior of the pinwheel strings. In fact, the average pinwheel string length is unaffected by the variable orientation selectivity profile $\sigma(z)^{2}$ as the same previously derived identities of Eq. (6.46) hold for the pinwheel string length as validated numerically in Fig. 6.5E.

\section{Orientation domains in gyri-type geometries}

The neocortex is intrinsically curved and in many species folded resulting in a relative inflation of deep cortical layers at gyri and deflation of deep layers at silci. How does a curved cortex affect the spatial organization of orientation selectivity? In order to examine the impact of a 
A

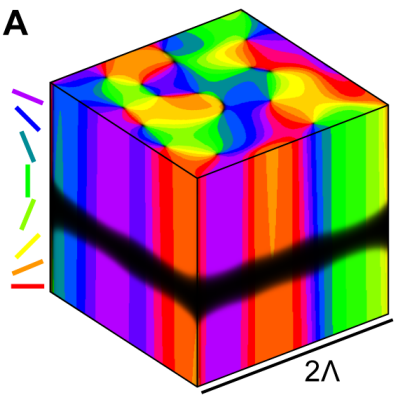

B

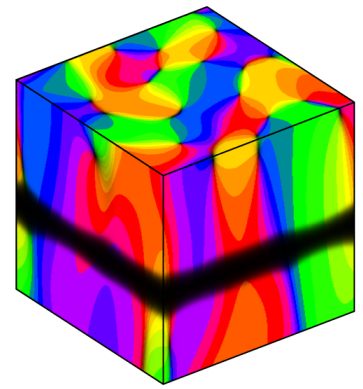

C

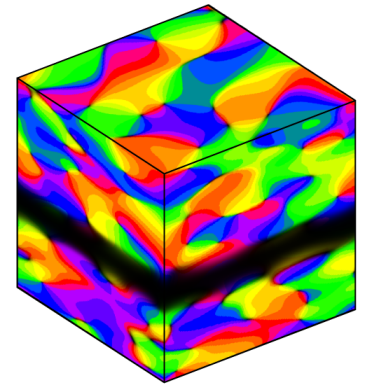

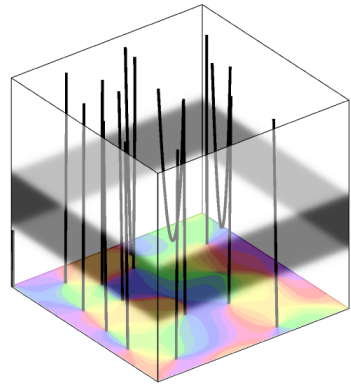
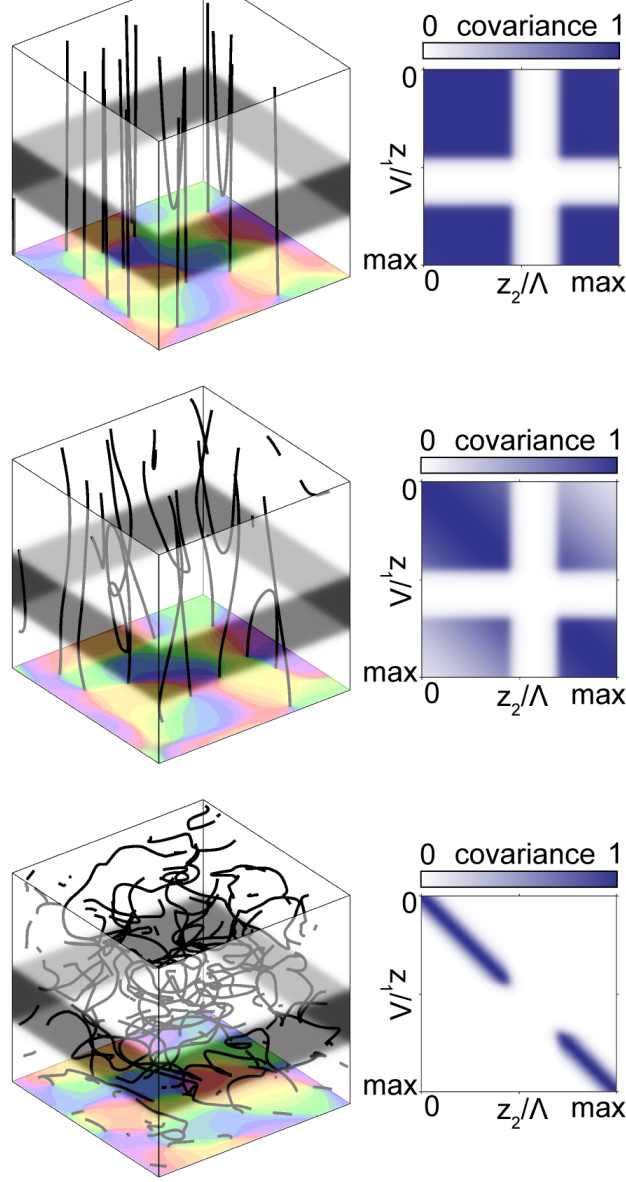

D

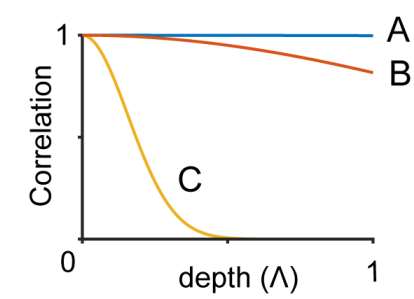

$\mathrm{E}$

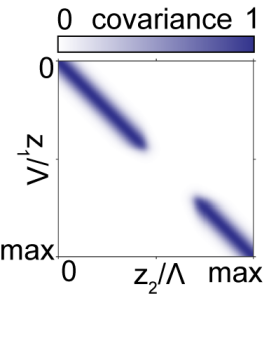

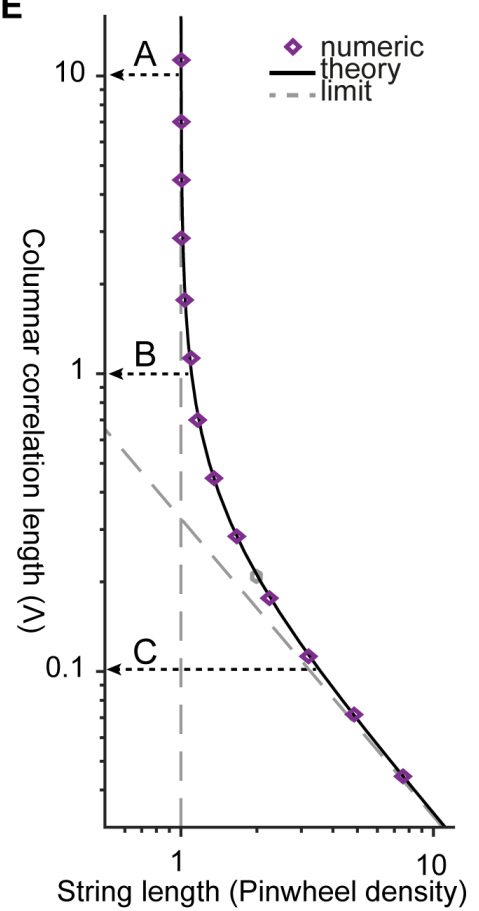

Figure 6.5: Orientation domains in three-dimensional flat geometries with finite columnar correlation length and nearly orientation unselective layer 4. A-C Left: Synthesized example orientation domains (see Eq. (6.47) and Eq. (6.51)) with finite horizontal typical scale $\Lambda$, cortical thickness $2 \Lambda$, nearly orientation unselective layer 4 (black stripe) and varying finite columnar correlation lengths of $\lambda=10 \Lambda, \lambda=\Lambda$ and $\lambda=0.1 \Lambda$, respectively. Center: Pinwheel strings from left orientation domain examples. Right: Columnar covariance of orientation selectivity $C_{c}\left(z_{1}, z_{2}\right)$ from Eq. (6.47). Selectivity profile is given by $\sigma(z)=1-\left(1+\exp \left(-\left(z-z_{0}\right) / \gamma\right)\right)^{-1}+\left(1+\exp \left(-\left(z-z_{1}\right) / \gamma\right)\right)^{-1}, \gamma=0.5 \cdot 10^{-2} \Lambda$, $z_{0} \approx 1.49 \Lambda$ and $z_{1} \approx 0.9 \Lambda$. D Columnar correlation functions $C_{c}(0, z)$ for synthesized orientation domain examples of A-C. E Pinwheel string density or string length in units of pinwheel density versus columnar correlation length in units of typical scale $\Lambda, \beta=10$ (solid black line: theory from Eq. (6.43), diamonds: measured pinwheel string length of synthesized example orientation domains, see methods, dashed gray lines and gray circle: analytical limits, see text). 
A
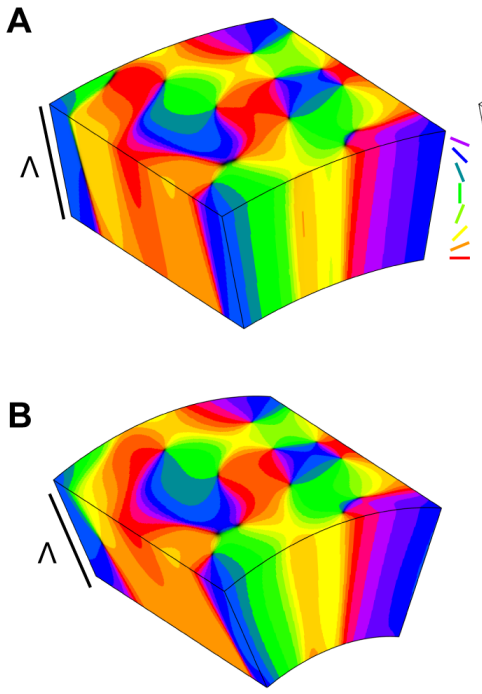

C

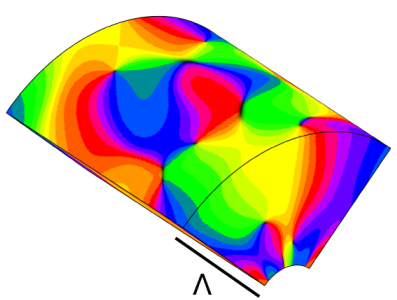

D

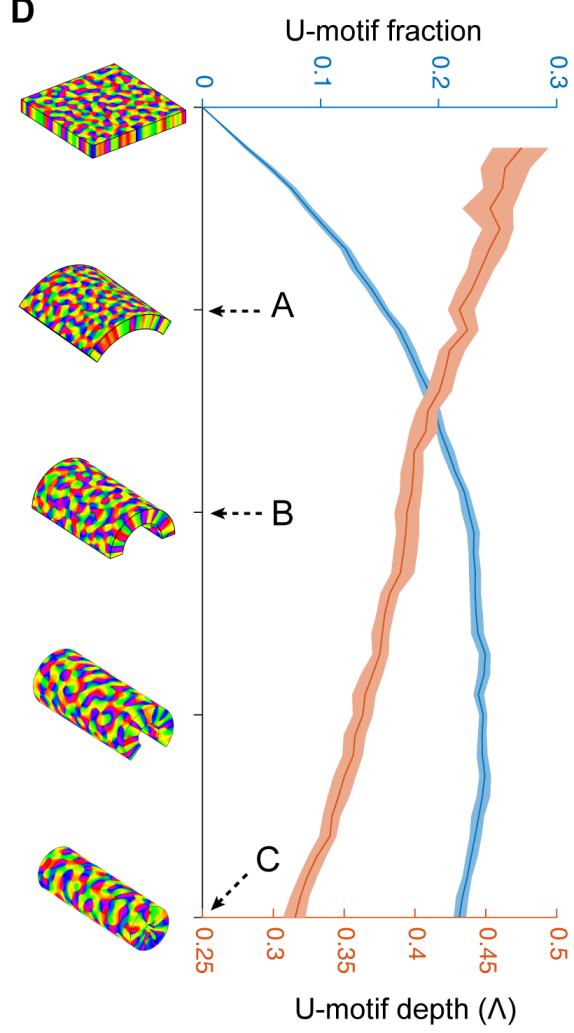

Figure 6.6: Three-dimensional orientation domains in cylindrical, gyri-type geometries. AC Synthesized example orientation domains (see Eq. (6.54)) in cylindrical geometry with fixed typical scale $\Lambda$, columnar correlation length $\lambda=10 \Lambda$ and different curvatures. The area of the outer shell is fixed to $2 \Lambda \times 2 \Lambda$ in all examples. The opening angles are $\mathbf{A} \phi=\pi / 8, \mathbf{B} \phi=\pi / 4, \mathbf{C} \phi=\pi / 2$. D Means (solid lines) and $90 \%$ confidence intervals (shaded areas) of U-motif (see inset of $\mathbf{A}$ ) fraction and their penetration depth in 100 orientation domains of cylindrical geometry versus varying curvature with a cortical thickness of $\Lambda$, columnar correlation length $\lambda=10 \Lambda$, outer shell area of $8 \Lambda \times 8 \Lambda$ and opening angles $\phi \in[0,2 \pi]$

curved cortex, a gyri-type cortex in shape of a cylindrical geometry is utilized as a paradigm model.

The correlation function in each horizontal layer equals the previously chosen correlation function if the curved cortex is flattened to a horizontal plane. The typical scale in each layer is fixed such that the number of typical scales shrinks for deeper layers. Thus, perfect columnar correlation becomes impossible due to the shrinking cortical area with depth. In the limit of large cylindrical radii or equivalently vanishing curvature the correlation functions are equal to the previously used flat cortex with constant columnar orientation selectivity profile (for details see methods). Synthesized examples of large columnar correlation combined with three different curved cortices are depicted in Fig. 6.6A-C. Although orientation domains seem to change only weakly on the surface, pinwheel strings follow more intricate paths for increased curvature. One particular frequently observed pinwheel string path is the U-motif (see inset of Fig. 6.6A) that proliferates and migrates towards the surface for increasing curvature, see Fig. 6.6D. 
A
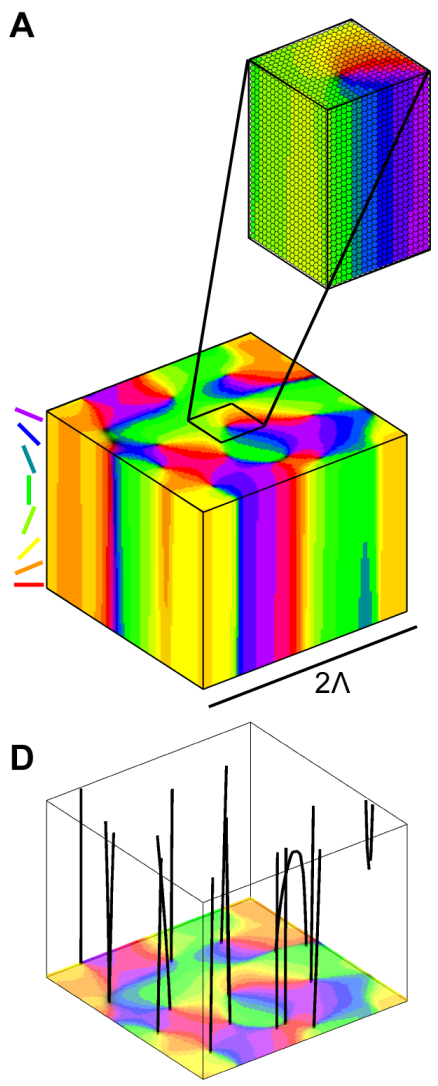

B

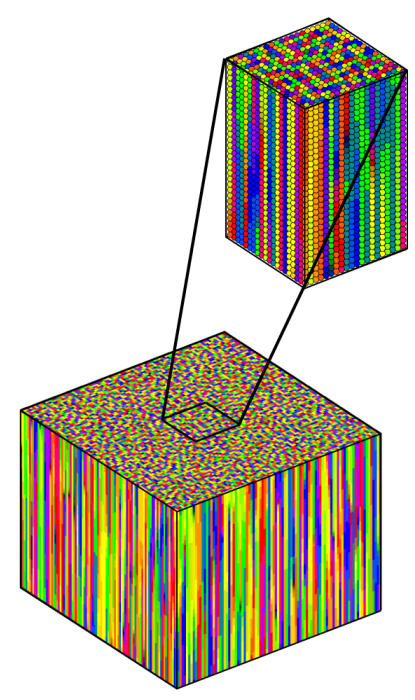

E

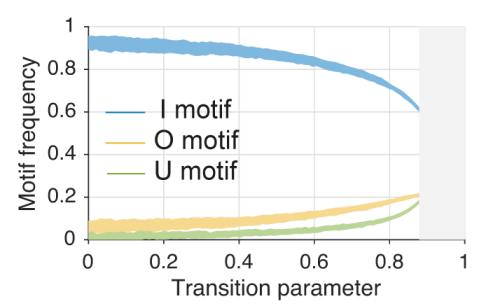

C

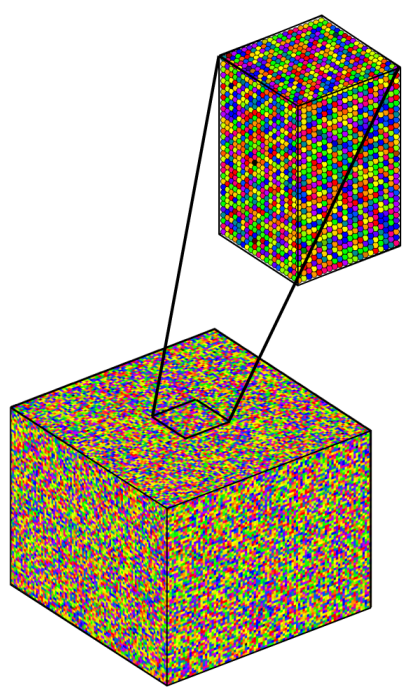

$\mathbf{F}$

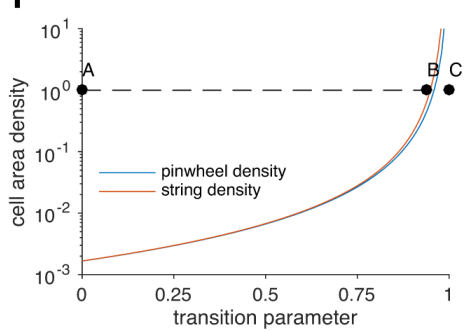

Figure 6.7: Transition between orientation domains and interspersed layout by shrinking correlation lengths. A Synthesized orientation domain layout with typical scale $\Lambda$ and columnar correlation length $\lambda=10 \Lambda$, see Eq. (6.48) with $s=0$. B Synthesized intermediate orientation domain layout with clusters due to decreased correlation lengths, see Eq. (6.48) with $s=0.95$. C Synthesized interspersed orientation layout, see Eq. (6.48) with $s \rightarrow 1$. D Pinwheel strings corresponding to A. E The 95\% confidence interval of different pinwheel motif fractions (see Fig. 6.4 and Fig. 6.6) depending on the transition parameter $s$ in 100 different realizations per $s$. Grey area indicates the region that was computationally too exhaustive due to pinwheel string proliferation. F Pinwheel string and pinwheel density (see Eq. (6.43), Eq. (6.46) and Eq. (6.53)) in units of cell density of $0.002 / \mathrm{\mu m}^{2}$ and $\Lambda=1 \mathrm{~mm}$ versus the transition parameter $s$, see Eq. (6.48). In all examples, the cortical thickness is $1.6 \Lambda_{0}$.

\section{Transitions between orientation domains and interspersed layout}

Spatially organized orientation domains are a feature of the primary visual cortex of primates and placental carnivores [49,60-62]. In contrast, orientation selective neurons in the primary visual cortex of rodents are not spatially organized referred to as interspersed or salt-and-pepper layout [41]. The distinct spatial organizations of orientation preference in these species raises the question of what kind of transitions may have occurred between these organizations in evolution?

Mathematically, a transition between these distinct layouts corresponds to a continuous change of the spatial correlation lengths $\Lambda$ and $\lambda$ from a finite value (orientation domains) to zero (interspersed layout). Despite the vast number of possible transitions, two paradigm transitions appear to be idealized extremes: (i) Decay of spatial correlation lengths and (ii) Invasion of an interspersed layout.

In the first scenario, Fig. 6.7, the transition parameter $s \in[0,1)$ linearly controls the spatial correlation lengths by

$$
\lambda(s)=(1-s) \lambda_{0}, \quad \Lambda(s)=(1-s) \Lambda_{0}
$$


A
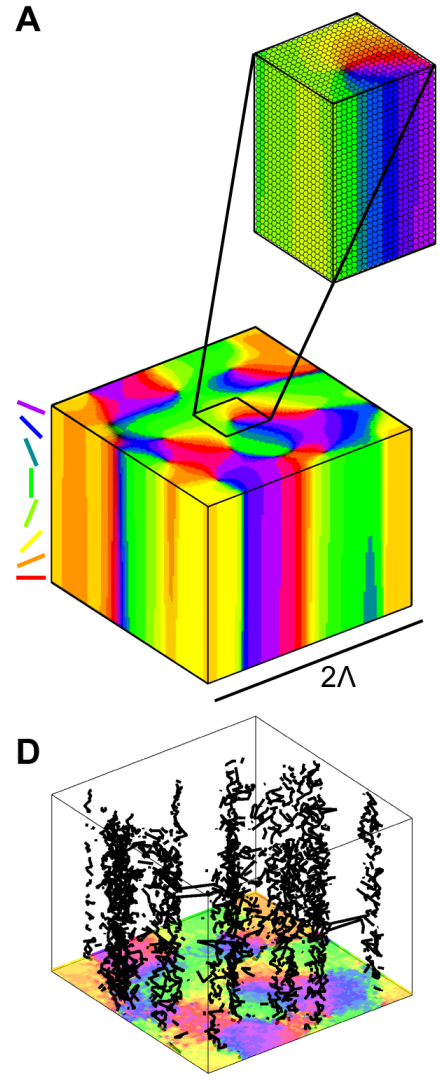

B

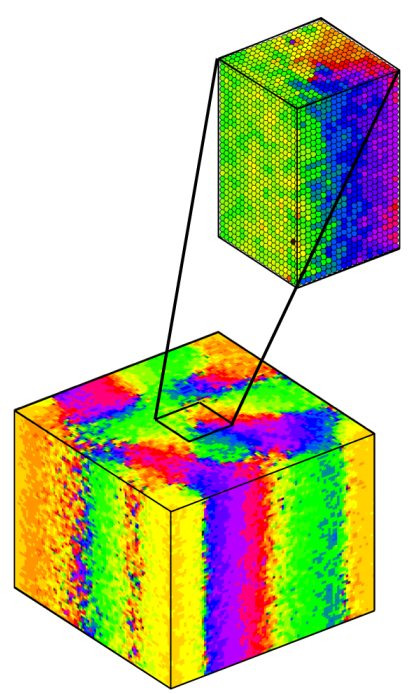

E

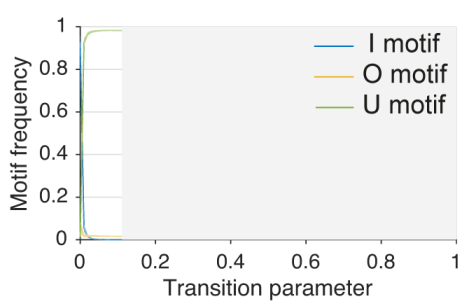

C

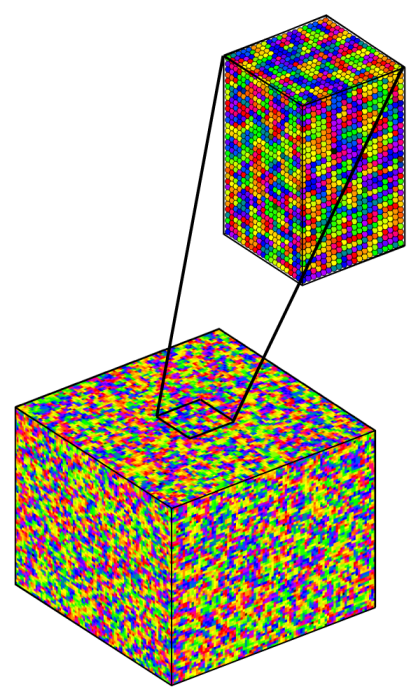

$\mathbf{F}$

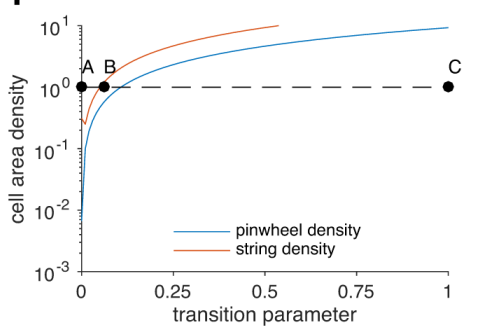

Figure 6.8: Transition between orientation domains and interspersed layout by invasion. A Synthesized orientation domain layout with typical scale $\Lambda$ and columnar correlation length $\lambda=10 \Lambda$, see Eq. (6.49) with $s=0$. B Synthesized intermediate orientation domain layout with clusters due to decreased correlation lengths, see Eq. (6.49) with $s=0.07$. C Synthesized interspersed orientation layout, see Eq. (6.49) with $s \rightarrow 1$. D Pinwheel strings corresponding to B. E The $95 \%$ confidence interval of different pinwheel motif fractions (see Fig. 6.4 and Fig. 6.6) depending on the transition parameter $s$ in 100 different realizations per $s$. Grey area indicates the region that was computationally too exhaustive due to pinwheel string proliferation. F Pinwheel string and pinwheel density (see Eq. (6.43), Eq. (6.46) and Eq. (6.53)) in units of cell density of $0.002 / \mathrm{\mu m}^{2}$ and $\Lambda=1 \mathrm{~mm}$ versus the transition parameter $s$, see Eq. (6.49). In all examples, the cortical thickness is $1.6 \Lambda_{0}$.

with $\lambda_{0}=10 \Lambda_{0}$ and $\Lambda_{0}=1$. Increasing the transition parameter $s$ leads to a decline of orientation domain size and a clustering along the columnar direction that eventually reaches sub-cellular scale and thus is equal to an interspersed layout, see Fig. 6.7A-C. Initial pinwheel strings with strong columnar organization, Fig. 6.7D, are typically penetrating the entire cortex. The frequency of this I-motif decreases with increasing transition parameter $s$. In contrast, the U-motif (pinwheel strings with both ends at vertical boundaries) and O-motif (closed pinwheel strings) fraction increase with the transition parameter $s$, Fig. 6.7E. Pinwheel and pinwheel string density increase monotonically with the transition parameter until orientation domains reach sub-cellular sizes, Fig. 6.7F.

In the second scenario, Fig. 6.8, the transition parameter $s \in[0,1)$ interpolates between both layouts by

$$
C(r, \Delta z, s)=(1-s) C_{\text {domains }}(r, \Delta z)+s C_{\text {intersp. }}(r, \Delta z)
$$

with $C_{\text {domains }}$ the previous correlation function with $\lambda_{0}=10 \Lambda_{0}, \Lambda_{0}=1$ and $C_{\text {intersp. }}(r, \Delta z)=0$ except for $C_{\text {intersp. }}(0,0)=1$. Initial orientation domains with columnar organization are invaded 
A
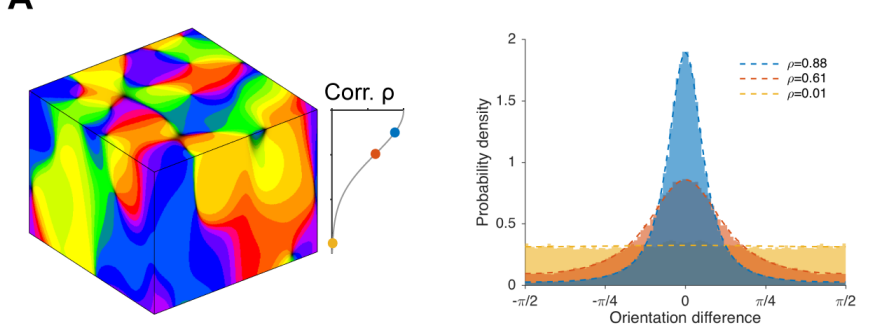

C
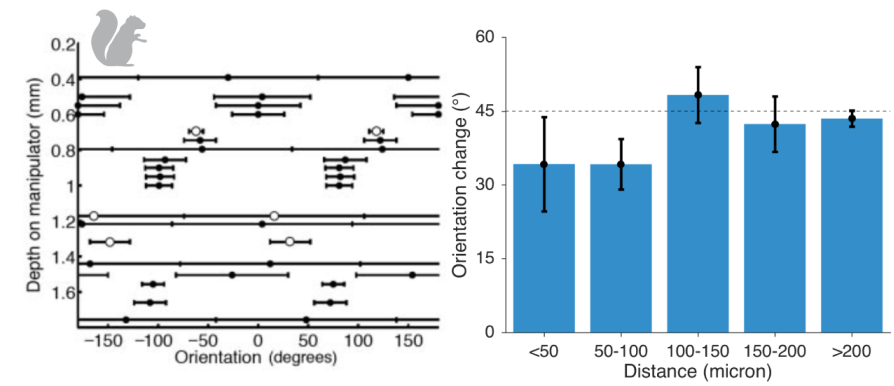

B

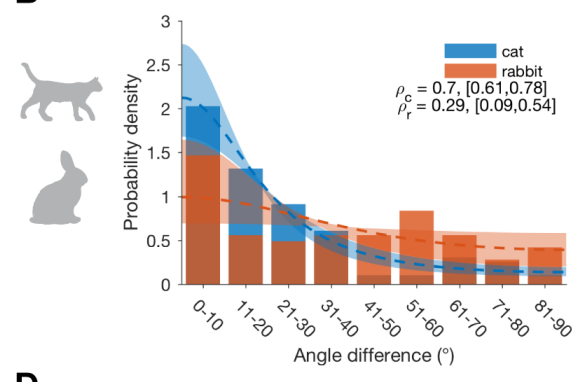

D

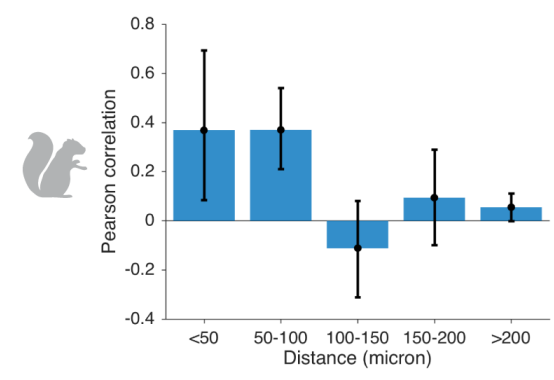

Figure 6.9: Is there evidence for intermediate orientation domain layouts based on orientation difference distributions of laminar layers? A Left: Synthesized orientation domain example, where the Pearson correlation between surface and deeper layers is given by a Gaussian function. Right: Pair-wise orientation difference distributions between surface neurons and at deeper laminar layers with specified Pearson correlations sampled numerically (bins) and analytically (dashed lines). B Orientation difference distributions of surface neurons and $200 \mu \mathrm{m}$ deep neurons recorded by electrode penetrations along a vertical tract indicated by the bars $(n=115$ for cat and $n=83$ for rabbit). Figure based on [48]. Dashed lines indicate the mean of least square fits of the analytical distribution in Eq. (6.50) to bootstrapped data. Shaded areas are the corresponding 95\% confidence intervals. C Left: Small isoorientation clusters were found in gray squirrels. Reprinted with permission from [42]. Right: Average pair-wise preferred orientation difference as a function of vertical distance. Figure based on [42]. D Estimated Pearson correlation coefficients obtained from average orientation change in $\mathbf{C}$ (Eq. (6.50)).

by an interspersed layout. The invaded regions are at low orientation selectivity domains and increase in size with the transition parameter until the total absence of spatial organization, see Fig. 6.8A-C. Ordered pinwheel strings, penetrating the entire columnar direction, form a pinwheel string foam in regions with low orientation selectivity, Fig. 6.8D. The frequency of the I-motif correspondingly decays strongly with the increase of the transition parameter $s$. For finite spatial resolutions, pinwheel strings are typically not closed such that the U-motif frequency strongly increases, while the O-motif frequency decreases with $s$, Fig. 6.8E. Pinwheel and pinwheel string density increase monotonically with the transition parameter and approach cell area density in a regime, where orientation domains are still discernible, Fig. 6.8F.

\section{Is there evidence for intermediate orientation layouts?}

In the framework of orientation field ensembles, the question of intermediate orientation layouts can be posed more precisely. For instance, what shape has the distribution of the pair-wise difference in preferred orientation at two different positions $\theta=\vartheta\left(\boldsymbol{x}_{1}\right)-\vartheta\left(\boldsymbol{x}_{2}\right)$ and which parameters define this distribution? This question can be answered precisely (for details see methods) 
for Gaussian random fields and leads to the distribution of orientation change

$$
\begin{aligned}
P(\theta)= & \frac{\left(1-\rho^{2}\right)}{2 \pi\left(1-\rho^{2} \cos ^{2}(2 \theta)\right)^{3 / 2}} \\
& \left(2 \sqrt{1-\rho^{2} \cos ^{2}(2 \theta)}+2 \rho \cos (2 \theta) \tan ^{-1}\left(\frac{\rho \cos (2 \theta)}{\sqrt{1-\rho^{2} \cos ^{2}(2 \theta)}}\right)+\pi \rho \cos (2 \theta)\right),
\end{aligned}
$$

which depends solely on the Pearson correlation coefficient between the considered neurons $\rho=C\left(\boldsymbol{x}_{1}, \boldsymbol{x}_{2}\right) / \sqrt{C\left(\boldsymbol{x}_{1}, \boldsymbol{x}_{1}\right) C\left(\boldsymbol{x}_{2}, \boldsymbol{x}_{2}\right)}$. The spatial dependence of $\rho$ is omitted for the sake of brevity. A synthesized example of orientation domains with decaying correlation in the columnar direction together with the corresponding distribution of orientation change between the surface and three marked laminar layers is depicted in Fig. 6.9A. The formula can be used to analyze a comparative electrode penetration study of cats and rabbits, where the orientation difference of $200 \mu \mathrm{m}$ vertically separated neurons was recorded [48]. Least square fits to the measurements result in Pearson correlation coefficients of $\rho=0.71$ with the $95 \%$ bootstrapped confidence interval $[0.61,0.78]$ for cats and $\rho=0.28$ with the $95 \%$ bootstrapped confidence interval $[0.09,0.54]$ for rabbits as shown in Fig. 6.9B. The corresponding columnar correlation lengths are $\lambda=0.24 \Lambda(0.20 \Lambda, 0.28 \Lambda)$ for cats and $\lambda=0.13 \mathrm{~mm}(0.09 \mathrm{~mm}, 0.18 \mathrm{~mm})$ for rabbits for a columnar Gaussian correlation profile (see Eq. (6.45)) and a typical scale of $\Lambda=1 \mathrm{~mm}$ (see Fig. 6.11C for typical scale estimation). A similar study of gray squirrels in [42] reported a rarely occurring clustering of orientation selectivity in the columnar direction from which a weak shift of mean orientation change remained for small distances along vertical electrode tracts as shown in Fig. 6.9C. The corresponding Pearson correlation coefficients can be obtained from the mean orientation change and are depicted in Fig. 6.9D.

\subsection{Discussion}

In this article, we presented a framework for the systematic examination of orientation selectivity and their intrinsic statistical properties in three-dimensional geometries. We show explicitly that maximum entropy models of orientation domain ensembles describe random wiring and frozen noise models in a broad range of parameters. For flat cortices, we derived an analytical expression for the average pinwheel string length and its dependence on the columnar correlation length. We showed explicitly that a varying columnar orientation selectivity profile has no influence on the pinwheel string length. In a gyri-type cylindrical geometry, we synthesized orientation domains numerically and found that the U-pinwheel string motif occurred more frequently for large curvatures than for flat cortices. Finally, we presented possible scenarios for the transition from orientation domains to an interspersed rodent-like orientation layout.

The assumption of orientation domains possessing Gaussian statistics brings the advantage of being valid for a model class in its transient and a random wiring scheme, but neglects long term optimization mechanisms of the visual cortex. Supposedly, orientation domains optimize the fine structure of their layout and are thus not accurately described by Gaussian random fields. Assigning a probability for an orientation domain $\Psi$ by a functional $\mathcal{P}[\Psi]$ is still a valid approach for an optimization scheme. However, the probability functional might depend on more moments than merely the first two as in the case of Gaussian statistics. Furthermore, the assumed symmetries of orientation domains might be too idealized. In particular shiftsymmetry requires an average equal representation of all orientations, which is incompatible with the observed cardinal bias [67, 68, 100, 108-114]. Shift-symmetry is also not expected to retain natural scene statistics [126] such as an increased probability of collinear contours [402], see $[138,139,171]$ for more details. 
Previously, orientation domains were only treated statically in two-dimensional planes [135, $145,147,171,173]$ and dynamically in self-organizing models $[27,118,122,128,130,131,263$, 266] except for one mechanistic three-dimensional model in [120]. In the static two-dimensional models a simple scheme for constructing orientation domains was provided for Euclidean [145] and non-Euclidean geometry [173] and an analytical formula for the pinwheel density was derived in $[135,147,171]$. In two-dimensional dynamical models orientation domains produced hexagonal $[27,118,122,130,131,263,266]$ and quasiperiodic patterns $[22,128]$. In the threedimensional model [120] a mechanistic model was used that minimizes a specific energy functional. We additionally contribute a scheme for constructing three-dimensional orientation domains in flat and curved geometries and provide an analytical formula for their mean pinwheel string length in flat geometries. For the dynamical models, we contribute the behavior of the models in the linear regime not only for two dimensions but for three. Finally, this models generalized the results obtained in the mechanistic three-dimensional model to an entire model class in the linear regime and random wiring models.

The proliferation of U-pinwheel string motifs in cylindrical geometries can be experimentally dissected preferably in highly convoluted cortices. The assumption of a constant interlaminar typical scale is crucial for this observation as for a variable typical scale proportional to the laminar layer area is equivalent to deformed flat cortices. In the case of a curved cortex in two directions with constant typical scale, i.e. spherical instead of cylindrical geometry, the U-motif is expected to occur more frequently as well, but with no favored orientation of the motifs. The conservation of the typical scale across layers can be best investigated in gyrencephalic cortices (strongly folded) rather than in lissencephalic cortices (smooth). As the cortical folding correlates with brain weight and cortical surface area [403], species with large brains are favored for that enterprise. More specifically, sheep, macaques, cats and ferrets are highly gyrencephalic, whereas tree shrews, rabbits, gray squirrels and galagos are rather lissencephalic, see [404, 405]. Finally, the cortical folding can be quantified by a gyrification index, which shows that ungulates is the mammalian clade with the highest investigated cortical folding [406].

In [147], it was shown that experimentally observed pinwheel densities are smaller than the lower bound $\pi$ of isotropic random orientation domains with Gaussian statistics suggesting pinwheel annihilation to occur during development. More generally, it is assumed that correlation lengths increase during development. If this is true, then in the three-dimensional framework pinwheel annihilation corresponds to a straightening of lines and, in particular, to the movement of pinwheel strings. An intriguing question arising from moving pinwheel strings is whether there are dedicated columnar layers in which this movement is reduced? Such regions are thus organizational layers, which would suggest these are the originating layers of orientation selectivity and that other layers align according to them.

Where is it expected to find a transition between an interspersed and orientation domain layout? Developmental studies of orientation domains such as [68] suggest that a transition from interspersed to orientation domain layout is unlikely to occur on a developmental time scale. However, on the timescale of million years it was shown that such a transition likely occurred at least twice [22] and all the intermediate organizations must have had a functional advantage. If the transition was continuous and not discontinuous, there should be species with mixture states of orientation domains with columnar organization and an interspersed layout. One putative candidate for a mixture state is the rabbit for which a clustered organization of orientation selectivity was found $[44,46,47]$ that clearly differed from the functional columnar organization of cats and macaques [48]. The reevaluation of vertical electrode penetrations in rabbits, Fig. 6.9B, resulted in small columnar correlation lengths. This is probably due to nonvertically aligned electrodes, since small columnar correlation length were also found for cats that have rather strong columnar organization. Unfortunately, the examination of orientation selectivity in rabbits do not allow conclusive statements of their organization. Independent of 
species, intermediate states should be best discernible in highly curved cortical areas.

For future work, it would be constructive to extend the simplified Gaussian assumption of the ensemble functional $\mathcal{P}[\Psi]$ to more realistic functionals. Based on experimentally observed orientation domains of adult animals belonging to one species the ensemble probability $\mathcal{P}[\Psi]$ could be estimated by including higher moments, which would shed light on the fine structure of orientation domains. The experimental accessibility of three-dimensional orientation domains with 2PLSM/3PLSM, OCT and high-resolution fMRI opens an exciting new research field of functional columnar organization for which this theoretical study provides a generic model.

\subsection{Methods}

\section{Model correlation function in the horizontal plane}

A phenomenogically motivated correlation function for orientation domains in a two-dimensional layer was proposed in [171] that is determined by a typical scale $\Lambda=2 \pi /\langle k\rangle=2 \pi$ and a powerspectral density width $\sim 1 / \beta$. The correlation function in real and Fourier space is defined by

$$
C_{h}(r)={ }_{1} F_{1}\left(\frac{2+\beta}{2}, 1,-\frac{r^{2}}{4 B}\right) \quad \text { and } \quad P_{h}(\|\boldsymbol{k}\|)=A\|\boldsymbol{k}\|^{\beta} e^{-\|\boldsymbol{k}\|^{2} B},
$$

respectively, with the normalization constants

$$
A=\frac{2 \Gamma\left(\frac{2+\beta}{2}\right)^{1+\beta}}{\Gamma\left(\frac{1+\beta}{2}\right)^{2+\beta}} \text { and } B=\frac{\Gamma\left(\frac{2+\beta}{2}\right)^{2}}{\Gamma\left(\frac{1+\beta}{2}\right)^{2}} .
$$

In Fig. 6.10A the correlation function and its Fourier transform is depicted with three synthesized examples shown in Fig. 6.10B. The pinwheel density per typical scale area for this model is shown in Fig. 6.10D and given by the formula

$$
\hat{\rho}_{2}=\Lambda^{2} \rho_{2}=\pi \frac{(\beta+2) \Gamma\left(\frac{\beta+1}{2}\right)^{2}}{2 \Gamma\left(\frac{\beta+2}{2}\right)^{2}} .
$$

A value of $\beta=10$ corresponds approximately to the power-spectral density of galagos, Fig. 6.11, and is therefore used throughout this article.

\section{Three-dimensional model in cylindrical geometry}

In order to account for orientation domains on a gyrus, we idealize a gyrus by a cylindrical geometry. The laminar distance has to be redefined to a distance on the shell of a cylinder. Thus, two points on the same shell of a cylinder $\boldsymbol{x}_{i}=\left(r \cos \phi_{i}, r \sin \phi_{i}, z_{i}\right)$ with $i=1,2$ have a laminar distance of $\sqrt{r^{2}\left(\phi_{1}-\phi_{2}\right)^{2}+\left(z_{1}-z_{2}\right)^{2}}$. This motivates the separation of the cylindrical correlation function into a horizontal (laminar) and a radial (columnar) part

$$
C\left(r_{1}, r_{2}, \phi_{1}, \phi_{2}, z_{1}, z_{2}\right)=C_{h}\left(\sqrt{\bar{r}^{2} \Delta \phi^{2}+\Delta z^{2}}\right) C_{c}(\Delta r)
$$

with the mean position $\bar{r}=\left(r_{1}+r_{2}\right) / 2$, the radial distance $\Delta r=r_{1}-r_{2}$, the phase difference $\Delta \phi=\phi_{1}-\phi_{2}$ and the axial distance $\Delta z=z_{1}-z_{2}$. The horizontal and columnar correlation functions are chosen as in the flat cortex (see Eq. (6.45) and Eq. (6.51)) to

$$
C_{c}(\Delta r)=e^{-\frac{\Delta r^{2}}{2 \lambda^{2}}}, \quad C_{h}(r)={ }_{1} F_{1}\left(\frac{2+\beta}{2}, 1,-\frac{r^{2}}{4 B}\right) .
$$




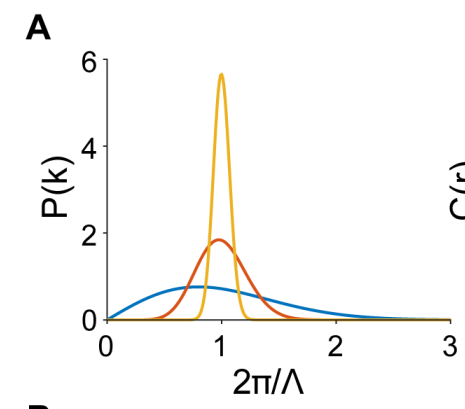

B
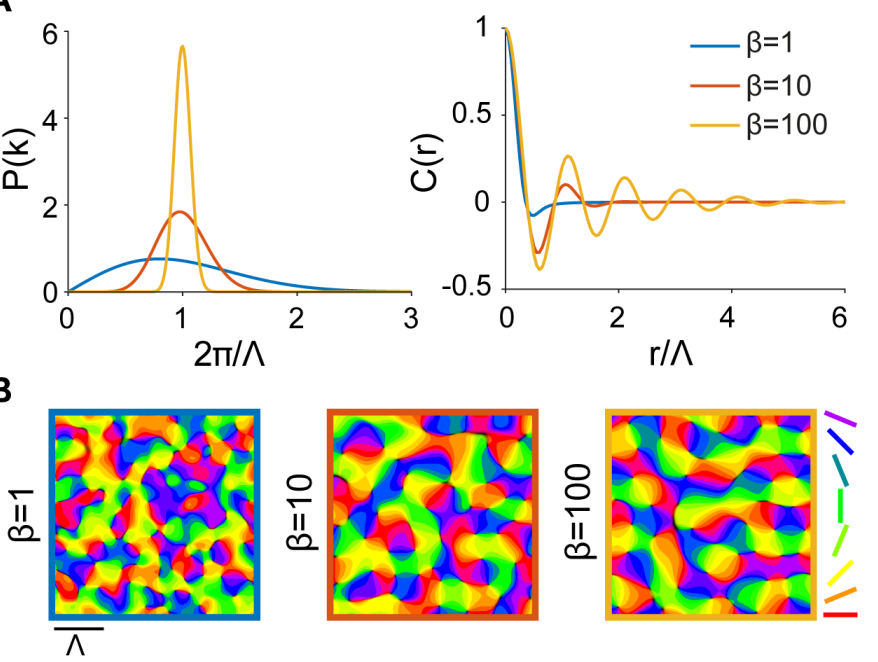

C

D

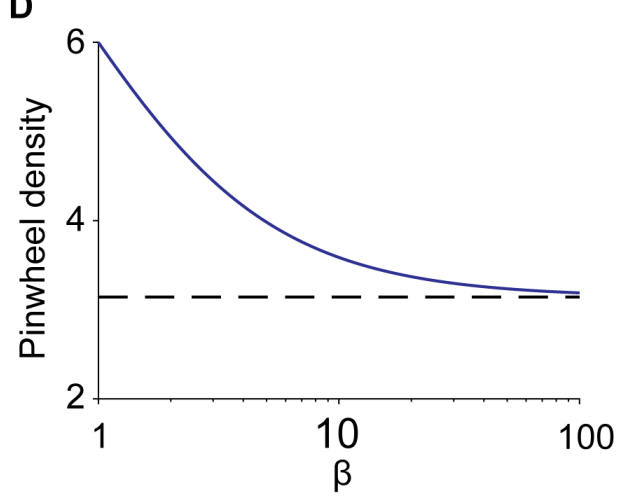

Figure 6.10: Gaussian random field model for two-dimensional orientation domains. A Right: Correlation function $C(r)=\langle\Psi(0) \bar{\Psi}(r)\rangle$ of the orientation field $\Psi$ is shown for different powerspectral density widths $\sim 1 / \beta$, see Eq. (6.51). Left: Fourier transform of the correlation function is depicted, showing that the parameter $\beta$ tunes the power-spectral density widths, while retaining the typical scale. B Synthesized example orientation domains from the correlation function in $\mathbf{A}$ for different power-spectral density widths. The power-spectral density width of $\beta=10$ is the default parameter for all other illustrations, see methods. C Two different types of pinwheels indicated by the gray and black dot, which are surrounded by all orientations in their vicinity. The pinwheel indicated by the gray dot changes preferred orientations clockwise, whereas the other pinwheel (black dot) changes them counterclockwise. D The analytically derived pinwheel density $\hat{\rho}_{2}$ for this orientation domain model from Eq. (6.53).

Notice that the eigenfunctions of the correlation function are given by

$$
\Phi_{l k}(\phi, z, \Delta r, \bar{r})=e^{i l \phi} e^{i k z} \psi_{l k}(\Delta r, \bar{r}) .
$$

The correlation function is hence diagonal in Fourierspace of $\phi$ and $z$

$$
\begin{aligned}
\left\langle e^{i l \phi} e^{i k z}, C_{h}\right\rangle_{\phi_{1}, z_{1}} & =\int_{-\infty}^{\infty} \mathrm{d} z_{1} \int_{-\pi}^{\pi} \mathrm{d} \phi_{1} e^{-i l \phi_{1}} e^{-i k z_{1}} C_{h}\left(\sqrt{\bar{r}^{2} \Delta \phi^{2}+\Delta z^{2}}\right) \\
& =e^{-i l \phi_{2}} e^{-i k z_{2}} \int_{-\infty}^{\infty} \mathrm{d} z \int_{-\pi}^{\pi} \mathrm{d} \phi e^{-i l \phi} e^{-i k z} C_{h}\left(\sqrt{\bar{r}^{2} \phi^{2}+z^{2}}\right) \\
& =\frac{e^{-i l \phi_{2}} e^{-i k z_{2}}}{\bar{r}} \int_{-\infty}^{\infty} \mathrm{d} z \int_{-\bar{r} \pi}^{\bar{r} \pi} \mathrm{d} \phi e^{-i \frac{l}{\bar{r}} y} e^{-i k z} C_{h}\left(\sqrt{y^{2}+z^{2}}\right) \\
& \approx e^{-i l \phi_{2}} e^{-i k z_{2}} \frac{1}{\bar{r}} P_{h}\left(\sqrt{\left(\frac{l}{\bar{r}}\right)^{2}+k^{2}}\right),
\end{aligned}
$$

where in the last step it is assumed that the correlation length of $C_{h}$ is much smaller than $\pi \bar{r}$ and $P_{h}$ denotes the Fourier transform of $C_{h}$. With that we identify the total non-diagonal eigenfunction as

$$
\psi_{l k}(\Delta r, \bar{r})=\frac{1}{\bar{r}} P_{h}\left(\sqrt{\left(\frac{l}{\bar{r}}\right)^{2}+k^{2}}\right) C_{c}(\Delta r) .
$$



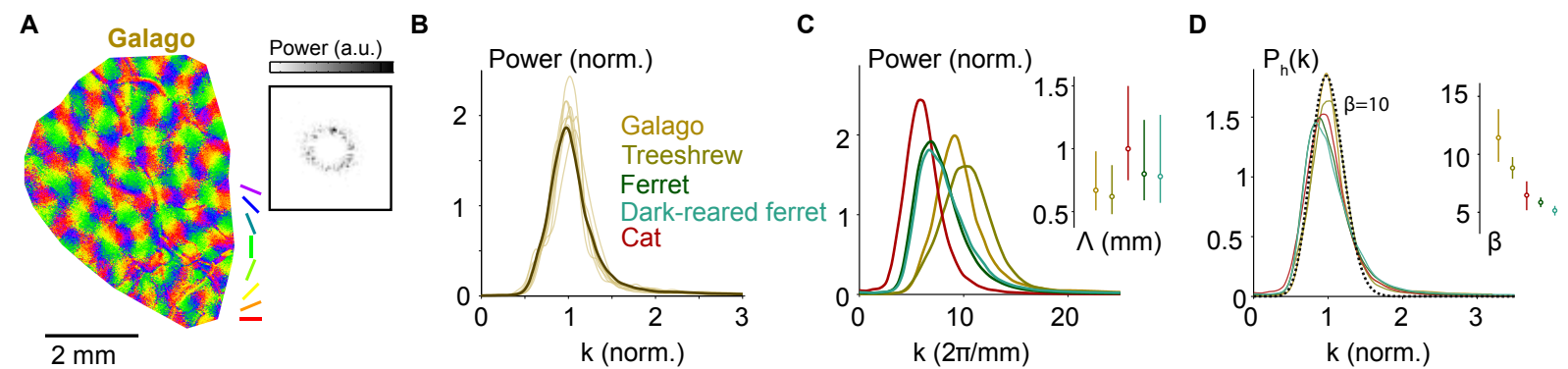

Figure 6.11: Power-spectral density width estimation of orientation domains. A High-pass filtered orientation domain from galago and its power-spectral density. B Radially averaged powerspectral densities of individual galagos (light lines) and their mean (dark line). C Means of radially averaged power-spectral densities from different species. Inset: Mean typical scales $\Lambda$ and $95 \%$ confidence intervals of animals in species. D Power-spectral densities from $\mathbf{C}$ with normalized area in rescaled length scale (solid lines) together with model power-spectral density from Eq. (6.51) with $\beta=10$ (dotted line). Inset: Average $\beta$ and $95 \%$ confidence intervals from least square fits of model to individual animals.

\section{Orientation difference distribution}

The orientation difference distribution at two different positions are defined by

$$
P(\theta)=\left\langle\delta\left(\frac{1}{2} \tan ^{-1}\left(\frac{\xi\left(\boldsymbol{x}_{1}\right)}{\eta\left(\boldsymbol{x}_{1}\right)}\right)-\frac{1}{2} \tan ^{-1}\left(\frac{\xi\left(\boldsymbol{x}_{2}\right)}{\eta\left(\boldsymbol{x}_{2}\right)}\right)-\theta\right)\right\rangle .
$$

For shift-symmetric fields with vanishing first moment the probability distribution is equal for real and imaginary part $p\left(\xi\left(\boldsymbol{x}_{1}\right), \xi\left(\boldsymbol{x}_{2}\right)\right)=p\left(\eta\left(\boldsymbol{x}_{1}\right), \eta\left(\boldsymbol{x}_{2}\right)\right)$ and given by

$$
p\left(\xi\left(\boldsymbol{x}_{1}\right), \xi\left(\boldsymbol{x}_{2}\right)\right)=\frac{1}{\sqrt{(2 \pi)^{2} \operatorname{det} \boldsymbol{\Sigma}\left(\boldsymbol{x}_{1}, \boldsymbol{x}_{2}\right)}} \exp \left(-\frac{1}{2}\left(\xi\left(\boldsymbol{x}_{1}\right), \xi\left(\boldsymbol{x}_{2}\right)\right) \boldsymbol{\Sigma}^{-1}\left(\boldsymbol{x}_{1}, \boldsymbol{x}_{2}\right)\left(\xi\left(\boldsymbol{x}_{1}\right), \xi\left(\boldsymbol{x}_{2}\right)\right)^{T}\right)
$$

with the covariance matrix

$$
\boldsymbol{\Sigma}\left(\boldsymbol{x}_{1}, \boldsymbol{x}_{2}\right)=\frac{1}{2}\left(\begin{array}{ll}
C\left(\boldsymbol{x}_{1}, \boldsymbol{x}_{1}\right) & C\left(\boldsymbol{x}_{1}, \boldsymbol{x}_{2}\right) \\
C\left(\boldsymbol{x}_{1}, \boldsymbol{x}_{2}\right) & C\left(\boldsymbol{x}_{2}, \boldsymbol{x}_{2}\right)
\end{array}\right)
$$

and the correlation function $C\left(\boldsymbol{x}_{1}, \boldsymbol{x}_{2}\right)=2\left\langle\xi\left(\boldsymbol{x}_{1}\right) \xi\left(\boldsymbol{x}_{2}\right)\right\rangle=2\left\langle\eta\left(\boldsymbol{x}_{1}\right) \eta\left(\boldsymbol{x}_{2}\right)\right\rangle$. Evaluating the integral leads to

$$
\begin{aligned}
P(\theta)= & \frac{\left(1-\rho^{2}\right)}{2 \pi\left(1-\rho^{2} \cos ^{2}(2 \theta)\right)^{3 / 2}} \\
& \left(2 \sqrt{1-\rho^{2} \cos ^{2}(2 \theta)}+2 \rho \cos (2 \theta) \tan ^{-1}\left(\frac{\rho \cos (2 \theta)}{\sqrt{1-\rho^{2} \cos ^{2}(2 \theta)}}\right)+\pi \rho \cos (2 \theta)\right),
\end{aligned}
$$

where the spatial dependence of the Pearson correlation coefficient

$$
\rho=C\left(\boldsymbol{x}_{1}, \boldsymbol{x}_{2}\right) / \sqrt{C\left(\boldsymbol{x}_{1}, \boldsymbol{x}_{1}\right) C\left(\boldsymbol{x}_{2}, \boldsymbol{x}_{2}\right)}
$$

is omitted for the sake of brevity.

\section{Gaussian ensembles are maximum entropy models}

The maximum entropy principle goes back to the seminal work of Jaynes [141, 142] and seeks for the least structured probability distribution that fulfills a set of statistical properties. The 
probability distribution has maximal entropy, while in our case reproduces a given covariance. In discrete space with the abbreviations $\Psi_{i}=\Psi\left(\boldsymbol{x}_{i}\right), \boldsymbol{\Psi}=\left(\Psi_{1}, \Psi_{2}, \ldots, \Psi_{N}\right)$ and the correlation function $(\boldsymbol{\Sigma})_{i j}=C\left(\boldsymbol{x}_{i}, \boldsymbol{x}_{j}\right)$, the following Lagrangian

$$
\begin{aligned}
\mathcal{L}= & -\int_{-\infty}^{\infty}\left(\prod_{k=1}^{N} \mathrm{~d} \Psi_{k}\right) p(\boldsymbol{\Psi}) \log p(\boldsymbol{\Psi})-\mu\left[\int_{-\infty}^{\infty}\left(\prod_{k=1}^{N} \mathrm{~d} \Psi_{k}\right) p(\boldsymbol{\Psi})-1\right] \\
& -\sum_{i, j} \lambda_{i j}\left[\int_{-\infty}^{\infty}\left(\prod_{k=1}^{N} \mathrm{~d} \Psi_{k}\right) p(\boldsymbol{\Psi}) \Psi_{i} \Psi_{j}-(\boldsymbol{\Sigma})_{i j}\right]
\end{aligned}
$$

needs to be maximized with respect to the multivariate distribution $p(\boldsymbol{\Psi})$ and the Lagrangian multipliers $\mu$ and $\lambda_{i j}$ for normalization and the correlation function, respectively. Optimizing the expression with respect to the probability distribution $\delta \mathcal{L} / \delta p=0$ gives

$$
p(\Psi)=e^{-1-\mu-\sum_{i j} \lambda_{i j} \Psi_{i} \Psi_{j}} .
$$

The normalization conditions $\partial_{\mu} \mathcal{L}=0$ can be evaluated by noticing that $(\boldsymbol{\lambda})_{i j}=\lambda_{i j}$ is a symmetric matrix. Then by the unitary transformation of the field onto the eigenbasis of the matrix $\boldsymbol{U} \boldsymbol{\lambda} \boldsymbol{U}^{T}=\operatorname{diag}\left(\tilde{\lambda}_{1}, \tilde{\lambda}_{2}, \ldots, \tilde{\lambda}_{N}\right)$ and $\tilde{\boldsymbol{\Psi}}=\boldsymbol{U} \boldsymbol{\Psi}$ the normalization condition becomes

$$
\begin{aligned}
1 & =\int_{-\infty}^{\infty}\left(\prod_{k=1}^{N} \mathrm{~d} \Psi_{k}\right) p(\boldsymbol{\Psi})=\int_{-\infty}^{\infty}\left(\prod_{k=1}^{N} \mathrm{~d} \tilde{\Psi}_{k}\right) e^{-1-\mu-\sum_{i} \tilde{\lambda}_{i} \tilde{\Psi}_{i}^{2}} \\
& =e^{-1-\mu} \int_{-\infty}^{\infty}\left(\prod_{k=1}^{N} \mathrm{~d} \tilde{\Psi}_{k} e^{-\tilde{\lambda}_{k} \tilde{\Psi}_{k}^{2}}\right)=e^{-1-\mu}\left(\prod_{k=1}^{N} \sqrt{\frac{\pi}{\tilde{\lambda}_{k}}}\right) \\
& =\frac{\pi^{N / 2} e^{-1-\mu}}{\sqrt{\operatorname{det} \boldsymbol{\lambda}}} .
\end{aligned}
$$

This defines the first Lagrange multiplier to $\mu=\log \pi^{N / 2}-\log \sqrt{\operatorname{det} \boldsymbol{\lambda}}-1$. The remaining Lagrange multipliers are defined by $\partial_{\lambda_{i j}} \mathcal{L}=0$. This can be solved again by the rotation onto the eigenbasis of $\boldsymbol{\lambda}$

$$
\begin{aligned}
& (\boldsymbol{\Sigma})_{i j}=\int_{-\infty}^{\infty}\left(\prod_{k=1}^{N} \mathrm{~d} \Psi_{k}\right) p(\boldsymbol{\Psi}) \Psi_{i} \Psi_{j} \\
& =\frac{\sqrt{\operatorname{det} \lambda}}{\pi^{N / 2}} \sum_{l m} \int_{-\infty}^{\infty}\left(\prod_{k=1}^{N} \mathrm{~d} \tilde{\Psi}_{k}\right) U_{l i} U_{m j} \tilde{\Psi}_{l} \tilde{\Psi}_{m} e^{-\sum_{i} \tilde{\lambda}_{i} \tilde{\Psi}_{i}^{2}} \\
& =\frac{\sqrt{\operatorname{det} \lambda}}{\pi^{N / 2}} \sum_{l m} U_{l i} U_{m j} \int_{-\infty}^{\infty}\left(\prod_{k=1}^{N} \mathrm{~d} \tilde{\Psi}_{k} e^{-\tilde{\lambda}_{k}} \tilde{\Psi}_{k}^{2}\right) \tilde{\Psi}_{l} \tilde{\Psi}_{m} \\
& =\sum_{l} U_{l i} U_{l j} \frac{1}{2 \tilde{\lambda}_{l}} \text {. }
\end{aligned}
$$

This equation can be solved by $\boldsymbol{\lambda}=\frac{1}{2} \boldsymbol{U} \boldsymbol{\Sigma}^{-1} \boldsymbol{U}^{T}$ with $(\boldsymbol{U})_{i j}=U_{i j}$ yielding the final shape of the distribution

$$
p(\Psi)=\frac{1}{\sqrt{(2 \pi)^{N} \operatorname{det} \boldsymbol{\Sigma}}} e^{-\frac{1}{2} \boldsymbol{\Psi}^{T} \boldsymbol{\Sigma}^{-1} \boldsymbol{\Psi}}
$$


that defines a Gaussian random field in the limit of continuous space $(N \rightarrow \infty)$. Notice that in the continuous limit $N \rightarrow \infty$, the integrals become path integrals

$$
\int_{-\infty}^{\infty}\left(\prod_{k=1}^{N} \mathrm{~d} \Psi_{k}\right) \rightarrow \int \mathcal{D} \Psi
$$

and limits have to be taken after computing an expectation value.

\section{Numerical methods}

For translation invariant fields the correlation function $C\left(\boldsymbol{x}_{1}-\boldsymbol{x}_{2}\right)$ is diagonal in Fourier space and given by $P(\boldsymbol{k})$. The Fourier transform of a field with the specified correlation function is synthesized by drawing Gaussian random numbers $u, v \sim \mathcal{N}(0,1)$ for all $\boldsymbol{k}$ and scaling them by $\tilde{\Psi}(\boldsymbol{k})=\sqrt{P(\boldsymbol{k})}(u+i v)$.

If the correlation function is not diagonalizable, a brute-force method was used for field synthesis. In discrete space, the correlation function $C\left(\boldsymbol{x}_{1}, \boldsymbol{x}_{2}\right)$ becomes a covariance matrix $(\boldsymbol{\Sigma})_{m n}=C\left(x_{1}^{m}, x_{2}^{n}\right)$ with the spatial variable $\boldsymbol{x}_{i}=\left(x_{i}^{1}, x_{i}^{2}, \ldots, x_{i}^{N}\right)$ for $i=1,2$. A Gaussian random field with the specified covariance matrix is obtained by transforming Gaussian random vectors $\boldsymbol{u}, \boldsymbol{v} \sim \mathcal{N}(0, \mathbf{1})$ by $\Psi(\boldsymbol{x})=\sqrt{\boldsymbol{\Sigma}}(\boldsymbol{u}+i \boldsymbol{v})$.

For a $d$ dimensional field with $N$ points per dimension, the computational complexity for the first method is $\mathcal{O}\left(N^{d} \log \left(N^{d}\right)\right)$ due to the fast Fourier transform and for the second method $\mathcal{O}\left(N^{3 d}\right)$ due to the diagonalization required for $\sqrt{\Sigma}$.

Orientation domains were synthesized by $256 \times 256 \times\left(128 \cdot L_{\text {ctx }}\right)$ pixels (length $\mathrm{x}$ width $\mathrm{x}$ depth) with an aspect ratio of $2 \Lambda \times 2 \Lambda \times L_{\mathrm{ctx}} \Lambda$ and a cortical depth of $L_{\mathrm{ctx}}=1.6$ and $L_{\mathrm{ctx}}=2$ for Fig. 6.4A-C and Fig. 6.5A-C, respectively. Numerical computations of the pinwheel string density, Fig. 6.4E , were conducted with periodic boundary conditions and required a refined discretization of $256 \times 256 \times 512$ pixels in an aspect ratio of $8 \Lambda \times 8 \Lambda \times L_{z} \Lambda$ with $L_{z}=160 \lambda / \Lambda$. The variable cortical thickness allowed a calculation of the pinwheel string density in a single synthesized orientation domain. For mathematical convenience, the same procedure was applied to the case of an unselective layer 4C, Fig. 6.5E, where the relative size of the unselective layers to the cortical depth remained fixed, while the depth was varied. Thus the actual size of the unselective layer $4 \mathrm{C}$ varied that should lead to an amplified difference to the analytical pinwheel string density if such a difference is present. However, no deviation between the numerical and analytical pinwheel string density are observed that justifies this approach. In cylindrical geometries, Fig. 6.6A-C, the resolution was $256 \times 256 \times 128$ pixels (height $\mathrm{x}$ azimuth $\mathrm{x}$ radius) with an aspect ratio of the outer shell $2 \Lambda \times 2 \Lambda$ and a depth of $\Lambda$. The calculation of U-motif frequencies and their depth was conducted with the same parameters except for the aspect ratio of the outer shell that was adjusted to $8 \Lambda \times 8 \Lambda$. In order to depict realistic cellular sizes in the synthesized orientation fields of the transitions, Fig. 6.7A-C and Fig. 6.8A-C, the length of a pixel was adjusted to cell densities of $10^{5}$ cells $/ \mathrm{mm}^{2}$ with a typical scale of $\Lambda=1 \mathrm{~mm}$. The resolution hence became $93 \times 93 \times 75$ pixels with an aspect ratio of $2 \Lambda \times 2 \Lambda \times 1.6 \Lambda$. For the calculation of the pinwheel string motifs, Fig. 6.7E and Fig. 6.8E, an enhanced spatial resolution of $256 \times 256 \times 102$ pixels with an aspect ratio of $4 \Lambda \times 4 \Lambda \times 1.6 \Lambda$ was used. 


\section{Discussion}

In this thesis, we theoretically examined the dynamic emergence, three-dimensional layout and the susceptibility to measurement noise of visual cortical architectures with analytical and numerical techniques from dynamical systems, pattern formation theory [28, 29] and statistical physics [30, 31].

The relative importance of genes and environments is a long-standing question in various fields. The answer to this question is unknown in general and in the nervous system perhaps most advanced at the first visual processing stage, the primary visual cortex (V1). Developmental mechanisms of visual cortical architecture are debated and both experimental and theoretical research is mostly focused on neuronal activity. Therefore, it is crucial to fill the theoretical gap and investigate possible genetic mechanisms for the determination of visual cortical architecture. In order to assess the quality of models during this endeavor, it is inevitable to quantitatively examine their functional architecture.

The core theoretical finding of this thesis is that a biologically plausible genetic mechanism can quantitatively encode visual cortical architectures by a small number of morphogens. This is the first time to our knowledge that such a mechanism has been identified. Importantly, it circumvents the alleged information bottleneck of the genome for hardwiring the cortex by a small number of self-organizing morphogens. The encoded functional architectures were found to be in good quantitative agreement with experimental data based on the comparison of their pinwheel configurations. Species-invariant pinwheel configuration statistics, constituting a common design, were theoretically examined in maximum entropy models of two-dimensional orientation domains. We further established a theoretical framework for high-precision measurements of pinwheel configurations under measurement noise. This paves the way for an improved model benchmarking framework for functional visual cortical architectures and informs future experiments. Finally, orientation domains in three-dimensional geometries pointed to cortical regions of high curvature that are prone to non-columnar organizations and are thus expected to be informative about evolutionary transitions of the functional architecture.

In the following, the results of the chapters will be recapitulated and related to previous work. Subsequently, future fruitful research directions that originated from or are related to this work will be discussed.

\subsection{Summary and relation to previous work}

\section{Genetic assimilation of visual cortical architecture}

In Chapter 3, we devised a transcellular genetic network that can quantitatively encode functional visual cortical architecture. In this model, the spatial organization of orientation pref- 
erence is encoded in the difference of morphogen concentrations. We identified active neural transport and trans-neuronal signaling as well as joined dynamics of morphogens and the connectome as key ingredients for such a genetic mechanism. The mechanism might support visual cortical development caused by neuronal activity and can serve as a redundant plan in the absence of instructive neuronal activity. This theory provides predictions for experimental tests of morphogen focal and dualsite overexpressions to differentiate between distinct genetic mechanisms based on self-organization and gradient read-out. The results thus help to clarify the relative importance of genes and environmental factors.

This work shows that the apparent information bottleneck of the genome for wiring up the cortex [27] can be circumvented by a small number of self-organizing morphogens. It is thus conceivable that complex cortical circuits are supported or even shaped by genes. In the presence of instructive neuronal activity, the genetic network might support targeting and pruning of axons similar to synapse formation by chemoaffinity $[2,83,204]$. In the absence of instructive neuronal activity, the mechanism might serve as a redundant plan for visual cortical development and thus provides a novel view on the extreme robustness of visual cortical architecture under dark-rearing [14]. Hence, genetic information potentially extends the repertoire of synapse formation by chemoaffinity $[2,83,204]$ and neuronal activity [407, 408]. Along that line of thought that genetic information reach further in cortical development is the experimental discovery of (i) a molecular correlate for ocular dominance bands [25] and (ii) the homeoprotein Otx2 that is activity-dependent transported from the retina to V1 and involved in regulating the critical period, a period of high cortical plasticity [26]. More generally, the feasibility of transcellular genetic networks generating complex patterns supports the idea of an important role of contactdependent long-range transport not only in the brain but also in organ development and function [212]. The basis of the devised genetic network model is a universality model class for visual cortical development $[22,128-131,136-139,262]$ that we extended by a biologically plausible, genetic mechanism. Since the orientation encoding part of the genetic network is equivalent to this universality class, previous work such as a coupling to ocular dominance $[129-131,137]$ and a transition to an interspersed orientation layout [140] can be easily incorporated. We found that joined dynamics of the connectome and morphogens are essential for the emergence of functional architectures. This finding transfers directly to the universality class [128] by substituting morphogen dynamics with orientation preference dynamics. Therefore, joined dynamics of orientation preference and the connectome appears to be a general requirement for visual cortical development.

\section{Pinwheel configuration in maximum entropy models of orientation domains}

In Chapter 4, we reviewed previous work on the link between pinwheel configuration statistics and orientation domain ensembles and extended it to all pinwheel configuration statistics of the common design. This examination highlights informative measures of pinwheel configurations and their link to orientation domain ensembles.

This research generalized the pinwheel (topological defect) density of orientation fields (Gaussian random fields) with vanishing mean that was previously only derived for ensembles with a specific set of symmetries [30, 31, 135, 147, 170, 171]. Pinwheel correlation functions of isotropic and shift-symmetric orientation fields derived earlier in another context [30] were combined with an approximated scheme of the nearest neighbor distribution, see [159]. The pinwheel correlation was further used to derive an exact expression for the pinwheel density fluctuations in subregions extending previous work [278, 279]. 


\section{The pinwheel configuration: theoretical significance and precision measurement}

In Chapter 5, we reviewed theoretical arguments for the complexity reduction of the functional visual cortical architecture to the pinwheel configuration and subsequently examined its susceptibility to measurement noise. The results provide a formalism for an extrapolation method of the pinwheel density to the zero noise limit. They also provide an approximated analytical expression for confidence regions of pinwheel centers. Finally, the results enable better estimations from available data, provide a framework to discuss measurement strategies and highlight recording techniques capturing spiking activity for precise pinwheel configuration measurements. The precise quantitative determination of pinwheel positions and their confidence regions will provide an enhanced framework for model benchmarking.

Mathematically, this work extends previous studies on maximum entropy ensembles with vanishing means [31] to ensembles with means given by stationary orientation fields. It extends specific calculations of pinwheel densities $[135,147,170,171]$ to a general analytical formula. The finding of pinwheel confidence regions and more generally the pinwheel observation probability can be further used to identify informative regions of transitions between distinct states of functional architecture presented in Chapter 6. The pinwheel observation probability directly transfers to orientation domain regions that may initially be invaded by an interspersed layout.

\section{Three-dimensional organization of orientation selectivity in cortical tissue}

In Chapter 6, we systematically examined maximum entropy models of three-dimensional functional visual cortical architectures in different geometries. In three dimensions, pinwheels generalize to pinwheel strings and are generically curved for finite columnar correlation lengths. In flat geometries, we derived an analytical expression for the average pinwheel string length and its dependence on the columnar correlation length. We showed explicitly that a varying columnar orientation selectivity profile has no influence on the pinwheel string length. In gyritype cylindrical geometries, we synthesized orientation domains numerically and found that the U-pinwheel string motif emerges more frequently for large curvatures than for flat cortices. Finally, we presented scenarios for the transition from orientation domains to an interspersed rodent-like orientation layout (salt-and-pepper). This work describes universal transient states of entire model classes in a broad parameter regime and thus provides a thorough theoretical understanding of three-dimensional functional architectures. The theory enables examining possible evolutionary transitions between different functional architectures. Intermediate organizations that are neither organized smoothly in orientation columns nor in an interspersed layout, but instead are composed of, for instance, iso-orientation clusters may still exist.

Although two-dimensional upper layer functional architectures adhere to the common design in primates and placental carnivores, their three-dimensional architectures differ substantially, most notably, by distinct columnar orientation selectivity profiles. While two-dimensional models have been investigated extensively, only one model examined the three-dimensional architecture to our knowledge [120]. We generalized this specific model of three-dimensional functional architectures [120] to maximum entropy ensembles. We corroborate their finding of a frequently occurring U-motif in curved geometries and extend the numerical results for the change of preferred orientations along vertical tracts to an exact analytical expression. The exact calculation of pinwheel string densities for isotropic field ensembles in the horizontal plane with a separable vertical dimension extends previous work that only evaluated this quantity for isotropic field ensembles [30, 31]. The work further extends two-dimensional maximum entropy models for orientation domains $[135,147,171]$ to a third dimension and confirms their pinwheel density for isotropic field ensembles. 


\subsection{Outlook}

The results from this thesis encourage further experimental and theoretical investigations of visual cortical architectures to clarify their underlying developmental mechanisms and advance their precise theoretical modeling.

\section{Screening for genetic mechanisms of functional architecture}

The proposed genetic network model of this thesis shows that the functional architecture of the primary visual cortex can be genetically encoded by a biological plausible mechanism comprising only a small number of morphogens. Therefore, the next logical step in this endeavor is to experimentally investigate whether such a mechanism is implemented in primates and placental carnivores. The most promising approach is transcriptome sequencing in combination with the recording of neuronal response properties. A feasible experimental setup would be to determine orientation preference by intrinsic signal imaging and apply transcriptome sequencing on cells from domains that differ the most in orientation preference. A similar experimental paradigm was used previously, where a molecular correlate for ocular dominance bands was found [25]. If a molecular correlate for orientation preference exists, further studies of focal or dualsite overexpression of these morphogens could be used to identify the type of genetic mechanism. In these experiments, genetic networks exhibiting self-organization, as proposed in this thesis, globally reorganize their functional architecture, while genetic networks based on gradient readout reorganize only locally.

Screening for genetic mechanisms of the development of functional architecture could be further extended to rodents. Since the orientation encoding part of the genetic network is equivalent to a universality class of visual cortical development [22, 128-131, 136-139, 262], previous work of a transition to an interspersed orientation layout [140] can be easily incorporated. Due to the interspersed layout of orientation preference, screening requires a more elaborate experimental setup, where orientation preference is first determined on a cellular level by, e.g. calcium imaging. Afterwards, the transcriptome of the recorded cells can be sequenced following established protocols [32-36].

\section{Dichotomy of functional architectures}

Comparative studies of various mammalian species suggest a dichotomy of function visual cortical architectures. On the one hand an ordered arrangement of orientation preference into orientation columns and on the other hand an interspersed layout without or only minimal columnar order [41, 71, 383]. The insight gained from our study of three-dimensional functional architectures indicates a possible modification of this view that suggests to revisit the experiments from rabbits [44-48] and gray squirrels [42] in order to identify possible mixture states. These experimental studies found small columnar clusters of orientation preference that might constitute intermediate evolutionary states. The advent of three-photon calcium imaging makes this endeavor more exciting than ever as the columnar structure can be investigated with cellular resolution [37]. Of course, other functional architectures of mammalian branches such as marsupials should be precisely quantified as well to clarify how functional architecture evolved as already pointed out $[23,136]$.

\section{Developmental model of orientation domain emergence}

Devising a genetic mechanism for the development of visual cortical architecture is only one aspect of visual cortical development. As shown in several experiments, neuronal activity can alter visual cortical architecture, which suggests an interaction between neuronal activity and 
genetic mechanisms. It is therefore worthwhile to explore an analytically tractable neuronal circuit that is driven by spontaneous neuronal activity and develops orientation domains that are in agreement with the common design. A first step towards this direction was already pioneered, but only analyzed numerically [121]. After establishing a neuronal activity based model and identifying their necessary ingredients such as statistics of spontaneous activity, these models can be coupled to genetic networks in order to gain a comprehensive understanding of the development of visual cortical architecture. A possible coupling candidate is activity-dependent transport as observed for the homeodomain transcription factor Otx2 [26]. The mechanisms based on neuronal activity and on genes might run in parallel and act synergistically. Axons that establish specific synapses by targeting and pruning can get more information by collusion of both mechanisms and are therefore expected to be faster in circuit selection.

\section{Pinwheel configurations in confined cortical areas}

Pinwheel configurations of primates and placental carnivores follow species invariant quantitative rules summarized under the term common design [22-24]. Remarkably, the long-range interaction model predicted a pinwheel density that is virtually identical to the observed pinwheel density [128, 135]. Provoked by this quantitative discovery and the theoretical success, the common design was subsequently used as model benchmark in [22-24, 121, 130, 131, 266] and further extended in this thesis. However, experimentally observed pinwheel configurations are confined by the boundaries of the visual cortical area and exhibit typically pinwheel sparse zones close to the boundaries [12]. In this thesis and in a variety of other models, boundaries are often excluded mainly due to their mathematical complexity and under the assumption that their contribution can be negligible in large domains. In the long-range interaction model, boundary effects were numerically explored and revealed a significant change of the orientation domain layout, albeit the pinwheel configuration was not systematically investigated [267]. A first glimpse of boundary effects can be appreciated by previous studies of Gaussian random fields. In these studies, the effect of a single boundary with Dirichlet and Neumann condition on the topological defect density, i.e. pinwheel density for orientation domains, was examined $[160,161]$, see also [163]. Surprisingly, a single boundary leads to a total excess of pinwheels for large areas despite low pinwheel densities close to the boundary. This approach was extended to compact systems in numerical [162] and experimental quantum billiard systems [150, 151], where similar effects have been observed. Therefore, the pinwheel density of models in confined cortical areas would be expected to increase or decrease depending on the size of the area. It is also plausible that other pinwheel configuration measures, such as nearest neighbor distributions, change through boundary effects. Perhaps, confined cortical areas also partially resolve the open challenge of model class orientation domain solutions to have finite power-spectral density widths [409]. Reviews for analytical approaches in dynamical systems can be found in $[28,410]$. Hence, in order to establish a more stringent benchmarking framework, it will be important to analytically and numerically dissect the qualitative and quantitative boundary effects on the pinwheel configuration.

\section{Finite power-spectral density width}

The common design of primates and placental carnivores is a remarkable mammalian brain trait discovered in a large-scale comparative analysis. The pinwheel configuration of closed-form solutions from the long-range interaction model [128] and the proposed genetic network model are in good quantitative agreement with the common design for sufficiently large interaction and transport ranges [22], respectively. However, discrepancies remain that are so far not explained by this model. One discrepancy was already pointed out and concerns the power-spectral 
density width that is much thinner for stable model solutions than in experimentally observed orientation domains [409]. Another perhaps related discrepancy is the invariance of the average pinwheel density to phase shuffling of closed-form model solutions in contrast to an increased pinwheel density in experimentally observed orientation domains [22, 411]. The dependence of the pinwheel density on phase shuffling is a clear deviation to orientation domains that are modeled by Gaussian random fields. This indicates that higher order correlation moments are present in experimentally observed orientation domains. Possible explanations for both discrepancies are, for instance, the heterogeneity of the typical scale and the aforementioned confined cortical area. While the treatment of confined cortical areas was described in the previous section, heterogeneity of the typical scale can be tackled in various ways. Dynamical models could be extended by a heterogeneous connectome that can result in a spatially variable typical scale. The analysis might then be pursuit by (i) an analytical approach with a spatial dependence of amplitude equations, (ii) numerical studies of corresponding field equations and (iii) spatially non-stationary Gaussian random fields. The understanding of the emergence of finite power-spectral density widths will endow the universality class of visual cortical development and related models with more theoretical depth and breadth.

\section{Functional architecture in visually deprived humans}

A more distant but very exciting research direction that might clarify the functional role of visual cortical architecture is suggested by findings in visually deprived humans. Monocular deprivation became an animal model for amblyopia almost immediately after the discovery of orientation selectivity in the primary visual cortex [69,412]. This experimental paradigm was extended to binocular visual deprivation, stripe rearing and dark rearing that lead to the discovery that visual experience can impair, prevent or even destroy the functional architecture $[14,66,100]$. The extension of monocular deprivation in humans used for the treatment of amblyopia lack a deep biological justification. Intriguingly, only recently a humanitarian mission was initiated that generated numerous new observations without any ethical concerns. In the humanitarian project Prakash [413], blind children with treatable eye problems, but without sufficient financial resources, are cured. Due to their condition, the children experienced an extended period of early-onset blindness starting before the first year of life and lasting for 8-17 years due to bilateral cataracts. Over 6 month after surgical cataract removal, the patients showed a surprising improvement of contrast sensitivity [413, 414]. Before cataract removal, their visual acuity was poor of at most finger counting at a distance of a meter. Postsurgical contrast sensitive assessments demonstrated an up to thirty-fold improvement of peak contrast thresholds and an enlarged sensitivity for higher spatial frequencies. How is this visual deprivation and a subsequent improvement of visual acuity reflected in the functional architecture of humans? Fortunately, the answer to that question can be non-invasively examined by high-field fMRI that was already used previously to examine orientation domains in cats and humans [58, 120, 396]. This may constitute a unique opportunity to dissect the functional architecture in visually deprived humans. A glimpse on the outcome of the experiments can be appreciated by comparison to dark rearing [14] and binocular deprivation experiments in animals [66]. In both cases, orientation domains emerge initially despite the lack of visual experience, but their orientation selectivity decrease after an extended period of visual deprivation. The increase in visual acuity after cataract removal is hence surprising and might be similar to natural eye-opening. A genetic mechanism as proposed in this thesis might again serve as a redundant or supportive mechanism for visual cortical development. 


\section{Appendix}

\subsection{Derivation of pair and charge pinwheel correlation function}

In this section a detailed analytical derivation for the pinwheel pair correlation function

$$
g(r)=\frac{1}{\rho^{2}}\left\langle\delta(\eta(\boldsymbol{x})) \delta(\xi(\boldsymbol{x}))|\nu(\boldsymbol{x})| \delta\left(\eta\left(\boldsymbol{x}^{\prime}\right)\right) \delta\left(\xi\left(\boldsymbol{x}^{\prime}\right)\right)\left|\nu\left(\boldsymbol{x}^{\prime}\right)\right|\right\rangle
$$

with $r=\left\|\boldsymbol{x}-\boldsymbol{x}^{\prime}\right\|$ and $\nu(\boldsymbol{x})=\xi_{x}(\boldsymbol{x}) \eta_{y}(\boldsymbol{x})-\xi_{y}(\boldsymbol{x}) \eta_{x}(\boldsymbol{x})$ of isotropic and shift-symmetric Gaussian random fields $\Psi(\boldsymbol{x})=\xi(\boldsymbol{x})+i \eta(\boldsymbol{x})$ will be derived following [30].

In order to evaluate the expectation value, the marginal distribution of the field components

$$
\begin{aligned}
\chi^{T} & =\left(\xi(\boldsymbol{x}), \xi\left(\boldsymbol{x}^{\prime}\right), \partial_{x} \xi(\boldsymbol{x}), \partial_{x^{\prime}} \xi\left(\boldsymbol{x}^{\prime}\right), \partial_{y} \xi(\boldsymbol{x}), \partial_{y^{\prime}} \xi\left(\boldsymbol{x}^{\prime}\right)\right) \\
\boldsymbol{\nu}^{T} & =\left(\eta(\boldsymbol{x}), \eta\left(\boldsymbol{x}^{\prime}\right), \partial_{x} \eta(\boldsymbol{x}), \partial_{x^{\prime}} \xi\left(\boldsymbol{x}^{\prime}\right), \partial_{y} \eta(\boldsymbol{x}), \partial_{y^{\prime}} \eta\left(\boldsymbol{x}^{\prime}\right)\right)
\end{aligned}
$$

is required. For isotropic orientation fields, the analysis simplifies by choosing the $x$-axis to lie along the difference vector $\boldsymbol{x}-\boldsymbol{x}^{\prime}$. As the off-diagonal elements of the covariance matrix vanish $\left\langle\boldsymbol{\nu} \boldsymbol{\chi}^{T}\right\rangle=0$ due to shift-symmetry, the probability density function is given by

$$
p(\boldsymbol{\chi}, \boldsymbol{\nu})=\frac{1}{\sqrt{(2 \pi)^{12} \operatorname{det} \boldsymbol{\Sigma}^{2}}} \exp \left(-\frac{1}{2}\left(\boldsymbol{\chi}^{T} \boldsymbol{\Sigma}^{-1} \boldsymbol{\chi}+\boldsymbol{\nu}^{T} \boldsymbol{\Sigma}^{-1} \boldsymbol{\nu}\right)\right)
$$

with the covariance matrix $\boldsymbol{\Sigma}=\left\langle\chi \chi^{T}\right\rangle=\left\langle\boldsymbol{\nu} \boldsymbol{\nu}^{T}\right\rangle$. The coefficients of the covariance matrix are related to the correlation function of the orientation field ensemble $C(r)=\left\langle\Psi\left(\boldsymbol{x}_{1}\right) \bar{\Psi}\left(\boldsymbol{x}_{2}\right)\right\rangle$ with $r=\left\|\boldsymbol{x}_{1}-\boldsymbol{x}_{2}\right\|$ by

$$
\begin{aligned}
& c_{1}=\langle\xi(\boldsymbol{x}) \xi(\boldsymbol{x})\rangle=\frac{1}{2} C(0) \\
& c_{2}=\left\langle\xi(\boldsymbol{x}) \xi\left(\boldsymbol{x}^{\prime}\right)\right\rangle=\frac{1}{2} C(r) \\
& c_{3}=\left\langle\xi(\boldsymbol{x}) \partial_{x^{\prime}} \xi\left(\boldsymbol{x}^{\prime}\right)\right\rangle=-\left\langle\partial_{x} \xi(\boldsymbol{x}) \xi\left(\boldsymbol{x}^{\prime}\right)\right\rangle=\left\langle\eta(\boldsymbol{x}) \partial_{x^{\prime}} \eta\left(\boldsymbol{x}^{\prime}\right)\right\rangle=-\left\langle\partial_{x} \eta(\boldsymbol{x}) \eta\left(\boldsymbol{x}^{\prime}\right)\right\rangle=\frac{1}{2} \partial_{r} C(r) \\
& c_{4}=\left\langle\partial_{x} \xi(\boldsymbol{x}) \partial_{x^{\prime}} \xi(\boldsymbol{x})\right\rangle=\left\langle\partial_{y} \xi(\boldsymbol{x}) \partial_{y^{\prime}} \xi(\boldsymbol{x})\right\rangle=\left\langle\partial_{x} \eta(\boldsymbol{x}) \partial_{x^{\prime}} \eta(\boldsymbol{x})\right\rangle=\left\langle\partial_{y} \eta(\boldsymbol{x}) \partial_{y^{\prime}} \eta(\boldsymbol{x})\right\rangle=-\frac{1}{2} \partial_{r}^{2} C(0) \\
& c_{5}=\left\langle\partial_{x} \xi(\boldsymbol{x}) \partial_{x^{\prime}} \xi\left(\boldsymbol{x}^{\prime}\right)\right\rangle=\left\langle\partial_{x} \eta(\boldsymbol{x}) \partial_{x^{\prime}} \eta\left(\boldsymbol{x}^{\prime}\right)\right\rangle=-\frac{1}{2} \partial_{r}^{2} C(r) \\
& c_{6}=\left\langle\partial_{y} \xi(\boldsymbol{x}) \partial_{y^{\prime}} \xi\left(\boldsymbol{x}^{\prime}\right)\right\rangle=\left\langle\partial_{y} \eta(\boldsymbol{x}) \partial_{y^{\prime}} \eta\left(\boldsymbol{x}^{\prime}\right)\right\rangle=-\frac{1}{2 r} \partial_{r} C(r),
\end{aligned}
$$


where the spatial dependence of the moments are omitted for the sake of brevity. The covariance matrix is then given by

$$
\boldsymbol{\Sigma}=\left(\begin{array}{cc}
\boldsymbol{M}_{1} & 0 \\
0 & \boldsymbol{M}_{2}
\end{array}\right) \quad \text { with } \quad \boldsymbol{M}_{1}=\left(\begin{array}{cccc}
c_{1} & c_{2} & 0 & -c_{3} \\
c_{2} & c_{1} & c_{3} & 0 \\
0 & c_{3} & c_{4} & c_{5} \\
-c_{3} & 0 & c_{5} & c_{4}
\end{array}\right), \quad \boldsymbol{M}_{2}=\left(\begin{array}{cc}
c_{4} & c_{6} \\
c_{6} & c_{4}
\end{array}\right) \text {. }
$$

The inverse of the covariance matrix then reads

$$
\boldsymbol{\Sigma}^{-1}=\left(\begin{array}{cc}
\boldsymbol{M}_{1}^{-1} & 0 \\
0 & \boldsymbol{M}_{2}^{-1}
\end{array}\right), \quad \boldsymbol{M}_{1}^{-1}=\left(\begin{array}{cc}
\boldsymbol{N}_{2} & \boldsymbol{N}_{3} \\
\boldsymbol{N}_{3}^{T} & \boldsymbol{N}_{1}
\end{array}\right), \quad \boldsymbol{M}_{2}^{-1}=\boldsymbol{N}_{4}
$$

and

$$
\begin{aligned}
& \boldsymbol{N}_{1}=\frac{1}{\operatorname{det} \boldsymbol{M}_{1}}\left(\begin{array}{cc}
c_{4}\left(c_{1}^{2}-c_{2}^{2}\right)-c_{1} c_{3}^{2} & -c_{5}\left(c_{1}^{2}-c_{2}^{2}\right)+c_{2} c_{3}^{2} \\
-c_{5}\left(c_{1}^{2}-c_{2}^{2}\right)+c_{2} c_{3}^{2} & c_{4}\left(c_{1}^{2}-c_{2}^{2}\right)-c_{1} c_{3}^{2}
\end{array}\right) \\
& \boldsymbol{N}_{2}=\frac{1}{\operatorname{det} \boldsymbol{M}_{1}}\left(\begin{array}{cc}
c_{1}\left(c_{4}^{2}-c_{5}^{2}\right)-c_{4} c_{3}^{2} & -c_{2}\left(c_{4}^{2}-c_{5}^{2}\right)+c_{5} c_{3}^{2} \\
-c_{2}\left(c_{4}^{2}-c_{5}^{2}\right)+c_{5} c_{3}^{2} & c_{1}\left(c_{4}^{2}-c_{5}^{2}\right)-c_{4} c_{3}^{2}
\end{array}\right) \\
& \boldsymbol{N}_{3}=\frac{1}{\operatorname{det} \boldsymbol{M}_{1}}\left(\begin{array}{cc}
c_{3}\left(c_{2} c_{4}-c_{1} c_{5}\right) & c_{3}\left(c_{3}^{2}-c_{1} c_{4}+c_{2} c_{5}\right) \\
-c_{3}\left(c_{3}^{2}-c_{1} c_{4}+c_{2} c_{5}\right) & -c_{3}\left(c_{2} c_{4}-c_{1} c_{5}\right)
\end{array}\right) \\
& \boldsymbol{N}_{4}=\frac{1}{\operatorname{det} \boldsymbol{M}_{2}}\left(\begin{array}{cc}
c_{4} & -c_{6} \\
-c_{6} & c_{4}
\end{array}\right) .
\end{aligned}
$$

The next part of the calculation consists of performing the average in Eq. (8.1) with the given probability density function. The delta distributions change the probability density function to a product of a scaling term and a modified probability density function $\tilde{p}$

$$
\begin{aligned}
& p(\chi)=\frac{\tilde{p}(\hat{\boldsymbol{\chi}})}{(2 \pi)^{2}\left(c_{1}^{2}-c_{2}^{2}\right)} \\
& \tilde{p}(\hat{\boldsymbol{\chi}})=\frac{1}{(2 \pi)^{4} \sqrt{\operatorname{det} \tilde{\boldsymbol{\Sigma}}}} \exp \left(-\frac{1}{2} \hat{\boldsymbol{\chi}}^{T} \tilde{\boldsymbol{\Sigma}}^{-1} \hat{\boldsymbol{\chi}}\right)
\end{aligned}
$$

with the newly introduced random variable

$$
\hat{\chi}=\left(\partial_{x} \xi(\boldsymbol{x}), \partial_{x^{\prime}} \xi\left(\boldsymbol{x}^{\prime}\right), \partial_{y} \xi(\boldsymbol{x}), \partial_{y^{\prime}} \xi\left(\boldsymbol{x}^{\prime}\right), \partial_{x} \eta(\boldsymbol{x}), \partial_{x^{\prime}} \eta\left(\boldsymbol{x}^{\prime}\right), \partial_{y} \eta(\boldsymbol{x}), \partial_{y^{\prime}} \eta\left(\boldsymbol{x}^{\prime}\right)\right)^{T}
$$

and the covariance matrix

$$
\tilde{\mathbf{\Sigma}}^{-1}=\operatorname{diag}\left(\boldsymbol{N}_{1}, \boldsymbol{N}_{4}, \boldsymbol{N}_{1}, \boldsymbol{N}_{4}\right) \text {. }
$$

The pair correlation function can thus be simplified to an average over the modified probability density function

$$
g\left(\boldsymbol{x}, \boldsymbol{x}^{\prime}\right)=\frac{1}{(2 \pi)^{2}\left(c_{1}^{2}-c_{2}^{2}\right) \rho^{2}}\left\langle|\nu(\boldsymbol{x})|\left|\nu\left(\boldsymbol{x}^{\prime}\right)\right|\right\rangle_{\tilde{p}}
$$

The Gaussian average over an absolute value is a typical problem in statistical theory [30, 277] and can be solved by utilizing an identity of the absolute value that leads in the case considered here to

$$
\left\langle|\nu(\boldsymbol{x})|\left|\nu\left(\boldsymbol{x}^{\prime}\right)\right|\right\rangle_{\tilde{p}}=\frac{1}{\pi^{2}} \int \frac{\mathrm{d} t}{t^{2}} \int \frac{\mathrm{d} s}{s^{2}}\left\langle(1-\cos (\nu(\boldsymbol{x}) t))\left(1-\cos \left(\nu\left(\boldsymbol{x}^{\prime}\right) s\right)\right)\right\rangle_{\tilde{p}}
$$


The solution of the expectation value can be obtained by rewriting the orthogonality measure as

$$
\begin{gathered}
\nu(\boldsymbol{x}) t=\hat{\chi}^{T} \boldsymbol{A}(t, 0) \hat{\chi} \\
\nu\left(\boldsymbol{x}^{\prime}\right) s=\hat{\chi}^{T} \boldsymbol{A}(0, s) \hat{\chi}
\end{gathered}
$$

with the anti-diagonal matrix

$$
\boldsymbol{A}(t, s)=\left(\begin{array}{cccc}
0 & 0 & 0 & \boldsymbol{B}(t, s) \\
0 & 0 & -\boldsymbol{B}(t, s) & 0 \\
0 & -\boldsymbol{B}(t, s) & 0 & 0 \\
\boldsymbol{B}(t, s) & 0 & 0 & 0
\end{array}\right), \quad \boldsymbol{B}(t, s)=-\frac{1}{2}\left(\begin{array}{cc}
t & 0 \\
0 & s
\end{array}\right) .
$$

The solution of the expectation value is given by

$$
\left\langle(1-\cos (\nu(\boldsymbol{x}) t))\left(1-\cos \left(\nu\left(\boldsymbol{x}^{\prime}\right) s\right)\right)\right\rangle_{\tilde{p}}=T(0,0)-T(t, 0)-T(0, s)+\frac{1}{2}(T(t, s)+T(t,-s))
$$

with

$$
T(t, s)=\Re\left\{\left\langle e^{i\left(\nu(\boldsymbol{x}) t+\nu\left(\boldsymbol{x}^{\prime}\right) s\right)}\right\rangle_{\tilde{p}}\right\}=\Re\left\{\left\langle\exp \left(i \hat{\boldsymbol{\chi}}^{T} \boldsymbol{A}(t, s) \hat{\boldsymbol{\chi}}\right)\right\rangle_{\tilde{p}}\right\}=\Re\left\{\frac{1}{\sqrt{\operatorname{det}(\mathbf{1}-2 i \tilde{\boldsymbol{\Sigma}} \boldsymbol{A}(t, s))}}\right\}
$$

The covariance matrix is given by

$$
\begin{aligned}
\tilde{\boldsymbol{\Sigma}} & =\operatorname{diag}\left(\boldsymbol{N}_{1}^{-1}, \boldsymbol{N}_{4}^{-1}, \boldsymbol{N}_{1}^{-1}, \boldsymbol{N}_{4}^{-1}\right), \\
\boldsymbol{N}_{1}^{-1} & =\left(\begin{array}{cc}
c_{4}-\frac{c_{1} c_{3}^{2}}{c_{1}^{2}-c_{2}^{2}} & c_{5}-\frac{c_{2} c_{3}^{2}}{c_{1}^{2}-c_{2}^{2}} \\
c_{5}-\frac{c_{2} c_{3}^{2}}{c_{1}^{2}-c_{2}^{2}} & c_{4}-\frac{c_{1} c_{3}^{2}}{c_{1}^{2}-c_{2}^{2}}
\end{array}\right), \quad \boldsymbol{N}_{4}^{-1}=\boldsymbol{M}_{2}=\left(\begin{array}{ll}
c_{4} & c_{6} \\
c_{6} & c_{4}
\end{array}\right) .
\end{aligned}
$$

The determinant becomes

$$
\begin{aligned}
\sqrt{\operatorname{det}(\mathbf{1}-2 i \tilde{\boldsymbol{\Sigma}} \boldsymbol{A}(t, s))}= & \left|\operatorname{det}\left(\mathbf{1}+4 \boldsymbol{N}_{1}^{-1} \boldsymbol{B}(t, s) \boldsymbol{N}_{4}^{-1} \boldsymbol{B}(t, s)\right)\right| \\
= & 1+c_{4}\left(c_{4}-\frac{c_{1} c_{3}^{2}}{c_{1}^{2}-c_{2}^{2}}\right)\left(s^{2}+t^{2}\right)+2 c_{6}\left(c_{5}-\frac{c_{2} c_{3}^{2}}{c_{1}^{2}-c_{2}^{2}}\right) s t \\
& +\frac{\operatorname{det} \boldsymbol{M}_{1} \operatorname{det} \boldsymbol{M}_{2}}{c_{1}^{2}-c_{2}^{2}} s^{2} t^{2} .
\end{aligned}
$$

Notice that due to Silvester's criterion, the upper sub-covariance matrices are positive semidefinite and the prefactors of $t^{2}$ are positive. By rescaling the integration variables $t$ and $s$ the solution becomes

$$
\begin{aligned}
g(r)= & \frac{c_{4}^{2}\left(c_{1}^{2}-c_{2}^{2}\right)-c_{1} c_{3}^{2} c_{4}}{4 \pi^{4}\left(c_{1}^{2}-c_{2}^{2}\right)^{2} \rho^{2}} \\
& \int \frac{\mathrm{d} t}{t^{2}} \int \frac{\mathrm{d} s}{s^{2}}\left(1-\frac{1}{1+t^{2}}-\frac{1}{1+s^{2}}+\frac{1+s^{2}+t^{2}+Z s^{2} t^{2}}{\left(1+s^{2}+t^{2}+Z s^{2} t^{2}\right)^{2}-Y^{2} s^{2} t^{2}}\right)
\end{aligned}
$$

with

$$
Y=\frac{2 c_{6}\left(c_{5}\left(c_{1}^{2}-c_{2}^{2}\right)-c_{2} c_{3}^{2}\right)}{\left(c_{1}^{2}-c_{2}^{2}\right) c_{4}^{2}-c_{1} c_{3}^{2} c_{4}}, \quad Z=\frac{\operatorname{det} \boldsymbol{M}_{1} \operatorname{det} \boldsymbol{M}_{2}\left(c_{1}^{2}-c_{2}^{2}\right)}{\left(\left(c_{1}^{2}-c_{2}^{2}\right) c_{4}^{2}-c_{1} c_{3}^{2} c_{4}\right)^{2}}
$$


One of the integrals can be evaluated by residuum theory yielding

$$
g(r)=\frac{2 c_{1}^{2}\left(c_{4}\left(c_{1}^{2}-c_{2}^{2}\right)-c_{1} c_{3}^{2}\right)}{\pi c_{4}\left(c_{1}^{2}-c_{2}^{2}\right)^{2}} \int_{0}^{\infty} \mathrm{d} t \frac{2 t^{4} Z+t^{2}\left(-\frac{Y^{2}}{2}+Z+3\right)+\frac{Y^{2}}{2}-Z+3}{\left(t^{2}+1\right)^{3} \sqrt{t^{4} Z+t^{2}\left(-\frac{Y^{2}}{4}+Z+1\right)+1}} .
$$

The solution of the last integral is provided in [154], but is rather cumbersome and therefore not shown here.

The charge correlation function can be similarly defined by

$$
g_{Q}\left(\boldsymbol{x}, \boldsymbol{x}^{\prime}\right)=\frac{1}{\rho^{2}}\left\langle\delta(\eta(\boldsymbol{x})) \delta(\xi(\boldsymbol{x})) \nu(\boldsymbol{x}) \delta\left(\eta\left(\boldsymbol{x}^{\prime}\right)\right) \delta\left(\xi\left(\boldsymbol{x}^{\prime}\right)\right) \nu\left(\boldsymbol{x}^{\prime}\right)\right\rangle .
$$

The derivation is the same until the averaging that can be rewritten as

$$
\left\langle\nu(\boldsymbol{x}) \nu\left(\boldsymbol{x}^{\prime}\right)\right\rangle_{\tilde{p}}=\left.\partial_{\alpha} \partial_{\beta}\left\langle e^{\alpha \nu(\boldsymbol{x})+\beta \nu\left(\boldsymbol{x}^{\prime}\right)}\right\rangle_{\tilde{p}}\right|_{\alpha=\beta=0} .
$$

For a vanishing static field the result reads

$$
g_{Q}\left(\boldsymbol{x}, \boldsymbol{x}^{\prime}\right)=\left.\frac{1}{(2 \pi)^{2}\left(c_{1}^{2}-c_{2}^{2}\right) \rho^{2}} \partial_{\alpha} \partial_{\beta} T(\alpha, \beta)\right|_{\alpha=\beta=0}=\frac{2 c_{6}\left(c_{5}\left(c_{1}^{2}-c_{2}^{2}\right)-c_{2} c_{3}^{2}\right)}{(2 \pi)^{2}\left(c_{1}^{2}-c_{2}^{2}\right)^{2} \rho^{2}}
$$




\section{Bibliography}

[1] T. J. C. Polderman et al. "Meta-analysis of the heritability of human traits based on fifty years of twin studies". Nature Genetics 47 (2015).

[2] L. C. Greig et al. "Molecular logic of neocortical projection neuron specification, development and diversity". Nature Reviews Neuroscience 14 (2013).

[3] Y. Arai and A. Pierani. "Development and evolution of cortical fields". Neuroscience Research 86 (2014).

[4] P. Berthold. "Genetic basis and evolutionary aspects of bird migration". Advances in the Study of Behavior 33 (2003).

[5] J. Morton and M. Johnson. "CONSPEC and CONLERN: a two-process theory of infant face recognition." Psychological Review 98 (1991).

[6] J. Lidz and A. Gagliardi. "How Nature Meets Nurture: Universal Grammar and Statistical Learning". Annual Review of Linguistics 1 (2015).

[7] K. Rockland and J. Lund. "Widespread periodic intrinsic connections in the tree shrew visual cortex". Science 215 (1982).

[8] R. Malach et al. "Relationship between intrinsic connections and functional architecture revealed by optical imaging and in vivo targeted biocytin injections in primate striate cortex." Proceedings of the National Academy of Sciences of the USA 90 (1993).

[9] C. D. Gilbert and T. N. Wiesel. "Clustered intrinsic connections in cat visual cortex." Journal of Neuroscience 3 (1983).

[10] E. M. Callaway and L. C. Katz. "Emergence and refinement of clustered horizontal connections in cat striate cortex". Journal of Neuroscience 10 (1990).

[11] Z. F. Kisvarday et al. "Orientation-specific relationship between populations of excitatory and inhibitory lateral connections in the visual cortex of the cat". Cerebral Cortex 7 (1997).

[12] W. H. Bosking et al. "Orientation selectivity and the arrangement of horizontal connections in tree shrew striate cortex." Journal of Neuroscience 17 (1997).

[13] L. C. Sincich and G. G. Blasdel. "Oriented axon projections in primary visual cortex of the monkey". Journal of Neuroscience 21 (2001).

[14] L. E. White, D. M. Coppola, and D. Fitzpatrick. "The contribution of sensory experience to the maturation of orientation selectivity in ferret visual cortex." Nature 411 (2001).

[15] M. Weliky and L. C. Katz. "Functional mapping of horizontal connections in developing ferret visual cortex: experiments and modeling." Journal of Neuroscience 14 (1994). 
[16] D. E. Wilson et al. "Orientation selectivity and the functional clustering of synaptic inputs in primary visual cortex." Nature Neuroscience 19 (2016).

[17] S. Löwel and W. Singer. "Selection of intrinsic horizontal connections in the visual cortex by correlated neuronal activity." Science 255 (1992).

[18] D. R. Muir et al. "Embedding of cortical representations by the superficial patch system." Cerebral Cortex 21 (2011).

[19] M. Kaschube et al. "Genetic influence on quantitative features of neocortical architecture." Journal of Neuroscience 22 (2002).

[20] M. Kaschube et al. "The pattern of ocular dominance columns in cat primary visual cortex: Intra- and interindividual variability of column spacing and its dependence on genetic background". European Journal of Neuroscience 18 (2003).

[21] F. Sengpiel and P. C. Kind. "The role of activity in development of the visual system". Current Biology 12 (2002).

[22] M. Kaschube et al. "Universality in the evolution of orientation columns in the visual cortex." Science $\mathbf{3 3 0}$ (2010).

[23] W. Keil et al. "Response to Comment on "Universality in the Evolution of Orientation Columns in the Visual Cortex"”. Science 336 (2012).

[24] M. Schottdorf et al. "Random wiring, ganglion cell mosaics, and the functional architecture of the visual cortex". PLoS Computational Biology 11 (2015).

[25] K. Tomita et al. "A Molecular Correlate of Ocular Dominance Columns in the Developing Mammalian Visual Cortex." Cerebral Cortex 23 (2013).

[26] S. Sugiyama et al. "Experience-dependent transfer of Otx2 homeoprotein into the visual cortex activates postnatal plasticity." Cell 134 (2008).

[27] C. von der Malsburg. "Self-organization of orientation sensitive cells in the striate cortex." Kybernetik 14 (1973).

[28] M. C. Cross and P. C. Hohenberg. "Pattern formation outside of equilibrium". Reviews of Modern Physics 65 (1993).

[29] P. Manneville. Dissipative Structures and Weak Turbulence. Ed. by H. Araki, A. Libchaber, and G. Parisi. 1st ed. Perspectives in Physics. Academic Press, 1990.

[30] M. V. Berry and M. R. Dennis. "Phase singularities in isotropic random waves". Proceedings of the Royal Society A 456 (2000).

[31] M. R. Dennis. "Nodal densities of planar gaussian random waves". European Physical Journal: Special Topics 145 (2007).

[32] T. Hashimshony et al. "CEL-Seq: Single-Cell RNA-Seq by Multiplexed Linear Amplification". Cell Reports 2 (2012).

[33] D. Ramsköld et al. "Full-length mRNA-Seq from single-cell levels of RNA and individual circulating tumor cells." Nature Biotechnology 30 (2012).

[34] F. Tang et al. "mRNA-Seq whole-transcriptome analysis of a single cell". Nature Methods 6 (2009).

[35] S. Islam et al. "Characterization of the single-cell transcriptional landscape by highly multiplex RNA-seq". Genome Research 21 (2011).

[36] Y. Sasagawa et al. "Quartz-Seq: a highly reproducible and sensitive single-cell RNA-Seq reveals non-genetic gene expression heterogeneity". Genome Biology 14 (2013). 
[37] D. G. Ouzounov et al. "In vivo three-photon imaging of activity of GCaMP6-labeled neurons deep in intact mouse brain". Nature Methods 14 (2017).

[38] S. Hecht, S. Shlaer, and M. H. Pirenne. "Energy at the threshold of vision." Science $\mathbf{9 3}$ (1941).

[39] E. R. Kandel et al. Principles of Neural Science. 5. 2014.

[40] D. H. Hubel and T. N. Wiesel. "Receptive Fields of Cells in Striate Cortex of Very Young, Visually Inexperienced Kittens." Journal of Neurophysiology 26 (1963).

[41] K. Ohki et al. "Functional imaging with cellular resolution reveals precise microarchitecture in visual cortex". Nature 433 (2005).

[42] S. D. Van Hooser et al. "Orientation selectivity without orientation maps in visual cortex of a highly visual mammal". Journal of Neuroscience 25 (2005).

[43] C. M. Niell and M. P. Stryker. "Highly selective receptive fields in mouse visual cortex." Journal of Neuroscience $\mathbf{2 8}$ (2008).

[44] K. L. Chow, R. H. Masland, and D. L. Stewart. "Receptive field characteristics of striate cortical neurons in the rabbit". Brain Research 33 (1971).

[45] A. Hughes. "Topographical relationships between the anatomy and physiology of the rabbit visual system". Documenta Ophthalmologica 30 (1971).

[46] R. C. Van Sluyters and D. L. Stewart. "Binocular neurons of the rabbit's visual cortex: effects of monocular sensory deprivation". Experimental Brain Research 19 (1974).

[47] J. . Bousfield. "Columnar organisation and the visual cortex of the rabbit". Brain Research 136 (1977).

[48] E. H. Murphy and N. Berman. "The rabbit and the cat: a comparison of some features of response properties of single cells in the primary visual cortex." Journal of Comparative Neurology 188 (1979).

[49] G. G. Blasdel and G. Salama. "Voltage-sensitive dyes reveal a modular organization in monkey striate cortex". Nature $\mathbf{3 2 1}$ (1986).

[50] D. Y. Ts'o et al. "Functional organization of primate visual cortex revealed by high resolution optical imaging". Science 249 (1990).

[51] K. Obermayer and G. G. Blasdel. "Geometry of orientation and ocular dominance columns in monkey striate cortex". Journal of Neuroscience 13 (1993).

[52] G. Blasdel, K. Obermayer, and L. Kiorpes. "Organization of ocular dominance and orientation columns in the striate cortex of neonatal macaque monkeys". Visual Neuroscience 12 (1995).

[53] G. G. Blasdel and D. Campbell. "Functional retinotopy of monkey visual cortex". Journal of Neuroscience 21 (2001).

[54] X. Xu et al. "Functional organization of visual cortex in the prosimian bush baby revealed by optical imaging of intrinsic signals." Journal of Neurophysiology 94 (2005).

[55] R. Malach, R. B. Tootell, and D. Malonek. "Relationship between orientation domains, cytochrome oxidase stripes, and intrinsic horizontal connections in squirrel monkey area V2." Cerebral Cortex 4 (1994).

[56] K. Obermayer and G. G. Blasdel. "Singularities in primate orientation maps". Neural Computation 9 (1997).

[57] X. Xu et al. "Functional organization of visual cortex in the owl monkey". Journal of Neuroscience $\mathbf{2 4}$ (2004). 
[58] E. Yacoub, N. Harel, and K. Ugurbil. "High-field fMRI unveils orientation columns in humans." Proceedings of the National Academy of Sciences of the USA 105 (2008).

[59] A. Grinvald et al. "Functional architecture of cortex revealed by optical imaging of intrinsic signals". Nature 324 (1986).

[60] N. V. Swindale, J. A. Matsubara, and M. S. Cynader. "Surface organization of orientation and direction selectivity in cat area 18." Journal of Neuroscience 7 (1987).

[61] T. Bonhoeffer and A. Grinvald. "Iso-orientation domains in cat visual cortex are arranged in pinwheel-like patterns". Nature 353 (1991).

[62] T. Bonhoeffer and A. Grinvald. "The layout of iso-orientation domains in area 18 of cat visual cortex: optical imaging reveals a pinwheel-like organization". Journal of Neuroscience 13 (1993).

[63] I. Gödecke and T. Bonhoeffer. "Development of identical orientation maps for two eyes without common visual experience". Nature 379 (1996).

[64] I. Gödecke et al. "Development of orientation preference maps in area 18 of kitten visual cortex". European Journal of Neuroscience 9 (1997).

[65] S. Löwel et al. "The layout of orientation and ocular dominance domains in area 17 of strabismic cats". European Journal of Neuroscience 10 (1998).

[66] M. C. Crair, D. C. Gillespie, and M. P. Stryker. "The role of visual experience in the development of columns in cat visual cortex". Science 279 (1998).

[67] T. Müller et al. "An analysis of orientation and ocular dominance patterns in the visual cortex of cats and ferrets." Neural Computation 12 (2000).

[68] B. Chapman, M. P. Stryker, and T. Bonhoeffer. "Development of orientation preference maps in ferret primary visual cortex." Journal of Neuroscience 16 (1996).

[69] D. H. Hubel and T. N. Wiesel. "Receptive fields, binocular interaction and functional architecture in the cat's visual cortex". Journal of Physiology 160 (1962).

[70] V. B. Mountcastle. "Modality and topographic properties of single neurons of cat's somatic sensory cortex." Journal of Neurophysiology 20 (1957).

[71] K. Ohki et al. "Highly ordered arrangement of single neurons in orientation pinwheels." Nature 442 (2006).

[72] A. Zepeda, C. Arias, and F. Sengpiel. "Optical imaging of intrinsic signals: recent developments in the methodology and its applications". Journal of Neuroscience Methods 136 (2004).

[73] J. R. Polimeni et al. "Physical limits to spatial resolution of optical recording: clarifying the spatial structure of cortical hypercolumns." Proceedings of the National Academy of Sciences of the USA 102 (2005).

[74] P. Tian et al. "Monte Carlo simulation of the spatial resolution and depth sensitivity of two-dimensional optical imaging of the brain". Journal of Biomedical Optics 16 (2011).

[75] S. C. Rao, L. J. Toth, and M. Sur. "Optically imaged maps of orientation preference in primary visual cortex of cats and ferrets". Journal of Comparative Neurology 387 (1997).

[76] J. H. Kaas. "Evolution of columns, modules, and domains in the neocortex of primates." Proceedings of the National Academy of Sciences of the USA 109 (2012).

[77] B. Scholl et al. "Emergence of orientation selectivity in the Mammalian visual pathway." Journal of Neuroscience 33 (2013). 
[78] S. D. Van Hooser et al. "Transformation of receptive field properties from lateral geniculate nucleus to superficial V1 in the tree shrew". Journal of Neuroscience 33 (2013).

[79] H. J. Chisum, F. Mooser, and D. Fitzpatrick. "Emergent properties of layer 2/3 neurons reflect the collinear arrangement of horizontal connections in tree shrew visual cortex." Journal of Neuroscience 23 (2003).

[80] D. H. Hubel and T. N. Wiesel. "Shape and arrangement of columns in cat's striate cortex". Journal of Physiology 165 (1963).

[81] L. C. Katz and J. C. Crowley. "Development of Cortical Circuits : Lessons From Ocular Dominance Columns". Nature Reviews Neuroscience 3 (2002).

[82] L. E. White and D. Fitzpatrick. "Vision and cortical map development." Neuron $\mathbf{5 6}$ (2007).

[83] A. D. Huberman, M. B. Feller, and B. Chapman. "Mechanisms underlying development of visual maps and receptive fields." Annual Review of Neuroscience 31 (2008).

[84] P. O. Kanold and H. J. Luhmann. "The subplate and early cortical circuits". Annual review of neuroscience $\mathbf{3 3}$ (2010).

[85] A. Ghosh and C. J. Shatz. "Pathfinding and target selection by developing geniculocortical axons." Journal of Neuroscience 12 (1992).

[86] C. J. Shatz and M. B. Luskin. "The relationship between the geniculocortical afferents and their cortical target cells during development of the cat's primary visual cortex." Journal of Neuroscience 6 (1986).

[87] K. Herrmann, A. Antonini, and C. J. Shatz. "Ultrastructural Evidence for Synaptic Interactions between Thalamocortical Axons and Subplate Neurons". European Journal of Neuroscience 6 (1994).

[88] C. Chiu and M. Weliky. "Spontaneous activity in developing ferret visual cortex in vivo." Journal of Neuroscience 21 (2001).

[89] M. Weliky and L. C. Katz. "Correlational structure of spontaneous neuronal activity in the developing lateral geniculate nucleus in vivo." Science 285 (1999).

[90] B. Chapman and M. P. Stryker. "Development of orientation selectivity in ferret visual cortex and effects of deprivation". Journal of Neuroscience 3 (1993).

[91] J. C. Durack and L. C. Katz. "Development of horizontal projections in layer $2 / 3$ of ferret visual cortex". Cerebral Cortex 6 (1996).

[92] K. Krug, C. J. Akerman, and I. D. Thompson. "Responses of neurons in neonatal cortex and thalamus to patterned visual stimulation through the naturally closed lids". Journal of Neurophysiology $\mathbf{8 5}$ (2001).

[93] E. S. Ruthazer and M. P. Stryker. "The role of activity in the development of long-range horizontal connections in area 17 of the ferret." Journal of Neuroscience 16 (1996).

[94] K. R. Duffy, K. M. Murphy, and D. G. Jones. "Analysis of the postnatal growth of visual cortex." Visual Neuroscience 15 (1998).

[95] J. R. Villablanca et al. "The growth of the feline brain from fetal into adult life. II. A morphometric study of subcortical nuclei". Developmental Brain Research 122 (2000).

[96] S. Rathjen et al. "The growth of cat cerebral cortex in postnatal life: A magnetic resonance imaging study". European Journal of Neuroscience 18 (2003).

[97] S. Rathjen, K. E. Schmidt, and S. Löwel. "Postnatal growth and column spacing in cat primary visual cortex." Experimental Brain Research 149 (2003). 
[98] M. Kaschube et al. "Interareal coordination of columnar architectures during visual cortical development." Proceedings of the National Academy of Sciences of the USA 106 (2009).

[99] W. Keil et al. "Reorganization of columnar architecture in the growing visual cortex." Proceedings of the National Academy of Sciences of the USA 107 (2010).

[100] F. Sengpiel, P. Stawinski, and T. Bonhoeffer. "Influence of experience on orientation maps in cat visual cortex." Nature Neuroscience 2 (1999).

[101] E. M. Callaway and L. C. Katz. "Effects of binocular deprivation on the development of clustered horizontal connections in cat striate cortex." Proceedings of the National Academy of Sciences of the USA $\mathbf{8 8}$ (1991).

[102] A. S. Ramoa et al. "Suppression of cortical NMDA receptor function prevents development of orientation selectivity in the primary visual cortex." Journal of Neuroscience $\mathbf{2 1}$ (2001).

[103] P. O. Kanold et al. "Role of subplate neurons in functional maturation of visual cortical columns." Science 301 (2003).

[104] S. M. Catalano and C. J. Shatz. "Activity-Dependent Cortical Target Selection by Thalamic Axons". Science 281 (1998).

[105] M. Weliky and L. C. Katz. "Disruption of orientation tuning in visual cortex by artificially correlated neuronal activity." Nature 386 (1997).

[106] S. Schuett, T. Bonhoeffer, and M. Hübener. "Pairing-induced changes of orientation maps in cat visual cortex". Neuron 32 (2001).

[107] B. Godde et al. "Plasticity of orientation preference maps in the visual cortex of adult cats." Proceedings of the National Academy of Sciences of the USA 99 (2002).

[108] B. Li, M. R. Peterson, and R. D. Freeman. "Oblique Effect : A Neural Basis in the Visual Cortex". Journal of Neurophysiology 90 (2003).

[109] V. Dragoi, C. M. Turcu, and M. Sur. "Stability of Cortical Responses and the Statistics of Natural Scenes". Neuron 32 (2001).

[110] G. Wang, S. Ding, and K. Yunokuchi. "Difference in the representation of cardinal and oblique contours in cat visual cortex". Neuroscience Letters 338 (2003).

[111] B. Chapman and T. Bonhoeffer. "Overrepresentation of horizontal and vertical orientation preferences in developing ferret area 17." Proceedings of the National Academy of Sciences of the USA 95 (1998).

[112] D. M. Coppola et al. "Unequal representation of cardinal and oblique contours in ferret visual cortex." Proceedings of the National Academy of Sciences of the USA 95 (1998).

[113] D. M. Coppola and L. E. White. "Visual experience promotes the isotropic representation of orientation preference." Visual Neuroscience 21 (2004).

[114] C. S. Furmanski and S. A. Engel. "An oblique effect in human primary visual cortex". Nature Neuroscience 3 (2000).

[115] R. Linsker. "From basic network principles to neural architecture: emergence of orientation columns." Proceedings of the National Academy of Sciences of the USA 83 (1986).

[116] R. Linsker. "From basic network principles to neural architecture: emergence of orientation-selective cells." Proceedings of the National Academy of Sciences of the USA 83 (1986). 
[117] R. Linsker. "From basic network principles to neural architecture: emergence of spatialopponent cells." Proceedings of the National Academy of Sciences of the USA 83 (1986).

[118] K. D. Miller. "A model for the development of simple cell receptive fields and the ordered arrangement of orientation columns through activity-dependent competition between ON- and OFF-center inputs." Journal of Neuroscience 14 (1994).

[119] K. Kang, M. Shelley, and H. Sompolinsky. "Mexican hats and pinwheels in visual cortex." Proceedings of the National Academy of Sciences of the USA 100 (2003).

[120] S. Tanaka et al. "Three-dimensional visual feature representation in the primary visual cortex". Neural Networks 24 (2011).

[121] J.-L. R. Stevens et al. "Mechanisms for stable, robust, and adaptive development of orientation maps in the primary visual cortex." Journal of Neuroscience 33 (2013).

[122] N. V. Swindale. "A Model for the Formation of Orientation Columns". Proceedings of the Royal Society B $\mathbf{2 1 5}$ (1982).

[123] P. C. Bressloff et al. "Geometric visual hallucinations, Euclidean symmetry and the functional architecture of striate cortex." Proceedings of the Royal Society B 356 (2001).

[124] P. C. Bressloff and J. D. Cowan. "An amplitude equation approach to contextual effects in visual cortex." Neural Computation 14 (2002).

[125] P. C. Bressloff and J. D. Cowan. "SO3 symmetry breaking mechanism for orientation and spatial frequency tuning in the visual cortex". Physical Review Letters 88 (2002).

[126] H. Y. Lee, M. Yahyanejad, and M. Kardar. "Symmetry considerations and development of pinwheels in visual maps." Proceedings of the National Academy of Sciences of the USA 100 (2003).

[127] P. Thomas and J. Cowan. "Symmetry Induced Coupling of Cortical Feature Maps". Physical Review Letters 92 (2004).

[128] F. Wolf. "Symmetry, Multistability, and Long-Range Interactions in Brain Development". Physical Review Letters 95 (2005).

[129] L. Reichl, S. Löwel, and F. Wolf. "Pinwheel stabilization by ocular dominance segregation". Physical Review Letters 102 (2009).

[130] L. Reichl et al. "Coordinated optimization of visual cortical maps (I) symmetry-based analysis." PLoS Computational Biology 8 (2012).

[131] L. Reichl et al. "Coordinated optimization of visual cortical maps (II) numerical studies." PLoS Computational Biology 8 (2012).

[132] N. V. Swindale. "The development of topography in the visual cortex: a review of models." Network: Computation in Neural Systems 7 (1996).

[133] E. M. Palmer and R. W. Robinson. "Enumeration of self-dual configurations". Pacific Journal of Mathematics 110 (1984).

[134] M. Voit. "Exact Multiplicity and Existence Results for Solutions to the Long Range Interaction Model". Master thesis. Georg-August University Göttingen, 2017.

[135] M. Kaschube, M. Schnabel, and F. Wolf. "Self-organization and the selection of pinwheel density in visual cortical development". New Journal of Physics 10 (2008).

[136] W. Keil. "Optimization principles and constraints shaping visual cortical architecture". PhD thesis. Georg-August University Göttingen, 2012.

[137] L. Reichl. "Symmetry breaking and pattern delection in models of visual development". PhD thesis. Georg-August University Göttingen, 2010. 
[138] W. Keil. "Shift-Twist-Symmetrie und Musterbildung im visuellen Kortex". Diploma thesis. Friedrich-Schiller-Universität Jena, 2006.

[139] M. Schnabel. "A symmetry of the visual world in the architecture of the visual cortex". PhD thesis. Georg-August University Göttingen, 2008.

[140] J. D. F. Weidinger. "Modeling the origins of spatial and temporal variability in visual cortical representations". PhD thesis. Georg-August University Göttingen, 2013.

[141] E. T. Jaynes. "Information theory and statistical mechanics. II". Physical Review 108 (1957).

[142] E. T. Jaynes. "Information theory and statistical mechanics". Physical Review 106 (1957).

[143] R. J. Adler and J. E. Taylor. Random fields and geometry. 1st ed. Springer, 2007.

[144] A. S. Rojer and E. L. Schwartz. "Cat and monkey cortical columnar patterns modeled by bandpass-filtered 2D white noise". Biological Cybernetics 62 (1990).

[145] E. Niebur and F. Worgotter. "Design Principles of Columnar Organization in Visual Cortex". Neural Computation 6 (1994).

[146] E. L. Schwartz. "Computational studies of the spatial architecture of primate visual cortex". Cerebral Cortex 10 (1994).

[147] F. Wolf and T. Geisel. "Spontaneous pinwheel annihilation during visual development". Nature 395 (1998).

[148] J. D. Cowan and A. E. Friedman. "Simple spin models for the development of ocular dominance columns and iso-orientation patches". NIPS 3 (1990).

[149] M. R. Dennis, K. O'Holleran, and M. J. Padgett. "Singular Optics- Optical Vortices and Polarization Singularities.pdf". Progress in Optics 53 (2009).

[150] Y. Kim et al. "Current and Vorticity Auto Correlation Functions in open microwave billiards". Progress of Theoretical Physics 150 (2003).

[151] R. Höhmann et al. "Density and correlation functions of vortex and saddle points in open billiard systems". Physical Review E 79 (2009).

[152] L. De Angelis et al. "Spatial Distribution of Phase Singularities in Optical Random Vector Waves". Physical Review Letters 117 (2016).

[153] J. F. Nye and M. V. Berry. "Dislocations in Wave Trains". Proceedings of the Royal Society A 336 (1974).

[154] M. Dennis. "Local properties and statistics of phase singularities in generic wavefields". Singular Optics (Optical Vortices): Fundamentals and Applications. 4403. 2001.

[155] G. Foltin. "Signed zeros of Gaussian vector fields-density, correlation functions and curvature". Journal of Physics A: Mathematical and General 36 (2003).

[156] M. R. Dennis. "Correlations and screening of topological charges in Gaussian random fields". Journal of Physics A: Mathematical and General 36 (2003).

[157] S. Torquato, B. Lu, and J. Rubinstein. "Nearest-neighbor distribution functions in manybody systems". Physical Review A 41 (1990).

[158] A. I. Saichev, K. F. Berggren, and A. F. Sadreev. "Distribution of nearest distances between nodal points for the Berry function in two dimensions". Physical Review E 64 (2001). 
[159] B. Bhattacharjee. "nth-nearest-neighbor distribution functions of an interacting fluid from the pair correlation function: A hierarchical approach". Physical Review E 67 (2003).

[160] M. V. Berry and H. Ishio. "Nodal densities of Gaussian random waves satisfying mixed boundary conditions". Journal of Physics A: Mathematical and General 35 (2002).

[161] M. V. Berry. "Statistics of nodal lines and points in chaotic quantum billiards: perimeter corrections, fluctuations, curvature". Journal of Physics A: Mathematical and General 35 (2002).

[162] M. V. Berry and H. Ishio. "Nodal-line densities of chaotic quantum billiard modes satisfying mixed boundary conditions". Journal of Physics A: Mathematical and General 38 (2005).

[163] C. T. Wheeler. "Curved boundary corrections to nodal line statistics in chaotic billiards". Journal of Physics A: Mathematical and General 38 (2005).

[164] H. Ishio. "Nodal Statistics of Billiards and Boundary Conditions". AIP Conference Proceedings $\mathbf{8 3 4}$ (2006).

[165] H. Ishio. "Nodal structures of wave functions in chaotic billiards". Frontiers of Computational Science: Proceedings of the International Symposium on Frontiers of Computational Science 2005. Ed. by K. Yukio, K. Hiroshi, and S. Masaki. Springer Berlin Heidelberg, 2007.

[166] A. Klein and O. Agam. "Critical point correlations in random Gaussian fields". Journal of Physics A: Mathematical and Theoretical 45 (2011).

[167] G. Foltin. "The distribution of extremal points of Gaussian scalar fields". Journal of Physics A: Mathematical and General 36 (2003).

[168] M. V. Berry and M. R. Dennis. "Topological events on wave dislocation lines: birth and death of loops, and reconnection". Journal of Physics A: Mathematical and Theoretical 40 (2007).

[169] M. Rossi. "The Defect of Random Hyperspherical Harmonics". arXiv (2016).

[170] M. Kaschube. "Neuronale Selektivitätsmuster im visuellen Kortex". Diploma. GeorgAugust University Göttingen, 2000.

[171] M. Schnabel et al. "Random waves in the brain: Symmetries and defect generation in the visual cortex". The European Physical Journal Special Topics 145 (2007).

[172] A. Afgoustidis. "Monochromaticity of Orientation Maps in V1 Implies Minimum Variance for Hypercolumn Size". Journal of Mathematical Neuroscience 5 (2015).

[173] A. Afgoustidis. "Orientation Maps in V1 and Non-Euclidean Geometry". Journal of Mathematical Neuroscience 5 (2015).

[174] B. Alberts et al. Molecular Biology of the Cell. 6th ed. Taylor \& Francis Ltd., 2014.

[175] D. S. Latchman. Eukaryotic Transcription Factors. 4th ed. Academic Press, 2003.

[176] P. Theresa. "Regulation of transcription and gene expression in eukaryotes". Nature Education 1 (2008).

[177] C. Vogel and E. M. Marcotte. "Insights into the regulation of protein abundance from proteomic and transcriptomic analyses." Nature Reviews Genetics 13 (2012).

[178] A. Reményi, H. R. Schöler, and M. Wilmanns. "Combinatorial control of gene expression." Nature Structural \& Molecular Biology 11 (2004). 
[179] S. B. Carroll. "Evo-Devo and an Expanding Evolutionary Synthesis: A Genetic Theory of Morphological Evolution". Cell 134 (2008).

[180] K. Struhl. "Fundamentally different logic of Gene Regulation in Eukaryotes and Prokaryotes". Cell 98 (1999).

[181] G. A. Wray et al. "The evolution of transcriptional regulation in eukaryotes". Molecular Biology and Evolution 20 (2003).

[182] M. T. Laub et al. "Global analysis of the genetic network controlling a bacterial cell cycle". Science 290 (2000).

[183] S. Kalir. "Ordering Genes in a Flagella Pathway by Analysis of Expression Kinetics from Living Bacteria". Science 292 (2001).

[184] A. Turing. "The chemical basis of morphogenesis". Proceedings of the Royal Society B 237 (1952).

[185] F. Crick. "Diffusion in embryogenesis". Nature 225 (1970).

[186] T. Hofer and P. Maini. "Turing patterns in fish skin?" Nature 380 (1996).

[187] J. Howard, S. W. Grill, and J. S. Bois. "Turing's next steps: the mechanochemical basis of morphogenesis." Nature Reviews Molecular Cell Biology 12 (2011).

[188] T. Bollenbach et al. "Morphogen transport in epithelia". Physical Review E 75 (2007).

[189] S. Kondo and R. Asai. "A reaction-diffusion wave on the skin of the marine angelfish Pomacanthus". Nature 376 (1995).

[190] S. Kondo. "The reaction-diffusion system: a mechanism for autonomous pattern formation in the animal skin". Genes to Cells 7 (2002).

[191] S. Kondo, M. Iwashita, and M. Yamaguchi. "How animals get their skin patterns: fish pigment pattern as a live Turing wave." The International Journal of Developmental Biology 53 (2009).

[192] S. Kondo and T. Miura. "Reaction-diffusion model as a framework for understanding biological pattern formation." Science 329 (2010).

[193] J. D. Murray. Mathematical Biology. 3rd ed. Springer, 2013.

[194] H. Frohnhöfer and C. Nüsslein-Volhard. "Organization of anterior pattern in the Drosophila embryo by the maternal gene bicoid". Nature 324 (1986).

[195] J. Gurdon et al. "Activin signalling and response to a morphogen gradient". Nature 371 (1994).

[196] J. Jaeger, Manu, and J. Reinitz. "Drosophila blastoderm patterning." Current Opinion in Genetics and Development 22 (2012).

[197] A. Minelli and G. Fusco. "Evo-devo perspectives on segmentation: Model organisms, and beyond". Trends in Ecology and Evolution 19 (2004).

[198] D. L. Richmond and A. C. Oates. "The segmentation clock: Inherited trait or universal design principle?" Current Opinion in Genetics and Development 22 (2012).

[199] N. Gregson et al. "A guide to drug discovery: Pricing medicines: theory and practice, challenges and opportunities". Nature Reviews Drug Discovery 4 (2005).

[200] A. D. Blest. "The rapid synthesis and destruction of photoreceptor membrane by a dinopid spider: a daily cycle". Proceedings of the Royal Society B 200 (1978).

[201] J. Tomita et al. "No transcription-translation feedback in circadian rhythm of KaiC phosphorylation." Science $\mathbf{3 0 7}$ (2005). 
[202] S. J. Chou et al. "Geniculocortical Input Drives Genetic Distinctions Between Primary and Higher-Order Visual Areas". Science 340 (2013).

[203] G. Pouchelon et al. "Modality-specific thalamocortical inputs instruct the identity of postsynaptic L4 neurons". Nature 511 (2014).

[204] R. Sperry. "Chemoaffinity in the orderly growth of nerve fiber patterns and connections". Proceedings of the National Academy of Sciences of the USA 50 (1963).

[205] U. Drescher et al. "In vitro guidance of retinal ganglion cell axons by RAGS, a 25 kDa tectal protein related to ligands for Eph receptor tyrosine kinases." Cell 82 (1995).

[206] H. J. Cheng et al. "Complementary gradients in expression and binding of ELF-1 and Mek4 in development of the topographic retinotectal projection map." Cell 82 (1995).

[207] C. Logan et al. "Rostral optic tectum acquires caudal characteristics following ectopic engrailed expression." Current Biology 6 (1996).

[208] Y. Shigetani, J. I. Funahashi, and H. Nakamura. "En-2 regulates the expression of the ligands for Eph type tyrosine kinases in chick embryonic tectum." Neuroscience Research 27 (1997).

[209] S. Retaux and W. Harris. "Engrailed and retinotectal topography". Trends in Neurosciences 19 (1996).

[210] N. Itasaki and H. Nakamura. "A role for gradient en expression in positional specification on the optic tectum." Neuron 16 (1996).

[211] I. Brunet et al. "The transcription factor Engrailed-2 guides retinal axons." Nature 438 (2005).

[212] T. B. Kornberg and S. Roy. "Communicating by touch-neurons are not alone." Trends in Cell Biology 24 (2014).

[213] P. Smolen, D. A. Baxter, and J. H. Byrne. "Mathematical Modeling of Gene Networks". Neuron 26 (2000).

[214] H. de Jong. "Modeling and simulation of genetic regulatory systems: a literature review." Journal of Computational Biology 9 (2002).

[215] C. J. Tomlin and J. D. Axelrod. "Biology by numbers: mathematical modelling in developmental biology." Nature Reviews Genetics 8 (2007).

[216] G. Karlebach and R. Shamir. "Modelling and analysis of gene regulatory networks." Nature Reviews Molecular Cell Biology 9 (2008).

[217] N. Vijesh, S. K. Chakrabarti, and J. Sreekumar. "Modeling of gene regulatory networks: A review". Journal of Biomedical Science and Engineering 6 (2013).

[218] M. D. Biggin. "Animal Transcription Networks as Highly Connected, Quantitative Continua". Developmental Cell $\mathbf{2 1}$ (2011).

[219] L. Bintu et al. "Transcriptional regulation by the numbers: Models". Current Opinion in Genetics and Development 15 (2005).

[220] N. E. Buchler, U. Gerland, and T. Hwa. "On schemes of combinatorial transcription logic." Proceedings of the National Academy of Sciences of the USA 100 (2003).

[221] A. V. Hill. "The possible effects of the aggregation of the molecule of hemoglobin on its dissociation curves." Journal of Physiology 40 (1910).

[222] C. H. Yuh, H. Bolouri, and E. H. Davidson. "Genomic cis-regulatory logic: experimental and computational analysis of a sea urchin gene". Science 279 (1998). 
[223] J. Monod, J. Wyman, and J. P. Changeux. "On the Nature of Allosteric Transitions: A Plausible Model." Journal of Molecular Biology 12 (1965).

[224] A. M. Walczak, G. Tkacik, and W. Bialek. "Optimizing information flow in small genetic networks. II. Feed-forward interactions". Physical Review E 81 (2010).

[225] G. Tkacik. "From statistical mechanics to information theory: understanding biophysical information-processing systems". arXiv (2010).

[226] M. C. Crair. "Neuronal activity during development: permissive or instructive?" Current Opinion in Neurobiology 9 (1999).

[227] C. D. Gilbert and T. N. Wiesel. "Columnar Specificity of Intrinsic Horizontal and Corticocortical Connections in Cat Visual Cortex". Journal of Neuroscience 9 (1989).

[228] M. Weliky et al. "Patterns of excitation and inhibition evoked by horizontal connections in visual cortex share a common relationship to orientation columns". Neuron 15 (1995).

[229] B. Roerig and J. P. Kao. "Organization of intracortical circuits in relation to direction preference maps in ferret visual cortex." Journal of Neuroscience 19 (1999).

[230] G. L. Ming et al. "Electrical activity modulates growth cone guidance by diffusible factors". Neuron 29 (2001).

[231] M. G. Hanson and L. T. Landmesser. "Normal patterns of spontaneous activity are required for correct motor axon guidance and the expression of specific guidance molecules". Neuron 43 (2004).

[232] A. Sahay et al. "Secreted Semaphorins Modulate Synaptic Transmission in the Adult Hippocampus". Journal of Neuroscience 25 (2005).

[233] F. Bouzioukh et al. "Semaphorin3A regulates synaptic function of differentiated hippocampal neurons". European Journal of Neuroscience 23 (2006).

[234] S. Roy et al. "Cytoneme-mediated contact-dependent transport of the Drosophila decapentaplegic signaling protein." Science 343 (2014).

[235] J. O. Dubuis et al. "Positional information, in bits". Proceedings of the National Academy of Sciences of the USA 110 (2013).

[236] M. D. Petkova et al. "Optimal decoding of information from a genetic network". arXiv (2016).

[237] L. J. Cox et al. "Intra-axonal translation and retrograde trafficking of CREB promotes neuronal survival." Nature Cell Biology 10 (2008).

[238] S. Maday et al. "Review Axonal Transport : Cargo-Specific Mechanisms of Motility and Regulation". Neuron 84 (2014).

[239] M. Sajic et al. "Impulse Conduction Increases Mitochondrial Transport in Adult Mammalian Peripheral Nerves In Vivo". PLoS Biology 11 (2013).

[240] M. Pigliucci, C. J. Murren, and C. D. Schlichting. "Phenotypic plasticity and evolution by genetic assimilation." Journal of Experimental Biology 209 (2006).

[241] J. Sharma, A. Angelucci, and M. Sur. "Induction of visual orientation modules in auditory cortex". Nature 404 (2000).

[242] K. B. Godfrey and N. V. Swindale. "Modeling development in retinal afferents: retinotopy, segregation, and ephrinA/EphA mutants". PLoS ONE 9 (2014).

[243] A. Kicheva et al. "Investigating the principles of morphogen gradient formation: from tissues to cells." Current Opinion in Genetics and Development 22 (2012). 
[244] K. E. Cosker, S. L. Courchesne, and R. A. Segal. "Action in the axon: generation and transport of signaling endosomes." Current Opinion in Neurobiology 18 (2008).

[245] E. Oztas. "Neuronal tracing". Neuroanatomy 2 (2003).

[246] C. F. Ibáñez. "Message in a bottle: long-range retrograde signaling in the nervous system." Trends in Cell Biology 17 (2007).

[247] J. D. Bronzino and D. R. Peterson. Tissue Engineering and Artificial Organs. The Biomedical Engineering Handbook, Third Edition. Taylor \& Francis Ltd., 2006.

[248] J. Ha et al. "A neuron-specific cytoplasmic dynein isoform preferentially transports TrkB signaling endosomes." Journal of Cell Biology 181 (2008).

[249] E. A. van Niekerk et al. "Sumoylation in axons triggers retrograde transport of the RNAbinding protein La." Proceedings of the National Academy of Sciences of the USA $\mathbf{1 0 4}$ (2007).

[250] R. Phillips et al. Physical Biology of the Cell. 2nd ed. Taylor \& Francis Ltd., 2012.

[251] R. V. Barkus et al. "Identification of an axonal kinesin-3 motor for fast anterograde vesicle transport that facilitates retrograde transport of neuropeptides." Molecular Biology of the Cell 19 (2008).

[252] S. E. Ahmari, J. Buchanan, and S. J. Smith. "Assembly of presynaptic active zones from cytoplasmic transport packets." Nature Neuroscience 3 (2000).

[253] H. V. Friedman et al. "Assembly of new individual excitatory synapses: time course and temporal order of synaptic molecule recruitment." Neuron 27 (2000).

[254] J. Dubnau and G. Struhl. "RNA recognition and translational regulation by a homeodomain protein". Nature 379 (1996).

[255] R. Rivera-Pomar and D. Niessing. "RNA binding and translational suppression by bicoid". Nature 379 (1996).

[256] S. Mayfield. "Double agent: translational regulation by a transcription factor." Chemistry E Biology 3 (1996).

[257] A. Prochiantz. "Signaling with homeoprotein transcription factors in development and throughout adulthood." Current Genomics 14 (2013).

[258] M. Piques et al. "Ribosome and transcript copy numbers, polysome occupancy and enzyme dynamics in Arabidopsis". Molecular Systems Biology 5 (2009).

[259] L. Brocchieri and S. Karlin. "Protein length in eukaryotic and prokaryotic proteomes". Nucleic Acids Research 33 (2005).

[260] J. E. Pérez-Ortín, P. M. Alepuz, and J. Moreno. "Genomics and gene transcription kinetics in yeast". Trends in Genetics 23 (2007).

[261] R. Milo and R. Phillips. Cell Biology by the Numbers. 1st ed. Taylor \& Francis Ltd., 2016.

[262] F. Wolf. "Symmetry breaking and pattern selection in visual cortical development". Les houches Session LXXX. Elsevier, 2003. Chap. 12.

[263] U. A. Ernst et al. "Intracortical origin of visual maps." Nature Neuroscience 4 (2001).

[264] A. A. Koulakov and D. B. Chklovskii. "Orientation preference patterns in mammalian visual cortex: A wire length minimization approach". Neuron 29 (2001).

[265] N. M. Mayer, J. M. Herrmann, and T. Geisel. "Pinwheel Stability in a Non-Euclidean Model of Pattern Formation in the Visual Cortex". Journal of the Korean Physical Society 50 (2007). 
[266] W. Keil and F. Wolf. "Coverage, continuity, and visual cortical architecture." Neural Systems 8 Circuits 1 (2011).

[267] D. Heide. "Nonlinear Dynamics of Large Scale Patterns in the Visual Cortex". Diploma thesis. Georg-August University Göttingen, 2005.

[268] P. N. Brown and Y. Saad. "Hybrid Krylov methods for nonlinear systems of equations". SIAM Journal on Scientific and Statistical Computing 11 (1990).

[269] J. E. Dennis and R. B. Schnabel. Numerical Methods for Unconstrained Optimization and Nonlinear Equations. 16. Society for Industrial and Applied Mathematics, 1987.

[270] W. H. Press et al. Numerical recipes in C (2nd ed.): the art of scientific computing. 29. 1992.

[271] J. H. Kaas. "Why is Brain Size so Important:Design Problems and Solutions as Neocortex Gets Bigger or Smaller". Brain and Mind 1 (2000).

[272] D. H. Hubel, T. N. Wiesel, and M. P. Stryker. "Anatomical demonstration of orientation columns in macaque monkey". Journal of Comparative Neurology 177 (1978).

[273] J. H. Kaas. "Why does the brain have so many visual areas?" Journal of Cognitive Neuroscience 1 (1989).

[274] C. Igel and M. Hüsken. "Empirical evaluation of the improved Rprop learning algorithms". Neurocomputing 50 (2003).

[275] M. Riedmiller and H. Braun. "A direct adaptive method for faster backpropagation learning: The RPROP algorithm". IEEE International Conference on Neural Networks - Conference Proceedings. 1993.

[276] C. Igel and M. Hüsken. "Improving the Rprop learning algorithm". Proceedings of the Second International Symposium on Neural Computation (2000).

[277] W. V. Li and A. Wei. "Gaussian integrals involving absolute value functions". High Dimensional Probability V: The Luminy Volume 5 (2009).

[278] S. Torquato and F. H. Stillinger. "Local density fluctuations, hyperuniformity, and order metrics." Physical Review E 68 (2003).

[279] C. E. Zachary. "Characterizing Fluctuations in the Structures of Many-Particle Distributions and Random Heterogeneous Media". PhD thesis. Princeton University, 2011.

[280] J. Beck. "Irregularities of distribution". Acta Mathematica 159 (1987).

[281] J. Beck. "Randomness in lattice point problems". Discrete Mathematics 229 (2001).

[282] D. G. Kendall. "On the number of lattice points inside a random oval". Quarterly Journal of Mathematics 19 (1948).

[283] D. G. Kendall and R. A. Rankin. "On the number of points of a given lattice in a random hypersphere". Quarterly Journal of Mathematics 4 (1953).

[284] D. Stoyan and H. Stoyan. Fractals, random shapes, and point fields: methods of geometrical statistics. 37. 1994.

[285] P. M. Dixon. "Ripley's K function". Encyclopedia of Environmetrics 3 (2002).

[286] D. Fairthorne. "The Distances Between Random Points in Two Concentric Circles". Biometrika 51 (1964).

[287] A. M. Mathai. An Introduction to Geometrical Probability: Distributional Aspects with Applications. Statistical distributions and models with applications. Taylor \& Francis Ltd., 1999. 
[288] P. Hertz. "Über den gegenseitigen durchschnittlichen Abstand von Punkten, die mit bekannter mittlerer Dichte im Raume angeordnet sind". Mathematische Annalen 67 (1909).

[289] J. H. Kaas. "Reconstructing the areal organization of the neocortex of the first mammals". Brain, Behavior and Evolution 78 (2011).

[290] B. L. Finlay and R. B. Darlington. "Linked regularities in the development and evolution of mammalian brains." Science 268 (1995).

[291] R. A. Barton and P. H. Harvey. "Mosaic evolution of brain structure in mammals". Nature 405 (2000).

[292] C. F. Stevens. "An evolutionary scaling law for the primate visual system and its basis in cortical function". Nature 411 (2001).

[293] E. C. Bush and J. M. Allman. "The scaling of frontal cortex in primates and carnivores". Proceedings of the National Academy of Sciences of the USA 101 (2004).

[294] P. M. Kaskan et al. "Peripheral variability and central constancy in mammalian visual system evolution." Proceedings of the Royal Society B 272 (2005).

[295] R. J. Douglas and K. A. C. Martin. "Neuronal Circuits of the Neocortex". Annual Review of Neuroscience 27 (2004).

[296] K. D. Harris and T. D. Mrsic-Flogel. "Cortical connectivity and sensory coding." Nature 503 (2013).

[297] D. H. Hubel and T. N. Wiesel. "Receptive Fields and Functional Architecture of Monkey Striate Cortex". Journal of Physiology 195 (1968).

[298] H. Ko et al. "Functional specificity of local synaptic connections in neocortical networks." Nature 473 (2011).

[299] P. Berkes et al. "Spontaneous cortical activity reveals hallmarks of an optimal internal model of the environment." Science 331 (2011).

[300] H. B. Barlow. "Visual experience and cortical development". Nature 258 (1975).

[301] P. J. Thomas and J. D. Cowan. "Generalized spin models for coupled cortical feature maps obtained by coarse graining correlation based synaptic learning rules". Journal of Mathematical Biology 65 (2012).

[302] R. Temam. "Inertial manifolds". The Mathematical Intelligencer 12 (1990).

[303] G. Leuba and R. Kraftsik. "Changes in volume, surface estimate, three-dimensional shape and total number of neurons of the human primary visual cortex from midgestation until old age". Anatomy and Embryology 190 (1994).

[304] Y. Li, D. Fitzpatrick, and L. E. White. "The development of direction selectivity in ferret visual cortex requires early visual experience." Nature Neuroscience 9 (2006).

[305] Y. Li et al. "Experience with moving visual stimuli drives the early development of cortical direction selectivity." Nature 456 (2008).

[306] G. B. Smith et al. "The development of cortical circuits for motion discrimination." Nature Neuroscience 18 (2015).

[307] B. S. Wang, R. Sarnaik, and J. Cang. "Critical Period Plasticity Matches Binocular Orientation Preference in the Visual Cortex". Neuron 65 (2010).

[308] N. L. Rochefort et al. "Development of direction selectivity in mouse cortical neurons". Neuron $\mathbf{7 1}$ (2011). 
[309] J. S. Espinosa and M. P. Stryker. "Development and plasticity of the primary visual cortex." Neuron 75 (2012).

[310] S. J. Kuhlman, E. Tring, and J. T. Trachtenberg. "Fast-spiking interneurons have an initial orientation bias that is lost with vision." Nature Neuroscience 14 (2011).

[311] Y. Li et al. "Broadening of Cortical Inhibition Mediates Developmental Sharpening of Orientation Selectivity." Journal of Neuroscience 32 (2012).

[312] B. S. Wang et al. "Environmental Enrichment Rescues Binocular Matching of Orientation Preference in Mice that Have a Precocious Critical Period". Neuron 80 (2013).

[313] J. L. Hoy and C. M. Niell. "Layer-Specific Refinement of Visual Cortex Function after Eye Opening in the Awake Mouse". Journal of Neuroscience 35 (2015).

[314] H. Ko et al. "The emergence of functional microcircuits in visual cortex". Nature 496 (2013).

[315] H. Ko, T. D. Mrsic-Flogel, and S. B. Hofer. "Emergence of feature-specific connectivity in cortical microcircuits in the absence of visual experience." Journal of Neuroscience $\mathbf{3 4}$ (2014).

[316] K. M. Hagihara et al. "Neuronal activity is not required for the initial formation and maturation of visual selectivity". Nature Neuroscience 18 (2015).

[317] M. Sato and M. P. Stryker. "Distinctive features of adult ocular dominance plasticity." Journal of Neuroscience $\mathbf{2 8}$ (2008).

[318] K. Lehmann and S. Löwel. "Age-dependent ocular dominance plasticity in adult mice". PLoS ONE 3 (2008).

[319] F. Greifzu et al. "Environmental enrichment extends ocular dominance plasticity into adulthood and protects from stroke-induced impairments of plasticity." Proceedings of the National Academy of Sciences of the USA 111 (2014).

[320] Y. Kremer et al. "Late emergence of the vibrissa direction selectivity map in the rat barrel cortex." Journal of Neuroscience 31 (2011).

[321] F. Wolf, K. Pawelzik, and T. Geisel. "Linear and nonlinear dynamics in development of orientation preference maps". The Neurobiology of Computation. Springer US, 1993. Chap. 59.

[322] G. Toulouse and M. Kléman. "Principles of a classification of defects in ordered media". Journal de Physique Lettres 37 (1976).

[323] N. V. Swindale. "Visual cortex: Looking into a Klein bottle". Current Biology 6 (1996).

[324] S. Tanaka. "Topological analysis of point singularities in stimulus preference maps of the primate visual cortex". Proceedings of the Royal Society B 261 (1995).

[325] M. Weliky, W. H. Bosking, and D. Fitzpatrick. "A systematic map of direction preference in primary visual cortex". Nature 379 (1996).

[326] A. Shmuel and A. Grinvald. "Functional organization for direction of motion and its relationship to orientation maps in cat area 18." Journal of Neuroscience 16 (1996).

[327] N. V. Swindale, A. Grinvald, and A. Shmuel. "The spatial pattern of response magnitude and selectivity for orientation and direction in cat visual cortex". Cerebral Cortex $\mathbf{1 3}$ (2003).

[328] G. C. DeAngelis et al. "Functional micro-organization of primary visual cortex: receptive field analysis of nearby neurons." Journal of Neuroscience 19 (1999). 
[329] K. A. C. Martin and S. Schroder. "Functional heterogeneity in neighboring neurons of cat primary visual cortex in response to both artificial and natural stimuli". Journal of Neuroscience 33 (2013).

[330] K.-S. Lee, X. Huang, and D. Fitzpatrick. "Topology of ON and OFF inputs in visual cortex enables an invariant columnar architecture". Nature 533 (2016).

[331] Y. Wang et al. "Columnar organization of spatial phase in visual cortex." Nature Neuroscience 18 (2015).

[332] J. Kremkow et al. "Principles underlying sensory map topography in primary visual cortex." Nature $\mathbf{5 3 3}$ (2016).

[333] A. J. Ziskind et al. "Neurons in Cat V1 show significant clustering by degree of tuning." Journal of Neurophysiology (2015).

[334] M. Miyashita and S. Tanaka. "A mathematical model for the self-organization of orientation columns in visual cortex". Neuroreport 3 (1992).

[335] K. D. Miller. "Development of orientation columns via competition between ON- and OFF-center inputs." Neuroreport 3 (1992).

[336] F. Wolf et al. "Map structure from pinwheel position". ICANN '93: Proceedings of the International Conference on Artificial Neural Networks Amsterdam, The Netherlands 13-16 September 1993. Ed. by S. Gielen and B. Kappen. Springer London, 1993.

[337] F. Wolf et al. "Organization of the visual cortex." Nature 382 (1996).

[338] S.-B. Paik and D. L. Ringach. "Retinal origin of orientation maps in visual cortex". Nature Neuroscience 14 (2011).

[339] V. R. A. Hore, J. B. Troy, and S. J. Eglen. "Parasol cell mosaics are unlikely to drive the formation of structured orientation maps in primary visual cortex." Visual Neuroscience 29 (2012).

[340] M. Schottdorf et al. "Can retinal ganglion cell dipoles seed iso-orientation domains in the visual cortex?" PLoS ONE 9 (2014).

[341] P. O'Herron et al. "Neural correlates of single-vessel haemodynamic responses in vivo". Nature 534 (2016).

[342] R. D. Frostig et al. "Cortical functional architecture and local coupling between neuronal activity and the microcirculation revealed by in vivo high-resolution optical imaging of intrinsic signals." Proceedings of the National Academy of Sciences of the USA $\mathbf{8 7}$ (1990).

[343] J. E. Mayhew et al. "Cerebral vasomotion: a 0.1-Hz oscillation in reflected light imaging of neural activity." NeuroImage 4 (1996).

[344] V. A. Kalatsky and M. P. Stryker. "New paradigm for optical imaging: Temporally encoded maps of intrinsic signal". Neuron 38 (2003).

[345] E. Bartfeld and A. Grinvald. "Relationships between orientation-preference pinwheels, cytochrome oxidase blobs, and ocular-dominance columns in primate striate cortex." Proceedings of the National Academy of Sciences of the USA 89 (1992).

[346] A. Grinvald et al. "In-vivo optical imaging of cortical architecture and dynamics". Modern Techniques in Neuroscience Research. Ed. by U. Windhorst and H. Johansson. Springer Berlin Heidelberg, 1999. Chap. 34.

[347] H. S. Orbach and L. B. Cohen. "Optical monitoring of activity from many areas of the in vitro and in vivo salamander olfactory bulb: a new method for studying functional organization in the vertebrate central nervous system." Journal of Neuroscience $\mathbf{3}$ (1983). 
[348] B. Q. Mao et al. "Dynamics of spontaneous activity in neocortical slices". Neuron 32 (2001).

[349] C. Stosiek et al. "In vivo two-photon calcium imaging of neuronal networks." Proceedings of the National Academy of Sciences of the USA 100 (2003).

[350] J. N. D. Kerr et al. "Imaging input and output of neocortical networks in vivo." Proceedings of the National Academy of Sciences of the USA 102 (2005).

[351] C. Grienberger and A. Konnerth. "Imaging Calcium in Neurons". Neuron 73 (2012).

[352] R. Homma et al. "Wide-field and two-photon imaging of brain activity with voltage- and calcium-sensitive dyes". Dynamic Brain Imaging. 489. Humana Press, 2009. Chap. 3.

[353] A. Grinvald et al. "High-resolution optical imaging of functional brain architecture in the awake monkey." Proceedings of the National Academy of Sciences of the USA 88 (1991).

[354] G. G. Blasdel. "Orientation selectivity, preference, and continuity in monkey striate cortex". Journal of Neuroscience 12 (1992).

[355] P. E. Maldonado et al. "Orientation selectivity in pinwheel centers in cat striate cortex." Science 276 (1997).

[356] J. Schummers, J. Marino, and M. Sur. "Synaptic integration by V1 neurons depends on location within the orientation map". Neuron 36 (2002).

[357] J. Mariño et al. "Invariant computations in local cortical networks with balanced excitation and inhibition." Nature Neuroscience 8 (2005).

[358] I. Nauhaus et al. "Neuronal Selectivity and Local Map Structure in Visual Cortex". Neuron 57 (2008).

[359] F. Wolf and T. Geisel. "Universality in visual cortical pattern formation." Journal of Physiology 97 (2003).

[360] I. Freund. "Optical vortices in Gaussian random wave fields: statistical probability densities". Journal of the Optical Society of America A 11 (1994).

[361] L. F. Shampine. "Vectorized adaptive quadrature in MATLAB". Journal of Computational and Applied Mathematics 211 (2008).

[362] L. F. Shampine. "Matlab program for quadrature in 2D". Applied Mathematics and Computation 202 (2008).

[363] R. Dawkins and Y. Wong. The Ancestor's Tale: A Pilgrimage to the Dawn of Life. Orion, 2010.

[364] J. J. Hutsler, D.-G. Lee, and K. K. Porter. "Comparative analysis of cortical layering and supragranular layer enlargement in rodent carnivore and primate species". Brain Research 1052 (2005).

[365] J. C. Horton and D. L. Adams. "The cortical column: a structure without a function". Proceedings of the Royal Society B $\mathbf{3 6 0}$ (2005).

[366] N. M. da Costa and K. A. C. Martin. "Whose cortical column would that be?" Frontiers in Neuroanatomy 4 (2010).

[367] K. Albus. "14C-Deoxyglucose mapping of orientation subunits in the cats visual cortical areas". Experimental Brain Research 37 (1979).

[368] B. B. Lee et al. "The depth distribution of optimal stimulus orientations for neurones in cat area 17". Experimental Brain Research 27 (1977). 
[369] P. C. Murphy and A. M. Sillito. "Continuity of orientation columns between superficial and deep laminae of the cat primary visual cortex". Journal of Physiology 381 (1986).

[370] W. Singer. "Topographic organization of orientation columns in the cat visual cortex". Experimental Brain Research 44 (1981).

[371] A. Schoppmann and M. P. Stryker. "Physiological evidence that the 2-deoxyglucose method reveals orientation columns in cat visual cortex". Nature 293 (1981).

[372] I. D. Thompson, M. Kossut, and C. Blakemore. "Development of orientation columns in cat striate cortex revealed by 2- deoxyglucose autoradiography". Nature 301 (1983).

[373] D. Hubel, T. Wiesel, and M. Stryker. "Orientation columns in macaque monkey visual cortex demonstrated by the 2-deoxyglucose autoradiographic technique". Nature $\mathbf{2 6 9}$ (1977).

[374] B. Chapman, K. R. Zahs, and M. P. Stryker. "Relation of cortical cell orientation selectivity to alignment of receptive fields of the geniculocortical afferents that arborize within a single orientation column in ferret visual cortex." Journal of Neuroscience 11 (1991).

[375] A. L. Humphrey and T. T. Norton. "Topographic organization of the orientation column system in the striate cortex of the tree shrew (Tupaia glis). I. Microelectrode recording." Journal of Comparative Neurology 192 (1980).

[376] A. L. Humphrey, C. S. Skeen, and T. T. Norton. "Topographic organization of the orientation column system in the striate cortex of the tree shrew (Tupaia glis). II. Deoxyglucose Mapping." Journal of Comparative Neurology 192 (1980).

[377] S. LeVay, S. K. McConnell, and M. B. Luskin. "Functional organization of primary visual cortex in the mink (Mustela vison), and a comparison with the cat". Journal of Comparative Neurology 257 (1987).

[378] P. G. H. Clarke, I. M. L. Donaldson, and D. Whitteridge. "Binocular visual mechanisms in cortical areas I and II of the sheep". Journal of Physiology 256 (1976).

[379] D. Hubel and T. Wiesel. "Functional architecture of macaque monkey visual cortex". Proceedings of the Royal Society B 198 (1977).

[380] G. G. Blasdel and D. Fitzpatrick. "Physiological organization of layer 4 in macaque striate cortex." Journal of Neuroscience 4 (1984).

[381] D. L. Ringach, R. M. Shapley, and M. J. Hawken. "Orientation selectivity in macaque V1: diversity and laminar dependence." Journal of Neuroscience 22 (2002).

[382] M. Gur, I. Kagan, and D. M. Snodderly. "Orientation and Direction Selectivity of Neurons in V1 of Alert Monkeys: Functional Relationships and Laminar Distributions". Cerebral Cortex 15 (2004).

[383] D. L. Ringach et al. "Spatial clustering of tuning in mouse primary visual cortex." Nature Communications 7 (2016).

[384] A. Cruz-Martín et al. "A dedicated circuit links direction-selective retinal ganglion cells to the primary visual cortex." Nature 507 (2014).

[385] S. Scholpp and A. Lumsden. "Building a bridal chamber: Development of the thalamus". Trends in Neurosciences 33 (2010).

[386] M. Chatterjee and J. Y. H. Li. "Patterning and compartment formation in the diencephalon". Frontiers in Neuroscience 6 (2012).

[387] E. Grant, A. Hoerder-Suabedissen, and Z. Molnár. "Development of the corticothalamic projections". Frontiers in Neuroscience 6 (2012). 
[388] D. J. Price et al. "The importance of combinatorial gene expression in early mammalian thalamic patterning and thalamocortical axonal guidance". Frontiers in Neuroscience 6 (2012).

[389] P. Theer, M. T. Hasan, and W. Denk. "Two-photon imaging to a depth of 1000 microm in living brains by use of a Ti:Al2O3 regenerative amplifier." Optics Letters 28 (2003).

[390] F. Helmchen and W. Denk. "Deep tissue two-photon microscopy". Nature Methods 2 (2005).

[391] J. N. D. Kerr and W. Denk. "Imaging in vivo: watching the brain in action". Nature Reviews Neuroscience 9 (2008).

[392] W. Mittmann et al. "Two-photon calcium imaging of evoked activity from L5 somatosensory neurons in vivo". Nature Neuroscience 14 (2011).

[393] D. Huang et al. "Optical Coherence". Science 254 (1991).

[394] D. J. Faber et al. "Light absorption of (oxy-)hemoglobin assessed by spectroscopic optical coherence tomography." Optics Letters 28 (2003).

[395] J. G. Fujimoto. "Optical coherence tomography for ultrahigh resolution in vivo imaging". Nature Biotechnology 21 (2003).

[396] D. S. Kim, T. Q. Duong, and S. G. Kim. "High-resolution mapping of iso-orientation columns by fMRI." Nature Neuroscience 3 (2000).

[397] D. L. Ringach. "Haphazard wiring of simple receptive fields and orientation columns in visual cortex." Journal of Neurophysiology 92 (2004).

[398] J. M. Alonso, W. M. Usrey, and R. C. Reid. "Rules of connectivity between geniculate cells and simple cells in cat primary visual cortex." Journal of Neuroscience 21 (2001).

[399] J. Jin et al. "Population receptive fields of ON and OFF thalamic inputs to an orientation column in visual cortex." Nature Neuroscience 14 (2011).

[400] R. B. Illing and H. Wässle. "The retinal projection to the thalamus in the cat: a quantitative investigation and a comparison with the retinotectal pathway." Journal of Comparative Neurology 202 (1981).

[401] A. Peters and B. R. Payne. "Numerical relationships between geniculocortical afferents and pyramidal cell modules in cat primary visual cortex". Cerebral Cortex 3 (1993).

[402] M. Sigman et al. "On a common circle: natural scenes and Gestalt rules." Proceedings of the National Academy of Sciences of the USA 98 (2001).

[403] G. F. Striedter, S. Srinivasan, and E. S. Monuki. "Cortical Folding: When, Where, How, and Why?" Annual Review of Neuroscience 38 (2015).

[404] http://brainmuseum.org/.

[405] J. Neal et al. "Insights into the gyrification of developing ferret brain by magnetic resonance imaging." Journal of Anatomy 210 (2007).

[406] P. Pillay and P. R. Manger. "Order-specific quantitative patterns of cortical gyrification". European Journal of Neuroscience $\mathbf{2 5}$ (2007).

[407] D. O. Hebb. The Organization of Behavior. 911. Wiley \& Sons, 1949.

[408] N. Caporale and Y. Dan. "Spike timing-dependent plasticity: a Hebbian learning rule." Annual Review of Neuroscience 31 (2008).

[409] K. D. Miller. " $\pi$ = Visual cortex." Science 330 (2010). 
[410] M. Cross and H. Greenside. Pattern Formation and Dynamics in Nonequilibrium Systems. 1st ed. Cambridge University Press, 2009.

[411] M. Schottdorf. "The reconstitution of visual cortical feature selectivity in-vitro". PhD thesis. Georg-August University Göttingen, 2017.

[412] T. Wiesel and D. Hubel. "Single-Cell response in striate cortex of kittens deprived of vision in one eye". Journal of Neurophysiology 26 (1963).

[413] P. Sinha. "NeuroScience and Service". Neuron 92 (2016).

[414] A. Kalia et al. "Development of pattern vision following early and extended blindness." Proceedings of the National Academy of Sciences of the USA 111 (2014). 



\section{Acknowledgements}

Last but not least, I am pleased to acknowledge all people who supported the compilation of this thesis both explicitly and implicitly over the last couple of years.

The first acknowledgments are due to Theo Geisel, who established and retained this unique working environment. He supported me with talk training at the Dynamics Symposia and brought regularly Jazz to my Christmas time. Thank you!

I would like to express my special gratitude to Fred Wolf, who gave me the opportunity to conduct research in theoretical neuroscience and supervised me through all the years of my $\mathrm{PhD}$. He provided my research with excellent feedback and facilitated my interactions with researchers all around the world through extensive visits of conferences.

I am grateful for my thesis committee members Jörg Enderlein, Siegrid Löwel and Fred Wolf, who supported me in the compilation of this project.

My office mate Rainer Engelken deserves credits for loads of discussions about science, news and all other important subjects that exist in the world. It was a blast to spend all that time with him in our nearly aseptic little office.

I thank Manuel Schottdorf for scientific discussions, exploration of remote places around the world and a joint successful commitment to challenges in science, sports and other things. It was a pleasure.

Thank you, Juan Daniel Florez-Weidinger a.k.a. Chepe for scientific discussions, your critical mindset and the best coffee in the world.

I thank all remaining people from the second floor of the MPI DS building for their helpful answers to questions, discussions, critics and the working atmosphere.

A decisive factor for the excellent working environment was the smoothly working computer infrastructure including the computer cluster. I want to thank Denny Fliegner, Hecke Schrobsdorff and Yorck-Fabian Beensen for their fabulous support.

I am grateful for the administrative work from Victoria Novak, Regina Wunderlich and Ayse Bolik, who facilitated the daily routine and special events such as the advanced computational neuroscience summer school.

Many eyes see better than two. Therefore, I want to thank all people, who proof-read the thesis or parts of it: Fred Wolf, Manuel Schottdorf, Johannes Zierenberg, Rainer Engelken, Jonas Franz, Juan Daniel Florez-Weidinger, Alexander Schmidt, Sonja Sommerfeldt and Ralf Riedinger.

Special thanks to Sonja and Harry who laid the foundation for my logical thinking and efficient working, respectively.

I would like to thank Jessica Grebe for supporting me non-scientifically during the entire time of my PhD.

Finally, I want to thank all my friends and my family for the support. 



\section{Curriculum Vitae}

\section{Personal Information}

Name

Date of birth

Place of birth

Citizenship

\section{Education}

06/2014-06/2017

01/2014-06/2017

10/2011-09/2013

08/2010-03/2011

03/2008-08/2011

10/2007-03/2008

$06 / 2007$

\section{Fellowships}

01/2015-06/2017

03/2012-09/2013
Joscha Liedtke

$17^{\text {th }}$ March 1988

Marburg (Wehrda)

German
Georg-August University, Göttingen, Germany

Doctoral studies in Physics, Theoretical and Computational Neuroscience at the GGNB

MPI for Dynamics and Self-Organization, Göttingen, Germany

Research fellow

Georg-August University, Göttingen, Germany

M. Sc. (graduate) studies in Physics

Gothenburg University/Chalmers, Gothenburg, Sweden

Exchange student (Erasmus) in Physics

Philipps University Marburg

B. Sc. (undergraduate) studies in Physics

TU Darmstadt, Darmstadt, Germany

Undergraduate studies in Machine Engineering

Adolf-Reichwein-Schule, Marburg, Germany

High school graduation ("Abitur")

GGNB Excellence Stipend

German National Academic Foundation 